\title{
International Harmonization of Nomenclature and Diagnostic Criteria (INHAND): Non-proliferative and Proliferative Lesions of the Non-human Primate (M. fascicularis)
}

\author{
Karyn Colman (Chair) $)^{1 *}$, Rachel N. Andrews ${ }^{2}$, Hannah AtKins $^{3}$, Theresa Boulineau 4 , Alys Bradley 5 , \\ Annamaria Braendli-Baiocco ${ }^{6}$, Raffaella Capobianco ${ }^{7}$, David Caudell ${ }^{8}$, Mark Cline $^{8}$, TakuYa Doi $^{9}$, \\ Rainer Ernst ${ }^{10}$, Eric van Esch ${ }^{11}$, Jeffrey Everitt ${ }^{12}$, Pierluigi Fant ${ }^{13}$, Margarita M. Gruebbel ${ }^{14}$, \\ Lars Mecklenburg ${ }^{15}$, Andew D. Miller ${ }^{16}$, Kristen J. Nikula ${ }^{17}$, Shigeru Satake ${ }^{18}$, Julie Schwartz ${ }^{19}$, \\ Alok Sharma ${ }^{20}$, Akihito Shimoi ${ }^{21}$, Cécile Sobry ${ }^{22}$, Ian Taylor ${ }^{23}$, Vimala Vemireddi ${ }^{24}$, Justin Vidal ${ }^{25}$, \\ Charles Wood ${ }^{4}$, John L. VAhle ( GESC Liaison) ${ }^{26}$ \\ ${ }^{1}$ Novartis Institutes for BioMedical Research, Cambridge, MA, USA \\ 2 Wake Forest School of Medicine, Department of Radiation Oncology, Winston-Salem, NC, USA \\ 3 Penn State College of Medicine, Department of Comparative Medicine, Hershey, PA, USA \\ ${ }_{4}$ Boehringer Ingelheim Pharmaceuticals, Inc., Ridgefield, CT, USA \\ ${ }_{5}^{5}$ Charles River Laboratories Edinburgh Ltd., Tranent, Scotland, UK \\ ${ }^{6}$ Roche Pharma Research and Early Development, Pharmaceutical Sciences, Roche Innovation Center Basel, Switzerland \\ 7 Janssen Research \& Development, a Division of Janssen Pharmaceutica NV, Beerse, Belgium \\ ${ }^{8}$ Department of Pathology, Section on Comparative Medicine, Wake Forest School of Medicine, Winston-Salem, NC, USA \\ ${ }^{9}$ LSIM Safety Institute Corporation, Ibaraki, Japan \\ 10 Hennigsdorf, Germany \\ ${ }^{11}$ InSight Pathology BV, Oss, the Netherlands \\ 12 Department of Pathology, Duke University School of Medicine, Durham, NC, USA \\ 13 Charles River Laboratories, Lyon, France \\ 14 Experimental Pathology Laboratories, Inc., Research Triangle Park, NC, USA \\ 15 Covance Preclinical Services GmbH, Münster, Germany \\ ${ }^{16}$ Cornell University College of Veterinary Medicine, Ithaca, NY, USA \\ 17 Inotiv, Maryland Heights, MO, USA \\ 18 Shin Nippon Biomedical Laboratories, Ltd., Kagoshima and Tokyo, Japan \\ 19 Charles River Laboratories, Reno, NV, USA \\ 20 Covance Laboratories, Inc., Madison, WI, USA \\ ${ }^{21}$ Ina Research Inc, Nagano, Japan \\ 22 Charles River Laboratories, Evreux, France \\ ${ }^{23}$ Covance Laboratories, Ltd., Eye, UK \\ ${ }_{24}$ Covance Laboratories, Inc., Chantilly, VA, USA \\ ${ }_{25}$ Charles River, Mattawan, MI, USA \\ ${ }^{26}$ Lilly Research Laboratories, Indianapolis IN, USA
}

\begin{abstract}
The INHAND (International Harmonization of Nomenclature and Diagnostic Criteria for Lesions Project (www.toxpath.org/inhand. asp) is a joint initiative of the Societies of Toxicologic Pathology from Europe (ESTP), Great Britain (BSTP), Japan (JSTP) and North America (STP) to develop an internationally accepted nomenclature for proliferative and nonproliferative lesions in laboratory animals. The purpose of this publication is to provide a standardized nomenclature for classifying microscopic lesions observed in most tissues and organs from the nonhuman primate used in nonclinical safety studies. Some of the lesions are illustrated by color photomicrographs. The standardized nomenclature presented in this document is also available electronically on the internet (http://www.goreni.org/). Sources of material included histopathology databases from government, academia, and industrial laboratories throughout the world. Content includes spontaneous lesions as well as lesions induced by exposure to test materials. Relevant infectious and parasitic lesions are included as well. A widely accepted and utilized international harmonization of nomenclature for lesions in laboratory animals will provide a common language among regulatory and scientific research organizations in different countries and increase and enrich international exchanges of information among toxicologists and pathologists. (DOI: 10.1293/tox.2021-I002; J Toxicol Pathol 2021; 34: 1S-182S)
\end{abstract}

Keywords: nonhuman primate, pathology, toxicopathology, nomenclature, background findings, INHAND, cynomolgus macaque

\footnotetext{
*Address correspondence to: Karyn Colman, Novartis Institutes for BioMedical Research, Cambridge, MA, USA.

e-mail: Karyn.colman@novartis.com

C2021 The Japanese Society of Toxicologic Pathology

This is an open-access article distributed under the terms of the Creative Commons Attribution Non-Commercial No Derivatives (by-nc-nd) License

$<\mathrm{htt}$ ://creativecommons.org/licenses/by-nc-nd/4.0/>.
} 


\section{INTRODUCTION}

The INHAND Project (International Harmonization of Nomenclature and Diagnostic Criteria) is a joint initiative of the societies of toxicologic pathology from Europe (European Society of Toxicologic Pathology - ESTP), United Kingdom (British Society of Toxicological Pathologists - BSTP), Japan (Japanese Society of Toxicologic Pathology - JSTP), and North America (Society of Toxicologic Pathology - STP) to update the existing World Health Organization/International Agency for Research on Cancer (WHO/IARC) and Society of Toxicologic Pathology/ Standardized System of Nomenclature and Diagnostic Criteria (STP/SSNDC) nomenclature systems. The INHAND nomenclature and the related diagnostic criteria represent a consensus of experienced toxicologic pathologists and were reviewed by the INHAND-Global Editorial and Steering Committee (INHAND-GESC) for compliance with INHAND principles. Members of the Societies of Toxicologic Pathology had the opportunity to comment on the draft version during a 60 -day period. The initial series of nomenclature publications were focused on findings in rats and mice. With the interest of the United States Food and Drug Administration (FDA) in the use of published terminology and the decision of the Clinical Data Interchange Standards Consortium (CDISC) initiative on Standard for the Exchange of Non-clinical Data (SEND) to model the controlled terminology (CT) based on the INHAND nomenclature, the INHAND project was extended to other laboratory animal species including the nonhuman primate, rabbit, mini-pig, dog, and fish.

Although the INHAND nomenclature and diagnostic criteria represent a preferred international standard nomenclature for lesions identified in nonclinical studies, recommendations for diagnostic criteria and preferred terminology may not be applicable in all situations. The purpose of specific experiments or the specific context of a given study may require modifications to this standardized nomenclature and diagnostic criteria. The appropriate diagnoses are ultimately based upon on the scientific judgement of the study pathologist.

The present publication provides standardized terms and diagnostic criteria for histopathology observations to be used in nonclinical toxicology studies conducted in cynomolgus monkeys (Macaca fascicularis). Throughout this publication, findings applicable for use in general toxicology studies in monkeys are tabulated by organ system. The terms and tables build on the existing INHAND rodent nomenclature. In most instances, the description and definition of the rodent finding applies to the monkey and is not further described. This publication focuses on findings that are unique to the monkey and are not observed in rodents, and findings in monkeys that share the same terminology with a rodent finding but display different morphologic features. The tabulated findings are categorized according to the following characteristics: "Common", "Uncommon", "Not Observed but Potentially Relevant", and "Not Applicable".

The distinction between common and uncommon findings is based on the occurrence in untreated, relatively young cyno- molgus monkeys in the authors' experience, and is not based on published references. The uncommon category is reserved for changes that are observed only sporadically as spontaneous findings in nonhuman primate toxicology studies, or those that are induced almost exclusively by xenobiotics. "Not Observed but Potentially Relevant" are changes that have not been described or observed in cynomolgus monkeys; however, the use of these terms has been considered permissible, should a finding meet the diagnostic criteria. The category "Not Applicable" refers to rodent specific findings or terminology, such as chronic progressive nephropathy, and the use of these terms in monkeys is considered not appropriate. It should be kept in mind that the monkeys used in toxicology studies are usually of young age and are only on study for a relatively short time, a fraction of their normal life span. The health status of individual monkeys is usually checked carefully and the individuals selected for a study are in excellent condition. For these reasons, the spectrum and frequency of changes can be different from those observed in long-term research-based primate colonies, and common age-related findings including neoplasms are rarely seen in these animals. Accordingly, most documented age-related findings are categorized as "Uncommon". Neoplasms represent a very rare event in nonclinical toxicology studies and thus have been excluded for the most part. Only those neoplastic findings that have occurred in toxicology studies in the authors' institutes \&/or been reported in literature to occur in toxicology studies ${ }^{1}$ are included. For more complete descriptions and diagnostic criteria of tumors occurring in cynomolgus monkeys, the reader is referred to Chapter 6: Neoplasia and Proliferative Disorders of Nonhuman Primates in Nonhuman Primates in Biomedical Research: Diseas$\mathrm{es}^{2}$ and/or current literature. Whenever possible the equivalent rodent term/SEND terminology should be used for any tumors not specifically addressed in this manuscript, as appropriate.

In addition to the journal publication, the nomenclature and diagnostic criteria for the cynomolgus monkey are also available online (www.goreni.org). The online version contains additional images and useful links to differential diagnoses characterizing it as a practical tool for diagnostic work. In addition, all INHAND publications are available at www.toxpath.org. Several manuscripts and texts have been published on background lesions in non-human primates and the reader is directed to the general bibliography at the end for further reading.

The recommended nomenclature is generally descriptive rather than diagnostic, based on standard hematoxylin and eosin stained paraffin embedded sections only. Histochemical or immunohistochemical staining characteristics may be addressed in the comments section of the respective finding. Such special techniques may be required in some situations, but a comprehensive discussion of these methods is outside the scope of this publication. Systemic non-proliferative findings that occur across organs systems and are not specific to an organ are reviewed in the section on systemic pathology. Instead of "synonyms" for each term, the non-rodent publications have used the notation "other terms". While these synonyms or other terms have been used historically, the primary listed term is 
the preferred term and will link to the controlled terminology in SEND.

Findings included in this nomenclature system may be further specified by modifiers. Criteria are given for modifiers that are of particular relevance. These modifiers should be consistently applied. Additional modifiers not provided in this nomenclature system may describe the location, tissue type, or duration, among others. General principles of the INHAND nomenclature have been published separately ${ }^{3}$. As new information becomes available, new terms will be needed from time to time and a request for this new term will be applied by "change control" (see goRENI and STP websites). 


\section{Systemic Pathology}

Microscopic findings that may be observed in multiple organs and/or tissues are listed in the following table with associated diseases and conditions as well as tissues in which they are usually observed. An associated comment or description is available in this chapter for terminology marked with an asterisk. For description of other terminology, the reader is referred to the chapter of the corresponding organs. Metabolic/ systemic syndromes, along with the terminology to be used for associated findings within affected organs, are discussed in the section following this table (Table 1).

Syndromes included:

- Fatal Fasting Macaque Syndrome

- Hemophagocytic Syndrome

- Phospholipidosis

\section{Ectopic tissue: Multiple tissues (Figure 1-6)}

\section{Diagnostic features}

- Histologically normal tissue in an aberrant location.

Comments: Ectopic thymic tissue is commonly identified in or adjacent to the thyroid or parathyroid glands in cynomolgus monkeys. Adrenal cortical tissue can also be identified in or adjacent to the liver ${ }^{6}$. Accessory spleens, also referred to as ectopic spleens, spleniculi, or splenic nodules, are occasionally observed in cynomolgus monkeys. These are often embedded in or attached to the pancreas?. Several other ectopic locations for tissue are described under individual organs.

\section{Amyloid: Multiple tissues (Figure 7-9)}

\section{Pathogenesis/Cell of Origin}

Amyloid is a pathologic proteinaceous substance deposited between cells in various tissues and organs of the body in a wide variety of clinical settings.

In AA-amyloidosis, there is an increased level of serum amyloid-A (SAA), which is common in inflammatory states. It is formed mainly in the liver upon stimulation by proinflammatory cytokines and normally plays a role in cholesterol transport and as a chemoattractant in the inflammatory processes ${ }^{8}$. When the concentration of this molecule is increased, typically as a result of chronic inflammation, certain isoforms of SAA are partially cleaved into fragments that have an increased propensity to form fibrillar aggregates of amyloid deposited systemically. Deposition of insoluble amyloid fibrils occurs either due to a defect in SAA-degrading enzyme in the system, or consequent to the synthesis of abnormal SAA protein resistant to the enzymatic degradation.

In AL-amyloidosis, unstable monoclonal immunoglobulin light chains, produced by a plasma cell dyscrasia, lead to the formation and deposition of fibrils.

\section{Diagnostic features}

- Grossly, affected organs appear enlarged, moderately firm, and abnormally discolored (pale gray or yellowish orange).

- Microscopically, amyloid is deposited extracellularly in various affected tissues.

- Amyloid is a homogeneous, extracellular, hyaline to finely fibrillar waxy material.

- Can interfere with normal tissue function and eventually may produce pressure atrophy of adjacent cells.

\section{Differential Diagnoses}

Other similar-appearing extracellular deposits such as collagen and fibrin:

- Necrosis:

- Congo red, Thioflavin $\mathrm{T}$ and immunohistochemical staining negative.

- Disruption of cellular architecture

- Nuclear pyknosis, karyolysis, or karyorrhexis.

- Fibrosis:

- Fibrillary appearance with small numbers of fibroblasts

- Congo red, Thioflavin $\mathrm{T}$ and immunohistochemical staining negative.

- Positive collagen staining (van Gieson and Massontrichrome).

\section{Special Techniques for Diagnostics}

- Congo red staining orange to red with green birefringence under polarized light.

- Other special stains: crystal violet (amyloid stains purpleviolet) and thioflavin T (brilliant orange fluorescence).

- Immunohistochemistry to identify amyloid deposits and the specific constituents composing the deposits such as the anti $\beta$-light chain antibodies.

- TEM: non-branching fibrils of indefinite length and 0.7$1 \mathrm{~mm}$ diameter that form single to laterally aggregated bundles or interlocking mesh-like ribbons lacking periodicity.

Comments: Different types and clinical forms of amyloidosis are known based on the deposition in tissues and organs of various domestic and wild animals, as well as humans. Systemic amyloid deposition can be due to AL-amyloidosis, AA-amyloidosis, or familial amyloidosis. Amyloid substance may be confined at a given area in the body in the form of localized amyloidosis. Amyloidosis involving several tissues and organs throughout the body is referred to as systemic amyloidosis9.

AA-amyloidosis is the most common form of amyloidosis in NHP. Affected animals present weight loss, diarrhea and malabsorption. Common sites of amyloid deposition in NHP include spleen, lymph nodes, kidney, liver, pancreatic islets and lamina propria or submucosa of the small intestine; especially in aged macaques, often due to chronic enterocolitis or other inflammatory conditions ${ }^{10-12}$. 
Table 1. Microscopic Findings: Systemic Pathology (Generally Used Preferred Terms): Non-Human Primate

\begin{tabular}{|c|c|c|c|c|}
\hline Lesion & Common & Uncommon & Associated Diseases/Conditions & Tissues reported in: \\
\hline \multicolumn{5}{|l|}{ Congenital } \\
\hline $\begin{array}{l}\text { Aplasia/hypoplasia (Absence/failed development of } \\
\text { organ or compartment) }\end{array}$ & & $\mathrm{x}$ & Developmental anomaly & $\begin{array}{l}\text { Kidney (unilateral), } \\
\text { Ovary } \\
\text { Oviduct } \\
\text { Uterus } \\
\text { Thyroid gland (unilateral) }\end{array}$ \\
\hline Ectopic tissue * & $\mathrm{x}$ & & Developmental anomaly & $\begin{array}{l}\text { Adrenal cortex } \\
\text { Ovary } \\
\text { Spleen } \\
\text { Thyroid } \\
\text { Thymus } \\
\end{array}$ \\
\hline \multicolumn{5}{|l|}{ Non-neoplastic } \\
\hline Abscess & & $\mathrm{x}$ & & Multiple tissues \\
\hline Accumulation, adipocytes ${ }^{1}$ & $\mathrm{x}$ & & & $\begin{array}{l}\text { Endocrine glands (multiple) } \\
\text { Heart } \\
\text { Lymph nodes } \\
\text { Pancreas } \\
\text { Salivary gland } \\
\text { Skeletal muscle } \\
\text { Thymus } \\
\end{array}$ \\
\hline Amyloid * & & $\mathrm{x}$ & Amyloidosis & $\begin{array}{l}\text { Colon } \\
\text { Jejunum } \\
\text { Kidney } \\
\text { Liver } \\
\text { Lymph node } \\
\text { Pancreas } \\
\text { Spleen } \\
\end{array}$ \\
\hline Apoptosis ${ }^{2}$ & $\mathrm{x}$ & & & Multiple tissues \\
\hline Apoptosis/single cell necrosis ${ }^{2}$ & $\mathrm{x}$ & & & Multiple tissues \\
\hline Bacteria * & & $\mathrm{x}$ & $\begin{array}{l}\text { Sepsis } \\
\text { Immune compromise }\end{array}$ & Multiple tissues \\
\hline Basophilic granules \# & & $\mathrm{x}$ & Oligonucleotide treatment & $\begin{array}{l}\text { Kidney } \\
\text { Liver } \\
\text { Lymph node } \\
\text { Phagocytic cells } \\
\end{array}$ \\
\hline Congestion & $\mathrm{x}$ & & $\begin{array}{l}\text { Inflammation } \\
\text { Hypertension } \\
\text { Cardiac decompensation }\end{array}$ & Multiple tissues \\
\hline Edema & & $\mathrm{x}$ & Increased vascular permeability & Multiple tissues \\
\hline Extramedullary hematopoiesis $(\mathrm{EMH}){ }^{* 3}$ & $\begin{array}{c}\mathrm{x} \\
\text { (endogenous } \\
\text { and induced) }\end{array}$ & & Anemia & $\begin{array}{l}\text { Adrenal gland } \\
\text { Mandibular lymph node } \\
\text { Spleen } \\
\text { Kidney } \\
\end{array}$ \\
\hline Fibrosis & & $\mathrm{x}$ & Repair process & Multiple tissues \\
\hline Fungus * & & $\mathrm{x}$ & Immune compromise & Multiple tissues \\
\hline Hemorrhage & $\mathrm{x}$ & & $\begin{array}{l}\text { Increased permeability of blood vessels; } \\
\text { Trauma }\end{array}$ & Multiple tissues \\
\hline Inclusions, intranuclear or cytoplasmic * & & $\mathrm{x}$ & Viral infection & Multiple tissues \\
\hline Infiltrate, [insert appropriate cell type] ${ }^{*}$ & $\mathrm{x}$ & & & Multiple tissues \\
\hline Inflammation * & & $\mathrm{x}$ & Response to injury & Multiple tissues \\
\hline Metaplasia, osseous & & $\mathrm{x}$ & $\begin{array}{l}\text { Chronic inflammation } \\
\text { Ischemia } \\
\text { Hematoma } \\
\text { Degenerative changes }\end{array}$ & $\begin{array}{l}\text { Endocrine organs } \\
\text { Kidney } \\
\text { Lung } \\
\text { Lymph node }\end{array}$ \\
\hline Mineralization & $\mathrm{x}$ & & $\begin{array}{l}\text { Local injury } \\
\text { Generalized mineral imbalance }\end{array}$ & $\begin{array}{l}\text { Blood vessels } \\
\text { Brain (Cerebellum, Cerebrum) } \\
\text { Endocrine organs } \\
\text { Kidney } \\
\text { Leptomeninges } \\
\text { Lymph node } \\
\text { Ovary } \\
\text { Salivary glands } \\
\text { Seminal vesicle } \\
\end{array}$ \\
\hline Necrosis & & $\mathrm{x}$ & & Multiple tissues \\
\hline Parasite/Granuloma, parasitic * & & $\mathrm{x}$ & & $\begin{array}{l}\text { Heart } \\
\text { Gastrointestinal tract } \\
\text { Liver } \\
\text { Lung } \\
\text { Nervous system } \\
\end{array}$ \\
\hline Pigment* & $\mathrm{x}$ & & $\begin{array}{l}\text { Accumulation of endogenous or exogenous pigment, e.g.: } \\
\text { Hemosiderin } \\
\text { Hematoidin } \\
\text { Hemozoin (malaria pigment) } \\
\text { Lipofuscin } \\
\text { Ink (tattoo) } \\
\text { Melanin (melanosis) } \\
\text { Pneumoconiosis/anthracosis } \\
\end{array}$ & Multiple tissues \\
\hline Serous atrophy of fat $\mathrm{t}^{4,5}$ & & $\mathrm{x}$ & $\begin{array}{l}\text { Chronic emaciation and/or inanition } \\
\text { Fatal fasting Macaque syndrome }\end{array}$ & $\begin{array}{l}\text { Bone marrow } \\
\text { Heart (coronary band) } \\
\text { Mesentery } \\
\text { Perirenal tissue } \\
\text { Soft tissue } \\
\end{array}$ \\
\hline Single cell necrosis ${ }^{2}$ & $\mathrm{x}$ & & & Multiple tissues \\
\hline Vacuolation & $\begin{array}{c}\mathrm{x} \\
\text { (endogenous } \\
\text { and induced) } \\
\end{array}$ & & $\begin{array}{l}\text { Associated with various conditions and administration of } \\
\text { xenobiotics, including phospholipidosis }\end{array}$ & Multiple tissues \\
\hline \multicolumn{5}{|l|}{ Neoplastic } \\
\hline Lymphoma $^{3}$ & 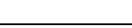 & $\mathrm{x}$ & 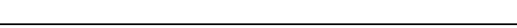 & \\
\hline
\end{tabular}


Similar to humans, aging NHP can develop cerebrovascular amyloid angiopathy, within vessels of the cortex and leptomeninges or cerebral plaques composed of amyloid- $\beta$, which correlate with neurocognitive decline ${ }^{13}$.

\section{Bacteria: Multiple tissues (No image provided)}

\section{Diagnostic features}

- Intracellular or extracellular cocci, bacilli ,coccobacilli, rods or other less common forms visualized by light microscopy.

- Often associated with inflammation, tissue degeneration, and/or necrosis and can be obscured by cellular debris.

- Histochemical methods suchs as Gram, Acid Fast or Silver (Warthin-Starry) stains aid in visualization.

- Culture results and molecular diagnostics.aid in diagnosis.

\section{Differential Diagnoses}

- Necrosis, of other known or unknown causes.

- inflammation, of other known or unknown causes.

Comments: Primary bacterial infections are rare in laboratory macaques. However, like many other mammalian species, NHP are susceptible to opportunistic infection. A complete review of bacterial lesions in macaques is beyond the scope of this manuscript. The reader is referred to the literature. Relevant emerging and reemerging bacterial pathogens of laboratory NHP are reviewed in Bailey and Mansfield 2010 14 .

\section{Extramedullary hematopoiesis (EMH): Multiple Tissues}

(Figure 10-11)

\section{Modifiers}

Increased

\section{Diagnostic features}

- Varying proportions of marture and immature myeloid, erythroid and megakaryocyte lineages, depending on the etiology.

- Common extramedullary hematopoiesis sites.

- Medullary cords in lymph nodes

- Sinusoids in the liver

- Adrenal cortex

- Renal interstitium

\section{Differential Diagnoses:}

- Infiltrate [insert appropriate cell type].

- Infiltration of mature leukocytes into the tissue.

- Presence of leukocytes but no other histologic criteria of inflammation.

- Hematopoietic neoplasms

- Clonal disorders involving hematopoietic blast cells of myeloid lineage and their progenitors.

$->20 \%$ blasts in bone marrow, neoplastic cells in circula- tion or infiltrating parenchyma tissue (refer to Hematolymphoid General Terms).

- Lymphoma

- Distinguished by cell morphology, tissue distribution and clonality.

Comments: Extrameduallary hematopoiesis (EMH) is rarely noted in the spleen in healthy control cynomolgus macaques ${ }^{7}$, yet is commonly seen in the mandibular lymph nodes, medullary cords as well as sporadically in other lymph nodes. When bone marrow is the target of toxicity, EMH can be seen in tissues such as the adrenal gland, liver (sinusoids) and kidneys (interstitium) ${ }^{15}$. Extramedullary hematopoiesis may be recorded when it is increased above background levels and the modifier "increased" may be used in such cases.

Fungus: Multiple tissues (Figure 12-13)

\section{Diagnostic features}

- Host response to fungal infection can vary from neutrophilic to granulomatous inflammation in miliary to mass-like lesions. Multinucleate giant cells may also be present.

- Distinct fungal morphologic features are often visualized by light microscopy.

- Special histochemical stains (Grocott or Gomori methenamine silver [GMS] and periodic acid Schiff [PAS] being most commonly used), culture results, antigen detection and molecular diagnostics aid in diagnosis.

Comments: Primary fungal infections are extremely rare in laboratory macaques. However, like many other mammalian species, NHP are susceptible to opportunistic infection. NHP are often temporarily housed or originate in geographic locations that are endemic for specific fungal pathogens, and thus can be exposed to a variety of fungal agents, most of which remain latent in immunocompetent animals. Reported fungal infections observed in immunosuppressed macaques include Pneumocystis carinii in the lung, Histoplasma capsulatum, Cryptococcus neoformans and non-neoformans spp., Aspergillus spp., and Coccidioides spp. ${ }^{16-18}$ Disseminated Talaromyces (Penicillium) marneffei infection was also recently reported in a laboratory cynomolgus macaque and directly linked to the immunosuppressive pharmacology of therapeutic agent given ${ }^{19}$. Candida albicans, while considered a normal saprophytic inhabitant of mucous membranes of the alimentary and genital tract in NHP, can also cause localized infection (thrush) or disseminated disease in neonates and immunocompromised animals ${ }^{17}$.

Inclusions: Multiple tissues (Figure 14-16)

Other term(s)

Viral infection 


\section{Modifiers}

Cytoplasm; intranuclear

\section{Diagnostic features}

- Intranuclear inclusions are typically round, may partially or almost completely fill the nucleus leading to margination of host nuclear chromatin.

- Contents of intranuclear inclusion bodies vary from eosinophilic, amphophilic to basophilic and may be granular or flocculent.

- Intracytoplasmic inclusions are typically eosinophilic, round to oval, homogenous, vary in size and occur as single or multiple structures in the cytoplasm.

Comments: Laboratory cynomolgus monkeys are the natural hosts for several well-described retroviruses, herpesviruses (e.g. cytomegalovirus, lymphocryptovirus and simian varicella virus), small DNA viruses (parvovirus and SV40) and adenovirus. The majority of viral infections in laboratory cynomolgus monkey colonies are clinically silent, since host-virus coevolution has allowed for lifelong viral persistence, often with a limited detrimental effect on the immunocompetent natural host. While complete elimination of infection is not possible, present strategies for pathogen control in laboratory non-human primate colonies including pre-study viral screening for known NHP retroviruses, Herpes B and measles virus have effectively decreased the likelihood that viral infection will confound toxicology study results. However, these viruses do pose a risk of opportunistic infection under conditions of immunosuppression or immunomodulation 17, 20,21. Cytomegalovirus, lymphocryptovirus and adenovirus are among the most common latent viral infections in macaques ${ }^{22}$. Viral inclusions should be recorded as "inclusion, intranuclear and/or cytoplasmic" when observed, further specifications can be provided in observation comments or the pathology narrative. Inclusions may be recorded as "present" or graded, depending on pathologist discretion. Non-viral causes of inclusion bodies such as those due to lead exposure are less common, but have been reported in macaques ${ }^{23}$ and should be considered.

\section{Infiltrate, [insert appropriate cell type]: Multiple tissues} (No image provided)

\section{Modifiers}

Neutrophil, eosinophil, lymphocyte, plasma cell, mast cell, macrophage, mononuclear cell, lympho-histiocytic, lymphoplasmacytic, mixed cell

\section{Other term(s)}

Inflammatory cell infiltrate; aggregates, inflammatory cell

\section{Pathogenesis/Cell of Origin}

The pathogenesis of inflammatory cell infiltrate is undetermined; however it is hypothesized that infiltrates may occur as a self-limiting immune response or as part of a tissue repair process.

\section{Diagnostic features}

- Usually manifests as variably sized focal to multifocal aggregates of inflammatory or immune cells in diverse tissues and body organs

- In general, lack of concomitant edema, congestion, hemorrhage, necrosis, and/or fibrosis, helps to differentiate from active or resolving inflammation

Differential Diagnoses

- Inflammation:

- In addition to inflammatory cell infiltrates, additional features include edema, tissue damage, hemorrhage, and/or fibrosis

- Mucosa-associated lymphoid tissue (MALT)

- MALT structures are normal anatomical features of the mucosal immune system that are histologically identified as nonencapsulated subepithelial lymphoid follicles with parafollicular areas and overlying specialized follicle-associated epithelium (FAE). The reader is referred to the hematopoietic and lymphoid system section for a detailed discussion of MALT and MALTlike structures such as duct-associated lymphoid tissue (DALT).

\section{Special Techniques for Diagnostics}

- Infiltrates are easily identifiable in H\&E stained tissue sections

- Immunohistochemistry may be performed to identify the lineage of leukocytes involved

Comments: Mononuclear cell infiltrates in various tissues are among the most common spontaneous pathology findings in control cynomolgus monkeys 6 , but need to be differentiated from naturally occurring mucosa-associated lymphoid tissue (MALT) and duct-associated lymphoid tissue (DALT). Within the conjunctiva, mononuclear or lymphoid cell infiltrates may not be indicative of an inflammatory process but may instead represent conjunctiva-associated lymphoid tissue (CALT), a normal structure of the conjunctival mucosa. With chronic antigenic stimulation, lymphoid hyperplasia may occur in CALT structures.

\section{Inflammation: Multiple tissues (No image provided)}

\section{Modifiers}

Granulomatous, mixed cell, neutrophil, eosinophil, lymphocyte, mast cell, macrophage, mononuclear cell

\section{Other term(s)}

"-itis", specific to the organ affected, e.g. encephalitis, gastritis, etc.

\section{Pathogenesis/Cell of Origin}

Inflammation in a tissue may be evoked as a response of 
resident or systemic inflammatory cells in response to nocuous stimuli.

\section{Diagnostic features}

- Presence of a single cell lineage or multiple types of leukocytic infiltrates in tissues along with other features of tissue damage and/or repair such as edema, congestion, hemorrhage, necrosis, and fibrosis.

- Based on the cell type, inflammation can be classified as suppurative (mainly neutrophilic), mononuclear or mixed cell (consisting of neutrophils, eosinophils, lymphocytes, plasma cells, and macrophages), granulomatous (mainly consisting of macrophages with or without multinucleated giant cells), or pyogranulomatous (consisting of both macrophages and neutrophils).

\section{Differential Diagnoses}

- Infiltrate, [insert appropriate cell type]

- Abscess

- Leukocytic neoplasms

\section{Special Techniques for Diagnostics}

- Inflammation is easily identifiable in H\&E stained tissue sections

- Immunohistochemistry may be performed to identify the lineage of leukocytes involved

Comments: Inflammation can occur as a normal host defense or a repair process in response to tissue injury. It should be differentiated from an "inflammatory cell" infiltrate as the latter does not have other features of tissue damage and/or repair such as edema, congestion, hemorrhage, necrosis, and fibrosis. Inflammation is one of the most common spontaneous pathology findings reported in control cynomolgus monkeys 6 . Differentiation of systemic tumors e.g. by accumulation of "inflammatory cells" without a discernible stimulus, morphology of cells such as mitosis or pleomorphism.

For organ specific, detailed characteristics refer to the description in the respective organ section.

Various approaches to further characterize inflammation are used by pathologists, including chronicity (peracute, acute, subacute, etc.), location (perivascular, peribiliary, etc.), and others. To implement a descriptive terminology, the use of the term "inflammation" is preferred over the "-itis" terminology, and the indication of the predominant cell type(s) in the diagnosis is recommended over the conventional use of chronicity, e.g.:

- Inflammation, neutrophil

- Inflammation, lymphocyte

- Inflammation, plasma cell

- Inflammation, monocyte/macrophage

When applicable the terms are combined, e.g.:

- Inflammation, lympho-plasmocytic

- Inflammation, lympho-histiocytic

Further description by location and distribution is recom- mended.

\section{Parasite/Parasitic granuloma: Multiple tissues}

(Figure 17-27)

\section{Diagnostic features}

- Distinct helminth or protozoal morphologic features are often visualized by light microscopy.

- Host response to tissue invasion can vary from neutrophilic, eosinophilic to granulomatous inflammation in miliary to mass-like nodular lesions.

- Heminth tissue invasion commonly presents as organized, focal, well demarcated, nodular lesions. Parasite profiles are often encapsulated by fibroblasts, lymphocytes, and plasma cells, with limited to no active inflammation.

- Epithelioid macrophages and multinucleated giant cells (Langhans or foreign body types) are often present.

- Granulomas may distort normal tissue architecture.

Comments: Many parasitic pathogens are endemic in NHP colonies and remain clinically inapparent in immunocompetent animals. Treatment with anti-helmintics prior to importation and during quarantine has significantly reduced or eliminated many parasitic infestations; however, a low incidence is still noted on post mortem examination. In healthy immunocompetent animals, most parasitic infections present as non-invasive disease with low numbers of organisms present. For example, Balantidium coli is commonly identified within the gastrointestinal tract lumen, specifically in the cecum and colon without mucosal invasion or alteration. Similarly, Sarcocystis spp. cysts are occasionally observed in skeletal muscle, and less frequently in the cardiac and smooth muscle ${ }^{24}$ in the absence of active disease.

Common helminths identified in cynomologus macaques include species of Strongyloides, Oesophagostomum, Anatrichosoma, Trichostrongylus, Trichuris, Gongylonema, larval stages of nematodes (ascarids, spirurids), cestodes (Echinococcosis spp.) and trematodes (the lung fluke Paragonimus westermanii) ${ }^{25}$. Cerebral Baylisascaris larva migrans has also recently been reported in cynomolgus macaques $^{26}$. In most instances of infection, only a few organisms are observed and tissue damage is limited with parasitic granulomas being a common presentation.

Protozoal infections encountered in macaques include Cryptosporidium parvum, Enterocytozoon bieneusi, Plasmodium spp., Trichomonas spp., Acanthamoeba spp., Toxoplasma gondii ${ }^{27}$ and Trypanosoma cruzi ${ }^{28}$. Toxoplasma gondii systemic infection can be seen spontaneously in macaques ${ }^{29}$ and is characterized by necrosis and inflammation in the brain, lungs, mesenteric lymph node, liver and/ or heart. Trypanosoma cruzi infection can be the cause of inflammation in the heart and/or brain of cynomolgus monkeys.

Lastly, pulmonary acariasis (Pneumonyssus simicola) was once prevalent, yet due to current pathogen control measures is now rarely identified in laboratory colonies. 
A complete review of parasitic lesions in macaques is beyond the scope of this manuscript. The reader is referred to the literature.

Pigment: Multiple tissues (Figure 28-34)

\section{Modifiers}

May include the pigment type, if known

\section{Other term(s)}

Endogenous pigments

- Lipofuscin

- Melanin

- Hemosiderin

- Mite pigment

- Hematoidin

- Hemozoin (malaria pigment)

- Bilirubin

Exogenous pigments

- Carbon (coal dust), anthracosis

- Tattoo ink

\section{Pathogenesis/Cell of Origin}

The pathogenesis varies based on the type of pigment observed in tissue sections. Pigments are generally colored based substances that arise from either exogenous sources or endogenous processes. Endogenous pigments can be further classified as either hematogenous or nonhematogenous. In most cases, these products develop as a pathological consequence; however some may occur as normal physiological products.

\section{Diagnostic features}

- Lipofuscin or lipochrome is an insoluble pigmented byproduct of lipid peroxidation ${ }^{30}$. It appears as yellowbrown, finely granular cytoplasmic material in histological sections of tissue. Accumulates in macrophages in the heart, liver, spleen, gastrointestinal tract and brain. Ceroid is a golden acid-fast, sudanophilic pigment, probably a type of lipofuscin, although differing from true lipofuscins by failing to stain with Schmorl ferric-ferricyanide reduction $\operatorname{stain}^{30}$.

- Melanin is a naturally occurring substance formed the oxidation of tyrosine to dihydroxyphenylalanine in melanocytes $^{30}$. The result is a brown-black pigment that is deposited mainly in the skin, meninges, uvea, iris, and reproductive tissues where it is sometimes known as melanosis ${ }^{31}$.

- Hemosiderin appears as golden brown granules within macrophages. Hemosiderin results when the intracellular protein ferritin binds with free iron ${ }^{30}$. Ferritin is highly abundant in the liver, spleen and bone marrow; therefore these tissues may contain numerous hemosiderin-laden macrophages. Hemosiderin may be observed in a variety of tissues subsequent to hemorrhage. Accumulation of perivascular hemosiderin-laden macrophages is a common finding in the lung ${ }^{32}$. Application of the Perls' Prus- sian blue histochemical stain will detect this pigment in tissues.

- Mite pigment. This pigment results from Pneumonyssus simicola infections ${ }^{33}$. Although it is seen primarily in the lungs in proximity to the mite or bronchial lesions, it may also extend to the tracheobronchial lymph nodes. Histologically macrophages contain a golden brown to black pigment that is refractile.

- Acid hematin. This pigment develops in tissues fixed in unbuffered formalin ${ }^{31}$. It is most abundant in regions rich in erythrocytes or hemoglobin including regions of hemorrhage and congested blood vessels. Acid hematin presents as a granular to sometimes crystaline black to dark brown substance. The pigment can be bleached with the Kadasewitsch-method to avoid interference with endogenous pigments.

- Hematoidin is a golden-brown crystalline pigment. Morphologically the crystals may appear in a variety of forms including: 1) thread-like filaments that form star-shaped clusters (Medusa's head); 2) small, irregular crystals; 3) spheroid crystals; and 4) rhomboid crystals ${ }^{34}$. Closed tissue compartments have low oxygen tension, which are suitable for the formation of hematoidin following degradation of hemoglobin from extravasated erythrocytes.

- Hemozoin also known as malaria pigment is the result of the Plasmodium parasite converting free heme to the insoluble b-hematin ${ }^{35}$. The pigment can be detected in blood films by light microscopy as intracytoplasmic yellow to green-brown pigment within granulocytes and/or monocytes. Hemozoin is recognized as granular birefringent material with polarization.

- Bilirubin is a breakdown product of erythrocytes and is also excreted by the hepatocytes as a bile pigment. Grossly it imparts a yellow discoloration to tissues also known as icterus. Bilirubin is a pigment found within bile and is therefore associated with cholestasis and observed histologically as yellow brown plugs or casts within the bile canaliculi.

- Carbon (coal dust). Anthracosis is the accumulation of inhaled carbon particles by alveolar and interstitial macrophages in the lungs. The carbon pigment may also extend to the tracheobronchial lymph nodes.

- Tattoo ink. This form of exogenous pigment is localized to the skin and draining lymph node. Dermal macrophages, as well as those located in nearby lymph nodes phagocytize this pigment where it remains indefinitely.

\section{Differential Diagnoses}

- Fine granular material:

- May resemble bacteria, which can be differentiated by Gram stain.

- Calcium salts:

- Impart a basophilic granular appearance, can be differentiated from pigmented substances using von Kossa histochemical staining. 
- Copper:

- May not be readily detected in hematoxylin and eosin stained tissues but can be differentiated from other granular material using rhodanine or rubeanic acid.

- Hematoidin:

- Should be distinguished from other pigments based on its negative Perls' Prussian blue staining.

\section{Special Techniques for Diagnostics}

- Light microscopy, special stains (Perls Prussian blue), electron microscopy, polarization

\section{Infectious Diseases}

Nonhuman primates used within routine toxicology studies may be subclinically infected with a variety of infectious organisms acquired from their breeding colony or holding facilites en-route to the testing facility. Evidence of these organisms or infections may be an incidental finding at necropsy, or they may become clinically activated or be transmitted while the animal is on a nonclinical study, particularly when one or more animals become immunologically compromised. Infectious agents seen within macaques include fungal, parasitic, bacterial and viral diseases, which should be recorded within the data capture system at a high level under the visible etiologic agent (fungus, parasite, bacteria) or inclusions (virus), and not under individual organisms or disease diagnosis (e.g. Plasmodium spp.). Any pathologic response(s) or finding(s) associated with the infectious agent should be recorded separately using appropriate INHAND terms where possible. Additional diagnostic tools such as in situ hybridization and immunohistochemistry can aid in identifying and investigating the impact of known or novel NHP pathogens on nonclinical studies 22 .

A complete review of infectious agents in macaques is beyond the scope of this manuscript. The reader is referred to the extensive literature and to the individual INHAND terms (fungus, bacteria, parasite, inclusions).

\section{Syndromes}

Syndromes that may occur in the nonhuman primates include fatal fasting macaque syndrome, hemophagocytic syndrome and phospholipidosis, which are described below. The syndrome should not be recorded as a finding but should be mentioned as an observation comment in the data capture system and/or in the pathology narrative. The individual findings that together make the syndrome should be recorded seperately using INHAND terms where possible.

\section{Fatal fasting macaque syndrome: Systemic Conditions} (Figure 35-37)

\section{Other term(s)}

Obese macaque syndrome; fatal fatty liver-kidney syndrome in obese monkeys; fatal fatty liver syndrome

\section{Pathogenesis/Cell of Origin}

Although still poorly understood, the pathogenesis of this syndrome is considered multifactorial ${ }^{36-43}$. Stress-inducing conditions such as transportation, recaging, changes in husbandry and others, may cause decreased food consumption up to anorexia and subsequent rapid and/or repeated weight variation/losses in previously overfed/obese adult monkeys. This finally causes a negative energy/nutritional imbalance. The subsequent mobilization of lipid and fatty acids from adipose tissue throughout the body disrupts lipid metabolism and finally causes lipid accumulation in several organs, especially the liver and kidneys. Raised level of circulating triglycerides may be associated with morphological changes, but blood chemistry profile generally does not suggest liver failure.

\section{Diagnostic features}

- Macroscopically, the liver and kidneys may appear enlarged and pale, and multiple pale-tan foci can be observed in the fat tissue of the abdominal cavity at necropsy.

- Various degrees of cellular swelling due to cytoplasmic vacuolation are noted in several organs especially the liver (hepatocytes) and kidneys (epithelial lining of the proximal convoluted tubules). The vacuoles are of variable size but generally large and round-oval and contain lipids. They appear empty in HE-stained section and are positive to Oil Red-O stain and other lipidic special stains.

- Acute degenerative and inflammatory changes can be observed in the pancreas, such as decreased zymogen content in acinar cells, acinar cell necrosis, acute celltype infiltration and steatonecrosis in the surrounding fat tissue.

- In some cases, adrenocortical hyperplasia and vacuolation, and thyroid follicular enlargement associated with flattening of the follicular epithelium have been reported in affected monkeys ${ }^{37}$.

\section{Differential Diagnoses}

- Xenobiotic-induced cellular degeneration in the liver and kidneys (with fatty change), pancreas and fat tissue.

- Traumatic events causing degenerative/inflammatory changes in fat tissue.

- Pancreatic cell degeneration associated with inflammation:

- Adenoviral-related pancreatitis has been reported in $M$. mulatta $^{44}$. Hypothyroidism and diabetes mellitus may be associated with fatty change in the liver.

- Serous atrophy of fat:

- Generally not associated with steatonecrosis and lipidic accumulations in other organs.

\section{Special Techniques for Diagnostics}

- Special stains for lipid content such as Oil Red-O and Sudan Black. 
Comments: This syndrome has been reported in several species of non-human primates such as $M$. fascicularis, M. mulatta, M. arctoides, M. nemestrina and M. radia$t^{36,}, 37,39,42$. It has been generally described in adult monkeys, and females seem to be predisposed.

\section{Hemophagocytic syndrome: Systemic Conditions}

(No image provided)

\section{Other term(s)}

HPS

\section{Pathogenesis/Cell of Origin}

HPS is a nonneoplastic condition characterized by proliferation of activated macrophages ${ }^{45}$. HPS is mediated by hyperinflammatory state comprising uncontrolled CD8+ T-cell activation leading to marked production by interferon-gamma $(\text { IFN- } \gamma)^{46}$. This is followed by histiocytic proliferation and production of cytokines including tumour necrosis factor-alpha (TNF- $\alpha$ ), IL-1, IL-6, IL-10, IL-18, and granulocyte colonystimulating factor $(\mathrm{G}-\mathrm{CSF})^{47}$. Together this leads to multiorgan dysfunction.

\section{Diagnostic features}

- Bone marrow cytopenia

- Increased numbers of hemophagocytic macrophages in peripheral tissues.

\section{Differential Diagnoses}

- Epstein-Barr infections:

- Often present with monocytosis, and Epstein-Barr Nuclear Antigen (EBNA) can be detected in sections of tissue by immunohistochemistry.

- Simian Parvovirus infections:

- May present with a normocytic, normochromic, nonregenerative anemia, or bone marrow dysmaturation with the presence of large intranuclear inclusion bodies within bone marrow hematopoietic cells ${ }^{14}$.

- Infection with Simian Retrovirus:

- A betaretrovirus, presents with cytopenic abnormalities that includes anemia, leukopenia and thrombocytopenia ${ }^{48}$. Earlier phases of infection may consist with bone marrow hyperplasia involving granulocytic and erythroid lineages ${ }^{49}$; however late stage infection typically present as severe pancytopenia associated with bone marrow depletion.

\section{Special Techniques for Diagnostics}

- Light microscopy, complete blood counts, clinical history, viral titers

Comments: The veterinary literature is lacking information regarding HPS in monkeys. A recent report described HPS in rhesus ${ }^{50}$ associated with SRV infection. Much of what is known about hemophagocytic syndrome (HPS) comes from the human medical literature and scant reports in the veterinary medical literature including cats and dogs ${ }^{51,52}$. HPS is an often-fatal nonneoplastic proliferative disorder characterized by activated macrophages, multi lineage cytopenias in the bone marrow and profound phagocytosis occurring in the bone marrow and liver. Both genetic $^{53}$ and acquired ${ }^{54}$ forms with Epstein-Barr infections have been reported.

Phospholipidosis: Systemic Conditions (No image provided)

Comments: Similar to the other laboratory species, the macaque is susceptible to phospholipidosis ${ }^{55}$. Drug-induced phospholipidosis is characterized by cytoplasmic vacuolation seen in multiple tissues on light microscopic examination. The descriptive term vacuolation is the preferred terminology. A diagnosis of phospholipidosis is confirmed by demonstrating the accumulation of lamellar bodies in lysosomes on TEM. 


\section{Cardiovascular System}

For detailed general considerations on the cardiovascular system, refer to the INHAND rodent publication ${ }^{56}$.

In nonclinical studies, morphological and functional endpoints are essential in the identification of any potential interaction between xenobiotics and cardio-vascular structures. As a minimum, post-mortem standard morphological evaluation of the cardio-vascular system includes heart weight measurement and light conventional histopathology of the myocardium and valvular leaflets in the heart ${ }^{57}$, aorta and blood vessels within tissues. Electron microscopy and immunohistochemistry may help to identify the pathological process caused by xenobiotics. In addition, the evaluation of serum levels of heart-specific biomarkers and functional assessments, such as blood pressure and echocardiography, can provide valuable in-life information correlating with morphological alterations observed at the post-mortem examination.

\section{A. Heart}

At necropsy, similar to other lab animal species, the heart is sampled with the root of the large vessels and fixed in buffered-formalin solution. Although not considered necessary, the heart may be opened prior to immersion in the fixative in order to ensure adequate fixation and eliminate large blood clots occupying the cardiac chambers.

Similar to all other laboratory animal species, light conventional histopathology of the heart is based on a thorough and consistent microscopic evaluation of hematoxylin and eosinstained sections of all relevant compartments and structures of the heart, including the ventricular, atrial and interventricular septal wall, the valves and the coronary vessels following published guidelines 58,59 . This allows an accurate identification of changes in the cardiomyocytes, extracellular matrix, conduction system and the vascular structures within the myocardium and the adjacent epicardial tissue. Occasionally, a more detailed evaluation of the conduction system may be required, and specific dissection techniques are necessary ${ }^{60}$, although this is not a standard procedure in routine toxicology studies. Special dissection procedures may be required to systematically evaluate the coronary arteries (Table 2).

\section{Cyst/plaque, squamous/epithelial cell: Heart}

(Figure 38-40)

\section{Other $\operatorname{term}(\mathrm{s})$}

Squamous cyst; squamous epithelial cyst; keratinized/nonkeratinized cyst; epithelial cyst; squamous plaque; squamous cyst/plaque; squamous metaplasia; ectopic epithelial structure; ectopic epithelium

\section{Pathogenesis/Cell of Origin}

The origin is unknown. Morphologic features are consistent with an embryonic remnant.

\section{Diagnostic features}

- Usually observed on the epicardial surface or sub-epicardially, rarely sub-endocardially, throughout the heart surface. Non-keratinized cysts are found primarily at the base of the heart, coronary groove and atria.

- Squamous cysts are lined by a keratinized or non-keratinized squamous epithelium and may contain concentric layers of keratin, inflammatory cells, cellular debris and/ or eosinophilic colloid-like material. They may be surrounded by a foreign-body inflammatory reaction if the epithelial wall has been broken.

- The epithelial lining is usually surrounded by a layer of fibrous connective tissue of varying thickness.

- Squamous plaques can be found alone or near squamous cysts $^{6,11,61-63}$.

\section{Differential Diagnoses}

- Ectopic thyroid tissue or thyroid follicle-like structures:

- Consist of variably sized single or clusters of colloidfilled follicles at the epicardial or mid-myocardial areas, generally at the base of the heart near the atrial appendages and large vessels.

Comments: Squamous atrial plaques can be associated with focal mesothelial proliferation suggesting that they could also represent a reactive change to physical irritation of the epicardium.

Squamous cysts and plaques are considered to represent ectopic foregut epithelium that was sequestered in the dorsal mesocardium prior to closure of the pleuro-pericardial fold during early embryonic development.

\section{Accumulation adipocytes, myocardium: Heart}

(Figure 41-42)

\section{Other term(s)}

Fat infiltration; increased adipose tissue; fatty replacement of cardiomyocytes; heart lipomatosis

\section{Pathogenesis/Cell of Origin}

It is uncertain whether the presence of adipose tissue in the heart is caused by an infiltrative process or a metaplastic phenomenon.

\section{Diagnostic features}

- Segmental or diffuse transmural loss of myocardium replaced by normally appearing white adipose tissue.

- Adipose tissue may extend from the epicardium towards the endocardium.

- Most commonly seen in the right ventricular wall, although may also be observed in left ventricle and interventricular septum.

- Infiltrating adipocytes displace/replace cardiomyocytes. Remaining cardiomyocytes in the adjacent myocardium may be decreased in size, suggesting a concurrent atrophic change. 
Table 2. Microscopic Findings of the Cardiovascular System: Heart; Non-human Primate

\begin{tabular}{|c|c|c|c|c|}
\hline Heart & Common & Uncommon & $\begin{array}{c}\text { Not Observed but } \\
\text { Potentially Relevant }\end{array}$ & Not Applicable \\
\hline \multicolumn{5}{|l|}{ Congenital } \\
\hline Cyst/plaque, squamous/epithelial cell * & & $\mathrm{x}$ & & \\
\hline \multicolumn{5}{|l|}{ Non-proliferative } \\
\hline Accumulation adipocyte, myocardium $* \wedge \#$ & $\mathrm{x}$ & & & \\
\hline Amyloid, myocardium ${ }^{\wedge}$ & & $\mathrm{x}$ & & \\
\hline Cardiomyopathy, rodent progressive & & & & $\mathrm{x}$ \\
\hline Degeneration, cardiomyocyte & & $\mathrm{x}$ & & \\
\hline Degeneration/necrosis, cardiomyocyte & & $\mathrm{x}$ & & \\
\hline Ectopic tissue, thyroid ${ }^{\wedge}$ & & $\mathrm{x}$ & & \\
\hline Edema, myocardium & & $\mathrm{x}$ & & \\
\hline Inclusions, cytoplasmic, cardiomyocyte $* \wedge$ & $\mathrm{x}$ & & & \\
\hline Inclusions, intranuclear, eosinophilic *^ & & $\mathrm{x}$ & & \\
\hline Infarct, myocardium & & $\mathrm{x}$ & & \\
\hline Infiltrate, [insert appropriate cell type], myocardium $* \wedge$ & $\mathrm{x}$ & & & \\
\hline Infiltrate/fibrosis & $\mathrm{x}$ & & & \\
\hline Inflammation, pericardial * & & $\mathrm{x}$ & & \\
\hline Inflammation, granulomatous; pericardial adipose tissue * & $\mathrm{x}$ & & & \\
\hline Karyomegaly/karyocytomegaly, cardiomyocyte * & $\mathrm{x}$ & & & \\
\hline 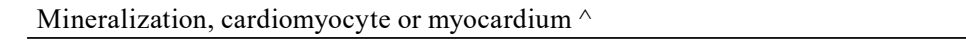 & & $\mathrm{x}$ & & \\
\hline Mononuclear cell infiltrate/fibrosis, myocardium & $\mathrm{x}$ & & & \\
\hline Hyperplasia, mesothelium* & $\mathrm{x}$ & & & \\
\hline Hyperplasia, Schwann cell, subendocardium & & & & $\mathrm{x}$ \\
\hline
\end{tabular}

* Terminology with diagnostic criteria and/or comments described below. \# Finding more frequent as an induced change. ${ }^{\wedge}$ Terminology addressed in the General/Systemic Pathology Section.

- This change may be associated with an inflammatory response, primarily lymphocytic infiltration 63,64 .

\section{Differential Diagnoses}

- Lipoma:

- This diagnosis should be taken into consideration, despite the lack of report on cardiac lipoma in monkey, to date. Lipoma may present as a lobulated mass composed of mature adipocytes supported by a fine fibrovascular stroma.

Comments: Adipose tissue replacement of the myocardium may be an incidental finding in healthy cynomolgus monkeys used in nonclinical studies, and generally without de- generative changes of the surrounding myocardium. This change has been associated with obesity and its incidence appears to increase with age. It is generally not accompanied by any evidence of cardiac dysfunction. If not an induced change, typically this background observation is not recorded.

Hemorrhage, subendocardium: Heart (No image provided)

Comments: Subendocardial hemorrhage is a common finding in the heart of cynomolgus monkeys derived from Mauritius $^{63}$ and various sources in Asia ${ }^{11}$.

The range of severity varies from small foci of erythrocytes to distinct hemorrhages, dissecting the cardiac myofibers. 
Hemosiderosis and erythrophagocytosis are often associated with subendocardial hemorrhage ${ }^{63}$.

The reason for subendocardial hemorrhage in nonhuman primates is not known, whereas a variety of mechanisms has been identified in humans ${ }^{65}$. It was believed that the early stages represent an agonal phenomenon. In man, it has been demonstrated that even minor subendocardial hemorrhages might have an influence on cardiac function ${ }^{66}$.

\section{Hypertrophy, cardiomyocyte: Heart (Figure 43-45)}

\section{Pathogenesis/Cell of Origin}

Focal or diffuse, ventricular cardiomyocyte hypertrophy can develop in response to various conditions such as exercise, blood pressure/volume overload or injury, and is a compensatory change. However, cardiomyocyte hypertrophy can also be induced by certain xenobiotics.

\section{Diagnostic features}

- Focal, or diffuse increase of diameter in individual cardiomyocytes in the ventricle wall.

- Nuclei of hypertrophied cardiomyocytes are often enlarged, irregularly shaped and hyperchromatic.

- Cardiomyocyte alignment may be distorted in more severe cases (e.g. myocardial remodeling).

- Diffuse cardiomyocyte hypertrophy can result in cardiac hypertrophy and increased heart weight.

- Modifiers: focal/diffuse, ventricular (left/right), apical.

\section{Differential Diagnoses}

- Foci of hypertrophic cardiomyocytes can be associated with degenerative myocardial changes in idiopathic myocardial cardiomyopathy.

Comments: Rhesus macaques appear to be prone to develop spontaneous left ventricular cardiac hypertrophy, as suggested by the description of several cases in a colony of rhesus monkeys, where concentric hypertrophy of the left ventricle associated with reduction of the ventricular lumen was observed in young adults of both sexes presented for sudden death. There appeared to be a strong genetic predisposition at pedigree analyses ${ }^{67}$. Cardiomyocyte hypertrophy has also been described in a Rhesus monkey bearing a functional pheochromocytoma ${ }^{68}$. The epinephrine produced by this tumor induced cardiomyocyte hypertrophy near areas of fiber atrophy and degeneration and bands of fibrous connective tissue disrupting the normal orientation. Examples of compounds inducing ventricular cardiomyocyte hypertrophy resulting in cardiac hypertrophy include Muraglitazar and certain synthetic androgens. Muraglitazar, a peroxisome proliferator-activated receptor (PPAR) agonist given orally to cynomolgus monkeys over a period of 1 year induced cardiac hypertrophy in the left ventricular wall due to increased cardiac workload secondary to plasma volume expansion ${ }^{69}$. Supra-physiological androgen exposure also can cause cardiomyocyte hypertrophy in ani- mals ${ }^{70}$. Hypertrophy of cardiomyocytes by testosterone and dihydrotestosterone (Figure 44) was determined to be directly androgen receptor-dependent ${ }^{71}$. Focal cardiomyocyte hypertrophy has also been reported in cynomolgus monkeys treated with 2-amino-3-methylimidazo[4,5-f]quinolone $^{72}$. The cardiomyocyte hypertrophy was observed near foci of fiber necrosis and atrophy, chronic inflammation, fibrosis and vasculitis.

Cardiomyocyte hypertrophy has been reported in aging male cynomolgus monkeys, and this was associated with increased cardiomyocyte apoptosis ${ }^{73}$.

\section{Inclusion, intracytoplasmic, cardiomyocyte: Heart}

(No image provided)

\section{Pathogenesis/Cell of Origin}

Ceroid and lipofuscin are known to accumulate in several tissues as a function of age.

\section{Diagnostic features}

- Presence of eosinophilic granules in cytoplasm of cardiomyocytes, generally located near the poles of the nuclei.

- Ceroid-lipofuscinosis has been reported in a cynomolgus monkey and was characterized by cytoplasmic accumulation of bright, eosinophilic cytoplasmic granules in multiple tissues and organs, including the cardiac muscle cells ${ }^{74}$.

- Ceroid and lipofuscin granules are approximately $1.0 \mu \mathrm{m}$ in diameter and show irregular contours. Special stains are needed in order to distinguish ceroid and lipofuscin pigments.

- Other types of inclusions have been described, such as hyaline granules, generally round and varying in size, up to $3.5 \mathrm{pm}$ in diameter.

\section{Special Techniques for Diagnostics}

- Special stains including PAS, Ziehl-Neelsen, Luxol Fast Blue, Oil-Red-O, Schmorl's, Mallory's and others, may be of great help to distinguish ceroid, lipofuscin, and hyaline-type intracytoplasmic granules.

Comments: Ceroid, lipofuscin and hyaline-type intracytoplasmic granules have been described in cardiomyocytes of cynomolgus monkeys ${ }^{74-76}$. Ceroid granules are considered to contain an insoluble lipid and are stainable with Luxol fast blue and Mallory's phloxine stain for hyaline. Lipofuscin granules represent age-related pigments. Hyaline granules appear to be devoid of lipid component.

\section{Inclusion, intranuclear, eosinophilic, cardiomyocyte:} Heart (No image provided)

\section{Pathogenesis/Cell of Origin}

Considered to be invagination of cytoplasm and cytoplasmic organelles into the nucleus of the cardiomyocyte. 


\section{Diagnostic features}

- Eosinophilic inclusions in the nucleus of cardiomyocytes

Comments: Reported as a rare event, sometimes associated with severe cases of cardiomyopathy ${ }^{6}$.

Infiltrate, [insert appropriate cell type], myocardium: Heart (No image provided)

Comments: Focal inflammatory cell infiltrations and focal myocarditis are other terms that have been used to describe different degrees of severity of the same pathological phenomenon ${ }^{77}$, which are commonly observed in cynomolgus monkeys as a spontaneous finding6, 78, 79. The term "myocarditis" should not be used, however, and more descriptive terminology would be preferred, according to INHAND guidance and norms. Lower grades are not associated with necrosis or degeneration of cardiomyocytes or subsequent increase in troponin ${ }^{80}$, and the term infiltrate with relevant cell type(s) is appropriate. Higher grades are associated with myocardial degeneration and necrosis or other evidence of inflammation such as edema, karyomegaly or fibrin deposition $^{77}$. In cases where more extensive inflammatory changes are observed, the term inflammation is appropriate.

The myocardium at sub-endocardial and sub-epicardial locations is most affected. The lesions are much more common in the ventricular than the atrial myocardium without preference for the left or right ventricle, heart base or apex ${ }^{6,61}$.

Inflammation, pericardial: Heart (No image provided)

\section{Other term(s)}

Pericarditis; epicarditis; epicardial inflammation

\section{Pathogenesis/Cell of Origin}

Infiltration of the pericardium/epicardium by inflammatory cells occasionally associated with myocardial inflammation.

\section{Diagnostic features}

- Infiltration of the pericardial tissues with inflammatory cells, predominantly lymphocytic, although mixed inflammatory infiltrates can be seen in more severe cases associated with focal myocardial inflammation.

- May be associated with degenerative/inflammatory lesions of the myocardium, and necrotic debris in the pericardium.

Comments: Pericardial inflammatory changes have been reported as secondary changes to diseases in wild caught monkeys. Toft and Eberhard ${ }^{81}$ reported pericarditis associated with ruptured lung mite lesions in rhesus monkeys, and April and Keith ${ }^{82}$ reported acute purulent pericarditis associated with severe myocarditis. Osborn et al. ${ }^{83}$, reported pericarditis as a significant finding in small number of animals in a colony of rhesus monkeys epizootic of apparent acquired immunodeficiency syndrome.
Inflammation, granulomatous, pericardial fat: Heart

(Figure 46)

\section{Other term(s)}

Fat tissue granuloma.

\section{Pathogenesis/Cell of Origin}

The cause is unknown.

\section{Diagnostic features}

- Granuloma(s) composed of macrophages with large cytoplasm (epithelioid cells), which may be surrounded by scattered inflammatory cells including lymphocytes, plasma cells and eosinophils, seldom accompanied by fibrosis ${ }^{61}$.

- Multinucleated giant cells may also be present.

- This change is more likely to occur in adipose tissue around the right coronary artery.

Differential Diagnoses

- Chronic inflammation of fat tissue (steatitis)

- Metastatic hematopoietic neoplasms

Special Techniques for Diagnostics

- Ziehl-Neelsen stain and Gram stains to exclude the presence of mycobacteria or other bacterial colonies

- PAS to exclude the presence of mycotic colonies

Comments: This is a relatively common finding in cynomolgus monkeys, although the incidence is generally low. Sometimes, similar granulomatous foci can be observed in the fat tissue associated with other organs, such as in mesenteric fat. This change may be associated with decrease in food intake and/or fat atrophy.

\section{Karyomegaly/karyocytomegaly, cardiomyocyte: Heart} (Figure 47-48)

\section{Other term(s)}

Anisokaryosis and karyomegaly; idiopathic myocardial degeneration; cardiomyopathy

\section{Pathogenesis/Cell of Origin}

Degenerative change of cardiomyocytes.

\section{Diagnostic features}

- Cardiomyocyte karyomegaly is generally observed at the apex of the heart, the interventricular septum, the papillary muscle and the subendocardial areas in both ventricles.

- Individual to small groups of myofibers, rarely up to extensive area of myocardium, may appear enlarged (i.e. hypertrophic).

- Histological changes may be limited to enlargement (karyomegaly) and irregular shape (anisokaryosis) of the nucleus. The nuclei may show intranuclear cytoplasmic invaginations. These changes are commonly seen in purposed-bred non-human primates. 
- Less frequently, the severity is higher and changes in affected fibers may include cytoplasmic vacuolation and higher degrees of karyomegaly and anisokaryosis. The nuclei show weakly stained chromatin and prominent nucleolus.

- In addition, anisokaryosis and karyomegaly have been described in association with interstitial thickening due to edema and/or collagen bundles, with mineralization in chronic lesions, accompanying cardiomyocyte disarray.

- The inflammatory component is generally sparse and consists of scattered lymphocytes, plasma cells and macrophages.

\section{Differential Diagnoses}

- Necrosis of cardiomyocyte:

- Generally not associated with karyomegaly, although scattered foci of degenerating fibers can be observed.

- Cellular infitrates:

- May be observed without karyomegaly and anisokaryosis. An inflammatory cell infiltration is generally not a primary feature associated with karyomegaly and anisokaryosis $6,63,78,84$.

\section{Special Techniques for Diagnostics}

- Masson's trichrome may help to identify collagen deposits.

Comments: Anisokaryosis and karyomegaly are considered to reflect polyploidy.

The term "spontaneous cardiomyopathy" has been recently used to describe the condition in cynomolgus monkeys $6,84,85$; however, a descriptive terminology of the histological changes is currently recommended in order to avoid any potential confusion with human-specific cardiac syndromes.

The pathogenesis of this myocardial change is poorly understood. However, it is considered as reminiscent of chronic catecholamine-induced experimental cardiomyopathy and stress cardiomyopathy in cynomolgus monkeys and humans ${ }^{84,86}$.

Parasite, protozoa: Heart (Figure 49-50)

\section{Pathogenesis/Cell of Origin}

Parasitic infestation of the heart.

\section{Diagnostic features}

- Presence of intracellular protozoan parasites (e.g. Trypanozoma cruzi amastigotes). The parasite-related inflammation is generally characterized by random, focal to diffuse, primarily lymphocytic myocardial infiltrates associated with amastigotes. In addition, cardiomyocyte degeneration, hyper-eosinophilia and loss of cross striations, and mixed cell and eosinophil-rich inflammatory cell infiltration may occur.

\section{Differential Diagnoses}

- Non-infectious cardiomyocyte degeneration and/or inflammation

Comments: Trypanosoma cruzi has been reported sporadically in cynomolgus monkey colonies in the USA and can be the cause of mononuclear cell infiltration in the heart of cynomolgus monkeys 28,87 . Intracellular clusters of amastigotes, however, can be very difficult to find microscopically. Other protozoal parasites such as Sarcocystis or Toxoplasma spp. have been reported in rhesus and cynomolgus macaques; however, these organisms are very rarely associated with myocardial inflammation in immunocompetent animals.

\section{Hyperplasia, mesothelium: Heart (Figure 51)}

\section{Other term(s)}

Benign mesothelial proliferation; reactive mesothelium

\section{Pathogenesis/Cell of Origin}

An excessive non-neoplastic growth of mesothelium perceived to be a response to local irritation, which may be a result of inflammation, infection or toxicity. No or few inflammatory cells may be observed.

\section{Diagnostic features}

- Papillary (villous) thickening of the mesothelium lining the pericardium, epicardium and tunica adventitia of vessels.

- Macroscopically, small white nodules or flat plaques may be visualized at necropsy on the epicardial, pericardial or adventitial vascular surface.

- Histologically, inflammatory changes may accompany the hyperplastic mesothelium.

- Unlike the normally flat simple squamous mesothelial cells, hyperplastic mesothelial cells may be cuboidal or columnar with abundant cytoplasm. Cells may occasionally be binucleate but cellular atypia is usually low and mitoses are infrequent.

- Outgrowing hyperplastic mesothelium (papillary or villous) are usually lined by single layer of cells supported by loose fibro-vascular and/or edematous stroma, more frequently observed in atrial wall.

- Occasionally, cells may be shed and subsequently surrounded by inflammatory exudate or tissue.

\section{Differential Diagnoses}

- Mesothelioma:

- Increased anisocytosis, anisokaryosis, increased numbers of multinucleate cells, mitotic figures and stromal or parenchymal invasion

- Carcinomas and sarcomas:

- Lack of immunoreactivity to both cytokeratin and vimentin 
- Fibrosis:

- Bundling of cells and presence of collagen deposits

\section{Special Techniques for Diagnostics}

- Special stains such as Alcian blue able to detect cytoplasmic mucins

- Immunohistochemistry. Mesothelial cells are immunoreactive to both cytokeratin and vimentin, but negative for CEA, S-100.

- Electron microscopy. Lining mesothelial cells may show numerous desmosomes and tight junctions, long slender microvilli on cell surface, and circumferential bundles of perinuclear intermediate microfilaments (tonofilaments).

Comments: In non-human primates, cardiovascular mesothelial hyperplasia may be seen as a background change associated with epicardial and/or pericardial inflammation, presence of fluid/exudate in the pericardial sac, therapeutic implants, aberrant helminth migration, protozoal infections (e.g. Chagas), and bacterial diseases involving pericardium or epicardium (e.g. tuberculosis). The change can also be associated with fibromatosis accompanying retrovirus infection. Vascular adventitial reactions in non-human primates may be seen accompanying atherosclerosis and arteritis ${ }^{11,63,88}$.

\section{B. Heart Valves}

Recent observation of drug-induced valvulopathy in postmarketing surveillance in man has led to withdrawal of some commercialized compounds, such as appetite suppressant drugs. This has increased the interest in the evaluation of heart valves during nonclinical studies.

Histopathological examination of the cardiac valves in all laboratory animal species, including non-human primates, may suffer from inconsistencies and artifacts related to both the technical approach and the inherent small size of the valves. The trimming/sectioning of the heart may greatly influence the type, number and correct orientation of valves within the histological sections, which finally affects the accuracy of the microscopic examination. Therefore, in those studies where drugrelated effects are suspected to occur in the cardiac valves, the pathologist may need to adapt the preparation of the heart and trimming of valves to ensure the best representation of most or all four cardiac valves.

Valvular thrombosis, inflammation and atherosclerosis can be seen in aging, hypercholesterolemic macaques. For more specific comments and diagnostic criteria on atherosclerotic lesions, please refer to the section on blood vessels (Table 3).

\section{Degeneration, myxomatous: Heart Valves}

(No image provided)

\section{Other term(s)}

Endocardial myxomatous change; spontaneous valvulopathy; valvular endocardiosis

\section{Pathogenesis/Cell of Origin}

The pathogenesis of degenerative valvular disease of cynomolgus monkeys is unknown. It likely involves activation of valvular interstitial cells leading to either increased production or decreased remodeling of interstitial matrix.

\section{Diagnostic features}

- Diffuse or nodular thickening of valve leaflets

- Expansion of the spongiosa of the valve leaflet with hypocellular extracellular matrix composed predominately of glycosaminoglycans

- Incidence increases with age

\section{Differential Diagnoses}

- Drug-induced valvulopathy:

- Has not definitively been recognized in cynomolgus monkeys used in nonclinical studies

- Valvular endocarditis:

- Would be distinguished by greater cellularity with infiltrates of inflammatory cells as well as frequently including hemorrhage and cellular necrosis

Comments: Spontaneous valvular disease is uncommon in cynomolgus monkeys in survey pathology studies 6 , although this is likely due to the young age of animals used in toxicity testing. Myxomatous valvular changes are known to be an age-associated lesion ${ }^{89}$. Mitral valve prolapse in human patients is the result of an age-related myxomatous degeneration of atrioventricular valves ${ }^{90}$. The valvular lesions

Table 3. Microscopic Findings of the Cardiovascular System: Heart Valves; Non-human Primate

\begin{tabular}{|c|c|c|c|c|}
\hline Heart Valve & Common & Uncommon & $\begin{array}{c}\text { Not Observed but } \\
\text { Potentially Relevant }\end{array}$ & Not Applicable \\
\hline \multicolumn{5}{|l|}{ Non-proliferative } \\
\hline Angiectasis & $\mathrm{x}$ & & & \\
\hline Degeneration, myxomatous *\# & & $\mathrm{x}$ & & \\
\hline Inflammation $^{\wedge}$ & & $\mathrm{x}$ & & \\
\hline \multicolumn{5}{|l|}{ Proliferative (non-neoplastic) } \\
\hline Proliferation, stroma & & $\mathrm{x}$ & & \\
\hline
\end{tabular}


that can result may allow systolic regurgitant blood flow (valvular insufficiency) and myocardial disease/remodeling from increased hemodynamic preload. Morphologically similar changes have been described as a spontaneous condition in atrioventricular valves of rhesus monkeys in a breeding colony ${ }^{91}$, although this is not a common finding and the etiology of the high incidence in this colony remained unknown.

\section{Blood Vessels}

The microscopic evaluation of the vasculature in organs is generally performed as part of the evaluation of each specific organ. This may reveal the presence of either organ-specific vascular changes (possibly as part of an organ-specific toxicity) or widespread vascular changes visible in several organs pointing toward a systemic vascular injury. Specific collection of larger vessels may be required in particular when intravascular administration of xenobiotics is performed (e.g. through bolus injection or slow infusion), with potential for local and/ or systemic vascular injury. Large arteries that can be easily collected in non-human primates include the thoracic and abdominal aorta, carotid arteries and iliac arteries. Some lesions occur in vessel-specific progression (e.g. atherosclerosis). Strategies for the systemic evaluation of the arterial tree have been published ${ }^{58}$. Vascular injury in non-human primates, as well as in other laboratory animal species, can occur as a spontaneous pathological change or be drug-induced. In nonclinical drug development, vascular injury can be caused by a wide array of compounds encompassing small and large molecules (such as monoclonal antibodies) and antisense oligonucleotides. Several papers have been published to better characterized drug-induced vascular injury and associated biomarkers in nonclinical studies $^{92-94}$. However, the distinction between the spontaneous and drug-induced vascular injury may still be challenging and a correct identification of the histological pattern of vascular changes may be crucial.

The observation of a vascular injury may reflect an organspecific toxicity or represent a widespread change visible in several organs and, therefore, suggesting the occurrence of a systemic vascular disorder.

The histopathological evaluation of the heart and the other organs according to standard guidelines for organ trimming is generally considered sufficient to ensure the thorough evaluation of the vascular tree (Table 4).

\section{Amyloid, media or wall: Blood Vessels (No image provided)}

Comments: Cynomolgus monkey are considered prone to develop systemic amyloidosis following chronic inflammatory processes. Several organs may be affected, most frequently the spleen and liver. An age-related endocrine amyloidosis has been described in the pancreas often associated with diabetes ${ }^{95}$.

It is seldom observed as a primary vascular lesion but typically occurs if there is amyloid deposition in the adjacent tissues. In such cases, it should not be recorded as a separate vascular-specific diagnosis.

\section{Atherosclerosis: Blood Vessels (Figure 52)}

\section{Other $\operatorname{term}(\mathbf{s})$}

Arteriosclerosis; fatty streak; intimal plaque

\section{Pathogenesis/Cell of Origin}

Atherosclerosis is a degenerative change that occurs along the tunica intima of large and small arteries and has been described in several non-human primate species including cynomolgus monkey, as well as in other animal species ${ }^{96}$. The pathogenesis is complex, is related to lipoprotein metabolism and includes progressive accumulation of lipids, lipoproteins and free cholesterol in the intima and media, with accumulation of macrophages and lymphocytes within and around the arterial wall. Normal vascular wall architecture can be disrupted and the intima, internal elastic lamina, media, and adventitia may be effaced by inflammatory cells and fibrosis. Necrosis may occur with accumulation of free cholesterol crystals and mineralization. Chronic lesions are progressively remodeled due to ongoing/progressive collagen accumulation, which may finally result in either stenosis or enlargement of the lumen6, 97 .

\section{Diagnostic features}

- Regions of intimal thickening that are composed of smooth muscle cells, mucopolysaccharies, and hypocellular collagen deposits. The mucopolysaccharides can predominate in some lesions conferring a loose appearance.

- Lesions are typically segmental. Early lesions may consist only of slight elevation of the intima by lipid-laden macrophages. Can partially or completely occlude the vessel lumen.

- No inflammatory component is generally noted.

\section{Differential Diagnosis}

- Intramural plaque:

- Fibrous response to indwelling catheter or at infusion sites within a blood vessel

\section{Special Techniques for Diagnostics}

- Plaques can be highlighted with a variety of immunohistochemical and histochemical stains.

- Verhoff-Van Gieson staining may be used to distinguish muscular, fibrous, and elastic elements of the lesion, and to identify disruption or splitting of the internal elastic lamina.

- The plaques can be highlighted with a Masson's trichrome as well as immunohistochemistry for smooth muscle actin.

Comments: Arteriosclerosis encompasses a spectrum of lesions in man, but most often refers to atherosclerosis. Arteriosclerosis in man is an age-related change in blood vessels which are characterized by thick and stiff wall and 
Table 4. Microscopic Findings of the Cardiovascular System: Blood Vessels; Non-human Primate

\begin{tabular}{|c|c|c|c|c|}
\hline Blood vessels & Common & Uncommon & $\begin{array}{c}\text { Not Observed but } \\
\text { Potentially Relevant }\end{array}$ & Not Applicable \\
\hline \multicolumn{5}{|l|}{ Non-proliferative } \\
\hline Amyloid, media or wall, artery $* \wedge$ & & $\mathrm{x}$ & & \\
\hline Aneurysm, artery or aorta & & $\mathrm{x}$ & & \\
\hline Angiectasis & & $\mathrm{x}$ & & \\
\hline Atherosclerosis * & & $\mathrm{x}$ & & \\
\hline Cytomegaly, endothelial cell *\# & & $\mathrm{x}$ & & \\
\hline Degeneration/necrosis, media or wall, artery *\# & $\mathrm{x}$ & & & \\
\hline Embolus (hair, foreign material) * & $\mathrm{x}$ & & & \\
\hline Fibrosis, perivascular/adventitial & $\mathrm{x}$ & & & \\
\hline Hemorrhage, medial or wall, artery ${ }^{\wedge}$ & $\mathrm{x}$ & & & \\
\hline Hypertrophy, endothelium & $\mathrm{x}$ & & & \\
\hline Hypertrophy, media or wall, artery & $\mathrm{x}$ & & & \\
\hline Infiltrate, [insert appropriate cell type], perivascular ${ }^{\wedge}$ & $\mathrm{x}$ & & & \\
\hline Inflammation, media or wall, artery ${ }^{\wedge}$ & $\mathrm{x}$ & & & \\
\hline Intimal thickening, acellular & & $\mathrm{x}$ & & \\
\hline Mineralization, media or wall, artery ${ }^{* \wedge}$ & & $\mathrm{x}$ & & \\
\hline Mucinous change, aorta * & $\mathrm{x}$ & & & \\
\hline Necrosis/inflammation, media or wall, artery $^{\wedge}$ & & $\mathrm{x}$ & & \\
\hline Parasite $^{\wedge}$ & & $\mathrm{x}$ & & \\
\hline Plaque, intramural ${ }^{\# 1}$ & & $\mathrm{x}$ & & \\
\hline SIV-related arteriopathy * & & & $\mathrm{x}$ & \\
\hline Thrombus & & $\mathrm{x}$ & & \\
\hline Vacuolation, media or adventitia, artery $^{\wedge}$ & & $\mathrm{x}$ & & \\
\hline \multicolumn{5}{|l|}{ Proliferative (non-neoplastic) } \\
\hline Hyperplasia, angiomatous & & $\mathrm{x}$ & & \\
\hline Hyperplasia, endothelial cell & & $\mathrm{x}$ & & \\
\hline Hyperplasia, hemangioendothelium & & $\mathrm{x}$ & & \\
\hline Proliferation, intimal, artery or vein & $\mathrm{x}$ & & & \\
\hline \multicolumn{5}{|l|}{ Neoplastic } \\
\hline Hemangioma * & & $\mathrm{x}$ & & \\
\hline Hemangiosarcoma & & $\mathrm{x}$ & & \\
\hline
\end{tabular}

luminal dilatation in large arteries. Atherosclerosis is a different pathological condition; however, these two terms are sometimes used interchangeably ${ }^{98}$. Atherosclerosis is primarily an arterial disorder and refers to the accumulation of fats, cholesterol and lipid-laden macrophages in the artery wall with formation of plaques, potentially restricting blood flow. In man, it is considered that the earliest change of atherosclerosis occurs in the endothelium resulting in endothelial dysfunction. This can result in increased permeability to lipids, macrophage migration with formation of foam cells, and infiltration by T-lymphocytes and platelets in the vessel wall. These early inflammatory changes are accompanied by accumulation of extra cellular matrix and stimulate smooth muscle cells in the vascular media which then migrate and proliferate ${ }^{99-101}$.

Fibrous intramural plaques are infrequent and while they have been referred to as "Other Terms" with arterioscle- rotic lesions, this term should not be used as a synonym for atherosclerotic lesions in the non-human primate. Intramural plaques can be induced at sites of chronic catheter implantation as well as infusion sites, and the term should be restricted to these observations.

It has been demonstrated that atherosclerosis in the coronary arteries can cause myocardial infarction in rhesus and cynomolgus monkeys fed an atherogenic diet (low-fiber, high-carbohydrate and high-fat diet) for more than one year ${ }^{102}$. Additionally, it appears that cynomolgus monkeys are more prone to develop atherosclerosis than rhesus macaques, and that the social status may play a role in females, with competitive female cynomolgus monkeys having less atherosclerosis than submissive females ${ }^{96}$. 


\section{Cytomegaly, endothelial cell: Blood Vessels (Figure 53)}

\section{Pathogenesis/Cell of Origin}

Infection by Cytomegaloviruses spp. of the family of the Betaherpesvirinae.

\section{Diagnostic features}

- Hypertrophy of endothelial cells, bulging in the vascular lumen,

- generally associated with vascular/perivascular inflammation.

- Large intranuclear Cowdry-type A inclusion bodies may be observed whereas smaller amphiphilic, intra-cytoplasmic inclusions are less frequent.

- In addition to endothelial cells, many other cell types (such as epithelial cells, cardiomyocytes etc) can be affected, particularly in the lungs, heart and kidneys. Intravascular cytomegalic cells can occasionally be seen.

\section{Differential Diagnoses}

- Other cytomegalic viruses (e.g. Adenovirus).

\section{Special Techniques for Diagnostics}

- Immunohistochemistry and in situ hybridization may be useful diagnostic tools, especially when inclusions are rare and cytomegaly is mild.

Comments: Cytomegaloviruses are highly host-specific viruses of human, non-human primates and other species. The sero-prevalence of simian cytomegaloviruses in adult monkeys is about $100 \%$ in most colonies. Infection is most commonly asymptomatic. Latent infections tend to persist in glandular tissue, lympho-reticular cells and kidneys, with periodic shedding in body secretions. Clinically apparent disease occurs in immunodeficient animals, after viral infection (simian immunodeficiency virus, retroviruses) or treatment with immunosuppressive agents ${ }^{95}$.

\section{Degeneration/necrosis, media or wall: Blood Vessels}

(Figure 54)

\section{Other term(s)}

Fibrinoid vascular necrosis; vasculitis; polyarteritis nodosa

\section{Pathogenesis/Cell of Origin}

Unknown.

\section{Diagnostic features}

- Segmental necrosis of the entire vascular media, accompanied by inflammatory cell infiltrates and collagen deposits which may disrupt the vascular wall architecture.

- Segmental or diffuse loss of cellular architecture and replacement of the vessel wall by bright eosinophilic fibrinoid material.

\section{Differential Diagnoses}

- Infectious vasculitides such as the hemorrhagic fevers (filoviruses and flaviviruses).

\section{Special Techniques for Diagnostics}

- PTAH staining may be used to demonstrate the presence of fibrin in the lesion

Comments: This lesion has been associated with simian immunodeficiency virus infection (SIV), but also occurs in monkeys with no history of SIV exposure. The cause remains unknown, and the relative significance of the lesion in the context of a study should be considered; low numbers of sporadic lesions can be considered a "background" lesion, whereas highly prevalent lesions would be unusual and would suggest a study-related cause.

Vascular inflammation characterized by prominent mural degeneration/necrosis can be induced by treatment with a large spectrum of molecules such as monoclonal antibodies and proteins causing formation of immune complex which deposit onto the endothelial surface triggering endothelial activation followed by chemotaxis of inflammatory cells. A similar morphological pattern can also be rarely observed with certain antisense oligonucleotides, as a consequence of drug-related complement activation (which is a monkeyspecific phenomenon), which triggers the inflammatory cascade.

Polyarteritis nodosa has been described in cynomolgus monkeys and can affect small- to medium-sized arteries in several organs (including kidney, gut, heart, spleen, mesentery, and others), showing varying severity and stage of development. The lesion is considered to progress from a segmental infiltration of the tunica adventitia and media by lymphocytes, macrophages, and few neutrophils and eosinophils. Intimal thickening due to accumulation of mucinous stroma can also occur. Some vessels may show marked intimal proliferation and only few inflammatory cells. In severe lesions, fibrinoid necrosis of the tunica media can be prominent and accompanied by loss of the internal elastic lamina and luminal occlusion due to thrombus formation. The mural inflammatory changes are generally associated with inflammatory cell infiltration in the tunica adventitia and surrounding tissues $6,59,77,103-105$.

\section{Embolus: Blood Vessels (No image provided)}

Comments: Foreign material and fragments of keratin or hair shaft can be observed in cynomolgus monkeys in continuous infusion studies within the inflammatory reaction surrounding the cannulated vessels and/or in distant vessels such as in the lungs, generally associated with granulomatous inflammation ${ }^{106,107 .}$ 
Mineralization, media or wall: Blood Vessels

(No image provided)

\section{Pathogenesis/Cell of Origin}

Unknown.

\section{Diagnostic features}

- Mineralization in the wall of the aorta or in the media layer of small- or medium-sized arteries and arterioles

\section{Special Techniques for Diagnostics}

- Intensely basophilic material in the H\&E stain which gives a positive reaction with von Kossa stain.

Comments: Mineralization of various degrees has been reported in the brain of cynomolgus monkeys with no evident age-relationship in incidence or severity and no macroscopic correlate. The lesions were observed in the medial medullary lamina dividing the globus pallidus into the medial and lateral segments, always bilaterally. No mineralization was reported in other regions of the brain. The lesions were characterized by the presence of globoid bodies with prominent concentric lamellar structures in the arteriolar and vein wall (type A) or fine granules in the media of small or mediumsized arteries (type B), appearing as dense deposits in the media of small- or medium-sized arteries and arterioles, at electron microscopy. X-ray analysis demonstrated the presence of calcium and other minerals. The pathogenesis was unclear, although there appeared that degenerative change in the vessel wall preceded the formation of mineralized deposits. These changes were not accompanied by any abnormalities in growth, weight gain, or neurologic signs ${ }^{108}$.

Mucinous change, aorta: Blood Vessels (Figure 55-56)

\section{Pathogenesis/Cell of Origin}

Unknown.

\section{Diagnostic features}

- Thickening of the wall of the aorta and/or small- or medium-sized arteries due to intimal accumulation of loose material with sparse fibroblast-like cells surrounded by eosinophilic extracellular substance.

- May accompany intimal fibrosis and atherosclerotic lesions.

\section{Special Techniques for Diagnostics}

- PAS and/or other stains specific for mucopolysaccharides may help in identifying the extracellular substance.

Comments: This can be a striking although infrequent change in cynomolgus monkeys used in nonclinical studies and can lead to local swelling of the affected blood vessel, especially in the aorta near the coronary sinus and/or the carotid arteries. Similar lesions can occur in the subendocardial regions of the atria (Figure 57-58) and ventricles as well as heart valves where it is often referred to as myxomatous degeneration ${ }^{6}$.

\section{SIV-related arteriopathy: Blood Vessels}

(No image provided)

\section{Pathogenesis/Cell of Origin}

Infection by Simian immunodeficiency virus (SIV).

\section{Diagnostic features}

- Intimal and medial thickening due to fibrosis characterized by collagen deposits, extracellular matrix, smooth muscle cells and macrophage infiltration.

- May be associated with luminal occlusion and/or thrombosis

- Fragmentation of the internal elastic laminae

- At early (absence of intimal thickening) and late stages, the endothelial cells may appear plump, vacuolated, and/ or detached from the subendothelial space.

- Pulmonary arteries seem to be preferentially affected; vessels in other organs appear less frequently affected.

\section{Differential Diagnoses}

- Atherosclerosis

- Mucinous change

Comments: This disease has been reported in rhesus and cynomolgus macaques ${ }^{109,110}$. Cases of systemic arteriopathy additionally affecting the intestine, pancreas, liver, heart, lymph nodes, spleen and testis have been described in SIVinfected rhesus macaques with concurrent Cytomegalovirus infection ${ }^{108}$.

Hemangioma: Blood Vessels (No image provided)

\section{Pathogenesis/Cell of Origin}

Unknown.

\section{Diagnostic features}

- Presence of multiple vascular lacunae filled by blood and lined by well-differentiated endothelial cells.

\section{Differential Diagnoses}

- hemangiosarcoma

Comments: A case of hemangioma was reported in the adrenal glands in an adult female cynomolgus monkey and appeared to dissect the parenchyma in the adrenal cortical region'.

Another case of vascular tumor has been reported in an adult rhesus macaque and was identified as an epithelioid hemangioendothelioma ${ }^{111}$. 


\section{Digestive System}

For detailed general considerations on the digestive system, please refer to the rodent publication ${ }^{112}$. The terminology regarding "vascular lesions" and "infectious diseases" are covered elsewhere (cardiovascular and systemic chapters).

\section{A. Upper Alimentary Tract \\ (Oral Cavity, Tongue, Pharynx, and Esophagus)}

Most commonly, microscopic lesions in the upper alimentary tract are observed in tissues that are required to be examined microscopically, based on regulatory guidelines and the laboratories preferences, e.g., esophagus, and tongue. In most instances, other lesions in the oral cavity and pharynx are examined only when gross lesion are recorded at necropsy (Table 5).

\section{Cyst, esophagus: Upper Alimentary Tract}

(No image provided)

\section{Other $\operatorname{term}(\mathbf{s})$}

Inclusion cyst; developmental cyst

\section{Pathogenesis/Cell of Origin}

Congenital lesion from abnormal primitive foregut tissue, whose remnants are transferred during the normal develop-

Table 5. Microscopic Findings of the Digestive System: Upper alimentary tract; Non-human Primate

\begin{tabular}{|c|c|c|c|c|}
\hline Upper Alimentary Tract & Common & Uncommon & $\begin{array}{c}\text { Not observed but } \\
\text { potentially relevant }\end{array}$ & Not Applicable \\
\hline \multicolumn{5}{|l|}{ Congenital } \\
\hline Cleft, palate & & $\mathrm{x}$ & & \\
\hline Cyst, esophagus * & & $\mathrm{x}$ & & \\
\hline Dilatation, esophagus & & $\mathrm{x}$ & & \\
\hline Diverticulum, esophagus & & $\mathrm{x}$ & & \\
\hline Ectopic tissue, sebaceous gland & & & $\mathrm{x}$ & \\
\hline Malformation & & & $\mathrm{x}$ & \\
\hline \multicolumn{5}{|l|}{ Non-proliferative } \\
\hline Amyloid ${ }^{\wedge}$ & & $\mathrm{x}$ & & \\
\hline Apoptosis, squamous epithelium \#^ & & $\mathrm{x}$ & & \\
\hline Apoptosis/single cell necrosis, squamous epithelium $\# \wedge$ & & $\mathrm{x}$ & & \\
\hline Atrophy, squamous epithelium & & $\mathrm{x}$ & & \\
\hline Cyst & & $\mathrm{x}$ & & \\
\hline Degeneration/necrosis, muscle & & $\mathrm{x}$ & & \\
\hline Edema $^{\wedge}$ & & $\mathrm{x}$ & & \\
\hline Erosion/ulcer \# & & $\mathrm{x}$ & & \\
\hline Hemorrhage $^{\wedge}$ & & $\mathrm{x}$ & & \\
\hline Hyperkeratosis & & $\mathrm{x}$ & & \\
\hline 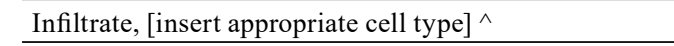 & $\mathrm{x}$ & & & \\
\hline Inflammation $^{\wedge}$ & & $\mathrm{x}$ & & \\
\hline Inflammation, foreign body & & $\mathrm{x}$ & & \\
\hline Inflammation, mixed cell & & $\mathrm{x}$ & & \\
\hline Inflammation, mononuclear cell & & $\mathrm{x}$ & & \\
\hline Inflammation, vessel & & $\mathrm{x}$ & & \\
\hline Metaplasia, sebaceous gland * & & $\mathrm{x}$ & & \\
\hline Mineralization $^{\wedge}$ & & $\mathrm{x}$ & & \\
\hline Necrosis, squamous epithelium \# & & $\mathrm{x}$ & & \\
\hline Necrosis/ulcer * & & $\mathrm{x}$ & & \\
\hline Pigment $^{\wedge}$ & & $\mathrm{x}$ & & \\
\hline \multicolumn{5}{|l|}{ Proliferative (non-neoplastic) } \\
\hline Hyperplasia, basal cell & & $\mathrm{x}$ & & \\
\hline Hyperplasia, squamous cell & & $\mathrm{x}$ & & \\
\hline Hyperplasia, gingiva *\# & & $\mathrm{x}$ & & \\
\hline \multicolumn{5}{|l|}{ Neoplastic } \\
\hline Leiomyoma & & $\mathrm{x}$ & & \\
\hline
\end{tabular}


mental process into other sites (tracheobronchial tree, oesophagus, stomach, intestine, mediastinum) ${ }^{113}$.

\section{Diagnostic features}

- Grossly, soft, whitish area with a smooth, glossy surface

- Cyst located within the submucosa of the esophagus with no communication with the esophageal lumen

- Cyst filled with serous fluid

- Cyst lined by a pseudostratified ciliated epithelium

\section{Metaplasia, sebaceous gland cell: Upper Alimentary Tract} (Figure 59)

\section{Other term(s)}

Fordyce's spots; Fordyce's granules

\section{Pathogenesis/Cell of Origin}

Sebaceous gland cell metaplasia most likely originates from pluripotential basal cells within the squamous esophageal (or buccal) epithelium ${ }^{114}$.

\section{Diagnostic features}

- Presence of nests of cells, exhibiting characteristic sebaceous cell differentiation, within the intra-epithelial cell-nests mucosal epithelium, primarily located at the stratum basale, showing characteristic sebaceous cell differentiation.Within the cell-nest, differentiation from basally oriented seboblasts to centrally orientated, mature sebocytes with finely cytoplasmic vacuolation and a centrally placed nucleus is evident.

- Individual or small clusters of mature, highly vacuolated sebocytes can be found ascending within the overlying normal stratified squamous, non-keratinized epithelium.

- An excretory duct is lacking.

Comments: Sebaceous gland cell metaplasia within the esophageal mucosa, which is of endodermal origin is an extremely rare finding in man and monkey. In contrast, in man, sebaceous gland metaplasia is very commonly found in organs of ectodermal origin, such as the inner surface of the lips and buccal mucosa. This however seems not the case in monkeys ${ }^{115}$. The term ectopic sebaceous glands described in the rodents as a congenital finding is not considered appropriate in these specific sites (buccal and esophageal mucosa), since this observation appears to be acquired in primates, and is not observed in younger animals.

\section{Necrosis/ulcer: Upper Alimentary Tract}

(No image provided)

\section{Other $\operatorname{term}(\mathrm{s})$}

Necrotizing and ulcerative gingivitis and stomatitis (Noma)

\section{Pathogenesis/Cell of Origin}

Localized disruption of the squamous epithelium in the oral cavity with full penetration of the squamous epithelium (ulcer) associated with coagulative necrosis and mixed bacteria.

Ulceration is usually is the result of epithelial necrosis.

\section{Diagnostic features}

- Focal or locally extensive loss of surface epithelium in the oral cavity

- Homogenous amorphous eosinophilic and karyorrhectic debris (necrosis)

- Acute/chronic inflammatory response (neutrophilic or lymphocytic-plasmacytic inflammatory cell infiltrate)

- Hemorrhage may be seen with larger ulcers

\section{Differential diagnoses}

- Squamous cell carcinoma:

- May present with erosion/ulcer on the mucosal surface. The presence of this neoplasm would preclude diagnosis of an associated erosion/ulcer.

- Atrophy, epithelial:

- Reduced thickness of all layers of the squamous epithelium or, in severe cases, absence of basal germinal layers; usually no inflammatory cell infiltrate.

- Erosion/ulcer:

- Focal or focally extensive absence of superficial or, in severe cases, of all epithelial layers with extension of the alteration into the muscularis.

Comments: Noma is a rapidly developing, necrotizing gingivitis and stomatitis which frequently progresses to include both underlying bone and overlying skin. Noma is microscopically characterized by mucosal ulcer covered by necrotic debris and mixed bacteria. Intense neutrophilic infiltration, mixed bacteria, spirochetes and vascular thrombi are present in areas of necrosis. In more severe cases, there is osteonecrosis ${ }^{116}$.

The precise etiology of this lesion remains unclear, but a variety of microorganisms are isolated from lesions (Fusobacterium necrophorum, Sighella flexneri, Spirochetal organisms, Streptococcus spp., Staphylococcus aureus) $)^{117,118 .}$ The presence of noma in a macaque colony is strongly suggestive of endemic type D retrovirus infection, though severe malnutrition should be considered a possible cause.

\section{Hyperplasia, gingiva: Upper Alimentary Tract}

(No image provided)

\section{Other $\operatorname{term}(\mathbf{s})$}

Gingival fibromatosis; fibrous gingival hyperplasia; gingival overgrowth

\section{Pathogenesis/Cell of Origin}

Gingival hyperplasia is a benign proliferation of gingival tissue. The etiology is unknown, but gingival hyperplasia may be a sequela to chronic gingivitis. There is a possibility that it is familial in macaques ${ }^{117}$.

In both humans and non-human primates, this condition has also been associated with the use of some anticonvulsants such 
as diphenylhydantoin.

\section{Diagnostic features}

- Grossly, bright red/pink tissue, with granular surface

- Fusiform-shaped fibroblasts admixed with collagen

- Squamous epithelium is extended into rete pegs

- Inflammatory cells may be present in the submucosa but are rarely associated with the fibrous tissue

\section{Differential Diagnoses}

- Hyperplasia, squamous cell:

- Proliferation and thickening of the stratum spinosum

- Fibrosis

- Poorly cellular fibrous tissue containing few fibroblasts; very little if any leukocyte infiltration.

- Fibroplasia

- Cellular, containing numerous active fibroblasts which although may be plump, show no atypical features.

Comments: There are usually no clinical signs. There is mild to marked firm enlargement of the marginal and alveolar gingiva, including the interdental papillae. The hyperplastic tissue may completely cover the teeth.

\section{B. Stomach}

Unlike the rodent, the stomach of the cynomolgus monkey consists of glandular mucosa only and is devoid of a nonglandular mucosa; therefore, the terminology for the nonglandular stomach of rodents is not applicable for the NHP and was omitted.

The cynomolgus monkey has a simple stomach comprising cardiac, fundic and pyloric glandular regions. To assure a thorough examination of the stomach, examination of representative sections of all regions is mandatory. Vidal et al. ${ }^{119}$ developed a reliable method for routine sampling of the stomach for microscopic evaluation in nonclinical safety studies, allowing for correlation of the gross subdivisions (fundus, cardia, body, and antrum) with the histologic distribution of parietal, chief, and mucus cells.

The cynomolgus monkey has a prominent fundus that extends to the level of the gastroesophageal junction. Macroscopically, the fundus can be distinguished from the body, as the fundus is a lighter shade of tan. The fundus of the cynomolgus monkey has prominent gastric pits, lacks parietal cells, and has extensive mucous-secreting cells. The body of the cynomolgus monkey is histologically similar to that reported in the human, and although similar to the described "fundic mucosa", care must be taken in using this term, as the mucosa of the true fundus in cynomolgus monkeys is distinct. The proximal portions of body contained more chief cells than the distal portions, as has been reported in the human, dog, and cat. The cardia was similar to that of humans in size and histology. The antrum was similar in size and consistent in histologic appearance with that reported in other $\operatorname{species}^{119}$ (Table 6).
Infiltrate, [insert appropriate cell type]: Stomach

(No image provided)

\section{Diagnostic features}

- High incidence in Cynomolgus monkeys

- Commonly observed as lymphoplasmacytic infiltrates in the lamina propria

- In fundus and antrum

- Occasionally accompanied by atrophy of epithelial cells and dilation of gastric glands, but without the presence of ulceration and hemorrhage.

Comments: The cause of lymphoplasmacytic infiltrations in cynomolgus monkey stomachs is not known and it is possible that environmental antigens such as food antigens may cause an immunological reaction within the lamina propria of the stomach. One of the proposed causes is thought to be Helicobacter spp. Infection $61,120-122$. Lymphoplasmacytic infiltrates, even without additional features characteristic of inflammation (e.g. necrosis, ulceration), has sometimes been referred to as inflammation or "gastritis". This approach is not recommended and the term infiltrate, lymphoplasmacytic is preferred. The presence of inflammatory cell infiltrates in the absence of other features of inflammation should be diagnosed as an 'infiltrate' with predominate/ appropriate cell type(s) identified. Only if other prominent morphologic features (congestion, hyperemia, hemorrhage, edema, necrosis, fibrosis, foreign material, bacterial organisms, etc.) are present, it is preferable to characterize the change as an inflammatory process (inflammation) with specific descriptors.

Hyperplasia, polypoid: Stomach (No image provided)

\section{Other $\operatorname{term}(\mathbf{s})$}

Polyp; adenomatous polyp; gastric polyposis

\section{Diagnostic features}

- Circumscribed areas of gastric hyperplasia with prominent stroma

- Well-differentiated tall, columnar, mucus-secreting cells perpendicular to papillary projections of stroma

- Stroma can be branching and radiate from the underlying submucosa

- Deep, mucus-filled crypts present between adjacent papillary projections

- Most often seen associated with presence of nematode infection ( $P$. tumefaciens)

\section{Differential Diagnoses}

- Adenoma

- Nodular lesions growing exophytic (polypoid, papillary); distortion of glandular architecture and in case of non-papillary adenomas also compression of surrounding tissue.

Comments: This finding is extremely rarely observed as 
Table 6. Microscopic Findings of the Digestive System: Stomach; Non-human Primate

\begin{tabular}{|c|c|c|c|c|}
\hline Stomach & Common & Uncommon & $\begin{array}{c}\text { Not observed but } \\
\text { potentially relevant }\end{array}$ & Not Applicable \\
\hline \multicolumn{5}{|l|}{ Congenital } \\
\hline Cyst, squamous & & $\mathrm{x}$ & & \\
\hline Ectopic tissue $^{\wedge}$ & & $\mathrm{x}$ & & \\
\hline \multicolumn{5}{|l|}{ Non-proliferative } \\
\hline Amyloid ^ & & $\mathrm{x}$ & & \\
\hline Apoptosis $\wedge^{\#}$ & & $\mathrm{x}$ & & \\
\hline Apoptosis/single cell necrosis, mucosa ${ }^{\wedge \#}$ & & $\mathrm{x}$ & & \\
\hline Atrophy \# & & $\mathrm{x}$ & & \\
\hline Cyst, glandular & $\mathrm{x}$ & & & \\
\hline Dilatation, gland & & $\mathrm{x}$ & & \\
\hline Edema $^{\wedge}$ & & $\mathrm{x}$ & & \\
\hline Eosinophilic globules & & & & $\mathrm{x}$ \\
\hline Erosion/ulcer \# & & $\mathrm{x}$ & & \\
\hline Hemorrhage $^{\wedge}$ & & $\mathrm{x}$ & & \\
\hline Hypertrophy, mucous cell & & $\mathrm{x}$ & & \\
\hline Infiltrate, [insert appropriate cell type] ${ }^{* \wedge}$ & $\mathrm{x}$ & & & \\
\hline Inflammation $^{\wedge}$ & & $\mathrm{x}$ & & \\
\hline Mineralization $^{\wedge}$ & $\mathrm{x}$ & & & \\
\hline Necrosis $\wedge \#$ & & $\mathrm{X}$ & & \\
\hline Necrosis, mucosa ${ }^{\wedge}$ & & $\mathrm{x}$ & & \\
\hline Secretion, decreased & & $\mathrm{x}$ & & \\
\hline Vacuolation, epithelium \# & & $\mathrm{x}$ & & \\
\hline \multicolumn{5}{|l|}{ Proliferative (non-neoplastic) } \\
\hline Diverticulum & & $\mathrm{x}$ & & \\
\hline Hyperplasia, mucosa, focal & & $\mathrm{x}$ & & \\
\hline Hyperplasia, mucosa, diffuse & & $\mathrm{x}$ & & \\
\hline Hyperplasia, neuroendocrine cell & & $\mathrm{x}$ & & \\
\hline Hyperplasia, polypoid * & & $\mathrm{x}$ & & \\
\hline
\end{tabular}

a spontaneous observation in the absence of nematode infestation. A spontaneous case can be differentiated by the absence of any parasites or ova123.

\section{Small and Large Intestine (Duodenum, Jejunum, Ileum, Cecum, Colon, Rectum) (Table 7)}

\section{Herniation, gland: Small and Large Intestine}

(No image provided)

\section{Other term(s)}

Glandular herniation; glandular micro-herniation; crypt herniation

\section{Pathogenesis/Cell of Origin}

Herniation is thought to result from a defect in the overlying muscularis mucosa, causing the glands to protrude through the gap into the underlying GALT in the sub mucosa. The increased intraluminal pressure, as part of normal peristalsis is thought to be a contributing factor to the herniation of the mu- cosal glands ${ }^{117}$.

\section{Diagnostic features}

- Extension of crypts through muscularis mucosa, into the submucosa

- Basement membrane integrity always maintained

- Occurs mainly in the colon, but can be seen also in small intestine and the stomach

- Glands are frequently observed within in the gut-associated lymphoid tissue (GALT)

- Often accompanied by inflammation and regenerative/ reparative processes.

\section{Differential Diagnosis}

- Diverticulum, diverticulosis:

- Diverticula are blind, mucosal lined pouches of the alimentary tract that communicate with the lumen. Usually they occur in the colon. They may be congenital or acquired. Congenital diverticula contain all layers of the intestinal wall, while acquired diverticula either 
Table 7. Microscopic Findings of the Digestive System: Small and Large Intestine; Non-human Primate

\begin{tabular}{|c|c|c|c|c|}
\hline Small and Large Intestines & Common & Uncommon & $\begin{array}{c}\text { Not observed but } \\
\text { potentially relevant }\end{array}$ & Not Applicable \\
\hline \multicolumn{5}{|l|}{ Congenital } \\
\hline Cyst, squamous & & & $\mathrm{x}$ & \\
\hline Ectopic tissue $^{\wedge}$ & & $\mathrm{x}$ & & \\
\hline \multicolumn{5}{|l|}{ Non-proliferative } \\
\hline Amyloid & & $\mathrm{x}$ & & \\
\hline Apoptosis $^{1}$ & & $\mathrm{x}$ & & \\
\hline Apoptosis/single cell necrosis, mucosa \# & & $\mathrm{x}$ & & \\
\hline Atrophy & & $\mathrm{x}$ & & \\
\hline Atrophy, Brunner's gland & & $\mathrm{x}$ & & \\
\hline Congestion $^{\wedge}$ & $\mathrm{x}$ & & & \\
\hline Degeneration/necrosis & & $\mathrm{x}$ & & \\
\hline Degeneration/necrosis, Brunner's glands & & $\mathrm{x}$ & & \\
\hline Degeneration, neuron, myenteric plexus & & & $\mathrm{x}$ & \\
\hline Dilatation & & $\mathrm{x}$ & & \\
\hline Edema $^{\wedge}$ & & $\mathrm{x}$ & & \\
\hline Erosion/ulcer \# & & $\mathrm{x}$ & & \\
\hline Hemorrhage $^{\wedge}$ & $\mathrm{x}$ & & & \\
\hline Herniation, gland * & & $\mathrm{x}$ & & \\
\hline Hypertrophy & & $\mathrm{x}$ & & \\
\hline Hypertrophy, Paneth cell & & $\mathrm{x}$ & & \\
\hline Increased karyorrhectic debris, villi ${ }^{1}$ & $\mathrm{x}$ & & & \\
\hline Infiltrate, [insert appropriate cell type $]^{\wedge}$ & $\mathrm{x}$ & & & \\
\hline Inflammation $^{\wedge}$ & $\mathrm{x}$ & & & \\
\hline Intussusception & & $\mathrm{x}$ & & \\
\hline Lymphangiectasis & & $\mathrm{x}$ & & \\
\hline Metaplasia, osseous ^ & & & $\mathrm{x}$ & \\
\hline Metaplasia, Paneth cell & & $\mathrm{x}$ & & \\
\hline Mineralization $^{\wedge}$ & & $\mathrm{x}$ & & \\
\hline Necrosis, mucosa \# & & $\mathrm{x}$ & & \\
\hline Parasite $^{\wedge}$ & $\mathrm{x}$ & & & \\
\hline Parasite granuloma $^{\wedge}$ & $\mathrm{x}$ & & & \\
\hline Pigment $^{\wedge}$ & $\mathrm{x}$ & & & \\
\hline Paneth cell, decreased & & $\mathrm{x}$ & & \\
\hline Syncytia, epithelium & & $\mathrm{x}$ & & \\
\hline Vacuolation, mucosa & & $\mathrm{x}$ & & \\
\hline \multicolumn{5}{|l|}{ Proliferative (non-neoplastic) } \\
\hline Diverticulum & & $\mathrm{x}$ & & \\
\hline Hyperplasia, mucosa & & $\mathrm{x}$ & & \\
\hline Hyperplasia, Brunner's glands & & $\mathrm{x}$ & & \\
\hline Hyperplasia, follicle-associated epithelium *^ & $\mathrm{x}$ & & & \\
\hline Metaplasia, squamous cell & & & $\mathrm{x}$ & \\
\hline \multicolumn{5}{|l|}{ Neoplastic } \\
\hline Adenocarcinoma & & $\mathrm{x}$ & & \\
\hline
\end{tabular}


lack or have an attenuated muscularis propria. Colonic diverticula generally occur alongside the taenia coli. Hypertrophy of the colonic musculature is commonly observed.

- The mechanism of diverticulum development is unknown but two factors appear to be involved: an increase in intraluminal pressure and areas of relative weakness in the colonic wall. Contributing factors are low fiber diet and old age.

Comments: Micro-hernias of colonic mucosal glands through the muscularis mucosae are common in both humans and nonhuman primates ${ }^{124}$. A retrospective study of Chamanza et al. ${ }^{6}$ indicated that the incidence of glandular herniation was of $1-1.5 \%$.

Micro-herniations play an important role in the spread of pathogens from the lamina propria to the submucosa by allowing the infective agents (e.g. Balantidium coli or bacteria) to pass through the muscularis mucosae. Parasites and fecal matter trapped within these herniation or diverticula can incite a chronic inflammatory cell reaction with foreign-body giant cells.

Complications include hemorrhage, ulceration, inflammation, abscess formation, perforation, fistulas, stricture formation, and phlebitis.

\section{Salivary Glands}

The major salivary glands are examples of exocrine glands and include the parotid, submandibular and sublingual glands. The parotid, submandibular, and sublingual glands are all branched, tubulo-alveolar (acinar) glands with a merocrine type of secretion. The secretory product is either serous (parotid) or mucous (sublingual gland) or both (submandibular gland).

All major salivary glands should be sampled for routine toxicological examination (Table 8).

Table 8. Microscopic Findings of the Digestive System: Salivary glands; Non-human Primate

\begin{tabular}{|c|c|c|c|c|}
\hline Salivary Glands & Common & Uncommon & $\begin{array}{c}\text { Not observed but } \\
\text { potentially relevant }\end{array}$ & Not Applicable \\
\hline \multicolumn{5}{|l|}{ Congenital } \\
\hline Ectopic tissue $^{\wedge}$ & & $\mathrm{x}$ & & \\
\hline \multicolumn{5}{|l|}{ Non-proliferative } \\
\hline Accumulation, adipocytes ${ }^{\wedge}$ & & $\mathrm{x}$ & & \\
\hline Amyloid ^ & & $\mathrm{x}$ & & \\
\hline Apoptosis ^\# & & $\mathrm{x}$ & & \\
\hline Apoptosis/single cell necrosis ${ }^{\wedge} \#$ & & $\mathrm{x}$ & & \\
\hline Atrophy & & $\mathrm{x}$ & & \\
\hline Calculus, duct & $\mathrm{x}$ & & & \\
\hline Ectasia, duct & & $\mathrm{x}$ & & \\
\hline Edema $^{\wedge}$ & & $\mathrm{x}$ & & \\
\hline 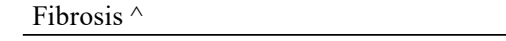 & & $\mathrm{x}$ & & \\
\hline Focus, hypertrophic, basophilic & & & & $\mathrm{x}$ \\
\hline Granules increased, granular duct & & & & $\mathrm{x}$ \\
\hline Hemorrhage $^{\wedge}$ & & $\mathrm{x}$ & & \\
\hline Hypertrophy, acinar cell & & $\mathrm{x}$ & & \\
\hline Infiltrate, [insert appropriate cell type] ${ }^{\wedge}$ & $\mathrm{x}$ & & & \\
\hline Inflammation $^{\wedge}$ & & $\mathrm{x}$ & & \\
\hline Metaplasia, acinar cell & & $\mathrm{x}$ & & \\
\hline Metaplasia, squamous cell & & $\mathrm{x}$ & & \\
\hline 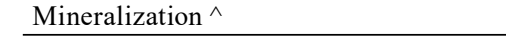 & & $\mathrm{x}$ & & \\
\hline Necrosis & & $\mathrm{x}$ & & \\
\hline Pigment $^{\wedge}$ & & $\mathrm{x}$ & & \\
\hline Secretion decreased, acinar cell ${ }^{1}$ & & $\mathrm{x}$ & & \\
\hline Secretion decreased, granular duct & & & & $\mathrm{x}$ \\
\hline Vacuolation & & $\mathrm{x}$ & & \\
\hline \multicolumn{5}{|l|}{ Proliferative (non-neoplastic) } \\
\hline Hyperplasia & & $\mathrm{x}$ & & \\
\hline
\end{tabular}




\section{E. Exocrine Pancreas (Table 9)}

Hyperplasia, acinar cell: Exocrine Pancreas (No image provided)

Comments: Fujisawa et al. ${ }^{125}$ describe this observation in cynolgous macaques.

\section{Hyperplasia, ductal cell: Exocrine Pancreas (Figure 60)}

\section{Pathogenesis/Cell of Origin}

Non-neoplastic proliferative lesion associated with micro- sporidian parasites (Enterocytozoan bieneusi-like) in immunosuppressed rhesus macaques. The lesion is noted in association with hepatobiliary infection or inflammation ("cholangiohepatitis", "cholecystitis", or "choledochitis")126.

\section{Diagnostic features}

- Grossly, thickened, firm, dilated pancreatic ducts

- Proliferation of pancreatic duct epithelial cells forming papilliferous lumenal projections

- Circumscribed by mild to moderate periductular fibrosis

- Can be associated with mild non-suppurative inflammation

Table 9. Microscopic Findings of the Digestive System: Exocrine Pancreas; Non-human Primate

\begin{tabular}{|c|c|c|c|c|}
\hline Pancreas & Common & Uncommon & $\begin{array}{l}\text { Not Observed but Po- } \\
\text { tentially Relevant }\end{array}$ & Not Applicable \\
\hline \multicolumn{5}{|l|}{ Congenital } \\
\hline Ectopic tissue $^{\wedge}$ & & $\mathrm{x}$ & & \\
\hline \multicolumn{5}{|l|}{ Non-proliferative } \\
\hline 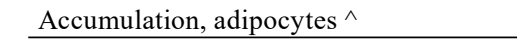 & & $\mathrm{x}$ & & \\
\hline Amyloid ^ $^{\wedge}$ & & $\mathrm{x}$ & & \\
\hline Apoptosis $\wedge$ & & $\mathrm{x}$ & & \\
\hline Apoptosis/single cell necrosis ${ }^{\wedge} \#$ & & $\mathrm{x}$ & & \\
\hline Atrophy, acinar cell & & $\mathrm{x}$ & & \\
\hline Autophagic vacuoles, acinar cell & & $\mathrm{x}$ & & \\
\hline Ectasia, duct & & $\mathrm{x}$ & & \\
\hline Edema $^{\wedge}$ & & $\mathrm{x}$ & & \\
\hline Eosinophilic globules & & $\mathrm{x}$ & & \\
\hline Fibrosis $^{\wedge}$ & & $\mathrm{x}$ & & \\
\hline Focus, basophilic & & $\mathrm{x}$ & & \\
\hline Halos, peri-insular, decreased & & & & $\mathrm{x}$ \\
\hline Halos, peri-insular, increased & & & & $\mathrm{x}$ \\
\hline Hemorrhage $^{\wedge}$ & & $\mathrm{x}$ & & \\
\hline Hypertrophy, acinar cell & & $\mathrm{x}$ & & \\
\hline 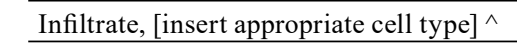 & & $\mathrm{x}$ & & \\
\hline Inflammation $^{\wedge}$ & & $\mathrm{x}$ & & \\
\hline Inflammation, vessel & & $\mathrm{x}$ & & \\
\hline Metaplasia, ductule & & $\mathrm{x}$ & & \\
\hline Metaplasia, hepatocyte & & & $\mathrm{x}$ & \\
\hline Mineralization $^{\wedge}$ & & $\mathrm{x}$ & & \\
\hline Necrosis & & $\mathrm{x}$ & & \\
\hline Pigment $^{\wedge}$ & & $\mathrm{x}$ & & \\
\hline Secretion decreased, acinar cell ${ }^{1}$ & & $\mathrm{x}$ & & \\
\hline Single cell necrosis & & $\mathrm{x}$ & & \\
\hline Vacuolation, acinar cell & & $\mathrm{x}$ & & \\
\hline \multicolumn{5}{|l|}{ Proliferative (non-neoplastic) } \\
\hline Hyperplasia, acinar cell * & & $\mathrm{x}$ & & \\
\hline Hyperplasia, ductal cell $*$ & & $\mathrm{x}$ & & \\
\hline \multicolumn{5}{|l|}{ Neoplastic } \\
\hline Adenoma, acinar cell & & $\mathrm{x}$ & & \\
\hline Adenoma, ductal cell & & $\mathrm{x}$ & & \\
\hline Adenocarcinoma, acinar cell & & $\mathrm{x}$ & & \\
\hline Adenocarcinoma, ductal cell & & $\mathrm{x}$ & & \\
\hline
\end{tabular}




\section{ENDOCRINE SYSTEM}

For detailed general considerations on the endocrine system, refer to the INHAND rodent publication ${ }^{127}$.

\section{A. Pituitary Gland}

The pituitary gland is structurally and functionally the most complex of the endocrine glands and exhibits a typical mammalian morphology in the non-human primate (Table 10).

\section{Infiltrate, [insert appropriate cell type]: Pituitary Gland}

(No image provided)

\section{Pathogenesis/Cell of Origin}

Mononuclear cell infiltrates can be seen in many tissues in non-human primates including pituitary.

Comments: Mononuclear cell infiltrates (lymphocytes, plasma cells, macrophages) are not uncommon in the pituitary in cynomolgus monkeys in toxicity studies. They are generally seen focally or multifocally in the pars intermedia or pars nervos $a^{11}$. As for other locations in the body, their incidence and severity can be increased compared to controls after administration of immunostimulating compounds such as proinflammatory oligonucleotides ${ }^{104}$.

\section{Adenoma, pars distalis: Pituitary Gland}

(No image provided)

Comments: Remick et al. ${ }^{128}$ have described the spontaneous occurrence of pituitary adenoma observed in cynomlogus macaques.

\section{B. Pineal Gland}

The pineal gland in the primate is located on the posterior wall of the third ventricle, between the hemispheres, rostral to the cranial colliculi (Table 11).

\section{Thyroid and Parathyroid Glands}

The thyroid consists of two lobes connected by a narrow isthmus, below and anterior to the larynx. The C-cells of the cynomolgus monkey are less apparent than in other species, and focal accumulations of $\mathrm{C}$ cells are rare ${ }^{11}$.

The primate usually has two pairs of parathyroids, em-

Table 10. Microscopic Findings of the Endocrine System: Pituitary gland; Non-human primate

\begin{tabular}{|c|c|c|c|c|}
\hline Pituitary Gland & Common & Uncommon & $\begin{array}{c}\text { Not observed but } \\
\text { potentially relevant }\end{array}$ & Not applicable \\
\hline \multicolumn{5}{|l|}{ Congenital } \\
\hline Aplasia/Hypoplasia & & $\mathrm{x}$ & & \\
\hline Craniopharyngeal structures, aberrant & & $\mathrm{x}$ & & \\
\hline Persistent Rathke's pouch & $\mathrm{x}$ & & & \\
\hline \multicolumn{5}{|l|}{ Non-proliferative } \\
\hline Angiectasis & & $\mathrm{x}$ & & \\
\hline Atrophy & & $\mathrm{x}$ & & \\
\hline Cyst & $\mathrm{x}$ & & & \\
\hline Fibrosis $\wedge^{\wedge}$ & & $\mathrm{x}$ & & \\
\hline Gliosis, pars nervosa & & & $\mathrm{x}$ & \\
\hline Hemorrhage $^{\wedge}$ & & $\mathrm{x}$ & & \\
\hline Hypertrophy, pars distalis & & $\mathrm{x}$ & & \\
\hline Hypertrophy, pars intermedia & & & $\mathrm{x}$ & \\
\hline Infiltrate, [insert appropriate cell type] ${ }^{* \wedge}$ & & $\mathrm{x}$ & & \\
\hline Inflammation $^{\wedge}$ & & $\mathrm{x}$ & & \\
\hline Metaplasia, osseous ${ }^{\wedge}$ & & $\mathrm{x}$ & & \\
\hline Pigment $^{\wedge}$ & & $\mathrm{x}$ & & \\
\hline Pseudocyst & $\mathrm{x}$ & & & \\
\hline Thrombus & & $\mathrm{x}$ & & \\
\hline Vacuolation \# & & $\mathrm{x}$ & & \\
\hline \multicolumn{5}{|l|}{ Proliferative (non-neoplastic) } \\
\hline Hyperplasia, pars distalis & & $\mathrm{x}$ & & \\
\hline Hyperplasia, pars intermedia & & $\mathrm{x}$ & & \\
\hline \multicolumn{5}{|l|}{ Neoplastic } \\
\hline Adenoma, pars distalis * & & $\mathrm{x}$ & & \\
\hline
\end{tabular}

* Terminology with diagnostic criteria or comments described below. \# Findings more frequently observed as an induced change.

$\wedge$ Terminology addressed in the Systemic/General Pathology section. 
Table 11. Microscopic Findings of the Endocrine System: Pineal gland; Non-human primate

\begin{tabular}{|c|c|c|c|c|}
\hline Pineal Gland & Common & Uncommon & $\begin{array}{c}\text { Not observed but } \\
\text { potentially relevant }\end{array}$ & Not applicable \\
\hline \multicolumn{5}{|l|}{ Congenital } \\
\hline Striated muscle fibers & & & $\mathrm{x}$ & \\
\hline \multicolumn{5}{|l|}{ Non-proliferative } \\
\hline Fibrosis $^{\wedge}$ & & $\mathrm{x}$ & & \\
\hline Infiltrate, [insert appropriate cell type $]^{\wedge}$ & & $\mathrm{x}$ & & \\
\hline Mineralization $^{\wedge}$ & & $\mathrm{x}$ & & \\
\hline Vacuolation & & $\mathrm{x}$ & & \\
\hline
\end{tabular}

bedded in the posterior connective tissue of the thyroid. The parathyroid in non-human primates is composed of small chief cells and larger oxyphil cells. The chief cell is the predominant cell type. Oxyphil cells are larger cells with granular eosinophilic cytoplasm, containing abundant mitochondria, but no secretory granules. Small numbers may be seen throughout the parathyroid gland, and their incidence increases with age. The function of the oxyphil cell is yet to be determined (Table 12, $13)$.

\section{Ectopic tissue, salivary gland: Thyroid / Parathyroid Gland (Figure 61)}

\section{Pathogenesis/Cell of Origin}

Congenital foci of ectopic salivary gland

\section{Diagnostic features}

- Foci of salivary gland acini and ducts in the thyroid or parathyroid gland.

Comments: Ectopic salivary gland in the thyroid or parathyroid gland is rare in monkeys ${ }^{11}$. In humans ectopic salivary gland is reported in the oropharyngeal region including thyroid and parathyroid gland.

\section{Accumulation, adipocytes: Thyroid / Parathyroid Gland} (Figure 62)

\section{Other term(s)}

Fatty infiltration; lipomatosis of the thyroid gland

\section{Pathogenesis/Cell of Origin}

Unknown/Mature adipocytes

\section{Diagnostic features}

- Focal or diffuse adipocyte infiltration of the interstitium of thyroid or parathyroid gland.

Comments: Infiltration of adipocytes in the thyroid or parathyroid gland is rare ${ }^{11}$ and is also described in human.
Colloid alteration: Thyroid / Parathyroid Gland (No image provided)

Comments: Colloid alteration characterized by granular or clumped colloid with mineralized material is also rarely observed in cynomolgus monkey. Variations in the staining characteristics of the colloid can be seen in the absence of other morphological changes and are considered a background change and not a finding to be reported.

\section{Degeneration, vacuolar: Thyroid / Parathyroid Gland} (Figure 63)

\section{Other $\operatorname{term}(\mathbf{s})$}

Hydropic degeneration of follicular cells; vacuolar change

\section{Pathogenesis/Cell of Origin}

Degenerative change

\section{Diagnostic features}

- Thyroid gland without macroscopic changes

- Enlarged follicular cells.

- Apically displaced nuclei.

- Large intracellular vacuole with homogeneous eosinophilic content located at the base of follicular epithelial cells.

\section{Differential Diagnosis}

- Hypertrophy, follicular cells

- The nucleus is not apically displaced.

- No vacuoles at the base of the follicular epithelial cells.

Comments: A lesion with similar morphologic features is observed in in dwarf and normal-sized Hannover Wistar GALAS rats and diagnosed as thyroid dysplasia ${ }^{129}$.

By electron microscopy, the rough endoplasmic reticulum was dilated ${ }^{130}$, which displaced the normally basally located nucleus to an apical position. 
Table 12. Microscopic Findings of the Endocrine System: Thyroid gland; Non-human primate

\begin{tabular}{|c|c|c|c|c|}
\hline Thyroid Gland & Common & Uncommon & $\begin{array}{c}\text { Not observed but } \\
\text { potentially relevant }\end{array}$ & Not applicable \\
\hline \multicolumn{5}{|l|}{ Congenital } \\
\hline Ectopic tissue, salivary gland $*$ & & $\mathrm{x}$ & & \\
\hline Ectopic tissue, thymus & $\mathrm{x}$ & & & \\
\hline Ectopic tissue, thyroid & & $\mathrm{x}$ & & \\
\hline Persistent thyroglossal duct & & $\mathrm{x}$ & & \\
\hline Cyst, ultimobranchial & $\mathrm{x}$ & & & \\
\hline Thyroid dysplasia & & & & $\mathrm{x}$ \\
\hline \multicolumn{5}{|l|}{ Non-proliferative } \\
\hline Accumulation, adipocyte $* \wedge$ & $\mathrm{x}$ & & & \\
\hline Amyloid & & $\mathrm{x}$ & & \\
\hline Atrophy & & $\mathrm{x}$ & & \\
\hline Colloid alteration * & & $\mathrm{x}$ & & \\
\hline Cystic follicle & $\mathrm{x}$ & & & \\
\hline Degeneration, vacuolar * & & $\mathrm{x}$ & & \\
\hline Dilatation, follicle, diffuse & $\mathrm{x}$ & & & \\
\hline Hypertrophy, follicular cell & & $\mathrm{x}$ & & \\
\hline Infiltrate, [insert appropriate cell type] *^ & $\mathrm{x}$ & & & \\
\hline Inflammation $^{\wedge}$ & $\mathrm{x}$ & & & \\
\hline Mineralization $^{\wedge}$ & $\mathrm{x}$ & & & \\
\hline Pigment $^{\wedge}$ & & $\mathrm{x}$ & & \\
\hline \multicolumn{5}{|l|}{ Proliferative (non-neoplastic) } \\
\hline Hyperplasia, C-cell & & $\mathrm{x}$ & & \\
\hline Hyperplasia, Follicular cell & & $\mathrm{x}$ & & \\
\hline
\end{tabular}

Table 13. Microscopic Findings of the Endocrine System: Parathyroid gland; Non-human primate

\begin{tabular}{|c|c|c|c|c|}
\hline Parathyroid Gland & Common & Uncommon & $\begin{array}{c}\text { Not observed but } \\
\text { potentially relevant }\end{array}$ & Not applicable \\
\hline \multicolumn{5}{|l|}{ Congenital } \\
\hline Ectopic tissue, parathyroid ${ }^{\wedge}$ & & $\mathrm{x}$ & & \\
\hline Ectopic tissue, salivary gland $* \wedge$ & & $\mathrm{x}$ & & \\
\hline Ectopic tissue, thymus $\wedge$ & & $\mathrm{x}$ & & \\
\hline \multicolumn{5}{|l|}{ Non-proliferative } \\
\hline Accumulation, adipocytes $* \wedge$ & & $\mathrm{x}$ & & \\
\hline Amyloid & & $\mathrm{x}$ & & \\
\hline Angiectasis & & $\mathrm{x}$ & & \\
\hline Atrophy & & $\mathrm{x}$ & & \\
\hline Cyst & $\mathrm{x}$ & & & \\
\hline Fibrosis $\wedge$ & & $\mathrm{x}$ & & \\
\hline Hypertrophy & & $\mathrm{x}$ & & \\
\hline Infiltrate, [insert appropriate cell type $]^{\wedge}$ & $\mathrm{x}$ & & & \\
\hline Inflammation $^{\wedge}$ & & $\mathrm{x}$ & & \\
\hline Giant cells, multinucleated & & & $\mathrm{x}$ & \\
\hline \multicolumn{5}{|l|}{ Proliferative (non-neoplastic) } \\
\hline Hyperplasia & & $\mathrm{x}$ & & \\
\hline
\end{tabular}


Infiltrate, [insert appropriate cell type]: Thyroid Gland

(No image provided)

\section{Other term(s)}

Inflammatory cell infiltrate; thyroiditis

\section{Pathogenesis/Cell of Origin}

Accumulation of inflammatory cells; uncertain pathogenesis or local immune response.

\section{Diagnostic features}

- Focal to multifocal lymphoplasmacytic infiltrate in the interstitium

- Lymphoid follicle formation may be present
- Macrophage infiltrate within the follicles

Comments: Focal to multifocal inflammatory cell infiltrate and focal to multifocal thyroiditis are terms used previously ${ }^{11}$. The latter terminology should be avoided in favor of more descriptive terminology and prevent any confusion with the conditions observed in clinical setting in humans.

\section{Adrenal Gland}

Special care is required when removing the right adrenal gland of the cynomolgus macaque, due to its anatomic location and close association with the right lateral lobe of the liver. It also has a very distinctive pyramidal shape (Table14).

Table 14. Microscopic Findings of the Endocrine System: Adrenal gland; Non-human primate

\begin{tabular}{|c|c|c|c|c|}
\hline Adrenal Gland & Common & Uncommon & $\begin{array}{c}\text { Not observed but } \\
\text { potentially relevant }\end{array}$ & Not applicable \\
\hline \multicolumn{5}{|l|}{ Congenital } \\
\hline Adhesion, adrenohepatic * & & $\mathrm{x}$ & & \\
\hline Ectopic tissue, adrenocortical $* \wedge$ & & $\mathrm{x}$ & & \\
\hline Involution, fetal cortex $*$ & & $\mathrm{x}$ & & \\
\hline \multicolumn{5}{|l|}{ Non-proliferative } \\
\hline Amyloid^^ & & $\mathrm{x}$ & & \\
\hline Angiectasis & & $\mathrm{x}$ & & \\
\hline Atrophy, cortex & & $\mathrm{x}$ & & \\
\hline Cyst, cortex & $\mathrm{x}$ & & & \\
\hline Degeneration, cortical * & & $\mathrm{x}$ & & \\
\hline Degeneration, cystic & & & & $\mathrm{x}$ \\
\hline Fibrosis $^{\wedge}$ & & $\mathrm{x}$ & & \\
\hline 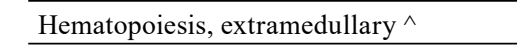 & $\mathrm{x}$ & & & \\
\hline Hemorrhage $^{\wedge}$ & $\mathrm{x}$ & & & \\
\hline Hypertrophy, cortex, diffuse & $\mathrm{x}$ & & & \\
\hline Hypertrophy, cortex, focal & & $\mathrm{x}$ & & \\
\hline Infiltrate, [insert appropriate cell type $]^{\wedge}$ & $\mathrm{x}$ & & & \\
\hline Inflammation $^{\wedge}$ & $\mathrm{x}$ & & & \\
\hline Metaplasia, osseous ${ }^{\wedge}$ & & $\mathrm{x}$ & & \\
\hline Mineralization *^ & $\mathrm{x}$ & & & \\
\hline Necrosis & & $\mathrm{x}$ & & \\
\hline Persistence, X Zone & & & & $\mathrm{x}$ \\
\hline Pigment $^{\wedge}$ & & $\mathrm{x}$ & & \\
\hline Thrombus & & $\mathrm{x}$ & & \\
\hline Vacuolation, cortex, decreased, diffuse & $\mathrm{x}$ & & & \\
\hline Vacuolation, cortex, decreased, focal & & $\mathrm{x}$ & & \\
\hline Vacuolation, cortex, increased, diffuse & $\mathrm{x}$ & & & \\
\hline Vacuolation, cortex, increased, focal & & $\mathrm{x}$ & & \\
\hline \multicolumn{5}{|l|}{ Proliferative (non-neoplastic) } \\
\hline Hyperplasia, cortex, diffuse & & $\mathrm{x}$ & & \\
\hline Hyperplasia, cortex, focal $*$ & & $\mathrm{x}$ & & \\
\hline Hyperplasia, medulla, diffuse & & $\mathrm{x}$ & & \\
\hline Hyperplasia, medulla, focal & & $\mathrm{x}$ & & \\
\hline Hyperplasia, subcapsular cell & & & & $\mathrm{x}$ \\
\hline
\end{tabular}


Adhesion, adrenohepatic: Adrenal Gland (Figure 64-65)

\section{Pathogenesis/Cell of Origin}

Spontaneous congenital lesion

\section{Diagnostic features}

- Adhesion of liver tissue and the right adrenal gland tissue with a connective septum in between and without intermingling of the respective parenchyma (adrenocortical adhesion).

- Union of liver tissue and the right adrenal gland with close intermingling of the respective parenchyma without a connective capsule in between (adrenocortical fusion) $6,11,131$.

\section{Differential Diagnoses}

- Ectopic tissue, adrenocortical

- Ectopic adrenal tissue is attached to the liver or lying within the liver capsule.

- Adhesion (inflammation in the abdominal cavity)

- Presence of inflammatory cell infiltrate or fibrosis.

Comments: This finding is observed in young cynomolgus monkey at necropsy. The distinction between adhesion and fusion is not considered essential ${ }^{132}$. This finding is also sporadically described in human and the pathogenesis is controversial, some authors suggest a developmental anomaly and other consider it as an acquired anomaly.

\section{Ectopic tissue, adrenocortical: Adrenal Gland}

(No image provided)

\section{Other term(s)}

Accessory cortical tissue; cortical herniation; extracapsular adrenal tissue.

Comments: This finding is morphologically similar to this described in rodents, but reported under multiple other terms as indicated above 6,11 .

Involution, fetal cortex: Adrenal Gland (Figure 66)

\section{Pathogenesis/Cell of Origin}

Remnants of fetal cortex

\section{Diagnostic features}

- Additional layer of solid sheets and columns of cells supported by vascular capillary bed that surrounds the entire circumference of the medulla

- Large polyhedral cells with abundant eosinophilic, slightly finely vacuolated cytoplasm

- All three zones of the cortex (glomerulosa, fascicularis, and reticularis) can be clearly identified

Comments: This finding is very rare in cynomolgus monkey and there is only one case described in literature ${ }^{133}$. In NHP the fetal cortex is gradually replaced with the three zones and the involution is accompanied by hemorrhage and necrosis with consequent dystrophic mineralization.

Degeneration, cortical: Adrenal Gland

(No image provided)

\section{Pathogenesis/Cell of Origin}

Sequela of altered steroidogenesis or metabolism in adrenal cortical epithelium.

\section{Diagnostic features}

- Cortical cell loss (decreased number of cortical epithelium in comparison to surrounding parenchyma).

- Zona fasciculata is more commonly affected

- Both increased cytoplasmic vacuolation and decreased vacuolization with increased cytoplasmic eosinophilia have been described.

\section{Differential Diagnoses}

- Atrophy, cortical:

- Decreased cortical thickness and individual epithelial cell size with no evidence of cell degeneration or loss.

- Vacuolation, Increased, Cortical

- Increased cytoplasmic vacuolization of cortical epithelium with little or no evidence of cell loss or degeneration.

Comments: Adrenal cortical degeneration is an uncommon xenobiotic-induced finding in cynomolgus monkeys although is reported that the adrenal is the endocrine gland most sensitive to compound-induced lesions ${ }^{134,135}$.

\section{Mineralization: Adrenal Gland (No image provided)}

Comments: Mineralization occurring at the corticomedullary junction of the adrenal gland in cynomolgus monkeys is a relatively common incidental observation $(6 \%$ incidence in male cynomolgus macaques) ${ }^{6,11}$. This finding is believed to be caused by dystrophic calcification of remnants of the fetal adrenal cortex and is considered to be of no pathological significance. It is much more frequently observed in rhesus macaques (up to $45 \%$ incidence in both males and females) 136,137 . The focal change can be quite extensive and may show up during in-vivo imaging techniques.

\section{Hyperplasia, cortical, focal: Adrenal Gland}

(No image provided)

Comments: Cortical focal hyperplasia has also been reported as hyperplasia, nodular ${ }^{11}$. The term hyperplasia, cortical, focal is preferred.

\section{E. Endocrine Pancreas (Islets of Langerhans)}

The endocrine pancreas consists of discrete aggregates of cells throughout the exocrine pancreas (islets of Langerhans) 
whose main function is the regulation of blood glucose. The islets are composed of pale staining polygonal cells of variable size, and in the primate show a mainly intra-lobular distribution. Large- to small-sized islets are present in all lobes of the pancreas. Two main distribution patterns of $\alpha$ - and $\beta$-cells are present, there are $\alpha$-cell-rich or $\beta$-cell-rich islets in all lobes of the pancreas. In the $\alpha$-cell-rich islet, $\alpha$-cells are distributed in all parts of the islet, and $\beta$-cells are scattered or accumulated in the periphery of the islet, forming an irregular ring. In the $\beta$-cell rich islet, $\beta$-cells are distributed in all parts of the islet. Relatively numerous $\delta$-cells are scattered in the center or periphery equally in both $\alpha$-cell-rich and $\beta$-cell-rich islets ${ }^{138}$. Characterization of the different cell types within the islets is not possible on H\&E staining. Application of immunohistochemical staining for the different hormones, or the use of electron microscopy is necessary to differentiate the structure of the secretory granules in each cell type (Table 15).

Table 15. Microscopic Findings of the Endocrine System: Endocrine pancreas; Non-human primate

\begin{tabular}{|c|c|c|c|c|}
\hline Endocrine Pancreas (Islets of Langerhans) & Common & Uncommon & $\begin{array}{c}\text { Not observed but } \\
\text { potentially relevant }\end{array}$ & Not applicable \\
\hline \multicolumn{5}{|l|}{ Non-proliferative } \\
\hline Amyloid, islet ${ }^{\wedge}$ & & $\mathrm{x}$ & & \\
\hline Angiectasis, islet & & $\mathrm{x}$ & & \\
\hline Apoptosis, islet cell & & $\mathrm{x}$ & & \\
\hline Atrophy, islet cell & & $\mathrm{x}$ & & \\
\hline Degranulation, islet cell & & $\mathrm{x}$ & & \\
\hline Fibrosis, islet ${ }^{\wedge}$ & & $\mathrm{x}$ & & \\
\hline Hemorrhage, islet ${ }^{\wedge}$ & & $\mathrm{x}$ & & \\
\hline Hypertrophy, islet cell & & $\mathrm{x}$ & & \\
\hline Infiltrate, [insert appropriate cell type $]^{\wedge}$ & & $\mathrm{x}$ & & \\
\hline Inflammation $^{\wedge}$ & & $\mathrm{x}$ & & \\
\hline Metaplasia, hepatocyte & & & & $\mathrm{x}$ \\
\hline Single cell necrosis $\wedge$ & & $\mathrm{x}$ & & \\
\hline Pigment, islet ${ }^{\wedge}$ & & $\mathrm{x}$ & & \\
\hline Vacuolation, islet cell & & $\mathrm{x}$ & & \\
\hline \multicolumn{5}{|l|}{ Proliferative (non-neoplastic) } \\
\hline Hyperplasia, islet cell & & $\mathrm{x}$ & & \\
\hline \multicolumn{5}{|l|}{ Neoplastic } \\
\hline Adenoma, islet cell & & $\mathrm{x}$ & & \\
\hline
\end{tabular}




\section{Hematopoietic and Lymphoid System}

For detailed general considerations on the hematopoietic and lymphoid system, refer to the INHAND rodent publication ${ }^{139}$. A tiered diagnostic approach has been used in the rodent nomenclature, however due to the smaller number of animals in general toxicology studies using cynomolgus macaques, the authors of this document recommend use of the descriptive terminology and not the more conventional terms. Accordingly, only the descriptive terms are included in the tables for this section.

Owing to their immunological, genetic, physiological, and anatomic similarities to humans, nonhuman primates (NHP) have shed significant insight into nonclinical pharmaceutical studies, as well as several translational models of human diseases including cancer, infectious diseases, immunology, and radiation toxicity. Of the NHPs used in research, cynomolgus and rhesus macaques are the species most widely used.

NHP are commonly the large animal species of choice to evaluate immunomodulatory compounds, and thus, a comprehensive evaluation of hematolymphoid tissues is critical. Specific recommendations for evaluating immune parameters including histopathology are addressed in regulatory documents (FDA Guidance for Industry: S8 Immunotoxicity Studies for Human Pharmaceuticals [ICH S8], 2006 ${ }^{140}$ ). Standard post-mortem morphological evaluation of the hematolymphoid system in NHP is similar to that of rodents which include evaluation of primary lymphoid organs including bone marrow and thymus where lymphocyte proliferation and maturation occur independent of exogenous antigen stimulation and secondary lymphoid organs including spleen, lymph nodes, and mucosal associated lymphoid tissue (MALT) where exogenous antigen-dependent lymphocyte development and maturation takes place. As a standard, spleen and thymus weight measurements, and microscopic evaluation of the bone marrow, spleen, thymus, Peyer's patches (typically included in section of ileum), mesenteric and mandibular lymph nodes are evaluated. Axillary and/or inguinal lymph nodes are often included among the lymphoid tissues collected. Depending on the study objectives, additional lymphoid tissues including lymph nodes draining sites of test article or antigen administration may be collected and examined. In general, MALT is examined in routine sections of lung (BALT) and gastrointestinal tract including stomach, small and large intestines (GALT). Mucosal associated lymphoid tissue surrounding the lacrimal and salivary gland ducts referred to as DALT ${ }^{141}$ are also observed in NHP. For drugs administered by inhalation or nasal route of exposure, specific mucosal associated lymphoid tissue from the nasal cavity (nasal-associated lymphoid tissues [NALT]) may be evaluated and requires special collection ${ }^{142}$. Tonsils are an important component of the oropharyngeal mucosal associated lymphoid tissues which were not discussed in the rodent manuscript as they are not present in rodent species but are often evaluated in NHP toxicology studies (anatomic details described in MALT section).

Each lymphoid organ has separate identifiable compart- ments that support specific immune functions and should be evaluated individually for changes on microscopic evaluation. Microscopic findings should be recorded accordingly, using locators/modifiers to describe the compartment (cortex, medulla, medullary sinus, etc.) that is affected ${ }^{143,}{ }^{144}$. Immunohistochemistry (IHC) may be incorporated into a toxicology study design as an adjunct diagnostic tool to further characterize a morphologic finding observed in hematoxylin and eosin (HE) stained tissue sections. IHC can be used to determine tissue distribution of an antigen, lymphocyte subset and/or drug target and be useful in identifying target versus off-target effects. A weight of evidence approach is particularly important in interpreting morphologic changes in hematolymphoid organs. Correlating in-life data such as immunophenotyping results, immune function assays, anti-drug antibody and exposure data with postmortem morphological alterations can aid in interpreting a xenobiotic effect (Table 16).

\section{Decreased number and size, germinal centers: Lymphoid Tissues (Figure 67-70)}

\section{Additional locator}

lymphoid follicle

Comments: This morphologic term falls under the general category of "lymphocytes, decreased"; however, because many immunomodulatory drugs have highly specific molecular targets using enhanced terminology with compartment specific modifiers can aid in elucidating target specific effects. To further characterize drug-related findings in the splenic white pulp, lymph node cortex, tonsil and mucosal associated lymphoid tissues the use of the descriptive term increased or decreased, number and size, lymphoid follicles or germinal centers has been recommended ${ }^{145}$. If follicular changes specifically involve the germinal center, the locator "germinal center" is used.

\section{Erythrophagocytosis: Lymphoid tissues (Figure 71)}

\section{Other term(s)}

Erythrophagia

\section{Pathogenesis/Cell of Origin}

This condition refers to the engulfment of RBCs by macrophages or neutrophils. It may be seen in local lymph nodes following trauma or surgical biopsy associated with draining lymphatic vessels. It can be seen in a variety of conditions including viral, bacterial, and parasitic infections. Specifically, it has been observed lymph nodes from SIV-infected monkeys. Klump et al. 146 reported the finding in a monkey infected with Salmonella spp. and it is also a feature of monkeys infected with Plasmodium spp. It can be seen in the late stages of Simian Retrovirus Type D infections whereby markedly depleted lymph nodes exhibit hemosiderosis, medullary histiocytosis, and erythrophagocytosis. Finally, erythrophagia has been observed in cynomolgus monkeys infected with Ebola virus. In 
Table 16. Microscopic Findings of the Hematopoietic and Lymphoid System: General Terms; Non-human Primate

\begin{tabular}{|c|c|c|c|c|}
\hline Hematolymphoid System - General Terms & Common & Uncommon & $\begin{array}{c}\text { Not Observed but } \\
\text { Potentially Relevant }\end{array}$ & Not Applicable \\
\hline \multicolumn{5}{|l|}{ Congenital } \\
\hline Aplasia/Hypoplasia & & $\mathrm{x}$ & & \\
\hline \multicolumn{5}{|l|}{ Non-proliferative } \\
\hline Amyloid $^{\wedge}$ & & $\mathrm{x}$ & & \\
\hline Apoptosis, increased, lymphocyte \# & $\mathrm{x}$ & & & \\
\hline Cellularity, decreased, lymphocyte & $\mathrm{x}$ & & & \\
\hline Cellularity, increased, mast cell & & $\mathrm{x}$ & & \\
\hline Decreased number and size, germinal centers $*$ & $\mathrm{x}$ & & & \\
\hline Erythrophagocytosis * & $\mathrm{x}$ & & & \\
\hline Extramedullary hematopoiesis, [increased] ${ }^{\wedge}$ & $\mathrm{x}$ & & & \\
\hline Immunoblasts, increased * & & $\mathrm{x}$ & & \\
\hline Increased number and size, germinal centers * & & $\mathrm{x}$ & & \\
\hline Infiltrate, [insert appropriate cell type] ${ }^{\wedge}$ & $\mathrm{x}$ & & & \\
\hline Inflammation $^{\wedge}$ & $\mathrm{x}$ & & & \\
\hline Lymphoid follicle formation (TLS) * & $\mathrm{x}$ & & & \\
\hline Metaplasia, osseous ${ }^{\wedge}$ & & $\mathrm{x}$ & & \\
\hline Mineralization & $\mathrm{x}$ & & & \\
\hline Necrosis & & $\mathrm{x}$ & & \\
\hline Pigment, increased, macrophages $\wedge$ & $\mathrm{x}$ & & & \\
\hline Tingible body macrophage, increased & $\mathrm{x}$ & & & \\
\hline Vacuolation, macrophage & $\mathrm{x}$ & & & \\
\hline \multicolumn{5}{|l|}{ Neoplastic } \\
\hline Hematopoietic neoplasms * & & $\mathrm{x}$ & & \\
\hline \multicolumn{5}{|l|}{ Histiocytic sarcoma * } \\
\hline \multicolumn{5}{|l|}{ Leukemia, erythroid } \\
\hline \multicolumn{5}{|l|}{ Leukemia, megakaryocytic } \\
\hline \multicolumn{5}{|l|}{ Leukemia, myeloid } \\
\hline \multicolumn{5}{|l|}{ Leukemia, NOS } \\
\hline \multicolumn{5}{|l|}{ Lymphoid neoplasms } \\
\hline Lymphoma * & & $\mathrm{x}$ & & \\
\hline
\end{tabular}

contrast to infectious diseases, it has been observed as an offtarget effect in spleens of cynomolgus monkeys dosed with a therapeutic monoclonal antibody. In humans, it has been reported in immune-mediated diseases, as well as, incompatible blood transfusions.

\section{Diagnostic features}

- Gross and histological findings may include an enlarged lymph node, abundant sinus macrophages, sinus erythrocytosis, or hemosiderosis. The cytological features are RBCs located within the cytoplasm of macrophages or neutrophils.

Special Techniques for Diagnostics

- Histology (Hematoxylin and Eosin) or blood smear (Wrights or Wright-Giemsa stain)

Comments: The presence of erythrophagocytosis suggests an underlying disorder but can be seen at a minimal level spontaneously within lymph nodes.

\section{Immunoblasts, increased: Lymphoid Tissues}

(Figure 72-73)

\section{Modifiers}

lymphoid

\section{Other term(s)}

Increased lymphoblasts, increased centroblasts, lymphoid hyperplasia

\section{Pathogenesis/Cell of Origin}

Physiologic response to antigen exposure

\section{Diagnostic features}

- The term immunoblasts is most commonly used to refer to proliferating lymphocytes; however, immunohistochemi- 
cal characterization is necessary for definitive identification. Immunoblasts are large, pale-staining polygonal cells with increase nuclear to cytoplasmic ratio, vesicular chromatin, often prominent nucleoli and moderate, pale to basophilic cytoplasm. They often have visible mitotic figures and are commonly present in the germinal center of secondary lymphoid follicles. Increased immunoblasts may represent a normal physiologic response to antigen presentation.

- Increased immunoblasts may be associated with clusters of plasma cells and occasional Reed-Sternberg (RS) cells. RS cells are large 30-50 microns EBV/LCV transformed immortalized B cells which often have either a multinucleated or bilobed nucleus with prominent eosinophilic inclusion-like nucleoli (an "owl's eye" appearance). Mitotic figures and atypical cells can be frequent in these clusters. These clusters are commonly found in periarteriolar lymphoid sheath (PALs) in the spleen as well as in the paracortex and medullary cords in lymph nodes. Adjacent to these clusters, normal $\mathrm{T}$ and $\mathrm{B}$ cell area architecture is preserved.

- Increased immunoblasts with plasmacytoid features (plasmablasts) can be admixed with mature plasma cells, identified in spleen in the marginal zone and red pulp as well as in lymph nodes in the paracortex and medullary cords.

\section{Differential Diagnoses}

- Lymphoma

- Diffuse infiltration, loss of distinction between normal tissue compartments and may extend beyond tissue capsule or border.

- Cell population is more homogeneous.

\section{Special Techniques for Diagnostics}

- Ki67, an immunohistochemistry (IHC) marker of proliferation, can be used to label immunoblasts

- IHC is useful to aid in identifying in RS cells. RS cells are typically $\mathrm{CD} 30$ and $\mathrm{CD} 15$ positive and usually negative for CD20 and CD45.

- In situ hybridization for EBV-encoded nuclear RNAs (EBERs), present in most infected B cells in relatively high copy numbers, can be used to verify LCV infection.

Comments: Lymphoproliferation, whether caused by exposure to an infectious agent or exogenous substance such as a therapeutic protein, is a normal physiologic response. Increased responses such as reactive plasmacy tosis have been described with antigen stimulation through vaccination or with severe infection. One of the most common infectious causes of lymphoproliferation in cynomolgus monkeys is simian lymphocryptovirus (LCV), a gammaherpes virus similar to Epstein-Barr virus (EBV). Most, if not all, Old World non-human primates, including cynomolgus macaques, are infected with LCV. Naturally acquired, primary LCV infection usually occurs within the first year of life in cynomolgus monkeys with nearly all individuals infected by 2 years of age 147,148 . LCV establishes a latent and asymptomatic infection in B cells which is mediated by $\mathrm{T}$ cell viral suppression. Immunomodulatory agents that cause immunosuppression can lead to LCV reactivaton, associated lymphoproliferative disorders ${ }^{149}$ and oncogeneis. Post-transplant lymphoproliferative disorders (PTLDs) are reported in NHP following solid organ, stem cell or other xenotransplants in NHP149, 150. Differentiation from malignant lymphoma is often difficult with the florid lymphoproliferation observed with PTLD and relies on molecular studies (restriction fragment length polymorphism [RFLP]) to determine clonality. Proliferative cells often have plasmacytoid features. Plasma or serum electrophoresis can aid in differentiation of a monoclonal spike seen with neoplasia vs. the lack of a spike with PTLD. Since kappa and lamda light chains in plasma cells are of similar frequency in NHPs, immunohistochemical or flow cytometric demonstration of enhanced distribution toward either light chain would be suggestive of malignancy.

\section{Increased number and size, germinal centers: Lymphoid} Tissues (No image provided)

\section{Additional locator}

lymphoid follicle

Comments: This morphologic term falls under the general category of "lymphocytes, increased". To further characterize drug-related findings the descriptive terminology with compartment specific modifiers can aid in elucidating target specific effects.

\section{Lymphoid follicle formation: Lymphoid Tissues} (Figure 74-76)

\section{Other term(s)}

Organized lymphoid aggregates; tertiary lymphoid structures (TLS)

\section{Pathogenesis/Cell of Origin}

May be related to persistent antigen exposure

\section{Diagnostic features}

- One or more organized primary or secondary lymphoid follicles noted in an ectopic or uncommon location.

- Occasionally present in the thymic medulla, bone marrow of the femur or sternum, and salivary glands $7,11,151$

\section{Differential Diagnoses}

- Infitrate, mononuclear cell (or lymphocyte)

- lack distinct germinal center formation and organized follicle architecture

- Mucosa-associated lymphoid tissue (MALT)

- Nonencapsulated subepithelial lymphoid structures with parafollicular areas and overlying follicle-associ- 
ated epithelium (FAE)

Comments: Increases in the number of lymphoid follicles above the normal variance may suggest immune stimulation. Tertiary lymphoid structures frequently occur in association with inflammation, tumors or chronic infections in non-lymphoid organs.Variably-organized lymphoid aggregates, with or without follicular development, observed in the bone marrow have been postulated to be related to type $\mathrm{D}$ retrovirus infection ${ }^{116}$. However, most non-human primates used in nonclinical studies are currently prescreened for simian retroviruses and thus other agents or non-specific immunosurveillance could be the cause. Increased secondary lymphoid follicles in the bone marrow have also been observed in cynomolgus monkeys in association with test article exposure.

\section{Hematopoietic Neoplasms: Lymphoid Tissues}

(No images provided)

\section{Other $\operatorname{term}(\mathrm{s})$}

Acute Myeloid Leukemia (AML); Acute Lymphocytic Leukemia (ALL); Chronic Myeloid Leukemia (CML); Chronic Lymphocytic Leukemia; Myeloma; Myelodysplastic Syndrome

\section{Pathogenesis/Cell of Origin}

Hematopoietic neoplasms are clonal disorders involving hematopoietic blast cells of myeloid lineage and their progenitors. In general, complete neoplastic transformation occurs due to DNA damage that often results in gross chromosomal damage (e.g., chromosomal translocations (CTs)). Complementary secondary mutations (e.g., point mutations) accumulate in DNA and together with CTs lead to neoplastic transformation. A variety of risk factors may predispose individuals to the development of hematological malignancies including accidental or therapy-related ionizing radiation, chemotherapeutic agents (e.g., alkylating agents and topoisomerase II inhibitors), and exposures to environmental carcinogens.

Reports of spontaneous hematopoietic malignancies in nonhuman primates are sparse compared to humans and rodents. A case report involving a rhesus macaque with granulocytic leukemia has been reported following exposure to whole body, fractionated low dose, neutron irradiation ${ }^{152}$. A second case of AML was reported in a monkey which developed delayed onset of disease following radiation exposure ${ }^{153}$. A larger study involving monkeys that received whole body proton irradiation found that one monkey out of 123 rhesus macaques developed AML two and a half years post exposure154.

The majority of reports relating to leukemia in NHPs are associated with radiation exposure. Gaps in our knowledge are due to a low prevalence of disease and a lack of suitable assays including cytogenetics and genomics.

\section{Diagnostic features}

- $>20 \%$ blasts in bone marrow, neoplastic cells in circula- tion or infiltrating parenchyma tissue.

- Presence of blasts with immature nuclear chromatin.

- Myeloblasts may have round or indented nuclei.

- Cyotplasmic granules may be present.

\section{Differential Diagnoses}

- Myelodysplastic Syndrome typically present with peripheral cytopenias despite hypercellular marrow. Both the hematopoietic cells in the marrow as well as those on the smear present with dysplastic features (e.g., large or giant platelets, basophilic stippling in erythrocytes and immature but abundant precursors in the marrow).

- Leukemoid reactions, are differentiated on the basis of orderly maturation of hematopoietic cells undergoing proliferation and have normal morphology. The marrow is hypercellular characterized by myeloid hyperplasia.

- Myelitis (Inflammation) may present as either acute or chronic. The duration will determine the morphology wich may present with abundant (degenerate) neutrophils with fibrin and necrosis or the presence of macrophages and well-formed abscesses. Infectious agents (bacteria, viral inclusions, or fungi) may or may not be present.

\section{Special Techniques for Diagnostics}

- Histology, Cytology, Flow Cytometry, Immunohistochemistry, PCR, Southern Blotting, In Situ Hybridization, Cytogenetics

Comments: Leukemia in contrast to lymphoma is a rare disease in nonhuman primates.

\section{Histiocytic sarcoma: Lymphoid Tissues}

(No image provided)

Comments: A single case has been reported in an 11-yearold male cynomolgus monkey fed a high-fat diet ${ }^{155}$.

\section{Lymphoma: Lymphoid Tissues (Figure 77-78)}

\section{Other $\operatorname{term}(\mathrm{s})$}

Leukemic lymphoma; lymphosarcoma; lymphoid leukemia

\section{Pathogenesis/Cell of Origin}

Lymphoma is a solid mass of neoplastic lymphocytes that arises from extramedullary sites such as spleen, thymus, lymph nodes, or mucosa immune structures. Many lymphomas in nonhuman primates are associated with viral infections including SIV, STLV, LCV, and Rhadinaviruses ${ }^{156,157 .}$

\section{Diagnostic features}

- A diagnosis is made when a large percentage (>50\%) of the cell population consists of homogenous atypical, large lymphocytes in contrast to a heterogenous population typically seen within lymphoid tissue.

- Lymphoma can be classified based on anatomic site involvement (e.g., lymph node vs thymus), histological lo- 
cation within the lymph node (e.g., follicular vs diffuse) and morphology of the cell (large cell vs lymphoblastic).

- Some authors have differentiated B-cell and T-cell lymphomas based on immunohistochemical staining.

- Demonstration of clonality is important to differentiate non-neoplastic lymphoproliferative disease from lymphoma.

\section{Differential Diagnoses}

- Leukemia can be differentiated on the basis of increased numbers of myeloblasts in bone marrow, invasion into parenchymal tissues and in circulation ${ }^{158}$.

- Lymphoid (Reactive) Hyperplasia is a benign condition wherein lymph node follicles are numerous with sharp demarcations outlining the germinal centers and mantle zones. Cells comprising these reactive regions comprise centroblasts, centrocytes, and tingible body macrophages ${ }^{159}$.

\section{Special Techniques for Diagnostics}

- Histology, Cytology, Flow Cytometry, Immunohistochemistry, PCR, Southern Blotting, In Situ Hybridization, Cytogenetics

Comments: Gammaherpesvirus-related lymphoma has been reported in non-human primates exposed to immunomodulatory biotherapeutics in non-clinical studies as well as in experimental xenotransplant models. An association with chronic pharmacologic T-lymphocyte depletion has been made.

\section{A. Bone Marrow}

Bone marrow is routinely examined in situ in a section of sternum. The quality of histologic sections of bone marrow is greatly influenced by the decalcification procedure, which involves a balance between preservation of marrow cell features versus adequate removal of mineral to allow histologic sectioning ${ }^{160}$. In nonclinical toxicity studies, hematology data in conjunction with microscopic examination of the bone marrow provide an assessment of overall hematopoietic cellularity. If the combined data is inadequate, then cytologic evaluation of the bone marrow smears (from rib or sternum) may be considered for assessing the effect of test articles on the hematopoietic system $^{143,160}$. Other techniques, such as flow cytometric evaluation, clonigenic assays and electron microscopy may also be helpful in certain situations ${ }^{160}$ (Table 17).

\section{Aggregate, lymphocyte: Bone Marrow (Figure 79) \\ Other $\operatorname{term}(\mathrm{s})$ \\ Infiltrate, lymphocyte \\ Pathogenesis/Cell of Origin \\ Unknown}

\section{Diagnostic features}

- A focal accumulation of lymphocytes in tissues which lacks organized follicular structure

Table 17. Microscopic Findings of the Hematopoietic and Lymphoid System: Bone Marrow; Non-human Primate

\begin{tabular}{|c|c|c|c|c|}
\hline Bone Marrow & Common & Uncommon & $\begin{array}{c}\text { Not Observed but } \\
\text { Potentially Relevant }\end{array}$ & Not Applicable \\
\hline \multicolumn{5}{|l|}{ Non-proliferative } \\
\hline Aggregate, lymphocyte * & $\mathrm{x}$ & & & \\
\hline Angiectasis & & $\mathrm{x}$ & & \\
\hline Cellularity, decreased & $\mathrm{x}$ & & & \\
\hline Cellularity, decreased, adipocyte & & $\mathrm{x}$ & & \\
\hline Dyshematopoiesis & & $\mathrm{x}$ & & \\
\hline Emperipolesis, increased, megakaryocyte* & & $\mathrm{x}$ & & \\
\hline Erythrophagocytosis ${ }^{1}$ & & $\mathrm{x}$ & & \\
\hline Fibrosis $\wedge^{\wedge}$ & & $\mathrm{x}$ & & \\
\hline Hypersegmentation, granulocyte & & & $\mathrm{x}$ & \\
\hline Serous atrophy of fat ${ }^{\wedge}$ & & $\mathrm{x}$ & & \\
\hline \multicolumn{5}{|l|}{ Proliferative (non-neoplastic) } \\
\hline Cellularity, increased, adipocyte \# & & $\mathrm{x}$ & & \\
\hline Cellularity, increased, bone marrow & & $\mathrm{x}$ & & \\
\hline Cellularity, increased, macrophage & & $\mathrm{x}$ & & \\
\hline Cellularity, increased, mast cell & & & $\mathrm{x}$ & \\
\hline Decreased M:E ratio & & $\mathrm{x}$ & & \\
\hline Increased M:E ratio & & $\mathrm{x}$ & & \\
\hline
\end{tabular}

* Terminology with diagnostic criteria or comments described below. \# Finding more frequent as an induced change. ${ }^{\wedge}$ Terminology addressed in the Systemic/General Pathology section. ${ }^{1}$ For a description and diagnostic criteria, refer to General Terminology within the Hematolymphoid System. 


\section{Differential Diagnoses}

- Lymphoid follicle formation

- organized primary or secondary lymphoid follicles in an ectopic location (e.g. tertiary lymphoid structures in non-lymphoid organs)

Comments: Commonly identified in the bone marrow in the femur or sternum.

\section{Emperipolesis, increased, megakaryocyte: Bone Marrow} (Figure 80)

\section{Pathogenesis/Cell of Origin}

The migration of blood cells, including lymphocytes, neutrophils, and erythrocytes, through the cytoplasm of megakaryocytes is referred to as emperipolesis. This is in contrast to phagocytosis, which involves the engulfment of cells that are targeted for killing and elimination. Emperipolesis has been observed in a variety of species including dogs, cats, rats, monkeys and humans. Emperipolesis is observed in healthy marrow and increases under conditions of hematopoietic stress or bone marrow disease ${ }^{161}$. In humans, increased emperipolesis has been observed with several types of bone marrow neoplasms ${ }^{162}$ and when stimulated by agents that cause thrombopoiesis including recombinant human IL-6163. The underlying mechanism and significance remains poorly understood.

\section{Diagnostic features}

- The presences of lymphocytes, neutrophils, and erythrocytes in the cytoplasm or proplatelet extensions of megakaryocytes.

\section{Differential Diagnoses}

- Phagocytosis of dead or dying cells and/or cellular debris. Depending on the orientation of the cells, emperipolesis should be distinguished from granules (which are considerably smaller) or inclusions.

\section{Special Techniques for Diagnostics}

- Histology, Cytology

Comments: The significance of emperiopolesis in normal megakaryocytes of monkey bone marrow is not clear.

\section{B. Thymus}

Non-human primates, have a bilobed thymus located in the anterior thoracic cavity just cranial to the heart and cardiac vessels. The thymus has a thin connective tissue capsule that surrounds the organ and penetrates into the lobes dividing it into multiple lobules. Lobules are easily identified ${ }^{7}$ (Table 18).

Table 18. Microscopic Findings of the Hematopoietic and Lymphoid System: Thymus; Non-human Primate

\begin{tabular}{|c|c|c|c|c|}
\hline Thymus & Common & Uncommon & $\begin{array}{c}\text { Not Observed but } \\
\text { Potentially Relevant }\end{array}$ & Not Applicable \\
\hline \multicolumn{5}{|l|}{ Non-proliferative } \\
\hline Apoptosis, increased, lymphocyte & $\mathrm{x}$ & & & \\
\hline Cellularity, decreased, lymphocyte * & $\mathrm{x}$ & & & \\
\hline Corticomedullary ratio, decreased & & $\mathrm{x}$ & & \\
\hline Corticomedullary ratio, increased & & $\mathrm{x}$ & & \\
\hline Cyst, epithelial & $\mathrm{x}$ & & & \\
\hline Ectopic tissue (parathyroid) $^{\wedge}$ & & $\mathrm{x}$ & & \\
\hline Ectopic tissue (specify tissue) $^{\wedge}$ & & $\mathrm{x}$ & & \\
\hline Ectopic tissue (thymus) $^{\wedge}$ & & $\mathrm{x}$ & & \\
\hline Hypoplasia & $\mathrm{x}$ & & & \\
\hline Involution, age-related & & & & $\mathrm{x}$ \\
\hline Loss of corticomedullary distinction & & $\mathrm{x}$ & & \\
\hline Myoid cell, increased * & & $\mathrm{x}$ & & \\
\hline Necrosis, lymphocyte & & $\mathrm{x}$ & & \\
\hline Tingible-body macrophage, increased & & $\mathrm{x}$ & & \\
\hline \multicolumn{5}{|l|}{ Proliferative (non-neoplastic) } \\
\hline Cellularity, increased, epithelial cell, medulla & & $\mathrm{x}$ & & \\
\hline Cellularity, increased, lymphocyte & & $\mathrm{x}$ & & \\
\hline Epithelium-free areas, increased & & & & $\mathrm{x}$ \\
\hline Thymic corpuscles, increased & & $\mathrm{x}$ & & \\
\hline \multicolumn{5}{|l|}{ Neoplastic } \\
\hline Thymoma, benign * & & $\mathrm{x}$ & & \\
\hline
\end{tabular}


Cellularity, decreased, lymphocyte: Thymus

(Figure 81-83)

\section{Other term(s)}

Thymic atrophy; thymic involution; lymphocytes decreased

\section{Pathogenesis/Cell of Origin}

Thymic involution can be divided into physiological or agerelated or stress-induced. Examples of stress related causes include metabolic derangements, pregnancy, or malnutrition ${ }^{164}$.

\section{Diagnostic features}

- Decreased cellularity of cortical region

- Interlobular fat infiltration

- Reduced or loss of delineation of the corticomedullary junction, with reduction in cortical and medullary $\mathrm{T}$ and B cells

- Reduced cortical-to-medullary ratio

- Decreased thymic weights

\section{Differential Diagnoses}

- Atrophy

- Age-associated and stress-induced thymic both result in loss of cortical and medullary thymocytes ${ }^{165}$.

- Determining the underlying cause is important to classification.

- Apoptotic lymphocytes can be differentiated from other mechanisms of cell death (e.g., necrosis) by cleaved caspase 3 and TUNEL.

\section{Special Techniques for Diagnostics}

- Histology, Flow cytometry, sjTREC, thymic weight

Comments: Stress, age, and sex differences should be considered when evaluating the thymus. Studies have shown that females do not exhibit the same degree of thymic change as seen in males; this may be attributed to the influence of pre/post menopause and the role of sex steroids.

Myoid cells, increased: Thymus (No image provided)

\section{Other term(s)}

Ectopic tissue, myoid cells

\section{Pathogenesis/Cell of Origin}

The normally occurring presence of myoid cells in the thymus has been well known since $1888^{166}$. Myoid cells are considered a source of acetylcholine receptors and play a key component in the pathogenesis of myasthenia gravis ${ }^{167}$. Recent studies suggest that myoid cells may protect the resident thymocytes from apoptosis and regulate their differentiation ${ }^{168}$.

\section{Diagnostic features}

- Myoid cells are located in the medulla and share morphological and functional features with skeletal muscle; they express myoid proteins and contain acetylcholine receptors.

\section{Differential Diagnoses}

- Hamartoma is a benign growth consisting of an abnormal collection of heterogenous cells or tissue types normally located in that particular region of the body.

- Choristoma is a benign growth consisting of normal tissue that is derived from germ cells that are not normally found in that region of the body.

- Rhabdomyoma is a benign tumor composed of striated muscle fibers that may appear as a discrete mass.

\section{Special Techniques for Diagnostics}

- Light microscopy, immunohistochemistry

Comments: It should be noted that myoid cells are part of the normal thymic tissue architecture. These cells are not readily observed on routine light microscopy and are best visualized using IHC.

Thymoma, benign: Thymus (Figure 84-86)

\section{Pathogenesis/Cell of Origin}

Considered to be of thymic epithelial cell origin

\section{Diagnostic features}

- Present as solitary, well encapsulated, thymic nodules, distinct from the surrounding normal thymic tissues.

- May have pale and dark zones noted on HE. Pale areas are typically composed of proliferations of large spindle or elongated cells, often densely packed and may form a storiform pattern. Dark areas typically represent areas composed primarily of lymphocytes like those in the thymic cortex.

- Relative proportions of epithelial cells and lymphocytes vary between tumors and within a given tumor.

- Additional features may include interspersed Hassall's corpuscles, individual, small clusters of small lymphocytes (mixed T and B cells), scattered eosinophils, necrosis, cavitation, mineralization, multinucleate giant cells (nearby keratin whorls) and cholesterol clefts.

- Neoplastic spindle cells have a relatively low mitotic index.

- No evidence of vascular invasion or metastasis.

\section{Differential Diagnoses}

- Thymoma, malignant

- Marked invasion of adjacent tissues

- Lymphoma

- No neoplastic epithelial component

- Other lymphoid organs often involved

\section{Special Techniques for Diagnostics}

- Useful IHC markers in published reports include pancytokeratin, vimentin, CD3, CD20, PCNA, and S100

- Pan-cytokeratin helps visualize the epithelial component.

- Neoplastic spindle cells can have variable cytokeratin and/or vimentin staining. 
Comments: Neoplastic lesions including thymomas are rare in cynomolgus monkeys used in toxicologic studies. Two published cases of benign thymomas have been reported $^{169,170}$. Both were diagnosed in relatively young monkeys and were subclinical in nature. Both tumors had benign features (well-encapsulated, low mitotic rate, and no evidence of metastasis), and prominent medullary differentiation including the presence of Hassall's corpuscles, an unusual occurrence in human tumors. The main differences were in their morphological characteristics. One was histologically categorized as a mixed thymoma (type AB) composed of proliferating spindle cells and a lymphoproliferative component and the other was compatible with the WHO Type A morphology in which the neoplastic component was limited to a neoplastic spindle cell population.

\section{Spleen}

Anatomical differences in the structure of the spleen in macaques as compared to rodents are worth mentioning. In macaques, the spleen is strongly sinusal with well-defined medullary cords (syn. cords of Billroth) and sinuses like humans. In humans and Old World monkeys, splenic sinuses are lined by modified sinus-lining cells called littoral cells (LC). LC have multilineage expressions patterns with endothelial and monocyte/macrophage/dendritic cell antigen expression patterns suggesting increased plasticity of function. LC play a major role in regulating the cellular composition of the systemic circulation by regulating which red blood cells (RBC) or leukocytes will pass back into venous circulation or be destroyed. When sinusoidal lining cells become reactive, for example during endotoxemia or sepsis, the cords appear almost epithelial116 (Table 19).

Table 19. Microscopic Findings of the Hematopoietic and Lymphoid System: Spleen; Non-human Primate

\begin{tabular}{|c|c|c|c|c|}
\hline Spleen & Common & Uncommon & $\begin{array}{c}\text { Not Observed but } \\
\text { Potentially Relevant }\end{array}$ & Not Applicable \\
\hline \multicolumn{5}{|l|}{ Non-proliferative } \\
\hline Accumulation, acidophilic material, germinal center * & $\mathrm{x}$ & & & \\
\hline Accumulation, adipocyte & & & $\mathrm{x}$ & \\
\hline Aggregates, macrophage, increased & $\mathrm{x}$ & & & \\
\hline Angiectasis & & $\mathrm{x}$ & & \\
\hline Apoptosis, increased, lymphocyte & $\mathrm{x}$ & & & \\
\hline Cellularity, decreased, lymphocyte & $\mathrm{x}$ & & & \\
\hline Cellularity, decreased, red pulp & $\mathrm{x}$ & & & \\
\hline Cellularity, decreased, white pulp & & $\mathrm{x}$ & & \\
\hline Cellularity, increased, macrophage & & $\mathrm{x}$ & & \\
\hline Congestion $^{\wedge}$ & & $\mathrm{x}$ & & \\
\hline Contraction & & & & $\mathrm{x}$ \\
\hline Ectopic tissue, spleen ${ }^{\wedge}$ & $\mathrm{x}$ & & & \\
\hline Erythrophagocytosis $^{1}$ & $\mathrm{x}$ & & & \\
\hline Fibrosis & & $\mathrm{x}$ & & \\
\hline Hypertrophy, macrophage & & & $\mathrm{x}$ & \\
\hline Necrosis, lymphocyte & & $\mathrm{x}$ & & \\
\hline Pigment, increased, macrophage & & $\mathrm{x}$ & & \\
\hline Tingible body macrophages, increased & & $\mathrm{x}$ & & \\
\hline \multicolumn{5}{|l|}{ Proliferative (non-neoplastic) } \\
\hline Cellularity, increased, adipocyte & & $\mathrm{x}$ & & \\
\hline Cellularity, increased, lymphocyte & & $\mathrm{x}$ & & \\
\hline Cellularity, increased, macrophage & & $\mathrm{x}$ & & \\
\hline Cellularity, increased, mast cell & & & $\mathrm{x}$ & \\
\hline Cellularity, increased, mesothelial cell & & $\mathrm{x}$ & & \\
\hline Cellularity, increased, plasma cell, red pulp & & $\mathrm{x}$ & & \\
\hline Cellularity, increased, plasma cell, white pulp & & $\mathrm{x}$ & & \\
\hline Cellularity, increased, stromal cell & & $\mathrm{x}$ & & \\
\hline Cellularity, increased, white pulp & & $\mathrm{x}$ & & \\
\hline Compound follicle * & $\mathrm{x}$ & & & \\
\hline Extramedullary hematopoiesis, increased $^{\wedge}$ & & $\mathrm{x}$ & & \\
\hline Hyperplasia, nodular & & $\mathrm{x}$ & & \\
\hline Hyperplasia, stromal cell * & & $\mathrm{x}$ & & \\
\hline
\end{tabular}


Accumulation acidophilic material, germinal center:

Spleen (Figure 87)

\section{Additional modifiers}

Eosinophilic, acidophilic, hyalinized

\section{Other term(s)}

Hyalinized germinal centers; acidophilic material ${ }^{145}$; centrofollicular hyalinosis ${ }^{61}$; amorphous eosinophilic material7.

\section{Pathogenesis/Cell of Origin}

Unknown, presumed to represent products of the immune reactions such as antigen-antibody complexes. Increased eosinophilic PAS-positive extracellular germinal center deposits, confirmed by electron microscopy to be non-amyloid, have been associated with follicle involution post-SIV-induced follicular hyperplasia ${ }^{171}$.

\section{Diagnostic features}

- Amorphous extracellular deposits within lymphoid follicle germinal centers stain eosinophilic in H\&E stained sections.

- PAS positive staining

- These deposits can have a granular or hyalinized appearance.

\section{Special Techniques for Diagnostics}

- Differentiated from amyloid with histochemical stains such as Congo red or Thioflavin $\mathrm{T}$ or with electron microscopy.

- Differentiated from collagen with Masson's trichrome stain.

- Immunohistochemical stains for kappa and lambda immunoglobulin light chains can be performed. This material often stains positive for $\operatorname{IgM}$.

Comments: Deposition of eosinophilic material is frequently seen in lymphoid follicle germinal centers in clinically normal juvenile cynomolgus monkeys used in general safety assessment studies $6,11,61$. The material is generally considered to represent products of the immune reactions such as immunoglobulin.

\section{Compound follicle: Spleen (Figure 88-89)}

\section{Modifiers}

Lymphoid

\section{Other $\operatorname{term}(\mathrm{s})$}

Giant lymphoid follicles ${ }^{116}$; atypical lymphoid follicles ${ }^{172}$; nodular hyperplasia; lymphoid follicle ${ }^{11}$; coalescing of lymphoid follicles

\section{Pathogenesis/Cell of Origin}

Unknown

\section{Diagnostic features}

- Large, variably-shaped, irregular lymphoid follicles and bizarre shaped germinal centers. Often appear to be the result of two coalescing lymphoid follicles ${ }^{145}$.

- In some giant follicles, the centers may contain amorphous eosinophilic or acidophilic extracellular material and/or small central pale areas of lymphocytic necrosis, fibrosis, and/or macrophage accumulation. Other follicles in the surrounding spleen are normal in size and structure.

- On gross evaluation, these giant irregular follicles can be recognized as single or multiple raised pale foci on cut surface. They can cause slight compression of surrounding tissue normal splenic tissue or bulging of the splenic capsule.

\section{Differential Diagnoses}

- Increased number and size, lymphoid follicle, germinal centers

- Diffuse, increase in the number and size of lymphoid follicle, most of which have normal architectural features, including well-defined germinal centers with a surrounding mantle/marginal zone.

Comments: These giant follicles occur in both wild-caught and purpose-bred cynomolgus monkeys ${ }^{116}$. While the pathogenesis is considered unknown, it has been suggested that these exuberant lymphoid follicles may be the result of chronic parasitic infestation (including malaria), bacterial or viral infection.

They occur in low frequency and are typically surrounded by normal lymphoid follicles and other normal splenic components. There is no evidence to support that these lesions progress to lymphoma ${ }^{11}$.

Hyperplasia, stromal cell: Spleen (No image provided)

\section{Modifiers}

Nodular

\section{Other $\operatorname{term}(\mathrm{s})$}

Reticular networks; fibroplasia; hamartoma; nodular hyperplasia (red pulp)

\section{Pathogenesis/Cell of Origin}

Much of what is known about nonhematopoietic splenic stromal cells is derived from developmental studies in rodents and humans, and to some degree macaques. A complete discussion on stromal cell biology is beyond the scope of this article. Stromal cells play an important role in regulating hematopoietic stem cell (HSC) niches, directing lymphoid traffic, modulating immune responses, and shaping the development and compartmentalization of the spleen ${ }^{173-175}$. Studies in humans have revealed phenotypically and morphologically distinct subsets of stromal cells that are uniquely different from those described in mice176. This suggests that species differences must be taken into account when assessing the spleen. 


\section{Diagnostic features}

- Dense aggregates of well differentiated plump to spindle cells with abundant cytoplasm and round to oval nuclei; mitoses may be frequent.

- Surrounded by normal red pulp

\section{Differential Diagnoses}

- Sarcomas are typically composed of unencapsulated to infiltrating bundles of poorly differentiated pleomorphic neoplastic cells often with a high mitotic index.

- Hamartoma is a benign growth consisting of an abnormal collection of heterogenous cells or tissue types normally located in that particular region of the body.

\section{Special Techniques for Diagnostics}

- Histology, Immunohistochemistry

Comments: Lymphoid-associated stromal cell hyperplasia is considered here as being distinct from reticuloendothelial hyperplasia, which is regarded as a separate entity that involves cells of the immune (monocyte) system in the splenic red pulp.

\section{Lymph Node}

Typically examined lymph nodes in toxicologic pathology studies include a section of mandibular and mesenteric lymph node but other nodes may be sampled as indicated by the study design or target biology. Ideally, a standardized midsagittal section of lymph node should be obtained to include all the lymph node compartments, which constitute the follicles and interfollicular cortex, the paracortex (or deep cortex) and the medulla, including medullary cords and medullary sinuses ${ }^{177}$ (Table 20).

\section{Cellularity, increased, dendritic cells: Lymph Node} (No image provided)

\section{Other term(s) [cell type]}

Antigen presenting cells; conventional type 1 DCs; conventional type 2 DCs; plasmacytoid DCs; interdigitating dendritic cell

\section{Pathogenesis/Cell of Origin}

Increased numbers of dendritic cells have been observed in a variety of conditions in nonhuman primates.

\section{Diagnostic features}

- Dendritic cells (DCs) are potent antigen-presenting cells found throughout the body in mucosal sites and skin. They are also abundant within lymph nodes, especially mesenteric lymph nodes where they play a pivotal role in linking innate and adaptive immunity.

- Dendritic cells are characterized by fine cytoplasmic extensions.

Table 20. Microscopic Findings of the Hematopoietic and Lymphoid System: Lymph node; Non-human Primate

\begin{tabular}{|c|c|c|c|c|}
\hline Lymph node & Common & Uncommon & $\begin{array}{c}\text { Not Observed but } \\
\text { Potentially Relevant }\end{array}$ & Not Applicable \\
\hline \multicolumn{5}{|l|}{ Non-proliferative } \\
\hline Aggregates, increased, macrophage & & $\mathrm{x}$ & & \\
\hline Apoptosis, increased, lymphocyte & & $\mathrm{x}$ & & \\
\hline Cellularity, decreased, lymphocyte & & $\mathrm{x}$ & & \\
\hline Dilatation, lymphatics & & $\mathrm{x}$ & & \\
\hline Dilatation, sinus & $\mathrm{x}$ & & & \\
\hline Erythrocytes, intrasinusoidal & $\mathrm{x}$ & & & \\
\hline Erythrophagocytosis $^{1}$ & $\mathrm{x}$ & & & \\
\hline 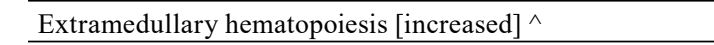 & $\mathrm{x}$ & & & \\
\hline Fibrosis $\wedge^{\wedge}$ & & $\mathrm{x}$ & & \\
\hline Hypertrophy, interdigitating cells & & & & $\mathrm{x}$ \\
\hline Increased/dilated, blood vessels & & & $\mathrm{x}$ & \\
\hline Necrosis, lymphoid & & $\mathrm{x}$ & & \\
\hline \multicolumn{5}{|l|}{ Proliferative (non-neoplastic) } \\
\hline Cellularity, increased, dendritic cell * & & $\mathrm{x}$ & & \\
\hline Cellularity, increased, interdigitating dendritic cells ${ }^{2}$ & & & & $\mathrm{x}$ \\
\hline Cellularity, increased, lymphocyte & $\mathrm{x}$ & & & \\
\hline Cellularity, increased, macrophage, intrasinusoidal & $\mathrm{x}$ & & & \\
\hline Cellularity, increased, mast cell & & & $\mathrm{x}$ & \\
\hline Cellularity, increased, plasma cell & $\mathrm{x}$ & & & \\
\hline Cellularity, increased, stromal cell & & $\mathrm{x}$ & & \\
\hline Hyperplasia, angiomatous & & $\mathrm{x}$ & & \\
\hline Hypertrophy/hyperplasia high endothelial venules (HEVs) & & $\mathrm{x}$ & & \\
\hline
\end{tabular}




\section{Differential Diagnoses}

- Macrophages

- Have phagocytic capabilities and often have cytoplasmic vacuoles.

- Macrophages can be detected using anti-CD163, antiCD68, and HAM56 antibodies.

\section{Special Techniques for Diagnostics}

- Using immunofluorescence, DCs were identified based on their morphology including dendritic morphology.

- Flow cytometry, immunohistochemistry (CD103, CD11c, CD163, CD68, HAM56)

- The debate over how best to adequately identify DCs in blood and tissue has evolved with improvements in monoclonal antibodies and flow cytometry strategies. One study evaluated DCs in rhesus lymph nodes using antihuman Fascin (p55), a marker for actin protein located within dendrites, in combination with the markers CD123, p55, and CD11c.

Comments: This diagnosis should only be used when dendritic cell origin is confirmed with immunohistochemical stains. Increased numbers of dendritic cells have been observed in a variety of conditions in nonhuman primates. For example, monkeys administered Flt3 ligand had increased numbers of pre-DC subsets in lymphoid tissue 178. A similar study showed a marked increase in nodal DCs following administration of supraphysiologic doses of Flt3-Ligand to SIV- and SHIV-infected macaques ${ }^{179}$. Other studies involving SIV-infected macaques have shown that acute SIV infection is followed by depletion of circulating myeloid DCs and a paradoxical accumulation of immature DC within lymph nodes ${ }^{180}$.

A complete review of DCs is beyond the scope of this manuscript. Recent studies using unsupervised high-end dimensional analysis ${ }^{181}$ have provided a new framework for identifying dendritic cells across tissues in several species including macaques. The reader is referred to these studies $^{182}$. In routine studies without special stains, the diagnosis of increased cellularity, macrophage should be used for this finding.

\section{E. Mucosa-Associated Lymphoid Tissues (MALT)}

The form and function of the mucosal immune system begins during prenatal development and changes dramatically with time in response to a number of environmental and physiological pressures 183,184 . Specific anatomic features that distinguish MALT structures histologically from other secondary lymphoid tissues include the lack of a defined capsule and afferent lymphatics, the presence of specialized follicle-associated epithelium (FAE) and high endothelial venules. FAE may appear flattened, lack cilia and contain few to no goblet cells. The characteristic histology of normal MALT $141,183,185$ should be differentiated from cellular infiltrates and inflammation. Mucosal immunity has been extensively studied in macaques and thus the anatomical features and cellular composition of major mucosal associated lymphoid tissue sites have been described ${ }^{141}$. Tonsils are also an important component of the oropharyngeal mucosal associated lymphoid tissues. Similar to humans, NHP have at least 3 sets of tonsils, the lingual, palatine, and pharyngeal (nasopharyngeal), and may also have tubal tonsils, but descriptions of tubal tonsils in NHP are lacking. The palatine and lingual tonsils are the most commonly evaluated, the pharyngeal tonsil is equivalent to the adenoid in humans. These are all classified as follicular tonsils which refers to tonsils with crypts as opposed to those without. Tonsilar crypts are invaginations of the surface epithelium into the submucosal lymphoid tissue $^{141}$. Studies describing the microscopic and ultrastructural anatomy of the lingual tonsil in cynomolgus monkeys have been published ${ }^{186}$. Just briefly, tonsillar units are arranged in a rosary fashion below a slit-like mucosal fold forming a blind-ended pouch. Crypt epithelium is generally non-keratinized and has moderate numbers of infiltrating intraepithelial lymphocytes and dendritic cells. The lumen contained a mixture of exfoliated epithelial cells, leukocytes and bacteria.

Unlike rodents, lymphoid tissue in mucosal and non-mucosal tissues is frequently identified in NHP, dogs and swine used in nonclinical studies. In nonclinical studies, MALT is generally recognized in the bronchus or bronchioles, small and large intestine, nasal cavity and tonsils, and is less frequently recognized as part of the normal MALT of the trachea, oral cavity, stomach, gallbladder, eye (uvea, sclera and conjunctiva also referred to as conjunctiva associated lymphoid tissue, CALT), eustachian tube, urinary bladder, urethra and vagina ${ }^{122,}$ 142, 183, 187, 188. Duct-associated (non-mucosal) lymphoid tissue (DALT) is routinely recognized in the monkey parotid salivary gland 189 but may also be found in all glandular tissues (Table 21).

\section{Multinucleate syncytial cell/lymphocytic syncytia: Muco- sal-Associated Lymphoid Tissue (Figure 90)}

\section{Other term(s)}

Warthin-Finkeldey cells

\section{Pathogenesis/Cell of Origin}

The origin of the multinucleate syncytial cells is not definitively known, but immunophenotyping suggests a lymphocytic or dendritic cell origin. It has been postulated that they form by cell fusion or abnormal nuclear division.

\section{Diagnostic features}

- Multinucleate giant cells of Warthin-Finkeldey type are frequently observed in the MALT of the larynx, BALT of the lung and the GALT of the large intestines. The syncytial cells are typically present in the lymphoid follicle germinal centers.

- Nuclei are typically round or oval and closely packed in the center of the cell. Individual nuclei are generally similar in size and appearance to those of surrounding lymphoid cells. The cells have relatively little cytoplasm. 
Table 21. Microscopic Findings of the Hematopoietic and Lymphoid System: Mucosal associated lymphoid tissue; Non-human Primate

\begin{tabular}{|c|c|c|c|c|}
\hline Mucosa-associated Lymphoid Tissue & Common & Uncommon & $\begin{array}{c}\text { Not Observed but } \\
\text { Potentially Relevant }\end{array}$ & Not Applicable \\
\hline \multicolumn{5}{|l|}{ Non-proliferative } \\
\hline Aggregates, macrophage & $\mathrm{x}$ & & & \\
\hline Apoptosis, increased, lymphocyte & & $\mathrm{x}$ & & \\
\hline Cellularity, decreased, lymphocyte & $\mathrm{x}$ & & & \\
\hline Degeneration, follicle-associated epithelium & & $\mathrm{x}$ & & \\
\hline Dilatation, lymphatics & & $\mathrm{x}$ & & \\
\hline High endothelial venules (HEV), decreased & & $\mathrm{x}$ & & \\
\hline Hyaline material & $\mathrm{x}$ & & & \\
\hline Hypertrophy, macrophage & $\mathrm{x}$ & & & \\
\hline Multinucleate syncytial cell/lymphocytic syncytia* & $\mathrm{x}$ & & & \\
\hline \multicolumn{5}{|l|}{ Proliferative (non-neoplastic) } \\
\hline Cellularity, increased, lymphocyte & $\mathrm{x}$ & & & \\
\hline Cellularity, increased, macrophage & & & $\mathrm{x}$ & \\
\hline Hyperplasia, follicle-associated epithelium & & $\mathrm{x}$ & & \\
\hline Hyperplasia, goblet cell, follicle-associated epithelium & & & $\mathrm{x}$ & \\
\hline Hypertrophy/hyperplasia high endothelial venule (HEV) & & $\mathrm{x}$ & & \\
\hline Metaplasia squamous, follicle-associated epithelium & & $\mathrm{x}$ & & \\
\hline
\end{tabular}

The nuclei occupy most of the space and mold together to take on various shapes.

- No lymphocyte necrosis or associated inflammation, or viral inclusions are present.

\section{Differential Diagnoses}

- Infectious lymphadenopathy due to various viral etiological agents (LCV, cytomegalovirus, Herpes B virus, measles morbillivirus) which exhibit increased lymphoid cellularity, and increased immunoblasts, Reed Sternberg cells and/or lymphocyte necrosis.

- Granulomatous inflammation with multinucleate giant cells of Langerhan's giant cell type of macrophage origin. Nuclei are arranged either around the periphery of the cell in the form of a horseshoe or ring or cluster are the two poles of the cell. Can be a response to a foreign body material, non-living (particulate) or living cells (fungi or bacteria), or endogenous material (cholesterol, keratin, or crystals).

- Other multinucleated or giant cells in tissues: circulating megakaryocytes, osteoclasts, cells with cytoplasmic inclusions.

\section{Special Techniques for Diagnostics}

- Immunohistochemistry can be used to further characterize the syncytial giant cells.

Comments: The immunophenotype of these lymphoid multinucleate giant cells has not been well studied in cynomolgus monkeys. In humans, immunohistochemistry studies have provided discordant results, with some reports of a T-helper cell phenotype and other of follicular dendritic cell origin. Lymphoid multinucleate giant cells of WarthinFinkeldey type have been experimentally produced with inoculation of wild type measles virus in monkeys and are observed in natural measles infections in humans. However, these syncytia are also found in apparently healthy cynomolgus monkeys in nonclinical studies. The cause of these syncytia in NHP are unknown although these animals are free of measles and other active viral diseases ${ }^{6}$.

\section{F. Other Lymphoid Tissues (Tertiary Lymphoid Structures [TLS] and Serosa-associated Lymphoid Clusters [SALCs]) (Table 22)}

Table 22. Microscopic Findings of the Hematopoietic and Lymphoid System: Other lymphoid tissues; Non-human Primate

\begin{tabular}{|c|c|c|c|c|}
\hline Other Lymphoid Structures & Common & Uncommon & $\begin{array}{l}\text { Not Observed but } \\
\text { Potentially Relevant }\end{array}$ & Not Applicable \\
\hline \multicolumn{5}{|l|}{ Non-proliferative } \\
\hline Cellularity, increased lymphocyte, adipose tissue & $\mathrm{x}$ & & & \\
\hline Lymphoid follicle formation (TLS) $^{1}$ & $\mathrm{x}$ & & & \\
\hline SALCs, increased $^{2}$ & & & & $\mathrm{x}$ \\
\hline Tertiary Lymphoid Structures (TLSs) ${ }^{3}$ & & & & $\mathrm{x}$ \\
\hline
\end{tabular}




\section{Hepatobiliary System}

For detailed general considerations on the hepatobiliary system, refer to the INHAND rodent publication ${ }^{190}$.

Liver and gall bladder pathology diagnostic terms for nonhuman primates have been covered in detail in other publications ${ }^{11,191,192}$. Non-proliferative abnormalities of the liver and gall bladder can be observed spontaneously in young cynomolgus macaques used in nonclinical toxicity studies, particularly mononuclear cell infiltrate, vacuolation, necrosis, and pigment as well as cellular infiltrates in the gall bladder.

\section{A. Liver}

The liver of the cynomolgus monkey has four lobes: the median (the largest), the right and left lateral, and the caudate lobes. The gall bladder is long and fusiform and lies within a fossa of the inferior surface of the right median lobe ${ }^{193}$. The microscopic and functional anatomy of the liver is similar across species used in nonclinical safety studies (rodents, dogs, and primates) as well as in humans ${ }^{194}$. Recommended routine sections include one sample from the right medial lobe to include gallbladder and adjacent liver parenchyma, and one additional section from the left lateral lobe (Table 23).

\section{Bile plug: Liver (Figure 91)}

\section{Other term(s)}

Cholestasis; icterus; pigment; pigment deposition

\section{Pathogenesis/Cell of Origin}

Obstruction of bile flow, Hepatocellular injury

\section{Diagnostic features}

- Green-brown bile pigment, primarily bilirubin, in hepatic bile caniculi

- Pigment may appear in Kupffer cells (after rupture of bile caniculi)

\section{Differential Diagnoses}

- Other pigments, including hemosiderin, lipofuscin, porphyrin, hematoxylin, and formaldehyde:

- A comprehensive discussion of differentiation is included in the rodent manuscript under pigmentation ${ }^{190}$.

- Proof of bile pigment can be achieved by special stains (e.g. iodine test or Hall's test).

Cytoplasmic alteration: Liver (No image provided)

\section{Other term(s)}

Granular change; ground glass cytoplasm

\section{Pathogenesis/Cell of Origin}

Typically xenobiotic-induced, and may be associated with hepatocellular hypertrophy

\section{Diagnostic features}

- Hepatocytes characterized by presence of abundant eosinophilic, amorphous cytoplasm, with uniformly granular appearance

- Affected hepatocytes may be enlarged, and exhibit a zonal distribution.

Comments: The modifier "ground glass" should be used to describe the characteristic light microscopic appearance of the cytoplasm in hypertrophic hepatocytes as a result of enzyme induction following administration of xenobiotics ${ }^{190}, 195$. Hypertrophic hepatocytes are characterized by enlargement with amorphous, eosinophilic cytoplasm. This characteristic appearance is caused by proliferation of the smooth endoplasmic reticulum (SER) which can be confirmed by electron microscopy or immunohistochemistry. Similar changes are more commonly observed in rodents, and xenobiotics which induce hepatocellular hypertrophy in rats rarely induce similar changes in non-human primates $^{194 .}$

\section{Dissociation, hepatocyte: Liver (Figure 92)}

\section{Pathogenesis/Cell of Origin}

Acute degenerative change

\section{Diagnostic features}

- Characterized by hepatocytes with rounded cell margins that have lost intercellular attachments ${ }^{196}$

- Centrilobular areas with acute circulatory failure

\section{Special Techniques for Diagnostics}

Cytoskeletal integrity can be assessed by staining liver sections with Gomori's method for reticulin fibers.

Comments: Hepatocellular dissociation can result from tissue hypoxia or direct damage to cytoskeletal filaments ${ }^{196}$ and has been observed in cases of acute circulatory failure. Euthanasia with barbiturates can also induce a similar change in monkeys which is reported as barbiturate lysis 197 . The term hepatocellular dissociation should be preferred, and the cause should be discussed in the narrative or as a comment.

\section{Erythrophagocytosis, Kupffer cell: Liver (Figure 93)}

\section{Pathogenesis/Cell of Origin}

Spontaneous background finding (only if present at very low incidence) or due to malaria infection.

\section{Diagnostic features}

- Multifocal Kupffer cells with intracytoplasmic erythrocytes or intravascular hemolysis-associated erythrocyte "ghosts" or presence of hemozoin (malaria pigment) in the Kupffer cells.

Comments: Kupffer cell erythrophagocytosis often results 
Table 23. Microscopic Findings of the Hepatobiliary System: Liver; Non-human primate

\begin{tabular}{|c|c|c|c|c|}
\hline Liver & Common & Uncommon & $\begin{array}{l}\text { Not Observed but } \\
\text { Potentially Relevant }\end{array}$ & Not Applicable \\
\hline \multicolumn{5}{|l|}{ Congenital } \\
\hline Hepatodiaphragmatic nodule & & & & $\mathrm{x}$ \\
\hline \multicolumn{5}{|l|}{ Non-proliferative } \\
\hline Amyloid^^ & & $\mathrm{x}$ & & \\
\hline Angiectasis & & $\mathrm{x}$ & & \\
\hline Apoptosis^\# & $\mathrm{x}$ & & & \\
\hline Apoptosis/single cell necrosis ${ }^{\wedge}$ & & $\mathrm{x}$ & & \\
\hline Atrophy, hepatocyte & & $\mathrm{x}$ & & \\
\hline Basophilic granules, Kupffer cells ${ }^{\wedge}$ & & $\mathrm{x}$ & & \\
\hline Bile plug *1 & & $\mathrm{x}$ & & \\
\hline Congestion $^{\wedge}$ & & $\mathrm{x}$ & & \\
\hline Crystals & & & $\mathrm{x}$ & \\
\hline Cyst, bile duct (hepatic cysts) & & & $\mathrm{x}$ & \\
\hline Cytoplasmic alteration, Modifier: ground glass * & & $\mathrm{x}$ & & \\
\hline Degeneration, cystic & & $\mathrm{x}$ & & \\
\hline Degeneration, hepatocyte, hydropic \# & & $\mathrm{x}$ & & \\
\hline Dissociation, hepatocyte* & & $\mathrm{x}$ & & \\
\hline Erythrophagocytosis, Kupffer cell * & & $\mathrm{x}$ & & \\
\hline Extramedullary hematopoiesis $\wedge$ & $\mathrm{x}$ & & & \\
\hline${\text { Fatty change (see Vacuolation })^{2}}^{2}$ & & & & $\mathrm{x}$ \\
\hline Fibrosis $\wedge$ & & $\mathrm{x}$ & & \\
\hline Helicobacter spp. Hepatitis & & & & $\mathrm{x}$ \\
\hline Hypertrophy, hepatocyte \# & & $\mathrm{x}$ & & \\
\hline Hypertrophy, Kupffer cells \# & & $\mathrm{x}$ & & \\
\hline Hypertrophy/karyomegaly, endothelial cell & & $\mathrm{x}$ & & \\
\hline Inclusions, intranuclear and cytoplasmic ${ }^{\wedge}$ & & $\mathrm{x}$ & & \\
\hline Infarct & & $\mathrm{x}$ & & \\
\hline${\text { Infiltrate, [insert appropriate cell type }]^{\wedge}}$ & $\mathrm{x}$ & & & \\
\hline Infiltrate, mixed cell & $x$ & & & \\
\hline Infiltration, mononuclear cell & $x$ & & & \\
\hline Infiltration, neutrophil & & $x$ & & \\
\hline Infiltration, peribiliary (intrahepatic) & $x$ & & & \\
\hline Inflammation $^{\wedge}$ & & $\mathrm{x}$ & & \\
\hline Mouse hepatitis virus hepatitis & & & & $\mathrm{x}$ \\
\hline Murine norovirus hepatitis & & & & $\mathrm{x}$ \\
\hline Inflammation, granuloma/microgranuloma $*$ & & $\mathrm{x}$ & & \\
\hline Intrahepatocellular erythrocytes & & & $\mathrm{x}$ & \\
\hline Intravascular hepatocytes & & & $\mathrm{x}$ & \\
\hline Karyocytomegaly & & $\mathrm{x}$ & & \\
\hline Metaplasia, glandular, hepatocyte & & & $\mathrm{x}$ & \\
\hline Metaplasia, pancreatic acinar cell & & & $\mathrm{x}$ & \\
\hline Mineralization ^^ & & $\mathrm{x}$ & & \\
\hline Multinucleated hepaptocytes & $\mathrm{x}$ & & & \\
\hline Necrosis, focal/multifocal & & $\mathrm{x}$ & & \\
\hline Single cell necrosis ${ }^{\wedge}$ & $\mathrm{x}$ & & & \\
\hline Necrosis, zonal \# (centrilobular, midzonal, periportal, diffuse) & & $\mathrm{x}$ & & \\
\hline Parasite $\wedge(\text { Echinococcus cyst *; Cryptosporidia } \text { spp } *)^{*}$ & & $\mathrm{x}$ & & \\
\hline Phospholipidosis^^ & & $\mathrm{x}$ & & \\
\hline Pigment $^{\wedge}\left(\right.$ protoporphyrin $\left.^{*}\right)$ & & $\mathrm{x}$ & & \\
\hline Rarefaction, cytoplasmic ${ }^{3}$ & $\mathrm{x}$ & & & \\
\hline Thrombus & & $\mathrm{x}$ & & \\
\hline Tyzzer's disease (Clostridium piliforme infection) & & & & $\mathrm{x}$ \\
\hline Vacuolation, hepatocyte *\# & & $\mathrm{x}$ & & \\
\hline Vacuolation, Ito cells & & & $\mathrm{x}$ & \\
\hline \multicolumn{5}{|l|}{ Proliferative (non-neoplastic) } \\
\hline Cholangiofibrosis & & & $\mathrm{x}$ & \\
\hline Focus of cellular alteration & & & $\mathrm{x}$ & \\
\hline Hyperplasia, bile duct & & $\mathrm{x}$ & & \\
\hline Hyperplasia, hemangioendothelial & & $\mathrm{x}$ & & \\
\hline Hyperplasia, hepatocyte, non-regenerative & & $\mathrm{x}$ & & \\
\hline Hyperplasia, hepatocyte, regenerative & & $\mathrm{x}$ & & \\
\hline Hyperplasia, Ito cell & & & $\mathrm{x}$ & \\
\hline Hyperplasia, oval cell & & $\mathrm{x}$ & & \\
\hline Hypertrophy/hyperplasia, Kupffer cell & & $\mathrm{x}$ & & \\
\hline \multicolumn{5}{|l|}{ Neoplastic } \\
\hline Adenoma, hepatocyte & & $\mathrm{x}$ & & \\
\hline Carcinoma, hepatocyte & & $\mathrm{x}$ & & \\
\hline
\end{tabular}

* indicates terminology with diagnostic criteria or comments described below. \# indicates findings that are more frequently observed as an induced change. $\wedge^{\wedge}$ indicates new terms that are addressed in the Systemic Pathology section. ${ }^{1}$ Bile plug is the preferred term, instead of pigment, to describe the pale green-brown plugs within the bile canaliculi. ${ }^{2}$ The descriptive term vacuolation is preferred to the term fatty change used in the rodent terminology since the diagnosis is normally based on evaluation of the H\&E stained sections. ${ }^{3}$ The term rarefaction is the preferred term to describe the irregularly and poorly defined clear spaces in the cytoplasm due to glycogen accumulation. 
when erythrocytic membranes become coated with antibodies and are thus targeted for removal by reticuloendothelial cells ${ }^{198}$.

This finding is of potential relevance in toxicologic studies since phagocytosis of erythrocytes by monocyte derived Kupffer cells is associated with depression of macrophage function including the ability to phagocytize and kill bacteria ${ }^{198,} 199$. The functional impairment likely occurs due to interactions between erythrocyte components (including hemoglobin and reactive products of oxygen metabolism) and reactive products of phagocytes resulting in inhibition of oxidative bactericidal mechanisms ${ }^{200}$. The consequence of such impairment is increased susceptibility to bacterial infection and endotoxic shock.

Erythrophagocytosis by Kupffer cells has been well documented and investigated in animal models ${ }^{201}$ and humans.

In cynomolgus monkey, erythrophagocytosis or presence of hematoidin was described in monkey infected with Plasmodium spp ${ }^{202}$.

Inflammation, granuloma/microgranuloma: Liver (Figure 94-95)

\section{Other term(s)}

Microgranuloma; granulomatous inflammation

\section{Pathogenesis/Cell of Origin}

Various etiologies: single cell necrosis of hepatocytes, tuberculosis, Hepatocystis kochi ${ }^{203}$

\section{Diagnostic features}

- Granulomas are characterized by central area of eosinophilic cellular and karyorrhectic debris (necrosis) admixed with degenerated neutrophils, and surrounded by macrophages, lymphocytes and plasma cells.

- Fibroblasts and fibrous connective tissue may circumscribe the granuloma.

- Epithelioid macrophages, Langhans and foreign body type multinucleated giant cells can be present.

- Microgranuloma are small and consist of presence of inflammatory cells, mainly macrophages, lymphocytes, and small numbers of plasma cells.

- Microgranuloma can be associated with single cell necrosis of hepatocytes.

Comments: Microgranulomas are of much lower incidence in cynomolgus monkeys than in other laboratory animals ${ }^{11}$. In some cases, microgranuloma is used as Other Term of inflammatory cell infiltrate. The term microgranuloma should be used in case of small aggregate with predominant macrophage and lymphocytic component.

\section{Parasite (Cryptosporidia spp.): Liver/Gall bladder}

(Figure 96-97)

\section{Pathogenesis/Cell of Origin}

A protozoal infestation of the digestive system which may extend to the gall bladder, common bile duct and/or intrahepatic bile ducts.

\section{Diagnostic features}

- Cryptosporidial organisms attached to the luminal surface of the mucosal epithelium.

- Organisms are approximately 2-4 $\mu \mathrm{m}$ in diameter, weakly eosinophilic with HE stain and deep blue with Giemsa stain.

- Infested bile ducts may be prominent with increased mucosal folds, increased periductal connective tissue, thickened lamina propria due to inflammatory cell infiltrates (primarily lymphocyte, plasma cells and monocytes).

- Epithelial hyperplasia may be present.

- Intraductal neutrophilic exudates and/or luminal cellular debris may be present.

\section{Differential Diagnosis}

- Enterocytozoon bieneusi infection

Comments: Cryptosporidium infestation is reported to occur at a relatively low incidence in laboratory macaques. Infection is often of limited or no clinical significance in the immunocompetent host; however, infection can disseminate and cause disease in immunosuppressed or juvenile animals ${ }^{204-207}$. Cryptosporidia are most commonly reported in the stomach; however, there are report of hepatobiliary infection in juveniles and macaques experimentally infected with acquired immunodeficiency syndrome (AIDS) $208,209$.

\section{Parasite (Echinococcus cyst) : Liver (Figure 98-100)}

\section{Other term(s)}

Hydatid disease; hydatidosis; echinococcal disease

\section{Pathogenesis/Cell of Origin}

Zoonotic infestation caused by the larval stage of the tapeworms Echinococcus (E.) multilocularis (alveolar echinococcosis) or E. granulosus (cystic echinococcosis).

\section{Modifiers}

Cystic echinococcosis or alveolar echinococcosis

\section{Diagnostic features}

- Unilocular (E. granulosus) or multilocular (E. multlocularis) cysts in the liver210, 211 .

- Cystic wall has three different layers: outer layer consisting of fibrous tissue and inflammatory cells, intermediate layer is an avascular laminated membrane, and the inner germinal layer gives rise to brood capsules with protoscolices attached by short stalk in infectious (fer- 
tile) cysts.

- Cysts may occur in liver, lung or vertebra.

Comments: Serodiagnosis (ELISA) is used to distinguish E. multilocularis from E. granulosus infections ${ }^{212}$. E. granulosus and multilocularis are endemic in Europe, E granulosus endemic in China. Both are important zoonoses.

Pigment ([proto]porphyrin): Liver (Figure 101)

\section{Modifier}

Intercanalicular

\section{Pathogenesis/Cell of Origin}

(Proto) porphyrias are rare pathologic conditions caused by inherited or induced enzyme defects at different stages of the heme biosynthesis causing porphyrin precursors to accumulate. Morphologically, this accumulation of protoporphyrins can become visible as a typical intra- and extracellular pigment.

\section{Diagnostic features}

- Multifocal globular pigment deposits within the bile canaliculi and within the hepatocellular cytoplasm.

- The pigment often is dark brown-red in color.

- This typical pigment sometimes can also be found within the cytoplasm of Kupffer cells and macrophages.

- Pigment deposits are birefringent and often show the characteristic "Maltese Cross"-like appearance under polarized light.

- True bile plugs can sometimes be found additionally.

- Mononuclear infiltrates may accompany the condition.

\section{Special Techniques for Diagnostics}

- Fluorescence microscopy of fresh liver tissue at wavelengths between 395-408 $\mathrm{nm}$ can produce red fluorescence (emitting between 620-630 nm).

- Electron microscopy reveals a typical pattern with radiating double-lined crystalline structures, called "starburst".

- Biochemical detection of changes in enzymes that play a role in the heme synthesis (e.g. ferrochelatase, hepatic $\delta$-aminolevulinic acid synthase (ALA-S), porphobilinogen deaminase (PBGD)).

Comments: Porphyria was induced in macaques by the administration of allylisopropylacetamide 213 . In treated animals, hepatic $\delta$-aminolevulinic acid synthase (ALA-S) was increased, while Cytochrome P-450 and associated monooxygenase activities and microsomal heme oxygenase activity were decreased. Clinically, the monkeys showed elevation of porphyrin levels in blood and urine.

In human, accumulation of high levels of porphyrins in the liver is known to cause liver damage, including cirrhosis, siderosis, focal necrosis and hyperplasia. In rat, mices and dogs can be observed following administration of a number of different xenobiotics ${ }^{195}$.
Vacuolation, hepatocyte: Liver (Figure 102-104)

\section{Other $\operatorname{term}(\mathbf{s})$}

Fatty change; lipidosis; lipid; macrovescicular and/or microvescicular steatosis; phospholipidosis

\section{Pathogenesis/Cell of Origin}

Perturbation in lipid metabolism and disposition resulting in excessive accumulation of vacuoles in the cytoplasm.

\section{Modifiers}

Macrovescicular and microvescicular:

- Macrovesicular: Hepatocytes contain a large well-defined single rounded vacuole within each cell. Nucleus and cytoplasm displaced to the periphery. A few hepatocytes may contain one or more smaller vacuoles.

- Microvesicular: Hepatocytes partially or completely filled with numerous small lipid vacuoles. Affected hepatocytes may have a "foamy" appearance. Small vacuoles do not normally displace the nucleus to the periphery.

\section{Differential Diagnoses}

- Cytoplasmic rarefaction

\section{Special Techniques for Diagnostics}

Frozen sections of unfixed or fixed tissue can be stained for fat (oil red O, Sudan IV) and are helpful in recognizing microvesicular lipidosis and in differentiating fat from other substances that may accumulate in vacuoles.

Comments: The descriptive term vacuolation is preferred to the term fatty change and other etiologic terms used in the rodent terminology ${ }^{190}$, since the diagnosis is normally based on evaluation of the H\&E stained sections. The main diagnostic features as well as the differential diagnosis are the same as these described in the rodent manuscript.

Diffuse and severe vacuolation of the hepatocytes is observed in the fatal fatty liver syndrome also known as fatal fasting syndrome or fat macaque syndrome. This syndrome is described in detail in the systemic/general pathology section since the condition is considered an acute metabolic syndrome with involvement of multiple organs.

\section{B. Gall Bladder (Table 24)}

\section{Infiltrate, [insert appropriate cell type]: Gall Bladder} (No image provided)

\section{Pathogenesis/Cell of Origin}

Spontaneous background (may represent MALT)

\section{Diagnostic features}

- Focal to diffuse, frequently lymphoplasmacytic, infiltrate in the gall bladder lamina propria61.

- May have germinal centers. 
Table 24. Microscopic Findings of the Hepatobiliary System: Gall Bladder; Non-human primate

\begin{tabular}{|c|c|c|c|c|}
\hline Gallbladder and Biliary Tree & Common & Uncommon & $\begin{array}{c}\text { Not Observed but } \\
\text { Potentially Relevant }\end{array}$ & Not Applicable \\
\hline \multicolumn{5}{|l|}{ Congenital } \\
\hline Ectopic tissue, pancreas & & & $\mathrm{x}$ & \\
\hline Ectopic tissue, hepatocytes & & & $\mathrm{x}$ & \\
\hline \multicolumn{5}{|l|}{ Non-proliferative } \\
\hline Calculus & & $\mathrm{x}$ & & \\
\hline Cholecystitis $^{1}$ & & & & $\mathrm{x}$ \\
\hline Metaplasia, glandular & & & $\mathrm{x}$ & \\
\hline Hyalinosis & & & & $\mathrm{x}$ \\
\hline Infiltrate, [insert appropriate cell type] *^ & $\mathrm{x}$ & & & \\
\hline Inflammation $^{\wedge}$ & & $\mathrm{x}$ & & \\
\hline Parasite $^{\wedge}($ Enterocytozoon bieneusi $*)$ & & $\mathrm{x}$ & & \\
\hline \multicolumn{5}{|l|}{ Proliferative (non-neoplastic) } \\
\hline Hyperplasia & & $\mathrm{x}$ & & \\
\hline
\end{tabular}

Comments: Inflammatory cell infiltrates of the gall bladder have been reported in New World NHPs in association with Helicobacter sp. Infections, which have also been reported in macaques.

\section{Parasite (Enterocytozoon bieneusi): Gall Bladder}

(Figure 105-106)

\section{Pathogenesis/Cell of Origin}

Enterocytozoon bieneusi is a microsporidian parasite of primarily macaque species that causes proliferative cholecystits, choledochitis, and serositis

\section{Diagnostic features}

- Non-suppurative inflammation of the biliary tract.

- Single, sloughed epithelial cells that contain the organism are diagnostic for this infection. It is very difficult to see the organism without special stains.

- Marked proliferation of bile ducts in the portal tracts with concurrent fibrosis.

- Proliferative serositis associated with mesothelial hyperplasia and non-suppurative inflammation of the small intestine.

\section{Differential Diagnoses}

- Cryptosporidium parvum

- Ascending biliary infections are associated with biliary hyperplasia and eosinophilic to neutrophilic inflammation admixed with mononuclear cells.

- Organisms are seen attached to the apical surface of the biliary epithelial cells or free in the lumen.

- Organisms can be very numerous.

\section{Special Techniques for Diagnostics}

- Immunohistochemistry for measles matrix protein will highlight the organism. A modified Weber's trichrome is also diagnostic.

- Additional diagnostic testing can be performed using electron microscopy or PCR.

Comments: This is an uncommon opportunistic infection in immunocompromised macaques ${ }^{126}$. Recently data suggest that $M$. fascicularis can also harbor the parasite although pathogenesis studies have not been performed to date ${ }^{214}$. The proliferative serositis lesion is an uncommon manifestation ${ }^{215}$. In severe cases of E. bieneusi, grossly the liver can have an accentuated lobular pattern due to the degree of biliary hyperplasia and accompanying fibrosis. 


\section{INTEGUMENT}

For detailed general considerations on the integument system, refer to the INHAND rodent publication ${ }^{216}$. Descriptions of "vascular lesions" and "infectious diseases" are covered elsewhere (cardiovascular and systemic chapters).

In nonclinical studies, correlations between clinical observations and microscopic observations are essential in the identification of any potential interaction between xenobiotics and integument structures. The entire skin should be carefully examined at the post-mortem evaluation and all abnormalities should be recorded. As a minimum, a sample from the chest skin overlying the mammary gland should be collected and examined by conventional histopathology. Electron microscopy and immunohistochemistry may help to identify pathological processes caused by xenobiotics.

\section{A. Skin}

Cynomolgus monkeys are born with a dense black hair coat. By the age of 2 to 3 months, this hair coat starts to change into an adult type of pelage, which covers backs, legs, and arms with brown to grayish fur. The hair coat shows lesser density on the ventral aspect of the trunk ${ }^{217-219}$. The face is pinkishbrown and the fur on the head sweeps back over the forehead, often creating a crest of hair on the top of the head. Males and females have facial hair and both have cheek whiskers and may white coloration on the eyelids near the nose ${ }^{219}$. The epidermis in cynomolgus monkeys is slightly thicker than in rodents, but thinner when compared to minipigs and man. Melanocytes are found in the epidermis and the dermis. Hair coat density varies between regions, but the basic structure of hair follicle complexes is similar across the body. Hair follicles are arranged in complexes with accessory glands, namely sebaceous glands and apocrine tubular glands. Eccrine sweat glands are found in the digital pads.

Skin structures characteristic of Old World monkeys, including cynomolgus macaques, are the ischial callosities. Callosities are greatly thickened areas of compact keratin on which the animal may sit for extended periods of time without damage to underlying soft tissue.

Skin swelling at the perineum and the base of the tail occurs in female cynomolgus macaques in association with the estrogenic (follicular) phase of their reproductive cycle and this region, when swollen, is frequently referred to as "sex skin"220,221. These swellings consist grossly of erythema and thickening of the skin by dermal and subcutaneous edema, fibroplasia, and hyaluronate deposition. They are most pronounced around the time of ovulation 220 . There is substantial individual variation in the amount of sex skin swelling in cynomolgus macaques; the presence or absence of sex skin is not a reliable indicator of reproductive status. There is potential for sex skin swelling to occur as a treatment effect in the case of hormonally active test agents.

Alopecia is very common in cynomolgus monkeys, especially if socially housed. It is often linked to behavioral abnor- malities, but alternative causes of alopecia include: endocrine disease, nutritional deficiencies, aging, immunologic disorders, allergic disease, and stress ${ }^{222}$. In order to determine the cause of alopecia, a diagnostic approach should be taken as in clinical veterinary dermatology 223 .

The skin immune system in non-human primates, specifically the cynomolgus monkey, shows distinct differences from that of rodents and humans. The epidermis of cynomolgus macaques contains a population of dendritic-shaped $\mathrm{T}$ cells expressing the $\gamma / \delta \mathrm{T}$ cell receptor. This population of skin immune cells plays a critical role in mice, but is of little importance in humans. The $\gamma / \delta$ T cells in cynomolgus macaque epidermis differ from rodents in that they appar to be inactivat-

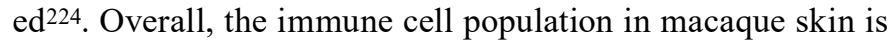
very similar to that in humans, with the epidermis containing Langerhans cells, $\mathrm{T}$ cells and putative NK cells. The dermis contains dermal dendritic cells, resident macrophages, $\mathrm{T}$ cells and putative NK cells. The skin may also contain polymorphonuclear cells ${ }^{225}$.

Cynomolgus monkey skin contains Merkel cells, i.e. tactile epithelial cells that make synaptic contacts with somatosensory afferent nerve fibers, particularly in highly sensitive skin like that of the fingertips ${ }^{226}$, and it contains Meissner and Pacinian corpuscles, i.e. mechanoreceptors in glabrous skin which are responsible for sensitivity to vibration and pressure227 (Table 25).

\section{Erosion/ulcer: Skin, Epidermis (No image provided)}

Comments: Wounds are commonly encountered in social housed NHP. They are typically recorded in the in-life phase and/or during necropsy and should be sampled for microscopic examination. Erosion/ulcer can be a consequence of bacterial infections and may progress to dermal/ subcutaneous abscesses. Cultures typically reveal Staphylococcus aureus, Klebsiella oxytoca, Actinomyces spp., Streptococcus spp., Enterobacter spp., or Pseudomonas spp.228. Increased incidence of skin infections has been described in association with immunomodulatory drugs 228 , and with dual blockade of interleukin (IL)-1 $\beta$ and IL-17A ${ }^{229}$.

\section{Infiltrate, [insert appropriate cell type] (epidermis, dermis): Skin, Epidermis, Dermis and Subcutis (No image provided)}

Comments: Inflammatory cell infiltrates into the epidermis occur in association with pustules. In addition, they are a component of interface dermatitis, i.e. a superficial perivascular dermatitis, in which lymphocytes approximate the dermo-epidermal junction, associated with epidermal necrosis $^{230}$. A typical case of interface dermatitis in NHP was described by Palanisamy et al. ${ }^{231}$. 
Table 25. Microscopic Findings of the Integument: Skin, Non-human Primate

\begin{tabular}{|c|c|c|c|c|}
\hline Skin & Common & Uncommon & $\begin{array}{c}\text { Not Observed but } \\
\text { Potentially Relevant }\end{array}$ & Not Applicable \\
\hline \multicolumn{5}{|l|}{ Non-proliferative } \\
\hline \multicolumn{5}{|l|}{ Skin - epidermis } \\
\hline Apoptosis ${ }^{\wedge}$ & & $\mathrm{x}$ & & \\
\hline Atrophy, epidermis & & $\mathrm{x}$ & & \\
\hline Edema, epidermis, intercellular & & $\mathrm{x}$ & & \\
\hline Edema, epidermis, intracellular & & $\mathrm{x}$ & & \\
\hline Erosion/ulcer * & $\mathrm{x}$ & & & \\
\hline Hyperkeratosis, epidermis & $\mathrm{x}$ & & & \\
\hline Infiltrate, [insert appropriate cell type], epidermis $* \wedge$ & $\mathrm{x}$ & & & \\
\hline Necrosis, epidermis * & & $\mathrm{x}$ & & \\
\hline Pigment, decreased * & & $\mathrm{x}$ & & \\
\hline Pigment, increased * & & $\mathrm{x}$ & & \\
\hline Pustule * & & $\mathrm{x}$ & & \\
\hline Vesicle * & & $\mathrm{x}$ & & \\
\hline \multicolumn{5}{|l|}{ Skin - cutaneous adnexa } \\
\hline Adnexal dysplasia & & $\mathrm{x}$ & & \\
\hline Atrophy, adnexa *\# & & $\mathrm{x}$ & & \\
\hline Hyperkeratosis, adnexa & & $\mathrm{x}$ & & \\
\hline Inflammation, adnexa ${ }^{\wedge}$ & & $\mathrm{x}$ & & \\
\hline Necrosis, adnexa & & $\mathrm{x}$ & & \\
\hline \multicolumn{5}{|l|}{ Skin - dermis and subcutis } \\
\hline Atrophy, dermis & & $\mathrm{x}$ & & \\
\hline Edema, dermis & & $\mathrm{x}$ & & \\
\hline Elastosis, dermis & & & $\mathrm{x}$ & \\
\hline Fibroplasia $^{1}$ & & $\mathrm{x}$ & & \\
\hline Fibrosis $^{\wedge 1}$ & & $\mathrm{x}$ & & \\
\hline Infiltrate, [insert appropriate cell type], dermis *^ & $\mathrm{x}$ & & & \\
\hline Inflammation $* \wedge$ & $\mathrm{x}$ & & & \\
\hline Mineralization $* \wedge$ & & $\mathrm{x}$ & & \\
\hline Necrosis ${ }^{1}$ & & $\mathrm{x}$ & & \\
\hline \multicolumn{5}{|l|}{ Proliferative (non-neoplastic) } \\
\hline \multicolumn{5}{|l|}{ Skin - epidermis } \\
\hline Cyst, squamous & & $\mathrm{x}$ & & \\
\hline Hyperplasia, epidermis & & $\mathrm{x}$ & & \\
\hline \multicolumn{5}{|l|}{ Skin - cutaneous adnexa } \\
\hline Hyperplasia, adnexa & & $\mathrm{x}$ & & \\
\hline \multicolumn{5}{|l|}{ Skin - dermis and subcutis } \\
\hline Hyperplasia, melanocytic * & & $\mathrm{x}$ & & \\
\hline \multicolumn{5}{|l|}{ Neoplastic } \\
\hline \multicolumn{5}{|l|}{ Skin - epidermis } \\
\hline Papilloma, squamous cell * & & $\mathrm{x}$ & & \\
\hline Carcinoma, squamous cell & & $\mathrm{x}$ & & \\
\hline \multicolumn{5}{|l|}{ Skin - dermis and subcutis } \\
\hline Lipoma $* 1$ & & $\mathrm{x}$ & & \\
\hline Melanoma, malignant* & & $\mathrm{x}$ & & \\
\hline
\end{tabular}

* Terminology with diagnostic criteria or comments described below. ${ }^{*}$ Finding more frequently observed as an induced change. ${ }^{\wedge}$ Terminology addressed in the Systemic/General Pathology section. ${ }^{1}$ indicates terminology not included in the rodent integument manuscript but included in the soft tissue manuscript and included in the table for completeness. 


\section{Necrosis, epidermis: Skin, Epidermis (Figure 107)}

Comments: Necrosis of keratinocytes is a well-known feature of cutaneous toxicities ${ }^{230}$. Necrosis may be of the single-cell type like in the clinical disease "Erythema multiforme", where individual necrotic keratinocytes are found at all epidermal levels, or they may become confluent to form full-thickness necrosis, which is the typical feature of the clinical disease "Toxic Epidermal Necrolysis". Other frequently used terms of clinical disease with similar histological features are "Stevens-Johnson syndrome" and "Fixed drug eruption"; a distinction based on histomorphology alone is not feasible. Epidermal necrosis is typically a feature of interface dermatitis, i.e. a superficial perivascular dermatitis, in which lymphocytes approximate the dermoepidermal junction, associated with vacuolar degeneration in the basal zone.

Drug-induced epidermal necrosis in NHP is rare. A typical example was described by Palanisamy et al231, who described keratinocyte degeneration/necrosis in association with lymphocytic interface dermatitis in relation to oral treatment with metabotropic glutamate receptor 5 modulators. Skin lesions were grossly characterized by swelling and erythema and developed 8 to 9 days after initiation of treatment. Lymphocytes were predominantely CD4 positive, and the authors hypothesized that the underlying pathomechanism was a delayed type-IV hypersensitivity reaction.

Epidermal necrosis in NHP was described in association with drug-induced peripheral vasoconstriction, likely resulting in ischemia ${ }^{232}$. Skin lesions were grossly characterized by blisters (syn. vesicle), ulceration and scabs (syn. crust), associated with medial hypertrophy/hyperplasia of arterioles in the superficial dermis.

Infection with the measles virus is a differential diagnosis for epidermal necrosis. The underlying clinical symptom is a skin rash ${ }^{233}$. Epidermal necrosis may lead to the formation of subepidermal vesicles, i.e. detachment of the epidermis from the underlying dermis, and subsequently to ulceration. Necrosis of the basal cell layer may lead to "pigmentary incontinence", which is characterized by the presence of melanin within macrophages in the upper dermis 230 .

\section{Pigment, decreased: Skin, Epidermis (No image provided)}

\section{Diagnostic features}

- Decreased melanin content in the epidermis

- Might be associated with an increased number of melanin-containing macrophages in the superficial dermis

Comments: Grossly visible loss of skin pigmentation in humans is known as albinism, leukoderma or vitiligo. The former two are genetic and have not been described in cynomolgus macaques. Vitiligo is an acquired patchy loss of epidermal melanocytes. Cases of vitiligo in cynomolgus monkeys have not been published but there are annecdotal reports that hypopigmentation has been observed in this species.

Pigment, increased: Skin, Epidermis (Figure 108)

\section{Diagnostic features}

- Increased melanin content in the epidermis, characterized by an increased amount of cytoplasmic melanin granules in basal and suprabasal keratinocytes

- The number of epidermal melanocytes might be increased; differentiation of melanocytes from pigmentcontaining keratinocytes requires investigation of cell ultrastucture (transmission electron microscopy) or immunohistochemical staining (e.g., melan A)

- Might be associated with an increased number of melanocytes or melanin-containing macrophages in the superficial dermis

Comments: Every species of primate has epidermal and dermal melanocytes ${ }^{234}$. Hyperpigmentation is clinically defined as the darkening of skin. Melanin granules are produced within epidermal melanocytes. They are transported along cytoplasmic dendrites and are then transferred to adjacent keratinocytes. Melanocye activity is stimulated by UV light.

In NHP, epidermal hyperpigmentation has been described as a consequence of treatment with antibody drug conjugates $^{235}$.

\section{Pustule: Skin, Epidermis (Figure 109)}

Comments: Pustules are not uncommon in social housed NHP, but they can be drug-induced.

Drug-induced pustules in NHP have been described with dual blockade of interleukin (IL) $1 \beta$ and IL 17A, which resulted in time-dependent spontaneous skin infections, consistent with a reduced host resistance ${ }^{229}$. Skin infections were also described in NHP studies investigating the pharmacokinetics and toxicity of a synergistic antibody mixture directed against epidermal growth factor receptor ${ }^{236}$, clinically presenting as rash (syn. erythema); however, microscopic details of the skin infection were not described.

\section{Vesicle: Skin, Epidermis (Figure 110)}

Comments: The formation of subepidermal vesicles, i.e. detachment of the epidermis from the underlying dermis, is a typical consequence of keratinocyte necrosis in the basal layer ${ }^{230}$.

Vesicles in NHP are rare. A typical case of vesicles as a consequence of epidermal necrosis was described by Palanisamy et al. ${ }^{231}$. Another case was described by Kikkawa ${ }^{237}$. Macroscopic skin lesions occurred after 7 consecutive days of oral dosing and were characterized by scabs (syn. crust). Vesicles were accompanied by pustules and epidermal necrosis. The inflammatory infiltrate in the superficial dermis 
was sparse and was dominated by neutrophils and macrophages rather than lymphocytes (which does not exclude the presence of an interface dermatits at earlier time points).

Vesicles can also develop as a consequence of contact dermatitis. Morris and Etheridge 238 described a case in cynomolgus monkeys, that developed vesicular dermatitis, associated with an eosinophilic infiltrate in the superficial dermis, epidermal spongiosis (syn. intercellular edema), and blood eosinophilia. The antigen causing this reaction was not identified.

Although mostly affecting the mucosal surface, vesicles in the skin can also be caused by Macacine Herpesvirus-1 (Herpes B Virus) ${ }^{239}$ and simian varicella virus ${ }^{240}$.

\section{Atrophy, adnexa: Skin, Cutaneous Adnexa (Figure 111)}

Comments: Atrophy of adnexal structures is a rare finding in NHP. Sebaceous gland atrophy has been described after oral treatment with an acyl-Coa:cholesterol acyltransferase (ACAT) inhibitor ${ }^{135}$. The authors describe a decreased size and number of differentiated sebocytes, associated with hypertrophy/hyperplasia of peripheral reserve cells. The depletion of tissue lipids was an expected physiologic sequela to ACAT inhibition, and similar changes were described in the adrenal glands and ovaries.

\section{Inflammation: Skin, Dermis and Subcutis}

(No image provided)

Comments: For NHP, the same principles apply as for rodents. Inflammation in the dermis, subcutis and the underlying soft tissue is predominantly a consequence of local trauma and is frequently observed at subcutaneous injection sites. Eosinophilic inflammation is a feature of contact dermatitis 238 .

\section{Mineralization: Skin, Dermis and Subcutis}

(No image provided)

Comments: Mineralization in the dermis and/or subcutis can occur as a syndrome previously described as dystrophic calcinosis circumscripta that occurs following tissue damage, without abnormalities in calcium and phosphorus homeostasis, and it is characterized by deposition of calcium salts in soft tissues. This syndrome has been reported in both humans and animals, including NHP. In nonhuman primates, calcinosis circumscripta has been reported in two female rhesus macaques ${ }^{241}$. Idiopathic calcinosis circumscripta has been described in two cynomolgus monkeys ${ }^{242}$ and in one female cynomolgus macaque from a 39-week oral toxicity study 243 . The descriptive term "mineralization" should be used rather than the syndrome term.

\section{Hyperplasia, melanocyte: Skin, Dermis and Subcutis}

(No image provided)

\section{Diagnostic features}

- Foci of nests and cords of pigmented cells in the dermis

Comments: Melanocyte hyperplasia in the dermis might be associated with epidermal hyperpigmentation. It can be difficult to differentiate from an accumulation of melanophages, i.e. macrophages that have phagocytosed melanin granules, for example in association with "melanin incontinence" occurring with interface dermatitis.

Melanocyte hyperplasia in the dermis can be a feature of neurocutaneous melanosis, which is a rare phakomatosis characterized by proliferation of melanin-producing cells in skin and brain ${ }^{244}$.

\section{Papilloma, squamous cell: Skin, Epidermis}

(No image provided)

\section{Other term(s)}

Wart

\section{Diagnostic features}

- Well circumscribed papilliform exophytic or endophytic nonpigmented mass.

- Composed of diffusely thickened epidermis separated by islands of dermal connective tissue.

- Basal cell layer markedly increased in thickness (4 to 8 cell layers) composed of monomorphic cells with distinct cell borders and little basophilic cytoplasm.

- Suprabasal cells show gradual squamous differentiation and keratinization with a thickened granular cell layer and irregularly enlarged (clumped) keratohyalin granules.

- Markedly thickened compacted stratum corneum (up to 1 $\mathrm{mm}$ in thickness).

- Mitotic figures are common.

- Suprabasal cells show varying degrees of cytomegaly with cytoplasmic pallor, nuclear clearing with peripheralized chromatin, and eccentric basophilic to magenta nucleoli (koilocytes).

- Suprabasal cells may show pale eosinophilic round to ovoid intranuclear inclusions (approximately $7 \mu \mathrm{m}$ in diameter) typically surrounded by a halo of cleared chromatin.

- Mild to moderate dermal mononuclear inflammation.

Comments: Spontaneous squamous cell papilloma in NHP are associated with papilloma virus infection. Papillomaviruses (PVs) are a group of small, non-enveloped DNA viruses that cause mucosal or cutaneous neoplasia in a variety of animals. Whilst most papillomas will regress spontaneously, some may persist or undergo malignant transformation. There was a case report of persistent and extensive warts observed on the hands and feet of a cynomolgus macaque ( $\mathrm{Ma}$ caca fascicularis). The genomic DNA of this PV was cloned and sequenced, and the PV was designated $M$. fascicularis papillomavirus type 1 (MfPV-1). Its genome was $7588 \mathrm{bp}$ 
in length and the organization of its putative open reading frames (E1, E2, E6, E7, L1, L2 and E4) was similar to that of other PVs. Molecular analysis of MfPV-1 genomic DNA classified it into the genus Betapapillomavirus, to which all epidermodysplasia verruciformis (EV)-type PVs belong245. Diseases caused by PVs of the genus Betapapillomavirus are usually associated with natural or iatrogenic immunosuppression.

\section{Lipoma: Skin, Dermis and Subcutis (No image provided)}

Comments: Lipoma in the subcutis of a female cynomolgus monkey from Chinese origin was reported by Kaspareit et al. ${ }^{1}$
Melanoma, malignant: Skin, dermis and subcutis (No image provided)

Comments: Malignant melanoma in NHP is very rare. A case has been described in a 3.3-year-old cynomolgus monkey, exhibiting a dermal mass on the dorsal surface of the hind limb ${ }^{246}$. In this case, the dermal melanoma was associated with lymphatic invasion. Neoplastic cells stain positive for S-100, Melan-A, HMB-45 and HMB-50. Transmission electron microscopy can confirm the presence of stage IV melanosomes (mature melanin granules) in the cytoplasm. 


\section{Mammary Gland}

For detailed general considerations and appropriate terminology, refer to the INHAND rodent publication ${ }^{247}$.

The mammary gland is a dynamic hormonally responsive organ that can be affected by pharmacologic, chemical, and dietary agents. Evaluation of the mammary gland is particularly important in toxicology studies of substances that may mimic endogenous sex steroids or otherwise disrupt endocrine pathways. Mammary gland tissue should always be examined in the context of reproductive age and hormonal status, including the cyclic phase of the primary reproductive organs. Evaluation of potential physiologic influences will provide a better understanding of possible mechanisms underlying any observed changes. In this section, we describe specific anatomic and physiologic details as well as pathologic changes of the non-human primate mammary gland, with an emphasis on the female cynomolgus macaque.

\section{Anatomy}

Macaques have two pectoral mammary glands with similar basic anatomical features as humans. In adult non-lactating macaques, flattened mammary glands consist of branching lactiferous ducts radiating from the nipple and ending in terminal ductal lobular units. The terminal lobular units are intralobular ducts surrounded by alveoli 248 . Alveoli and ducts have a single layer of luminal cuboidal to columnar glandular epithelium surrounded by a single layer of myoepithelium. Each nipple contains five to seven lactiferous ducts lined by columnar glandular epithelium transitioning to stratified squamous epithelium at their openings. The primary ductal/lobuloalveolar network is contained within the mammary pad, which is more fibrous than the adjacent subcutaneous fat. Glandular tissue is most prevalent cranial and lateral to the nipple, extending to the axilla ${ }^{249}$. The areola in macaques is indistinct. Normal non-lactating and quiescent macaque mammary glands commonly contain intraepithelial fat droplets. During lactation, the macaque breast becomes larger and often pendulous, hanging away from the pectoral muscle. The mammary gland of male macaques is similar to that of females but with a small nipple and a less developed ductal and lobular network. Mammary gland tissue collection in both sexes should include a full-thickness sample of skin and subcutaneous fat running from the nipple laterally towards the axilla. This orientation provides a cross-section of skin and the underlying mammary tissue.

\section{Development}

The macaque mammary gland undergoes dramatic changes in morphology across different life stages, from puberty to pregnancy and senescence. Mammary gland development begins in utero, due in part to maternal sex hormone exposure ${ }^{250}$. By birth, a nipple and branching ductal system, a few hundred micrometers in diameter are present 251 . The only con- genital mammary defects reported in macaques are supernumerary nipples ${ }^{250}$. The most substantial changes occur during the peripubertal period when rapid expansion of the mammary gland epithelium occurs ${ }^{252}$. Such postnatal changes highlight the need to understand reproductive status and endogenous hormone context when evaluating potential xenobiotic effects.

The peripubertal period is characterized by nipple enlargement followed by rapid elongation and branching of the rudimentary ductal system ${ }^{253}$. Nipple enlargement is often the first macroscopic sign of progression into the peripubertal period in macaques and may precede menstruation by several months. The rapid, extensive, and variable proliferation of the peripubertal mammary gland is often a challenge to assess in studies involving young macaques 248,253 . Female macaques at the beginning of menstruation often possess a mixture of well-differentiated and highly branched lobuloalveolar units with variable numbers of acini within each lobule249. Proliferating glandular epithelium terminal end-buds take the form of multilayered ducts and may resemble focal ductal hyperplasia or carcinoma in situ ${ }^{252}$. Immature or transitional ducts can be differentiated from true aberrant proliferative lesions by lack of well-differentiated lobular structures and the presence of other developmental features such as a loose peri-ductal stroma 248,252 . Young male macaques may also develop transient gynecomastia similar to that observed in peripubertal human males 254 .

The adult non-lactating macaque mammary gland consists primarily of mature lobules with variable levels of cell proliferation ${ }^{255}$. Mammary epithelium expresses estrogen and progesterone receptors (similar to the human mammary gland) and shows modestly higher rates of cell proliferation in the luteal phase of the menstrual cycle 256 . These biomarker profiles are described in detail elsewhere248. Significant mammary gland development occurs in pregnancy to prepare for lactation. During this time, a high concentration of endogenous estrogens, progesterone, prolactin, chorionic gonadotropin, and placental lactogen stimulate epithelial proliferation, secretory dilation of the ductal and alveolar lumens, and upregulation of pathways related to prolactin signaling and milk protein production 257,258 . The average length of lactation in macaques is twelve months ${ }^{259}$. Lactation suppresses ovulation in macaques, shifting energy from reproduction to milk production. The overall composition of breast milk is similar in women and macaques, although milk protein is almost two times higher in macaques ${ }^{260}$. Macaque milk also contains lower concentrations of secretory IgA, lactoferrin, polymeric immunoglobulin receptor, alpha-1 antichymotrypsin, vitamin D-binding protein, and haptocorrin compared to human milk ${ }^{261}$. After weaning, mammary gland tissue undergoes post-lactational involution, and glandular morphology returns to a state resembling a non-pregnant premenopausal animal.

The mammary gland in aged macaques is characterized by marked lobular atrophy with limited ductal and lobular proliferation. Expression of estrogen and progesterone receptors persists in ovariectomized macaques for at least six to seven years, and the breast remains responsive to hormonal stimulation during this time248, 262 (Table 26). 
Table 26. Microscopic Findings in the Mammary Gland; Non-human Primate

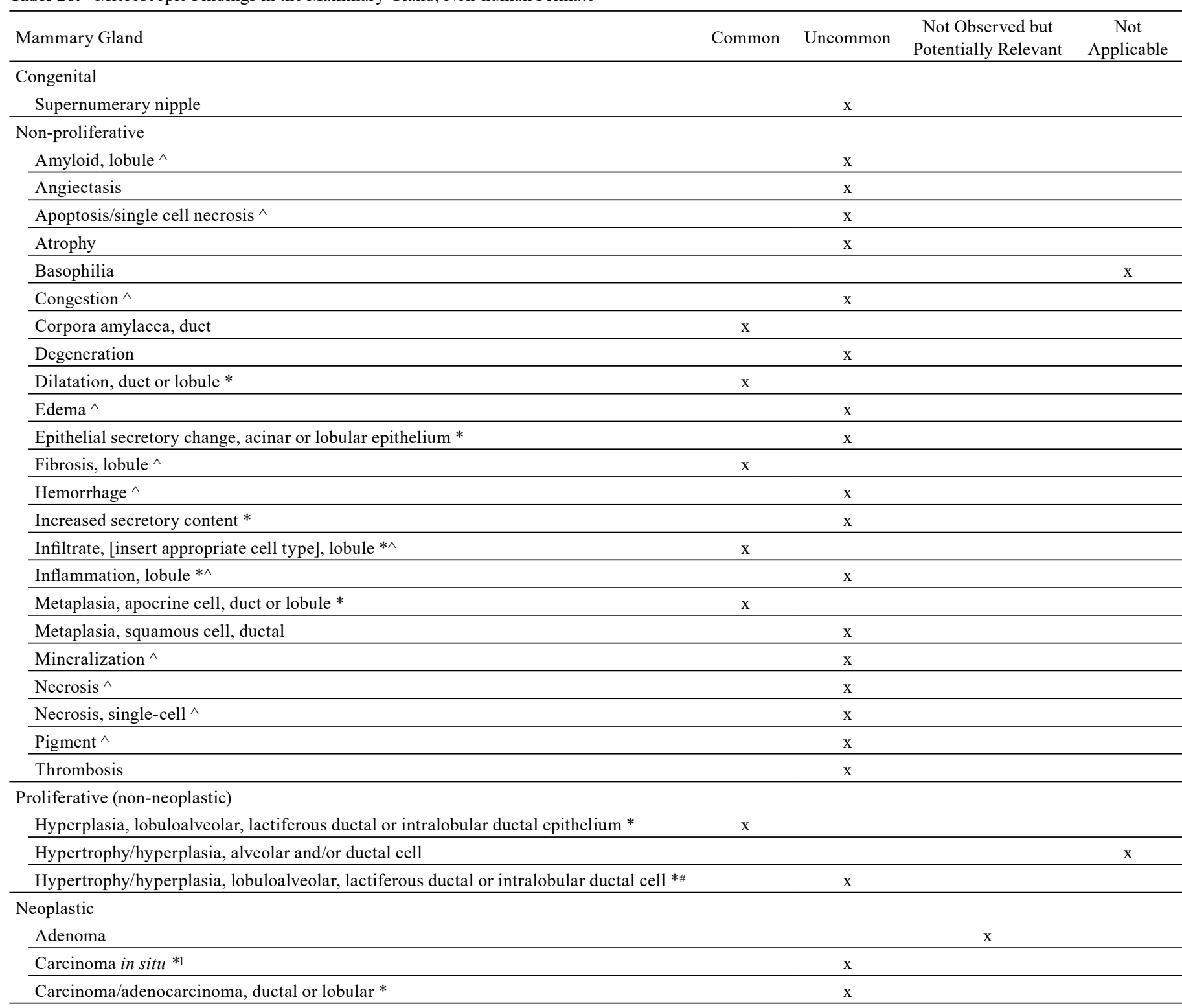

* Terminology with diagnostic criteria or comments described below. ${ }^{\wedge}$ Terms addressed in the Systemic/General Pathology section. ${ }^{\#}$ Findings more frequently observed as an induced change. ${ }^{1}$ Commonly observed in lifetime studies or those including aged colonies.

\section{Dilatation, duct or lobule: Mammary Gland}

(No image provided)

\section{Other term(s)}

Cystic change

\section{Pathogenesis/Cell of Origin}

Incidental finding of mammary gland ducts or acini

\section{Diagnostic features}

- Dilated ducts or acini with attenuated epithelium

- May or may not contain lightly eosinophilic intraluminal material with secretory droplets (protein)

\section{Differential Diagnoses}

- Increased secretory content (galactorrhea)

- additional changes of epithelial hypertrophy and hyperplasia are present

Comments: Cystic dilation of ducts or lobules is considered an incidental finding, particularly in older parous macaques $^{249,262}$. Dilated ducts may also be observed secondary to hyperplastic or neoplastic mammary gland lesions ${ }^{263}$. There is no reported association with hormonal treatment 249 . 
Epithelial secretory change, acinar or lobular cell: Mammary Gland (Figure 112)

\section{Pathogenesis/Cell of Origin}

Lobular or acinar epithelial cells

\section{Diagnostic features}

- Cytoplasmic vacuoles/lipid droplets, often with intraluminal lipid/protein secretion and luminal dilation

- Apical blebs (luminal "snouts")

- Bright red granules within the cytoplasm of mammary gland epithelial cells

\section{Differential Diagnoses}

- Apocrine metaplasia

- cuboidal glandular epithelium, apical blebs and bright red granules not common features

- Increased secretory content (galactorrhea)

- additional changes of epithelial hypertrophy and hyperplasia are present

- Physiologic secretory activity (lactation):

- dilated acini containing milk protein

Comments: Epithelial secretory change is a common background lesion in macaques associated with production and release of milk proteins, immunoglobulins, and fats into the ductal lumens ${ }^{248}$. It is important to distinguish this lesion from physiologic secretory activity, including lactation, in which lobuloalveolar units are diffusely increased in size and number.

\section{Increased secretory content: Mammary Gland}

(No image provided)

\section{Other term(s)}

Galactorrhea

\section{Pathogenesis/Cell of Origin}

Acinar epithelial cells are stimulated directly by prolactin to take on a secretory phenotype with increased intracytoplasmic lipid droplets.

\section{Diagnostic features}

- Lobular and acinar hyperplasia with epithelial cell hypertrophy

- Dilated acini contain variably-sized droplets of homogeneous, eosinophilic material (milk protein)

\section{Differential Diagnoses}

- Epithelial secretory change:

- no lobular or acinar hyperplasia

Comments: Increased secretory content is associated with prolactin-producing pituitary neoplasms in male and female macaques ${ }^{128,264}$. Chronic daily morphine administration also results in transient hyperprolactinemia and may induce mammary gland hyperplasia/hypertrophy in male macaques 265 .

Infiltrate, [insert appropriate cell type], lobule: Mammary Gland (No image provided)

\section{Pathogenesis/Cell of Origin}

Lymphocytes, plasma cells, neutrophils, eosinophils, macrophages, or a mixture of these cell types

\section{Diagnostic features}

- Presence of lymphocytes, plasma cells, neutrophils, eosinophils, macrophages, or a mixture of these cell types in the stroma surrounding lobules (most common site) or ducts

\section{Differential Diagnoses}

- Inflammation

- immune cells associated with other findings such as edema, hemorrhage, congestion, degenerative epithelial changes, and/or fibrosis

- Lymphoma

- infiltrative population of lymphocytes with neoplastic features such as pleomorphism and high mitotic index

Comments: The base term "infiltrate" should be followed by the predominant cell type, or "mixed" if there is not a dominant cell type. Infiltrates consisting only of lymphocytes or plasma cells may indicate an immunologic effect of a xenobiotic but can also be observed as a spontaneous change 266 .

\section{Inflammation, lobule: Mammary Gland}

(No image provided)

Other term(s)

Mastitis

\section{Pathogenesis/Cell of Origin}

Lymphocytes, plasma cells, neutrophils, eosinophils, macrophages, multinucleated giant cells, or a mixture of inflammatory cell types

\section{Diagnostic features}

- Infiltration of inflammatory cells within the mammary gland stroma, epithelium, and/or lumens, with associated degenerative changes, edema, hemorrhage, congestion, and/or fibrosis

\section{Differential Diagnoses}

- Infiltrate

- Infiltration without associated tissue changes

Comments: Mastitis in macaques has been rarely reported in the literature as a spontaneous finding262, 267. 
Metaplasia, apocrine cell, duct or lobule: Mammary

Gland (Figure 113)

\section{Pathogenesis/Cell of Origin}

Ductal or lobular epithelial cells

\section{Diagnostic features}

- Glandular epithelial cells are cuboidal with pale eosinophilic cytoplasm and round nuclei arranged in a discrete row (similar to an apocrine sweat gland).

- Variable features include apical blebbing and luminal dilatation.

- Lobular lesions may be present within one or more acini.

\section{Differential Diagnoses}

- Ductal or lobuloalveolar hyperplasia

- an increased number of ductal or lobular acinar cells

- Secretory change

- eosinophilic cytoplasmic granules in the luminal epithelium (without apocrine-type morphology)

Comments: Apocrine metaplasia is an incidental finding in older macaques 249,268 . There is no clear association with specific hormone treatment or higher-grade lesions in cynomolgus macaques ${ }^{262}$. In women, simple apocrine metaplasia is not associated with increased risk for breast cancer ${ }^{269}$. A common type of apocrine metaplasia in the human breast has strongly eosinophilic coarse granules in the apical cytoplasm of luminal cells. It is unclear whether this type of granular change is related to the red cytoplasmic granules noted above under secretory change. Here we have limited the diagnosis of apocrine metaplasia to glands with distinctive cuboidal apocrine-type morphology. Additional markers for apocrine cells in the human breast include periodic acid-Schiff after diastase digestion, epithelial membrane antigen, cytokeratins 8 and 18, androgen receptors, and gross cystic disease fluid protein (GCDFP) proteins 15, 24 and 44.4269 .

\section{Metaplasia, squamous cell, duct: Mammary Gland}

(Figure 114-115)

\section{Pathogenesis/Cell of Origin}

Ductal epithelial cells

\section{Diagnostic features}

- Presence of foci or areas of stratified squamous non-keratinizing epithelium

\section{Differential Diagnoses}

None

Comments: Squamous metaplasia can be observed in response to the direct cytotoxicity induced by certain antibody-drug conjugates (e.g., directed against HER2). Immunohistochemical markers of basal/squamous cells include cytokeratin 14 and p63.

\section{Hyperplasia, lobuloalveolar, lactiferous duct, or intralobular (terminal) duct epithelium: Mammary Gland (Figure 116-119)}

\section{Other term(s)}

Basophilia; hyperchromasia; columnar cell hyperplasia

\section{Pathogenesis/Cell of Origin}

Lobuloalveolar, lactiferous duct, or intralobular (terminal) duct epithelium may be affected. Hyperplastic lesions can occur as spontaneous findings, particularly in older macaques, but show increased incidence with different types of hormone treatment 268 . In one survey of rhesus and cynomolgus macaques, $47 \%$ of animals $(9 / 19)$ with hyperplastic breast lesions had received some form of hormone treatment ${ }^{263}$. A separate experiment in older ovariectomized macaques found a higher incidence of mammary epithelial hyperplasia in animals treated with oral $17 \beta$-estradiol (E2) compared to controls ${ }^{262}$. Benign proliferative lesions showed greater expression of estrogen receptor- $\alpha$ and greater expression of progesterone receptor in response to E2 than controls, suggesting enhanced sensitivity to estrogen exposure262.

\section{Diagnostic features}

- Hyperplastic lesions have increased numbers of densely packed glandular epithelial cells, often with large hyperchromatic nuclei, lining ducts or acini.

- Epithelial cells may form mounds, tufts, or micropapillae.

- Distribution can be focal, multifocal, or diffuse.

- Variable features include tall pseudostratified columnar cells, apocrine-type morphology, cystic dilated lumens, and secretory changes.

- Mitoses or proliferation markers such as Ki67 may be increased relative to adjacent tissue.

- Heterogeneous morphologies have been reported, but lesions are generally classified as conventional (non-atypical) or atypical:

- Non-atypical: Increased numbers of monomorphic cells without atypical cytologic or architectural features

- Atypical: Acini or ducts have at least two layers of luminal epithelial cells. Atypia can be cytologic (e.g., anisocytosis and anisokaryosis, irregular mitotic figures) or architectural (abnormal arrangement of cells or luminal shape). Micropapillary change is the most common atypical growth pattern. Atypical lesions may represent a precursor to ductal/lobular carcinoma in situ.

\section{Differential Diagnoses}

- Hormone-dependent lobular enlargement

- Carcinoma in situ:

- Carcinoma in situ is characterized by both architectural and cytologic atypia

- Normal development in peripubertal macaques

- multilayered ducts

Comments: Ductal hyperplasia has been reported in 3\% of 
middle-aged adult macaques and increases with age ${ }^{249}$. The severity of mammary gland hyperplasia can range from small focal micropapillae (most common type) to more diffuse bilateral lesions with atypia ${ }^{263,268}$. Focal lobular hyperplasia against a background of atrophic lobules is documented as a background finding in older macaques. The focal hyperplastic nodules in these cases may be large enough to compress adjacent tissues 249 . Certain types of atypical benign lesions have been associated with increased breast cancer risk in women ${ }^{270}$. In macaques, the relationship between hyperplasia and carcinoma is not established 262 .

\section{Hyperplasia/hypertrophy, lobuloalveolar, lactiferous} ductal, or intralobular duct epithelium: Mammary Gland (Figure 120-122)

\section{Other $\operatorname{term}(\mathbf{s})$}

Gynecomastia (males)

\section{Pathogenesis/Cell of Origin}

Ductal and lobular epithelial cells.

\section{Diagnostic features}

- Well-differentiated ducts and lobuloalveolar units with variable numbers of acini in each lobule

\section{Differential Diagnoses}

- None

Comments: Hyperplasia and hypertrophy of mammary gland elements can be observed in male macaques secondary to long-term estrogen treatment. The development of breast tissue in peripubertal males occurs analogously to that in females so that some degree of hyperplasia and hypertrophy may be present in the peripubertal period ${ }^{271}$. The morphology of exuberant mammary gland development in males generally resembles that of normal premenopausal females $^{249}$. This term should not be confused with the term in rodents.

Carcinoma in situ: Mammary Gland (Figure 123-124)

\section{Other term(s)}

Dysplasia

\section{Pathogenesis/Cell of Origin}

Ductal or lobular epithelial cells

\section{Diagnostic features}

- Growth patterns include comedo, micropapillary, solid, and cribriform architecture. Most common type (comedo) is characterized by marked expansion of ducts by epithelial cells with central necrosis.

- Other features include high mitotic rates, nuclear pleomorphism, prominent nucleoli, and lack of polarity and cohesion.

- Variable stromal fibrosis may be observed but no evidence of local invasion or metastasis.

\section{Differential Diagnoses}

- Invasive carcinoma or adenocarcinoma

- local/stromal invasion (with penetration of epithelial basement membrane), vascular invasion, and/or evidence of metastasis

Comments: The lifetime incidence of in situ and invasive mammary carcinoma in macaques ranges from $3.5-6.1 \%$. E-cadherin is a marker of ductal origin in carcinoma in situ lesions 263 . While most cancers occur in older animals, DCIS and invasive cancers have been reported in animals as young as 4 years of age.

\section{Carcinoma/adenocarcinoma, duct or lobule: Mammary \\ Gland (Figure 125-127)}

Other $\operatorname{term}(\mathrm{s})$

Invasive carcinoma

\section{Pathogenesis/Cell of Origin}

Ductal or acinar epithelial cells

\section{Diagnostic features}

- Neoplastic cells are arranged in sheets, with variable comedo, cribriform, and micropapillary growth patterns.

- Cellular features include large round to oval euchromatic nuclei, prominent nucleoli, abundant amphophilic to basophilic cytoplasm, and increased mitotic figures compared to non-neoplastic epithelium.

- Tubules have irregular morphology with decreased luminal size or loss of lumens.

- Central necrosis is common within neoplastic cells.

- Areas of squamous differentiation can be observed in some carcinomas.

- Higher grade carcinomas have high nuclear pleomorphism and mitotic rate, loss of tubular lumens, extensive stromal invasion, and vascular invasion.

\section{Differential Diagnoses}

Carcinoma in situ: neoplastic epithelial cells do not invade the basement membrane

Comments: Carcinomas may be observed macroscopically as raised subcutaneous masses adjacent to the nipple, with or without an ulcerated surface 263 . There is no clear association between the extent of necrosis and grade in macaque carcinomas $^{263}$. Mammary cancers in macaques variably express estrogen and progesterone receptors. A subset of ductal carcinomas may overexpress HER2/neu. Metastasis of invasive carcinomas may occur to the axillary lymph nodes and lungs 263 . 


\section{Nervous System}

For detailed general considerations, refer to the INHAND rodent publication ${ }^{272}$.

The neuroanatomy of the non-human primate is covered in detail elsewhere 273,274 . The large brain size of the monkey compared to rodents necessitates a different approach to trimming and embedding the brain; a recommended trimming scheme is presented in an STP position paper by Bolon et al. ${ }^{275}$ and a technical guide ${ }^{276}$. Non-proliferative spontaneous findings in the nervous system are not common in young cynomolgus monkeys used in routine nonclinical toxicity studies 11,191 . Due to the young age of the animals and the short duration of the studies, neoplastic lesions are rarely observed in routine nonclinical toxicity studies involving non-human primates ${ }^{1}$. The few published reports of neoplasms in young monkeys include primitive neuroectodermal tumor (PNET), astrocytoma and ependymoma (Table 27).

\section{Cyst, ependyma: General (No image provided)}

\section{Pathogenesis/Cell of Origin} esis

Aberrant migration of ependymal cells during embryogen-

\section{Diagnostic features}

- Intracranial cavitation lined by ciliated cuboidal to low columnar epithelium

- Immunohistochemically positive for cytokeratin, S-100, and GFAP

Comments: Ependymal cyst is rare in non-human primates with reports in cynomolgus and African green monkeys 277,278 .

Infiltrate, mononuclear cell: General (No image provided)

\section{Other term(s)}

Mononuclear cell infiltrate

\section{Pathogenesis/Cell of Origin}

Unknown.

\section{Diagnostic features}

- Multifocal perivascular aggregates of lymphocytes, plasma cells, and macrophages in the parenchyma of CNS (usually surrounding blood vessels), choroid plexus, meninges, cranial or peripheral nerves and/or ganglia

- The infiltrate in the CNS parenchyma may be admixed with glial cells such as microglia and oligodendrocytes

Comments: Perivascular infiltrates of mononuclear cells are the most common spontaneous finding in the nervous system of cynomolgus monkeys ${ }^{6,11}$. Other cell types such as microglial cells and or oligodendrocytes may be admixed with mononuclear cell infiltrates in the CNS parenchyma and may represent a glial response to the mononuclear cells transiting from the Virchow-Robin space into the parenchyma due to a breach in the blood-brain barrier ${ }^{279}$.

The presence of admixed neutrophils and/or eosinophils may represent tissue or vascular injury. Drug-induced vascular injury associated with biotherapeutics, particularly immune complex deposition, is frequently identified in the choroid plexus in NHP in safety toxicology studies ${ }^{33}$. Mononuclear cell infiltrates in the choroid plexus should be differentiated from mixed perivascular and/or vascular cell infiltrates or inflammation. Associated vascular changes such as endothelial activation, plump endothelial appearance with protrusion into the lumen, should be described.

Neural tissue loss, cerebrum: General (Figure 128-130)

\section{Other term(s)}

Porencephaly, brain tissue cavitation

\section{Pathogenesis/Cell of Origin}

Unknown

\section{Diagnostic features}

- Chronic unilateral or bilateral (generally asymmetrical) lesions characterized by loss of nervous tissue, neurons and neuropil, resulting in altered tissue architecture with cavitation and partial or full thickness defects in the cerebral hemisphere wall.

- Nervous tissue is replaced by a thin fibrovascular meshwork with multifocal clusters of pigmented macrophages (hemosiderin and/or lipofuscin laden). The surrounding cerebral parenchyma is often atrophic with variable gliosis. Prominent astrocytes and astrocytic fibrils can be observed around excavation areas.

- Often noted grossly as depressed areas with brown discoloration. These areas may be associated with cranial adhesions.

\section{Differential Diagnosis}

- Ependymal cyst

- Cystic dilatation within the white matter reported in the caudal cerebral hemisphere, partially or completely lined by flattened cuboidal to low columnar epithelium with a high percentage of ciliated cells. May be associated with acute or chronic hemorrhage, atrophy and/or gliosis in the surrounding neuropil.

Comments: The parenchymal loss/cavitary lesions are most commonly observed in the parietal and/or occipital lobes. While the pathogenesis is uncertain, the constellation of histologic findings is suggestive of a chronic ischemic lesion, resulting from an ischemic event (thrombus of unknown origin) or traumatic injury causing impaired cerebral circulation. Due to the young age of affected animals, it has been suggested that the damage in some cases could occur in utero during development ${ }^{280}, 281$. The change can rarely be associated with hydrocephalus and sometimes 
Table 27. Microscopic Findings of the Nervous System: Brain, Spinal Cord, and Peripheral nerve; Non-human Primate

\begin{tabular}{|c|c|c|c|c|}
\hline Brain, Spinal Cord, and Peripheral Nerve & Common & Uncommon & $\begin{array}{c}\text { Not Observed, but } \\
\text { Potentially Relevant }\end{array}$ & Not Applicable \\
\hline \multicolumn{5}{|l|}{$\begin{array}{l}\text { Non-proliferative } \\
\text { General }\end{array}$} \\
\hline \multicolumn{5}{|l|}{ General } \\
\hline$\frac{\text { Cholesterol clefts }}{\text { Cyst, ependyma }}$ & & $\frac{x}{x}$ & & \\
\hline Cyst, squamous & & $\frac{4}{x}$ & & \\
\hline Dilatation, ventricle or central canal, spinal cord & & $\mathrm{x}$ & & \\
\hline Hemorrhage^^$\wedge^{\wedge}$ & & $\mathrm{x}$ & & \\
\hline \multirow{2}{*}{\multicolumn{5}{|c|}{$\begin{array}{l}\text { Hydrocephalus } \\
\text { Infiltrate } \text { _insert anpropriate cell typel (mononuclear cell) }{ }^{* \wedge}\end{array}$}} \\
\hline & & & & \\
\hline Inflammation $^{\wedge}$ & & $\mathrm{x}$ & & \\
\hline $\begin{array}{l}\text { Mineralization } \wedge \\
\end{array}$ & & $\mathrm{x}$ & & \\
\hline Neural tissue loss, cerebrum * & & $\mathrm{x}$ & & \\
\hline Pigment, lipofuscin^^ & & $\frac{1}{x}$ & & \\
\hline Pigment, melanin ^ & & $\mathrm{x}$ & & \\
\hline Senile amyloid plaques & & $\mathrm{x}$ & & \\
\hline Syringomyelia/Hydromyelia & & & & $\mathrm{x}$ \\
\hline \multirow{2}{*}{\multicolumn{5}{|c|}{ Neuron-Cell Body }} \\
\hline & & & & \\
\hline Decreased cellularity, neuron & & $\mathrm{x}$ & & \\
\hline Chromatolysis & & $\mathrm{x}$ & & \\
\hline Necrosis, neuron & & $x$ & & \\
\hline $\begin{array}{l}\text { Neuronophagia } \\
\text { Neuronal ceroid-lipofuscinosis * }\end{array}$ & & $\frac{x}{x}$ & & \\
\hline Heterotopia, neuron & & $\frac{2}{x}$ & & \\
\hline \multirow{2}{*}{\multicolumn{5}{|c|}{ Neuron-Axon }} \\
\hline & & & & \\
\hline Atrophy, axon & & $\mathrm{x}$ & & \\
\hline Degeneration, axon & & $\frac{1}{x}$ & & \\
\hline Degeneration, nerve fiber * & $\mathrm{x}$ & & & \\
\hline \multirow{2}{*}{\multicolumn{5}{|c|}{ Glia-Cell Body }} \\
\hline & & & & \\
\hline $\begin{array}{l}\text { Astrocyte swelling/vacuolation } \\
\text { Astrocyte swelling }\end{array}$ & & $\frac{x}{x}$ & & \\
\hline Astrocytosis & $\mathrm{x}$ & 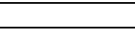 & & \\
\hline Gliosis (NOS) * & $\mathrm{x}$ & & & \\
\hline Globoid cell leukodystrophy * & & $\mathrm{x}$ & & \\
\hline Microgliosis & $\mathrm{x}$ & & & \\
\hline Satellitosis & & $\mathrm{x}$ & & \\
\hline Type II astrocytes & & $\mathrm{x}$ & & \\
\hline $\begin{array}{l}\text { Glia-Myelin } \\
\text { Accumulation, laminar, Schwann cell }\end{array}$ & & & & \\
\hline $\begin{array}{l}\text { Accumulation, laminar, Schwann cell } \\
\text { Demyelination }\end{array}$ & & $\mathrm{x}$ & $x$ & \\
\hline Intramyelinic edema & & $\frac{1}{x}$ & & \\
\hline Choroid Plexus & & & & \\
\hline Extramedullary hematopoiesis ^ & & $\mathrm{x}$ & & \\
\hline Infiltrate, mononuclear cell^ ${ }^{\wedge}$ & & $\mathrm{x}$ & & \\
\hline Vacuolation & & $\mathrm{x}$ & & \\
\hline Vascular $^{1}$ & & & & \\
\hline Necrosis/inflammation, media or wall, artery & & $\frac{x}{x}$ & & \\
\hline $\begin{array}{l}\text { Infarct } \\
\text { Thrombus }\end{array}$ & & $\frac{x}{x}$ & & \\
\hline Meninges & & & & \\
\hline$\frac{\text { Infiltrate, [insert appropriate cell type] (mononuclear cell) } * \wedge}{\text { Inflammation^^}}$ & $\mathrm{x}$ & $x$ & & \\
\hline Mineralization $^{\wedge}$ & & $\frac{x}{x}$ & & \\
\hline $\begin{array}{l}\text { Peripheral nervous system } \\
\text { Necrosis/inflammation, media or wall, artery } 1\end{array}$ & & $\mathrm{x}$ & & \\
\hline $\begin{array}{l}\text { Necrosis/inflammation, media or wall, artery }{ }^{1} \\
\text { Accumulation, matrix }\end{array}$ & & $\frac{x}{x}$ & & \\
\hline Atrophy, axon & & $\frac{\mathrm{x}}{\mathrm{x}}$ & & \\
\hline $\begin{array}{l}\text { Autophagy, neuron, dorsal root ganglia * } \\
\end{array}$ & & $\mathrm{x}$ & & \\
\hline Cell loss, neuron & & $\frac{x}{x}$ & & \\
\hline Chromatolysis & & $\mathrm{x}$ & & \\
\hline Degeneration, axon & & $\frac{2}{x}$ & & \\
\hline Degeneration, nerve fiber * & & $\frac{2}{x}$ & & \\
\hline Demyelination & & $\mathrm{x}$ & & \\
\hline Dystrophy, axon & & $\mathrm{x}$ & & \\
\hline Heterotopia, neuron & & $\mathrm{x}$ & & \\
\hline Infiltrate, [insert appropriate cell type]^ ${ }^{\wedge}$ & & $\frac{\mathrm{x}}{\mathrm{x}}$ & & \\
\hline Inflammation^^${ }^{\wedge}$ & & $\frac{x}{x}$ & & \\
\hline Intramyelinic edema & & $\frac{x}{x}$ & & \\
\hline Necrosis, neuronal & & $\mathrm{x}$ & & \\
\hline Neuronophagia & & $\mathrm{x}$ & & \\
\hline Vacuolation, neuronal & & $\frac{\mathrm{x}}{\mathrm{x}}$ & & \\
\hline Proliferative (non-neoplastic) & & & & \\
\hline Glia & & & & \\
\hline $\begin{array}{l}\text { Hyperplasia, glia NOS } \\
\text { Meninges }\end{array}$ & & & $\mathrm{x}$ & \\
\hline Aggregates, granular cell & & & $\mathrm{x}$ & \\
\hline Other cell lineages & & & & \\
\hline Hamartoma, lipomatous & & $\mathrm{x}$ & & \\
\hline Neoplastic & & & & \\
\hline Neuronal & & & & \\
\hline Ganglioneuroblastoma & & $\mathrm{x}$ & & \\
\hline Primitive neuroectodermal tumor * & & $\frac{x}{x}$ & & \\
\hline Astrocyte/Oligodendrocyte/Glial precursor cell & & & & \\
\hline Astrocytoma, malignant $*$ & & $\mathrm{x}$ & & \\
\hline Oligodendroglioma, malignant ${ }^{*}$ & & $\mathrm{x}$ & & \\
\hline Ependyma & & & & \\
\hline Ependymoma, benign & & $\frac{x}{x}$ & & \\
\hline
\end{tabular}

* Terminology with diagnostic criteria or comments described below. ${ }^{\#}$ Findings more frequently observed as an induced change. ${ }^{\wedge}$ Terms addressed primarily in the Systemic/General Pathology section. ' See "Cardiovascular system". 
due to bacterial infection following direct CNS injections. Lesions may be subclinical or present with associated neurologic signs.

\section{Autophagy, neuron, dorsal root ganglia (DRG): Neuron-Cell Body (Figure 131)}

\section{Other term(s)}

Lysis, neuron; degeneration, neuron; necrosis, neuron

\section{Pathogenesis/Cell of Origin}

Auto-programmed cell death, presumably following lethal cell injury to the sensory neurons (though the nature of the fatal damage is not known).

\section{Diagnostic features}

- Scattered neurons with typically stippled or granular pale eosinophilic neuroplasm

- Absent or lytic nucleus, with distribution of residual chromatin as irregular amphophilic to deeply eosinophilic central, often winding islands of coalescing globules and spicules.

- The affected neurons are interspersed amongst morphologically normal appearing neurons

- No inflammatory cell reaction is generally observed

\section{Special Diagnostic Techniques}

The appearance is distinctive on H\&E-stained sections. If special procedures are performed, the following features are evident:

- Bielschowsky's silver stain: The clumped chromatin residue appears as black debris.

- Fluoro Jade: Disintegrating cells are not stained.

- Immunohistochemistry: In monkeys, affected DRG neurons label positively for peripherin and ATB16L1, indicating autophagy as an underlying process in the DRG neurons. Peripherin is a type III intermediate filament protein expressed mainly in ganglion neurons and ATB16L1 is an autophagy-related protein ${ }^{282}$.

- Ultrastructure: By transmission electron microscopy, autophagic neurons are filled with phagolysomes, which gives the cells their granular cytoplasmic appearance by light microscopy; have disrupted nuclei comprised of angular to granular chromatin spicules often arranged in a vaguely circular array; and possess intact cell membranes 282

\section{Differential Diagnoses}

- Degeneration, neuron is a potentially reversible indication of substantial cell injury, which typically presents as cell and/or nuclear swelling.

- Necrosis, neuron is an end-stage change indicating cell death, which is apparent as slightly shrunken, hypereosinophilic cells with small, condensed ["pyknotic"] or fragmented ["karyorrhectic"] nuclei.

- Apoptosis is an end-stage finding indicating cell death, which presents as slightly shrunken cells possessing either nuclei with marginated crescents of chromatin or irregularly sized, globular pieces of the disintegrating nucleus.

Comments: Neuronal autophagy is a relatively common background finding in the dorsal root ganglia and to a lesser extent trigeminal and the autonomic ganglia of cynomolgus monkeys ${ }^{283}$. The change is less commonly seen in rats and dogs. As DRG's are uncommonly examined, existing historical control data for the incidence of this finding may be of limited value $273,282,283$. The change is noted frequently in untreated and vehicle-treated control animals (1 or occasionally 2 cells per affected ganglion). When present, the change is likely to be observed in multiple DRG's in the same animal. The ultrastructural features of this change suggest that it represents autophagy, but the initiating cause is unknown. To date, this finding has not been associated with in-life neurological signs or exposure to potentially neurotoxic test articles, though it is possible that a test article could increase the incidence of this background change. The change has been diagnosed historically as "necrosis, neuronal".

\section{Ceroid-lipofuscinosis, neuron: Neuron-Cell Body}

(Figure 132)

\section{Other term(s)}

Batten-disease; Kuf's disease; Parry's disorder; JanskyBielschowsky disease; Santavuori-Haltia disease

\section{Pathogenesis/Cell of Origin/Biological behavior}

Defects in cellular metabolism result in accumulation of intracellular lipopigment (lipofuscin), with or without neurologic impairment.

\section{Diagnostic features}

- Neuronal loss

- Neuronal swelling (ballooning), with intracytoplasmic, golden brown to pale amphophilic, autofluorescent finely granular material

- Astrocyte proliferation and hypertrophy

\section{Special Diagnostic Techniques:}

- Special stains ${ }^{284}$ :

- Stain positively for

- Periodic-acid Schiff

- Acid-fast (weak)

- Sudan black

- Schmorl's stain

- Aldehyde fuchsin

- Luxol-fast blue

- Ultrastructural analysis:

- Irregular, granular, electron-dense, membrane-bound intracytoplasmic inclusions within neurons (as seen in a Cynomolgus macaque) ${ }^{284}$. 
- Inclusions in humans vary according to disease subtype 285 :

- Electron-dense curvilinear profiles

- Electron-dense profiles resembling "finger-prints"

- Electron-dense haphazardly arranged rectilinear complexes

Comments: The neuronal ceroid lipofuscinoses are a group of lysosomal storage disorders characterized by the intracellular accumulation of lipopigments resulting in progressive neurodegeneration and neurologic decline. Cases of ceroid lipofuscinsosis are exceptionally rare in Cynomolgus macaques ${ }^{74}$. Neuronal ceroid lipofuscinoses should not be confused with age-associated intraneuronal lipofuscin accumulation, which occurs commonly in aged macaques without neurologic impairment.

\section{Degeneration, nerve fiber: Neuron-Axon (Figure 133)}

\section{Other term(s)}

Axonal spheroids; axonopathy

\section{Pathogenesis/Cell of Origin/Biological behavior}

Degeneration resultant from primary axonal injury with secondary myelin degredation, or primary myelin damage leading to secondary axonal disintegration.

\section{Diagnostic features}

- Early axonal lesion is characterized by swollen, eosinophilic axons (also known as spheroids) without any effect on myelin sheaths.

- As a primary lesion, myelin degeneration is usually observed as a clear space with ovoid-shaped myelin debris surrounding an intact centrally located axon.

- Late-stage changes are characterized by formation of "digestion chambers" containing macrophages (gitter cells), axon fragments and degenerated myelin ovoids.

Comments: "Spheroids" are often observed within the white matter tracts in the brain of aging cynomolgus macaques but rarely in young animals. The globus pallidus of the basal ganglia is the frequently affected site and the finding is generally thought to be a function of ageing 286,287 . Nerve fiber degeneration can occur in the absence of detectable functional neurologic impairment.

\section{Gliosis, not otherwise specified: Glia (Figure 134-135)}

\section{Other term(s)}

Astrocytosis; gemistocytosis; glia hypertrophy; microgliosis; oligodendroglial satellitosis; glial "scar"

\section{Pathogenesis/Cell of Origin}

This finding is occasionally observed in cynomolgus macaques and presumed to result from cerebral trauma and subsequent (attempted) repair by any of multiple cell lineages.

\section{Diagnostic features}

- Focal or multifocal aggregation of glial cells.

Comments: Scattered aggregates of glial cells are observed as spontaneous findings in the brains of group housed macaques in toxicity studies, and are presumed consequent to minor cerebral trauma incurred during play, aggression or housing transfer. The cell aggregates are mainly composed of microglial cells, oligodendrocytes, and/or astrocytes occasionally admixed with lymphocytes 279 . Numbers of microglial cells have been shown to increase with age in cynomolgus monkey brains ${ }^{287}$. When possible, the type of gliosis should be specified (e.g.; astrocytosis, oligodendroglial satellitosis) with cell types identified according to cytoarchitectural features. Identification criteria are similar to those for "gliosis NOS" in rodents 272 . This reactive, nonneoplastic change should be distinguished from pre-neoplastic changes (now termed "hyperplasia, glial cell NOS" in rodents $\left.{ }^{288}\right)$.

Globoid cell leukodystrophy: Glia (No image provided)

\section{Other term(s)}

Krabbe disease; Krabbe's disease; galactosylceramide lipidosis; galactocerebrosideosis

\section{Pathogenesis/Cell of Origin}

Inherited bi-allelic loss of function mutations in GALC gene result in deficient activity of galactosylceramidase leading to defective lipid metabolism. Subsequent accumulation of sphingolipids and psychosine results in widespread myelin degeneration and oligodendrocyte death.

\section{Diagnostic features}

- Distinctive "globoid" cells: swollen, often multinucleate macrophages containing flocculent eosinophilic cytoplasm. Globoid cells commonly form perivascular aggregates.

- Severe myelin degeneration

- Absence or necrosis of oligodendrocytes

- Neuronal loss in thalamus, cerebellum and brainstem

\section{Special Diagnostic Techniques:}

- Special stains ${ }^{289}$

- Periodic acid-Schiff (PAS): Globoid cells contain strongly PAS positive material

- Ultrastructural analysis 290

- Moderately electron-dense, variably sized, tubular, intracytoplasmic inclusions

Comments: Globoid cell leukodystrophy is an autosomal recessive, lysosomal storage disorder which occurs in Rhesus macaques ${ }^{291,292}$, and is a model for the human disease. Affected monkeys typically appear normal at birth, but become lethargic, dehydrated, fail to thrive and develop intention tremors in the weeks to months following birth 289 . 


\section{Primitive neuroectodermal tumor: Neuronal}

(Figure 136-137)

\section{Other $\operatorname{term}(\mathbf{s})$}

Supratentorial primitive neuroectodermal tumor; medulloblastoma; PNET; embryonal tumor

\section{Pathogenesis/Cell of Origin}

Malignant transformation of neuroectodermal embryonal progenitors

\section{Diagnostic features}

- Gross appearance:

- Invasive

- Soft to granular, gray parenchyma

- Variably cystic or hemorrhagic

- Histologic appearance:293

- Densely packed, small, oval cells with hyperchromatic nuclei

- High nucleus:cytoplasm ratio

- Mitotic figures are abundant

Comments: Central nervous system primitive neuroectodermal tumors (PNETs) are rare, malignant embryonal neoplasms, which occur most frequently in children ${ }^{294}$. In humans, intracranial PNET were historically classified as supratentorial PNETs or medulloblastomas. Although histologically similar, these subtypes are considered distinct due to anatomic 295 , immunohistochemical, genetic ${ }^{296-298}$, epigenetic 299 and prognostic differences ${ }^{300,301}$. Medulloblastomas most frequently arise within the cerebellum ${ }^{295}$ and leptomeningeal and fourth ventricular extension is common. Recent changes in the 2016 World Health Organization nomenclature have discarded PNET terminology in favor of the broader classification as "Embryonal tumors" with further subtyping, including particular distinction of medulloblastomas from other embryonal tumor subtypes (Louis et al., 2016). Medulloblastomas are further subclassified into genetic and histologically defined variants. The remaining embryonal tumor variants are classified by a combination of genetic and histopathologic characteristics.

\section{Astrocytoma, malignant: Astrocyte/Glial Precursor Cell} (No image provided)

\section{Other $\operatorname{term}(\mathbf{s})$}

Diffuse astrocytic glioma; grade II astrocytoma; fibrillary astrocytoma

\section{Pathogenesis/Cell of Origin}

Malignant transformation of astrocytes or associated glial precursor cells

\section{Diagnostic features}

- Gross appearance:

- Ill-defined with indistinct margins
- Light to dark tan

- May be spongy, gelatinous with cysts or calcified

- Histologic appearance:

- Stellate cells with fibrillar eosinophilic cytoplasm resembling astrocytes ${ }^{302}$

- Low to moderate cellularity, low mitotic rate, infrequent atypia ${ }^{303}$

\section{Special Diagnostic Techniques}

- Glial fibrillary acidic protein (GFAP): Astrocytes express GFAP, however, this does not distinguish neoplastic cells from reactive gliosis.

Comments: Astrocytomas are primary central nervous system tumors, which account for less than $5.6 \%$ of all primary central nervous system tumors in humans ${ }^{304}$. These tumors are rare in non-human primates, with one report in a cynomolgus macaque ${ }^{302}$. In humans, astrocytomas are classified by criteria set forth by the World Health Organization (WHO), and identified on the basis of associated genetic mutations and histologic features ${ }^{305}$. Astrocytomas are generally classified as diffuse (grade II, low grade) or anaplastic (grade III, high grade); and differentiated by degrees of atypia, infiltration and mitotic rate. By definition, necrosis and microvascular proliferation are not features of astrocytomas; both of these features are reserved for the diagnosis of glioblastoma ${ }^{303}$. Spontaneous glioblastomas have been described in a Rhesus macaque ${ }^{306}$, and female baboon ${ }^{307}$.

\section{Oligodendroglioma, malignant: Oligodendrocyte/Glial Precursor Cell (No image provided)}

\section{Other $\operatorname{term}(\mathbf{s})$}

Anaplastic oligodendroglioma, grade III oligodendroglioma, malignant oligodendroglioma, diffuse oligodendrocytic glioma

\section{Pathogenesis/Cell of Origin}

Malignant transformation of oligodendrocytes, or associated glial precursor cells

\section{Diagnostic features}

- Gross appearance $308-310$

- Generally well-defined, pink to grey

- Texture ranges from gelatinous to firm

- May contain scattered foci of hemorrhage, mucoid change, cystic degeneration and calcification

- Predilection for white matter

- Leptomeningeal invasion is common ${ }^{309-311}$

- Histologic appearance ${ }^{308,309}$

- Moderate cellularity

- Round cells with swollen, clear cytoplasm creating a "honeycomb" or "fried egg" appearance

- Round to oval nuclei containing finely granular chromatin

Comments: Oligodendrogliomas account for $1.5 \%$ of pri- 
mary brain and other CNS tumors in humans ${ }^{304}$. Cases in non-human primates are also rare. Oligodendrogioma has not been reported in Cynomolgus macaques published, however the authors have observed a single case in an aged cynomolgus monkey in an academic research setting.

Diagnosis of oligodendrogliomas in humans relies on genet- ic phenotyping and identification of both an IDH mutation and $1 \mathrm{p} / 19 \mathrm{q}$ chromosomal co-deletion status ${ }^{305}$. Differentiation between low (WHO grade II) and high grade oligodendroglioma (WHO grade III) is reliant upon histologic evidence of malignancy, including a high mitotic rate, nuclear atypia, necrosis and microvascular proliferation ${ }^{303,312}$. 


\section{Reproductive System - Female}

This section describes common background and treatmentinduced changes in the reproductive tract of female cynomolgus macaques (Macaca fascicularis). The scope is generally limited to findings reported in young animals in toxicology studies and aligned with those described for rodents when possible $^{313}$. In addition to the material provided here, we refer the reader to the 2008 monograph on the reproductive system of the female macaque published as a special supplement by the Society of Toxicologic Pathology 314-319, which includes tissue trimming guidelines ${ }^{317}$, and to additional works on the reproductive pathology of non-human primates (NHPs) (e.g. ${ }^{11,320,321) .}$

Macaques are Asian-origin NHPs with many biological similarities to humans, including a high degree of gene sequence identity (approximately 98\% for coding sequences of the cynomolgus macaque ${ }^{322}$, compared with $40 \%$ for the laboratory mouse (Mus musculus) ${ }^{323}$ ). Genetic variability among and within sub-populations of cynomolgus macaques is relatively high, with the lowest inter-individual variation seen in animals from the island of Mauritius ${ }^{324}$. Cynomolgus macaques interbreed with rhesus macaques (Macaca mulatta) in the wild, and rhesus lineage is present in commercially available cynomolgus macaques ${ }^{325}$. Notably, animals from different geographical regions may show distinct patterns of reproductive physiology and pathology. Seasonal effects on reproduction are insignificant in cynomolgus macaques. In contrast, the closely related rhesus macaque has strong seasonal suppression of reproductive function during the summer ${ }^{326}$.

\section{Menstrual Cycle}

From menarche to menopause, the menstrual cycle is the central physiological feature of the reproductive system in female cynomolgus monkeys. Accordingly, the toxicologic pathologist may encounter a variety of morphologic patterns within the reproductive tract depending on the phase of the cycle. This variability requires a sound understanding of the menstrual cycle phases, along with an awareness of how circulating ovarian hormones influence the appearance of the reproductive tract. While these normal cyclic changes are not routinely diagnosed in standard toxicology studies, documentation of the different phases of the menstrual cycle in females may be included if there is cause for concern based on the pharmacological mode of action, on suspicion of treatment-related effects interfering with the normal cycle-related events, on relevant findings in previous studies, or in cases where cycle monitoring is required.

The menstrual cycle of the cynomolgus monkey is generally divided into three major phases: the follicular phase, the luteal phase, and the menstrual phase. The follicular phase is characterized by the presence of an enlarging dominant follicle within the ovary. The rising levels of estradiol produced by this dominant follicle (in combination with low serum progesterone) increase glandular mitotic activity in the endometrium and, as such, the follicular phase is often referred to as the proliferative phase. Ovulation and the development of the corpus luteum is the hallmark of the luteal phase; however, in cynomolgus monkeys, there is considerable variability in the microscopic appearance of the ovary and endometrium. During the luteal or "secretory" phase, elevated levels of progesterone induce differentiation of a secretory phenotype within the endometrial glands. In the absence of maternal recognition of pregnancy, the corpus luteum begins to regress and the menstrual phase begins. Following menstruation, there is a short period of transition before the follicular phase begins in which there is repair and regeneration of the endometrium, often referred to as the regenerative phase. While these phases can be subdivided and may have multiple other terms, it is recommended here to use follicular, luteal, and menstrual phases. These terms are most closely aligned with the cycling changes in the ovary, which provides the primary hormonal stimulation for all other aspects of the cycle.

In the NHP, cyclic changes are most evident in the ovary and endometrium, which are the most useful organs for assessing cycle stage microscopically. The normally cycling endometrium can be staged to within a few days (Figure 138). A full description of macaque menstrual cycle features is beyond the scope of this review but can be referenced elsewhere $315,316,321,327-332$.

\section{Follicular Phase}

The follicular phase is characterized by the presence of a developing dominant follicle. Rising serum concentrations of estradiol along with lower progesterone concentrations drive characteristic features within the endometrium, including increasing numbers of mitoses, epithelial pseudostratification, an increasing proportion of straight or slightly spiraled parallel endometrial glands, and stromal edema in the superficial endometrium (zona functionalis). The thickness of the superficial endometrium increases, whereas the morphology of the basal endometrium (zona basalis) does not appreciably change.

\section{Luteal Phase}

The luteal phase is characterized by the presence of a developing corpus luteum. Elevated levels of progesterone induce glandular secretion, coiling, and dilation of endometrial glands, as well as proliferation and differentiation of the spiral arteries, as the endometrium becomes receptive to implantation of the blastocyst and supportive of early pregnancy. The endometrium reaches its maximal height at the beginning of the luteal phase and decreases towards the end. The increasing secretion causes glandular dilation and accumulation of secretory material in the glandular lumina. Towards the end of the luteal phase, signs of regression and glandular exhaustion become evident, including apoptotic cells among the glandular epithelium in the basal endometrium, accumulation of inspissated secretion and stromal edema with small hemorrhages and fibrin leakage. 


\section{Menstrual Phase}

Menstruation is the most clinically visible phase of the menstrual cycle, and the first day of bleeding is often designated as the first day of the cycle. The menstrual phase is characterized by a regressing corpus luteum on one of the ovaries. During menstruation, vasoconstriction and vascular thrombosis of the spiral arteries and disturbed circulation lead to tissue necrosis and disintegration and shedding of the superficial layer of the endometrium into the uterine lumen. Multifocal hemorrhage into the superficial stroma is the first hallmark of the menstrual phase. There is focal dense clumping of superficial endometrial stromal cell nuclei. Portions of the sloughed endometrial tissue, hemorrhage from stromal blood vessels, leukocytes, and cellular debris can be found in the uterine lumen, the endocervical canal, and the vagina. During menstruation, the entire superficial endometrium is shed and the endometrial stroma is partially denuded. The remaining epithelial cells are low to medium columnar. The basal endometrium is spared. The glands of the basal endometrium may still contain secretory material but remain quiescent.

\section{Recommendations for Documentation of Sexual Maturity in Female NHPs}

In addition to normal ovarian cyclicity, there is also considerable variability in the histologic features across the different stages of reproductive development. The toxicologic pathologist is often required to interpret these patterns and document whether the animal is considered sexually mature based on microscopic features. Histologic confirmation of sexual maturity is especially important when working with NHPs, as age alone is not an effective way to determine if animals are sexually mature. It is recommended to document for each animal in a study if it is considered immature, peripubertal, mature, or senescent. Immaturity may be documented in some cases, leading to the presumption of maturity if not stated otherwise ${ }^{333}$. This is not a recommended practice, as a study with all mature animals will have nothing documented, and unless the assumption of maturity is specifically included in the materials and methods, downstream readers will have no way to know if the animals were mature or not. Therefore, it is recommended here to document sexual maturity or immaturity for all animals under study.

When documentation of sexual maturity is needed, it is best to create or use a "summary tissue" in the data entry system such as "Reproductive Status" and to use this parameter to record the maturity status of all animals in the study. Depending on the data entry system limits, documentation of sexual maturity can also be made under "General Observations". This approach allows for a full assessment of all individual organs in the reproductive system, for individual histopathologic diagnoses under the reproductive organs to be used independent of maturity status, and for data (including reproductive status) to be easily pulled into tabular summaries and historical control data sets. In-life data, if available, may also be correlated to support morphological findings.

The terms for describing the maturity status recorded under the pseudo-tissue or general observations are immature, peripubertal, mature (Figure 139), and senescent. Awareness of the normal features of these developmental stages is important in the identification and interpretation of treatment effects on the reproductive system.

\section{Immature}

Due to absence or low levels of hormone stimulation from the hypothalamus and pituitary, there is no (cyclic) development of hormone responsive structures within the primary and secondary reproductive organs. This state is reflected in the following morphologic characteristics: the ovaries show varying degrees of early follicular development, but lack corpora lutea or luteal remnants; the uterus is small with inactive glands and condensed stroma and myometrium; the vagina is small and shows a low, cuboidal non-keratinized epithelial lining; and the mammary gland consists only of rudimentary ducts and lacks signs of development ${ }^{334}$.

\section{Peripubertal}

Initiated by the increased production and secretion of gonadotropin-releasing hormone ( $\mathrm{GnRH})$ in the hypothalamus, the pituitary starts to produce follicle stimulating hormone (FSH) and luteinizing hormone (LH). These gonadotropins are responsible for early development of hormone-responsive structures within the reproductive organs through stimulation of the ovary and early steroidogenesis. The ovaries may show varying degrees of follicular development (up to the stage of tertiary or preovulatory follicles) or the presence of an occasional corpus luteum, but, in general, show absence of multiple remnants of previous corpora lutea as observed in mature regularly cycling females. Although young females may have microscopic evidence of prior ovulation (presence of one or two remnants of corpora lutea), it should be recognized that many of the NHPs used in routine general toxicology studies are only recently post-menarche and are not likely fully mature or regularly cycling. Because of the production of estrogens originating from developing follicles within the ovaries, the secondary reproductive organs can show varying degrees of development including proliferative changes within the endometrium (mitoses, pseudostratification), epithelial maturation with keratinization in the vagina, and ductal expansion, branching, and differentiation into lobuloalveolar units in the mammary gland ${ }^{252}$.

\section{Mature}

A mature animal has a cycle length generally between 28 and 32 days ${ }^{315,316}$, although there is considerable intra- and inter-animal variability, in large part due to social and hierarchal influences ${ }^{318}$. The microscopic appearance of the ovary varies with the phase of menstrual cycle and may display varying degrees of follicular development (up to the stage of a large preovulatory, tertiary follicle) or a developing or mature corpus 
luteum and has multiple remnants of corpora lutea from prior cycles. Since mature NHPs have a relatively high incidence of anovulatory cycles, evidence of current cyclicity is not always possible and the presence of multiple remnants of corpora lutea is often the only histologic indicator of maturity in these animals ${ }^{335}$. Due to the cyclic changes in estradiol and progesterone, the endometrium exhibits alternating features of follicular, luteal, or menstrual phase characteristics (as described by van Esch et al.315). The vagina has a well-keratinized epithelium throughout the cycle and the mammary glands show signs of development and/or secretory activity.

\section{Senescent}

While most toxicologic pathologists work with juvenile or early adult animals, an understanding of age-related changes may be important in certain disease models or aging studies, requiring documentation of the reproductive status for proper study interpretation. Macaques of 20 or more years of age typically show signs of reproductive inefficiency and senescence. Menses may become irregular and eventually cease (menopause). Decreased serum estradiol, normal or reduced progesterone, and increased FSH concentrations are often observed with histologic evidence of reproductive senescence ${ }^{336,337}$. Anti-Müllerian hormone levels decrease with increasing age and may be useful as a marker of diminished ovarian reserve ${ }^{338}$. As a part of reproductive senescence, the ovaries exhibit a decrease in primordial, primary, and antral follicles with an increase in atretic follicles. The uterine endometrial stroma and glands are often inactive and appear similar to an early follicular phase endometrium without mitotic activity. Endometrial glands are narrow and straight with cuboidal to low columnar epithelium. The endometrial height is generally low and as the animal becomes older, the endometrium becomes more compact with endometrial glands often oriented parallel to the endometrial surface. Endometrial glands may become dilated. Estrogen receptor expression is high in the initial stages of senescence but eventually decreases and may ultimately vanish ${ }^{315}$. The cervical and vaginal epithelium will show evidence of atrophy ${ }^{319}$. The mammary glands of older macaques regress with age, showing lobular atrophy and low proliferative activity 248 . It is important to note that the term "senescent" is a general term and there are considerable species differences in the patterns and morphologic features observed as a part of reproductive aging and the features described for NHP here are distinct from those observed in rodents ${ }^{313}$.

\section{A. Ovary}

The ovary of an immature animal typically contains multiple secondary or tertiary follicles, without signs of ovulation or formation of corpora lutea. In mature macaques, one ovary ovulates in a given cycle. Thus, the single dominant ovarian structure (mature follicle, corpus hemorrhagicum, or corpus luteum) will only be evident on one side, and it is necessary to examine complete sections of both ovaries in order to determine the likely hormonal context for interpretation of the endometrium and the rest of the reproductive tract. Gross photographs of both sides of the ovaries are a useful adjunct to histology, when assessment of reproductive function is critical. In a normally cycling adult animal, follicles are present at all stages (primordial to antral). As the dominant follicle enlarges, other antral follicles become atretic, with apoptosis of granulosa cells. Follicles develop over 3-5 cycles, so small to mid-sized antral follicles can be seen at any cycle stage. Corpora lutea largely regress at the end of the menstrual cycle, but remnants are usually visible for several subsequent cycles. Fibrous corpora albicantia may arise from either ovulatory or non-ovulatory follicles. After regression of non-dominant follicles, acellular, semicircular, pale eosinophilic zona pellucida remnants persist within the ovarian stroma for months to years. The macaque ovary has a prominent rete ovarii at the hilus but no ovarian bursa. The fimbria is prominent macroscopically and may be larger than the ovary. Uncommon disorders of the ovary include polycystic ovarian syndrome ${ }^{339}$, a condition that can be mimicked by treatment with high-dose FSH agonist treatment (Figure 140). Male pseudohermaphroditism has also been reported ${ }^{340}$ (Table 28).

\section{Deciduosis: Ovary, Oviduct, Vagina}

(Figure 141-142 (Ovary); Figure 173 (Vagina))

\section{Other term(s)}

Extrauterine decidual change; ectopic decidual reaction

\section{Pathogenesis/Cell of Origin}

Subcoelomic mesenchyme-derived stromal cells within the ovarian cortex; stromal cells of the oviduct, cervix, or vagina

\section{Diagnostic features}

- Presence of a plaque or nodule(s) of decidualized cells, sometimes forming polypoid projection from the ovarian surface

- Decidualized cells are large polyhedral, cytoplasm-rich (epithelioid) pale basophilic stromal cells.

- Granulated lymphocytes with eosinophilic cytoplasmic globules are often interspersed within areas of decidualized stromal cells in the endometrium.

- The cytology of the cells is comparable to the decidualized cells observed within the endometrium during pregnancy or during high-dose progesterone treatment.

\section{Differential Diagnoses}

- Ectopic decidual reaction should not be mistaken for carcinoma or other neoplastic growths.

\section{Special Techniques for Diagnostics}

- With PAS, decidualized stromal cells are found to store detectable amounts of cytoplasmic glycogen.

Comments: "Deciduosis" is the presence of decidual tissue or tissue resembling the endometrium of pregnancy in an ectopic site $^{6,11,314}$. Deciduosis is a rare finding in nulliparous NHPs. 
Table 28. Microscopic Findings of the Female Reproductive System: Ovary; Non-human Primate

\begin{tabular}{|c|c|c|c|c|}
\hline OVARY & Common & Uncommon & $\begin{array}{c}\text { Not Observed but } \\
\text { Potentially Relevant }\end{array}$ & Not Applicable \\
\hline \multicolumn{5}{|l|}{ Congenital } \\
\hline Ectopic tissue $^{\wedge}$ & & $\mathrm{x}$ & & \\
\hline Ovotestis & & & $\mathrm{x}$ & \\
\hline \multicolumn{5}{|l|}{ Non-proliferative } \\
\hline Amyloid $^{\wedge}$ & & $\mathrm{x}$ & & \\
\hline Angiectasis & & $\mathrm{x}$ & & \\
\hline Atrophy & & $\mathrm{x}$ & & \\
\hline Atrophy, age-related & & $\mathrm{x}$ & & \\
\hline Atrophy, corpora lutea & $\mathrm{x}$ & & & \\
\hline Cyst, bursal & & & & $\mathrm{x}$ \\
\hline Cyst, epithelial & & $\mathrm{x}$ & & \\
\hline Cyst, follicular & & $\mathrm{x}$ & & \\
\hline Cyst, luteal & & $\mathrm{x}$ & & \\
\hline Cyst, mesonephric & & $\mathrm{x}$ & & \\
\hline Cyst, NOS & & $\mathrm{x}$ & & \\
\hline Cyst, paraovarian & $\mathrm{x}$ & & & \\
\hline Cyst, rete ovarii & $\mathrm{x}$ & & & \\
\hline Deciduosis * & & $\mathrm{x}$ & & \\
\hline Decreased number/absent corpora lutea & $\mathrm{x}$ & & & \\
\hline Decreased number/absent follicles & & $\mathrm{x}$ & & \\
\hline Degeneration, corpora lutea & & $\mathrm{x}$ & & \\
\hline Degeneration, oocyte & & $\mathrm{x}$ & & \\
\hline Edema $^{\wedge}$ & & $\mathrm{x}$ & & \\
\hline Follicle, luteinized & $\mathrm{x}$ & & & \\
\hline Follicle, polyovular* & $\mathrm{x}$ & & & \\
\hline Hypertrophy, corpora lutea & & $\mathrm{x}$ & & \\
\hline Hypertrophy, interstitial cell & & $\mathrm{x}$ & & \\
\hline Immature & $x$ & & & \\
\hline Increased number, atretic follicles & & $\mathrm{x}$ & & \\
\hline Increased number, corpora lutea & & & $\mathrm{x}$ & \\
\hline Infiltrate, [insert appropriate cell type]^ & & $\mathrm{x}$ & & \\
\hline Inflammation $^{\wedge}$ & & $\mathrm{x}$ & & \\
\hline Mineralization, follicle $^{\wedge}$ & $\mathrm{x}$ & & & \\
\hline Pigment (melanin $/$ melanosis) $^{\wedge}$ & $\mathrm{x}$ & & & \\
\hline Vacuolation, corpora lutea & & $\mathrm{x}$ & & \\
\hline Vacuolation, granulosa cell & & $\mathrm{x}$ & & \\
\hline Vacuolation, interstitial cell & & $\mathrm{x}$ & & \\
\hline Vacuolation, theca cell & & $\mathrm{x}$ & & \\
\hline \multicolumn{5}{|l|}{ Proliferative (non-neoplastic) } \\
\hline Hyperplasia, cystic/papillary & & $\mathrm{x}$ & & \\
\hline Hyperplasia, granulosa cell & & $\mathrm{x}$ & & \\
\hline Hyperplasia, interstitial cell & & $\mathrm{x}$ & & \\
\hline Hyperplasia, surface epithelium * & $\mathrm{x}$ & & & \\
\hline Hyperplasia, rete ovarii & $\mathrm{x}$ & & & \\
\hline Hyperplasia, Sertoli cell & & & $\mathrm{x}$ & \\
\hline Hyperplasia, sex cord stromal, mixed & & $\mathrm{x}$ & & \\
\hline Hyperplasia, smooth muscle, mesovarium & & & $\mathrm{x}$ & \\
\hline Hyperplasia, theca cell & & $\mathrm{x}$ & & \\
\hline Hyperplasia, tubulostromal & & & $\mathrm{x}$ & \\
\hline Metaplasia, smooth muscle cell & & $\mathrm{x}$ & & \\
\hline \multicolumn{5}{|l|}{ Neoplastic } \\
\hline Adenoma, rete ovarii & & $\mathrm{x}$ & & \\
\hline Adenoma, tubulostromal & & $\mathrm{x}$ & & \\
\hline Carcinoma, embryonal & & $\mathrm{x}$ & & \\
\hline Choriocarcinoma * & & $\mathrm{x}$ & & \\
\hline Cystadenoma & & $\mathrm{x}$ & & \\
\hline Cystadenocarcinoma & & $\mathrm{x}$ & & \\
\hline Tumor, granulosa cell, benign * & $\mathrm{x}$ & & & \\
\hline Tumor, granulosa cell, malignant $*$ & & $\mathrm{x}$ & & \\
\hline Teratoma, benign * & $\mathrm{x}$ & & & \\
\hline Teratoma, malignant & & $\mathrm{x}$ & & \\
\hline
\end{tabular}

* Terminology with diagnostic criteria or comments described below. \# Findings more frequently observed as an induced change.

$\wedge^{\wedge}$ Terms that are addressed in the Systemic/General Pathology section. 
Follicle, polyovular: Ovary (Figure 143)

\section{Pathogenesis/Cell of Origin}

Polyovular follicles appear to occur due to a failure of normal germ cell nest breakdown during the formation of primordial follicles in the late fetal and early neonatal period.

\section{Diagnostic features}

- Multiple (two or more) oocytes present within a single developing follicle.

\section{Differential Diagnoses}

- None

Comments: Polyovular follicles are commonly observed in young macaques ${ }^{314,}{ }^{341}$. The presence and number can vary between animals, with occasional animals having numerous polyovular follicles. Increased polyovular follicles have been reported in mice following xenobiotic exposure during the fetal or perinatal period that induces hormonal dysregulation, in particular disruption of estrogen signaling pathways $^{342-344}$. In rhesus macaques, the number of polyovular follicles increase following exposure to exogenous $\mathrm{FSH}^{341}$. While test article-related increases can occur, this can be difficult to evaluate in macaques due to the variability and presence of large numbers of polyovular follicles in normal control animals.

\section{Mineralization, follicle: Ovary (Figure 144)}

\section{Pathogenesis/Cell of Origin}

The change appears to arise from degenerate primordial oocytes within atretic primordial follicles undergoing dystrophic calcification.

\section{Diagnostic features}

- Calcified round bodies are present within the ovarian cortex.

- The mineralized bodies are often associated with primordial oocytes.

\section{Differential Diagnoses}

- None

Comments: Follicular mineralization is a common finding in the macaque ovary. The change appears to arise from degenerate primordial oocytes within atretic follicles that undergo dystrophic calcification. This change is most commonly observed in young adult animals 11,314 and can vary between individuals with occasional animals having extensive mineralization.

\section{Hyperplasia, surface epithelium: Ovary (Figure 145)}

\section{Other $\operatorname{term}(\mathbf{s})$}

Hyperplasia, ovarian serosal epithelium; hyperplasia, ovarian mesothelium

\section{Pathogenesis/Cell of Origin}

Cell of origin is the ovarian serosal epithelial cell.

\section{Diagnostic features}

- Focal or multifocal papillary, indented, or saw-toothed, non-mass-forming lesions consisting of a single layer of closely packed columnar ovarian surface epithelial cells with normal ovarian stroma beneath.

- Few/no mitoses

- No atypia or pleomorphism

\section{Differential Diagnoses}

- Ovarian surface epithelial tumor

- Mass-forming exophytic neoplasm arising from the ovarian surface epithelium

- Endometriosis

- Typically, cystic and accompanied by endometrial stroma, macrophages (often hemosiderin-laden), and hemorrhage

Comments: Ovarian surface epithelial hyperplasia is a common background finding, usually of no significance ${ }^{314}$.

\section{Choriocarcinoma: Ovary, Uterus}

(Figure 166-167 (Uterus))

\section{Pathogenesis/Cell of Origin}

The cell of origin is unknown but is considered to be a primitive cell of ovarian germ cell origin, with differentiation into cytotrophoblast, intermediate trophoblast, and syncytiotrophoblast lineages.

\section{Diagnostic features}

- Pleomorphic, anaplastic polygonal to round, large cells with an infiltrative/destructively invasive growth pattern and a high mitotic rate.

- Two distinct neoplastic cellular populations, uninucleate (cytotrophoblast/intermediate trophoblast-like) and multinucleated (syncytiotrophoblast)

- Scant fibrovascular stroma

\section{Differential Diagnoses}

- Ovarian granulosa cell tumor

- Arise from within the ovary, and have a more uniform cellular population and a distinct nodular/nest/cord growth pattern

\section{Special Techniques for Diagnostics}

- Immunostaining for human chorionic gonadotropin (HCG), mucin 4 (MUC4) and CD146 (Mel-CAM) ${ }^{345}$

Comments: Choriocarcinomas are uncommon in macaques $^{314,346}$, but have been reported in toxicology studies $^{347}$. A mixed germ cell tumor with elements of choriocarcinoma has also been reported in a 7-year-old cynomolgus macaque in a toxicology study ${ }^{348}$. 
Tumor, granulosa cell, benign or malignant: Ovary

(Figure 146)

\section{Pathogenesis/Cell of Origin}

The cell of origin is the ovarian granulosa cell.

\section{Diagnostic features}

- Solid or cystic nodules, nests, and thick cords of uniform polygonal epithelial cells within the ovary, with scant fibrovascular stroma. Usually well circumscribed and bounded by a distinct basement membrane zone (benign); rarely invasive (malignant).

- Uniform cellular features resembling granulosa cells; moderate amount of pale basophilic cytoplasm, oval vesicular nuclei with finely stippled chromatin.

\section{Differential Diagnoses}

- Hyperplastic follicular remnants

- Follicular remnants are typically solitary, small $(<1$ $\mathrm{mm})$, occur in older animals, and may contain zona pellucida remnants.

- Hyperplastic rete ovarii

- The rete ovarii has a distinct tubular to retiform contour with concave borders.

- Sex cord-stromal tumor

- This tumor is composed of more fusiform cells, with palisading arrays along basement membrane zones.

- Cystadenoma/Cystadenocarcinoma

- This surface-oriented tumor has an exophytic, papillary morphology.

\section{Special Techniques for Diagnostics}

- Clinical, gross, or histopathologic evidence of hyperestrogenism

Comments: Granulosa cell tumors are one of the two most common malignant neoplasms of the ovary in rhesus macaques, the other being cystadenocarcinoma 314,349 . Granulosa cell tumors are often hormonally active, producing elevated serum concentrations of estradiol and resulting in uterine enlargement, endometrial hyperplasia, endometrial polyps, and/or endocervical squamous metaplasia.

Teratoma, benign: Ovary (Figure 147-149)

\section{Other term(s)}

Dermoid cyst

\section{Pathogenesis/Cell of Origin}

Germ cell origin, presumably congenital.

\section{Diagnostic features}

- Gross: Solitary round mass, often pigmented and cystic

- Histologic presence of at least 3 different cell types derived from different embryonic tissue lineages (mesodermal, ectodermal, endodermal, or neural tube/neural crest)

- Tissues disorganized but differentiated into recognizable tissue types (e.g. skin, bone, neural, intestinal)

\section{Differential Diagnoses}

- Other common ovarian tumor types (granulosa cell tumor, ovarian surface epithelial tumor)

- These tumors arise from a single cell type; morphology is described elsewhere in this monograph.

- Endometriosis

- Endometriosis consists of endometrial glands and stroma, with hemorrhage and macrophage-rich inflammation with hemosiderosis, but not multiple mature lineages.

\section{Special Techniques for Diagnostics}

- None are required; histologic features are distinctive and diagnostic.

Comments: Teratomas are the most common benign neoplasm of the macaque ovary2, 314, 347, 349, 350. In human pathology benign teratomas are the most common neoplasm in juveniles, and are also termed "dermoid cysts" 351 .

\section{B. Oviduct (Table 29)}

Table 29. Microscopic Findings of the Female Reproductive System: Oviduct; Non-human Primate

\begin{tabular}{|c|c|c|c|c|}
\hline OVIDUCT & Common & Uncommon & $\begin{array}{c}\text { Not Observed but } \\
\text { Potentially Relevant }\end{array}$ & Not Applicable \\
\hline \multicolumn{5}{|l|}{ Non-proliferative } \\
\hline Atrophy & $\mathrm{x}$ & & & \\
\hline Cyst & & $\mathrm{x}$ & & \\
\hline Infiltrate, [insert appropriate cell type $]^{\wedge}$ & & $\mathrm{x}$ & & \\
\hline Inflammation $^{\wedge}$ & & $\mathrm{x}$ & & \\
\hline Salpingitis isthmica nodosa & & & & $\mathrm{x}$ \\
\hline \multicolumn{5}{|l|}{ Proliferative (non-neoplastic) } \\
\hline Hyperplasia, epithelium & & $\mathrm{x}$ & & \\
\hline Metaplasia, squamous cell & & $\mathrm{x}$ & & \\
\hline \multicolumn{5}{|l|}{ Neoplastic } \\
\hline Mullerian tumor & & $\mathrm{x}$ & & \\
\hline
\end{tabular}




\section{Uterus}

\section{Introduction to Non-Cyclic Hormone-mediated Endome- trial Changes}

Ovarian estrogens and progesterone mediate a range of glandular and stromal changes during the menstrual cycle. Administration of various activators or inhibitors of trophic factors (e.g. GnRH, FSH, and $\mathrm{LH}$ ) or steroid receptor agonists/antagonists can alter this normal pattern and result in morphologic abnormalities, including abnormal development/ maturation of glands, stroma, and/or spiral arteries. There may be overt alterations of these structures, or the changes may be within the range of what is observed during the normal cycle, just without the normal timing of events within the endometrium or without the expected development or structures (i.e., dominant follicle or corpus luteum in the ovary). Such changes can be challenging to identify and require thorough knowledge of the normal endometrial morphologic events for proper diagnosis and interpretation. For steroid receptor agonists/antagonists, the endometrium can be inactive or dyssynchronous and show non-physiologic secretory and proliferative patterns (preferred terms are described below) depending on pharmacologic profile of the test article, dose, treatment period, and/ or hormonal context.

\section{Inactive endometrium}

An inactive endometrium arises from decreased activation of estrogen and/or progesterone receptors, either because of reduced hormonal stimulation or because of a blockade/inhibition at the receptor or pathway level. Morphologically, the characteristics of an inactive endometrium consist of stromal compaction, inactive glands lined by low columnar to cuboidal epithelium with small, dark nuclei and minimal cytoplasm and a very low number or even absence of mitotic figures or absence of cytoplasmic vacuoles (Figure 150). Examples of compounds capable of inducing an inactive endometrium are gonadotropin-releasing hormone $(\mathrm{GnRH})$ antagonists, selective estrogen receptor modulators (SERMs) such as bazedoxifene acetate, and selective progesterone receptor modulators (SPRMs) (with predominantly antagonistic activity) such as mifepristone (RU 486). Long-standing suppression can finally result in an atrophic endometrium in which the number of estrogen and progesterone receptors are markedly reduced (e.g. Ethun et al. ${ }^{352}$ ).

\section{Dyssynchronous endometrium}

A dyssynchronous endometrium arises from a perturbation of the normal physiologic sequence of events that occurs within the endometrium during the menstrual cycle. It consists of a varying combination of overlapping morphologic changes consisting of inactive glands, glands showing low mitotic activity and/or nuclear pseudostratification, cystic dilated glands, gland tortuosity, varying grades of glandular secretory changes, apoptotic changes, hemorrhage, compact stroma, stromal decidual changes, and thick-walled spiral arteries (Figure 151153). SPRMs with mixed agonistic/antagonistic progesterone receptor activity such as asoprisnil and ulipristal acetate are examples of compounds that can induce a dyssynchronous endometrium.

\section{Asynchronous endometrium (normophysiologic secretory pattern)}

An asynchronous endometrium mimics a normal cyclic luteal phase (and can have the characteristics of an early, mid, and late luteal phase), but it is induced by exogenous administered hormones. The endometrium is called asynchronous because it does not correspond to the ovarian phase/morphology. Examples are estrogen/progestogen therapies including oral contraceptives.

\section{Non-physiologic patterns of endometrial histopathology}

A variety of non-physiologic patterns may be seen in the endometrium that reflect endogenous or exogenous influences on the reproductive system. The most common pattern occurring as a background effect is reduced endometrial proliferation and/or differentiation as a result of social, environmental, or treatment-related stressors. The impact of social status and stress on reproductive function is well-documented ${ }^{318,353}$. Follicular and luteal phase deficiencies may result from suppression of the hypothalamic-pituitary-gonadal (HPG) axis. Suppression of the HPG axis in socially subordinate animals is a normal facet of macaque reproductive physiology, resulting in non-ovulatory cycles and a corresponding lack of full lutealphase endometrium.

Other non-physiologic patterns can provide insight into class-specific effects. Examples include (1) the effects of selective estrogen receptor agonists such as tamoxifen, which produce glandular atrophy, cystic change, and endometrial stromal fibrosis ${ }^{354}$; and (2) the pattern produced by selective progesterone receptor agonists, consisting of overt luteal phase-type of changes of the glands, with saw-tooth appearance and varying grades of epithelial cytoplasmic vacuolation, prominent spiral arteries and (pseudo)decidualization of the stroma. These endometrial changes can mimic pregnancy-related endometrial changes.

Although it is tempting and sometimes useful to discuss class-specific changes in terms of "typical patterns" of estrogen and progestogen effects, the most useful approach for study interpretation is to simply indicate the presence of a nonphysiologic pattern, and to include a careful description of the morphologic features of that pattern, using concurrent control animals as comparators (Table 30 ).

\section{Adenomyosis: Uterus (Figure 154)}

\section{Other term(s)}

Myometrial endometriosis; endometriosis interna; endometrial diverticulosis

\section{Pathogenesis/Cell of Origin}

Adenomyosis pathogenesis is still largely unknown but multiple hypotheses exist including direct myometrial invasion by 
Table 30. Microscopic Findings of the Female Reproductive System: Uterus; Non-human Primate

\begin{tabular}{|c|c|c|c|c|}
\hline UTERUS & Common & Uncommon & $\begin{array}{c}\text { Not Observed but } \\
\text { Potentially Relevant }\end{array}$ & Not Applicable \\
\hline \multicolumn{5}{|l|}{ Congenital } \\
\hline Aplasia, segmental & & $\mathrm{x}$ & & \\
\hline Remnant, mesonephric duct & & $\mathrm{x}$ & & \\
\hline \multicolumn{5}{|l|}{ Non-proliferative } \\
\hline Abscess(es) & & $\mathrm{x}$ & & \\
\hline Adenomyosis * & & $\mathrm{x}$ & & \\
\hline Amyloid ^ & & $\mathrm{x}$ & & \\
\hline Angiectasis & & $\mathrm{x}$ & & \\
\hline Apoptosis $\wedge$ & & $\mathrm{x}$ & & \\
\hline Atrophy, (endometrial, epithelial, glandular, stromal) & & $\mathrm{x}$ & & \\
\hline Cyst, NOS & & $\mathrm{x}$ & & \\
\hline Decidual reaction (nodule) & & & & $\mathrm{x}$ \\
\hline Decidualization * & $\mathrm{x}$ & & & \\
\hline Decreased endometrial differentiation, luteal phase * & $\mathrm{x}$ & & & \\
\hline Decreased glandular development, follicular phase * & $\mathrm{x}$ & & & \\
\hline Degeneration, spiral arteries * & & $\mathrm{x}$ & & \\
\hline Dilatation, glandular, cystic & $\mathrm{x}$ & & & \\
\hline Dilatation, lumen & & $\mathrm{x}$ & & \\
\hline Dyssynchronous endometrium * & $\mathrm{x}$ & & & \\
\hline Ectopic tissue, ovary ${ }^{\wedge *}$ & $\mathrm{x}$ & & & \\
\hline Endometriosis* & $\mathrm{x}$ & & & \\
\hline Fibrosis & $\mathrm{x}$ & & & \\
\hline Granuloma & & $\mathrm{x}$ & & \\
\hline Hemorrhage, abnormal, endometrial * & $\mathrm{x}$ & & & \\
\hline Hyaline deposits, perivascular * & $\mathrm{x}$ & & & \\
\hline Hyalinization, stroma & & $\mathrm{x}$ & & \\
\hline Hypersecretion, glandular * & & $\mathrm{x}$ & & \\
\hline Hypertrophy, epithelium & & $\mathrm{x}$ & & \\
\hline Hypertrophy, myometrium & & $\mathrm{x}$ & & \\
\hline Hypoplasia & & $\mathrm{x}$ & & \\
\hline Immature & & $x$ & & \\
\hline Inactive endometrium * & $\mathrm{x}$ & & & \\
\hline Infarct * & & $\mathrm{x}$ & & \\
\hline 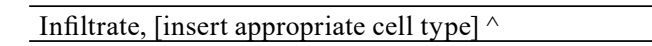 & $\mathrm{x}$ & & & \\
\hline Inflammation $\wedge^{\wedge}$ & & $\mathrm{x}$ & & \\
\hline Inflammation, endometrium & & $\mathrm{x}$ & & \\
\hline Inflammation, myometrium & & $\mathrm{x}$ & & \\
\hline Necrosis & & $\mathrm{x}$ & & \\
\hline 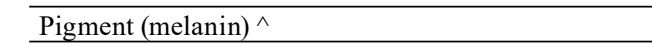 & & $\mathrm{x}$ & & \\
\hline Prolapse & & $\mathrm{x}$ & & \\
\hline Pyometra & & $\mathrm{x}$ & & \\
\hline Thrombus & & $\mathrm{x}$ & & \\
\hline Vacuolation, epithelial cell & & $\mathrm{x}$ & & \\
\hline \multicolumn{5}{|l|}{ Proliferative (non-neoplastic) } \\
\hline Epithelial plaque * & $\mathrm{x}$ & & & \\
\hline Hyperplasia, angiomatous & & & $\mathrm{x}$ & \\
\hline Hyperplasia, basal gland * & & $\mathrm{x}$ & & \\
\hline Hyperplasia, endometrium, diffuse & & $\mathrm{x}$ & & \\
\hline Hyperplasia, endometrial stroma & & $\mathrm{x}$ & & \\
\hline Hyperplasia, glandular, cystic & & $\mathrm{x}$ & & \\
\hline Hyperplasia, glandular, focal & & $\mathrm{x}$ & & \\
\hline Hyperplasia, granular cell & & & & $\mathrm{x}$ \\
\hline Hyperplasia, mesothelium, papillary & & $\mathrm{x}$ & & \\
\hline Hyperplasia, myometrium & & $\mathrm{x}$ & & \\
\hline Metaplasia, ciliated cell $*$ & & $\mathrm{x}$ & & \\
\hline Metaplasia, endocervical * & & $\mathrm{x}$ & & \\
\hline Metaplasia, squamous cell * & & $\mathrm{x}$ & & \\
\hline \multicolumn{5}{|l|}{ Neoplastic } \\
\hline Adenoma, endometrium & & $\mathrm{x}$ & & \\
\hline Papilloma, squamous cell & & $\mathrm{x}$ & & \\
\hline Polyp, glandular & & $\mathrm{x}$ & & \\
\hline Polyp, stromal & & $\mathrm{x}$ & & \\
\hline Leiomyoma & $\mathrm{x}$ & & & \\
\hline
\end{tabular}


the endometrium, uterine traumatization (e.g. surgery), (focal) myometrial weakening or rupture (e.g. related to pregnancy), developmental anomalies (misplaced endometrial precursors), or bone marrow-derived stem cells differentiating to glandular and stromal components 355 .

\section{Diagnostic features}

- Focal aggregate(s) of normal, well-differentiated glandular epithelium and stroma, reminiscent of endometrium, but present in the myometrium.

- Myometrium may be grossly thickened.

- Foci of adenomyosis may show cyclic changes in concordance to the endometrium.

- Foci of adenomyosis may show a connection to the luminal surface, suggesting endometrial diverticulosis.

- Adenomyosis may be focal and well-circumscribed (adenomyoma) or diffuse.

\section{Differential Diagnoses}

- Fallopian tube or tangential sections of endometrium within the myometrium

- Endometrial stromal sarcoma

- Nodule of neoplastic stromal tissue resembling proliferative phase endometrial stroma within the myometrium. Tumors may extend into the endometrium as a polyp.

- Lack of endometrial glands as observed in adenomyosis.

- May contain necrosis or hemorrhage depending on grade.

\section{Special Techniques for Diagnostics}

- Immunohistochemistry

- CD10/CALLA (membranous and cytoplasmic) - Adenomyosis stromal cells are positive, endometrial glands are negative

Comments: Adenomyosis is most commonly diagnosed in captive NHPs after hysterectomy or at necropsy with a prevalence of up to $24 \%$ in macaques ${ }^{356}$. The change can also can be induced by exposure to high levels of unopposed estrogenic compounds ${ }^{357}$. In rhesus monkeys, some cases of adenomyosis were found in conjunction with endometriosis $^{356}$. Macaques with adenomyosis may show clinical signs of abnormal uterine bleeding and abdominal pain. Histologic diagnosis should only be made from well-positioned, full thickness histology sections and not biopsy.

\section{Decidualization: Uterus (Figure 151)}

\section{Other term(s)}

Decidual reaction; pseudodecidualization

\section{Modifiers}

Focal, diffuse, perivascular

\section{Pathogenesis/Cell of Origin}

Endometrial stromal cell, that under progestogenic stimulation transform into large, polyhedral, cytoplasm-rich (epithelioid) cells storing large amounts of glycogen (the process of decidualization).

\section{Diagnostic features}

- Presence of endometrial periarterial areas of decidualized stromal cells to, in more progressed cases, presence of decidualized stromal cells occupying the whole of the stroma in the superficial endometrium and even part of the basalis.

- The decidualized cells are large polyhedral to spindleshaped, pale basophilic cytoplasm-rich (epithelioid) stromal cells.

- Intermingled within areas of decidualized stromal cells, often endometrial lymphocytes with their eosinophilic cytoplasmic globules (NK cells) can be observed additionally.

- In progressed cases, stromal decidualization can cause compression and obstruction and consequently dilation of the upper part of the glands.

- In cases of chronic stimulation, the decidualized stromal cells can become spindle-shaped and resemble smoothmuscle cells.

\section{Differential Diagnoses}

- Normal luteal-phase decidual change.

\section{Special Techniques for Diagnostics}

- With the PAS methods, decidualized stromal cells are found to store detectable amounts of cytoplasmic glycogen.

- Decidual cells stain positive for CD10, while the NK cells within the decidualized area stain positive for CD56

Comments: Decidualization is a progesterone-driven process in which the stromal cells transform to large, polyhedral, cytoplasm-rich cells storing large amounts of glycogen during pregnancy. In case of pregnancy, the process is temporary and will disappear after labor. Similar morphologic changes may be induced by (high doses/unopposed) progestogenic stimulation in non-pregnant non-human primates. In addition to the stromal marker CD10, decidualized cells can stain positive for vimentin, smooth muscle actin, progesterone receptor, and VCAM-1358.

\section{Decreased endometrial differentiation, luteal phase: Uterus (No image provided)}

\section{Other term(s)}

Luteal phase defect

\section{Pathogenesis/Cell of Origin}

Luteal phase defect may be associated with stress or illness ${ }^{359}$. In humans, it is characterized by decreased progester- 
one release from the corpus luteum.

\section{Diagnostic features}

- Clinical tests:

- Low serum progesterone

- Histologic characteristics (present in the majority of the endometrium):

- Decreased sacculation or decreased numbers of sacculated endometrial glands with low-medium columnar epithelial cells

- Low endometrium

- Compact stroma in the zona basalis

- Persistent mitoses in the zona functionalis glands

- A combination of the characteristics listed above plus the presence of a mature corpus luteum on ovary

\section{Differential Diagnoses}

- Normal endometrium

- Dyssynchronous endometrium (if changes are present focally/multifocally rather than diffusely)

Comments: In women, luteal phase defects are characterized by menstrual cycle lengths that are normal for the individual, with a long follicular phase and shortened luteal phase ${ }^{360,361}$. In cynomolgus macaques, animals with social stress-associated suppression of ovulation have decreased luteal phase progesterone 359 .

Decreased glandular development, follicular phase: Uterus (No image provided)

\section{Other term(s)}

Inactive endometrium; endometrial atrophy; proliferative phase defect

\section{Pathogenesis/Cell of Origin}

Uterine proliferative phase defects are often identified as decreased endometrial development without changes in associated hormone concentrations (e.g. anti-Mullerian hormone, inhibin $\mathrm{B}, \mathrm{FSH}, \mathrm{LH}$, and progesterone) ${ }^{362}$. In women, ultrasound measurements show decreased endometrial thickness, often with no difference in luteal phase length ${ }^{363}$.

\section{Diagnostic features (relative to expectations based on ovarian morphology)}

- Decreased endometrial thickness

- Decreased number/proportion of endometrial glands

- Decreased glandular mitoses in superficial endometrium

- Decreased glandular pseudostratification in superficial endometrium

- Compact endometrial stroma?

\section{Differential Diagnoses}

- Endometrial atrophy (senile)

- Normal endometrium

- Decreased endometrial differentiation, luteal phase - low progesterone, clinical evidence of a long follicular phase and with short luteal phase

Comments: This condition may correspond to a clinical follicular phase defect. Diagnosis of a follicular phase defect is often difficult and should be made in conjunction with histologic evaluation of the ovaries and when possible, in-life menstrual cycle monitoring and/or serum hormone levels.

\section{Degeneration, spiral arteries: Uterus (Figure 155)}

\section{Pathogenesis/Cell of Origin}

Spiral arteries are unique to the primate endometrium and are the key vessels that control menstruation. They are very sensitive to progesterone. Treatment with anti-progestins or progesterone receptor modulators can lead to degradation and degeneration of those vessels.

\section{Diagnostic features}

- Spiral arteries can show varying grades of degradation and/or atrophy of the mural smooth muscle cells.

- The tunica media can become replaced by hyaline deposits.

- The endometrial stroma often is very compact.

\section{Differential Diagnoses}

None

Comments: Degeneration and degradation of spiral arteries can be induced by progesterone (receptor) antagonists ${ }^{364,} 365$. Anti-progestin treatment such as with RU486 and ZK 137316 can lead to stromal cell atrophy, stromal compaction and hyalinizing degeneration of the spiral arteries, all in the presence of high serum concentrations of estradiol and increased ER ${ }^{364}$. Anti-progestin treatment causing degeneration and atrophy of spiral arteries may contribute to endometrial atrophy because of reduction in endometrial blood flow ${ }^{365}$.

\section{Dyssynchronous endometrium: Uterus (Figure 151-153)}

\section{Other term(s)}

Non-physiologic endometrial pattern

\section{Pathogenesis/Cell of Origin}

A dyssynchronous endometrium arises from perturbation of the normal physiologic sequence of events that occurs within the endometrium during the menstrual cycle. Estrogen- and progesterone-mediated glandular and stromal changes occur in a predictable cyclic fashion during the menstrual cycle and administration of various steroid receptor agonists/antagonists alone or in combination can alter the typical morphologic pattern. Changes in hormonal stimulation can result in the appearance of abnormal development/maturation of glands, stroma, and/or spiral arteries. There may be overt alteration of these components, or the changes may appear to be within the range of what can be observed during the normal cycle, just without 
the normal timing of events within the endometrium or occurring without the expected development/structures (i.e., dominant follicle or corpus luteum) in the ovary. This altered timing of development within the endometrium and/or lack of concordance with ovarian cyclicity represents a dyssynchronous endometrium.

\section{Diagnostic features}

- Depending on the compound's profile, the treatment period and dose, the endometrium exhibits a varying combination of simultaneously appearing morphologic changes that may include inactive glands, glands showing low mitotic activity, and/or nuclear pseudostratification; cystic dilated glands; excessive gland tortuosity; varying degrees of glandular secretory changes; apoptosis; compact stroma; stromal pseudodecidual changes; and/or thickwalled spiral arteries.

- Individual glands can display features of mixed estrogen (follicular/proliferative phase) and progesterone (luteal/ secretory phase) stimulation simultaneously.

- Squamous metaplasia of endometrial glands can be present (typically with estrogen receptor agonism).

- Despite some of the similarities to a typical secretory phase endometrium, the whole of selective progesteronereceptor modulator (SPRM) induced morphologic endometrial changes does not match any of the normal cyclic related morphologic events.

- Because of tissue selectivity, the ovaries can appear normal (morphologically as well as functionally) or display a lack of cyclicity.

- Depending on the ratio of agonistic and antagonistic influence(s), the endometrium can have an increased, decreased or normal thickness.

Comments: Selective progesterone receptor modulators mainly developed for the treatment of endometriosis and uterine leiomyomata represent a class of synthetic ligands that can exert partial agonistic, partial antagonistic or mixed agonistic and antagonistic effects on various progesterone target tissues. A few examples of compounds in this class that have a mixed agonistic/antagonistic profile and can induce endometrial dyssynchrony in cynomolgus monkeys (more or less comparable to human) are asoprisnil ${ }^{366}$ and ulipristal acetate ${ }^{367}$. In toxicity studies using sexually mature animals, the cyclic phase of the ovary (follicular or luteal phase) at the start or during of the study possibly plays a role in the development and progression of the changes, since endogenous estrogen or progesterone can interfere with the agonistic or antagonistic properties of the compounds on test: asoprisnil and other 11 $\beta$-benzaldoxime-substituted SPRMs, for instance, exert tissue-selective effects on the uterus in the presence of follicular phase estrogen concentrations ${ }^{366}$. Possibly, the physiologic progesterone-mediated downregulation of the expression of the estrogen receptor is perturbed resulting in simultaneous expression of both the typical estrogen- and progesterone-mediated endometrial changes ${ }^{368}$. SPRM dose may be important, as some effects may be dose-dependent 369,370 . Although not all histological features are present simultaneously, it is usually possible to identify several of the characteristic changes induced by SPRMs, as described for the human endometrium ${ }^{371}$. These complex changes can be challenging to identify in some cases, and knowledge of normal endometrial morphology is a prerequisite for the correct diagnosis.

\section{Ectopic tissue, ovary: Uterus (Figure 156)}

\section{Diagnostic features}

- Small focus of ovarian stroma with varying amounts of primordial, primary, and atretic follicles.

- Typically present on the serosal surface of the uterus, within the myometrium, or within the broad ligament.

- Not usually visible grossly and found incidentally during microscopic evaluation of the uterus

Comments: Ectopic ovarian tissue can occasionally be reported in macaques and has been observed in approximately $8 \%$ of animals ${ }^{372}$. Given that this change is only observed microscopically, incidence may vary with different trimming patterns of the uterus. When using ovariectomized macaques, completeness of ovariectomy should be confirmed using postsurgical serum estradiol, progesterone, or gonadotropin concentrations, or a combination of these hormones ${ }^{373}$.

\section{Endometriosis: Uterus (Figure 157)}

\section{Pathogenesis/Cell of Origin}

The pathogenesis of endometriosis is largely unknown. In women, the most cited theory is retrograde menstruation: endometrial fluid leakage from the fallopian tubes into the abdominal or pelvic cavity during menstruation ${ }^{374}$. This theory does not account for differences in the percentage of women with endometriosis $(10 \%)$ and women that experience retrograde menstruation $(90 \%)$. The theory of retrograde menstruation also fails to explain cases of endometriosis in newborns, pre-menarchal girls, and men undergoing prostate cancer treatment 375,376 . Additional factors likely predispose some women to endometriosis. A second theory involves embryonic development of endometriosis tissue. The peritoneum and pleural lining, ovarian germinal epithelium, and Müllerian ducts develop from the mesoderm. Estrogen stimulates the metaplastic transformation of these tissues following puberty 377 . Finally, endometrial and bone marrow-derived stem cells are implicated in endometriosis development ${ }^{378}$. The basal layer of endometrium contains stem cells necessary for regeneration following menses. Studies show endometrial stem cells may become distributed throughout the body by retrograde menstruation, blood vessels, or lymphatics ${ }^{379}$. 


\section{Diagnostic features}

- Gross Features:

- Dark red to brown cysts in the abdomen or pelvis that contain brown, viscous fluid

- Other morphologies include white scar-like lesions.

- Histopathology features:

- Glandular epithelium and stroma reminiscent of the endometrium

- Glands filled with hemorrhage with degenerate and viable macrophages

- Stromal hemorrhage and aggregates of hemosiderinladen macrophages

- Endometrial granular leukocytes

- Endometriosis stromal cells may decidualize under the influence of exogenous or endogenous progesterone ${ }^{380}$

- Often contains features of menstrual cyclicity similar to the eutopic endometrium.

\section{Differential Diagnoses}

- Fibroplasia

- Fibroplasia has dense bands of fibrous connective tissue surrounded by elongated cells with scant cytoplasm and oval, basophilic nuclei (reactive fibroblasts).

- Granulation tissue

- Granulation tissue consists of small caliber capillaries perpendicular to organ surface, spindle shaped (reactive) fibroblasts parallel to organ surface.

- Metastatic neoplasia

- Metastatic adenocarcinomas - Typically lack characteristic blood-filled cysts, endometrial stroma, and hemosiderin-laden macrophages.

- Squamous cell carcinoma - Polygonal neoplastic cells have intercellular junctions and eosinophilic, granular cytoplasm. Individual cells often become keratinized with brightly eosinophilic intracytoplasmic material.

- Malignant deciduoid mesothelioma - Neoplastic cells arranged in sheets, papilla, tubules, or cords. Mesothelial cell nuclei are larger and more vesicular than decidualized endometriosis stroma.

- Retroperitoneal fibromatosis

- Nodules consist of stellate cells in bundles and swirls with distinct borders, and oval nuclei supported by a fibrovascular stroma (but no endometrial glands).

- Atypical extra-placental trophoblastic invasion

- Observed in peritoneal cavity of pregnant animals. Sheets and cords of round to polygonal cells with round to oval nuclei infiltrate serosal surfaces (but no endometrial glands).

\section{Special Techniques for Diagnostics}

- Cyst fluid cytopathology

- Cellular debris

- Degenerate red and white blood cells (peripheral blood)

- Macrophages often contain hemosiderin, erythrocytes, and cellular debris
- Immunohistochemical markers

- CD10/CALLA (membranous and cytoplasmic) - Endometrial stromal cells are positive, endometrial glands are negative;

- Estrogen receptor (nuclear) - immunoreactivity extent and intensity varies depending on the phase of cycle and history of hormone-based treatments

- Progesterone receptor positive (nuclear) - immunoreactivity extent and intensity varies depending on the phase of cycle and history of hormone-based treatments

Comments: Spontaneous endometriosis prevalence may be as high as $30 \%$ in captive macaque populations ${ }^{381}$. Cases are common in colonies with a high rate of Cesarean section births. Endometriosis lesions can be induced in NHPs through injection or implantation of the endometrium into the peritoneal cavity ${ }^{382}$. Lesions are frequently found on uterine, ovarian, and colonic serosal surfaces but thoracic cavity and abdominal wall endometriosis are possible 383,384 .

\section{Hemorrhage, abnormal, endometrium: Uterus}

(Figure 152)

\section{Other term(s)}

Abnormal uterine bleeding; atypical endometrial bleeding

\section{Pathogenesis/Cell of Origin}

Abnormal endometrial bleeding can be attributed to disruption of the HPG axis and is often observed during anovulatory cycles. This may be due to administration of exogenous substances or normal cycle variation.

\section{Diagnostic features}

- Histologic characteristics:

- Endometrium or uterine lumen hemorrhage, usually in conjunction with follicular or periovulatory phase endometrium

- Ovaries may contain one or more, small or medium, variably atretic follicles and lack a regressing corpus luteum.

- Often associated with a sudden drop in serum hormone levels (e.g. due to stress).

\section{Differential Diagnoses}

- Menses - hemorrhage observed in tandem with menstrual phase endometrium, entire endometrial superficial endometrium zone usually sloughs, apoptosis present in glands, regressing corpus luteum often present on ovaries.

\section{Special Techniques for Diagnostics}

- Clinical assessment of serum progesterone and estradiol concentrations or vaginal swab data across several cycles is often helpful in distinguishing abnormal uterine bleeding (spotting) between true menses.

Comments: In humans, abnormal endometrial bleeding is 
very common in reproductive-aged women ${ }^{385}$. Abnormal bleeding is also common in macaques with increased incidence in pubertal and adolescent animals ${ }^{314}$.

\section{Hyaline deposits, perivascular: Uterus (Figure 158)}

\section{Other $\operatorname{term}(\mathbf{s})$}

Pregnancy-associated Vascular Remodeling

\section{Pathogenesis/Cell of Origin}

Hyaline perivascular deposits are nonpathologic changes associated with pregnancy. Extracellular matrix (ECM) and collagen is deposited around vessels in response to rapid uterine expansion and increased uterine blood flow during pregnancy. Placental trophoblasts invade and remodel endometrial and myometrial blood vessel walls and perivascular connective tissue in late pregnancy. The result is loose connective tissue surrounding myometrial blood vessels, especially veins. The change is primarily adventitial but may involve the tunica media.

\section{Diagnostic features}

- Eosinophilic, hypocellular, hyaline material surrounding myometrial veins and, less often, arteries

\section{Differential Diagnoses}

Arteriosclerosis: In contrast to hyaline perivascular deposits, arteriosclerosis is of intimal, not adventitial, origin.

Comments: Aged, nulliparous animals may also have focal ECM and collagen deposition ${ }^{314}$. Pregnancy-related change may persist for more than six years after the last pregnancy and is a persistent reliable indicator of prior pregnancy status in cynomolgus macaques ${ }^{386}$. These changes may be considered background findings of no significance in nonreproductive studies, but are informative in the context of hormonally-active agents.

\section{Hypersecretion, glandular: Uterus (No image provided)}

\section{Pathogenesis/Cell of Origin}

Perturbation of the normal physiologic progesterone-mediated glandular and stromal changes resulting in premature development and maturation of glands or overexpression of the characteristic morphologic changes.

\section{Diagnostic features}

- Depending on the compound's profile, the treatment period, and dose, the endometrium can exhibit a secretory type of morphology that is not in-line with the expected secretory physiologic phase of the endometrium based on ovarian morphology. The synchrony between the different endometrial elements, however, is normal.

- Decidualization, normally not present within the cynomolgus endometrium but can be pronounced.

- Spiral arteries are well developed.

\section{Differential Diagnoses}

- Dyssynchronous endometrium. Within a dyssynchronous endometrium perturbation of the normal, physiologic separation between estrogen- and progesterone-mediated glandular and stromal changes results in dyssynchronous development or maturation of glands mutually or in stromal-epithelial dyssynchrony.

Comments: Localized hypertrophy of secretory endometrial glands is also known as the Arias-Stella reaction. The hypertrophic cells have enlarged nuclei that are hyperchromatic and irregular. The Arias-Stella reaction is a non-specific finding that can be seen in patients with ectopic pregnancy, intrauterine pregnancy and after ovarian stimulation with ovulation inducing agents.

\section{Inactive endometrium: Uterus (Figure 150)}

\section{Pathogenesis/Cell of Origin}

Endometrial structures become inactive due to lack of estrogen and/or progesterone receptor stimulation. Decreased stimulation may be either because of sub-physiologic or absent hormone production or inhibition of receptors or signaling pathways.

\section{Diagnostic features}

- The thickness of the endometrium is decreased with compact stroma.

- The epithelium of the glands, as well as the surface epithelium, is low columnar to cuboidal with small, dark nuclei and minimal cytoplasm.

- There is marked decrease or even absence of glandular and stromal proliferative or secretory activity (indicated by a very low number or even absence of mitotic figures or absence of cytoplasmic vacuoles).

- Cystic dilated glands can be present.

\section{Differential Diagnoses}

- Atrophy, endometrium (senile)

Comments: In contrast to the state of real atrophy, characterized by a very low and compact endometrium with scattered gland profiles often oriented parallel to the endometrium surface ${ }^{315}$, the number of estrogen or progesterone receptors in an inactive endometrium is high. Examples of an inactive endometrium are immaturity, menopause and after ovariectomy, in which the sudden drop in reproductive hormones causes inactivation of the endometrial structures. Nevertheless, after administration of exogenous hormones the endometrial structures can become active again. GnRH antagonists suppress FSH or LH release from the pituitary gland and can induce endometrial inactivity. SPRMs such as mifepristone (RU 486), which have predominant antiprogestogenic activity, can also induce endometrial inactivity 387 . 
Infarct, uterus: Uterus (Figure 159)

\section{Pathogenesis/Cell of Origin}

Usually associated with systemic illness, such as sepsis or injury leading to disseminated intravascular coagulopathy.

\section{Diagnostic features}

- "Watershed" pattern of necrosis of the endometrium or myometrium or both, on midline at the extremes of vascular supply.

Comments: This lesion was reported in a necropsy case series of cynomolgus macaques, occurring at a rate of $2.8 \% 388$.

\section{Epithelial plaque: Uterus (Figure 160-161)}

\section{Pathogenesis/Cell of Origin}

The epithelial plaque response is a lesion composed of a florid proliferation of epithelial cells located in the neck of the endometrial glands and the adjacent superficial endometrial lining. Although it is a normal temporary structure that develops as a reaction to embryo implantation during pregnancy, it also can be induced experimentally (i.e., by combined estrogen and progestogen treatment accompanied by trauma to the endometrium).

\section{Diagnostic features}

- The change appears focally within the endometrium, often on the opposing walls (in the marmoset it is a more diffuse superficial change).

- The lesions start to appear at the basal side of the epithelium lining the neck of the endometrial glands and form nodular cell nests that merge into a plaque-like lesion.

- The change has an abrupt border with absence of deeper endometrial invasion.

- The epithelial cells composing the plaque are large, pleomorphic, and polygonal, with sometimes anaplastic nuclear features. Giant and binucleated nuclei are often present.

- The cells within the plaque can be highly vacuolated because of storage of glycogen.

-Within the plaque, mitosis can be numerous.

\section{Differential Diagnoses}

- Endometrial carcinoma in situ

- Trophoblastic neoplasm

Comments: In primates, the true epithelial plaque is a common early endometrial response to implantation of the blastocys $\mathrm{t}^{389}$. During pregnancy, the epithelial plaque response is a temporary change that lasts for only a few weeks: it is highly proliferative during the first phase, but finally regresses via apoptosis. Immunohistochemical proliferation markers such as Ki67 are positive during the developmental phase of the change, while those for cleaved caspase 3, indicating apoptosis becomes positive during regression. An epithelial plaque also can be induced in non-pregnant rhe- sus monkeys by various stimuli, such as combined estrogen and progestogen treatment 390,391 , sometimes accompanied by trauma to the endometrium. The induced lesions show many similarities to the true, pregnancy-related epithelial plaque. Kaspareit et al. ${ }^{392}$ described the appearance of spontaneous epithelial plaques in non-pregnant cynomolgus monkeys. Because of the cellular and nuclear atypia and high mitotic index this lesion was believed to represent carcinoma in situ in the past ${ }^{391}$. Although the primate endometrium shares many similarities to the human endometrium, the epithelial plaque is unique to primates and is not present in women.

\section{Hyperplasia, basal gland: Uterus (Figure 162) \\ Pathogenesis/Cell of Origin}

Proliferation of the epithelial (progenitor) cells lining the blind ends of the endometrial glands, the most basal parts of the glands within the basal endometrium (zone IV). This is a distinctive treatment-related lesion of macaques.

\section{Diagnostic features}

- Intraepithelial hyperplasia starting at the basal site of the gland epithelium forming a multilayered epithelium that lifts-up or replaces the original cuboidal/columnar epithelial cell-lining

- In general, the change appears zonal (zone IV), not focal

- In more progressed cases hyperplastic areas can show a cribriform pattern, with many secondary lumina

- Mitotic figures as signs of ongoing proliferation can be observed, but also signs of apoptosis of the cells involved can be a feature

- Slight atypia sometimes can be observed

- Signs of hypersecretory activity of the cells can be a feature of the lesion

\section{Differential Diagnoses}

- Endometrial (adeno) carcinoma

Comments: Some synthetic hormones (including a compound that exhibited both SPRM and SERM activity) or combinations of hormones administrated at high dosage can cause unique hyperplasia of the epithelium lining the most basal part of the glands in the basal endometrium (zone IV). This change may be caused by the local expression patterns of estrogen and/or progesterone receptors. Even during the (early and mid) luteal phase of a normal cycle under relative progesterone dominance, mitotic activity increases in the glands in the deep basalis, while proliferation is inhibited in the rest of the gland portions ${ }^{315,393,394}$. This enhancement of proliferative activity in the deep basalis glands is thought to reflect the process of replenishing the pool of stem and progenitor cells ${ }^{394}$. Under continued hormonal stimulation, the balance between precursor cell proliferation and apoptosis can be lost and shifted in the direction of hyperplasia. 
Hyperplasia, glandular: Uterus (Figure 163-165)

\section{Modifiers}

Simple; complex; with atypia; focal; diffuse; cystic

\section{Pathogenesis/Cell of Origin}

Endometrial hyperplasia is induced in macaques by endogenous or exogenous estrogens $354,373$.

\section{Diagnostic features}

- Gross morphology: Thickened, soft, tan endometrium

- Histologic morphology:

- Glandular proliferation with an increased gland-to-stroma ratio either without glandular crowding (hyperplasia, simple) or with endometrial gland crowding "back to back glands" (hyperplasia, complex)

- Disordered glandular architecture relative to normal follicular phase

- Usually edema of the superficial endometrium

- Pseudostratification of glandular epithelium

- Increased glandular epithelial mitoses

- May or may not exhibit epithelial atypia (loss of polarity, increased nuclear-cytoplasm ratio, nuclear clearing with clumped, marginated chromatin, and round or irregular nuclei)

\section{Differential Diagnoses}

- Normal, proliferative endometrium in the follicular phase - parallel straight or spiral glands with superficial endometrium edema

- Dyssynchrony - Variable glandular patterns of mixed morphology, without increased mitoses or increased glandular area

- Cystic glandular (endometrial) hyperplasia - cystic dilation of glands in the endometrium without glandular proliferation

- Endometrial polyp - focal, pedunculated mass of endometrial stroma with variable scattered glands supported by a vascularized or fibrotic center; may contain hyperplasia, metaplasia, or malignant changes

Comments: Endometrial hyperplasia is associated with a slightly increased risk of endometrial cancer in women. Women with complex endometrial hyperplasia with atypia have an increased risk of developing endometrial cancer. In fact, up to $25 \%$ of women diagnosed with atypical complex endometrial hyperplasia will be diagnosed with a welldifferentiated carcinoma within one month of endometrial biopsy ${ }^{395}$. In macaques it may be observed in conjunction with other endometrial changes such as polyps or adenomyosis $^{357}$. This lesion is also associated with granulosa cell tumors, and polycystic ovarian syndrome ${ }^{339}$. Epithelial atypia should be noted, if present.
Metaplasia, ciliated cell: Uterus/Cervix (Figure 168)

Other term(s)

Tubal metaplasia

\section{Pathogenesis/Cell of Origin}

The normal endometrial or cervical columnar secretory epithelium is replaced by ciliated, tubal epithelium (both epithelia are of Müllerian origin).

\section{Diagnostic features}

- The endometrial or cervical secretory epithelium is replaced by ciliated cells.

- Ciliated epithelial cells clearly bear recognizable cilia on the surface

- The nuclei of the ciliated cell often are situated above the nuclei of the secretory cells.

- Atypia is absent.

Comments: Ciliated cell metaplasia is a common finding in the NHP endometrium. During estrogen dominance the number of ciliated cells within the glands increase ${ }^{315}$. The so-called clear cell is believed to represent a progenitor of the ciliated cell.

Metaplasia, endocervical cell: Uterus (No image provided)

\section{Other term(s)}

Endometrial mucinous metaplasia (EMM); endocervical cell metaplasia

\section{Pathogenesis/Cell of Origin}

The normal endometrial columnar secretory epithelium is replaced by mucus-producing epithelial cells that show a high similarity to the mucous cells lining the endocervix.

\section{Diagnostic features}

- The endometrial gland epithelium is focally replaced by columnar mucinous epithelial cells resembling the mucus cells of the endocervix.

- The cells in the lesion are tall and columnar and secrete mucins, which differentiate them histochemically from the normal glandular secretory cells.

- Atypia is absent.

Comments: Mucous cell metaplasia is an uncommon finding in the non-human primate endometrium. Sections including the endocervix will normally include mucus-producing cells. Mucous cells stain positive for Alcian-Blue (AB) and/or Periodic-Acid Shiff (PAS, AB-PAS).

\section{Metaplasia, squamous cell: Uterus/Cervix (Figure 169)}

\section{Pathogenesis/Cell of Origin}

The normal endometrial or cervical columnar secretory epithelium is replaced by squamous epithelial cells. 


\section{Diagnostic features}

- The endometrial or cervical secretory epithelium is replaced by squamous cells

- Squamous cells often lie beneath the endocervical

- Atypia is absent.

Comments: Squamous cell metaplasia is a common finding in the NHP endocervix during estrogen dominance, either physiologic (e.g. during puberty before first ovulation) or pharmacologic (due to administration of estrogen agonists).

\section{Cervix (Table 31)}

Adenosis: Cervix, Vagina (Figure 170)

\section{Other term(s)}

Glandular metaplasia

\section{Pathogenesis/Cell of Origin}

Basal epithelial cells of the ectocervical and vaginal mucosa; abnormal glandular rather than squamous differentiation.

\section{Diagnostic features}

- Adenosis is defined by the presence of simple columnar glandular epithelium in areas of the ectocervix and vagina normally lined by stratified squamous mucosa.

Table 31. Microscopic Findings of the Female Reproductive System: Cervix; Non-human Primate

\begin{tabular}{|c|c|c|c|c|}
\hline CERVIX & Common & Uncommon & $\begin{array}{c}\text { Not Observed but } \\
\text { Potentially Relevant }\end{array}$ & Not Applicable \\
\hline \multicolumn{5}{|l|}{ Congenital } \\
\hline Remnants, mesonephric duct & & $\mathrm{x}$ & & \\
\hline \multicolumn{5}{|l|}{ Non-proliferative } \\
\hline Aggregate, granular cells & & & & $\mathrm{x}$ \\
\hline Atrophy, epithelium & $\mathrm{x}$ & & & \\
\hline Cyst, NOS & $\mathrm{x}$ & & & \\
\hline Decidualization $^{1}$ & & $\mathrm{x}$ & & \\
\hline Degeneration, epithelium & & $\mathrm{x}$ & & \\
\hline Edema $^{\wedge}$ & $\mathrm{x}$ & & & \\
\hline Erosion/ulcer & & $\mathrm{x}$ & & \\
\hline Infiltrate, [insert appropriate cell type $]^{\wedge}$ & $\mathrm{x}$ & & & \\
\hline Inflammation $^{\wedge}$ & & $\mathrm{x}$ & & \\
\hline Keratinization, increased & $\mathrm{x}$ & & & \\
\hline Menstrual debris ${ }^{2}$ & $\mathrm{x}$ & & & \\
\hline Mucification, increased & $\mathrm{x}$ & & & \\
\hline Necrosis, epithelium & & $\mathrm{x}$ & & \\
\hline Prolapse, uterus & & $\mathrm{x}$ & & \\
\hline Vacuolation, epithelium & & $\mathrm{x}$ & & \\
\hline \multicolumn{5}{|l|}{ Proliferative (non-neoplastic) } \\
\hline Adenomyosis $^{1}$ & & $\mathrm{x}$ & & \\
\hline Adenosis* & & $\mathrm{x}$ & & \\
\hline Hyperplasia, epithelium & & $\mathrm{x}$ & & \\
\hline Hyperplasia, granular cell & & & & $\mathrm{x}$ \\
\hline Hyperplasia, stroma & & $\mathrm{x}$ & & \\
\hline Hypertrophy stroma & & $\mathrm{x}$ & & \\
\hline Metaplasia, ciliated cell ${ }^{1}$ & & $\mathrm{x}$ & & \\
\hline Metaplasia, reserve cell & & $\mathrm{x}$ & & \\
\hline Metaplasia, squamous cell ${ }^{1}$ & $\mathrm{x}$ & & & \\
\hline \multicolumn{5}{|l|}{ Neoplastic } \\
\hline Dysplasia, epithelial * & & $\mathrm{x}$ & & \\
\hline Carcinoma, squamous cell * & & $\mathrm{x}$ & & \\
\hline Polyp & & $\mathrm{x}$ & & \\
\hline
\end{tabular}

* Terminology with diagnostic criteria and/or comments below. ${ }^{\wedge}$ Terminology addressed in the Systemic/General Pathology Section. ${ }^{1}$ For a description and diagnostic criteria, refer to Uterus. ${ }^{2}$ Menstrual debris, consisting of fragmented, often degenerate epithelial and stromal elements and blood, is a common finding in the menstrual phase. While normal, it should be documented. 
- Glandular epithelium may be present as discrete islands within squamous mucosa or more generally as a "posteriorization effect" in which columnar cells extend diffusely past the os and along the ectocervix, fornix, and anterior vaginal wall.

- Abnormally located glandular structures may also be present in the submucosa of the ectocervix and vagina.

- Glandular epithelial cell morphology is often normal.

- Squamous epithelium may replace heterotopic glandular epithelium with age.

- Progression to neoplasia has not been reported.

\section{Differential Diagnoses}

- Normal transformation zone (see comment below)

\section{Special Techniques for Diagnostics}

- Immunohistochemical markers

- Cytokeratin 18 (cytoplasmic and membranous) - This marker is highly expressed in glandular but not squamous epithelial cells.

- Cytokeratin 14 (cytoplasmic and membranous) - This marker is highly expressed in squamous but not glandular epithelial cells.

Comments: Adenosis of the vaginal and ectocervical epithelium has been described in macaques as a developmental abnormality associated with perinatal exposure to certain estrogenic compounds, notably diethylstilbestrol (DES) ${ }^{396-398}$. Aberrant early-life estrogen treatment blocks proper transformation of the Müllerian epithelium into normal vaginal or ectocervical epithelium. Macaques exhibit a wide range of natural variation in the degree of eversion of the glandular cervix, which can extend close to the fornix in some cases ${ }^{319}$; this normal morphology should not be confused with adenosis.

\section{Dysplasia, epithelial: Cervix, Vagina (Figure 171)}

\section{Other term(s)}

Cervical/vaginal intraepithelial neoplasia; carcinoma in situ

\section{Pathogenesis/Cell of Origin}

Basal/squamous epithelial cells of the cervical and vaginal mucosa

\section{Diagnostic features}

- Hallmark is nuclear atypia of squamous epithelial cells.

- The growth pattern is often endophytic (invaginating).

- The extent of basal cell expansion determines lesion grade (low, moderate, and high):

- Low-grade dysplasia - acanthosis with large atypical nuclei and perinuclear clearing (koilocytosis) but limited expansion of the basal epithelium (less than onethird of the total epithelial thickness);

- Moderate-grade dysplasia - increased thickness of basal cell layers (one-third to two-thirds of the total epithe- lial thickness); and

- High-grade dysplasia (carcinoma in situ) - composed almost entirely of basal-type cells with hyperchromatic nuclei and scant cytoplasm; can be laterally invasive within the epithelium but do not penetrate the basement membrane.

\section{Differential Diagnoses}

- Papilloma, vagina (wart, condyloma acuminata)

- Papillomas show exophytic growth, often with a stromal core, and no or limited basal cell expansion.

- Squamous cell carcinoma, cervix/vagina

- Carcinomas show clear evidence of submucosal and/or vascular invasion.

- Squamous metaplasia, endocervix

- Metaplastic lesions do not show nuclear atypia, koilocytic change, or basal cell expansion.

- Hyperkeratosis, vagina

- Hyperkeratotic lesions have a normal maturation lineage of squamous epithelium and no atypia or basal expansion.

- Ectopic decidual reaction, cervix/vagina

- Decidual reactions are composed of plump stromal cells located subjacent to mucosal epithelium.

\section{Special Techniques for Diagnostics}

- Immunohistochemical markers

- Ki67 (nuclear) - Proliferation in normal mucosal epithelium is limited to basal/suprabasal layers. Dysplastic lesions show cell proliferation in more superficial layers.

- P16INK4A (nuclear and cytoplasmic) - This tumor suppressor protein is absent in normal mucosal epithelium but increased in dysplastic lesions.

- Cytologic markers

- Cells with large atypical nuclei and koilocytic change can be seen on Pap-stained cell preps obtained using cervicovaginal swabs.

- Genetic testing

- DNA testing (PCR) can be used to identify papillomavirus sequences.

- In situ hybridization is preferable to immunohistochemistry for viral antigen

Comments: Cervical and vaginal dysplasia is a common background lesion in female macaques, with a reported prevalence ranging from $5 \%$ to $19 \% 319$. Cervical dysplasia has also been reported in baboons ${ }^{399}$. This lesion is highly associated with genital papillomavirus infection, which is sexually transmitted 400,401 . Lower-grade dysplasias occur throughout the squamous epithelium of the cervix and vagina, while higher-grade dysplasias typically involve the squamocolumnar junction or transition zone of the cervix and may progress to squamous cell carcinoma ${ }^{402}$. 
Carcinoma, squamous cell: Cervix (Figure 172)

\section{Pathogenesis/Cell of Origin}

Basal/squamous epithelial cells of the cervical mucosa

\section{Diagnostic features}

- Neoplastic cells have the following features:

- Arranged in sheets and nests with a predominantly endophytic growth pattern;

- Highly atypical with large, often bizarre nuclei and high mitotic index; and

- Basal/squamous phenotype with variable degrees of squamous cell maturation and keratinization and no glandular elements.

- Basement membrane is breached by neoplastic cells.

- Keratinizing forms include whorls with central foci of sloughed keratin.

- Carcinomas may be found on a background of cervical dysplasia.

- Carcinomas may include focal areas of necrosis.

\section{Differential Diagnoses}

- Adenocarcinoma, endometrial

- Adenocarcinomas have extensive glandular elements.

- Papilloma, vagina

- Squamous papillomas show exophytic growth with no evidence of invasion.

- Dysplasia, cervix/vagina

- Cervical/vaginal dysplasias have atypia with or without basal cell expansion but no clear evidence of local or vascular invasion.

- Squamous metaplasia, endocervix

- Metaplastic lesions of the endocervical epithelium do not show atypia or evidence of local or vascular invasion.

- Hyperkeratosis, vagina

- Hyperkeratotic lesions have a normal maturation lineage of squamous epithelium and no atypia or invasion.

- Ectopic decidual reaction, cervix/vagina

- Decidual reactions are composed of plump stromal cells located subjacent to mucosal epithelium.

\section{Special Techniques for Diagnostics}

- Immunohistochemical markers

- Ki67 (nuclear) - Expression of this cell proliferation marker in normal mucosal epithelium is limited to basal/suprabasal layers. Neoplastic lesions show high rates of labeling throughout all layers.

- P16INK4A (nuclear and cytoplasmic) - This tumor suppressor protein is absent in normal mucosal epithelium but expressed in papillomavirus-associated neoplastic lesions.

- Cytologic markers

- Cells with large atypical nuclei can be seen on Papstained cell preps obtained using cervicovaginal swabs.

- Genetic testing
- DNA testing can be used to identify papillomavirus sequences.

Comments: Cervical carcinoma is a rare lesion in macaques associated with dysplasia and genital papillomavirus infec$\operatorname{tion}^{319,402}$. Carcinomas typically involve the squamocolumnar junction or transition zone of the cervix ${ }^{402}$. Cervical adenocarcinoma has not been reported in macaques.

\section{E. Vagina (Table 32)}

\section{Condyloma, inflammatory: Vagina (Figure 174-175)}

\section{Pathogenesis/Cell of Origin}

Pathogenesis is unknown, but it is distinct from "Dysplasia/Vaginal Intraepithelial Neoplasia" which is associated with papillomavirus infection. Papillomaviruses have not been found in this lesion.

\section{Diagnostic features}

- Exophytic polypoid lesion of the vagina or vulva

- Composed of hyperplastic squamous epithelium supported by fibrous stroma

- Submucosal and intraepithelial infiltrates of eosinophils.

- No basal hyperplasia, atypia, or dysplasia

- No invasion

\section{Differential Diagnoses}

- Vaginal dysplasia (intraepithelial neoplasia)

- Dysplastic lesions usually lack exophytic papillary growth pattern.

- Higher grade dysplasias have basal cell expansion.

- Dysplasia does not have eosinophilic infiltrates.

- Squamous metaplasia

- Metaplastic lesions are associated with glandular endocervical epithelium and lack exophytic growth and eosinophilic infiltrates.

- Vaginal hyperkeratosis

- Hyperkeratotic lesions have a normal maturation lineage of squamous epithelium and no atypia, basal cell expansion, or papillary morphology.

- Hyperkeratosis alone does not have eosinophilic infiltrates.

- Papilloma

- Composed of hyperplastic squamous epithelium supported by a fibrovascular core

- No evidence of invasion into adjacent structures

- Epithelial features may include acanthosis, perinuclear clearing (koilocytosis), and hyperkeratosis

- Papillomavirus-associated lesions also show nuclear atypia of squamous epithelial cells

- Limited expansion of the basal epithelium

Comments: This lesion has only been reported in Mauritian-origin macaques ${ }^{403}$. These lesions were characterized by hyperplastic squamous epithelium with acanthosis (but no atypia), prominent collagenous stroma, and marked eo- 
Table 32. Microscopic Findings of the Female Reproductive System: Vagina; Non-human Primate

\begin{tabular}{|c|c|c|c|c|}
\hline VAGINA & Common & Uncommon & $\begin{array}{c}\text { Not Observed but } \\
\text { Potentially Relevant }\end{array}$ & Not Applicable \\
\hline \multicolumn{5}{|l|}{ Congenital } \\
\hline Imperforate vagina & & & $\mathrm{x}$ & \\
\hline Prostatic rudiment & & & & $\mathrm{x}$ \\
\hline Remnant, mesonephric duct & & $\mathrm{x}$ & & \\
\hline \multicolumn{5}{|l|}{ Non-proliferative } \\
\hline Aggregate, granular cell & & & & $\mathrm{x}$ \\
\hline Atrophy, epithelium & $\mathrm{x}$ & & & \\
\hline Cyst, epithelial & & $\mathrm{x}$ & & \\
\hline Cyst, NOS & & $\mathrm{x}$ & & \\
\hline${\text { Decidualization, stromal cell }{ }^{1}}$ & & $\mathrm{x}$ & & \\
\hline Degeneration, epithelium & & $\mathrm{x}$ & & \\
\hline Edema $^{\wedge}$ & & $\mathrm{x}$ & & \\
\hline Erosion/ulcer & & $\mathrm{x}$ & & \\
\hline Immature & & $x$ & & \\
\hline Infiltrate, [insert appropriate cell type ${ }^{\wedge}$ & $\mathrm{x}$ & & & \\
\hline Inflammation $^{\wedge}$ & & $\mathrm{x}$ & & \\
\hline Keratinization, increased & $\mathrm{x}$ & & & \\
\hline Mucification, increased & & & & $\mathrm{x}$ \\
\hline Necrosis, epithelium & & $\mathrm{x}$ & & \\
\hline Prolapse, uterus & & $\mathrm{x}$ & & \\
\hline Vacuolation, epithelium & & $\mathrm{x}$ & & \\
\hline \multicolumn{5}{|l|}{ Proliferative (non-neoplastic) } \\
\hline Adenosis $^{2}$ & & $\mathrm{x}$ & & \\
\hline Condyloma, inflammatory * & $\mathrm{x}$ & & & \\
\hline Hyperplasia, epithelium & & $\mathrm{x}$ & & \\
\hline Hyperplasia, granular cell & & & & $\mathrm{x}$ \\
\hline Hyperplasia, stroma & & $\mathrm{x}$ & & \\
\hline Hypertrophy stroma & & $\mathrm{x}$ & & \\
\hline \multicolumn{5}{|l|}{ Neoplastic } \\
\hline \multicolumn{5}{|l|}{ Dysplasia $^{2}$} \\
\hline Papilloma * & $\mathrm{x}$ & & & \\
\hline Polyp & & $\mathrm{x}$ & & \\
\hline
\end{tabular}

sinophilic and lymphoplasmacytic inflammation; no association with papillomavirus was identified. Normal vaginal keratin in macaques can form distinct ridges that should not be confused with papillomas ${ }^{319}$.

\section{Papilloma: Vagina (Figure 176)}

\section{Other term(s)}

Vaginal wart; condyloma acuminata

\section{Pathogenesis/Cell of Origin}

Squamous epithelial cells of the vaginal mucosa; may be caused by papillomavirus infection.

\section{Diagnostic features}

- Exophytic polypoid growth protruding into the vaginal lumen

- Composed of hyperplastic squamous epithelium supported by a fibrovascular core

- No evidence of invasion into adjacent structures

- Epithelial features may include acanthosis, perinuclear clearing (koilocytosis), and hyperkeratosis

- Papillomavirus-associated lesions also show nuclear atypia of squamous epithelial cells

- Limited expansion of the basal epithelium

- Not known to progress to squamous cell carcinoma

\section{Differential Diagnoses}

- Vaginal dysplasia (intraepithelial neoplasia)

- Dysplastic lesions usually lack exophytic papillary growth pattern.

- Higher grade dysplasias have basal cell expansion. 
- Squamous metaplasia

- Metaplastic lesions are associated with glandular endocervical epithelium and lack atypia, koilocytic change, and exophytic growth.

- Vaginal hyperkeratosis

- Hyperkeratotic lesions have a normal maturation lineage of squamous epithelium and no atypia, basal cell expansion, or papillary morphology.

- Condyloma, inflammatory

- Hyperplastic squamous epithelium with acanthosis (but no atypia), prominent collagenous stroma, and marked eosinophilic and lymphoplasmacytic inflammation; no association with papillomavirus identified.

\section{Special Techniques for Diagnostics}

- Immunohistochemical markers:

- P16/INK4A and papillomavirus antigens (nuclear and cytoplasmic) - papillomavirus-associated lesions may show increased expression of these markers.

- Genetic testing:

- DNA testing can be used to identify papillomavirus sequences.

Comments: Vaginal papillomas can be observed as a rare background lesion in female macaques ${ }^{319}$, 402. In Indonesian-origin macaques, this lesion has been associated with genital papillomavirus infection ${ }^{402}$. Normal vaginal keratin in macaques can form distinct ridges that should not be confused with papillomas ${ }^{319}$. 


\section{Reproductive System - Male}

For detailed general considerations on the male reproductive system and tabulated terminologies not described in this chapter, please refer to the INHAND rodent publication ${ }^{404}$.

Anatomical features of the macaque male reproductive tract that are specific to these species and microscopic findings unique or applicable to cynomolgus monkey (M. fasicularis) and/or rhesus monkey (M. mulatta) are discussed. The cynomolgus monkey is a "non-seasonal breeding" macaque and is one of the most commonly used non-human primate in biomedical research. The Rhesus macaque, another commonly used non-human primate is a seasonal breeder with testes undergoing seasonal regression and recrudescence of spermatogenesis 405 , which may complicate the findings or interpretation in a toxicity study. Most non-clinical toxicity studies conducted in Cynomolgus monkeys are in groups that are immature or a mixture of immature and peripubertal to mature animals. Thus a challenge in many studies for evaluation of potential effects in the male reproductive tract is often related to notable differences in histoanatomical features in the reproductive tract as a result of normal variability of maturation in the groups of animals on study. These differences related to maturity must be separated from histopathologic findings that may represent either background pathology or test article-related effects.

\section{Sexual Maturation in the Male Macaque}

Male Cynomolgus monkeys are typically sexually mature at approximately 4.5-5 years of age and with a body weight of $>5 \mathrm{~kg}^{406}$; however, most toxicity studies are $\leq 6$ months in duration and use monkeys that are under 4 years of age, hence these animals are often sexually immature or a mix of immature, peripubertal, and occasionally mature at the study start and/or the terminal necropsy. To avoid possible misinterpretation of normal age/maturity-related morphology from test article-related effects, it is recommended that sexual maturity be routinely documented for each animal, using the category of immature, peripubertal, or mature.

Briefly, the three categories for sexual maturation are:

\section{Immature}

The seminiferous tubules are undeveloped, lacking a lumen and any evidence of spermatogenesis. Seminiferous tubules are lined by only Sertoli cells and quiescent spermatogonia with rare luminal germ cell debris and multinucleated cells. No mature spermatids and rare to absent germ cell debris are present in epididymis. Secondary sex organs are undeveloped.

\section{Peripubertal}

Seminiferous tubules are in varying stages of development. Mature spermatids may be present in some seminiferous tubules, with some seminiferous tubules containing mature spermatids and/or occasionally germ cell debris and multinucleated cells. A few mature sperm and/or germ cell debris may be seen in the ductular lumen of the epididymis that may also contain multinucleated giant cells and spermatids. Secondary sex or- gans development may be variable.

\section{Mature}

Mature spermatozoa predominate in all/most seminiferous tubules and ducts of the epididymis. Fully developed seminiferous tubules contain mature spermatids and a full complement of germ cells (spermatogonia, spermatocytes, round spermatids, and elongating spermatids). Epididymis contains abundant spermatozoa in the ductular lumen with rare to absent rounded germ cells. Secondary sex organs development may be variable.

\section{Morphologic Evaluation of Male Reproductive System}

Ability to assess effects of test compounds in the reproductive tract of mature NHPs is important and morphologic evaluation of the testis is one of the most sensitive methods for identifying many male reproductive toxicants ${ }^{407}$. An understanding of spermatogenesis whereby stem cell spermatogonia develop into highly specialized spermatozoa is essential for an accurate evaluation of the testis. Specifically, morphologic changes in germ cell morphology or appropriate cell numbers, types and cell associations need to be recognized in particular stages of the spermatogenic cycle. Several published guidelines for comprehensive qualitative evaluation of spermatogenesis in the NHP testis are available which can be used by pathologists for "stage-aware" evaluation of the testes to help identify subtle changes ${ }^{408-410}$.

A standard set of tissues sampled and evaluated in routine monkey toxicity studies include testes, epididymis, seminal vesicles, and prostate. Additional tissues related to the reproductive tract and sexual maturity that may be added for specific studies include efferent ducts, pampiniform plexus, bulbourethral glands, penis, prepuce, and mammary glands.

This manuscript provides a standardized nomenclature for classifying microscopic findings observed in the reproductive system of male cynomolgus monkeys including the testes, epididymides, seminal vesicles (or vesicular glands), prostate, prepuce, and penis. Spontaneously occurring background findings, as well microscopic findings resulting from administration of compounds are included. The diagnostic criteria used for these terms can generally be seen with standard hematoxylin and eosin-stained paraffin sections. Other terms in common use for the INHAND term ("Other Term(s)") are usually given as an aid to understanding the INHAND term, but are not to be used in preference to the INHAND term.

Incidental background microscopic findings are occasionally observed in the reproductive tract of male macaques used in general toxicity studies. When findings are present they may be test article-induced, or due to procedure-related injury or are a result of degenerative changes frequently associated with aging or systemic conditions. Modern laboratory animal management practices in nonhuman primate facilities have resulted in infrequent occurrence of spontaneous infectious disease thus lesions related to infectious reproductive tract diseases are not described in detail in this document.

Due to young age and the short duration of routine pre-clin- 
ical toxicity studies involving macaques, proliferative neoplastic lesions in the male reproductive tract are rarely observed. Most of the available literature about male reproductive tract tumors is limited to individual case reports and include prostate basal cell adenoma, prostate carcinoma in cynomolgus and rhesus monkey ${ }^{411-414 ;}$; bilateral interstitial cell tumor ${ }^{415}$ and seminal vesicle adenoma in a cynomolgus monkeyl. Rhesus papillomavirus type 1 and lymphocryptovirus have been identified in a few squamous cell papillomas and carcinomas of the penis and/or prepuce noted in cynomolgus and rhesus macaques $401,403,416$.

\section{A. Testis, Epididymides, Efferent Ducts}

The testes are ovoid in shape and comprised of lobules of seminiferous tubules that empty into the more centrally located rete testis within the mediastinum testis ${ }^{407}$. The rete testis is located along the lateral aspect of the testis, adjacent to the epididymis and comprises a series of interconnected channels, lined by simple cuboidal or columnar epithelium. The rete testis leads into a series of efferent ducts ${ }^{407}$. Efferent ducts have variably sized lumens lined by columnar ciliated epithelium; and connect via multiple entry points into the initial segment of the epididymis. The epididymis is crescent shaped, attached to the posterolateral border of the testis and is comprised of the initial segment, caput, corpus and cauda. The epididymis carries sperm from the testis to the vas deferens (ductus deferens), which is a thick walled convoluted tube that transfers sperm from the epididymis, to the prostatic urethra. The vas deferens is lined by pseudostratified epithelium with stereocilia ${ }^{407}$ (Table 33).

\section{Embryonic remnants: Testis, Epididymidis}

(Figure 177-178)

\section{Other term(s)}

Appendix testis; appendix epididymides; developmental abnormality; embryologic remnants; vestigial embryonic duct remnants; appendages; hydatids of Morgagni; rudimental tissue; cyst, embryonic; aberrant ductules; vasa aberrantia

\section{Pathogenesis/Cell of Origin}

Testicular and epididymal appendages are vestigial remnants of the embryonic genital primordia, the paramesonephric (Muellerian) duct and the mesonephric (Wolffian) duct, respectively. Aberrant ductules are blind ending persistent mesonephric tubules.

\section{Diagnostic features}

Appendages:

- Sessile or pedunculated structures (macroscopic)

- Simple to pseudostratified ciliated columnar surface epithelium with occasional gland or tubule-like invaginations

- A central fibromuscular stromal core

- Occasional congested or hemorrhagic fibrovascular stroma
- Appendix testes located at the upper pole of the testis beneath or attached to the epididymal body or, more rarely, epididymal head

- Appendix epididymidis is typically attached to epididymal head

- Unilateral or bilateral

Aberrant ductules:

- Small duct-like or cystic structures lined with a simple cuboidal to columnar, partly ciliated epithelium

- Thin smooth muscle wall with peripheral fibrous connective tissue layer

- Lumen may contain eosinophilic, floccular proteinaceous material

- Typically attached to tunica albugenia or vaginalis with a fibrous stalk

- Unilateral or bilateral

\section{Differential Diagnosis}

- Efferent ducts: Columnar ciliated and non-ciliated epithelium, lined by single layer of smooth muscle cells, lumen contain sperm in sexually mature monkeys, bilateral

Comments: The incidence of testicular and epididymal appendages, and aberrant ductules in macaques may be higher than the number of documented cases as these appendages are mostly asymptomatic, often only detected microscopically, may be unintentionally removed during the trimming/embedding, and/or may not be in the plane of section of routinely processed tissues 417 . In general, appendages are regarded as incidental developmental findings, but may have clinical relevance when they become macroscopically visible cystic structures filled with blood or undergo torsion with subsequent hemorrhagic infarction similar to that in humans ${ }^{417}$. In addition, appendages may be of toxicological significance with administration of estrogen or estrogen analogues; Zuckerman and $\mathrm{Krohn}^{418}$ showed that estrogen administration resulted in increased size as well as an increased stromal and epithelial mitotic activity in testicular and epididymal appendages in rhesus macaques. The actual incidence of cyst or vasa aberrantia in macaques is not well established as there is only one available documented report of aberrant ductules in Cynomolgus monkeys ${ }^{417}$.

\section{Hypoplasia, tubular: Testis, Epididymis (Figure 179)}

\section{Other term(s)}

Atrophy/hypoplasia; focal hypoplasia; focal subcapsular tubular atrophy or hypoplasia; segmental hypoplasia

\section{Pathogenesis/Cell of Origin}

The lobular distribution suggests that the group of tubular profiles belong to a single, coiled, seminiferous tubule never having been populated by germ cells, hence hypoplasia.

\section{Diagnostic features}

- Lobular distribution that can be wedge-shaped and situ- 
Table 33. Microscopic Findings of the Male Reproductive System: Testis, Epidiymis, Efferent ducts; Non-Human Primate

\begin{tabular}{|c|c|c|c|c|}
\hline Testis & Common & Uncommon & $\begin{array}{c}\text { Not Observed but } \\
\text { Potentially Relevant }\end{array}$ & Not Applicable \\
\hline \multicolumn{5}{|l|}{ Congenital } \\
\hline Aplasia $^{\wedge}$ & & & $\mathrm{x}$ & \\
\hline Embryonic remnants * & & $\mathrm{x}$ & & \\
\hline Cryptorchidism $^{1}$ & & & $\mathrm{x}$ & \\
\hline Hypoplasia, tubular* & & $\mathrm{x}$ & & \\
\hline Increased collagen, stromal $*$ & $\mathrm{x}$ & & & \\
\hline \multicolumn{5}{|l|}{ Non-proliferative } \\
\hline Amyloid^^ & & & $\mathrm{x}$ & \\
\hline Angiectasis & & $\mathrm{x}$ & & \\
\hline Atrophy, Leydig cell & & & $\mathrm{x}$ & \\
\hline Atrophy, tubule & & $\mathrm{x}$ & & \\
\hline Degeneration, germ cell & & $\mathrm{x}$ & & \\
\hline Degeneration, tubule \# & $\mathrm{x}$ & & & \\
\hline Degeneration/atrophy, tubule * & & $\mathrm{x}$ & & \\
\hline Depletion, germ cell \# & & $\mathrm{x}$ & & \\
\hline Dilatation, rete testis & & $\mathrm{x}$ & & \\
\hline Dilatation, tubular* & $\mathrm{x}$ & & & \\
\hline Edema $^{\wedge}$ & & $\mathrm{x}$ & & \\
\hline Exfoliation, germ cell & $\mathrm{x}$ & & & \\
\hline Fibrosis $\wedge^{\wedge}$ & & $\mathrm{x}$ & & \\
\hline Hypospermatogenesis * & $\mathrm{x}$ & & & \\
\hline 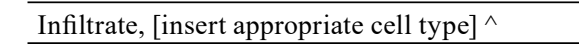 & $\mathrm{x}$ & & & \\
\hline Inflammation $\wedge^{\wedge}$ & & $\mathrm{x}$ & & \\
\hline Mineralization $\wedge^{\wedge}$ & & $\mathrm{x}$ & & \\
\hline Multinucleated giant cells & & $\mathrm{x}$ & & \\
\hline Necrosis/inflammation, vascular/perivascular ${ }^{2}$ & & $\mathrm{x}$ & & \\
\hline Necrosis, Leydig cell $\wedge^{\wedge}$ & & & $\mathrm{x}$ & \\
\hline Necrosis, testis $\wedge$ & & $\mathrm{x}$ & & \\
\hline Necrosis, tubule $^{\wedge}$ & & $\mathrm{x}$ & & \\
\hline Pigment $^{\wedge}$ & & $\mathrm{x}$ & & \\
\hline Residual bodies, atypical & & & & $\mathrm{x}$ \\
\hline Sperm granuloma & & $\mathrm{x}$ & & \\
\hline Sperm stasis & & $\mathrm{x}$ & & \\
\hline Spermatid retention \# & & $\mathrm{x}$ & & \\
\hline Spermatocele & & $\mathrm{x}$ & & \\
\hline Vacuolation, Leydig cell & & $\mathrm{x}$ & & \\
\hline Vacuolation, macrophage & & $\mathrm{x}$ & & \\
\hline Vacuolation, tubule ${ }^{3}$ & & $\mathrm{x}$ & & \\
\hline \multicolumn{5}{|l|}{ Proliferative (non-neoplastic) } \\
\hline Hyperplasia, Leydig cell & & & $\mathrm{x}$ & \\
\hline Hyperplasia, mesothelium & & & $\mathrm{x}$ & \\
\hline Hyperplasia, rete testis & & & $\mathrm{x}$ & \\
\hline
\end{tabular}

ated in a subcapsular location.

- Generally affects triangular clusters (segments) of varying numbers of seminiferous tubular profiles.

- Affected tubules are completely devoid of germ cells and are only lined by Sertoli cells.

- Tubule diameter smaller than normal tubules.
- Tubular lumina are empty.

- Affected tubules reside immediately adjacent to normal tubules.

- Unilateral, but may be bilateral. 


\section{Differential Diagnoses}

- Atrophy, tubular:

- Alternatively, seminiferous tubules that have sustained permanent loss of germ cells (atrophy) may result in Sertoli cell-only tubules. Basement membrane thickening may be a feature.

- Transitional areas (Tubuli recti):

- Germ cells are reduced or absent at transitional areas between seminiferous tubules and the rete testis. These areas are normal anatomy and not a finding to be diagnosed.

- Hypospermatogenesis

- A complete or partial loss of one or more germ cell layers that tends to have a patchy distribution throughout the testes.

Comments: Hypoplasia in monkey is similar to that in dogs but tend to be smaller areas and may not always be wedge shaped and can be confused with focal tubular atrophy. Tubules lined by only Sertoli cells are attributed to hypoplasia of entire tubules or tubular segments presumably never populated by germ cells during testicular development. The etiology is presumably due to a reduced migration of germ cells to the affected segment.

Increased, stromal collagen: Testis, Rete Testis (Figure 180)

\section{Other term(s)}

Fibrous hypoplasia; stromal hyperplasia

\section{Pathogenesis/Cell of Origin}

Congenital or developmental anomaly

\section{Diagnostic features}

- Excess collagen originating from mediastinum around rete testis expanding the rete and interlobular septa

- Dense collagen bundles with high cytoplasmic to nuclear ratio

- May have thickened capsule

- Replacement of testis parenchyma in moderate to severe lesions

- May be associated with tubular or rete tubule dilatation in mature monkeys

- Unilateral or bilateral

\section{Differential Diagnosis}

- Fibrosis:

- Sparse collagen fibers. May have inflammation, necrosis or be associated with distortion of normal anatomic structure

- Amyloid:

- Fibrillar eosinophilic, Congo red positive material

- Material deposited in perivascular and peritubular locations

Comments: Increased stromal collagen, also reported previously as fibrous hypoplasia, has been noted in the testes of sexually immature, maturing and mature Cynomolgus monkeys with higher prevalence and severity of the lesion in immature Asian Cynomolgus monkeys ${ }^{11,407,419}$. In addition, high incidence/severity of this lesion was noted in Cynomolgus monkeys of Chinese origin compared to the ones from Mauritius. The location (testis or rete testis) may be added as a locator or modifier, if deemed appropriate.

\section{Degeneration/atrophy, tubule: Testis (Figure 184, 181)}

Comments: This observation as a stand-alone diagnosis without tubular dilatation or hypospermatogenesis is not a common observation in control macaques. It is a frequent sequel to tubular dilatation and should not be recorded as a separate observation (see Dilatation, tubular). In macaque testis, individual seminiferous tubules are arranged within one lobule thus when an individual tubule is affected it results in a lobular pattern of degeneration. A few tubules or lobules with degeneration/atrophy can occur as a spontaneous finding in the macaque testis and in most cases of spontaneous tubular degeneration/atrophy, there will be some tubules that are undergoing active germ cell degeneration (tubular degeneration) or have no germinal epithelium (tubular atrophy) ${ }^{404}$. Typically, test article-related tubular degeneration/atrophy in Cynomolgus monkeys is multifocal or diffuse and may lack the stage specificity of degeneration commonly seen in the rat; and end-stage spermatogenic suppression is typically more pronounced in primates compared to rodents ${ }^{405}$. Other reported causes of tubular degeneration/atrophy in macaques include but are not limited to testicular hyperthermia secondary to inguinal hernia, senescence, prolonged restraint and generalized disease similar to SIV infection $415,420,421$. Rhesus monkey testes during non-breeding season show varying degrees of regression (degeneration/atrophy) with oligo/aspermia in the epididymidis $^{405}$. In addition, moderate calorie restriction for 5 years in aging rhesus macaques (24-30 year-old) resulted in testicular atrophy characterized by modest decrease in seminiferous tubule diameter and epithelium height, with a concomitant increase in the number of depleted germ cell lines ${ }^{422}$; however, moderate calorie restriction for 7 years did not cause any changes in adult rhesus macaque $(\sim 12$ year old) testis.

\section{Depletion, germ cell: Testis (Figure 182)}

Comments: Stage specific-depletion is not a common feature of test article-induced or spontaneous toxicity in macaque testis; however, one exception is diffuse type B spermatogonia depletion noted in early stages of gonadotropin or androgen reduction. Late stages of gonadotropin or androgen reduction results in more generalized depletion of multiple germ cells with variably present spermatid retention and is histologically similar to the maturation depletion that can occur with a direct spermatogonial toxicity $334,423,424$. 


\section{Dilatation, tubule or rete testis: Testis, Rete testis}

(Figure 186, 183)

\section{Pathogenesis/Cell of Origin}

Increased stromal collagen of testis or rete testis causing impaired outflow of the fluid; impaired fluid reabsorption in the efferent ducts/caput epididymis; distal obstruction in excurrent duct system (rete testis, efferent ducts, or initial segment)

\section{Diagnostic features}

- Increased luminal diameter of seminiferous tubules or rete testis ducts

- Sperm stasis may be present

- Accompanied by thinning or pressure atrophy of the seminiferous epithelium or duct epithelium

- Increased pressure could cause cystic dilatation and germ cell degeneration of seminiferous tubules

- May be associated with increased stromal collagen

- Focal to multifocal or segmental

- Unilateral or bilateral

\section{Differential Diagnosis}

- Tubular degeneration/atrophy:

- Degeneration and/or depletion of germ cells without increase in tubular luminal diameter

- No attenuation or thinning of the seminiferous epithelium

Comments: Outflow obstruction most frequently occurs at the level of the rete testis in macaques. Focal, unilateral or bilateral tubular dilation can be seen as a background incidental finding11, 419, 425 and can be particularly obvious during puberty407, 426. Moderate to severe increased stromal collagen (previously described as fibrous hypoplasia) may cause increased incidence and severity of tubular or rete testis dilatation in maturing or sexually mature animals 419,427 . This change frequently results in secondary tubular degeneration/atrophy and should be used as the appropriate diagnostic term where dilatation as a result of outflow obstruction is the primary change.

\section{Hypospermatogenesis: Testis (Figure 185)}

\section{Other $\operatorname{term}(\mathrm{s})$}

Segmental hypospermatogenesis

\section{Pathogenesis/Cell of Origin}

Hypospermatogenesis is attributed to transient failure of spermatogenesis affecting a segment of the seminiferous tubule resulting in an absence of one or more generations of germ cells.

\section{Diagnostic features}

- Affects scattered tubular profiles (segments) throughout the testes

- Focal spermatogenic interruption at various germ cell levels mixed with normal spermatogenesis

- Complete or partial absence of one or more generations of germ cells (spermatocytes, round spermatids or elongate spermatids)

- When affecting only some of the germ cells these tubular segments may be missing round spermatids and/or pachytene spermatocytes from the layers below the overlying elongate spermatids.

- May have a few sertoli-only cells tubules (atrophy)

- Generally occurs in the absence of any significant germ cell degeneration or apoptosis

- Not stage or cell specificity

- No accompanying increase in cell debris in the epididymidis

- Usually bilateral

\section{Differential Diagnosis}

- Immaturity:

- The absence of elongate spermatids

- Transitional areas (Tubuli recti)

- Transitional areas between seminiferous tubules and the rete testis

- Central location, adjacent to rete testis

- Partial sertoli-cell only lining without apparent degeneration

- Bilateral

- Tubular hypoplasia:

- Total lack of germinal cells with sertoli cell-only tubules

- Decreased tubular lumen or contracted tubules

- No luminal debris or content

- Depletion, Germ Cell:

- Generally germ cell specific

- Generally diffuse distribution throughout the testis

- Degeneration, Germ Cell:

- Active degeneration of germ cells including multinucleate giant cells

- Generally associated with increased cell debris in the epididymis

Comments: In macaques, spermatogenesis progresses at a different rate in all tubules, hence it is common for some groups of tubules to be significantly more or less advanced than others ${ }^{407}$, thus less developed tubules should not be diagnosed as having hypospermatogenesis. In addition to differential diagnoses discussed earlier, hypospermatogenesis can also histologically overlap with the appearance of seasonal involution and/or atrophy in rhesus macaques, but in general hypospermatogenesis is less uniform and does not follow a cell or stage specificity (Table 34 ).

\section{Dilatation, duct: Epididymis, Efferent Ducts}

(Figure 187-188)

\section{Other term(s)}

Dilatation, cystic 
Table 34. Microscopic Findings of the Male Reproductive System: Epididymis, Efferent ducts; Non-Human Primate

\begin{tabular}{|c|c|c|c|c|}
\hline Epididymis /Efferent Ducts & Common & Uncommon & $\begin{array}{c}\text { Not Observed but } \\
\text { Potentially Relevant }\end{array}$ & Not Applicable \\
\hline \multicolumn{5}{|l|}{ Congenital } \\
\hline Aplasia $^{\wedge}$ & & & $\mathrm{x}$ & \\
\hline Embryonic remnants $^{1}$ & & $\mathrm{x}$ & & \\
\hline Hypoplasia $^{\wedge}$ & & & $\mathrm{x}$ & \\
\hline \multicolumn{5}{|l|}{ Non-proliferative } \\
\hline Adenosis & & & $\mathrm{x}$ & \\
\hline Amyloid^^ & & & $\mathrm{x}$ & \\
\hline Atrophy duct & & & $\mathrm{x}$ & \\
\hline Cell debris, lumen & & $\mathrm{x}$ & & \\
\hline Cribriform change & & & $\mathrm{x}$ & \\
\hline Degeneration, epithelium & & $\mathrm{x}$ & & \\
\hline Dilatation, duct *\# & & $\mathrm{x}$ & & \\
\hline Ectopic tissue, adrenocortical ${ }^{\wedge}$ & & $\mathrm{x}$ & & \\
\hline Edema $^{\wedge}$ & & $\mathrm{x}$ & & \\
\hline Fibrosis $\wedge^{\wedge}$ & & $\mathrm{x}$ & & \\
\hline Infiltrate, [insert appropriate cell type] ${ }^{\wedge}$ & $\mathrm{x}$ & & & \\
\hline Inflammation $^{\wedge}$ & & $\mathrm{x}$ & & \\
\hline Karyomegaly & & & $\mathrm{x}$ & \\
\hline Metaplasia, squamous cell & & & $\mathrm{x}$ & \\
\hline Necrosis/inflammation, vascular/ perivascular ${ }^{3}$ & & $\mathrm{x}$ & & \\
\hline Single cell necrosis (epithelial) ${ }^{\wedge}$ & & $\mathrm{x}$ & & \\
\hline Sperm, decreased, lumen $\#$ & & $\mathrm{x}$ & & \\
\hline Sperm granuloma * & & $\mathrm{x}$ & & \\
\hline Sperm stasis & & $\mathrm{x}$ & & \\
\hline Spermatocele & & $\mathrm{x}$ & & \\
\hline Vacuolation, epithelium & & $\mathrm{x}$ & & \\
\hline \multicolumn{5}{|l|}{ Proliferative (non-neoplastic) } \\
\hline Hyperplasia, diffuse * & & $\mathrm{x}$ & & \\
\hline
\end{tabular}

Comments: Efferent duct dilatation can occur secondary to test article-induced inflammation 428 .

Hyperplasia, diffuse: Epididymis (No image provided)

\section{Other term(s)}

Physiologic hyperplasia; hyperplasia, functional

\section{Diagnostic features}

- Hypertrophy of ciliated cells and basal cells in the epididymal epithelium

- Piling or stratification of epithelial cells

- Irregular luminal epithelial border

- Increased diameter of epididymal ducts

- Increased mitosis

- Bilateral

- Diffuse change

Comments: This is a very uncommon change. Diffuse hy- pertrophy and hyperplasia of epididymal epithelium was noted in cynomolgus monkeys administered $\geq 100 \mu \mathrm{g} / \mathrm{kg}$ recombinant human epidermal growth factor (hEGF) for 2 weeks ${ }^{429}$. No changes were noted in the testes of monkeys administered hEGF. Focal hyperplasia or tumors of epididymides haven't been reported in macaques.

\section{Sperm granuloma: Epididymis, Efferent Ducts}

(No image provided)

Comments: In nonhuman primates, sperm granulomas in the epididymis, efferent ducts or vas deferens are primarily associated with vasectomy procedures ${ }^{430,431}$. 


\section{B. Prostate, Seminal vesicles, Bulbourethral Glands, Penis, and Prepuce (Accessory Sex Glands and External Genitalia)}

Male reproductive anatomy of the macaque differs from the rodent, most notably by the absence of coagulating and preputial glands, and the presence of external fibrovascular penis with a glans, and an os penis or baculum ${ }^{415}$.

The accessory sex glands in nearly all NHPs consist of single or two-lobed prostate gland and paired seminal vesicles and bulbourethral glands. In cynomolgus monkeys, the prostate has a prominent stromal component, indistinctly divided into a lobular cranial lobe and compact caudal lobe that incompletely envelops the urethra. These two lobes have been considered analogous to the central and peripheral zones of the human prostate, respectively. However, the cranial lobe is quite different functionally and histologically from the central zone of the human prostate ${ }^{415}$. The central portions of both lobes have large, irregularly arranged acini, which are more prominent in the cranial lobe. Acini in the peripheral portion of both lobes are smaller and more regularly arranged. The epithelium consists of columnar epithelial and basal cells on a basal lamina. Columnar epithelial cells are taller in the cranial lobe than in the caudal lobe. Cytoplasm and nucleus of epithelial cells are basally located and the apical region of the cell contains a large number of vacuoles and secretory material. The lumen of the acini contains eosinophilic-staining secretory material. Basal cells with clear cytoplasm are more numerous in the peripheral portion ${ }^{432}$.

Paired seminal vesicles are the largest of the accessory sex glands and produce a yellowish-white viscous fluid that makes up most of the ejaculate. These are highly convoluted glands consisting of rounded acini with a simple columnar epithelium, situated distal to the ampulla of the vas deferens, and empty via the ejaculatory duct into the urethra ${ }^{407}$ (Table 35 ).

Bulbourethral glands are paired compound tubuloalveolar glands that secrete a mucoid material into the penile urethra. The epithelial cells are pyramidal to columnar with a microvillous surface ${ }^{407}$.

Table 35. Microscopic Findings of the Male Reproductive System: Prostate, Seminal vesicles, and Bulbourethral glands; Non-Human Primate

\begin{tabular}{|c|c|c|c|c|}
\hline Prostate/Seminal Vesicles/Bulbourethral Gland & Common & Uncommon & $\begin{array}{c}\text { Not Observed but Po- } \\
\text { tentially Relevant }\end{array}$ & Not Applicable \\
\hline \multicolumn{5}{|l|}{ Congenital } \\
\hline Aplasia $^{\wedge}$ & & & $\mathrm{x}$ & \\
\hline Hypoplasia $^{\wedge}$ & & & $\mathrm{x}$ & \\
\hline \multicolumn{5}{|l|}{ Non-proliferative } \\
\hline Amyloid ^ & & & $\mathrm{x}$ & \\
\hline Angiectasis & & & $\mathrm{x}$ & \\
\hline Atrophy \#1 & & $\mathrm{x}$ & & \\
\hline Corpora amylacea $* 2$ & $\mathrm{x}$ & & & \\
\hline Dilatation, acinar/vesicle & $\mathrm{x}$ & & & \\
\hline Fibrosis, stroma ${ }^{\wedge}$ & & $\mathrm{x}$ & & \\
\hline Infiltrate, [insert appropriate cell type] ${ }^{\wedge}$ & $\mathrm{x}$ & & & \\
\hline Inflammation $^{\wedge}$ & $\mathrm{x}$ & & & \\
\hline Single cell necrosis ${ }^{\wedge}$ & & $\mathrm{x}$ & & \\
\hline Necrosis/inflammation, vascular/ perivascular ${ }^{3}$ & & $\mathrm{x}$ & & \\
\hline Metaplasia *\# & & $\mathrm{x}$ & & \\
\hline Necrosis ${ }^{\wedge}$ & & & $\mathrm{x}$ & \\
\hline Single cell necrosis, epithelium ${ }^{\wedge}$ & & $\mathrm{x}$ & & \\
\hline Sperm reflux $* 2$ & & $\mathrm{x}$ & & \\
\hline Vacuolation, epithelial & & $\mathrm{x}$ & & \\
\hline \multicolumn{5}{|l|}{ Proliferative (non-neoplastic) } \\
\hline Hyperplasia, atypical & & $\mathrm{x}$ & & \\
\hline Hyperplasia, basal cell $* 4$ & $\mathrm{x}$ & & & \\
\hline Hyperplasia, diffuse & & $\mathrm{x}$ & & \\
\hline Hyperplasia, reactive & & $\mathrm{x}$ & & \\
\hline Mesenchymal proliferative lesion & & & & $\mathrm{x}$ \\
\hline \multicolumn{5}{|l|}{ Neoplastic } \\
\hline Adenoma, basal cell ${ }^{4,5}$ & & $\mathrm{x}$ & & \\
\hline
\end{tabular}

* Terminology with diagnostic criteria and/or comments described below. ${ }^{*}$ Finding more frequently observed as an induced change. ${ }^{\wedge}$ Terminology addressed in the Systemic/General Pathology Section. ${ }^{1}$ Atrophy in the prostate is difficult to evaluate due to a small amount of stored secretion and the low height of the secretory epithelium. ${ }^{2}$ Prostate and seminal vesicles. ${ }^{3}$ Terminology addressed in the Cardiovascular Section. ${ }^{4}$ Prostate. ${ }^{5}$ Typically observed/reported in older non-human primates. 


\section{Corpora amylacea: Prostate, Seminal vesicles, Penis}

(Figure 189-190)

\section{Other term(s)}

Mineral; mineral calculi; concretions, semen; semen calculi; sperm cystoliths; semen matrix calculi; concretions

\section{Pathogenesis/Cell of Origin}

Spontaneous (common); much less frequently can be seen as a result of retrograde ejaculation (back flow) of sperm due to repeated transrectal electro-ejaculation

\section{Diagnostic features}

- Composed of numerous sperm embedded in a brightly eosinophilic matrix with/without mineralization.

- Could be intermixed with mineral, necrotic debris, epithelial cells and inflammatory cells

- Accompanying obstruction and inflammation/ulceration of the urethra and urinary bladder

Comments: Concretions, calcified concretions or mineral in the testis, seminal vesicles, prostate and distal urinary tract are typically composed of calcium carbonate11, 425, 433-437. Semen calculi have been reported in prostatic and penile urethra of macaques ${ }^{438-440}$. Retrograde sperm ejaculation due to repeated electro-ejaculation causes trapping of semen in the posterior urethra and back flow into urinary bladder causing obstruction and can result in corpora amylacea 438,439 , however the observation is commonly observed in monkeys as a spontaneous change, particularly within the seminal vesicles.

\section{Metaplasia: Prostate, Seminal Vesicles, Penis}

(No image provided)

Comments: Squamous metaplasia of the prostate and atrophy with stromal fibrosis secondary to administration of estrogenic compounds has been observed in prostate and other accessory sex glands ${ }^{432,441}$, including seminal vesicles (personal observation, Eric Van Esch).

\section{Sperm reflux: Prostate, Seminal Vesicles, Penis}

(Figure 191)

\section{Other term(s)}

Retrograde sperm ejaculation; sperm backflow

\section{Pathogenesis/Cell of Origin}

Spontaneous; Repeated electroejaculation-induced asynchronous flow or spontaneous sperm back flow

\section{Diagnostic features}

- Back reflux of sperm into seminal vesicles, prostatic or penile urethra

- Leads to semen concretions or calculi and obstruction of prostatic or penile urethra
Comments: In macaques, sperm reflux can occur spontaneously or associated with routine trans-rectal electroejaculation which induces a lack of synchronous flow potentially due to asynchronous stimulation of nerve tracts 438,439 . Sperm reflex when occurring as a spontaneous finding in macaques without any associated inflammatory or degenerative changes, does not need to be diagnosed routinely.

Hyperplasia, basal cell: Prostate (Figure 192)

\section{Other term(s)}

Hyperplasia; focal hyperplasia;

\section{Diagnostic features}

- Irregular, solid nests of basal cells displacing or replacing secretory columnar epithelium in one or more acini

- The cells have scant basophilic cytoplasm

- Nuclei are ovoid with fine chromatin and occasional indistinct nucleolus

- Scant but cellular fibromuscular stroma

- Rare mitosis

- No cellular atypia

\section{Differential Diagnosis}

- Hyperplasia, functional:

- All acini and ducts affected

- Frequent mitoses

- Prominent fibromuscular stroma

- Associated hyperplasia of prostatic urethra

- Adenoma:

- Larger, compressive, encapsulated masses that replace normal acini

- Typically has both basal and larger epithelial cells

- Areas of basal lamina deposition and squamous, neuroendocrine, transitional and goblet cell differentiation

- Loss of acinar architecture

- No metastasis

- Minimal cellular atypia

- Adenocarcinoma:

- Lacks basal cells

- Loss of acinar architecture

- Metastasis

\section{Special Techniques}

Immunohistochemical markers:

- Cytokeratin - Basal cells express cytokeratins recognized by the monoclonal antibody EAB 903 (34PE12), which can be used to distinguish basal cell lesions from acinar cell lesions.

Comments: Unlike in rodents, focal hyperplasia of the prostate is not often seen as a response to inflammation in the monkey and can occur as a spontaneous change in toxicity studies. Most reported prostate lesions were noted in the cranial lobe, represent a continuum of basal cell hyperplasia to adenoma and considered morphologically similar to 
benign basal cell lesions arising in the human prostate and thus macaque is considered a good animal model for studying prostatic diseases $412,432,433,442$. Functional or diffuse hyperplasia of prostate noted in rodents secondary to administration of exogenous androgens resulting in enlargement, weight increase, diffuse hyperplasia, and an increase in secretions $^{404}$ was also reported in rhesus macaques ${ }^{441}$. In addition, diffuse hyperplasia of prostate and seminal vesicles secondary to administration of recombinant human epidermal growth factor was noted in cynomolgus monkeys ${ }^{429}$ (Table 36).

Papilloma: Penis, Prepuce (No image provided)

\section{Other $\operatorname{term}(\mathrm{s})$}

Condyloma acuminatum; wart or verrucae

\section{Pathogensis}

Potential virus-induced proliferation of genital skin and mucous membranes

\section{Diagnostic features}

- Exophytic plaque, or cauliflower-like lesions (macroscopic) on shaft, glans penis and prepuce

- Irregular epidermal hyperplasia with anastomosing rete ridges, dyskeratosis, parakeratotic hyperkeratosis, hypergranulosis

- Prominent basal layer

- Fibrovascular stromal core

- Mixed cell inflammatory infiltrates in the superficial dermis

- Intranuclear viral particles in supra-basal keratinocytes

\section{Differential Diagnosis}

- Hyperplasia, squamous epithelium:

- Expansion of squamous cell layer
- No basal cell layer expansion

- No fibrovascular stromal core

- Squamous cell carcinoma:

- Clear evidence of submucosal and/or vascular invasion

- Cellular atypia

- Hyperkeratosis:

- Normal maturation lineage of squamous epithelium

- No basal cell layer expansion

- No cellular atypia

\section{Special Techniques}

- Immunohistochemical markers

- Ki67 (nuclear) - Proliferation in normal mucosal epithelium is limited to basal/suprabasal layers. Hyperplastic lesions show cell proliferation in more superficial layers.

- P16INK4A (nuclear and cytoplasmic) - This tumor suppressor protein is absent in normal mucosal epithelium but increased in hyperplastic or dysplastic lesions.

- Genetic testing

- DNA testing (PCR) can be used to identify virus sequences.

- In situ hybridization is preferable to immunohistochemistry for viral antigen.

Comments: Typically, monkeys with macroscopically visible lesions on external genitalia before the study start are culled. Papillomaviruses (PVs) are a group of small, nonenveloped DNA viruses that cause mucosal or cutaneous neoplasia in a variety of animals and humans. Human papilloma virus 11 and Lymphocryptovirus have been identified in a few reported genital skin papilloma lesions in macaques 403,416 . Whilst most papillomas will regress spontaneously, some may persist or undergo malignant transformation. A rhesus papillomavirus type 1 (RhPV-1)-related penile carcinoma was reported in a rhesus monkey ${ }^{401}$.

Table 36. Microscopic Findings of the Male Reproductive System: Penis, and Prepuce; Non-Human Primate

\begin{tabular}{|c|c|c|c|c|}
\hline Penis/Prepuce & Common & Uncommon & $\begin{array}{l}\text { Not Observed but } \\
\text { Potentially Relevant }\end{array}$ & Not Applicable \\
\hline \multicolumn{5}{|l|}{ Non-proliferative } \\
\hline Corpora amylacea $^{1}$ & & $\mathrm{x}$ & & \\
\hline Sperm reflux ${ }^{1}$ & & $\mathrm{x}$ & & \\
\hline \multicolumn{5}{|l|}{ Neoplastic } \\
\hline Papilloma * & & $\mathrm{x}$ & & \\
\hline
\end{tabular}




\section{RESPIRATORY SySTEM}

Standardized INHAND nomenclature of proliferative and nonproliferative respiratory tract lesions in rats and mice was previously published ${ }^{443}$ and that publication should be consulted for general respiratory tract nomenclature. This document follows a similar anatomical approach to that used previously although larynx, trachea, bronchi, and bronchioles have been combined to reduce redundancy, and respiratory bronchioles, alveolar ducts, and alveoli are combined as they represent the lung parenchyma.

Like humans, NHPs are microsmotic, and the NHP nasal cavity is anatomically more similar to that of humans than the more complex nasal cavities of rodents and dogs $444-446$ and NHPs, like humans, are oronasal breathers and may be more useful than rodents as a research model for toxic changes in the nasal cavity in inhalation studies. The nasopharyngeal tonsil, analogous to the adenoid in humans, is the primary and constitutive (present at birth) secondary lymphoid tissue of the posterior nasal cavity. Other small submucosal lymphoid tissues, sometimes called nasal associated lymphoid tissue (NALT) ${ }^{142}$, are part of the MALT system and are acquired postnatally after antigen exposure. Acquired MALT of the nasal cavity in NHP should not be confused with the NALT of the rodent nasal cavity which is constitutive (present at birth) but will mature further after postnatal antigen exposure.

Macaques have a single laryngeal sac, which is a thinwalled air-filled pouch anterior to the larynx, communicating with the main airway via a small opening at the base of the epiglottis 447,448 . This structure is lined by ciliated respiratory epithelium, and occasionally becomes infected ${ }^{449}$. The pharynx, larynx and carina in NHPs are anatomically and histologically more similar to humans and respond to xenobiotics in more similar (to humans) fashion than rats ${ }^{443,446,450}$. Macaques have 7 lung lobes, including left and right cranial, middle, and caudal lobes, and an accessory lobe arising from the right side. NHP lungs are anatomically more similar to humans than lungs of rats and mice based on the presence (in NHPs but not rats or mice) of cartilage and submucosal glands in intrapulmonary bronchi, of mucus cells as the primary cell type in conducting airways, and the presence of respiratory bronchioles ${ }^{451}$. Club cells are present mainly in terminal and respiratory bronchioles of humans, monkeys, and dogs. However, in contrast to the dichotomous branching pattern of human tracheobronchial airways, airways of monkeys, rodents, and dogs are monopodial. NHPs, like humans, have thin pulmonary veins with mostly fibrous walls ${ }^{452}$.

The respiratory system is susceptible to injury caused by blood borne or inhaled xenobiotics and thus should be routinely examined in all toxicity studies. For inhalation studies, it is recommended that tissues sampled include 4 to 6 transverse sections of the nasal cavity and nasopharynx to represent 4 levels from anterior to posterior, at least 2 transverse sections of the larynx, 1 or 2 transverse sections of the trachea, 1 section of the carina, samples of all lung lobes to include proximal and peripheral sections, and the tracheobronchial lymph nodes.
One transverse section of the epiglottis and 1 transverse section of the oropharynx bordered by the tonsils may be sampled as needed for specific studies. This sampling is illustrated in the Respiratory Collection Protocol of the Laboratory Monkey, published by GlaxoSmithKline, Charles River Laboratories, and EPL ${ }^{453}$ and more recently for the nasal cavities in Chamanza et al. ${ }^{142}$. Tissue sampling for non-inhalation studies may be less extensive but should include at least 2 sections of the lung (a proximal area near the mainstem bronchi and an area at the periphery) and one section of trachea.

Localized and generalized changes that can be observed within the respiratory tract, such as parasites/parasitic granulomas; intranuclear and cytoplasmic inclusions; cellular infiltrates and inflammatory changes; and thromboemboli are tabulated under the appropriate sections with comments added, but are discussed in more detail in the Systemic/General Pathology section.

\section{A. Nasal Cavity, Nasopharynx, Paranasal Sinuses}

(Table 37)

\section{Hyperplasia, polypoid: Nasal Cavity/Nasopharynx/Para- nasal Sinuses (No image provided)}

\section{Other term(s)}

Polyposis

\section{Pathogenesis/Cell of Origin}

Nasal polyps in nonhuman primates are inflammatory lesions of the nasal or paranasal sinus mucosa.

\section{Diagnostic features}

- Exophytic mucosal growth(s) on a fibrous or fibrovascular stalk.

- Typically associated with chronic inflammation.

- Frequently edematous and infiltrated with inflammatory cells. May have areas of erosion, ulceration, or hemorrhage.

- Epithelial cells do not exhibit nuclear atypia and mitotic figures are rare or absent.

- Basement membrane is intact.

- May block or partially block nasal passage or sinus.

- May be associated with nasal mites.

\section{Differential Diagnoses}

- Hyperplasia, epithelial

- No exophytic growth; does not form papillary projections. Lack of a fibrovascular stalk.

- Papilloma, squamous cell

- Papillary or filiform structures typically consisting of thick fronds of squamous cells or a mixture of squamous and mucus-producing cells without cilia. Relatively more epithelial proliferation and less inflammation than an inflammatory polyp.

Comments: Nasal mites (genus Rhinophaga) in the nasal 
Table 37. Microscopic Findings of the Respiratory System: Nasal Cavity, nasopharynx, paranasal sinuses. Non-Human Primate

\begin{tabular}{|c|c|c|c|c|}
\hline Nasal Cavity/Nasopharynx/Paranasal Sinuses & Common & Uncommon & $\begin{array}{c}\text { Not Observed, but } \\
\text { Potentially Relevant }\end{array}$ & Not Applicable \\
\hline \multicolumn{5}{|l|}{ Congenital } \\
\hline Cleft palate ${ }^{1}$ & & & $\mathrm{x}$ & \\
\hline Deviation of the nasal septum & & $\mathrm{x}$ & & \\
\hline \multicolumn{5}{|l|}{ Non-proliferative } \\
\hline Amyloid^^ & & $\mathrm{x}$ & & \\
\hline Angiectasis & & $\mathrm{x}$ & & \\
\hline Atrophy & $\mathrm{x}$ & & & \\
\hline Congestion $^{\wedge}$ & $\mathrm{x}$ & & & \\
\hline Corpora amylacea & $\mathrm{x}$ & & & \\
\hline Degeneration & $\mathrm{x}$ & & & \\
\hline Edema $^{\wedge}$ & & $\mathrm{x}$ & & \\
\hline Erosion/ulcer & $\mathrm{x}$ & & & \\
\hline Globules, eosinophilic & & $\mathrm{x}$ & & \\
\hline Hemorrhage $^{\wedge}$ & & $\mathrm{x}$ & & \\
\hline 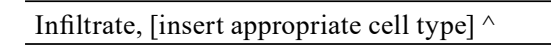 & $\mathrm{x}$ & & & \\
\hline Inflammation $^{\wedge}$ & $\mathrm{x}$ & & & \\
\hline Necrosis & & $\mathrm{x}$ & & \\
\hline Parasite/Parasitic granuloma $^{\wedge}$ & & $\mathrm{x}$ & & \\
\hline Perforation, septum & & & $\mathrm{x}$ & \\
\hline Regeneration $^{2}$ & $\mathrm{x}$ & & & \\
\hline Thrombus & & $\mathrm{x}$ & & \\
\hline \multicolumn{5}{|l|}{ Proliferative (non-neoplastic) } \\
\hline Hyperplasia, atypical & & $\mathrm{x}$ & & \\
\hline Hyperplasia, basal cell & & & $\mathrm{x}$ & \\
\hline Hyperplasia/metaplasia, mucous cell & $\mathrm{x}$ & & & \\
\hline Hyperplasia, neuroendocrine cell & & & $\mathrm{x}$ & \\
\hline Hyperplasia, olfactory epithelium & & $\mathrm{x}$ & & \\
\hline Hyperplasia, respiratory epithelium & & $\mathrm{x}$ & & \\
\hline Hyperplasia, squamous cell & $\mathrm{x}$ & & & \\
\hline Hyperplasia, transitional epithelium & & $\mathrm{x}$ & & \\
\hline Hyperplasia, polypoid * & & $\mathrm{x}$ & & \\
\hline Metaplasia, respiratory, olfactory/glandular & & $\mathrm{x}$ & & \\
\hline Metaplasia, squamous cell & $\mathrm{x}$ & & & \\
\hline
\end{tabular}

cavity and upper respiratory tract of Old World monkeys and apes and can cause inflammation and nasal polyp formation ${ }^{454}$. The term polyp should not be used since that is considered a neoplasm within the FDA's Standardization for Exchange of Nonclinical Data (SEND) criteria.

\section{B. Larynx (including Air Sacs), Trachea, Bronchi, and Bronchioles (Table 38)}

Hypertrophy/hyperplasia, Bronchial Smooth Muscle: Lung - Bronchi/Bronchioles (Figure 193-194)

\section{Pathogenesis/Cell of Origin}

Smooth muscle cells in the submucosa of bronchi and bron- chioles.

\section{Diagnostic features}

- Increased prominence of smooth muscle mass characterized by enlarged smooth muscle cells with or without increased nuclear density

- Smooth muscle cells are well differentiated and aligned appropriately

- Usually the thickened muscle layer is evenly distributed around the airways (depends on section)

- May be associated with an adjacent inflammatory cell infiltrate

- No disruption, compression or disturbance of adjacent tissues 
Table 38. Microscopic Findings of the Respiratory System: Larynx (including air sacs), Trachea, Bronchi, and Bronchioles. Non-Human Primate

\begin{tabular}{|c|c|c|c|c|}
\hline Larynx/Trachea/Bronchi/Bronchioles & Common & Uncommon & $\begin{array}{l}\text { Not Observed, but } \\
\text { Potentially Relevant }\end{array}$ & Not Applicable \\
\hline \multicolumn{5}{|l|}{ Non-proliferative } \\
\hline Alteration, epithelial & & & & $\mathrm{x}$ \\
\hline Bronchiectasis & & $\mathrm{x}$ & & \\
\hline Congestion $^{\wedge}$ & & $\mathrm{x}$ & & \\
\hline Degeneration & & $\mathrm{x}$ & & \\
\hline Ectasia, submucosal gland & & $\mathrm{x}$ & & \\
\hline Edema $^{\wedge}$ & & $\mathrm{x}$ & & \\
\hline Erosion/ulcer & & $\mathrm{x}$ & & \\
\hline Hemorrhage $^{\wedge}$ & & $\mathrm{x}$ & & \\
\hline Inclusions, cytoplasmic $^{\wedge}$ & & $\mathrm{x}$ & & \\
\hline 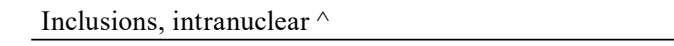 & & $\mathrm{x}$ & & \\
\hline Infiltrate, [insert appropriate cell type] ${ }^{\wedge}$ & $\mathrm{x}$ & & & \\
\hline Inflammation $^{\wedge}$ & & $\mathrm{x}$ & & \\
\hline Material, extracellular [insert morphology/color] \# & & $\mathrm{x}$ & & \\
\hline Necrosis & & $\mathrm{x}$ & & \\
\hline Parasite/parasitic granuloma $^{\wedge}$ & & $\mathrm{x}$ & & \\
\hline Regeneration $^{1}$ & & $\mathrm{x}$ & & \\
\hline Thrombus & & $\mathrm{x}$ & & \\
\hline \multicolumn{5}{|l|}{ Proliferative (non-neoplastic) } \\
\hline Hyperplasia, atypical & & $\mathrm{x}$ & & \\
\hline Hyperplasia, mucous cell & & $\mathrm{x}$ & & \\
\hline Hyperplasia, neuroendocrine cell & & & $\mathrm{x}$ & \\
\hline Hyperplasia, respiratory epithelium & & $\mathrm{x}$ & & \\
\hline Hyperplasia, squamous cell & & $\mathrm{x}$ & & \\
\hline Hypertrophy/hyperplasia, bronchial smooth muscle $* 2$ & & $\mathrm{x}$ & & \\
\hline Metaplasia, squamous cell & & $\mathrm{x}$ & & \\
\hline
\end{tabular}

\section{Differential Diagnoses}

- Tangential section through vessel:

- Other structures in section are cut tangentially, missing, or otherwise abnormal in shape

- Leiomyoma:

- Well circumscribed, nodular mass composed of interlacing bundles and whorls of uniform spindle cells arranged in criss-cross patterns of bundles

- NOTE: There are no reports of leiomyoma occurring in the respiratory tract of cynomolgus monkeys in routine toxicology studies

Comments: Smooth muscle cell hypertrophy/hyperplasia is uncommon as a spontaneous finding but can be seen in cases of lung inflammation, allergic disease and/or immune stimulation 455,456 , especially following inhalation administration of certain xenobiotics. Often it is observed as a fo$\mathrm{cal} /$ multifocal change rather than diffusely present throughout all areas of the parenchyma.

\section{Respiratory Bronchioles, Alveolar Ducts, and Alveoli (Table 39)}

\section{Accumulation, pigmented macrophage, perivascular:} Lung (Figure 195-196)

\section{Pathogenesis/Cell of origin}

Accumulation of bown pigment in macrophages in the perivascular interstitium. This is a specifc finding associated with vascular lesions and the pigment has been shown to be hemosiderin. Fragmentation or partial loss of the external or internal elastic laminae or arteries/arterioles can be detected using an elastin stain. Small- to medium-sized arteries and veins with prominent accumulation of brown pigment-laden macrophages may show degeneration and necrosis of smooth muscle of the tunica media.

\section{Diagnostic features}

- Accumulation of macrophages containing brown cytoplasmic pigment in the perivascular interstitium of small- 
Table 39. Microscopic Findings of the Respiratory System: Respiratory Bronchioles, Alveolar ducts, and Alveoli. Non-Human Primate

\begin{tabular}{|c|c|c|c|c|}
\hline Respiratory Bronchioles/Alveolar Ducts/Alveoli & Common & Uncommon & $\begin{array}{c}\text { Not Observed but } \\
\text { Potentially Relevant }\end{array}$ & Not Applicable \\
\hline \multicolumn{5}{|l|}{ Congenital } \\
\hline Cyst, congenital & & $\mathrm{x}$ & & \\
\hline Hypoplasia & & $\mathrm{x}$ & & \\
\hline \multicolumn{5}{|l|}{ Non-proliferative } \\
\hline Accumulation, pigmented macrophage, perivascular * & & $\mathrm{x}$ & & \\
\hline Atelectasis $^{1}$ & & $\mathrm{x}$ & & \\
\hline Congestion $^{\wedge 2}$ & $\mathrm{x}$ & & & \\
\hline Degeneration & & $\mathrm{x}$ & & \\
\hline Ectasia, acinus & & & & $\mathrm{x}$ \\
\hline Edema $^{\wedge}$ & & $\mathrm{x}$ & & \\
\hline Embolus & & $\mathrm{x}$ & & \\
\hline 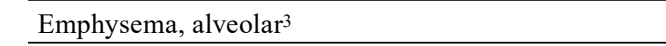 & & $\mathrm{x}$ & & \\
\hline Fibrosis $^{\wedge}$ & & $\mathrm{x}$ & & \\
\hline Hemorrhage $^{\wedge}$ & & $\mathrm{x}$ & & \\
\hline Hypertrophy, media, artery & & $\mathrm{x}$ & & \\
\hline Inclusions, cytoplasmic $^{\wedge}$ & & $\mathrm{x}$ & & \\
\hline 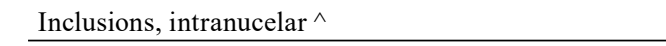 & & $\mathrm{x}$ & & \\
\hline Infiltrate, [insert appropriate cell type] ${ }^{\# \wedge}$ & $\mathrm{x}$ & & & \\
\hline Inflammation $\wedge$ & & $\mathrm{x}$ & & \\
\hline 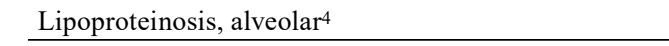 & & $\mathrm{x}$ & & \\
\hline Macrophages increased $* \# 5$ & $\mathrm{x}$ & & & \\
\hline Material, extracellular [insert morphology/color] \# & & $\mathrm{x}$ & & \\
\hline 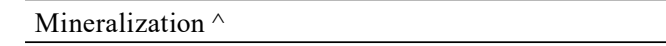 & $\mathrm{x}$ & & & \\
\hline Necrosis & & $\mathrm{x}$ & & \\
\hline Parasite $^{\wedge}$ & & $\mathrm{x}$ & & \\
\hline Pigment/foreign material ${ }^{\wedge} 6$ & $\mathrm{x}$ & & & \\
\hline Regeneration & & $\mathrm{x}$ & & \\
\hline Thromboembolism, pulmonary ${ }^{7}$ & & $\mathrm{x}$ & & \\
\hline Thrombus $^{7}$ & & $\mathrm{x}$ & & \\
\hline \multicolumn{5}{|l|}{ Proliferative (non-neoplastic) } \\
\hline Cyst, keratinizing & & & & $\mathrm{x}$ \\
\hline Hyperplasia, bronchiolo-alveolar & $\mathrm{x}$ & & & \\
\hline Metaplasia, mucous cell & & & & $\mathrm{x}$ \\
\hline Metaplasia, osseous ${ }^{\wedge}$ & & $\mathrm{x}$ & & \\
\hline Metaplasia, squamous cell & & $\mathrm{x}$ & & \\
\hline \multicolumn{5}{|c|}{$\begin{array}{l}\text { * Terminology with diagnostic criteria and/or comments described below. }{ }^{\wedge} \text { Terminology addressed in the Systemic/General Pathology } \\
\text { Section. \# Finding more frequent as an induced change in inhalation studies. }{ }^{1} \text { Usually not recorded as a finding in non-human primates. } \\
{ }^{2} \text { Common background finding. Describe only if greater severity/extent than commonly seen as an incidental background finding. }{ }^{3} \text { Alveo- } \\
\text { lar emphysema can be seen secondary to lung mite infections (and is sometimes referred to as bullous emphysema). The residual enlarged } \\
\text { air spaces can be seen after both mites and inflammation have subsided. }{ }^{4} \text { Diagnosis based on histochemical stains, electron microscopy } \\
\text { or immunohistochemistry. }{ }^{5} \text { Use of "Macrophage aggregation, alveolar" is not recommended and replaced by "Macrophages increased, al- } \\
\text { veolar". }{ }^{6} \text { Pigments such as anthracosis and "malaria pigment" are common background findings and should be described only if extensive. } \\
{ }^{7} \text { May be seen in large or small vessels including capillaries. Thromboemboli may be common in chronic infusion studies. }\end{array}$} \\
\hline
\end{tabular}

to medium-sized vessels.

- Affected vessels are interlobular or, in moderate to severe cases, also in the subpleural parenchyma.

- Hemorrhage may be present around vessels in severe cases.

- Pigment laden macrophages are not present in alveoli, or only negligible numbers can be found in alveoli.
Comments: Yamakawa et al. ${ }^{457}$ reported a $9 \%$ incidence among cynomolgus macaques bred in Cambodia. The affected animals were relatively young and the authors concluded that the findings were congenital lesions. 


\section{Macrophages increased, alveolar: Lung: Alveoli}

(Figure 197)

\section{Other $\operatorname{term}(\mathbf{s})$}

Alveolar macrophage aggregation; alveolar macrophage accumulation; alveolar histiocytosis

\section{Pathogenesis/Cell of origin}

Bone marrow-derived monocyte precursors; resident interstitial and alveolar macrophages proliferate locally in adults.

\section{Diagnostic features}

- Increased numbers of alveolar macrophages over and above the expected number of randomly scattered individual macrophages within the alveolar spaces.

- Often aggregated in multiple foci.

Comments: The term increased alveolar macrophages is intended to replace alveolar macrophage aggregation in the rat INHAND document, particularly for inhalation studies ${ }^{458}$. It is preferable to use increased alveolar macrophages as a stand-alone diagnosis and not as an umbrella term that includes other inflammatory, degenerative, or proliferative responses. The term also pertains to lesser numbers of interstitial macrophages that might accompany increased alveolar macrophages. The affected compartment should be addressed in the report narrative. Increased interstitial macrophages should only be diagnosed separately if the pulmonary interstitium represents the predominant location. If the macrophages from treated animals have cytoplasmic changes (pigment, vacuolation, foreign material, etc.) that are distinct from features in controls (historical or current), these changes should be part of the diagnostic term. The term alveolar phospholipidosis is not an "Other Term" for increased alveolar macrophages and should be reserved for accumulations of phospholipid-containing macrophages observed after treatment with cationic amphiphilic drugs ${ }^{459,} 460$.

\section{Pleura (Table 40)}

Fibrous Tag: Lung - Pleura (Figure 198)

\section{Pathogenesis/Cell of origin}

Mesenchymal/connective tissue originating from the pleural surface of the lung and/or thoracic cage.

\section{Diagnostic features}

- Increased amount of mature collagen extending as a ribbon from the pleural surface

- Low numbers of spindle cells (fibroblasts or fibrocytes) admixed

- Lined by a single layer of normal, flattened mesothelial cells

- May create adhesions between lung lobes or thoracic pleural surface

\section{Differential Diagnoses}

- Pleural fibrosis:

- Increased collagen and/or spindle cells along the pleural margin of the lung parenchyma

- Mesothelial hyperplasia:

- Focal or diffuse increase in layers of pleural mesothelium from normal one- to two-cell thickness, accompanied by inflammatory cells and an increase in underlying connective tissue

- Mesothelial cells are cuboidal with prominent nucleoli, abundant cytoplasm compared to flattened normal mesothelial lining cells.

Table 40. Microscopic Findings of the Respiratory System: Pleura. Non-Human Primate

\begin{tabular}{|c|c|c|c|c|}
\hline Pleura & Common & Uncommon & $\begin{array}{c}\text { Not Observed, but } \\
\text { Potentially Relevant }\end{array}$ & Not Applicable \\
\hline \multicolumn{5}{|l|}{ Congenital } \\
\hline Cyst, congenital & & $\mathrm{x}$ & & \\
\hline \multicolumn{5}{|l|}{ Non-proliferative } \\
\hline Effusion, noninflammatory & & & $\mathrm{x}$ & \\
\hline Fibrosis $^{\wedge}$ & $\mathrm{x}$ & & & \\
\hline Fibrous tag * & $\mathrm{x}$ & & & \\
\hline Hemorrhage $^{\wedge}$ & & $\mathrm{x}$ & & \\
\hline Infiltrate, [insert appropriate cell type $]^{\wedge}$ & & $\mathrm{x}$ & & \\
\hline Inflammation $^{\wedge}$ & & $\mathrm{x}$ & & \\
\hline Mineralization $^{\wedge}$ & & $\mathrm{x}$ & & \\
\hline Pigment/foreign material^ & & $\mathrm{x}$ & & \\
\hline Pyothorax & & & $\mathrm{x}$ & \\
\hline \multicolumn{5}{|l|}{ Proliferative (non-neoplastic) } \\
\hline Hyperplasia, mesothelium & $\mathrm{x}$ & & & \\
\hline
\end{tabular}


- Mesothelioma:

- As for mesothelial hyperplasia PLUS

- Evidence of cellular atypia and/or invasion of adjacent lung parenchyma or thoracic wall
Comments: Fibrous tag formation is a relatively common incidental observation in wild-caught and free-range purpose bred cynomolgus macaques, likely related to previous parasitic or inflammatory conditions. It is suggested that pathologists do not record these incidental observations unless a gross observation is made at necropsy and/or when particularly prominent. 


\section{Skeletal System AND ToOth}

For detailed general considerations, refer to the INHAND rodent publication ${ }^{461}$. Non-proliferative abnormalities of bones and growth plates can be observed spontaneously in young cynomolgus macaques used in nonclinical toxicity studies, particularly fractures and developmental cartilage abnormalities ${ }^{191}$. Spontaneous lesions of the joints and teeth are rare in young cynomolgus monkeys used in routine toxicity studies. Models of naturally occurring or induced diseases, such as osteoporosis, osteoarthritis and periodontal disease, are briefly addressed herein, although the widespread use of this species as a nonclinical model is precluded by disadvantages such as ethical issues, cost and availability ${ }^{462}$.

\section{A. Bone}

The skeletal maturation and bone turnover found in monkeys is generally considered to be most similar to humans, therefore studies in the non-human primate often play a prominent role in skeletal assessment ${ }^{463}$. In monkeys used in routine toxicity studies, which are usually $2-5$ years of age, the growth plates are generally open. The average time of physeal closure in cynomolgus monkeys in the proximal femur is 6 years in males, and 4 years and 9 months in females ${ }^{464,465}$. As in humans, bone remodeling in non-human primate occurs with a formation of osteons (Haversian systems) in the cortical bones, whereas cortical bone osteons do not usually form in rodents 466 .
The standard bone samples examined in non-human primates during a toxicity study are distal femur and proximal tibia, including the femoro-tibial joint. The proximal tibia may provide a more uniform physis for evaluation than the distal femur. Furthermore, the sternum is examined with the main purpose of evaluating bone marrow. Sometimes vertebrae are evaluated, particularly for compounds targeting the skeletal system. Rib samples may provide access to a more active site of endochondral growth ${ }^{467}$. The preparation of bones for optimal H\&E sections is a time-consuming procedure in a nonrodent species. Although several attempts have been made to optimize the decalcification procedure, the traditional method using a formic acid-based solution often gives the best results. As in rodents, special techniques can be performed in addition to routine histology and H\&E staining, such as bone densitometry, biomechanical testing, bone formation and resorption biomarkers, and histomorphometry (see review in: Vahle et al. ${ }^{463}$ ).

The terms below indicating the growth plate as it relates to skeletal maturity (growth plate open, closed and partially closed) may be recorded as a finding under the femur (or any other bone evaluated) or may be recorded under a "summary tissue" such as "Skeletal Maturity".

The only published report of spontaneous bone tumor in cynomolgus monkeys described a chondrosarcoma of the femur in a 4-year old individual ${ }^{2,468}$. One case of induced osteosarcoma was described in cynomolgus monkeys following chronic administration of aflatoxin $\mathrm{B}_{1}{ }^{469}$ (Table 41).

Table 41. Microscopic Findings of the Skeletal Tissues: Bone; Non-human primate

\begin{tabular}{|c|c|c|c|c|}
\hline Bone & Common & Uncommon & $\begin{array}{c}\text { Not Observed but } \\
\text { Potentially Relevant }\end{array}$ & Not Applicable \\
\hline \multicolumn{5}{|l|}{ Non-proliferative } \\
\hline Bone decreased, trabeculae and/or cortex $* \#$ & & $\mathrm{x}$ & & \\
\hline Bone increased, trabeculae and/or cortex *\# & & $\mathrm{x}$ & & \\
\hline Cyst, bone & & $\mathrm{x}$ & & \\
\hline Dysplasia, physeal * & & $\mathrm{x}$ & & \\
\hline Dysplasia, fibrous * & & $\mathrm{x}$ & & \\
\hline Eroded surface, increased & & $\mathrm{x}$ & & \\
\hline Fibrous osteodystrophy (FOD) & & $\mathrm{x}$ & & \\
\hline Fracture $^{1}$ & & & $\mathrm{x}$ & \\
\hline Fracture/Callus * & & $\mathrm{x}$ & & \\
\hline Growth plate closed & $\mathrm{x}$ & & & \\
\hline Growth plate partially closed $*$ & $\mathrm{x}$ & & & \\
\hline Growth plate open* & $\mathrm{x}$ & & & \\
\hline Lesion, fibro-osseus (FOL) & & & $\mathrm{x}$ & \\
\hline Necrosis & & $\mathrm{x}$ & & \\
\hline Osteoblastic surface, increased & & $\mathrm{x}$ & & \\
\hline Osteoclasts, increased & & $\mathrm{x}$ & & \\
\hline Osteoid, increased & & $\mathrm{x}$ & & \\
\hline Physis, decreased thickness & & $\mathrm{x}$ & & \\
\hline Physis, increased thickness, ${ }^{*}$ & & $\mathrm{x}$ & & \\
\hline \multicolumn{5}{|l|}{ Proliferative (non-neoplastic) } \\
\hline Hyperplasia, chondrocyte & & $\mathrm{x}$ & & \\
\hline Hyperplasia, osteoblast, focal & & $\mathrm{x}$ & & \\
\hline
\end{tabular}




\section{Bone decreased, trabeculae and/or cortex: Bone}

(No image provided)

Comments: In standard toxicity studies in monkeys, a reduction in trabecular or cortical bone is not a common observation, either as a spontaneous or test-article-related effect. It should be noted that the non-human primate is a commonly used model to evaluate the efficacy and safety of bone therapeutics ${ }^{470,471}$. A common model involves surgical ovariectomy in skeletally mature cynomolgus monkeys to induce a high bone turnover state and subsequent bone loss. In these models, quantitative measures such as densitometry and histomorphometry are used to assess the changes in bone mass. In those instances where routine histologic examination detects a decrease in trabecular and/or cortical bone, the descriptive term of decreased bone should be used.

\section{Bone increased, trabeculae and/or cortex: Bone}

(No image provided)

Comments: Diffuse increases in the relative amount of trabecular or cortical bone in toxicity studies are most often a result of a test-article-induced effect. For example, bone anabolic agents such as monoclonal antibodies that neutralize sclerostin and recombinant human parathyroid hormone cause increased bone formation 472,473 . Although quantitative analyses such as histomorphometry and bone densitometry are often used to characterize these changes, the increases in bone may be observed in routine histology sections in toxicity studies.

Increased cortical bone can sometimes be observed with hypertrophic osteopathy (HO), also called hypertrophic osteoarthropathy or hypertrophic pulmonary osteoarthropathy. This is an obscure pathogenesis syndrome reported in humans and various animal species, including non-human primates, which develops following a variety of severe, mostly intra-thoracic, neoplastic or non-neoplastic conditions. It is likely that the release of growth factors such as VEGF (vascular endothelial growth factor) and PDGF (platelet derived growth factor) is involved in the pathogenesis 474,475 . Bilateral symmetrical thickening of the long bones of distal extremities can be observed macroscopically along with irregular surface on the affected bones. Histologically, there is periosteal new bone formation involving the diaphysis of the long bones. HO should be differentiated from periosteal new bone formation associated with secondary hyperparathyroidism. There are only few reports of spontaneous HO in monkeys, and two cases of HO in cynomolgus monkeys were observed following severe opportunistic infections during a nonclinical study with an immunomodulator ${ }^{476}$.

Dysplasia, fibrous: Bone (No image provided)

\section{Pathogenesis/Cell of Origin}

Developmental disorder of bone in which all the compo- nents of normal bone are present but they do not differentiate into their mature structures (i.e. no rimming of trabeculae by osteoblasts and no evidence of lamellar replacement of woven bone).

\section{Diagnostic features}

- Well circumscribed intramedullary lesions that can expand and distort the bone.

- Fibrous dysplasia can involve a single bone (monostotic) or several bones (polyostotic).

- Characterized by disorganized trabeculae of woven bone that lack conspicuous osteoblastic rimming, surrounded by moderately cellular fibroblastic proliferation.

- Nodules of hyaline cartilage may be present.

- Cystic degeneration, hemorrhage and foamy macrophages can occur.

\section{Differential Diagnoses}

- Fibrous osteodystrophy:

- generalized condition; osteoblastic rimming present.

- Ossifying fibroma:

- located in the jaws; presence of osteoblastic rimming around trabeculae.

Comments: Polyostotic fibrous dysplasia has been reported in one 2.3 year old cynomolgus macaque with pathologic fractures 477 .

Dysplasia, physeal: Bone (No image provided)

Comments: Some cases of spontaneous metaphyseal dysplasia resembling rickets, often associated with growth plate (micro) fractures, have been described by Chamanza in a review of spontaneous findings in the cynomolgus monkey ${ }^{6}$. In this publication, the author also describes cases of cartilage abnormalities such as physeal osteochondrosis or dyschondroplasia.

Fracture/Callus: Bone (Figure 199-200)

Comments: Spontaneous fractures are not uncommon in cynomolgus monkeys kept under laboratory conditions, especially when they are group-housed (i.e. traumatic fractures). In a review of spontaneous pathology findings in cynomolgus monkeys, Chamanza reported digital fractures with an incidence of $7 \%$ in males, and $1.2 \%$ in females ${ }^{6}$. In the same publication, growth plate microfractures could be associated with metaphyseal dysplasia (see increased thickness, physis).

Increased susceptibility to fracture is observed as a consequence of decreased bone mass during aging and other models of osteoporosis 466 . 


\section{Growth plate open: Bone (Figure 201)}

Comments: The long bone epiphyseal growth plate is considered as "open" when the epiphyseal cartilage, regardless of thickness, spans the full width of the bone. The growth plate is thickest when bone growth is most rapid and narrows as the growth plate cartilage is replaced by bone (closed). Layers of cartilage are successively replaced by new layers of bone in an organized fashion, starting in the transitional metaphyseal region and extending away from the diaphysis to the epiphysis.

\section{Growth plate partially closed: Bone (Figure 202)}

Comments: As the epiphyseal growth plate cartilage is gradually replaced by bone, the growth plate narrows and partial segmental growth plate closure may be present. As in humans, the epiphyses of non-human primates close toward the end of adolescence $464,465,478$. Skeletal maturity will vary depending on the site, gender and species. In cynomolgus monkeys, subtle differences in the timing of appearance and fusion of secondary ossification centers have been reported. Zoetis et al. ${ }^{465}$ published an interspecies comparison of postnatal bone growth and development ${ }^{465}$. In their review, they reported the age of complete fusion of the femoral epiphyses in both species. In male cynomolgus monkeys complete fusion is reported at 6 and 5.25 years of age for the proximal and distal epiphyses, respectively, and in females both epiphyses are fused at 4.75 years of age.

\section{Physis, increased thickness: Bone (No image provided)}

Comments: Induced physeal thickening and dysplasia have been associated with several different drug classes ${ }^{479}$. Increased thickness and dysplasia of the physis have been observed in cynomolgus monkeys following treatment with angiogenesis inhibitors, such as an anti-angiogenic humanized monoclonal antibody, rhuMAbVEGF480, or a multi-targeted receptor tyrosine kinase (including VEGFR) inhibi- tor, Sunitinib ${ }^{481}$.

Rickets can be observed spontaneously in young monkeys. It is a disorder that affects growing bone and cartilage undergoing endochondral ossification, and is characterized by failure of bone mineralization with subsequent bone deformities and fractures. Dietary deficiencies and/or metabolic defects in vitamin $\mathrm{D}$, calcium or phosphate metabolism are predisposing conditions for rickets 466 . It has almost disappeared in modern facilities because of vitamin $\mathrm{D}$ supplementation in commercial diets ${ }^{191}$. Enlargement and deformity of the epiphyseal growth plate cartilage can be observed macroscopically. The bone is soft. At microscopic examination, the growth plate is elongated with a persistence of distorted, irregular masses of cartilage i.e. physeal dysplasia, which may project into the marrow. There is excess accumulation of non-mineralized matrix (i.e. increased osteoid). Transverse "pseudofractures" in the soft cartilage can be observed.

\section{B. Joint}

Although there are some differences in the gross morphology of joints in non-human primates, their microscopic, ultrastructural and biochemical features are very close to those in humans (for thorough literature review, see Pritzker and Kessler ${ }^{466}$. Differences in the joint biomechanics related to human adaptations for bipedal locomotion are smaller in monkeys compared to strict quadrupeds ${ }^{462}$.

General morphology of the joint can be assessed on routine decalcified H\&E sections. Additional sections of the joint may be stained with toluidine blue or safranin $\mathrm{O}$ to permit the grading of cartilage matrix integrity 467 .

Spontaneous lesions of the joints are rare in cynomolgus monkeys used in toxicity studies. To our knowledge, there are no published reports on tumors of the joint in cynomolgus macaques (Table 42).

Table 42. Microscopic Findings of the Skeletal Tissues: Joint; Non-human primate

\begin{tabular}{|c|c|c|c|c|}
\hline Joint & Common & Uncommon & $\begin{array}{c}\text { Not Observed but Po- } \\
\text { tentially Relevant }\end{array}$ & Not Applicable \\
\hline \multicolumn{5}{|l|}{ Non-proliferative } \\
\hline Degeneration, articular cartilage $* \#$ & & $\mathrm{x}$ & & \\
\hline Degeneration, chondromucinous & & & $\mathrm{x}$ & \\
\hline Degenerative joint disease * & & $\mathrm{x}$ & & \\
\hline 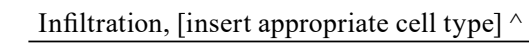 & & $\mathrm{x}$ & & \\
\hline Inflammation * & & $\mathrm{x}$ & & \\
\hline Osteophyte & & $\mathrm{x}$ & & \\
\hline \multicolumn{5}{|l|}{ Proliferative (non-neoplastic) } \\
\hline Hyperplasia, synovial cell & & $\mathrm{x}$ & & \\
\hline
\end{tabular}


Degeneration, articular cartilage: Joint (Figure 203)

\section{Other term(s)}

Arthropathy

\section{Pathogenesis/Cell of Origin}

Degeneration of the articular cartilage associated with various xenobiotics.

\section{Diagnostic features}

- Chondrocyte degeneration

- Focal loss of matrix

- Cavitations, fissures and/or erosion of the articular cartilage

- Generally, the inflammatory component (inflammation of synovial membranes) is not significant

\section{Differential Diagnoses}

- Spontaneous degenerative joint disease (older animals)

Comments: Drug-induced arthropathies have been observed with quinolones in juvenile animals of many laboratory species, including non-human primates ${ }^{482}$. The specific mechanism for the initiation of this arthropathy is not fully determined. The most likely primary mechanism is the chelation of magnesium ions by quinolones, resulting in impaired function of chondrocyte surface $\beta 1$ integrins and disturbed interaction between the matrix and cells ${ }^{483}$.

\section{Degenerative joint disease: Joint (No image provided)}

\section{Other term(s)}

Osteoarthritis

Comments: Degenerative joint disease (more commonly called osteoarthritis in the literature on non-human primates) is a naturally occurring entity in adult cynomolgus macaques that increases in prevalence and severity with increasing age. The knee joint is typically involved, and the lesion is characterized by articular cartilage microfissures, clefting and loss, with thickening of the subchondral bone ${ }^{484}$. Spontaneous cases are uncommon in cynomolgus macaques in routine toxicity studies due to the young age of the individuals.

Unilateral advanced degenerative joint disease, with full thickness loss of the articular cartilage on the femoral head and acetabulum, and replacement by a fibrous connective tissue that extended into the superficial subchondral bone marrow (pannus), was described in two presumptive cases of idiopathic chondrolysis in 3 year old, wild-caught, cynomolgus females of Vietnamese origin, reared in captivity ${ }^{485}$. Idiopathic chondrolysis is a human clinical entity typically reported in adolescents.

\section{Inflammation: Joint (No image provided)}

Comments: The monkey collagen-induced arthritis (CIA) model is used in the development of new drugs for the treatment of rheumatoid arthritis (RA) because non-human primates have the closest immunological and skeletal similarities to humans. Cynomolgus macaques may be used for this model ${ }^{486}$. This experimentally-induced auto-immune disease consists of immunizing monkeys with bovine type II collagen co-administered with complete Freund adjuvant. At histology, there is marked infiltration of $\mathrm{T}$ cells and macrophages in the synovium. Neutrophils are also present during the progression of the disease. Hyperplasia of the synovium and pannus formation overlying the cartilage precedes progressive destruction of the joints during the late phase of CIA, with loss of cartilage and subchondral bone, and bone remodeling $486-488$.

Takei described a case of spontaneous polyarthritis in a 5 year old female cynomolgus macaque from China ${ }^{489}$. This animal presented fever, swelling of the fingers, knees and tail, deformation of the pelvis, and loss of mobility. Although microscopy of the joints was not available, the presence of inflammatory cells in the synovial fluid obtained by arthrocentesis, and high counts of neutrophils, monocytes and large unstained cells along with increased serum CRP levels were consistent with polyarthritis of unknown pathogenesis.

\section{Tooth}

Non-human primates have teeth similar to those of humans. In Macaca fascicularis, the age of eruption of the decidual teeth occurs between two weeks and 6 months old, and between 1.38 and 5.75 years old for permanent teeth ${ }^{490}$.

Non-human primates have naturally occurring dental plaques, calculus, and periodontal disease ${ }^{491}$, and they are often used as experimental models for plaque formation, periodontal disease and caries ${ }^{116,492}$. However, reports and descriptions of teeth lesions are scarce in cynomolgus monkeys used in toxicity studies, because this tissue is not examined routinely (except during evaluation of oral implants), and probably also because of the generally young age of the animals.

Among neoplastic lesions, ameloblastic odontoma is the only odontogenic tumor described in the literature in cynomolgus monkey, in a single adult individual ${ }^{493}$, and an ossifying fibroma of the maxilla has been described in one 7 year old cynomolgus macaque ${ }^{468}$ (Table 43).

Inflammation, periodontium: Tooth (Figure 204)

\section{Other term(s)}

Periodontal disease; periodontitis

\section{Pathogenesis/Cell of Origin}

Periodontitis is a chronic, multifactorial immuno-inflammatory process that affects the supporting structures of the teeth 
Table 43. Microscopic Findings of the Skeletal Tissues: Tooth; Non-human primate

\begin{tabular}{|c|c|c|c|c|}
\hline Teeth & Common & Uncommon & $\begin{array}{l}\text { Not Observed but Po- } \\
\text { tentially Relevant }\end{array}$ & Not Applicable \\
\hline \multicolumn{5}{|l|}{ Congenital } \\
\hline Denticle & & & $\mathrm{x}$ & \\
\hline \multicolumn{5}{|l|}{ Non-proliferative } \\
\hline Cyst & & & $\mathrm{x}$ & \\
\hline Degeneration & & $\mathrm{x}$ & & \\
\hline Dentin, decreased & & & $\mathrm{x}$ & \\
\hline Dentin matrix alteration & & & $\mathrm{x}$ & \\
\hline Dentin niches & & & $\mathrm{x}$ & \\
\hline Dysplasia, dental & & $\mathrm{x}$ & & \\
\hline Fracture & & $\mathrm{x}$ & & \\
\hline Inflammation, periodontium * & & $\mathrm{x}$ & & \\
\hline Inflammation, pulp * & & $\mathrm{x}$ & & \\
\hline Necrosis & & $\mathrm{x}$ & & \\
\hline Periodontal pocket & & $\mathrm{x}$ & & \\
\hline Pulp concretion & & & $\mathrm{x}$ & \\
\hline Resorption * & & $\mathrm{x}$ & & \\
\hline Thrombus & & & $\mathrm{x}$ & \\
\hline
\end{tabular}

(periodontal ligaments) and alveolar bone. It is considered to be primarily due to infection by oral opportunistic bacteria, but individual variability in the host responses to bacterial infection contributes significantly to expression of the disease.

\section{Diagnostic features}

- Inflammatory cells, including neutrophils, within the gingival, periodontal spaces and/or adjacent connective tissue (i.e. periodontal pockets)

- Resorption of the periodontal ligament and bone

- Loosening and eventual loss of the teeth following destruction of the periodontal ligament

Comments: Non-human primates, including cynomolgus monkeys, are susceptible to naturally occurring periodontal disease and can be used as models of the disease in humans. To induce experimental periodontitis, plaque-accumulating devices such as orthodontic elastic ligatures or sutures are commonly placed ${ }^{491}$. Inoculation of monkeys with human pathogens such as Porphyromonas gingivalis also leads to plaque formation and bone loss 491,494 .

Inflammation, pulp: Tooth (No image provided)

Comments: Periodontal disease, dental fractures, implantation of biomedical devices, or endodontic procedures such as the cutting of canine teeth ${ }^{495}$, can lead to exposure of the pulp cavity or tooth root. The subsequent colonization by bacteria can result in chronic inflammation of the pulp and surrounding tissues, and development of abscesses (often characterized by facial swelling) and/or a draining tract ${ }^{117}$.

Resorption: Tooth (No image provided)

\section{Other term(s)}

Caries

Comments: Dental caries have been described in many species of non-human primate including the cynomolgus monkey ${ }^{496}$. Caries is a macroscopic term corresponding to a chronic infectious disease resulting from the resorption of dental hard tissues by oral bacteria ${ }^{497}$. Microorganisms subsequently trigger inflammatory and degenerative responses in the dental pulp. The development of caries is a multifactorial process ${ }^{117}, 498,499$ involving sugar-rich diets fed in captive environment, and microbial shifts towards highly acidogenic bacteria (e.g. some serotypes of Streptococcus mutans) among others. 


\section{Soft Tissues}

For detailed general considerations on soft tissues, skeletal muscle, and mesothelium refer to the INHAND rodent publication $^{5}$. This chapter includes lesions of soft tissues, skeletal and smooth muscle, adipose tissue, and mesothelium. Lesions of the synovium are described in the chapter on the skeletal system.

\section{A. Soft Tissue}

Soft tissue lesions observed in the non-human primate are generally identical to those observed in rodents and other domestic animals as well as in humans ${ }^{5}$. One additional non-human primate specific condition, retroperitoneal fibromatosis, is discussed (Table 44).

Retroperitoneal fibromatosis: Soft Tissue (Figure 205)

\section{Pathogenesis/Cell of Origin}

Retroperitoneal fibromatosis (RF) in macaques is caused by retroperitoneal fibromatosis herpesvirus (RPHV) and/or rhesus rhadinovirus (RRV) in the presence of retrovirus-induced immunodeficiency. RF has been reported in macaques that have concurrent natural infection with simian D-type retrovirus-2 (SRV-2) or experimental infection with SIV or SIV/HIV hybrid viruses ${ }^{500,501}$.

\section{Diagnostic features}

- Multifocal fibroproliferative syndrome that occurs subjacent to the peritoneum covering the ileocecal junction and associated mesenteric lymph nodes. There may also initially just be a diffuse opaque thickening of the mesentery.

- Macroscopically, two patterns occur with RF:

- Localized, in which fibroproliferative lesions occur only in solitary nodules.
- Progressive, in which fibromatosis occurs throughout the abdominal cavity as either discrete nodules of fibromatous tissue or as one large continuous mass that can circumscribe loops of intestines, interfering with peristalsis and gastrointestinal function (but which does not invade into or obstruct the intestinal lumen). The fibromatous tissue may even extend into the inguinal canal or invade through the diaphragm into the pleural cavity.

- Secondary subcutaneous fibromatous lesions also sometimes occur (referred to as subcutaneous fibromatosis).

- Histologically, two basic patterns occur with RF:

- Proliferative: This pattern has aggressive proliferation of highly vascular fibrous tissue with large, plump, spindleshaped fibroblasts in a disorganized stromal network of collagen and reticulum fibers. Mononuclear cell infiltrates and edema are also normally present in varying degrees.

- Sclerotic: This pattern has fewer fibroblasts and edema/ mononuclear cell infiltrates and can appear as a mass of mostly acellular collagen.

\section{Differential Diagnoses}

- Fibrosarcoma (differential for the proliferative histologic pattern of RF). The fibroblasts in RF tend to lack the densely cellular, anaplastic appearance of most fibrosarcomas. Also, with RF there is no metastasis by lymphatic or vascular routes or invasion of parenchymatous organs. - Fibroma (differential for the sclerotic histologic pattern).

Comments: This multifocal fibroproliferative syndrome in macaques was first recognized at the University of Washington National Primate Research Center in 1976 at which time it was linked to animals with concurrent SRV-2 associated immunosuppression ${ }^{502}$. Epidemic outbreaks occurred at that and other primate centers in the $1980 \mathrm{~s}^{503}$. Animals often presented clinically with chronic weight loss and diarrhea and had evidence of generalized lymphoid depletion. It

Table 44. Microscopic Findings of Soft Tissue: General terminology; Non-Human Primate

\begin{tabular}{|c|c|c|c|c|}
\hline Soft Tissue & Common & Uncommon & $\begin{array}{l}\text { Not Observed but } \\
\text { Potentially Relevant }\end{array}$ & Not Applicable \\
\hline \multicolumn{5}{|l|}{ Non-proliferative } \\
\hline Amyloid^^ & & $\mathrm{x}$ & & \\
\hline Fibroplasia $^{\wedge}$ & & $\mathrm{x}$ & & \\
\hline Fibrosis $^{\wedge}$ & & $\mathrm{x}$ & & \\
\hline Infiltrate, [insert appropriate cell type] ${ }^{\wedge}$ & $\mathrm{x}$ & & & \\
\hline Inflammation $^{\wedge}$ & & $\mathrm{x}$ & & \\
\hline Metaplasia & & $\mathrm{x}$ & & \\
\hline Mineralization $\wedge$ & & $\mathrm{x}$ & & \\
\hline Necrosis $\wedge$ & & $\mathrm{x}$ & & \\
\hline \multicolumn{5}{|l|}{ Proliferative (non-neoplastic) } \\
\hline Retroperitoneal fibromatosis * & & $\mathrm{x}$ & & \\
\hline
\end{tabular}


has since been observed in animals with immunosuppression caused by experimental infection with SIV retroviruses $^{500}$.

Localized RF syndrome can develop into the progressive RF syndrome, but even if it does not, animals with localized RF typically die of other infections associated with immunosuppression. The progressive syndrome of RF is considered uniformly fatal 504 .

RF is often discussed in the context of Kaposi's sarcoma (KS), another mesenchymal proliferative disorder that occurs in virally induced immunodeficient humans and caused by Kaposi's sarcoma herpesvirus, which is closely related to the RF herpesviruses ${ }^{501}$. Histologic findings of proliferative RF (highly vascularized, disorganized, proliferating fibrous tissue that is edematous with mononuclear cell infiltrates) resemble histologic features observed in $\mathrm{KS}^{505}$. Differences between the two diseases include that KS is characteristically a multicentric dermal disease compared to the typi- cally ileocecal regionality of RF (although secondary subcutaneous fibromatosis does sometimes occur with RF), and hemorrhage is a more prominent histologic feature with KS compared to RF504.

\section{B. Adipose Tissue and Smooth Muscle}

As noted in the rodent manuscript, careful recording of the nature, intensity, and duration of inflammatory responses to implants or injected substances in soft tissues is important for the assessment of their local tolerability 5 (Table 45, 46).

\section{Skeletal Muscle}

The special cellular structure of skeletal muscle, the different fiber types and their varying sensitivity in response to xenobiotics require a separate consideration of the pathological changes and the extent of sampling and careful selection of

Table 45. Microscopic Findings of Adipose Tissue; Non-Human Primate

\begin{tabular}{|c|c|c|c|c|}
\hline Adipose Tissue & Common & Uncommon & $\begin{array}{c}\text { Not Observed but } \\
\text { Potentially Relevant }\end{array}$ & Not Applicable \\
\hline \multicolumn{5}{|l|}{ Non-proliferative } \\
\hline Atrophy, adipocyte & & $\mathrm{x}$ & & \\
\hline Edema $^{\wedge}$ & & $\mathrm{x}$ & & \\
\hline Hemorrhage $^{\wedge}$ & & $\mathrm{x}$ & & \\
\hline Hypertrophy, adipocyte & & $\mathrm{x}$ & & \\
\hline Infiltrate, [insert appropriate cell type $]^{\wedge}$ & $\mathrm{x}$ & & & \\
\hline Inflammation $^{\wedge}$ & & $\mathrm{x}$ & & \\
\hline Inflammation, lipogranulomatous ${ }^{\wedge}$ & & $\mathrm{x}$ & & \\
\hline Necrosis ^ & & $\mathrm{x}$ & & \\
\hline Serous atrophy $^{\wedge}$ & $\mathrm{x}$ & & & \\
\hline \multicolumn{5}{|l|}{ Proliferative (non-neoplastic) } \\
\hline Hyperplasia, adipocyte & & $\mathrm{x}$ & & \\
\hline \multicolumn{5}{|l|}{ Neoplastic } \\
\hline Lipoma & & $\mathrm{x}$ & & \\
\hline Liposarcoma & & $\mathrm{x}$ & & \\
\hline
\end{tabular}

Table 46. Microscopic Findings of Smooth Muscle; Non-Human Primate

\begin{tabular}{|c|c|c|c|c|}
\hline Smooth Muscle & Common & Uncommon & $\begin{array}{l}\text { Not Observed but } \\
\text { Potentially Relevant }\end{array}$ & Not Applicable \\
\hline \multicolumn{5}{|l|}{ Non-proliferative } \\
\hline Edema $^{\wedge}$ & & $\mathrm{x}$ & & \\
\hline Hemorrhage $^{\wedge}$ & & $\mathrm{x}$ & & \\
\hline Necrosis $^{\wedge}$ & & $\mathrm{x}$ & & \\
\hline Regeneration & & $\mathrm{x}$ & & \\
\hline \multicolumn{5}{|l|}{ Proliferative (non-neoplastic) } \\
\hline Hyperplasia, smooth muscle & & $\mathrm{x}$ & & \\
\hline \multicolumn{5}{|l|}{ Neoplastic } \\
\hline Leiomyoma & & $\mathrm{x}$ & & \\
\hline Leiomyosarcoma & & $\mathrm{x}$ & & \\
\hline
\end{tabular}


sampling sites is important ${ }^{5}$. The ideal skeletal muscle choice has either entirely one type or a consistent mixture of fast and slow myofibers. Consistent orientation of myofibers is also important in order to produce true cross- and longitudinal sections. For routine toxicity studies, thigh muscles (biceps femoris and quadriceps) and hindlimb muscles (gastrocnemius, long digital extensor, and anterior tibialis) are most often used in NHP. Most of these routinely collected muscles have more fast than slow twitch fibers, especially superficially. In studies where evaluation of a particular muscle fiber type is desired, the soleus is recommended for type I fibers and the anterior tibialis muscle is recommended for type II fibers ${ }^{506}$ (Table 47).

\section{Mesothelium (Table 48)}

Table 47. Microscopic Findings of Skeletal Muscle; Non-Human Primate

\begin{tabular}{|c|c|c|c|c|}
\hline Skeletal Muscle & Common & Uncommon & $\begin{array}{c}\text { Not Observed but } \\
\text { Potentially Relevant }\end{array}$ & Not Applicable \\
\hline \multicolumn{5}{|l|}{ Non-proliferative } \\
\hline Atrophy & & $\mathrm{x}$ & & \\
\hline Degeneration & $\mathrm{x}$ & & & \\
\hline Edema $^{\wedge}$ & & $\mathrm{x}$ & & \\
\hline Hypertrophy & & $\mathrm{x}$ & & \\
\hline Infiltrate, [insert appropriate cell type] ${ }^{\wedge}$ & $\mathrm{x}$ & & & \\
\hline Inflammation $^{\wedge}$ & $\mathrm{x}$ & & & \\
\hline Mineralization $^{\wedge}$ & & $\mathrm{x}$ & & \\
\hline Necrosis & $\mathrm{x}$ & & & \\
\hline Regeneration & $\mathrm{x}$ & & & \\
\hline Vacuolation & & $\mathrm{x}$ & & \\
\hline
\end{tabular}

Table 48. Microscopic Findings of Mesothelium: Non-Human Primate

Mesothelium
$\begin{aligned} & \text { Proliferative (non-neoplastic) } \\ & \text { Hyperplasia }{ }^{1}\end{aligned}$
$\begin{aligned} & \text { Neoplastic } \\ & \text { Mesothelioma, malignant }\end{aligned}$
$\begin{aligned} & { }^{1} \text { Mesothelial hyperplasia is uncommon with the exception of epi/pericardium, where it can be commonly seen. } \\ & \text { Please refer to the Cardiovascular chapter for more information. }\end{aligned}$




\section{Special Senses (Eye And Ocular Adnexa)}

For detailed general considerations on the eyes and ocular adnexa, please refer to the rodent INHAND publication on Special Senses ${ }^{507}$. The suggested standardized nomenclature for the ocular system is subdivided into the eye and the glands of the eye. The ear is not examined routinely in toxicologic pathology studies and is not included in this manuscript. Recommended nomenclature for microscopic lesions in the nose is addressed in the respiratory chapter.

\section{A. Eye}

The macaque cornea is relatively small compared to the relatively larger corneas of many non-primate mammals ${ }^{508}$. A corneal Bowman's layer is present but rather poorly defined ${ }^{509-511}$. In macaques, the posterior corneal endothelium is poorly regenerative ${ }^{512,513}$. The corneal limbal epithelium (at the corneoscleral junction) is normally lightly pigmented (melanin) and has more cell layers than the central corneal epithelium ${ }^{514}$.

The macaque lens is small and flattened compared to the relatively larger, more spherical lens of rats and mice 515 . The lens provides about one-third of the total ocular refractive power while the cornea provides most of the remaining two-thirds (Ott, 2006). In primates such as macaques and humans, lens suture configuration in the adult has a multibranched "star" suture pattern ${ }^{516}$.

Many features of the macaque uvea (iris, ciliary body, and choroid) and aqueous filtration system are related to high accommodative ability ${ }^{517}$. The ciliary body consists of the anterior pars plicata (ciliary processes and ciliary muscle) and the posterior pars plana. In macaques, the ciliary body muscle (smooth muscle) is large and robust with melanin-laden cells normally scattered among the muscle fibers ${ }^{517}, 518$. The pars plana is flat and smooth and extends posteriorly to the junction with the retina ${ }^{519}$. Macaque and human eyes have a comparatively wide pars plana; the temporal pars plana is wider than the nasal and thus is a common site for intravitreal injection. The ora serrata (so called because of its wavy, indented outline) is the junction of the pars plana and the peripheral retina. Compared to many nonprimate mammals, the iridocorneal angle of macaques is relatively small and shallow and lacks well-developed pectinate ligaments ${ }^{517}, 518$. The anterior chamber is also relatively small ${ }^{515}$. The aqueous filtration system of macaques and humans has a canal of Schlemm, a single circumferentially oriented venous-like channel in the base of the ciliary body ${ }^{517}$.

The principal retinal phototransductive neurons are the two ciliary photoreceptors (rods and cones) ${ }^{520}$. The retina of macaques also contains intrinsically photosensitive retinal ganglion cells (ipRGC), a recently discovered third type of retinal photoreceptor ${ }^{521}$. As in humans and most mammalian species, rods greatly outnumber cones in the macaque retina ("rod-dominant") $520,522,523$. In most regions of the retina, the less numerous cones are spaced in orderly patterns ("mosaics") among the more abundant rods ${ }^{524}, 525$. The retinal fovea centralis (region of highest visual acuity, Figure 206) is a round depression created by centrifugal displacement of all blood vessels, rod and cone axons, and all cell bodies except those of the specialized foveal cones, and a few foveal Müller cells ${ }^{525-529}$. In the fovea, cones become centripetally more abundant than rods, such that the central fovea (foveola) is cone-dominant ${ }^{530,531}$. In foveate retinas, the widened perifoveal outer plexiform layer (OPL) is known as the Henle region and is composed of the obliquely oriented foveolar cone axonal processes (Henle fibers) which are greatly elongated to reach their synapses with bipolar and horiztonal cells displaced by the formation of the foveal pit $526,527,532,533$. The fovea centralis is surrounded by the macula lutea, a round to oval zone with a yellowish in vivo appearance due to high concentrations of yellow carotenoid pigments ${ }^{534-536}$. Histologically, the macula is distinguished by having more than one cell layer in the retinal ganglion cell layer (GCL) as well as increased bipolar cell density in the inner nuclear layer (IPL). Retinal ganglion cell axons comprise the retinal nerve fiber layer (NFL) and are normally unmyelinated in the macaque retina ${ }^{537}$. The numerous axons from the macular ganglion cells form a papillomacular bundle which extends into the temporal side of the optic nerve head ${ }^{538}$. Most retinal astrocytes are located in the retinal NFL 539.

The retinal pigment epithelium (RPE) is a monolayer of cuboidal, melanin-laden cells lining the subretinal space opposite the neurosensory retina. Bruch's membrane is a multilayered extracellular structure between the RPE and choroidal capillary bed (choriocapillaris). It is composed of the RPE and choriocapillaris capillary basement membranes and associated collagen and elastin layers ${ }^{540-542}$.

Except for the avascular foveal region, retinal blood vessels are distributed over the entire fundus ${ }^{528,543}$. The central retinal artery and vein are located within the optic nerve and enter the eye through the scleral lamina cribrosa at the optic nerve head ${ }^{536,544}$. The central retinal artery usually subdivides into four main branches, each of which supplies the one of the four retinal quadrants $535,543,544$. These vessels further branch to eventually create two interconnecting capillary beds in the inner retina ${ }^{544}$. In the normal macaque retina, capillaries extend only to the level of the inner nuclear layer (INL) and inner plexiform layer (IPL) ${ }^{543}$. Thus, the inner two-thirds of the retina is supplied by the central retinal artery and its branches while the outer, avascular one-third of the retina (rods and cones) is supplied by choroidal vessels ${ }^{536,541}$.

The optic nerve is composed of the axons of retinal ganglion cells which converge and exit the globe at the optic nerve head $(\mathrm{ONH})^{545}$. The optic nerve also contains glial cells (astrocytes, oligodendrocytes), and is surrounded by meninges (dura, arachnoid, and pia) continuous with those of the brain ${ }^{545,546 .}$ Supportive fibrous septa are intercalated between optic nerve fibers ${ }^{546}$. The scleral lamina cribrosa is very well-developed in macaques and humans ${ }^{547,548}$. Alterations in the lamina cribrosa and adjacent regions of the optic nerve/optic nerve head are important in the pathophysiology of various glaucomas ${ }^{548,549}$. In contrast to the white sclera of humans, the sclera of macaques and almost all other nonhuman primates is normally pale to dark brown in most adult individuals ${ }^{550-552}$. 
The macaque vitreous cavity is relatively large, with vitreous-to-globe area ratio of 0.7 in cynomolgus macaques, compared to 0.4 for the rabbit 515 . The vitreous is a transparent extracellular matrix gel composed of about $98-99 \%$ water with remainder being primarily collagen and hyaluronan ${ }^{553}$. In the normal vitreous, the only constituent cells are a few hyalocytes (bone marrow-derived mononuclear phagocytes) and fibroblasts ${ }^{553-555}$. The collagen fibrils of the central vitreous are condensed into funnel-like lamellae ${ }^{553,556}$. The vitreoretinal interface consists of the vitreous cortex, retinal inner limiting membrane (ILM), and the basal surfaces of retinal Müller glial cells 554 . Age-related vitreous liquefaction ("synchysis") has been documented in rhesus macaques ${ }^{557}$.

Tenon's capsule is an outer layer of loose fibrous connective tissue which invests the eye and extraocular muscles from the limbus to the optic nerve ${ }^{552}$. The sub-Tenon space is a potential cavity between Tenon's capsule and the adjacent episcleral connective tissue.

Within the conjunctiva, conjunctiva-associated lymphoid tissue (CALT) is normally present, especially in the palpebral and fornical conjunctivae $187,558-560$. The superior eyelid tends to have more lymphoid aggregates than the inferior eyelid ${ }^{187}$ (Table 49).

\section{Developmental Anomalies}

Various spontaneously occurring eye and ocular adnexal developmental anomalies have been noted in macaques, including remnants of persistent fetal hyaloid vessels (including Bergmeister's papilla); iridal and optic nerve colobomas; arteriovenous anastomoses; retinal dysplasia; and eyelid het-

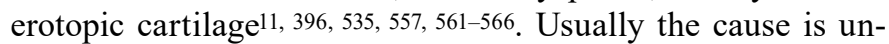
determined, though multiple ocular malformations in a rhesus fetus were attributed to experimental Zika virus infection of the pregnant dam ${ }^{567}$.

Small, discrete nodules of glial-like cell and fibers can extend from the retinal nerve fiber layer (NFL) or optic nerve head $(\mathrm{ONH})$ without associated inflammatory or degenerative changes, suggesting at least a partial astrocytic component. In otherwise unremarkable retinas, such glial nodules most likely represent minor developmental anomalies. These macaque glial nodules share some clinical and/or microscopic features with several proliferative but nonneoplastic entities in human retina such as astrocytic hamartomas ${ }^{568}$.

However, in response to disease or injury, retinal macroglia (astrocytes and Müller cells) can undergo reactive activation which if persistent or exacerbated, can result in excessive hypertrophy, proliferation, and nodular aggregation 569,570 . Thus, similar nodular glial aggregates can also occur macaque retinas in conjunction with degeneration, necrosis, inflammation, or other pathologic changes.

Foci of myelinated nerve fibers and myelin-forming cells (oligodendrocytes and/or Schwann cells) have been reported in the macaque retina and are generally regarded as minor developmental anomalies ${ }^{535,571-573}$.

\section{Retinal rosette; Retinal fold: Eye (Retina)}

(No image provided)

\section{Other $\operatorname{term}(\mathbf{s})$}

Pseudorosettes; photoreceptor rosettes; fleurettes; retinal dysplasia; outer retinal tubulations (ORT); Flexner-Wintersteiner rosettes; Homer-Wright rosettes; disarrangement of retinal structures

\section{Pathogenesis/Cell of Origin}

The term "retinal rosette" implies changes related to abnormal development and differentiation of the retina. Minor retinal rosettes or folds in otherwise normal macaque retinas are usually considered to arise during the fetal period due to random genetic anomalies. However, acquired insults to the developing fetal or immature perinatal retina could also result in such changes ${ }^{11}$. Rosettes and rosette-like structures can be morphologic features of retinoblastoma and other primitive neuropepithelial neoplasms in humans ${ }^{574,575}$.

In mature retinas in adult animals, fold-like bucklings or laminar disarray can be morphologic features of remodeling in extensive retinal degeneration (due to various acquired causes). For example, retinal fixed folds are common features of retinas detached by traction of contracted adhered epiretinal membranes ${ }^{576,577}$.

\section{Differential Diagnosis}

- Lange's fold

Comments: Various focal buckling, and/or disarray of retinal cell layers have been described by many different terms, depending on the constituent retinal cells, presence of a lumen or pseudolumen, and other morphologic fea-

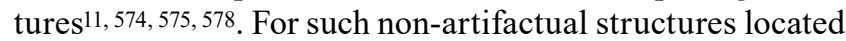
in any fundus region posterior to the ora serrata and attributable to abnormal retinal development or differentiaton, the recommended diagnostic term is retinal "rosette". The term "retinal fold" is recommended to denote acquired bucklings, projections or disruptions of the retina due to various causes, such as the fixed folds commonly seen in retinas detached by traction of contracted adhered epiretinal membranes ${ }^{576,577 .}$

"Lange's fold" (Figure 207) is a fixation artifact characterized as a raised, buckle-like or wave-like elevation of the peripheral retina at the ora serratain eyes of human and macaque fetuses, and is also common in the peripheral retina of young and adult macaques ${ }^{579}, 580$. Lange's folds, are considered fixation artifacts and should not be confused with non-artifactual retinal rosettes or folds. The peripheral location and distinct "undulated" form help differentiate Lange's fold from nonartifactual retinal rosettes or folds. 
Table 49. Microscopic Findings in Special Sense Organs: Eye; Non-human primate

\begin{tabular}{|c|c|c|c|c|}
\hline Eye & Common & Uncommon & $\begin{array}{c}\text { Not Observed but } \\
\text { Potentially Relevant }\end{array}$ & Not Applicable \\
\hline \multicolumn{5}{|l|}{ Congenital } \\
\hline \multicolumn{5}{|l|}{ Filtration Angle/Trabecular Meshwork } \\
\hline Malformation, filtration angle & & $\mathrm{x}$ & & \\
\hline \multicolumn{5}{|l|}{ Uvea, Iris and Ciliary body } \\
\hline Hypoplasia, ciliary body (iris and ciliary body) & & & $\mathrm{x}$ & \\
\hline Malformation, iris & & & $\mathrm{x}$ & \\
\hline Persistent pupillary membrane & & $\mathrm{x}$ & & \\
\hline \multicolumn{5}{|l|}{ Vitreous } \\
\hline Aplasia, vitreous & & & $\mathrm{x}$ & \\
\hline Persistent hyaloid vessels & & $\mathrm{x}$ & & \\
\hline Persistent hyperplastic primary vitreous & & $\mathrm{x}$ & & \\
\hline \multicolumn{5}{|l|}{ Retina } \\
\hline Arteriolar loop, pre-retinal (retina) & & & $\mathrm{x}$ & \\
\hline Retinal rosettes *1 & & $\mathrm{x}$ & & \\
\hline \multicolumn{5}{|l|}{ Non-proliferative } \\
\hline \multicolumn{5}{|l|}{ General Terms } \\
\hline Apoptosis^ & & $\mathrm{x}$ & & \\
\hline Atrophy & & $\mathrm{x}$ & & \\
\hline Fibroplasia & & $\mathrm{x}$ & & \\
\hline Fibrosis^ & & $\mathrm{x}$ & & \\
\hline${\text { Hemorrhage }{ }^{\wedge}}$ & & $\mathrm{x}$ & & \\
\hline Infiltrate, [insert appropriate cell type] ${ }^{* \wedge}$ & $\mathrm{x}$ & & & \\
\hline Inflammation ^^ & $\mathrm{x}$ & & & \\
\hline Mitotic figures, increased & & $\mathrm{x}$ & & \\
\hline$\underline{\text { Single cell necrosis } \wedge}$ & & $\mathrm{x}$ & & \\
\hline Neovascularization & & $\mathrm{x}$ & & \\
\hline \multicolumn{5}{|l|}{ Cornea / Conjunctiva } \\
\hline Atrophy, epithelium & & $\mathrm{x}$ & & \\
\hline Attenuation, endothelium (cornea) & & $\mathrm{x}$ & & \\
\hline Cyst, inclusion & & $\mathrm{x}$ & & \\
\hline Edema $^{\wedge}$ & & $\mathrm{x}$ & & \\
\hline Erosion/ulcer & & $\mathrm{x}$ & & \\
\hline Fibroplasia & & $\mathrm{x}$ & & \\
\hline Fibrosis, stroma ${ }^{\wedge}$ & & $\mathrm{x}$ & & \\
\hline Hypertrophy, Descemet's membrane & & $\mathrm{x}$ & & \\
\hline Infiltrate, [insert appropriate cell type $]^{\wedge}$ & $\mathrm{x}$ & & & \\
\hline Inflammation^^$\wedge^{\wedge}$ & $\mathrm{x}$ & & & \\
\hline Keratinization (cornea) & & $\mathrm{x}$ & & \\
\hline Keratoconus, cornea * & & $\mathrm{x}$ & & \\
\hline Mineralization (cornea) $^{\wedge}$ & & $\mathrm{x}$ & & \\
\hline Neovascularization (cornea) & & $\mathrm{x}$ & & \\
\hline${\text { Pigment }{ }^{\wedge}}$ & & $\mathrm{x}$ & & \\
\hline $\begin{array}{l}\text { Vacuolation, epithelium or endothelium } \\
\end{array}$ & & $\mathrm{x}$ & & \\
\hline \multicolumn{5}{|l|}{ Anterior chamber/Filtration Angle/Trabecular Meshwork } \\
\hline Narrowed filtration angle & & $\mathrm{x}$ & & \\
\hline Inflammation (Anterior chamber, Aqueous humor) ${ }^{\wedge}$ & & $\mathrm{x}$ & & \\
\hline Proteinaceous fluid (Anterior chamber, Aqueous humor) & & $\mathrm{x}$ & & \\
\hline \multicolumn{5}{|l|}{ Uvea, Choroid, Iris and Ciliary body } \\
\hline Adhesion, iris & & $\mathrm{x}$ & & \\
\hline Atrophy, iris & & $\mathrm{x}$ & & \\
\hline Atrophy, ciliary body & & $\mathrm{x}$ & & \\
\hline $\begin{array}{l}\text { Congestion } \wedge \\
\wedge\end{array}$ & & $\mathrm{x}$ & & \\
\hline Infiltrate, [insert appropriate cell type $]^{\wedge}$ & & $\mathrm{x}$ & & \\
\hline Inflammation^^ & & $\mathrm{x}$ & & \\
\hline Neovascularization (uvea: choroid) & & $\mathrm{x}$ & & \\
\hline Pigment, increased/decreased, iris $* \wedge$ & & $\mathrm{x}$ & & \\
\hline Vacuolation, cytoplasmic, epithelial & & $\mathrm{x}$ & & \\
\hline \multicolumn{5}{|l|}{ Lens } \\
\hline Degeneration, lens fiber* & & $\mathrm{x}$ & & \\
\hline Dislocation, lens, anterior or posterior & & & $\mathrm{x}$ & \\
\hline Fibroplasia, lens epithelium & & $\mathrm{x}$ & & \\
\hline Hypertrophy, lens capsule & & $\mathrm{x}$ & & \\
\hline Hypertrophy, lens epithelium & & $\mathrm{x}$ & & \\
\hline Hypertrophy, lens fiber & & $\mathrm{x}$ & & \\
\hline Inflammation, lens^^ & & $\mathrm{x}$ & & \\
\hline Mineralization, lens fiber^^ & & $\mathrm{x}$ & & \\
\hline Necrosis, lens epithelium & & & $\mathrm{x}$ & \\
\hline Rupture, lens capsule & & $\mathrm{x}$ & & \\
\hline Swelling, lens fiber * & & $\mathrm{x}$ & & \\
\hline Vacuolation, lens epithelium or lens fiber & & & $\mathrm{x}$ & \\
\hline
\end{tabular}


Table 49. (Continuation of table)

\begin{tabular}{|c|c|c|c|c|}
\hline Eye & Common & Uncommon & $\begin{array}{c}\text { Not Observed but } \\
\text { Potentially Relevant }\end{array}$ & Not Applicable \\
\hline \multicolumn{5}{|l|}{ Vitreous } \\
\hline Fibroplasia/fibrosis, vitreous & & $\mathrm{x}$ & & \\
\hline Hemorrhage $^{\wedge}$ & & $\mathrm{x}$ & & \\
\hline Hemosiderin-laden macrophages & & $\mathrm{x}$ & & \\
\hline 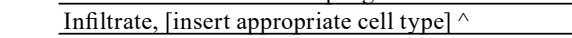 & & $\mathrm{x}$ & & \\
\hline Inflammation $^{\wedge}$ & & $\mathrm{x}$ & & \\
\hline Metaplasia, bone or cartilage & & & $\mathrm{x}$ & \\
\hline $\begin{array}{l}\text { Mineralization, vitreous } \wedge \\
\wedge\end{array}$ & & & $\mathrm{x}$ & \\
\hline \multicolumn{5}{|l|}{ Retina } \\
\hline Atrophy & & $\mathrm{x}$ & & \\
\hline Inner retinal atrophy & & $\mathrm{x}$ & & \\
\hline Outer retinal atrophy & & $\mathrm{x}$ & & \\
\hline Global retinal atrophy & & $\mathrm{x}$ & & \\
\hline Degeneration, microcystoid * & $\mathrm{x}$ & & & \\
\hline Detachment, retina & & $\mathrm{x}$ & & \\
\hline Displacement, photoreceptor nuclei $*$ & & $\mathrm{x}$ & & \\
\hline Edema, retina $*$ & & $\mathrm{x}$ & & \\
\hline Fibroplasia, retinal or epiretinal & & $\mathrm{x}$ & & \\
\hline 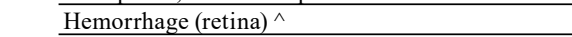 & & $\mathrm{x}$ & & \\
\hline Increased numbers, glial cells & & $\mathrm{x}$ & & \\
\hline Infiltrate, [insert appropriate cell type] ${ }^{\wedge}$ & & $\mathrm{x}$ & & \\
\hline Inflammation $^{\wedge}$ & & $\mathrm{x}$ & & \\
\hline Mineralization $^{\wedge}$ & & & $\mathrm{x}$ & \\
\hline Myelin, increased & & & $\mathrm{x}$ & \\
\hline Single cell necrosis & & $\mathrm{x}$ & & \\
\hline Neovascularization, (retina) & & $\mathrm{x}$ & & \\
\hline Retinal fold * & & $\mathrm{x}$ & & \\
\hline${\text { Pigment, increased }{ }^{\wedge}}$ & & $\mathrm{x}$ & & \\
\hline Vacuolation, cytoplasmic & & $\mathrm{x}$ & & \\
\hline Vacuolation, extracellular & & $\mathrm{x}$ & & \\
\hline \multicolumn{5}{|l|}{ Optic Nerve } \\
\hline Atrophy * & & $\mathrm{x}$ & & \\
\hline Degeneration, axon & & $\mathrm{x}$ & & \\
\hline Demyelination & & $\mathrm{x}$ & & \\
\hline Increased numbers, glial cells & & $\mathrm{x}$ & & \\
\hline Infiltrate, [insert appropriate cell type ${ }^{\wedge}{ }^{\wedge}$ & & $\mathrm{x}$ & & \\
\hline Inflammation $\wedge$ & & $\mathrm{x}$ & & \\
\hline Vacuolation & & $\mathrm{x}$ & & \\
\hline \multicolumn{5}{|l|}{ Retinal Pigment Epithelium (RPE) } \\
\hline Atrophy & & $\mathrm{x}$ & & \\
\hline Deposit, extracellular material, subretinal & & & & $\mathrm{x}$ \\
\hline Deposit, retinal pigment epithelium $*$ & & $\mathrm{x}$ & & \\
\hline Fibroplasia, subretina & & $\mathrm{x}$ & & \\
\hline Hypertrophy, RPE & & $\mathrm{x}$ & & \\
\hline Inclusions (intracytoplasmic accumulation), RPE & & $\mathrm{x}$ & & \\
\hline Necrosis & & $\mathrm{x}$ & & \\
\hline Pigment, decreased $^{\wedge}$ & & $\mathrm{x}$ & & \\
\hline Pigment, increased $^{\wedge}$ & & $\mathrm{x}$ & & \\
\hline Polarity, loss, RPE & & $\mathrm{x}$ & & \\
\hline \multicolumn{5}{|l|}{ Sclera } \\
\hline Atrophy & & $\mathrm{x}$ & & \\
\hline Infiltrate, [insert appropriate cell type] ${ }^{\wedge}$ & $\mathrm{x}$ & & & \\
\hline Inflammation $\wedge$ & & $\mathrm{x}$ & & \\
\hline \multirow{2}{*}{\multicolumn{5}{|c|}{ Proliferative (non-neoplastic) }} \\
\hline & & & & \\
\hline \multicolumn{5}{|l|}{ General terms } \\
\hline Mitotic figures, increased \# & & $\mathrm{x}$ & & \\
\hline \multicolumn{5}{|l|}{ Cornea / Conjunctiva } \\
\hline Dermoid, ocular & & & & $\mathrm{x}$ \\
\hline Hyperplasia/hypertrophy, endothelium & & $\mathrm{x}$ & & \\
\hline Hyperplasia, squamous cell & & $\mathrm{x}$ & & \\
\hline \multicolumn{5}{|l|}{ Filtration Angle/Trabecular Meshwork } \\
\hline Proliferation, trabecular meshwork (TM) & & $\mathrm{x}$ & & \\
\hline \multicolumn{5}{|l|}{ Uvea } \\
\hline Hyperplasia, melanocyte & & $\mathrm{x}$ & & \\
\hline \multicolumn{5}{|l|}{ Lens } \\
\hline Hyperplasia, lens epithelium & & $\mathrm{x}$ & & \\
\hline Retinal Pigment Epithelium (RPE) & & & & \\
\hline Hyperplasia, RPE & & $\mathrm{x}$ & & \\
\hline Neoplastic & & & & \\
\hline Melanoma (uvea, choroid, iris, ciliary body) & & $\mathrm{x}$ & & \\
\hline
\end{tabular}


Infiltrate, lymphoplasmacytic: Eye (General term)

(No image provided)

\section{Other term(s)}

Infiltrate, mononuclear cell; infiltrate, lymphoid cell; infiltrate, lymphocyte

\section{Pathogenesis/Cell of Origin}

Minor accumulations of lymphoplasmacytic cells are common incidental findings in many eye and ocular adnexal subsites in macaques, but their presence in other subsites is a pathologic change (inflammation and/or excess immunostimulation).

\section{Diagnostic features}

- Incidental accumulations are generally small foci of lymphocytes, often with plasma cells and/or small monocytes.

- Increases in size and numbers of cell accumulations (and distribution in atypical ocular subsites) can also occur.

\section{Differential Diagnosis}

- Lymphoma (neoplastic cell infiltrates).

Comments: Small foci of lymphoplasmacytic cells are common incidental "background" findings in several eye subsites, lacrimal gland, and conjunctiva of cynomolgus and other macaques $6,11,581,582$. Frequently affected subsites in the eye include the ciliary body, choroid, iris, and corneal limbus. Lymphoplasmacytic cell aggregates are also normally present in the eyelid and fornical conjunctivae (conjunctiva-associated lymphoid tissue (CALT) ${ }^{558,559}$. Increased numbers of lymphoplasmacytic cells in "typical background" sites can represent a reactive pathologic change (inflammation and/or excessive immunostimulation) due to various disease processes. Thus, in safetytesting studies, intergroup differences in severity of these accumulations can be highly relevant in distinguishing incidental levels from treatment-related effects.

The presence of even low numbers of other cell types (e.g., neutrophils, eosinophils, macrophages, epithelioid cells) within even the smaller lymphoplasmacytic infiltrates is abnormal. Thus, depending on the circumstances of a particular study, such cell admixtures (of any severity level) may warrant a diagnosis of "inflammation" (with appropriate modifiers) rather than "infiltrate" (see also Infiltrate and Inflammation sections in Chapter 2).

In addition, extremely pronounced increases in lymphoplasmacytic cell numbers in "typical background" subsites (with or without other cell types) seldom occur as isolated changes in otherwise apparently unremarkable eyes. Instead, very prominent accumulations of such cells are often just one morphologic component of complex regional or even pan-ocular inflammatory processes with concurrent interrelated pathologic changes in multiple ocular subsites (such as degeneration, necrosis, hemorrhage, fibrosis and architectural disruptions [e.g., retinal detachment, lens rupture, corneal perforation] $)^{583,584}$. The overall circumstances in such complex situations can be variable and even unique, so the question of whether or not to record the lymphoplasmacytic cell accumulations as separate diagnoses or to regard them only as part of a more general diagnosis of inflammation is most appropriately based the study pathologist's professional judgment after assessment of all relevant factors.

Lymphoplasmacytic cells are not typical "background" findings in certain other ocular subsites such as the anterior chamber, central cornea, lens, retina, subretinal space, retinal pigment epithelium (RPE), vitreous, optic nerve head, and optic nerve. In these sites, even low numbers of such cells are abnormal. As such, low-level accumulations (in otherwise unremarkable or minimally altered subsites) may warrant diagnosis.

In addition, lymphoplasmacytic cells in these "nontypical" subsites can also occur in much larger numbers when they too are components of complex regional or pan-ocular pathologic processes. In these complex situations, whether or not to record the lymphoplasmacytic cell accumulations as separate diagnoses or to regard them only as part of a more general diagnosis of inflammation is likewise most appropriately based the study pathologist's professional judgment after assessment of all relevant factors.

Keratoconus: Eye (Cornea) (No image provided)

\section{Pathogenesis/Cell of Origin}

Unknown

\section{Diagnostic features}

- Conical protrusion of central cornea (clinical).

- Corneal epithelial thinning.

- Corneal stromal thinning and lamellar disorganization.

- Corneal endothelial cell degeneration.

- Breaks or folds of Bowman's layer and Descemet's membrane.

\section{Differential Diagnosis}

- Artifactual distortion of the corneal surface during histologic processing.

Comments: Keratoconus has been reported in cynomolgus and rhesus macaques ${ }^{585,586}$.

\section{Pigment, increased (melanin): Eye: Iris}

(No image provided)

\section{Other $\operatorname{term}(\mathrm{s})$}

Hyperpigmentation

\section{Pathogenesis/Cell of Origin}

Chemically- induced increased production of melanin by existing iridal stromal melanocytes. 


\section{Diagnostic features}

- Clinically observed darker coloration of iris in treated eye compared to contralateral untreated eye, or to irides of control animals.

- Sometimes microscopically detectable by careful comparison of contralateral eyes in animals in which inciting test article was unilaterally administered.

Comments: Increased iridal coloration (hyperpigmentation) in humans and macaques has been reported as a sideeffect of treatment with certain prostaglandins, especially $\mathrm{PGF}_{2 \alpha}$ analogue latanoprost $587-590$. Prostaglandin-stimulated melanin overproduction by iridal stromal melanocytes is considered the underlying mechanism.

Degeneration, fiber: Eye (Lens) (No image provided)

\section{Other term(s)}

Cataract

\section{Pathogenesis/Cell of Origin}

Congenital lens fiber degeneration (cataracts) in macaques has been attributed to genetic defects, in utero infectious agents, concurrent ocular developmental anomalies, or is of unknown origin $581,591-594$.

Age-related spontaneous lens fiber degeneration (cataracts) has also been reported in older macaques ${ }^{11,546,581,595-597 .}$

Naturally occurring or experimentally induced acquired lens fiber degeneration (cataracts) in macaques can also develop due to many other causes including chemical toxins, irradiation, and diabetes mellitus ${ }^{591,598-602}$. Lens fiber degeneration (cataracts) in younger macaques in toxicity studies can also result from physical trauma to the lens or as sequelae of many spontaneous or induced ocular pathologies that secondarily affect the lens.

\section{Diagnostic features}

- Lens fiber swelling, ballooning, fragmentation, condensation, hypo- or hypereosinophilia.

- Anterior lens epithelium hyperplasia, posterior migration, fibrous metaplasia (epithelial-to-mesenchymal transition).

- Lens capsule thickening, thinning, and distortion.

- Mineralization.

\section{Differential Diagnosis}

- Fixation artifact, especially with acetic acid-containing fixatives (such as Davidson's solution).

Comments: Macaque lens fibers are quite susceptible to fixation artifact, especially after fixation with Davidson's or modified Davidson's solutions ${ }^{603-605}$. Distinguishing antemortem cataracts from this artifact can be challenging.
Degeneration, microcystoid: Eye (Retina) (Figure 208)

\section{Other term(s)}

Retinal peripheral cystoid degeneration; retinal cystic degeneration; Blessig-Iwanoff cysts; ora serrata cysts

\section{Pathogenesis/Cell of Origin}

Peripheral microcystoid degeneration is considered an aging-related change though it can also be present in young animals.

\section{Diagnostic features}

- Small to large, cyst-like, generally clear cavitations at the peripheral retina or pars plana (near the ora serrata)

- Can be single or multiple, often confluent.

\section{Differential Diagnosis}

- Lange's fold (artifact)

- distinct, often well-elevated buckling or crimped appearance rather than cystic cavitations.

- Retinal edema

- often located in more central retina (especially in and around the macula); often associated with other pathologic changes in retina and/or eye subsites.

- Retinoschisis

- true intraretinal expansion or "splitting" vs. artifactual splitting

Comments: Minor microcystoid formations in the peripheral retina and/or pars plana are common in macaque eyes and are considered innocuous incidental changes ${ }^{519,535,605}$.

\section{Displacement, photoreceptor nuclei: Eye (Retina Choroid)} (Figure 209)

\section{Other term(s)} cell

Photoreceptor displaced nuclei; subretinal photoreceptor

\section{Pathogenesis/Cell of Origin}

Spontaneous or pathologic change of rod and cone photoreceptors.

\section{Diagnostic features}

- Normal-appearing or pyknotic rod/cone nuclei in the subretinal space; displaced from their normal residence (outer nuclear layer [ONL]).

- Partly displaced nuclei can sometimes be seen spanning the outer limiting membrane (OLM).

- Low numbers of displaced nuclei may be present in the subretinal space as spontaneous incidental findings in otherwise normal retina.

\section{Differential Diagnosis}

- Infiltrates [insert appropriate cell type] in the subretinal space 
Comments: Rod and cone photoreceptor nuclei (and associated perikaryal) are normally located in the outer nuclear layer (ONL) but can be "displaced" into the subretinal space in various species including macaques $507,606,607$.

In otherwise unremarkable macaques, a few such subretinally displaced rod/cone nuclei are considered incidental spontaneous changes of no toxicological relevance. However, increased or excessive numbers of displaced rod/cone nuclei can be a feature of retinal degeneration and other pathologies.

\section{Edema, retina: Eye (Retina Choroid) (Figure 209)}

\section{Other term(s)}

Cystoid macular edema; cystic edema; macular cyst; retinoschisis; retinal cystoid degeneration

\section{Pathogenesis/Cell of Origin}

Abnormal intraretinal fluid accumulation (edema) occurs in many degenerative, inflammatory, and vascular retinal pathologies. The pathophysiology is not completely understood ${ }^{608-610}$.

\section{Diagnostic features}

- Variably sized, generally clear, often multiple cyst-like spaces or cavitations which can occur in any retinal region. In foveate retinas, edema cavitations are often localized or most prominent in the inner retina, and especially in the macular Henle fiber region (macular outer plexiform layer $[\mathrm{OPL}]$ and adjacent inner nuclear layer $[\mathrm{INL}])^{608,609}$.

- The cystic spaces are often crossed perpendicularly by the intact but attenuated trunks of Müller cells.

- Confluent cavitations can create the effect of internal retinal "splitting" (retinoschisis).

\section{Differential Diagnoses}

- Artifactual post-mortem "splitting" of the retinal outer plexiform layer (especially in the Henle fiber region)

- Peripheral retinal microcystoid degeneration - spontaneous and generally innocuous

Comments: Retinal edema is not a specific disease, but rather a feature of many degenerative, vascular, and inflammatory conditions, especially those that which have primary or most extensive effects in the inner retina.

\section{Atrophy, idiopathic, bilateral: Eye: Optic nerve}

(No image provided)

\section{Pathogenesis/Cell of Origin}

Unknown

\section{Diagnostic features}

- Temporal optic nerve has circumscribed minor to extensive loss of axons, often with associated gliosis (astrocyte proliferation) and apparent condensation/thickening of pial septa. The demarcation between the affected temporal side and the unaffected nasal side can be quite distinct.

- Frequently, overall shrinkage of the temporal-side optic nerve (most evident in cross-sections).

- Thinning (decreased axons) of temporal retina nerve fiber layer (NFL) (i.e., the papillomacular bundle).

- Decreased ganglion cells in the macula.

- Not always bilateral.

\section{Differential Diagnoses}

- Vitamin B12 deficiency

- Similar temporal optic nerve atrophy and temporal macular ganglion cell loss have also been noted, along with brain and spinal cord pathology following experimentally induced Vitamin B12 deficiency in "monkeys" (presumed to be rhesus macaques) ${ }^{611}$.

- Other causes of optic nerve and/or retinal degeneration/ atrophy

- usually do not exhibit such well-demarcated temporal localization

- often concurrent with other retinal and/or optic nerve pathologic changes such as inflammation.

Comments: Idiopathic bilateral optic atrophy is a specific optic nerve/retinal ganglion cell degenerative condition of unknown etiology that has been described in cynomolgus and rhesus macaques ${ }^{612-617}$.

\section{Deposit, retinal pigment epithelium: Eye (Retina RPE)} (No image available)

\section{Pathogenesis/Cell of Origin}

Deposits of amorphous material ("drusen") can be a feature of various age-related macular degeneration (AMD)-like syndromes in macaques. Genetic predisposition has been implicated in some cases of such accumulations and AMD-like disease in macaques, with some gene polymorphisms associated with human AMD also present in macaques 618,619 .

Later-onset deposits have a familial association in in some closed-colony rhesus populations $535,536,620,621$. Early-onset AMDlike disease in a cynomolgus colony was also demonstrated to be heritable $536,622,623$.

\section{Diagnostic features}

- Deposits are single to multiple, focal, amorphous extracellular eosinophilic aggregates typically located between Bruch's membrane and the retinal pigment epithelium (RPE).

- Aggregates often elevate the RPE in a "dome"-like manner.

\section{Differential Diagnoses}

- Lipofuscin accumulation in RPE cells

- has been noted as an aging-related change in rhesus ma-

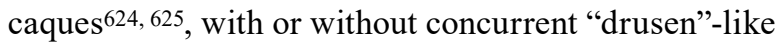
formations. 
- Neuronal ceroid lipofuscinosis

- Recently described as a naturally occurrence in a colony of Japanese macaques [Macaca fuscata] results in accumulations of abnormal glycoprotein storage materials in many central nervous system sites including the retina ${ }^{626}$

- "Pseudodrusen"-type material

- In human patients with AMD and certain other diseases, aggregates of drusen-like material ("reticular pseudorusen", "subretinal drusenoid deposits") can occur in the subretinal space (internal to the RPE) ${ }^{627}$.

- This material is similar though not identical to "classic" drusen in composition.

- Although apparently not described in macaques, such "pseudodrusen" -type material could potentially occur in these species.

Comments: In humans, drusen deposits are heterogenous mixtures of lipids, complement, immunoglobulins, proteins and other molecules 628 and are considered a hallmark of human $\mathrm{AMD}^{542}$.

Ophthalmoscopically visible "drusen" in macaques can correlate not just with the typical sub-RPE deposits but with vacuolated and/or lipid-filled RPE cells ${ }^{624,629-632}$.

Naturally occurring drusen-type deposits as well as retinal and RPE alterations suggestive of early-to intermediatestage human AMD have been noted in cynomolgus and rhesus macaques; in some but not all studies, these findings were characterized only clinically and not confirmed microscopically535, 620, 623, 628, 633 .

Spontaneous drusen-type deposits are not common in the typically younger macaques used in most toxicity safetytesting studies. However, the incidence in macaques tend to increase with age 535 , 619, 623, 632-634.

The descriptive terms "deposit" should be used when "drusen-type deposits" are observed in routine toxicology studies because such deposits are not usually biochemically analyzed. Diagnostic/intepretative terms used in the literature such as drusen, macular drusen, drusenoid leions, Aquired Macular Degeneration (AMD)-like lesions, drusenoid maculopathy, macular degeneration, and microcystic change should not be used.

\section{Melanoma: (Eye Uvea/Iris/Choroid) (No image provided)}

Comments: Proliferative lesions reported in macaques include choroidal melanomas with evidence of malig- nancy and local invasion of retina, in two cynomolgus macaques 635,636 . Circumscribed aggregates or nodules of pigmented (presumably melanocytic) spindle to round cells without evidence of malignancy can occur in the choroid and ciliary body of macaques, but it is unknown whether these are neoplasms versus hamartomatous and/or other nonneoplastic uveal proliferations ${ }^{637,638}$.

\section{B. Adnexa \\ (Eyelids, Extraocular Muscles and Lacrimal Glands)}

The caruncle (carcuncula lacrimalis) is an elevated, nodular fold of modified skin at the medial canthus. The plica semilunaris (vestigial nictitating membrane) is a small conjunctivalined fold closely applied to the contour of the lower medial globe, near the medial canthus 639,640 .

Macaque eyelids have a well-developed tarsal plate with embedded Meibomian sebaceous (tarsal) glands as well as smaller sebaceous glands (glands of Zeis), apocrine (sweat) glands (glands of Moll), and accessory lacrimal tissue ${ }^{641-643}$ A single lacrimal punctum is located at the medial canthus of each eyelid 644 .

Extraocular muscles include the superior and inferior rectus; superior and inferior oblique; and medial and lateral rectus. Cynomolgus and other macaque species also have an accessory lateral rectus muscle which is not routinely present in humans ${ }^{645-647}$. Macaque extraocular muscles are very well vascularized ${ }^{648}$. Eyelid superior levator palpebrae and orbicularis oculi muscles are also present in macaques $645,647,649$.

The macaque lacrimal gland is composed of serous acini and is located in the superior lateral orbit 645,650 . Multiple lacrimal gland ducts open into the superior lateral fornix ${ }^{187}$. In addition to the major lacrimal gland, microscopic clusters of lacrimal gland acini (accessory lacrimal tissue) are often scattered in the fornical conjunctival stroma (glands of Krause) and in the eyelid (glands of Wolfring) ${ }^{643}$ (Table 50, 51).

\section{Neoplastic lesions}

Comments: Other primary ocular neoplasms have been rarely noted, and include lacrimal gland myoepithelioma ${ }^{651}$, eyelid squamous cell papilloma ${ }^{652}$, and eyelid "dermoid carcinoma"653 in rhesus macaques. Retrovirus-induced lymphoma can spread into the eye and ocular adnexa. 
Table 50. Microscopic Findings in Special Sense Organs: Eyelid; Non-human primate

\begin{tabular}{|c|c|c|c|c|}
\hline Eyelid & Common & Uncommon & $\begin{array}{c}\text { Not Observed, but } \\
\text { Potentially Relevant }\end{array}$ & Not Applicable \\
\hline \multicolumn{5}{|l|}{ Non-proliferative } \\
\hline Infiltrate, [insert appropriate cell type], eyelid ${ }^{\wedge}$ & $\mathrm{x}$ & & & \\
\hline Inflammation, eyelid $^{\wedge}$ & $\mathrm{x}$ & & & \\
\hline Atrophy, Meibomian gland & & $\mathrm{x}$ & & \\
\hline $\begin{array}{l}\text { Infiltrate, [insert appropriate cell type], Meibomian gland } \\
\wedge\end{array}$ & & $\mathrm{x}$ & & \\
\hline 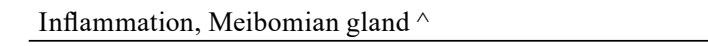 & & $\mathrm{x}$ & & \\
\hline Inflammation, Granulomatous, Meibomian gland $^{\wedge}$ & & $\mathrm{x}$ & & \\
\hline \multicolumn{5}{|l|}{ Proliferative (non-neoplastic) } \\
\hline Hyperplasia, Meibomian gland & & $\mathrm{x}$ & & \\
\hline \multicolumn{5}{|l|}{ Neoplastic* } \\
\hline Papilloma, squamous cell & & $\mathrm{x}$ & & \\
\hline Carcinoma, dermoid & & $\mathrm{x}$ & & \\
\hline
\end{tabular}

* Terminology with diagnostic criteria and/or comments described below. ${ }^{\wedge}$ Terminology addressed in the Systemic/General Pathology Section.

Table 51. Microscopic Findings in Special Sense Organs: Lacrimal glands; Non-human primate

\begin{tabular}{|c|c|c|c|c|}
\hline Lacrimal Glands & Common & Uncommon & $\begin{array}{l}\text { Not Observed, but } \\
\text { Potentially Relevant }\end{array}$ & Not Applicable \\
\hline \multicolumn{5}{|l|}{ Non-proliferative } \\
\hline Alteration, cytoplasmic, acinar & & & & $\mathrm{x}$ \\
\hline Apoptosis ^ & & $\mathrm{x}$ & & \\
\hline Atrophy & & $\mathrm{x}$ & & \\
\hline Cyst & & $\mathrm{x}$ & & \\
\hline Degeneration & & $\mathrm{x}$ & & \\
\hline Dilatation & & $\mathrm{x}$ & & \\
\hline Fibrosis $\wedge$ & $\mathrm{x}$ & & & \\
\hline Hemorrhage $^{\wedge}$ & & $\mathrm{x}$ & & \\
\hline Hypertrophy & & $\mathrm{x}$ & & \\
\hline Infiltrate, [insert appropriate cell type $]^{\wedge}$ & $\mathrm{x}$ & & & \\
\hline Inflammation $^{\wedge}$ & $\mathrm{x}$ & & & \\
\hline 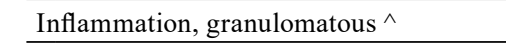 & & $\mathrm{x}$ & & \\
\hline Karyomegaly & & $\mathrm{x}$ & & \\
\hline Necrosis, single-cell ${ }^{\wedge}$ & & $\mathrm{x}$ & & \\
\hline $\mathrm{Necrosis}^{\wedge}$ & & $\mathrm{x}$ & & \\
\hline Porphyrin, increased & & & & $\mathrm{x}$ \\
\hline Regeneration & & $\mathrm{x}$ & & \\
\hline \multicolumn{5}{|l|}{ Proliferative (non-neoplastic) } \\
\hline \multicolumn{5}{|l|}{ Lacrimal Gland } \\
\hline Hyperplasia, acinar cell & & $\mathrm{x}$ & & \\
\hline \multicolumn{5}{|l|}{ Nasolacrimal Duct (NLD) } \\
\hline Hyperplasia, epithelium & & $\mathrm{x}$ & & \\
\hline \multicolumn{5}{|l|}{ Neoplastic* } \\
\hline Myoepithelioma & & $\mathrm{x}$ & & \\
\hline
\end{tabular}

* Terminology with diagnostic criteria and/or comments described below. ^ Terminology addressed in the Systemic/General Pathology Section. 


\section{URINARY TRACT}

For detailed general considerations on the Urinary system, refer to the INHAND rodent publication ${ }^{654}$. This chapter is limited to an overview of recommended terminology, diagnostic criteria, and histologic images for the urinary tract of Cynomolgus monkeys used in preclinical toxicity studies.

The range of spontaneous proliferative and non-proliferative urinary tract findings/lesions encountered in macaques is narrow when compared to rodents. Among the most common findings in macaques are renal and urinary bladder inflammatory cell infiltrates, urothelial cytoplasmic inclusions, and multinucleated cells in the medullary collecting ducts. These can sometimes vary by the geographical source of macaque ${ }^{61}$.

\section{A. Kidney (including Renal Pelvis)}

As in rodents, the kidney is a common target organ for therapeutic and diagnostic (e.g. imaging contrast media) agents. Injury may occur either as a result of direct effects on tubules or glomeruli, or indirectly via altered hemodynamics. Organs of the lower urinary tract (ureters, urinary bladder, and urethra) are less frequent targets of chemical-induced toxicity.

Exposure of the kidneys to drugs and chemicals is primarily through the bloodstream via paired renal arteries arising from the abdominal aorta. The kidneys are a major site of xenobiotic biotransformation, which can result in the formation of toxic metabolites from chemicals or drugs that may be present in the cytoplasm of tubular epithelial cells and/or excreted in the urine. Chemicals and/or metabolites are often concentrated in the urine and thus excretion may result in increased exposure of the lower urinary system. Deposition of biologic compounds (e.g., therapeutic antibodies, immune complexes, and/or proteins) commonly occurs in glomeruli.

Although the kidney is not a common site for spontaneous background lesions in macaques, the pathologist must be aware that changes associated with a variety of nonhuman primate microbial diseases that manifest lesions in multiple organ systems may also occur in the kidney. Unlike rodents, which are typically maintained in toxicology facilities with relatively less prevalent pathogens, nonhuman primates often carry potential pathogens that can result in spontaneous findings depending on species, age, gender, colony, and/or geographical source. Importantly, immunosuppression caused by either stress or test items can result in the clinical manifestation of spontaneous, newly acquired infections or the recrudescence of previously quiescent microbial associated lesions (viral and bacterial), some of which may result in renal findings (Table 52, 53).

Crystals: Kidney (Figure 210-211)

\section{Other term(s)}

Lithiasis; concretions; nephrocalcinosis

Comments: Crystalline deposits (nephrocalcinosis) have been observed sporadically in cynomolgus macaques, sometimes in clusters (i.e. seen in an apparent random distribution in multiple animals in a given study or within a batch of animals). The deposits are usually primarily interstitial in nature with classic foreign-body giant cells and other mononuclear cell infiltrates. The inciting cause is not understood but may be related to diet and/or water quality, and/or other environmental factors (such as excess phosphorus intake or an imbalance of calcium and phosphorus in the diet) resulting in secondary hyperparathyroidism at some point in the animals' lives ${ }^{655}$. Additionally, nutritional secondary hyperparathyroidism has been reported in Japanese macaques (Macaca fuscata) with no accompanying renal functional impact ${ }^{656,657}$.

Cline et al 1415 discusses this observation and cites literature stating this change to be relatively common in male baboons at $11.2 \%$ incidence ${ }^{658}$ and occurring as a spontaneous and induced lesion in squirrel monkeys 659,660 .

\section{Eosinophilic droplets, Glomerulus: Kidney (Figure 212)}

\section{Other term(s)}

Glomeruli, hyaline droplets

\section{Pathogenesis/Cell of Origin}

Visceral podocyte

Comments: Eosinophilic droplets are noted occasionally within the glomeruli of monkey kidneys. The droplets are generally bright pink with H\&E stains but are more easily visualized with PAS stains. These droplets are generally composed of albumin and/or other proteins that can pass through the podocyte slit diaphragms, and surprisingly the location is most often within visceral podocytes rather than in mesangial cells. Usually, only a small number of glomeruli within a kidney section are affected. Glomerular eosinophilic droplets may be a component of glomerular injury related to glomerulonephritis or glomerulosclerosis, but in some cases appear spontaneously without other evidence of glomerular dysfunction or damage. Ultrastructurally, the droplets are osmiophilic and surrounded by a limiting membrane which suggests they reside within endolysosomal compartments. The droplets are generally not associated with hyaline droplet nephropathy, which is limited to tubules and associated with drug:protein complexes within tubule lysosomes, therefore the term eosinophilic droplets is preferred to avoid confusion with tubule lesions. The finding should be differentiated from thrombi, which are typically solitary and larger and localized to capillary lumina, whereas eosinophilic droplets are almost always multiple and found within podocyte cytoplasm.

\section{Multinucleated cells: Kidney (Figure 213)}

Comments: Multinucleated cells are sometimes found in the renal tubular epithelium, usually in the collecting ducts in the renal papilla, in macaques. These are considered to be 
Table 52. Microscopic Findings in the Urinary System: Kidney; Non-human Primate

\begin{tabular}{|c|c|c|c|c|}
\hline Kidney & Common & Uncommon & $\begin{array}{c}\text { Not Observed but } \\
\text { Potentially Relevant }\end{array}$ & Not Applicable \\
\hline \multicolumn{5}{|l|}{ Congenital } \\
\hline Aplasia & & $\mathrm{x}$ & & \\
\hline Dysplasia, renal & & $\mathrm{x}$ & & \\
\hline Ectopic tissue, adrenal & & $\mathrm{x}$ & & \\
\hline Hypolasia & & $\mathrm{x}$ & & \\
\hline Immature glomerulus & & $\mathrm{x}$ & & \\
\hline \multicolumn{5}{|l|}{ Non-proliferative (renal parenchyma) } \\
\hline Accumulation, adipocytes, interstitial ${ }^{\wedge}$ & & $\mathrm{x}$ & & \\
\hline Accumulation, glycogen & & $\mathrm{x}$ & & \\
\hline Accumulation, hyaline droplets & & $\mathrm{x}$ & & \\
\hline Accumulation, lipid ${ }^{1}$ & & & & $\mathrm{x}$ \\
\hline Amyloid, glomerular $\wedge^{\wedge}$ & & $\mathrm{x}$ & & \\
\hline Amyloid, interstitial $\wedge^{\wedge}$ & & $\mathrm{x}$ & & \\
\hline Atrophy, glomerulus & & $\mathrm{x}$ & & \\
\hline Atrophy, tubule & & $\mathrm{x}$ & & \\
\hline Basophilia, tubule & & $\mathrm{x}$ & & \\
\hline Cast & $\mathrm{x}$ & & & \\
\hline Crystals* & & $\mathrm{x}$ & & \\
\hline Cyst & & $\mathrm{x}$ & & \\
\hline Degeneration, tubule & & $\mathrm{x}$ & & \\
\hline Dilatation, Bowman's space & & $\mathrm{x}$ & & \\
\hline Dilatation, tubule & $\mathrm{x}$ & & & \\
\hline Edema, interstitial ${ }^{\wedge}$ & & $\mathrm{x}$ & & \\
\hline Eosinophilic droplets, glomerulus * & & $\mathrm{x}$ & & \\
\hline Fibrosis, interstitial $\wedge$ & $\mathrm{x}$ & & & \\
\hline Glomerulonephritis & & $\mathrm{x}$ & & \\
\hline Glomerulopathy, hyaline & & $\mathrm{x}$ & & \\
\hline Glomerulopathy, mesangioproliferative & & $\mathrm{x}$ & & \\
\hline Glomerulosclerosis & & $\mathrm{x}$ & & \\
\hline Granules, basophilic $^{\wedge \#}$ & & & $\mathrm{x}$ & \\
\hline Extramedullary hematopoiesis ${ }^{\wedge}$ & & $\mathrm{x}$ & & \\
\hline Hemorrhage $^{\wedge}$ & & $\mathrm{x}$ & & \\
\hline Hypertrophy/hyperplasia, juxtaglomerular & & $\mathrm{x}$ & & \\
\hline Hypertrophy/hyperplasia, mesangial & & $\mathrm{x}$ & & \\
\hline Hypertrophy, tubule & & $\mathrm{x}$ & & \\
\hline Lipidosis, glomerular & & $\mathrm{x}$ & & \\
\hline Inclusion bodies ^ (Inclusions) & & $\mathrm{x}$ & & \\
\hline Infarct & & $\mathrm{x}$ & & \\
\hline 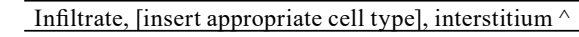 & $\mathrm{x}$ & & & \\
\hline Inflammation $^{\wedge} 2$ & $\mathrm{x}$ & & & \\
\hline Interstitial nephritis $^{2}$ & & & & $\mathrm{x}$ \\
\hline Karyomegaly & & $\mathrm{x}$ & & \\
\hline Mesangiolysis & & $\mathrm{x}$ & & \\
\hline Metaplasia, osseous ^ & & $\mathrm{x}$ & & \\
\hline Microabscess & & $\mathrm{x}$ & & \\
\hline 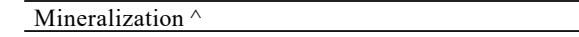 & $\mathrm{x}$ & & & \\
\hline Multinucleated cells * & $\mathrm{x}$ & & & \\
\hline $\mathrm{Necrosis}^{\wedge}$ & & $\mathrm{x}$ & & \\
\hline Necrosis, papilla & & $\mathrm{x}$ & & \\
\hline Single cell necrosis $\wedge \#$ & & $\mathrm{x}$ & & \\
\hline Nephropathy, alpha ${ }_{2 u}$-globulin nephropathy & & & & $\mathrm{x}$ \\
\hline Nephropathy, chronic progressive nephropathy & & & & $\mathrm{x}$ \\
\hline Nephropathy, obstructive & & $\mathrm{x}$ & & \\
\hline Nephropathy, retrograde & & $\mathrm{x}$ & & \\
\hline Pigment & & $\mathrm{x}$ & & \\
\hline Pyelonephritis $\wedge^{\wedge}$ & & $\mathrm{x}$ & & \\
\hline Regeneration, tubule & $\mathrm{x}$ & & & \\
\hline Vacuolation $^{1}$ & & $\mathrm{x}$ & & \\
\hline \multicolumn{5}{|l|}{ Proliferative (non-neoplastic) } \\
\hline Hyperplasia, juxtaglomerular & & $\mathrm{x}$ & & \\
\hline Hyperplasia, mesangium & & $\mathrm{x}$ & & \\
\hline Hyperplasia, oncocytic & & & $\mathrm{x}$ & \\
\hline Hyperplasia, tubule & & $\mathrm{x}$ & & \\
\hline Hypertrophy/hyperplasia, juxtaglomerular & & $\mathrm{x}$ & & \\
\hline Hypertrophy/hyperplasia, mesangial & & $\mathrm{x}$ & & \\
\hline Nephroblastematosis & & & $\mathrm{x}$ & \\
\hline Metaplasia/hyperplasia, Bowman's capsule & & & $\mathrm{x}$ & \\
\hline \multicolumn{5}{|l|}{ Neoplastic (renal parenchyma) } \\
\hline Nephroblastoma * & & $\mathrm{x}$ & & \\
\hline
\end{tabular}

* Terminology with diagnostic criteria and/or comments described below. ${ }^{*}$ Finding more frequently observed as an induced change. ${ }^{\wedge}$ Terminology addressed in the Systemic Pathology Section. ${ }^{1}$ Lipid accumulation within cells should use the descriptive term vacuolation. If special stains are performed identifying lipid, a modifier "lipid accumulation" may be used. ${ }^{2}$ See Systemic pathology section for the use of -itis terms . It is preferable to use the general term "Inflammation" with modifiers for location. 
Table 53. Microscopic Findings of the Urinary System: Renal Pelvis; Non-human Primate

\begin{tabular}{|c|c|c|c|c|}
\hline Renal Pelvis & Common & Uncommon & $\begin{array}{c}\text { Not Observed but } \\
\text { Potentially Relevant }\end{array}$ & Not Applicable \\
\hline \multicolumn{5}{|l|}{ Congenital } \\
\hline Aplasia, ureter & & $\mathrm{x}$ & & \\
\hline \multicolumn{5}{|l|}{ Non-proliferative } \\
\hline Calculus & & $\mathrm{x}$ & & \\
\hline Crystals & & $\mathrm{x}$ & & \\
\hline Dilatation, pelvis & & $\mathrm{x}$ & & \\
\hline Erosion & & $\mathrm{x}$ & & \\
\hline Edema $^{\wedge}$ & & $\mathrm{x}$ & & \\
\hline Inclusions, eosinophilic inctracytoplasmic $* \wedge 1$ & $\mathrm{x}$ & & & \\
\hline Infiltrate, [insert appropriate cell type $]^{\wedge}$ & & $\mathrm{x}$ & & \\
\hline Metaplasia, glandular & & $\mathrm{x}$ & & \\
\hline Metaplasia, squamous cell & $\mathrm{x}$ & & & \\
\hline Mineralization $^{\wedge}$ & & $\mathrm{x}$ & & \\
\hline Pyelonephritis & & $\mathrm{x}$ & & \\
\hline Ulceration & & $\mathrm{x}$ & & \\
\hline Vacuolation & & $\mathrm{x}$ & & \\
\hline \multicolumn{5}{|l|}{ Proliferative (non-neoplastic) } \\
\hline Hyperplasia, urothelium & & $\mathrm{x}$ & & \\
\hline
\end{tabular}

incidental, of unknown etiology, and have no known pathologic significance ${ }^{11,116,661 .}$

Nephroblastoma: Kidney (No image provided)

\section{Other term(s)}

Nephroblastomatosis; Wilms' tumor (human nomenclature); embryonal nephroma

\section{Pathogenesis/Cell of Origin}

Renal embryonic tissue

Comments: Nephroblastomas are uncommon in non-human primates but have been reported in young baboons, cotton-tailed tamarins, and cynomolgus macaques ${ }^{662,663}$. The nephroblastomas described in baboons and cottontailed tamarins were typically grossly and histologically very similar to those observed in rodents as described in the rodent INHAND Urinary System publication ${ }^{654}$. One macaque had a unilateral, markedly enlarged kidney in which normal kidney architecture was completely effaced by primitive tubules, occasional glomeruli, and edematous stromal tissue. The diffuse nature of this tumor and lack of any normal renal architecture is similar to the human pathology classification of "nephroblastomatosis" so this can be considered an alternative term. None of the nephroblastomas in non-human primates had chromosomal abnormalities equivalent to those seen in human Wilms' tumor.

\section{B. Ureter, Urethra and Urinary Bladder} (Table 54, 55, 56)

Inclusions, eosinophilic cytoplasmic: Renal pelvis/Ureter/ Urinary Bladder (Figure 214)

\section{Other term(s)}

Tonofilament pseudoinclusions

\section{Pathogenesis/Cell of Origin}

These pseudoinclusions consist of cytokeratin aggregates.

\section{Diagnostic features}

- Eosinophilic, intracytoplasmic bodies that resemble inclusion bodies present in the transitional cell epithelium of the urinary bladder and occasionally renal pelvis and/ or ureter.

Comments: These pseudoinclusions occur in macaques and are of no pathologic significance. It is important to recognize these as incidental findings and they should not be confused with viral inclusions ${ }^{116,664,665 .}$ 
Table 54. Microscopic Findings in the Urinary System: Ureter; Non-human Primate

\begin{tabular}{|c|c|c|c|c|}
\hline Ureter & Common & Uncommon & $\begin{array}{c}\text { Not Observed but } \\
\text { Potentially Relevant }\end{array}$ & Not Applicable \\
\hline \multicolumn{5}{|l|}{ Congenital } \\
\hline Aplasia, ureter & & $\mathrm{x}$ & & \\
\hline \multicolumn{5}{|l|}{ Non-proliferative } \\
\hline Calculus & & $\mathrm{x}$ & & \\
\hline Crystals & & $\mathrm{x}$ & & \\
\hline Dilatation & & $\mathrm{x}$ & & \\
\hline Edema $^{\wedge}$ & & $\mathrm{x}$ & & \\
\hline Erosion/ulceration & & $\mathrm{x}$ & & \\
\hline Inclusions, eosinophilic inctracytoplasmic *^ & $\mathrm{x}$ & & & \\
\hline 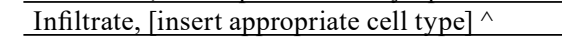 & & $\mathrm{x}$ & & \\
\hline Vacuolation, urothelium & & $\mathrm{x}$ & & \\
\hline \multicolumn{5}{|l|}{ Proliferative (non-neoplastic) } \\
\hline Hyperplasia, urothelium & & $\mathrm{x}$ & & \\
\hline Metaplasia, glandular & & $\mathrm{x}$ & & \\
\hline Metaplasia, squamous cell & & $\mathrm{x}$ & & \\
\hline
\end{tabular}

Table 55. Microscopic Findings in the Urinary System: Urethra; Non-human Primate

\begin{tabular}{|c|c|c|c|c|}
\hline Urethra & Common & Uncommon & $\begin{array}{c}\text { Not Observed but } \\
\text { Potentially Relevant }\end{array}$ & Not Applicable \\
\hline \multicolumn{5}{|l|}{ Non-proliferative } \\
\hline Infiltrate, [insert appropriate cell type] ${ }^{\wedge}$ & & $\mathrm{x}$ & & \\
\hline Obstruction & & $\mathrm{x}$ & & \\
\hline Plug, proteinaceous & & & $\mathrm{x}$ & \\
\hline \multicolumn{5}{|l|}{ Proliferative (non-neoplastic) } \\
\hline Hyperplasia, urothelium & & $\mathrm{x}$ & & \\
\hline Metaplasia, glandular & & $\mathrm{x}$ & & \\
\hline Metaplasia, squamous cell & & $\mathrm{x}$ & & \\
\hline
\end{tabular}

Table 56. Microscopic Findings in the Urinary System: Urinary Bladder; Non-human Primate

\begin{tabular}{|c|c|c|c|c|}
\hline Urinary Bladder & Common & Uncommon & $\begin{array}{c}\text { Not Observed but } \\
\text { Potentially Relevant }\end{array}$ & Not Applicable \\
\hline \multicolumn{5}{|l|}{ Congenital } \\
\hline Diverticulum & & $\mathrm{x}$ & & \\
\hline Remnant, umbilical artery & & $\mathrm{x}$ & & \\
\hline \multicolumn{5}{|l|}{ Non-proliferative } \\
\hline Angiectasis & & $\mathrm{x}$ & & \\
\hline Calculus & & $\mathrm{x}$ & & \\
\hline Crystals & & $\mathrm{x}$ & & \\
\hline Dilatation & & $\mathrm{x}$ & & \\
\hline Edema $^{\wedge}$ & & $\mathrm{x}$ & & \\
\hline Erosion & & $\mathrm{x}$ & & \\
\hline${\text { Hemorrhage }{ }^{\wedge}}^{\wedge}$ & & $\mathrm{x}$ & & \\
\hline Hypertrophy, urothelium & & $\mathrm{x}$ & & \\
\hline Inclusions, eosinophilic inctracytoplasmic $* \wedge$ & $\mathrm{x}$ & & & \\
\hline 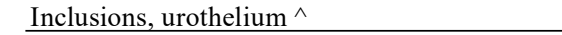 & & $\mathrm{x}$ & & \\
\hline Infiltrate, [insert appropriate cell type] ${ }^{\wedge}$ & & $\mathrm{x}$ & & \\
\hline Inflammation $^{\wedge}$ & & $\mathrm{x}$ & & \\
\hline Mineralization $^{\wedge}$ & & $\mathrm{x}$ & & \\
\hline Necrosis & & $\mathrm{x}$ & & \\
\hline Parasite $^{\wedge}$ & & & $\mathrm{x}$ & \\
\hline Plug, proteinaceous & & & $\mathrm{x}$ & \\
\hline Ulcer & & $\mathrm{x}$ & & \\
\hline Uropathy, obstructive & & $\mathrm{x}$ & & \\
\hline Vacuolation, urothelium & $\mathrm{x}$ & & & \\
\hline \multicolumn{5}{|l|}{ Proliferative (non-neoplastic) } \\
\hline Hyperplasia, urothelium & & $\mathrm{x}$ & & \\
\hline Mesenchymal proliferative lesion & & & $\mathrm{x}$ & \\
\hline Metaplasia, glandular & & $\mathrm{x}$ & & \\
\hline Metaplasia, squamous cell & & $\mathrm{x}$ & & \\
\hline
\end{tabular}




\section{Ethical Practices}

All procedures used to prepare macroscopic and microscopic images of animal specimens for this article were performed in accordance with regulations and established guidelines for humane treatment of research animals and were reviewed and approved in advance by an Institutional Animal Care and Use Committee.

\section{ACKNOWLEDGEMENTS}

The authors wish to express their sincere appreciation to multiple STP memberships (in particular STP, JSTP, ESTP, BSTP) for their comprehensive reviews and excellent comments. Photographs used in this document were provided by coauthors and by Drs. Cindy Farman (StageBio, Nevada), Jennifer Chilton (Charles River Laboraroties, Nevada), Ingrid Pardo (Pfizer, Connecticut), Sanjeev Gumber (Yerkes National Primate Research Center, Emory University, Georgia), Molly Boyle (Covance, California), Norimitsu Shirai (Pfizer, Connecticut) and Kendall Frazier (Florida). In addition, we acknowledge David Sabio (EPL Inc., North Carolina) and Ms. Beth Mahler (EPL, NIH/NIEHS, NC), for image collation, proofing and editing. Finally, we would like to acknowledge the work of Dr Rupert Kellner and his colleagues at the Fraunhofer Institute for Toxicology and Experimental Medicine, for his thorough review of the manuscript contents and images, and the insertion of nomenclature and images into goRENI. 

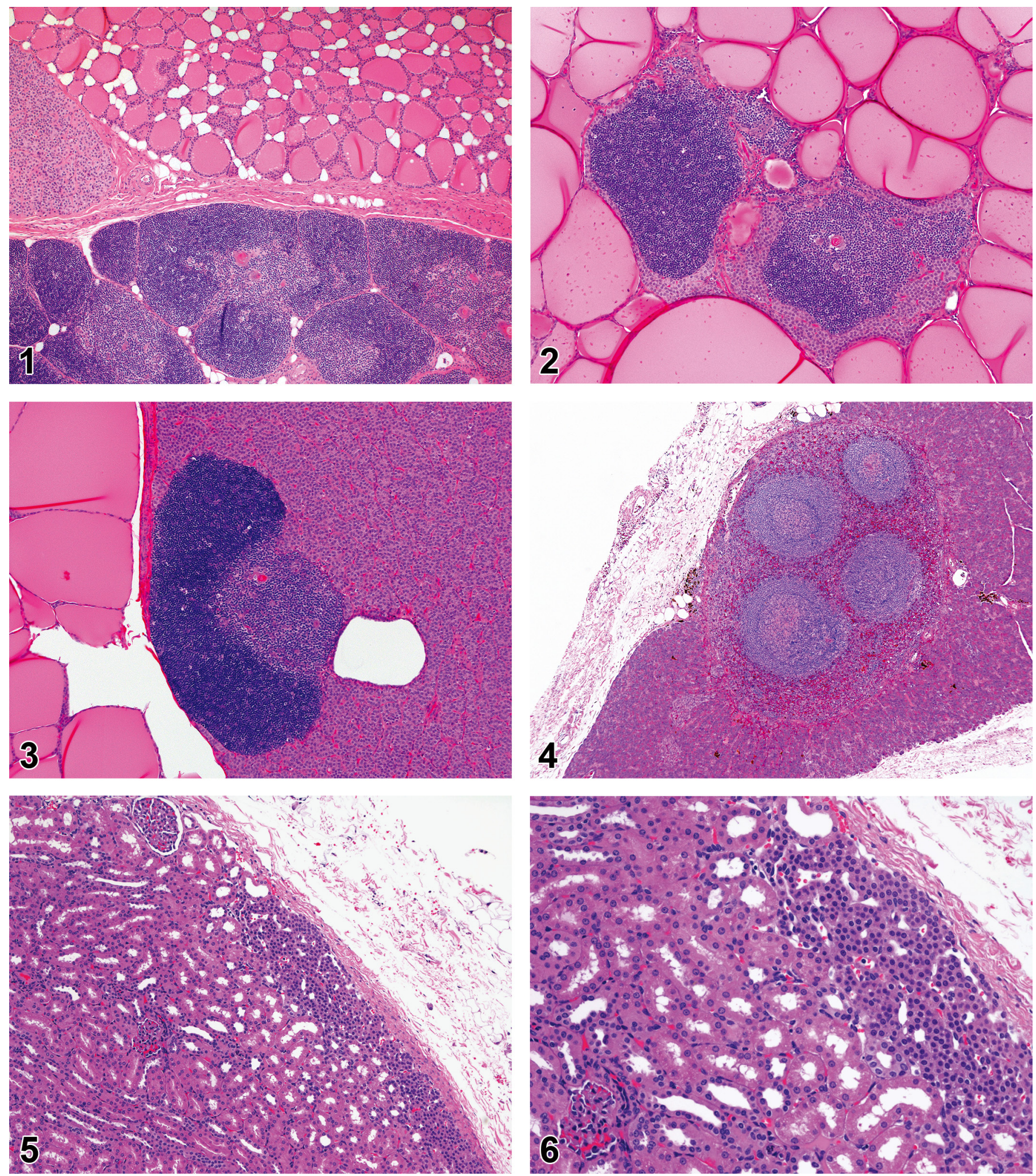

Figure 1.-Cynomolgus, Thyroid, Ectopic tissue, thymus, H\&E, Low magnification.

Figure 2.-Cynomolgus, Thyroid, Ectopic tissue, thymus, H\&E, High magnification.

Figure 3.-Cynomolgus, Parathyroid gland, Ectopic tissue, thymus, H\&E.

Figure 4.-Cynomolgus, Pancreas, Ectopic tissue spleen H\&E.

Figure 5. Cynomolgus, Kidney, Ectopic tissue, adrenal cortex, H\&E.

Figure 6.-Cynomolgus, Kidney, Ectopic tissue, adrenal cortex, H\&E. 

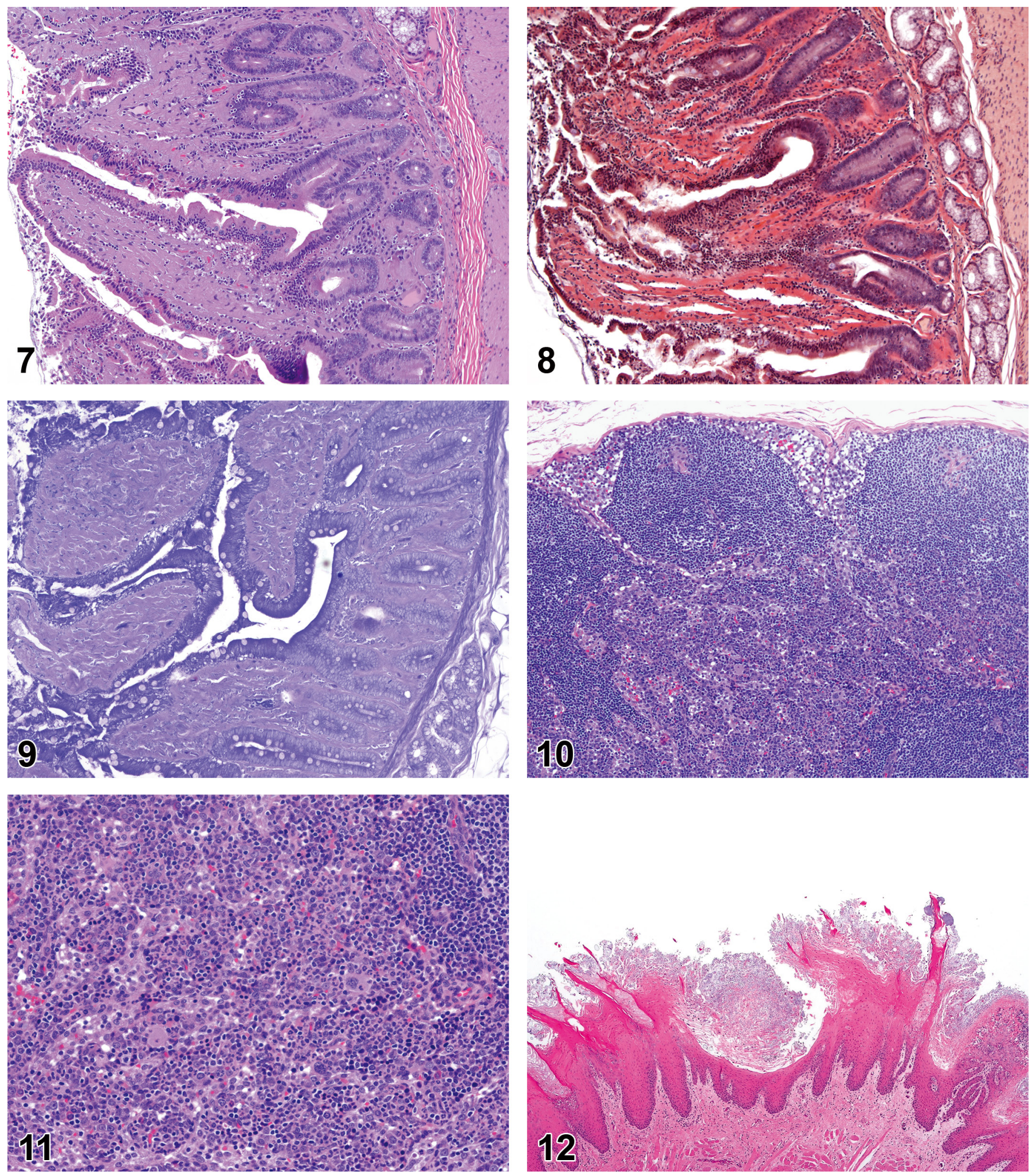

Figure 7.-Cynomolgus, Duodenum, Amyloid, H\&E.

Figure 8.-Cynomolgus, Duodenum, Amyloid, congo red.

Figure 9.-Cynomolgus, Duodenum, Amyloid, crystal violet.

FIGURE 10.-Cynomolgus, Lymph node, submandibular, Extramedullary hematopoiesis, H\&E, Low magnification.

Figure 11.-Cynomolgus, Lymph node, submandibular, Extramedullary hematopoiesis, H\&E, High magnification.

Figure 12.-Cynomolgus, Tongue, Fungus, Candida spp., H\&E, Low magnification; Courtesy of Dr. Cindy Farman. 


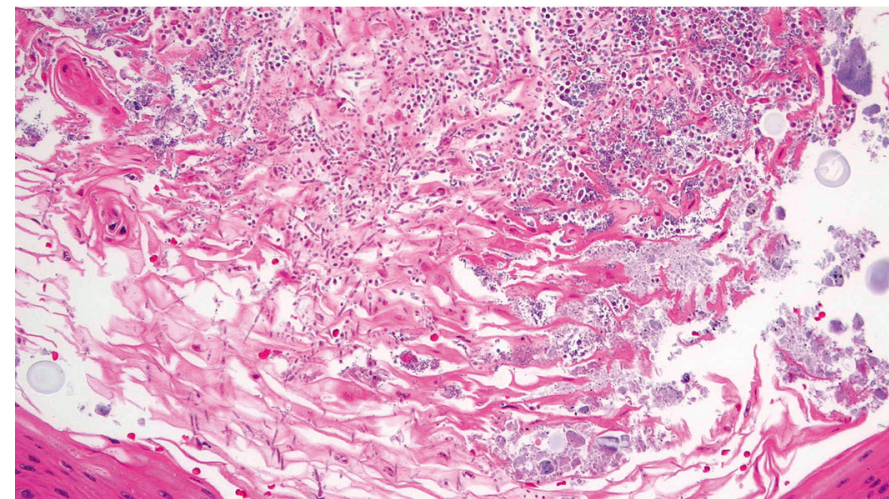

13
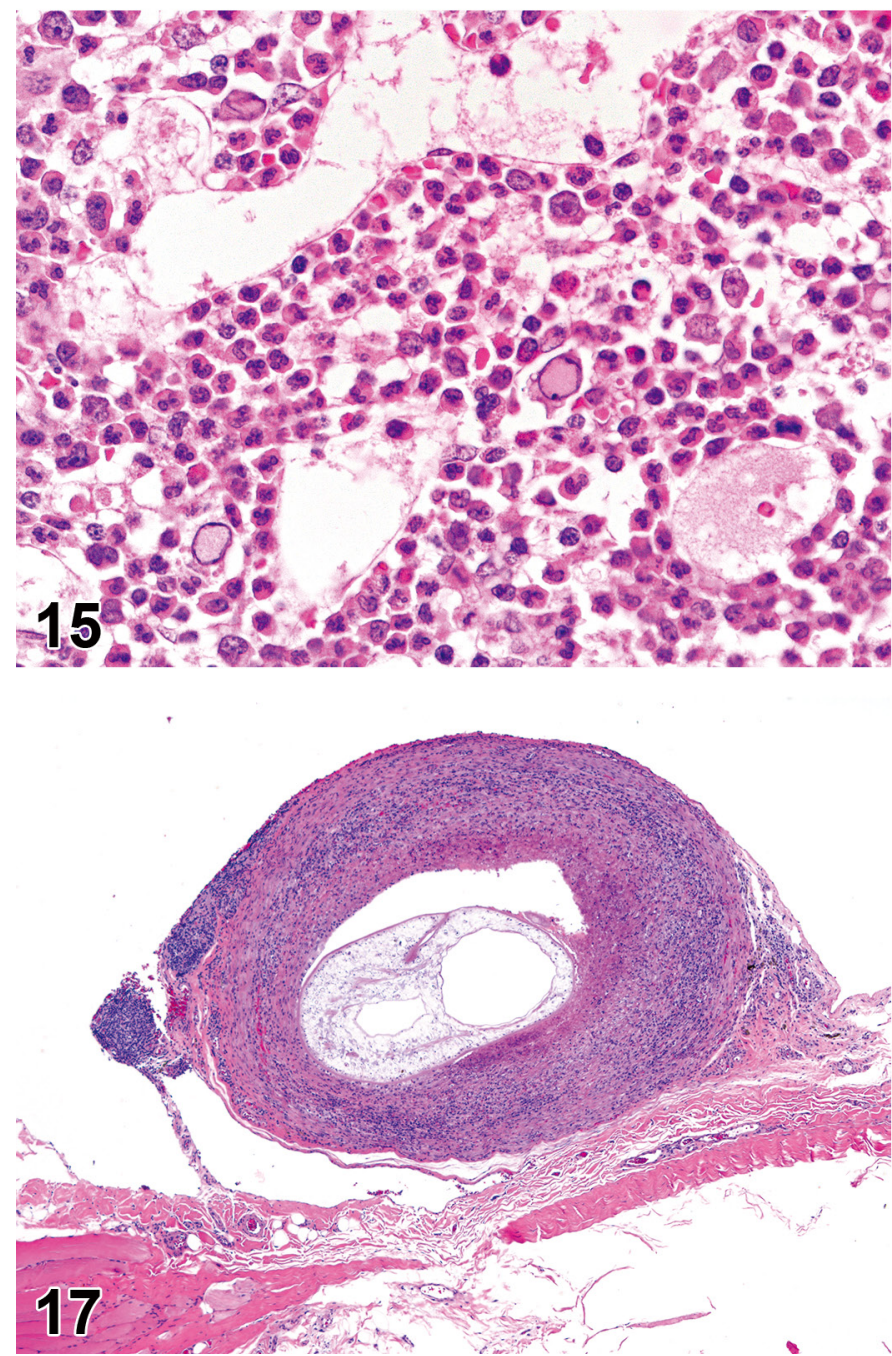
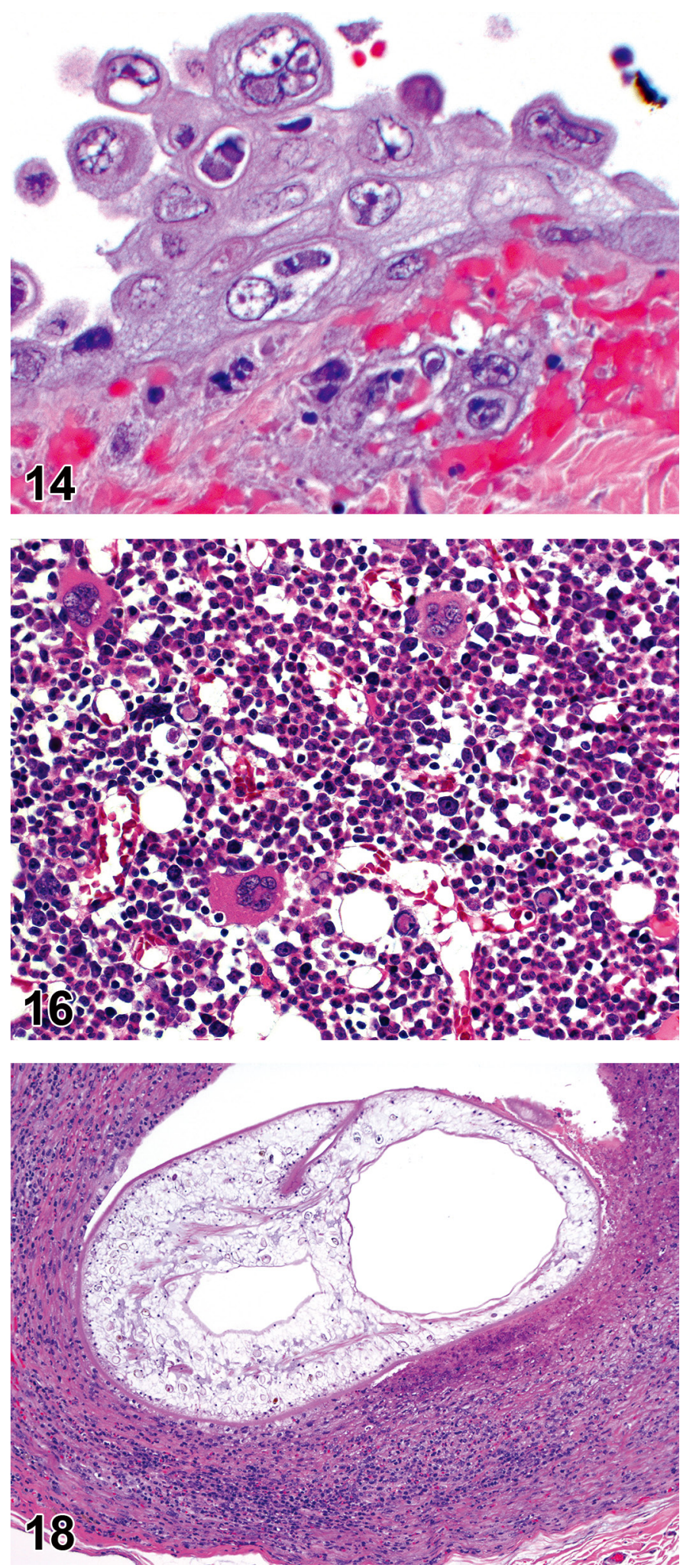

Figure 13.-Cynomolgus, Tongue, Fungus, Candida spp., H\&E, High magnification; Courtesy of Dr. Cindy Farman.

Figure 14.-Cynomolgus, Skin, Intranuclear inclusions, simian varicella virus, H\&E.

Figure 15.- Cynomolgus, Bone marrow, Intranuclear inclusions, simian parvovirus, H\&E.

Figure 16. - Cynomolgus, Bone marrow, Erythroid blasts with viral inclusions, simian parvovirus, H\&E.

Figure 17.-Cynomolgus, Diaphram, Parasitic granuloma, cestode, H\&E, Low magnification.

Figure 18.-Cynomolgus, Diaphram, Parasitic granuloma, cestode, H\&E, High magnification. 

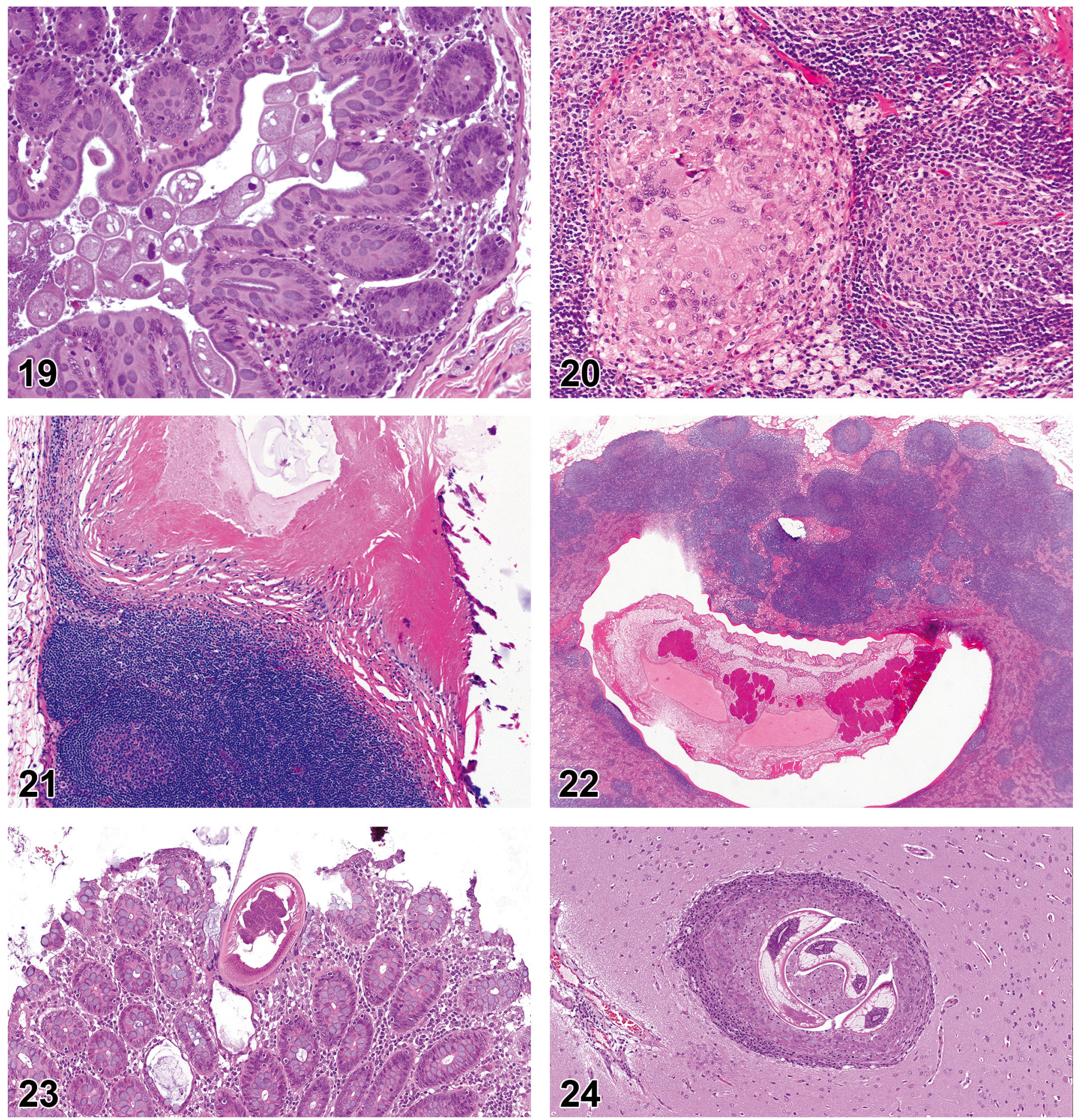

Figure 19.-Cynomolgus, Cecum, Parasite, Balantidium coli, H\&E. Figure 20.-Cynomolgus, Lymph node, Parasitic granuloma, H\&E. Figure 21.-Cynomolgus, Lymph node, Parasite, remnants H\&E. Figure 22.-Cynomolgus, Lymph node, mesenteric, Parasite, H\&E. Figure 23.-Rhesus, Cecum, Parasite, nematode H\&E.

FIgURE 24.-Rhesus, Brain, Parasitic granuloma, Baylisascaris spp. H\&E. 

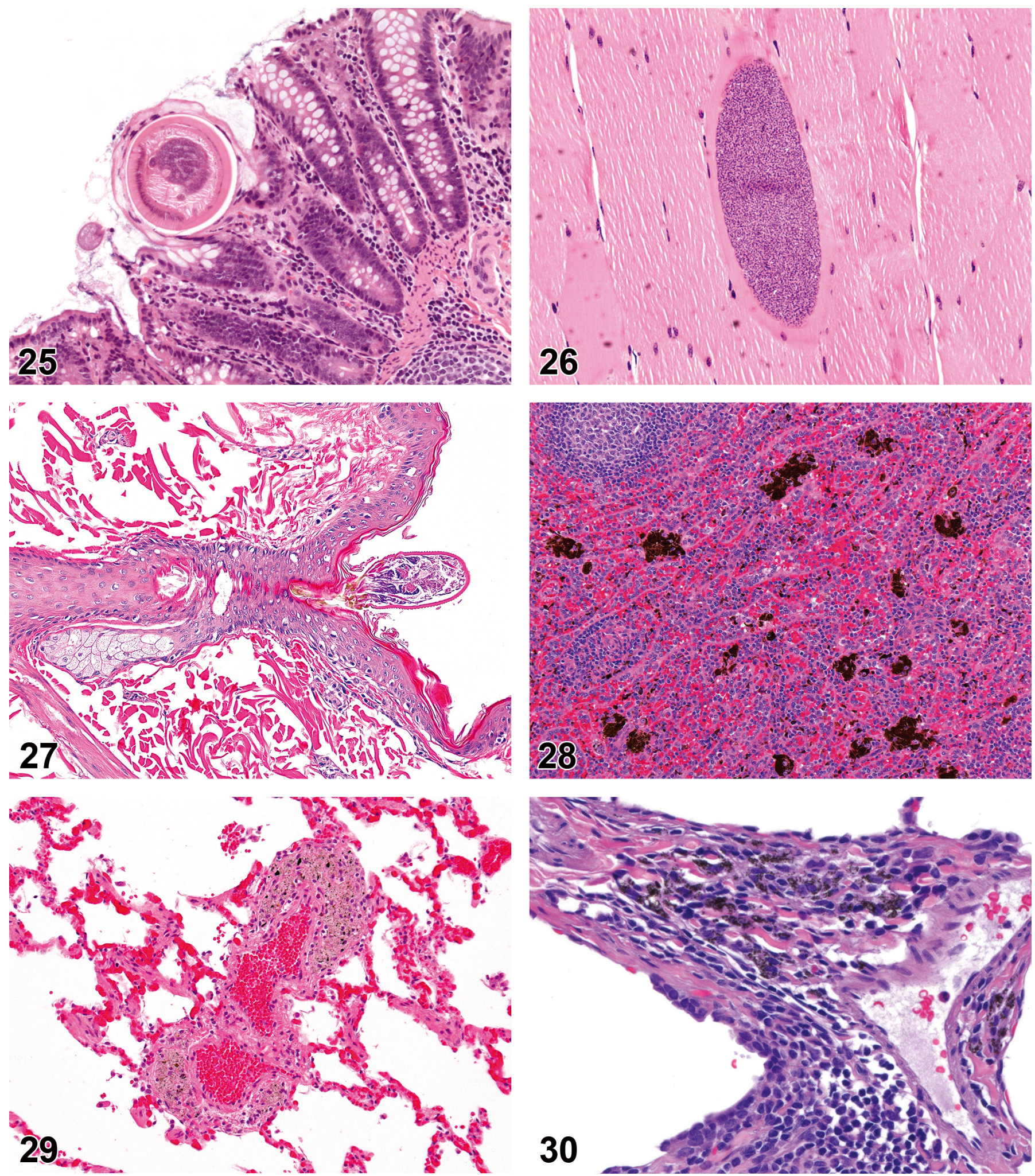

Figure 25.-NHP, Rectum, Parasite, Balantidium coli and Trichuris spp. H\&E.

Figure 26.- NHP, Skeletal muscle, Parasite, Sarcocystis H\&E.

Figure 27.-Cynomolgus, Skin, Parasite, acarid H\&E.

Figure 28.-Cynomolgus, Spleen, Pigment, malaria pigment, H\&E.

Figure 29. - Cynomolgus, Lung, Pigment, dust-like with birefringent crystals, H\&E.

Figure 30.-Cynomolgus, Lung, Pigment, peribronchiolar, H\&E. 

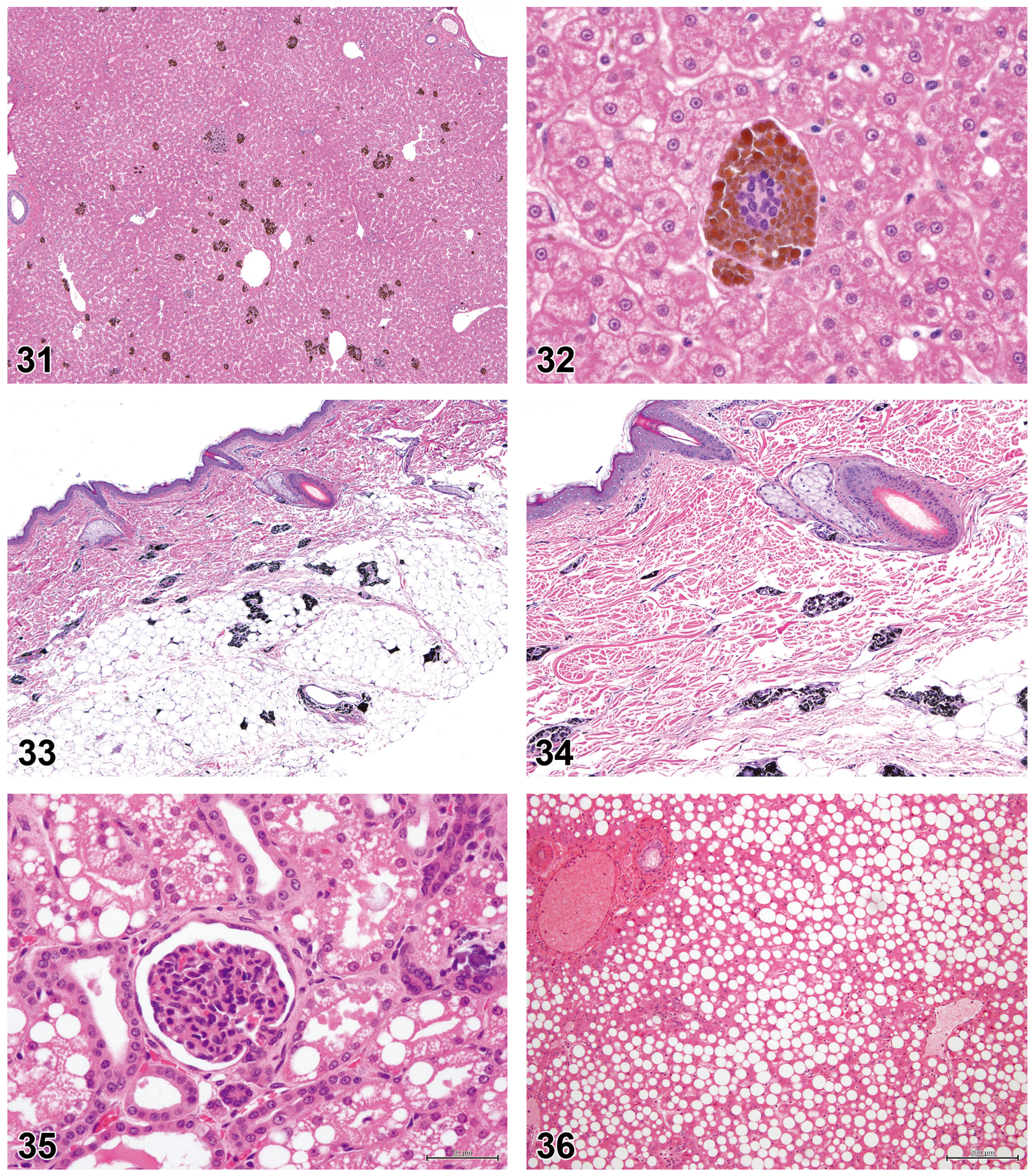

Figure 31.-Cynomolgus, Liver, Pigment, golden brown, H\&E, Low magnification.

Figure 32.-Cynomolgus, Liver, Pigment, golden brown, H\&E, High magnification.

Figure 33.-Cynomolgus, Skin, Skin/mammary gland, Pigment, tattoo ink, H\&E, Low magnification.

Figure 34.-Cynomolgus, Skin, Skin/mammary gland, Pigment, tattoo ink, H\&E, High magnification.

Figure 35.-Cynomolgus, Kidney, Tubular vacuolation, fatal fasting syndrome, H\&E.

Figure 36.-Cynomolgus, Liver, Vacuolation, fatal fasting syndrome, H\&E. 

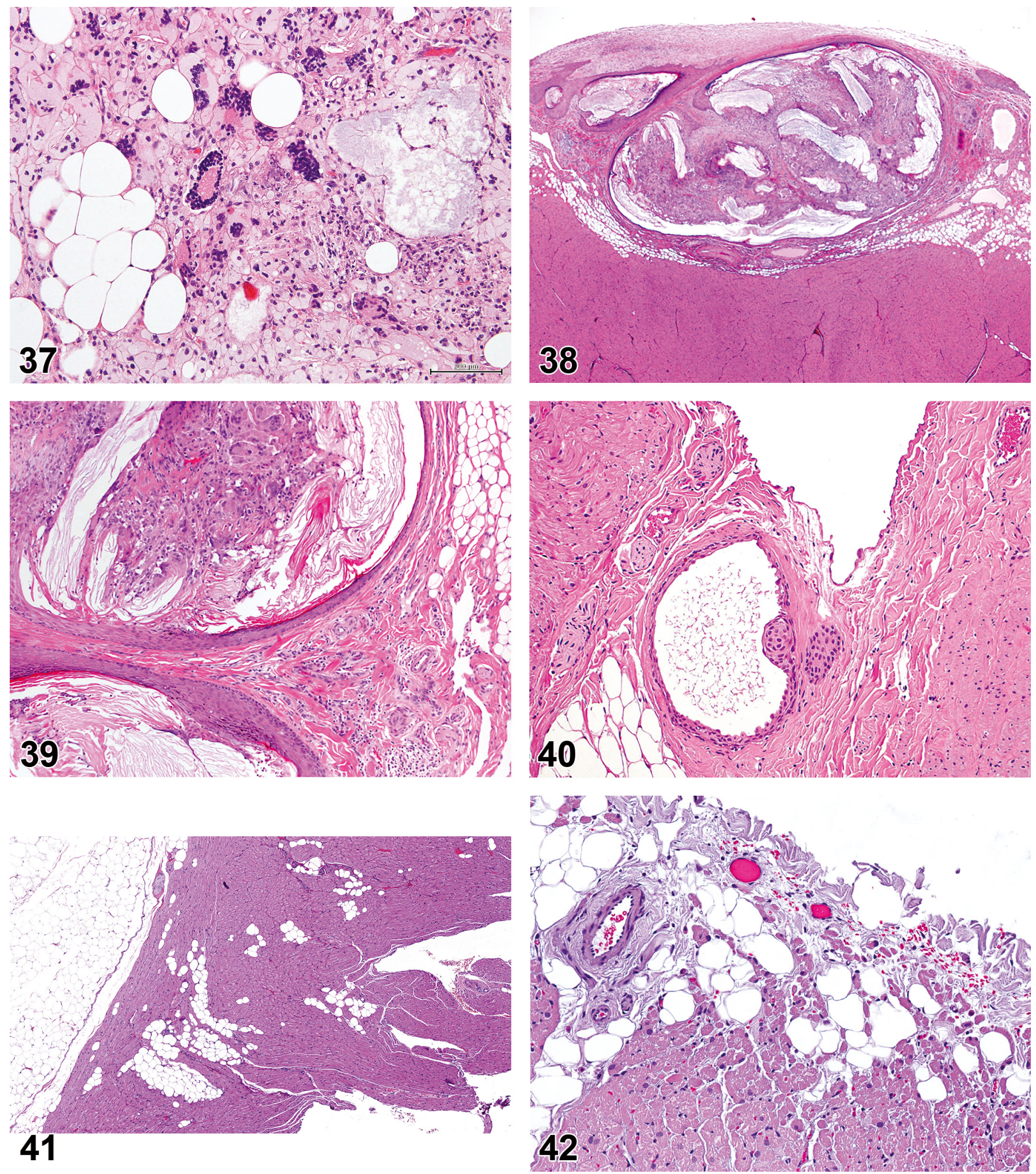

Figure 37.-Cynomolgus, Adipose tissue, Necrosis and inflammation, fatal fasting syndrome, H\&E.

Figure 38.-Cynomolgus, Heart, Cyst/plaque, squamous/epithelial cell, H\&E, Low magnification.

Figure 39.- Cynomolgus, Heart, Cyst/plaque, squamous/epithelial cell, H\&E, High magnification.

Figure 40.-Cynomolgus, Heart, Cyst/plaque, squamous/epithelial cell, H\&E, High magnification.

Figure 41.-Cynomolgus, Heart, Accumulation, adipocyte, myocardium, H\&E, Low magnification; Courtesy of Dr. Jennifer Chilton.

Figure 42.-Cynomolgus, Heart, Accumulation, adipocyte, subepicardium, H\&E, High magnification. 

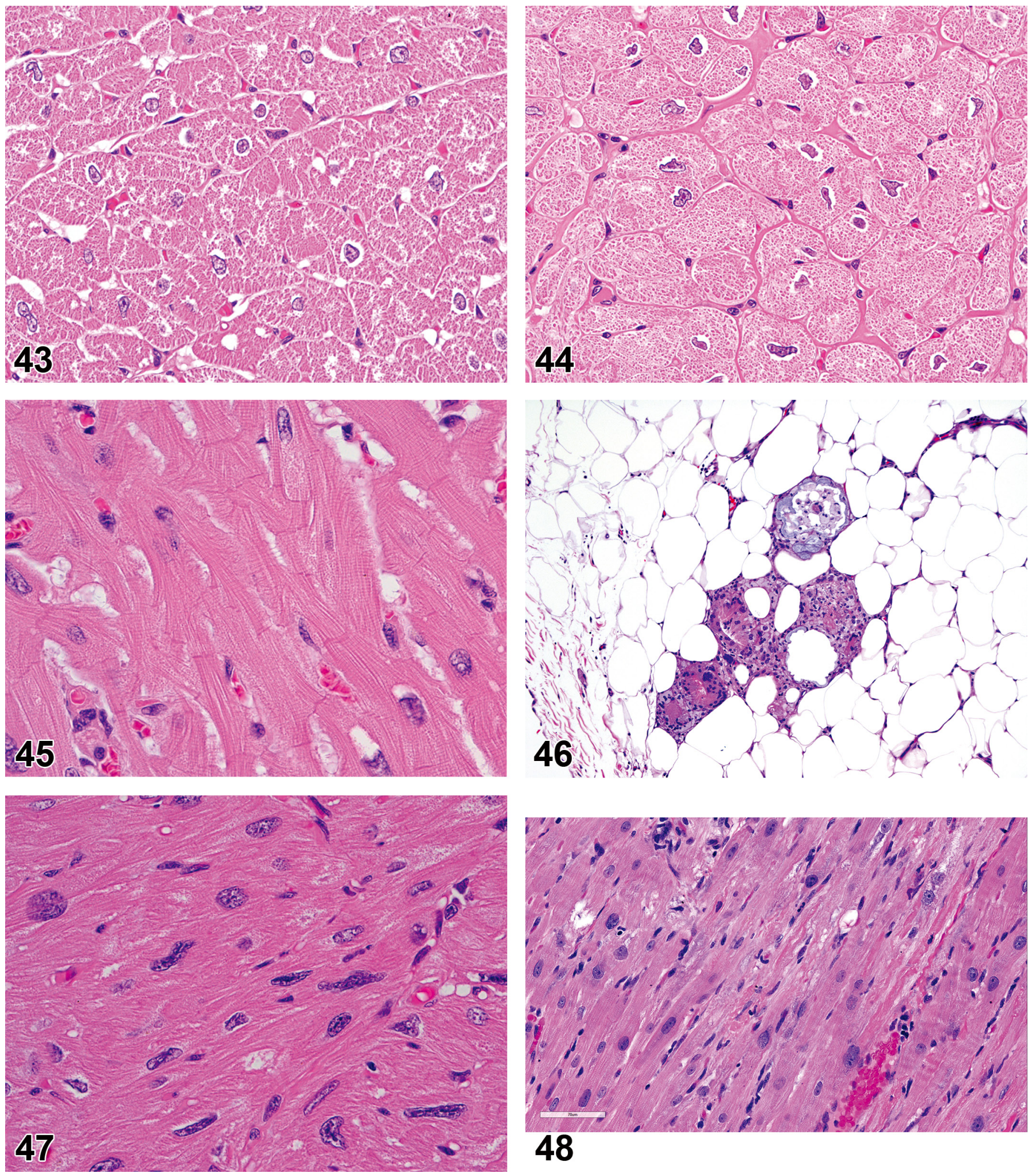

FiguRE 43.-Cynomolgus, Heart, Normal cardiomyocyte profile, H\&E.

Figure 44.-Cynomolgus, Heart, Hypertrophy, cardiomyocyte, H\&E.

FigURE 45.-Cynomolgus, Heart, Hypertrophy, cardiomyocyte with abnormal alignment, H\&E.

FigURE 46.-Cynomolgus, Heart, Inflammation, granulomatous, pericardial fat, H\&E.

FiguRE 47.-Cynomolgus, Heart, Karyomegaly, cardiomyocyte, H\&E.

FIGURE 48.-Cynomolgus, Heart, Karyomegaly/karyocytomegaly, cardiomyocyte, with degeneration, H\&E. 

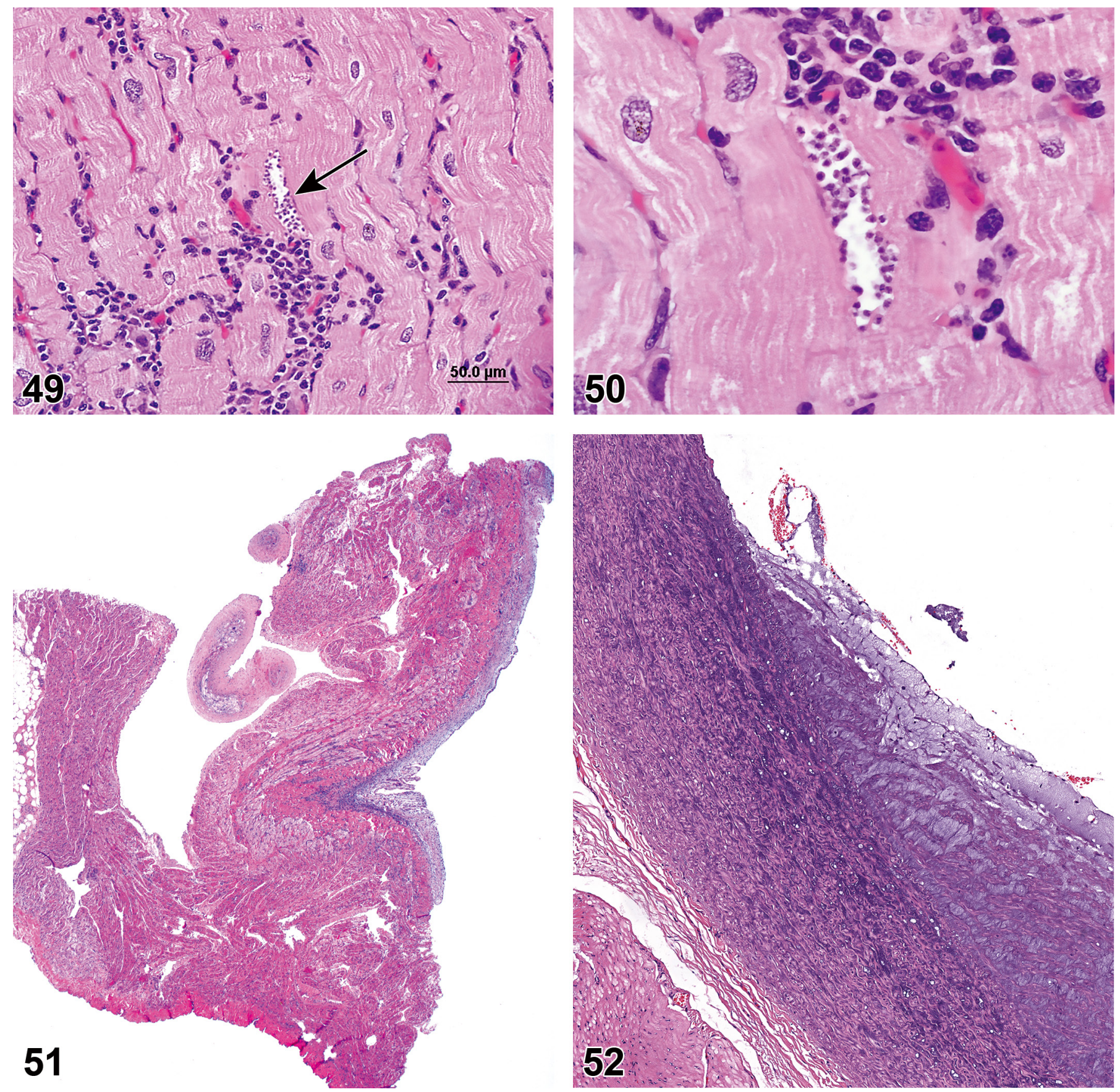

Figure 49.-Cynomolgus, Heart, Parasite, protozoa (T. cruzi) (arrow), H\&E, Low magnification.

Figure 50.-Cynomolgus, Heart, Parasite, protozoa (T. cruzi), H\&E, High magnification (oil).

Figure 51.-Cynomolgus, Heart, Hyperplasia, mesothelium, H\&E.

Figure 52.-Cynomolgus, Blood vessel/Aorta, Atherosclerosis, H\&E; Courtesy of Dr. Jennifer Chilton. 

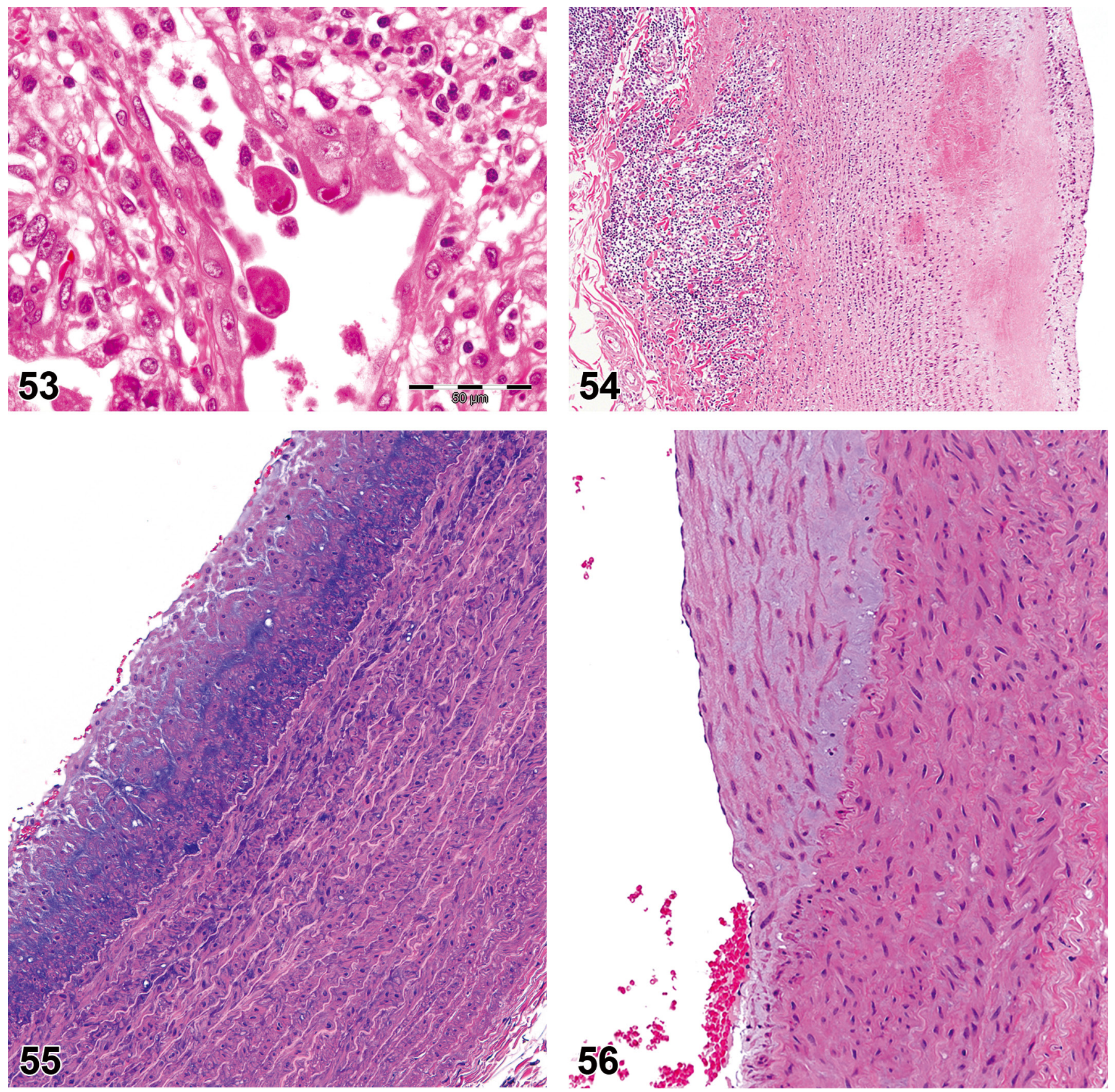

Figure 53.-Cynomolgus, Blood vessel, Cytomegaly, endothelium, H\&E.

FIGURE 54.-Cynomolgus, Blood vessels/Aorta, Degeneration/necrosis, medial/mural, H\&E.

Figure 55.-Cynomolgus, Blood vessels/Aorta, Mucinous change, H\&E, Low magnification.

Figure 56.-Cynomolgus, Blood vessels/Aorta, Mucinous change, H\&E, High magnification. 

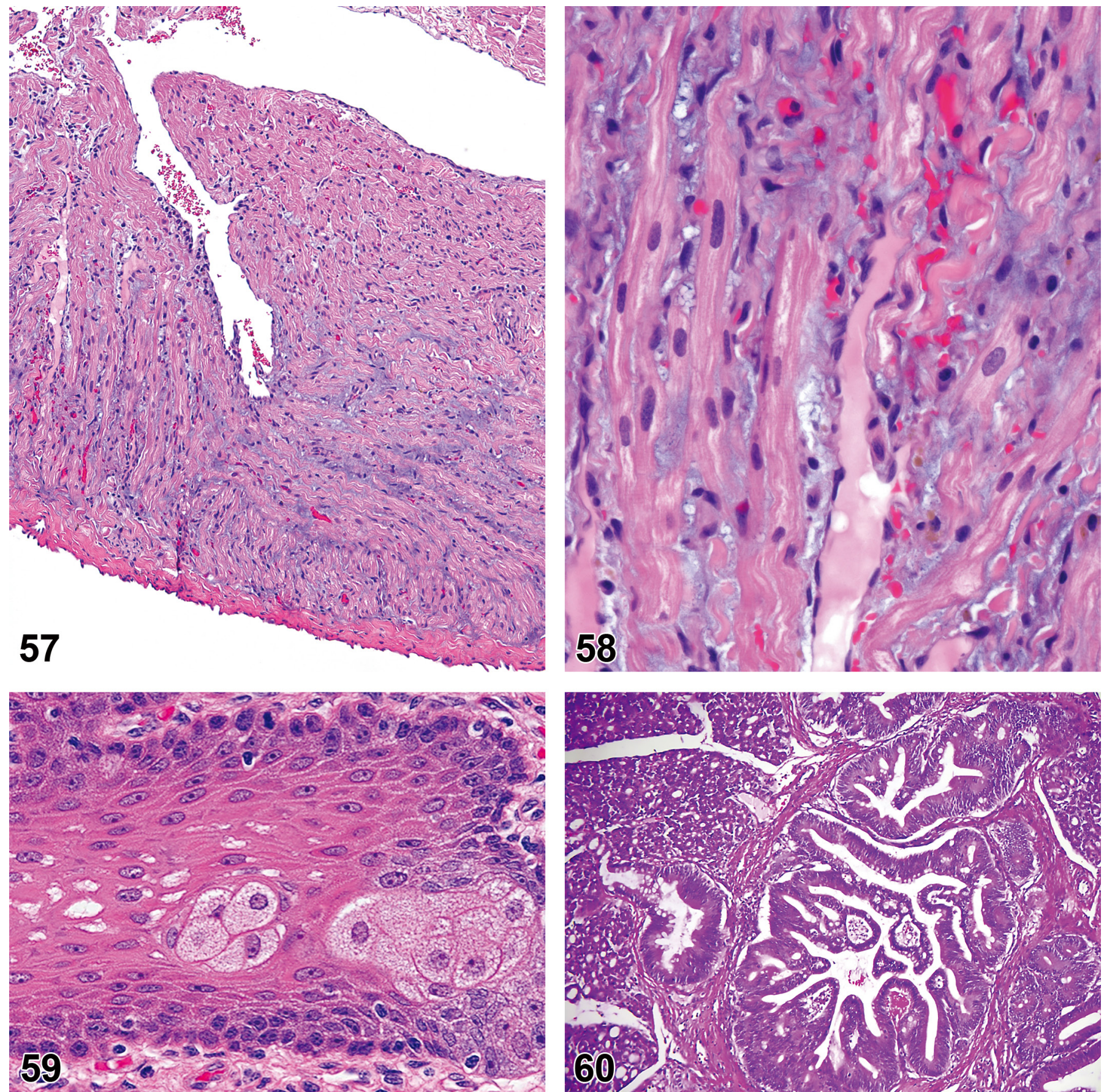

Figure 57.-Cynomolgus, Heart, Mucinous change, atrium, H\&E, Low magnification.

Figure 58. - Cynomolgus, Heart, Mucinous change, atrium, H\&E, High magnification.

Figure 59.-NHP, Esophagus, Metaplasia, sebaceous gland cell H\&E.

Figure 60.-Cynomolgus, Pancreas, Hyperplasia, ductal cell, H\&E. 

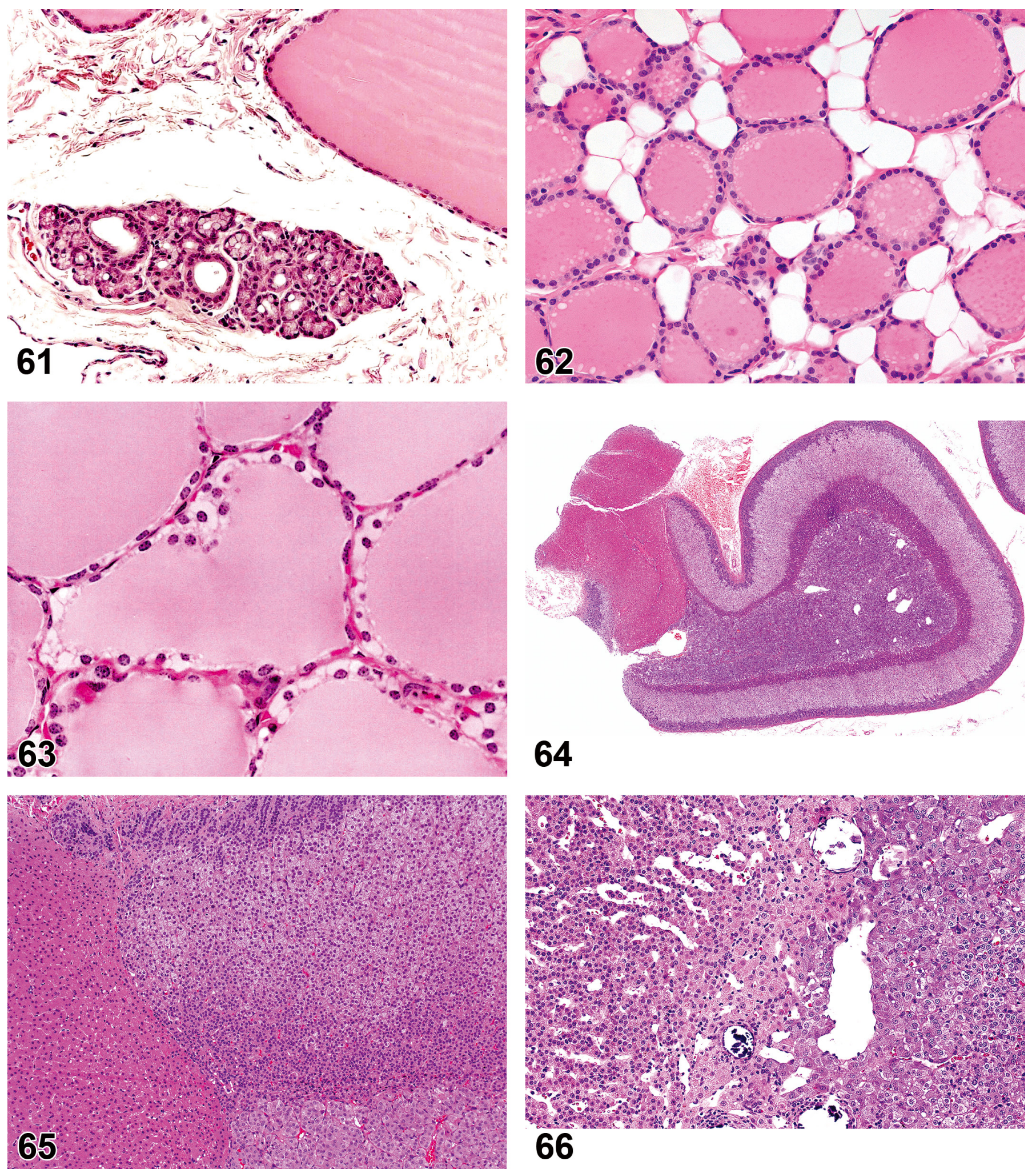

\section{6}

FIGURE 61.-Cynomolgus, Thyroid/parathyroid, Ectopic tissue, salivary gland, H\&E.

Figure 62.-Cynomolgus, Thyroid/parathyroid, Accumulation, adipocytes, H\&E.

Figure 63.-Cynomolgus, Thyroid, Degeneration, vacuolar, H\&E.

Figure 64.-Cynomolgus, Adrenal gland, Adrenohepatic fusion, H\&E, Low magnification.

Figure 65.-Cynomolgus, Adrenal gland, Adrenohepatic fusion, H\&E, High magnification.

FIgure 66.-Cynomolgus, Adrenal gland, Involution, fetal cortex, H\&E; Courtesy of Dr. Jennifer Chilton. 

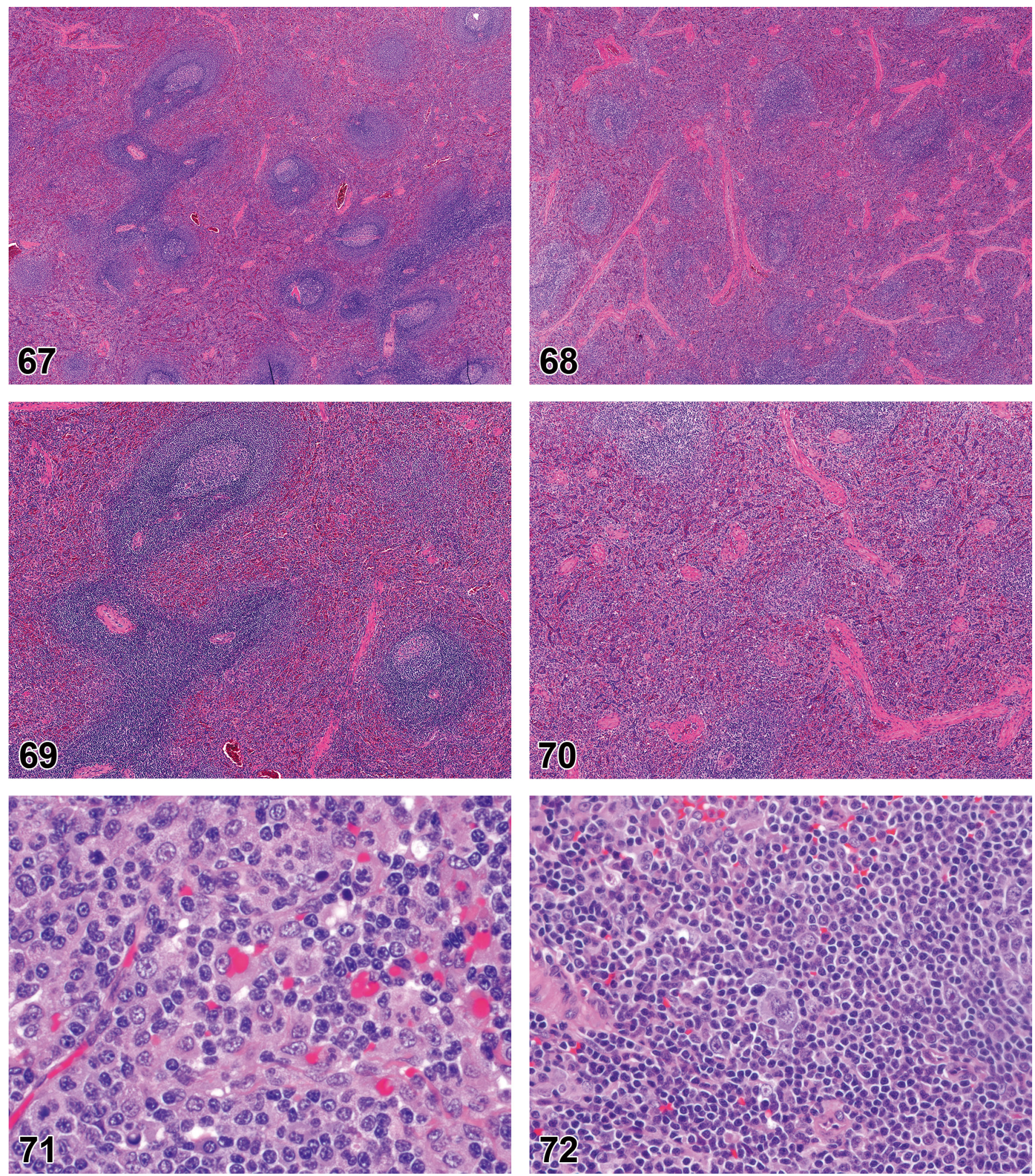

FIGURE 67.-Cynomolgus, Spleen, Lymphoid follicle germinal centers: replete for comparison, H\&E, Low magnification. Figure 68.-Cynomolgus, Spleen, Decreased number and size, germinal centers, H\&E, Low magnification.

Figure 69.- Cynomolgus, Spleen, Lymphoid follicle germinal centers: replete for comparison, H\&E, High magnification.

Figure 70.-Cynomolgus, Spleen, Decreased number and size, germinal centers, H\&E, High magnification.

Figure 71.- Cynomolgus, Lymph node, Erythrophagocytosis, medullary sinus, H\&E.

FiguRE 72.-Cynomolgus, Spleen, Immunoblasts, increased (Reed-sternberg [RS]-like cell), PAL, H\&E. 

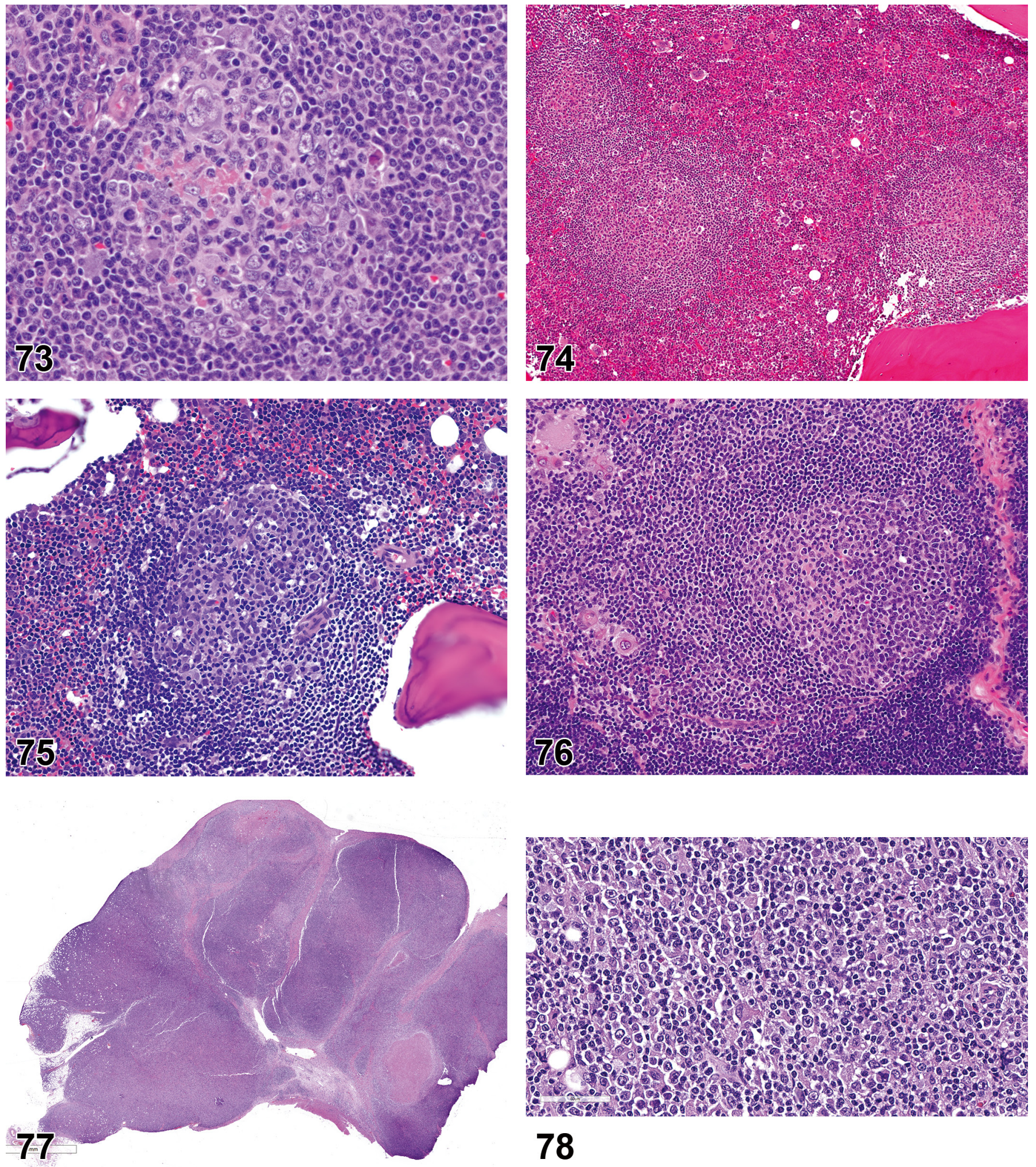

FIGURE 73.-Cynomolgus, Spleen, Immunoblasts, increased (Reed-sternberg [RS]-like cell), PAL, H\&E.

FIGURE 74.-Cynomolgus, Bone marrow, Lymphoid follicle formation, H\&E, Low magnification.

Figure 75.-Cynomolgus, Bone marrow, Lymphoid follicle formation, H\&E, High magnification.

Figure 76.-Cynomolgus, Thymus, medulla, Lymphoid follicle formation, H\&E.

Figure 77.-Cynomolgus, Lymph node, Lymphoma, H\&E, Low magnification.

Figure 78.-Cynomolgus, Lymph node, Lymphoma, H\&E, High magnification. 

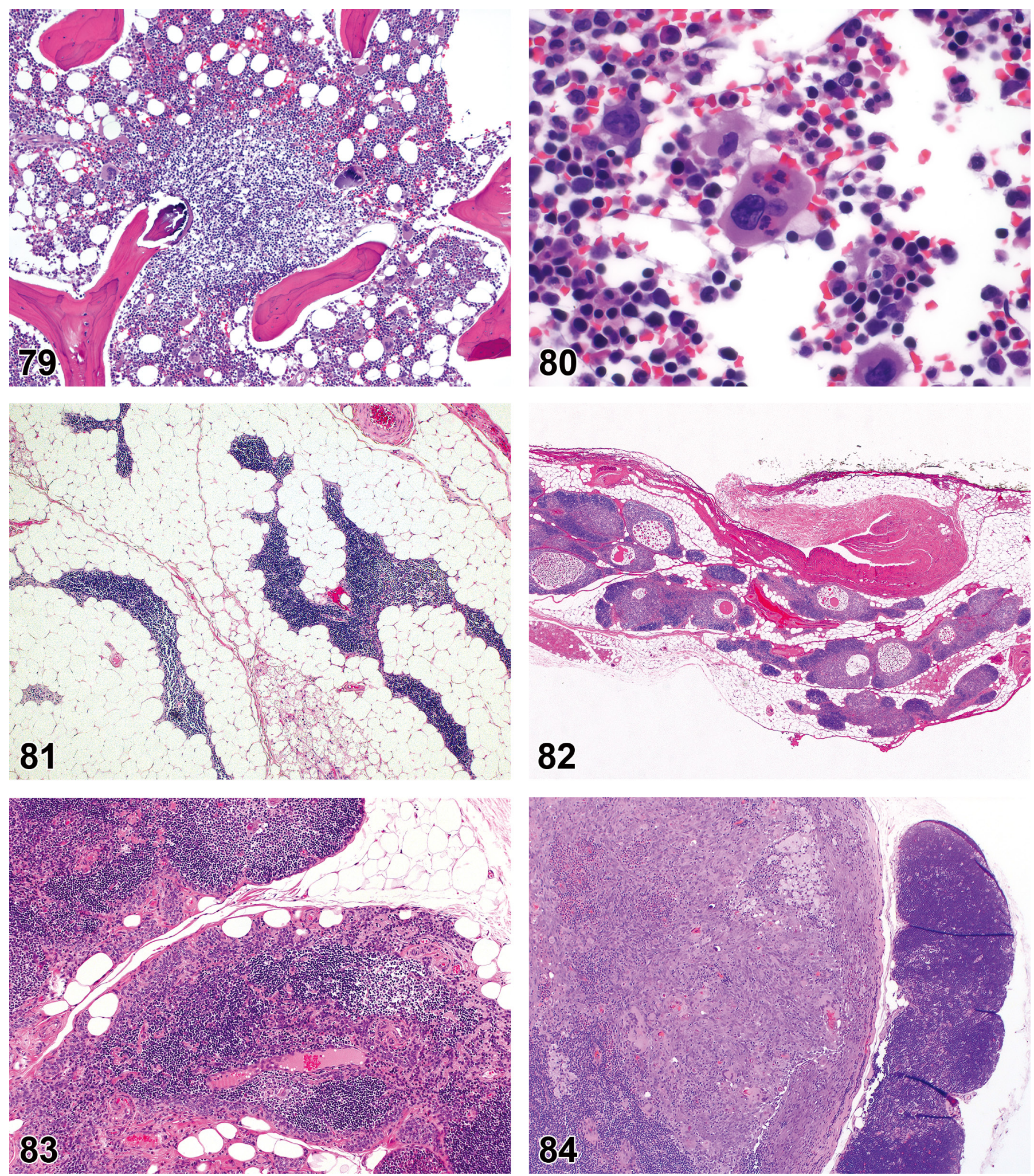

Figure 79.-Cynomolgus, Bone marrow, Lymphoid aggregate, H\&E.

Figure 80.-Cynomolgus, Bone marrow, Emperipolesis, increased, megakaryocyte, H\&E.

Figure 81.-Cynomolgus, Thymus, Cellularity decreased, lymphocytes, severe, H\&E, Low magnification.

Figure 82.-Cynomolgus, Thymus, Cellularity decreased, lymphocytes, with numerous epithelial cysts, H\&E, Low magnification.

Figure 83. - Cynomolgus, Thymus, Cellularity decreased, lymphocytes, H\&E, High magnification.

FIgure 84.-Cynomolgus, Thymus, Thymoma, benign, H\&E, Low magnification. 

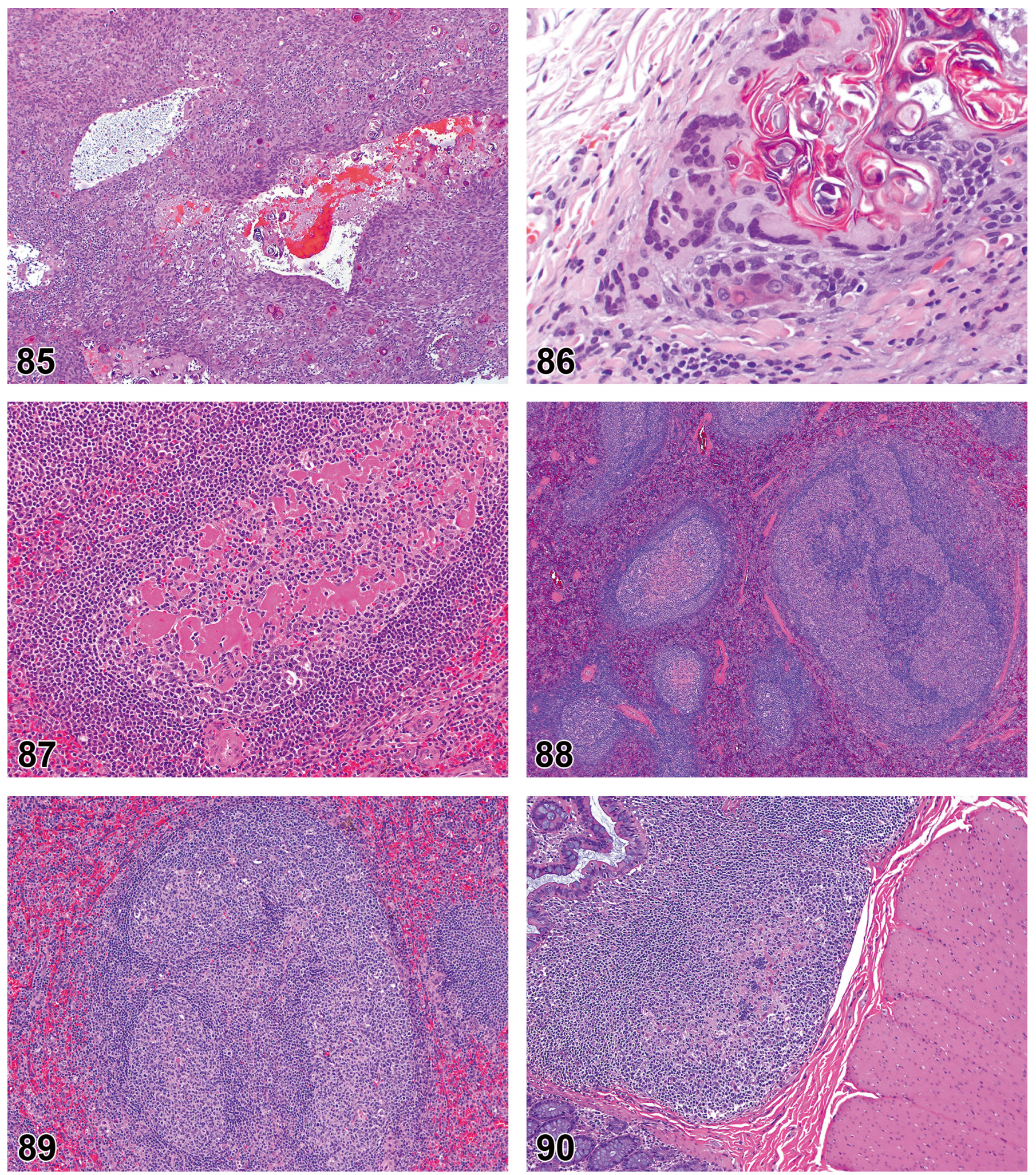

Figure 85.-Cynomolgus, Thymus, Thymoma, benign, H\&E, Higher magnification.

Figure 86.-Cynomolgus, Thymus, Thymoma, benign, H\&E, High magnification.

Figure 87.- Cynomolgus, Spleen, Accumulation acidophilic material, germinal centers, H\&E.

Figure 88.-Cynomolgus, Spleen, Compound follicle, H\&E, Low magnification.

Figure 89.-Cynomolgus, Spleen, Compound follicle, H\&E, Higher magnification.

Figure 90.-Cynomolgus, MALT [GALT], Multinucleate giant cell-lymphocytic syncytia, H\&E. 

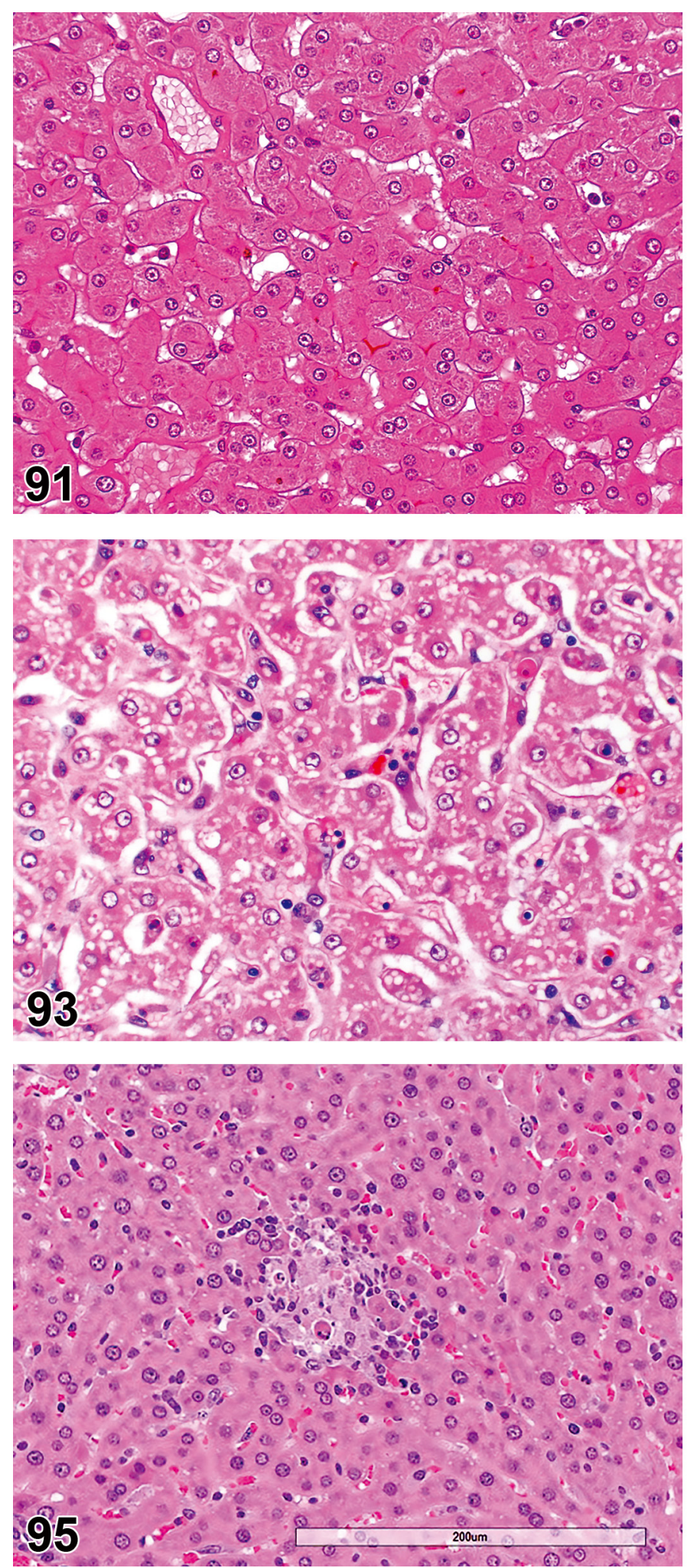
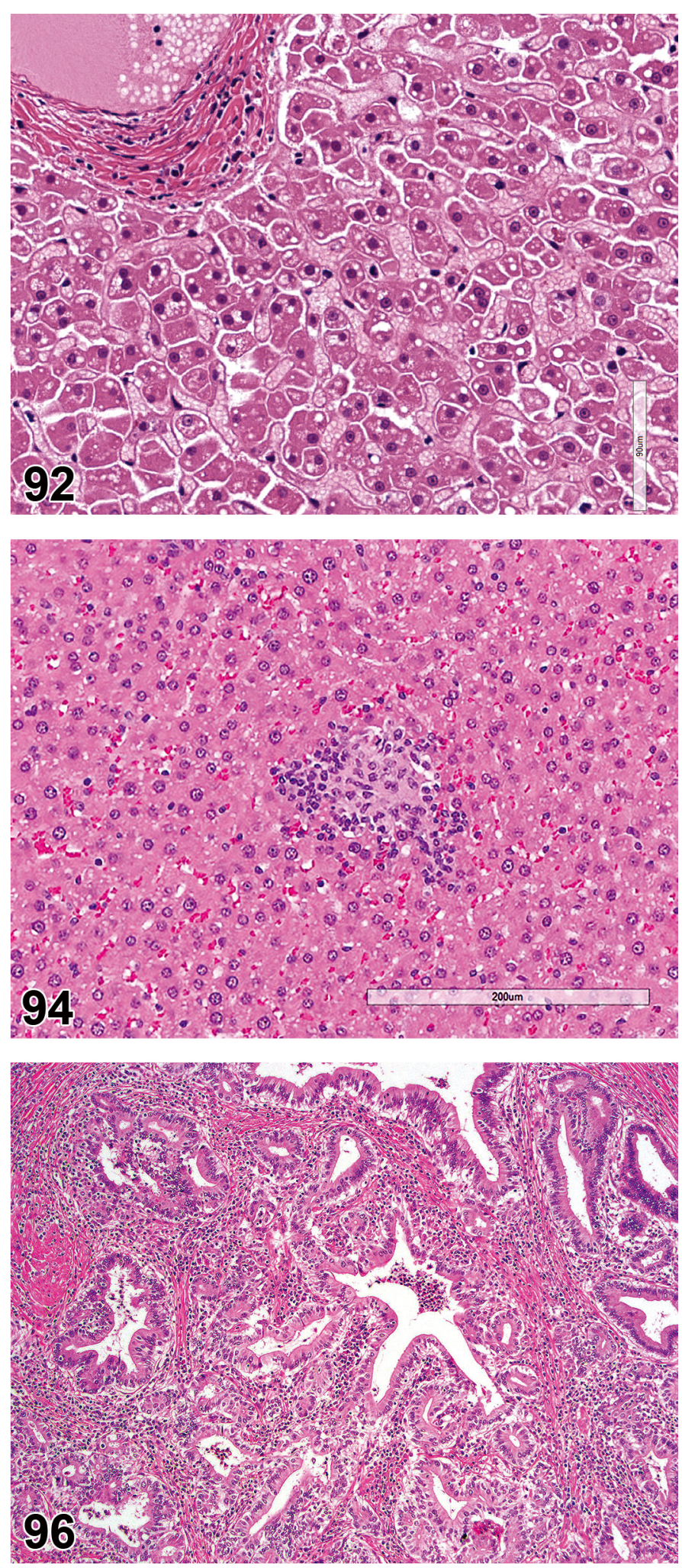

Figure 91.-Cynomolgus, Liver, Bile plugs, intracanalicular, H\&E.

Figure 92.-Cynomolgus, Liver, Dissociation, hepatocyte, H\&E.

Figure 93.-Cynomolgus, Liver, Erythrophagocytosis, Kupffer cell, H\&E.

Figure 94.-Cynomolgus, Liver, Granuloma/microgranuloma, H\&E.

Figure 95.-Cynomolgus, Liver, Granuloma/microgranuloma, with single cell necrosis, H\&E.

Figure 96.-Cynomolgus, Liver, Parasite, Cryptosporidia spp., H\&E, Low magnification. 

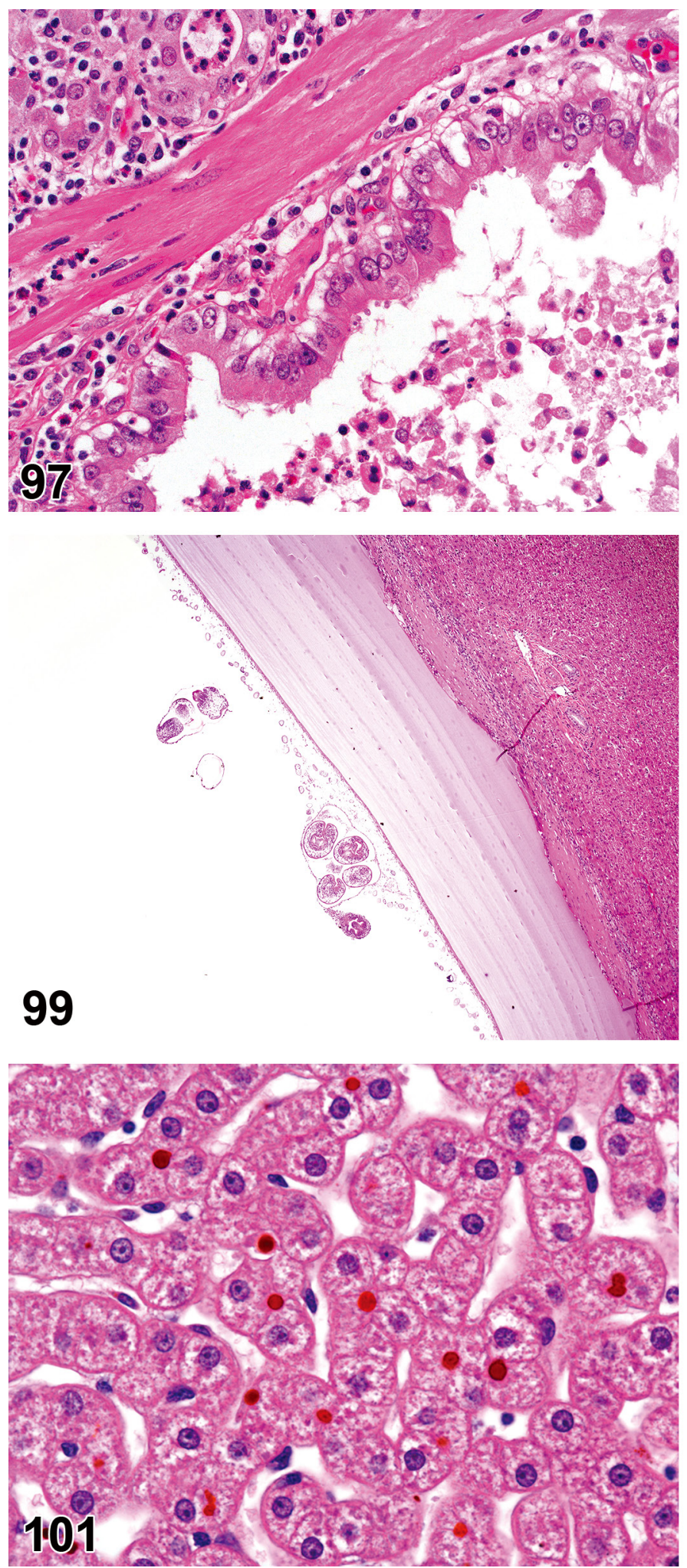
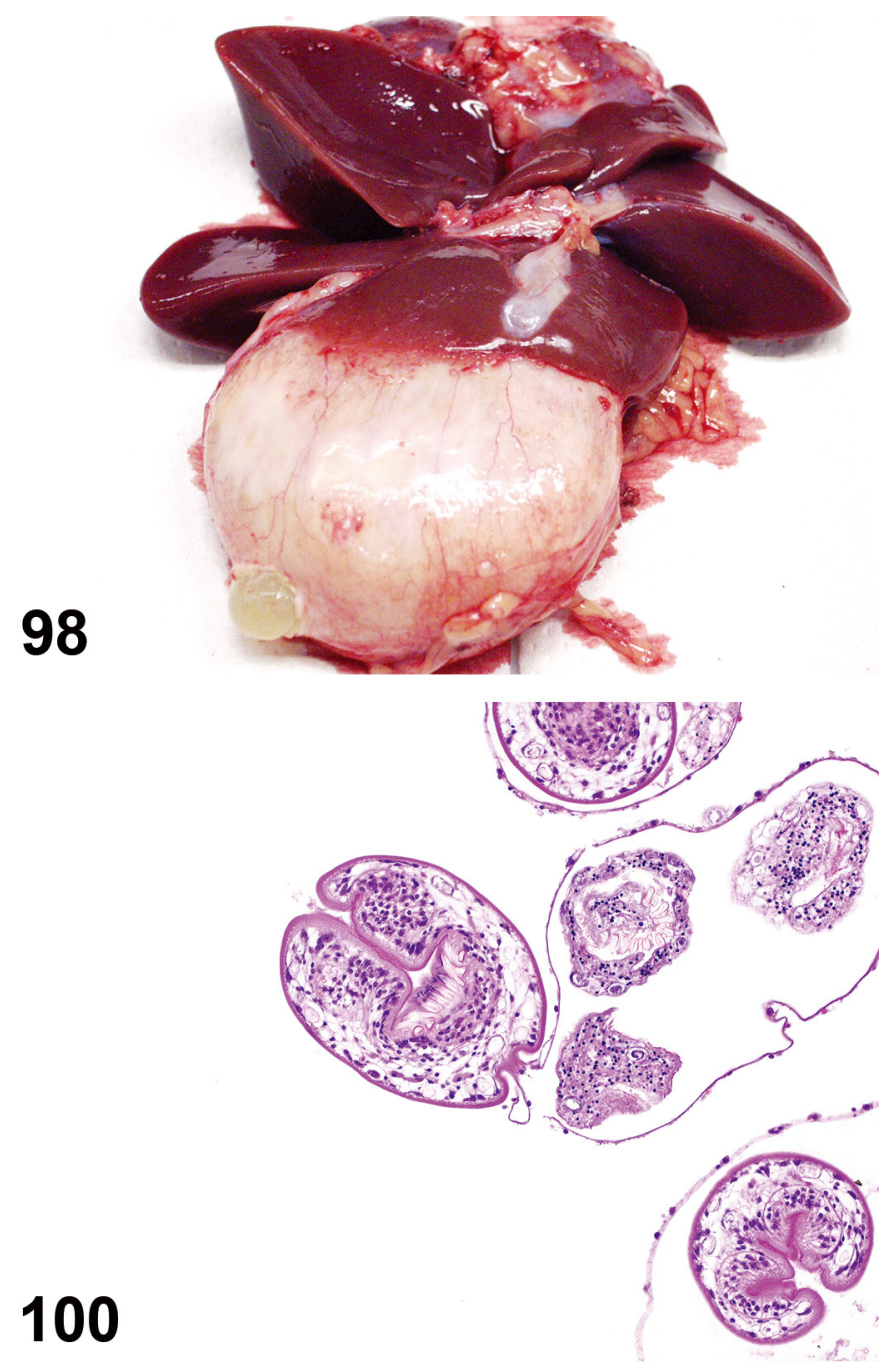

100

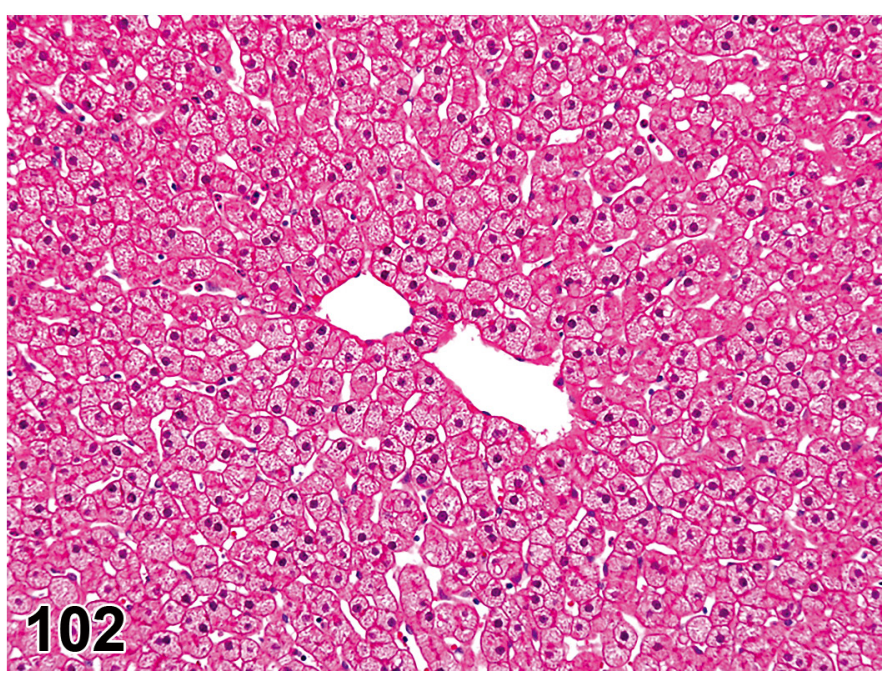

Figure 97.-Cynomolgus, Liver, Parasite, Cryptosporidia spp., H\&E, High magnification.

Figure 98.-Cynomolgus, Liver, Parasite, Echinococcus spp., gross observation.

Figure 99.-Cynomolgus, Liver, Parasite, Echinococcus spp., H\&E, Low magnification.

Figure 100.-Cynomolgus, Liver, Parasite, Echinococcus spp., H\&E, High magnification.

Figure 101.-Cynomolgus, Liver, Pigment, protoporphyrin, H\&E.

FIGURE 102.-Cynomolgus, Liver, Vacuolation, hepatocyte, microvesicular, H\&E. 

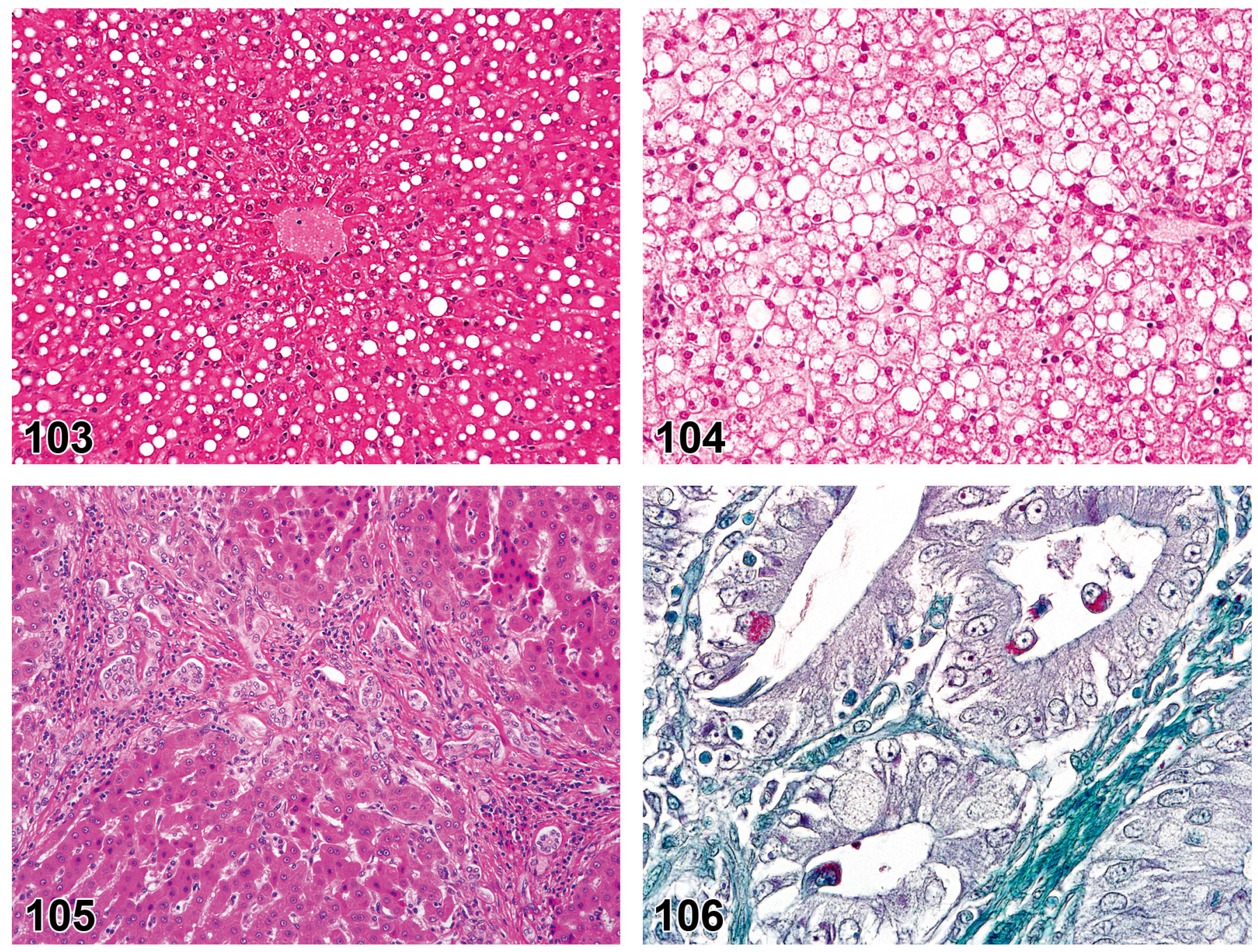

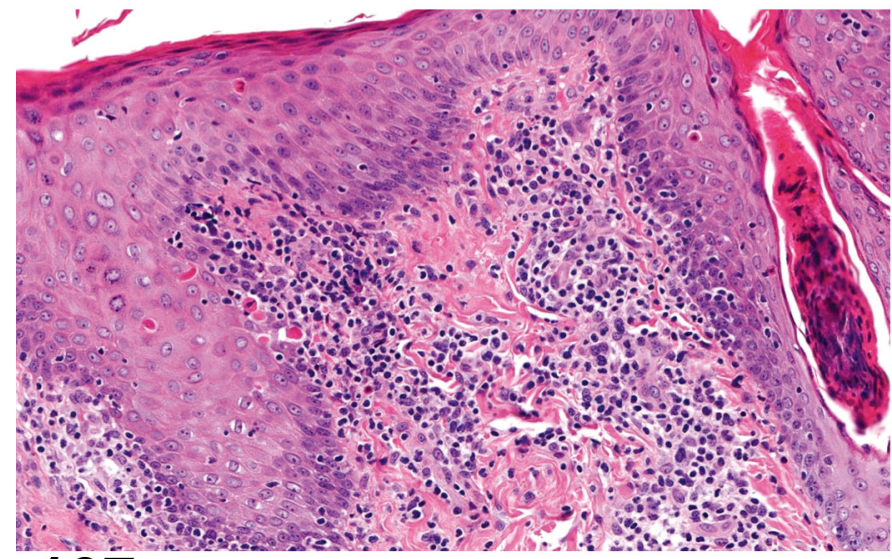

\section{7}

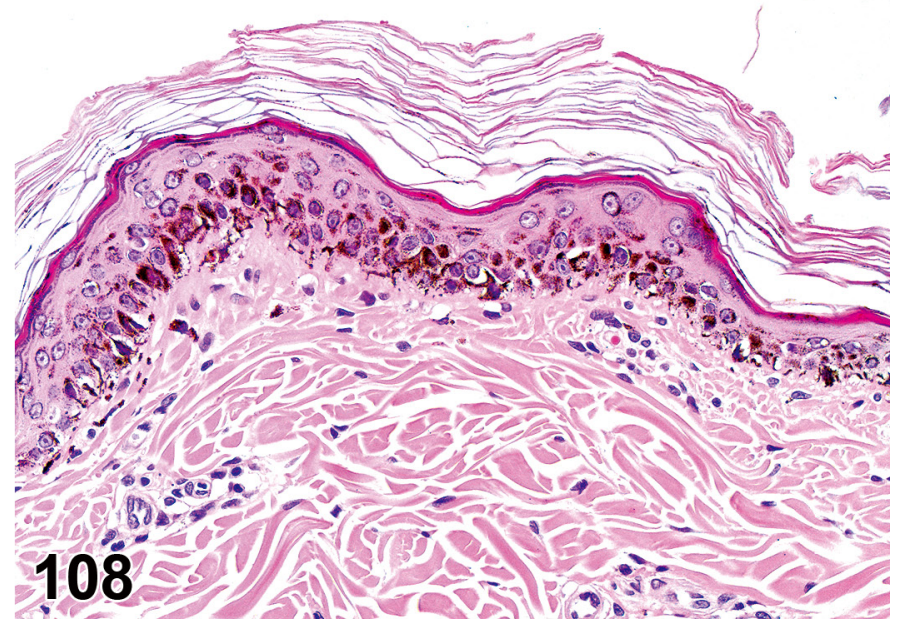

Figure 103.-Cynomolgus, Liver, Vacuolation, hepatocyte, macrovesicular, H\&E.

FIgURE 104.-Cynomolgus, Liver, Vacuolation, hepatocyte, macro- and microvesicular, H\&E.

Figure 105.-Cynomolgus, Gall bladder, Parasite, Enterocytozoon bieneusi, H\&E.

Figure 106.-Cynomolgus, Gall bladder, Parasite, Enterocytozoon bieneusi, IHC.

Figure 107.-Cynomolgus, Skin, Necrosis, single cell, epidermis, H\&E.

Figure 108.-Cynomolgus, Skin, Pigment increased, H\&E. 

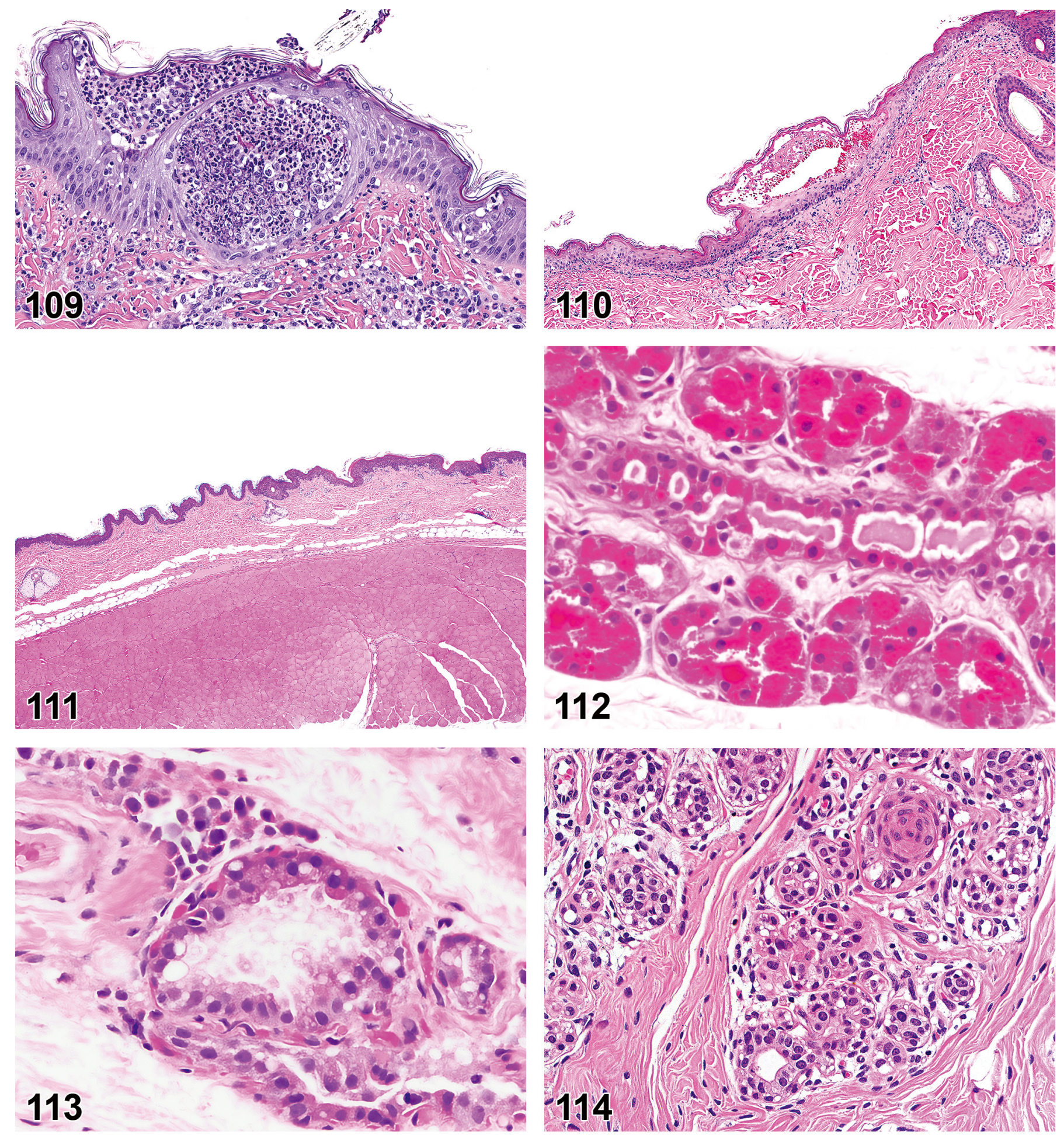

Figure 109.-Cynomolgus, Skin, Pustule, epidermis, H\&E; Courtesy of Dr. Jennifer Chilton. Figure 110.-Cynomolgus, Skin, Vesicle, epidermis, H\&E; Courtesy of Dr. Jennifer Chilton. Figure 111.-Cynomolgus, Skin, Atrophy, adnexal, H\&E; Courtesy of Dr. Jennifer Chilton. Figure 112.-Rhesus, Mammary gland, Epithelial secretory change, H\&E.

FIGURE 113.-Rhesus, Mammary gland, Metaplasia, apocrine cell, duct, H\&E.

Figure 114.-Cynomolgus, Mammary gland, Metaplasia, squamous cell, duct, H\&E, Low magnification. 

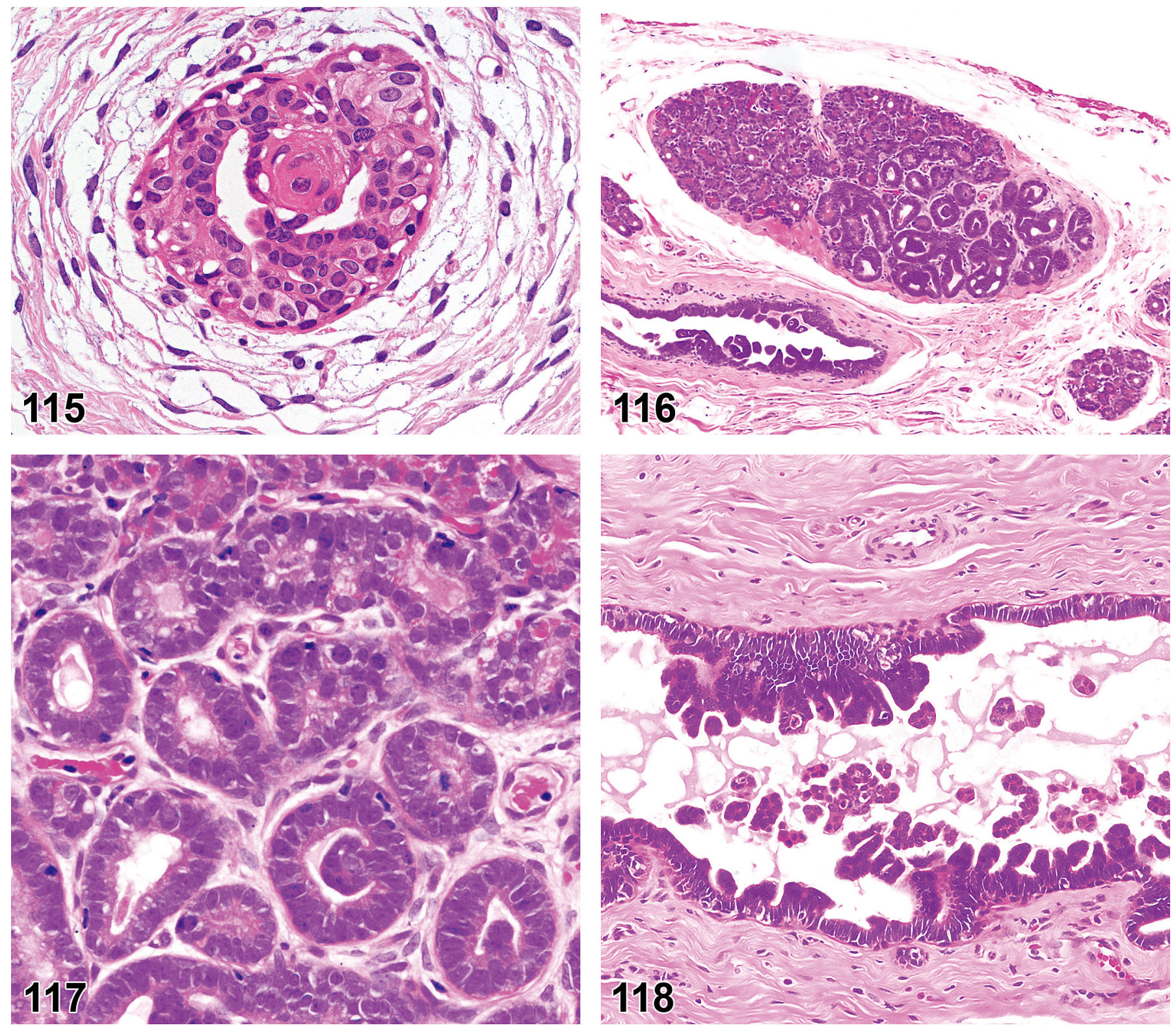

Figure 115.-Cynomolgus, Mammary gland, Metaplasia, squamous cell, duct, H\&E, High magnification.

Figure 116.-Rhesus, Mammary gland, Hyperplasia, lobuloalveolar, H\&E, Low magnification.

Figure 117.-Rhesus, Mammary gland, Hyperplasia, lobuloalveolar, H\&E, High magnification.

Figure 118.-Rhesus, Mammary gland, Hyperplasia, duct epithelium, H\&E, Low magnification. 

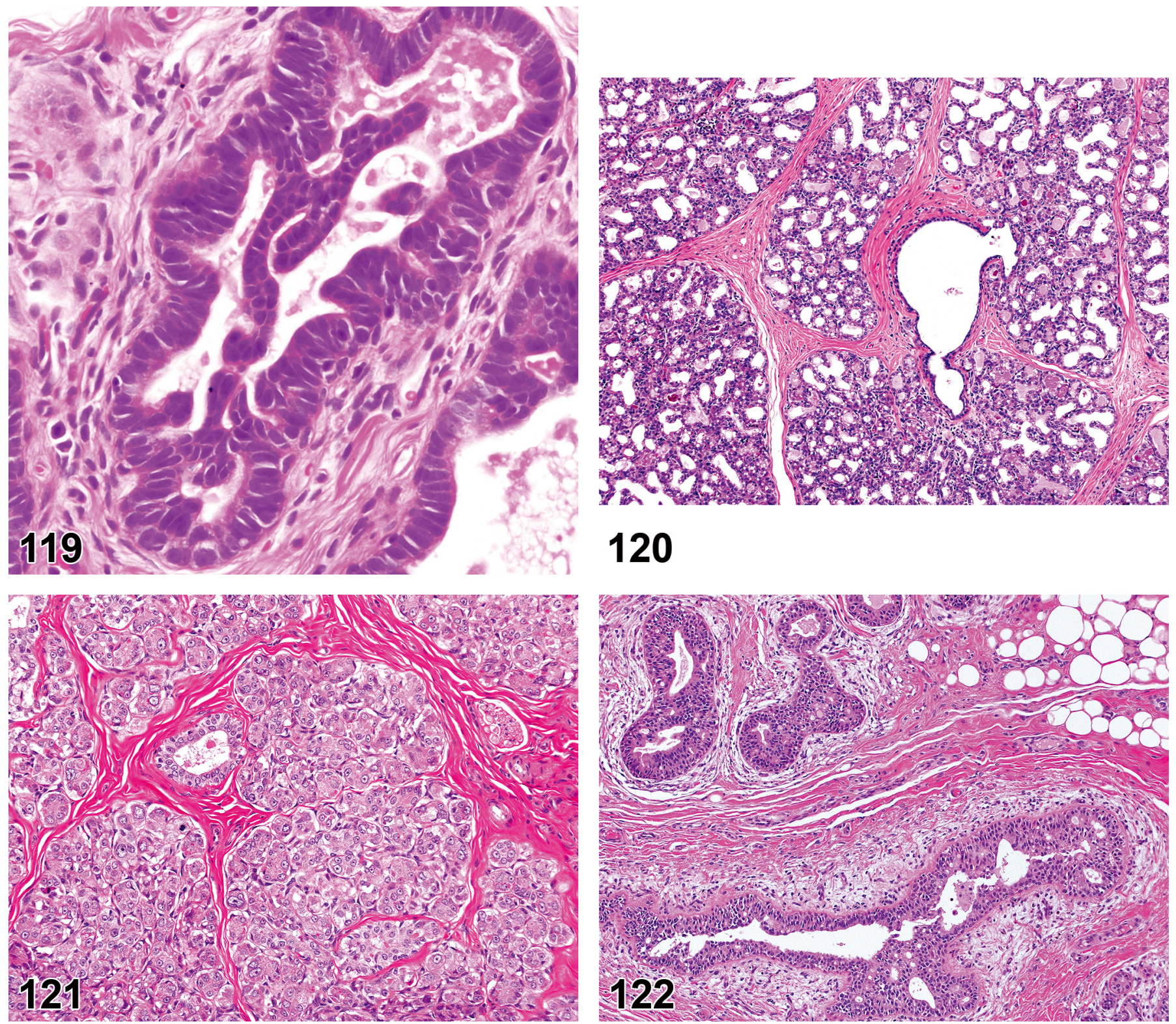

\section{0}

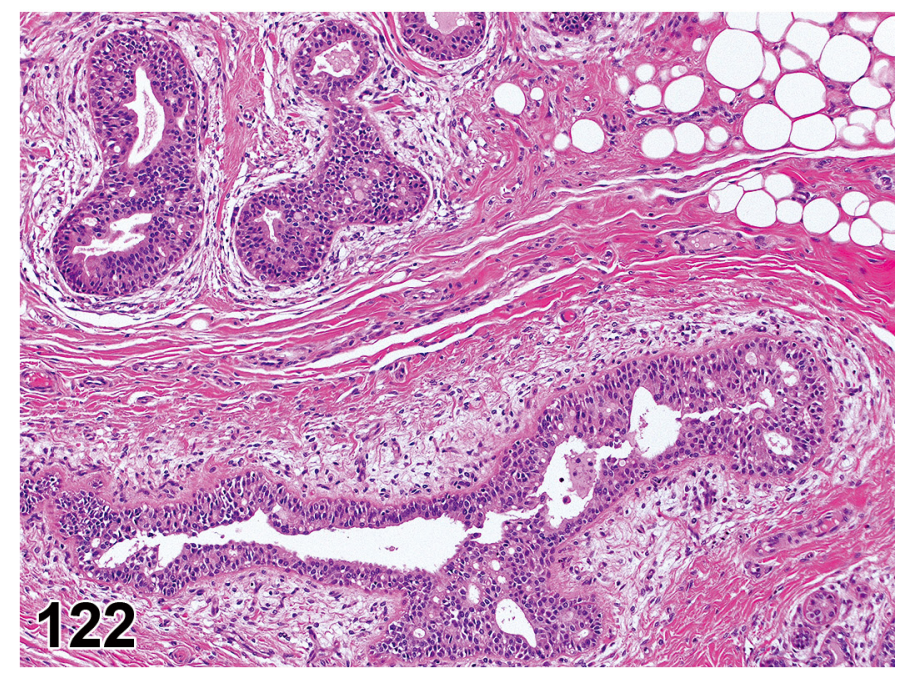

FigURE 119.-Rhesus, Mammary gland, Hyperplasia, duct epithelium, H\&E, High magnification.

FIGURE 120.-Cynomolgus, Mammary gland, Hyperplasia/hypertrophy, H\&E, Low magnification.

Figure 121.-Cynomolgus, Mammary gland, Hyperplasia/hypertrophy, H\&E, High magnification.

FiguRE 122.-Cynomolgus, Mammary gland, Hyperplasia/hypertrophy, ductal development, H\&E. 

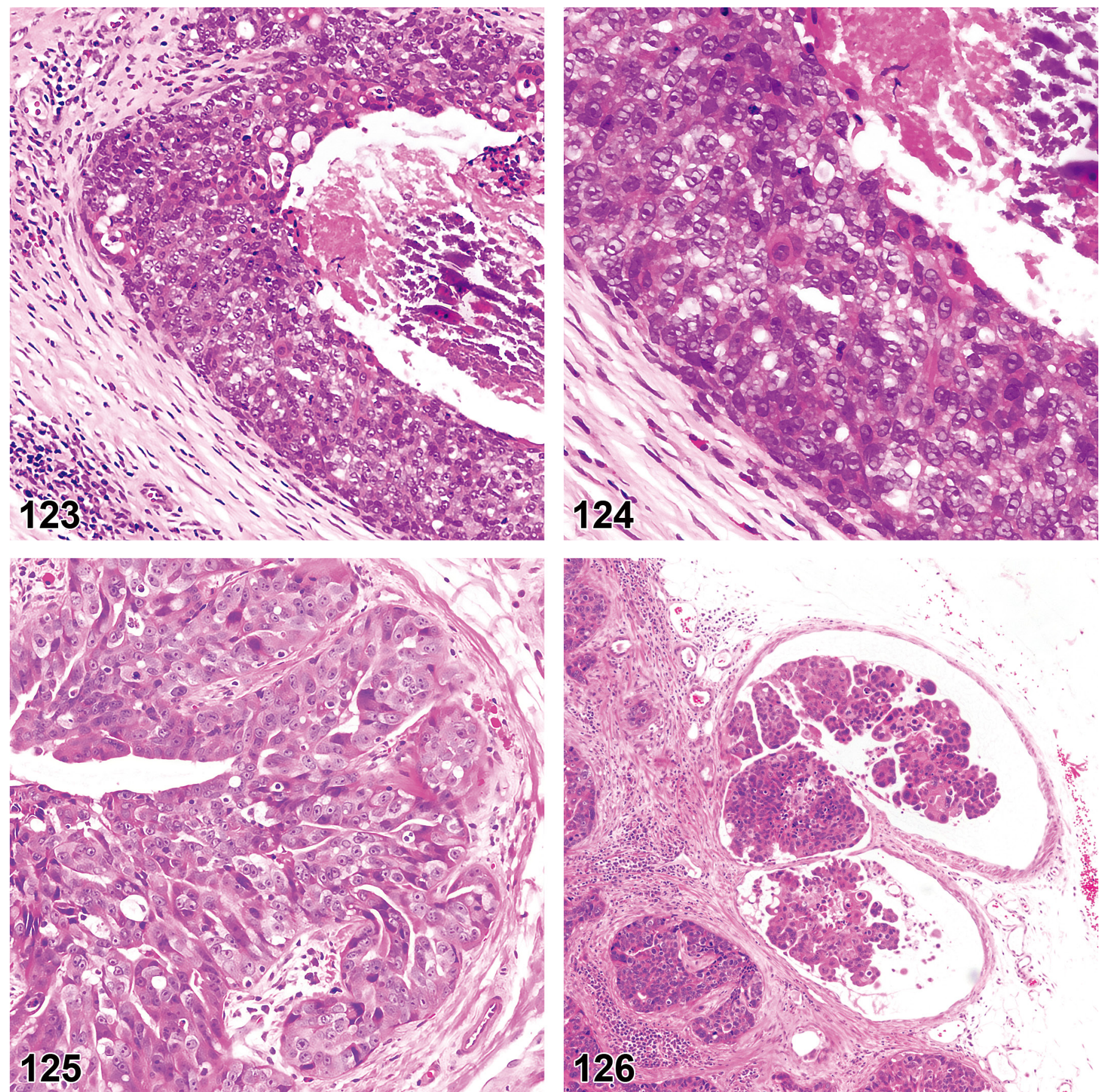

Figure 123.-Rhesus, Mammary gland, Carcinoma in situ, H\&E, Low magnification. Figure 124.-Rhesus, Mammary gland, Carcinoma in situ, H\&E, High magnification. Figure 125.-Rhesus, Mammary gland, Carcinoma, duct, H\&E, High magnification. Figure 126.-Rhesus, Mammary gland, Carcinoma, duct, H\&E, Low magnification. 

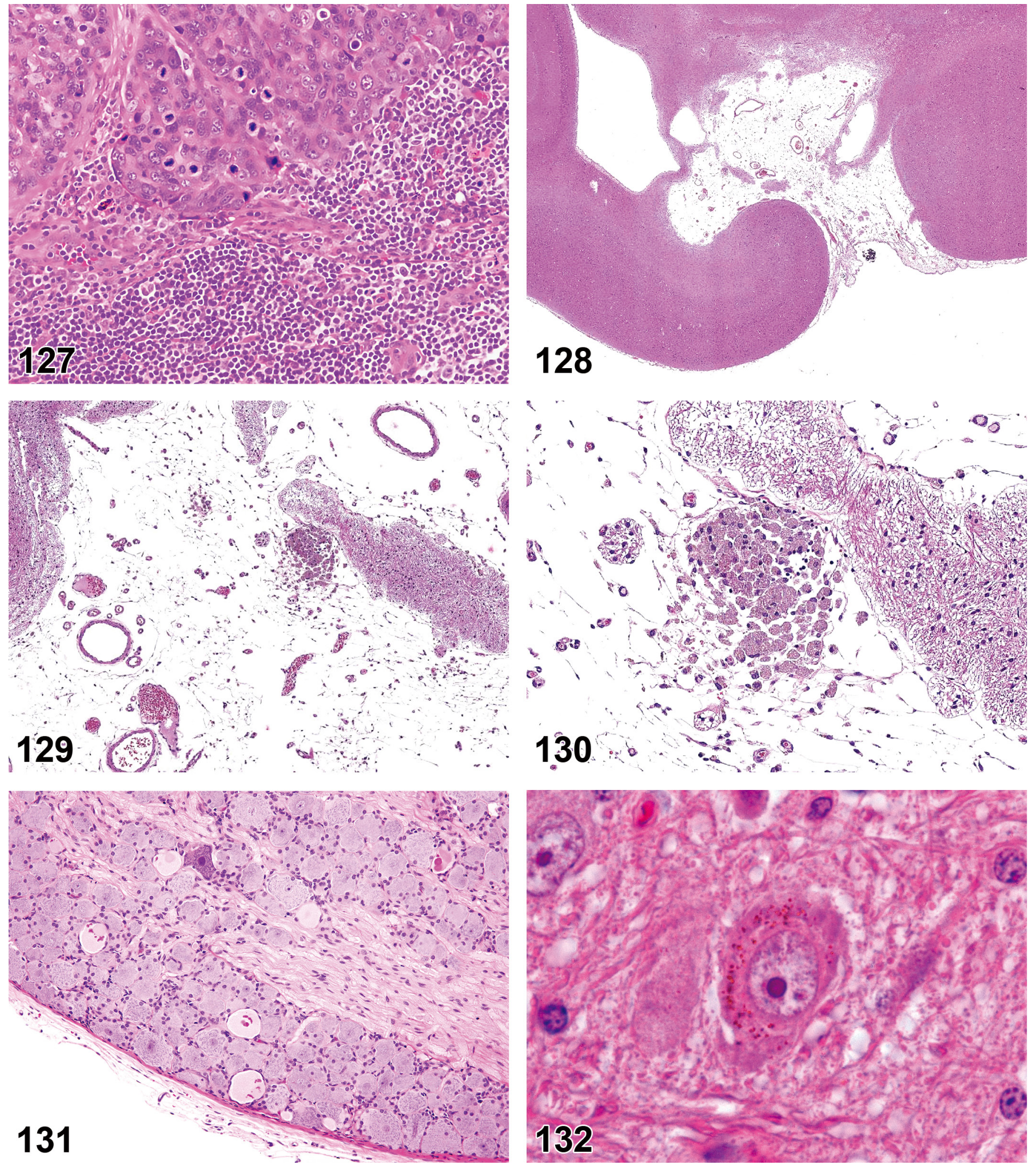

FIGURE 127.-Rhesus, Mammary gland, Carcinoma, duct, H\&E, High magnification.

Figure 128.-Cynomolgus, Brain, Neural tissue loss, cerebrum, H\&E, Low magnification.

Figure 129.-Cynomolgus, Brain, Neural tissue loss, cerebrum, H\&E, Higher magnification.

Figure 130.-Cynomolgus, Brain, Neural tissue loss, cerebrum, H\&E, High magnification.

Figure 131.-Cynomolgus, Dorsal root ganglia, autophagy, neuron, H\&E.

Figure 132.-Cynomolgus, Brain, Neuronal ceroid-lipofuscinosis, H\&E. 

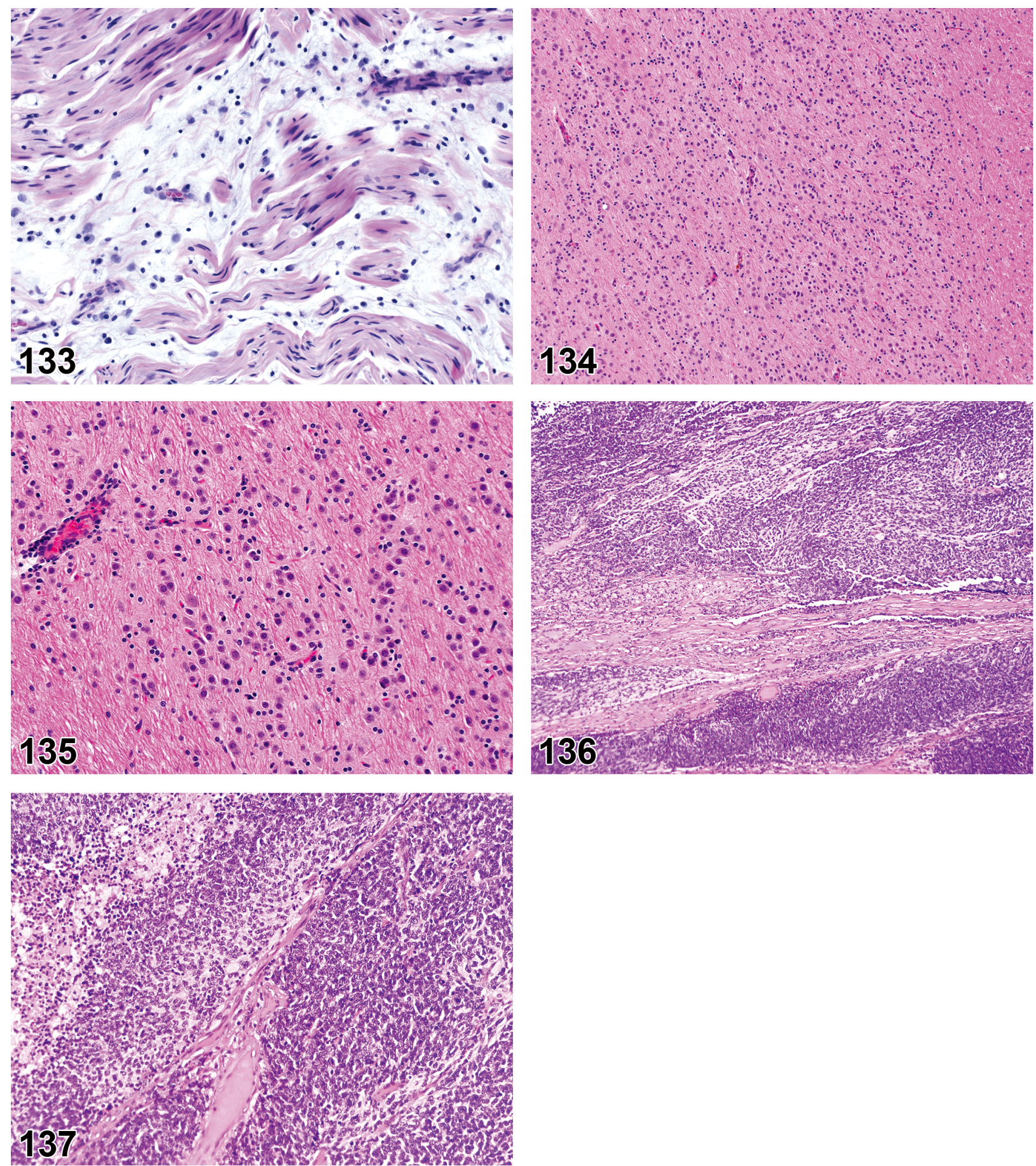

Figure 133.-Cynomolgus, Sciatic Nerve, Degeneration, nerve fiber, H\&E; Courtesy of Dr. Ingrid Pardo.

Figure 134.-Cynomolgus, CNS, Gliosis, H\&E, Low magnification.

Figure 135.-Cynomolgus, CNS, Gliosis, H\&E, High magnification.

Figure 136.-Cynomolgus, CNS, Primitive neurectodermal tumor, H\&E, Low magnification.

Figure 137.-Cynomolgus, CNS, Primitive neurectodermal tumor, H\&E, Higher magnification. 


\section{Histologic Changes in the Endometrium (Functionalis) Across the Menstrual Cycle in Cynomolgus Macaques}
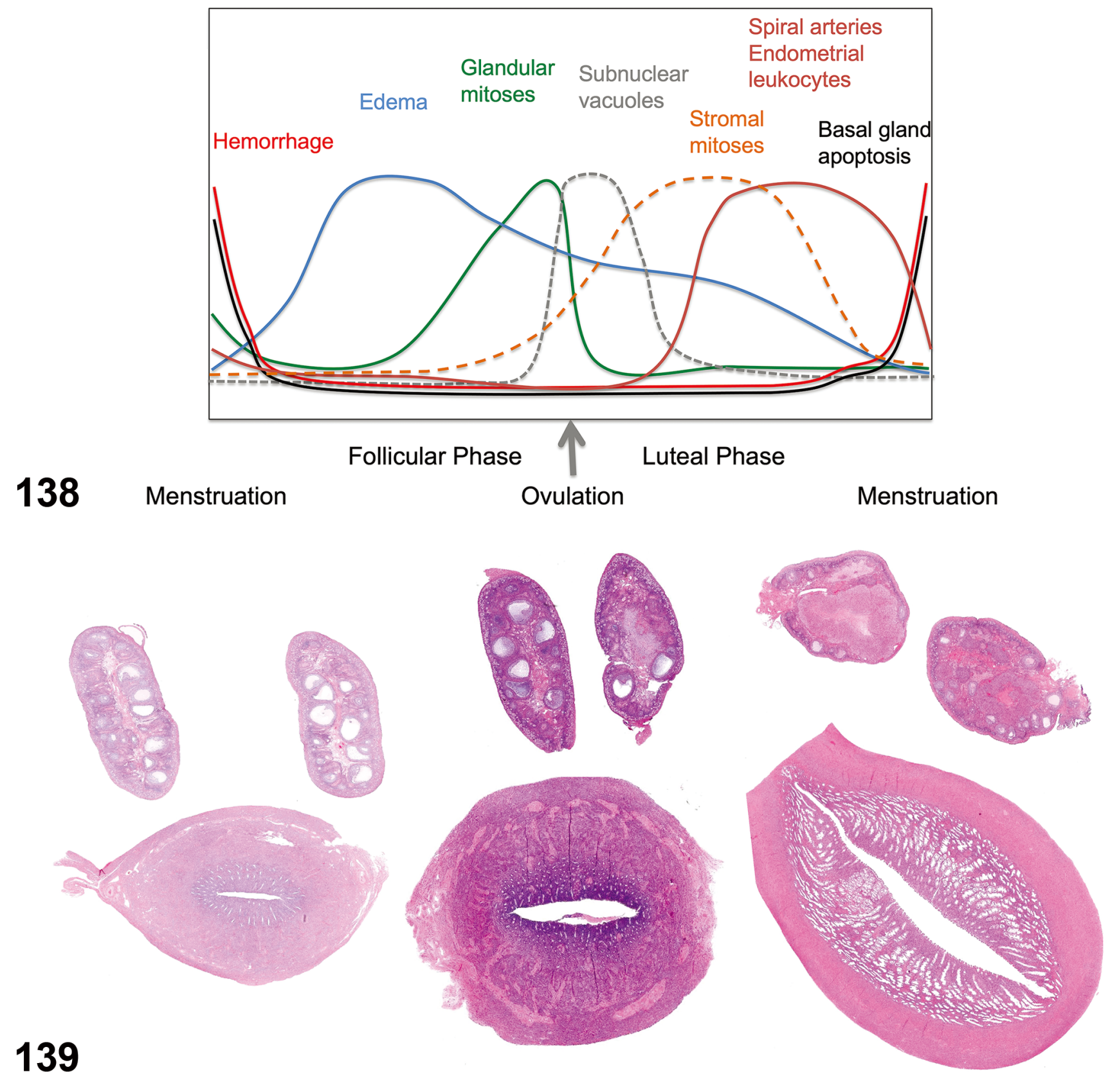

FIGURE 138.-Histologic changes in the endometrium across the menstrual cycle in macaques. Additional detail may be found in van Esch et al., 2008.

Figure 139.-Cynomolgus, Ovaries and uteri: Immature, peripubertal, and adult from right to left, H\&E. 

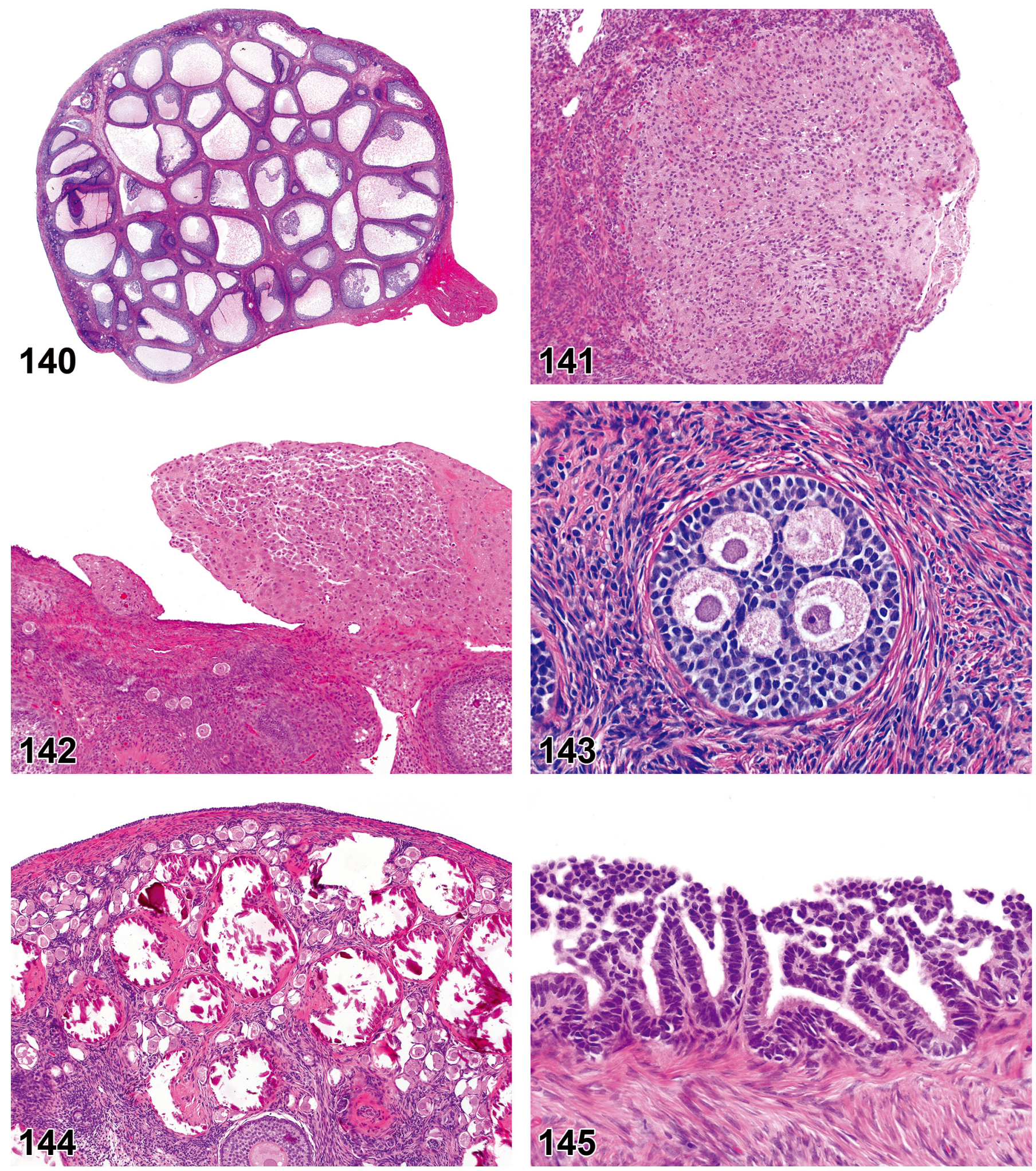

Figure 140.-Cynomolgus, Ovary, Cysts, follicular, multifocal, H\&E.

Figure 141.-Cynomolgus, Ovary, Deciduosis, focal, H\&E.

Figure 142.-Cynomolgus, Ovary, Deciduosis, focal, H\&E.

Figure 143.-Cynomolgus, Ovary, Follicle, polyovular, H\&E.

Figure 144.-Cynomolgus, Ovary, Mineralization, follicle, H\&E.

Figure 145.-Cynomolgus, Ovary, Hyperplasia, surface epithelium, H\&E. 

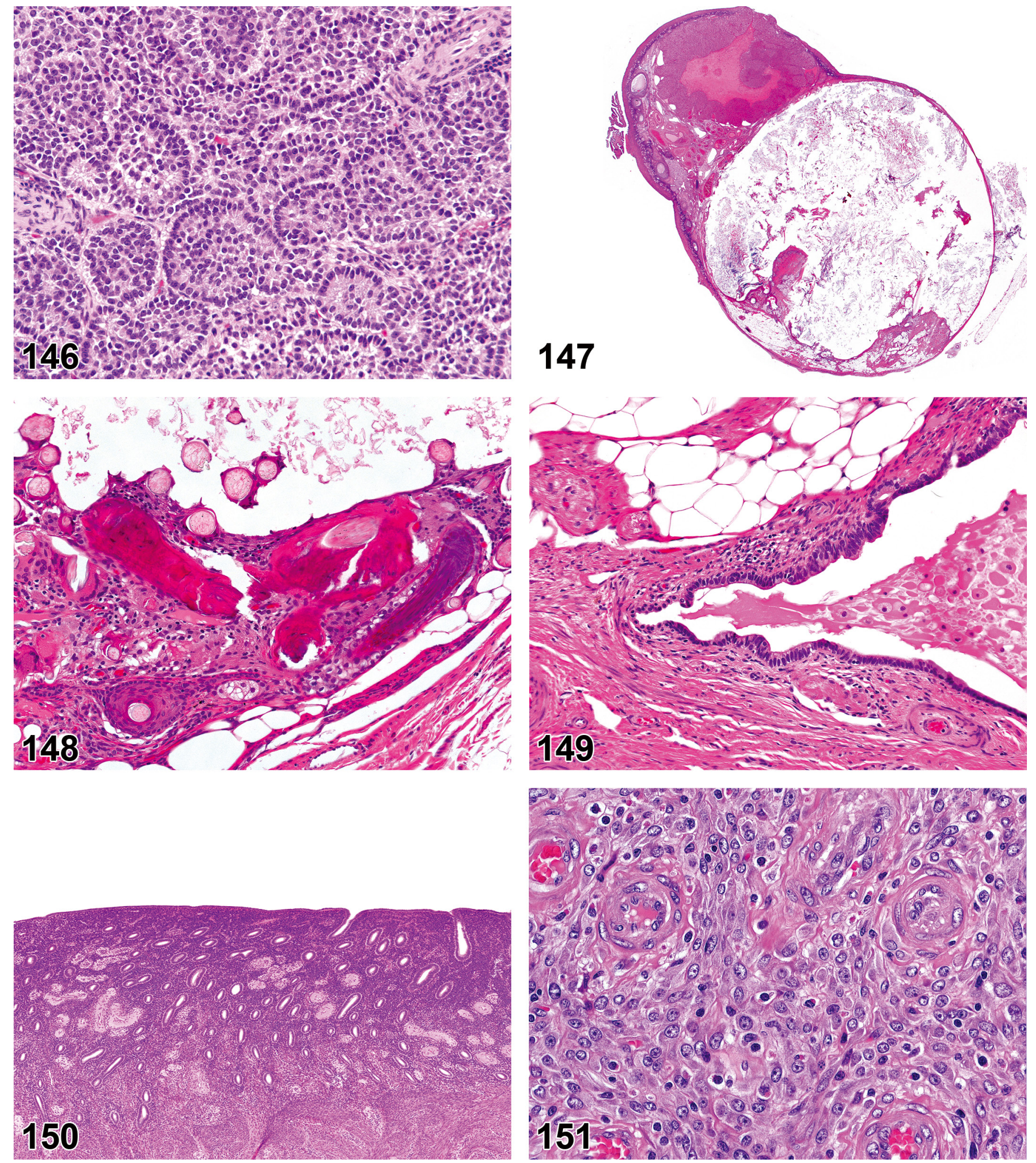

Figure 146.-Cynomolgus, Ovary, Granulosa cell tumor, H\&E.

Figure 147.-Cynomolgus, Ovary, Teratoma, benign. Entire ovary with cystic mass, H\&E, Low magnification. Modified from Cline et al., 2008.

FIGURE 148.-Cynomolgus, Ovary, Teratoma, benign, showing bone, hair and connective tissue, H\&E, High magnification. Modified from Cline et al., 2008.

FIgure 149.-Cynomolgus, Ovary, Teratoma, benign, showing connective tissue, fat, and gland, H\&E, High magnification. Modified from Cline et al., 2008.

Figure 150.-Cynomolgus, Uterus, Inactive endometrium, H\&E.

FIGURE 151.-Cynomolgus, Uterus, Decidualization, (Endometrial perivascular pseudodecidualization), H\&E. 

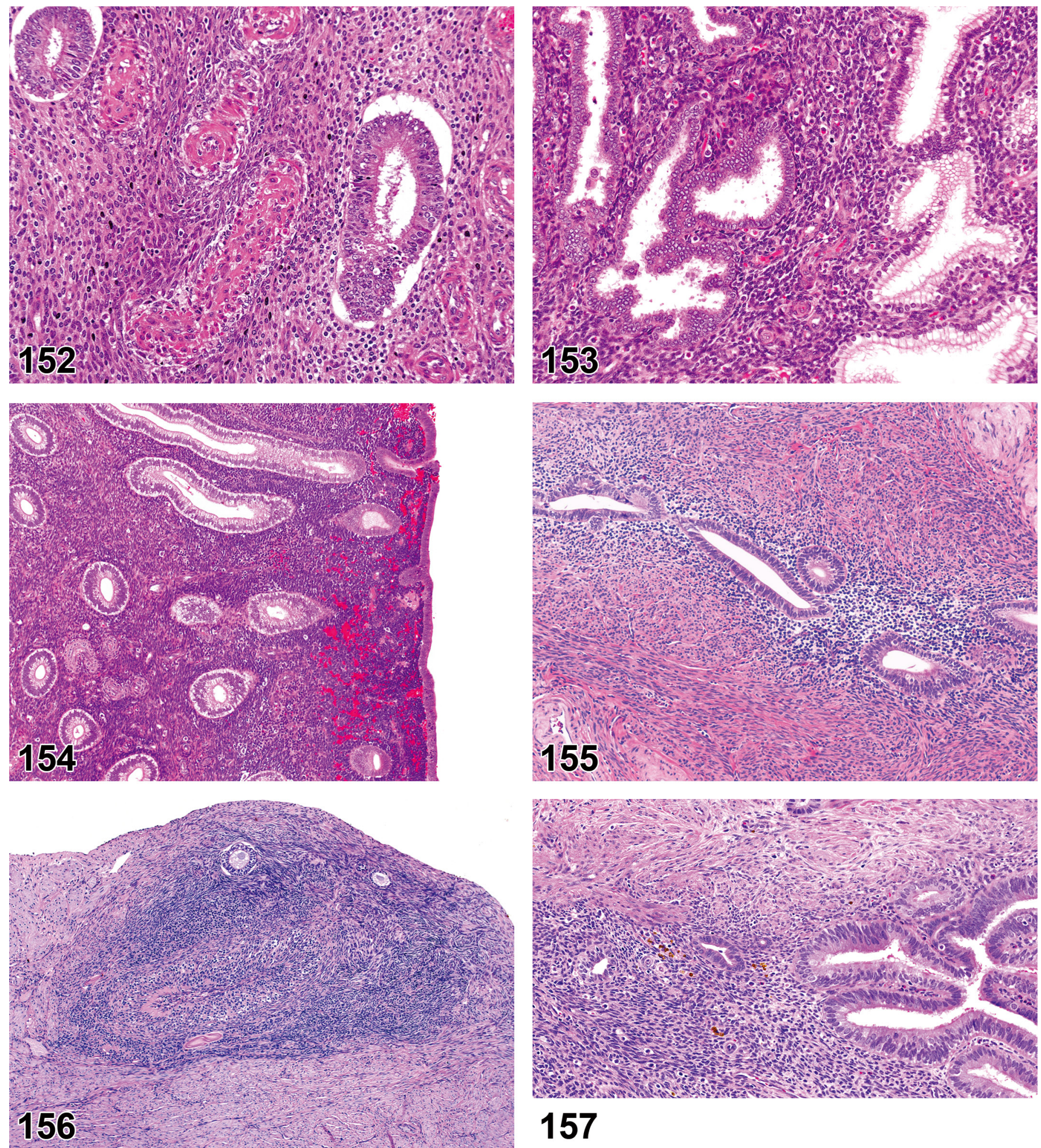

\section{7}

Figure 152.-Cynomolgus, Uterus, Hemorrhage, endometrial, abnormal, H\&E.

Figure 153.-Cynomolgus, Uterus, Dyssynchronous endometrium, H\&E.

Figure 154.-Cynomolgus, Uterus, Adenomyosis, myometrium, H\&E.

Figure 155.-Cynomolgus, Uterus, Degeneration, spiral arteries, H\&E.

Figure 156.-Cynomolgus, Uterus, Ectopic tissue, ovary, H\&E.

Figure 157.-Cynomolgus, Mesentery. Endometriosis, H\&E. 

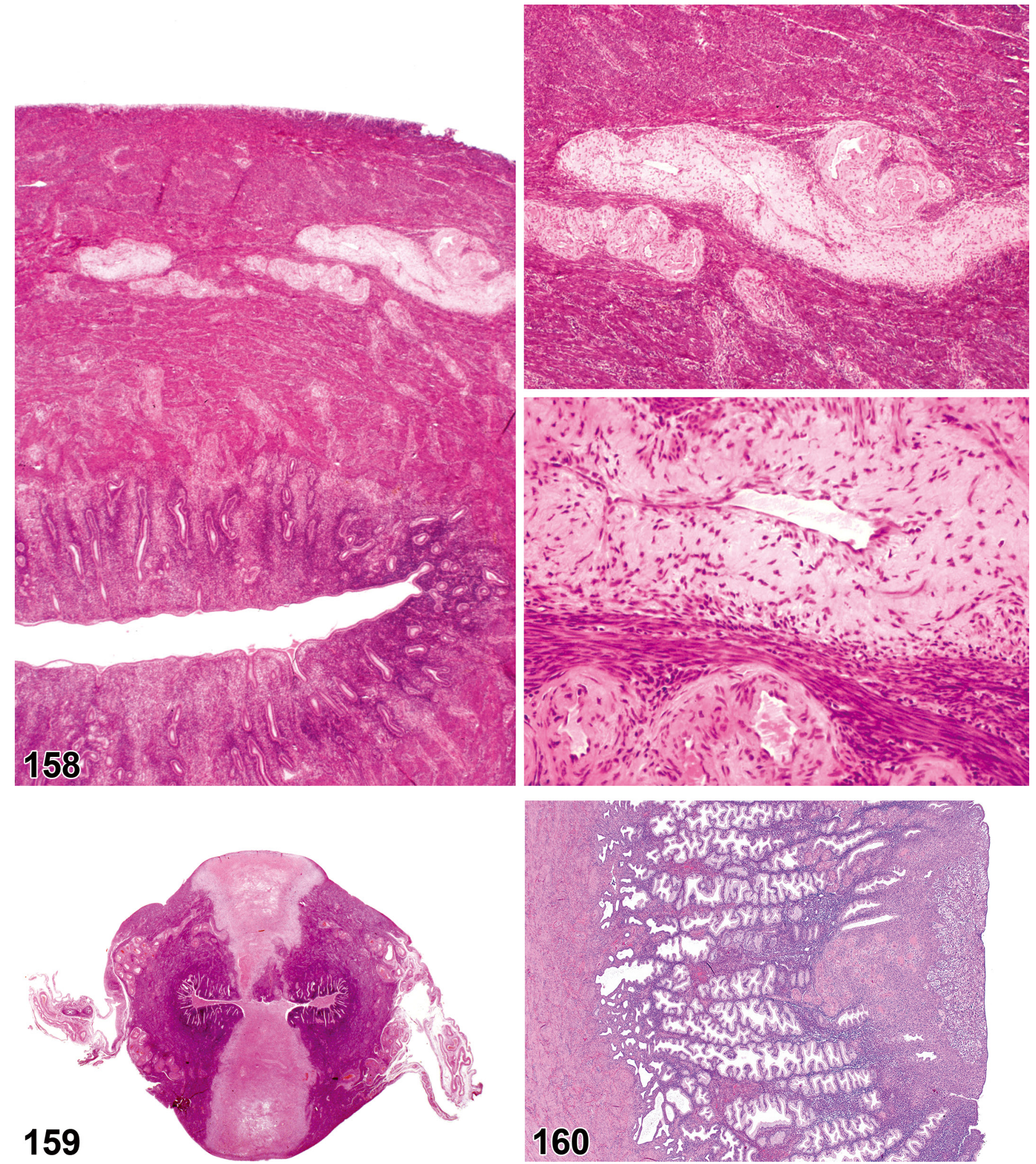

FiguRE 158.-Cynomolgus, Uterus, myometrium. Hyaline deposits, perivascular, H\&E.

Figure 159.-Cynomolgus, Infarct, uterus, H\&E (Trybus et al., 2007).

FIgURE 160.-Cynomolgus, Uterus, endometrium. Epithelial plaque, H\&E. 

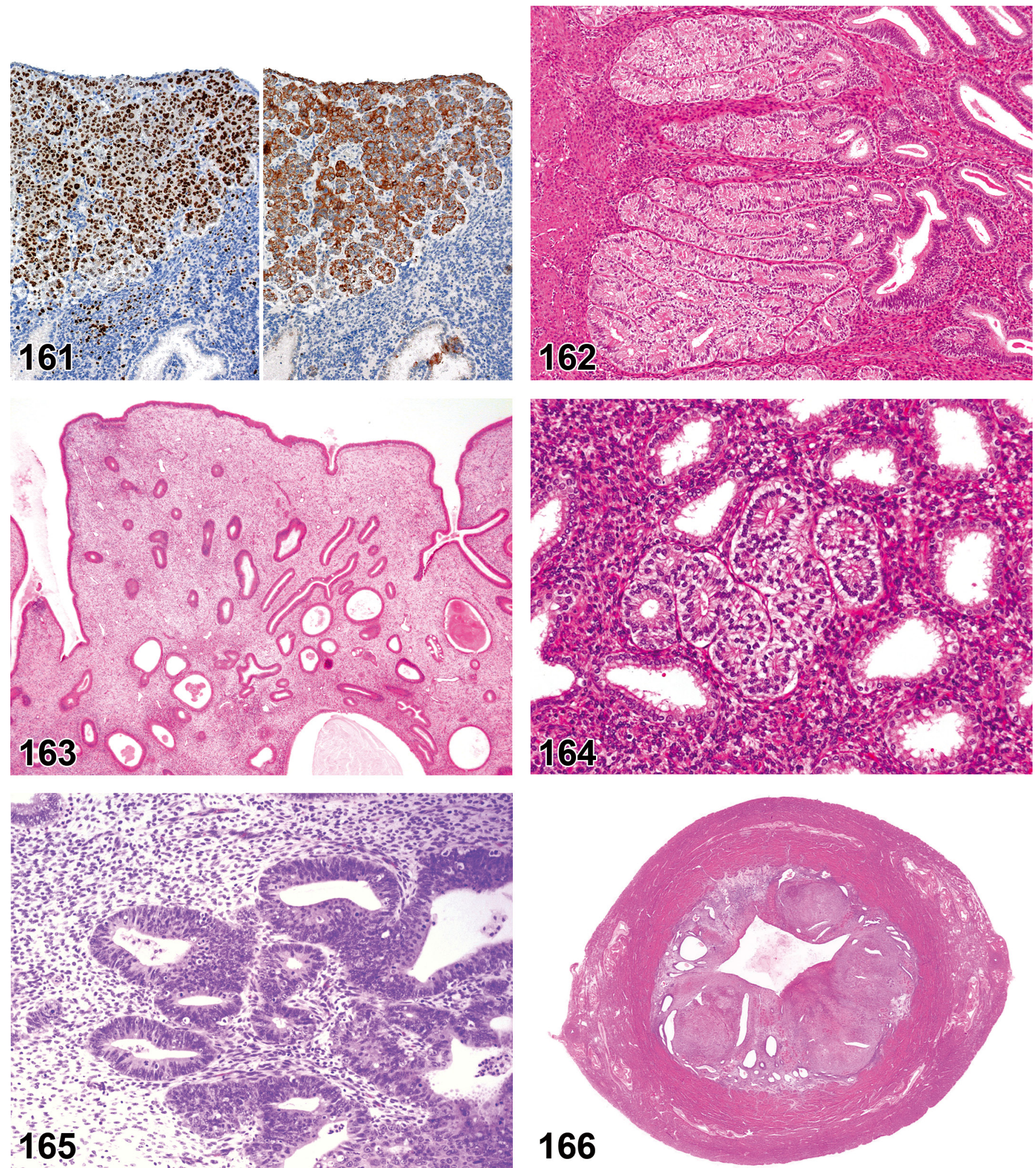

FIGURE 161.-Cynomolgus, Uterus, endometrium. Epithelial plaque, Ki67 (left) and pan-cytokeratin immunohistochemistry. Figure 162.-Cynomolgus, Uterus, endometrium. Hyperplasia, glandular, basal, H\&E.

Figure 163.-Cynomolgus, Uterus, endometrium. Hyperplasia, glandular, simple H\&E.

Figure 164.-Cynomolgus, Uterus, endometrium. Hyperplasia, glandular, complex, focal, H\&E.

FIGURE 165.-Cynomolgus, Uterus, endometrium. Hyperplasia, glandular, with atypia, H\&E.

Figure 166.-Cynomolgus Uterus, Choriocarcinoma, H\&E, Low magnification. 

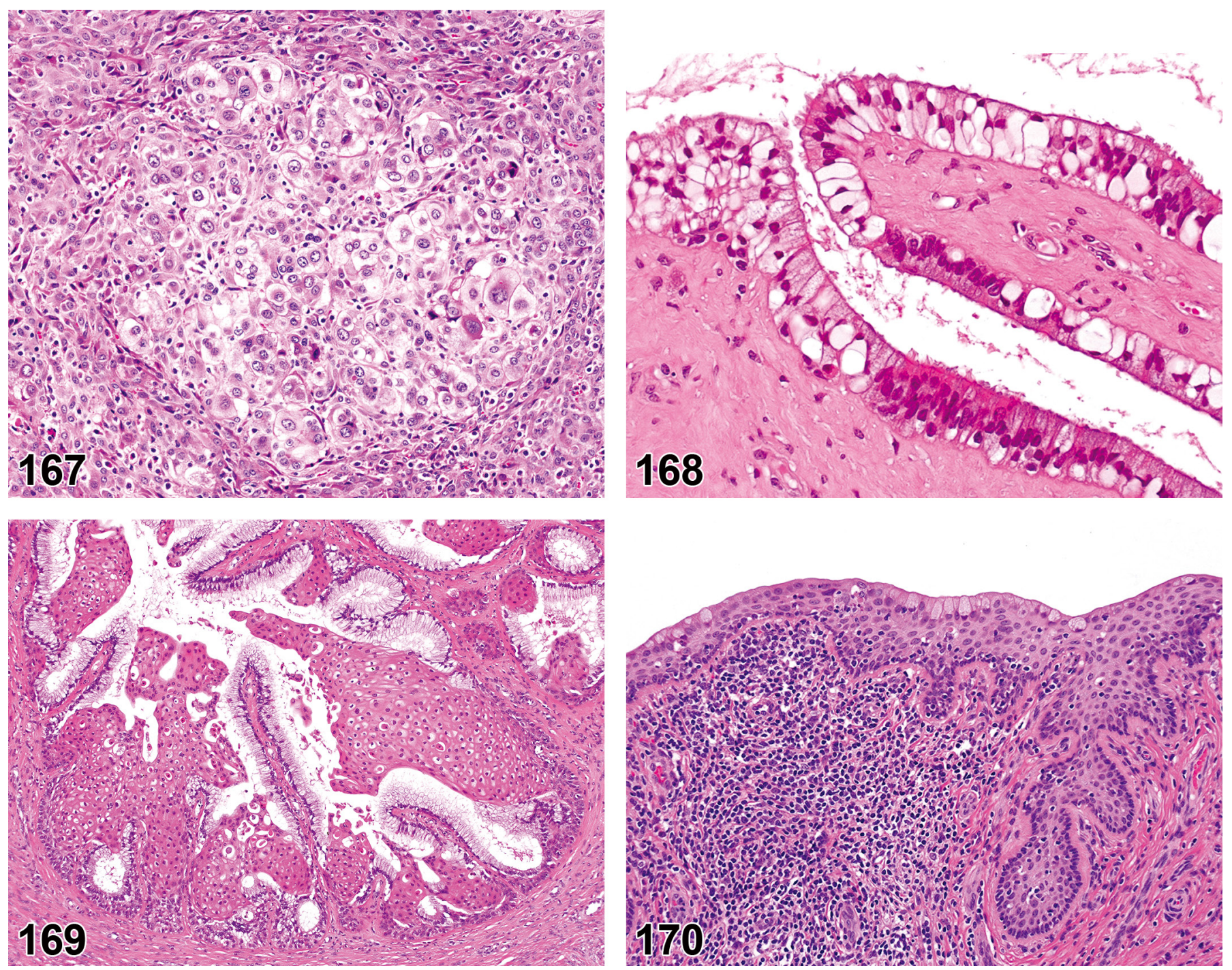

Figure 167.-Cynomolgus Uterus, Choriocarcinoma, H\&E, High magnification. Figure 168.-Cynomolgus, Cervix, Metaplasia, ciliary, H\&E.

Figure 169.-Cynomolgus, Cervix, Metaplasia, squamous, H\&E.

Figure 170.-Cynomolgus, Vagina, Adenosis, and infiltrate, lymphocytic, H\&E. 

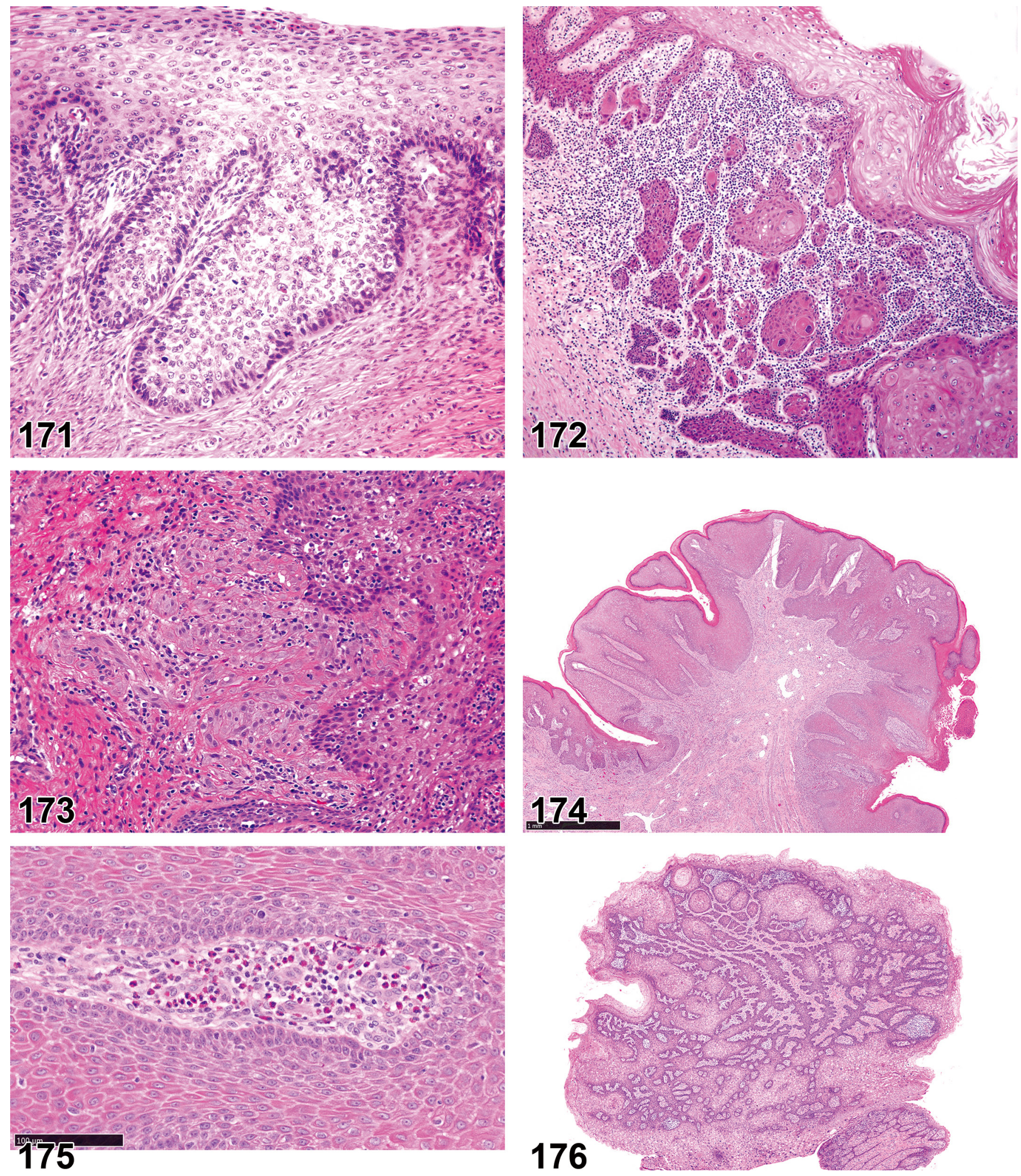

Figure 171.-Cynomolgus, Cervix (ectocervix). Epithelial dysplasia (cervical intraepithelial neoplasia), H\&E.

Figure 172.-Cynomolgus, Cervix (ectocervix). Carcinoma, squamous cell, H\&E.

Figure 173.-Cynomolgus Vagina, Deciduosis, focal, submucosal, H\&E.

Figure 174.-Cynomolgus, Vagina, Condyloma, inflammatory, H\&E, Low magnification.

Figure 175.-Cynomolgus, Vagina, Condyloma, inflammatory, H\&E, High magnification.

Figure 176.-Cynomolgus, Vagina, Papilloma, squamous, H\&E. 

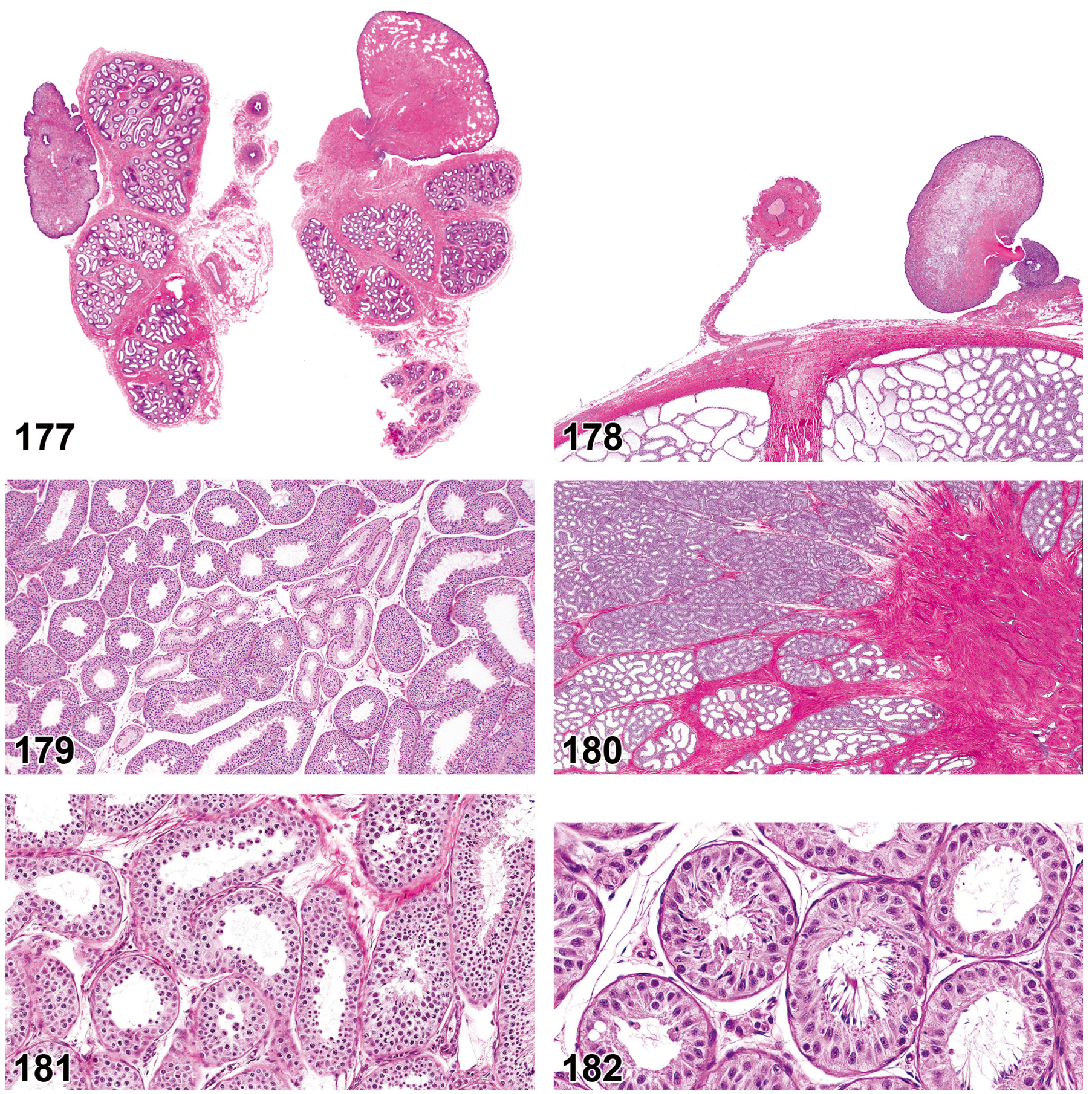

Figure 177.-Cynomolgus, Epididymis, Embryonic remnants (Appendix testis), bilateral, H\&E.

FiguRE 178.-Cynomolgus, Epididymis, Embryonic remnants (Appendix testis), with torsion (Testis also has tubular dilatation), H\&E.

Figure 179.-Cynomolgus, Testis, Hypoplasia, tubular, H\&E.

FIGURE 180.-Cynomolgus, Testis, Increased stromal collagen, with tubular dilatation, H\&E, Low magnification.

Figure 181.-Cynomolgus, Testis, Degeneration/atrophy, tubule, H\&E.

Figure 182.-Cynomolgus, Testis, Depletion, germ cell, H\&E. 

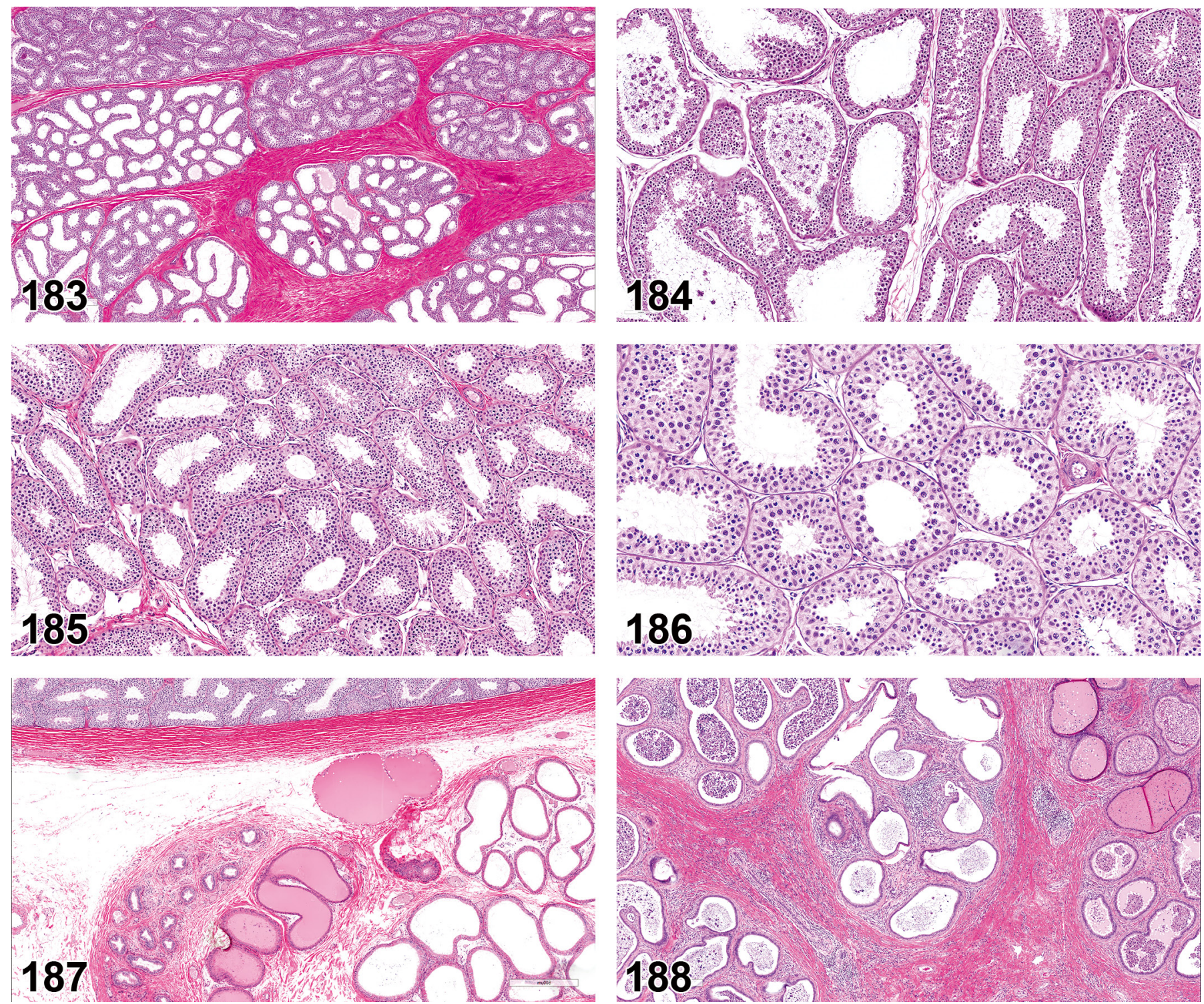

Figure 183.-Cynomolgus, Testis, Dilatation, tubule, multifocal, secondary to increased stromal collagen, H\&E, Low magnification.

Figure 184.-Cynomolgus, Testis, Dilatation, tubule, with sperm stasis and secondary tubular degeneration/atrophy, H\&E, High magnification.

Figure 185.-Cynomolgus, Testis, Hypospermatogenesis, H\&E, Low magnification.

Figure 186.-Cynomolgus, Testis, Hypospermatogenesis, H\&E, High magnification.

Figure 187.-Cynomolgus, Efferent ducts, dilatation, H\&E.

FIGURE 188.-Cynomolgus, Efferent ducts, dilatation, with inflammation, H\&E. 

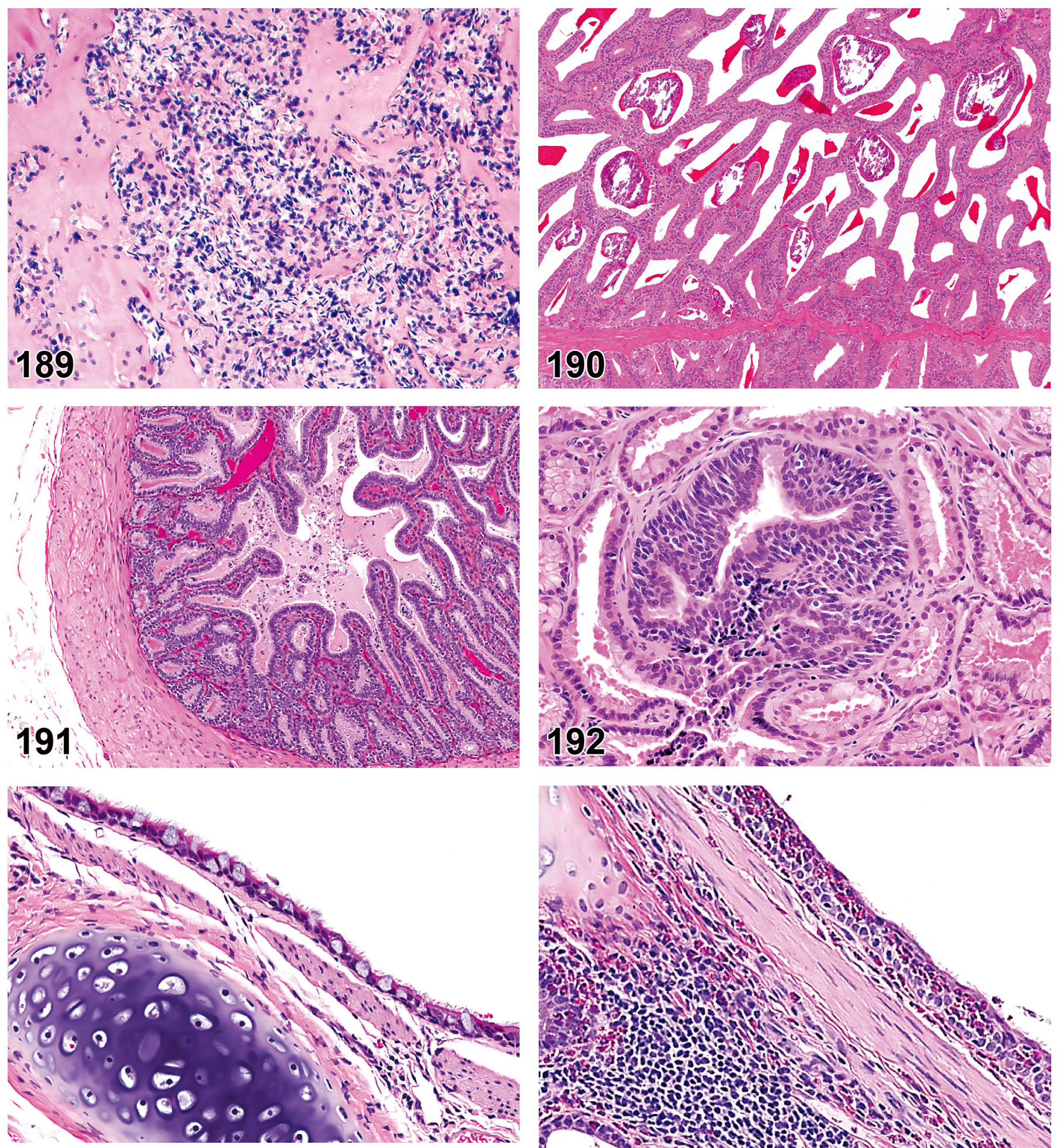

\section{3}

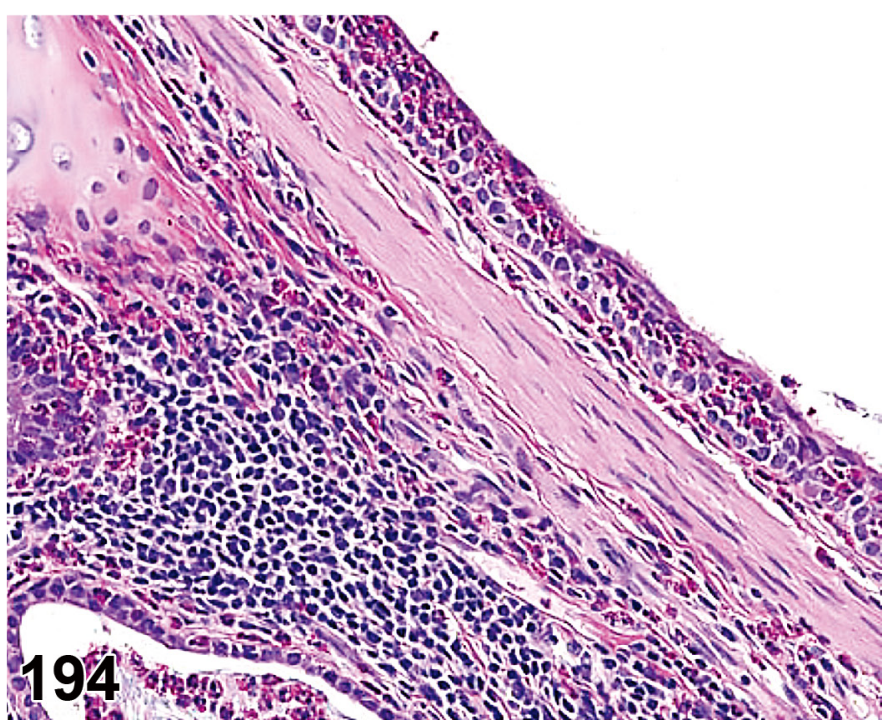

Figure 189.-Cynomolgus, Prostate, Corpora amylacea, semen, H\&E; Courtesy of Dr. Sanjeev Gumber.

Figure 190.-Cynomolgus, Seminal vesicle, Corpora amylacea, semen, H\&E.

Figure 191.-Cynomolgus, Seminal vesicle, Sperm reflux, H\&E.

Figure 192.-Cynomolgus, Prostate, Hyperplasia, basal cell, H\&E.

Figure 193.-Cynomolgus, Bronchus, normal thickness of smooth muscle, H\&E (Courtesy of Dr. Molly Boyle).

FIGURE 194.-Cynomolgus, Bronchus, Hypertrophy/hyperplasia, bronchial smooth muscle (in a case of eosinophilic airway inflammation), H\&E; Courtesy of Dr. Nori Shirai. 

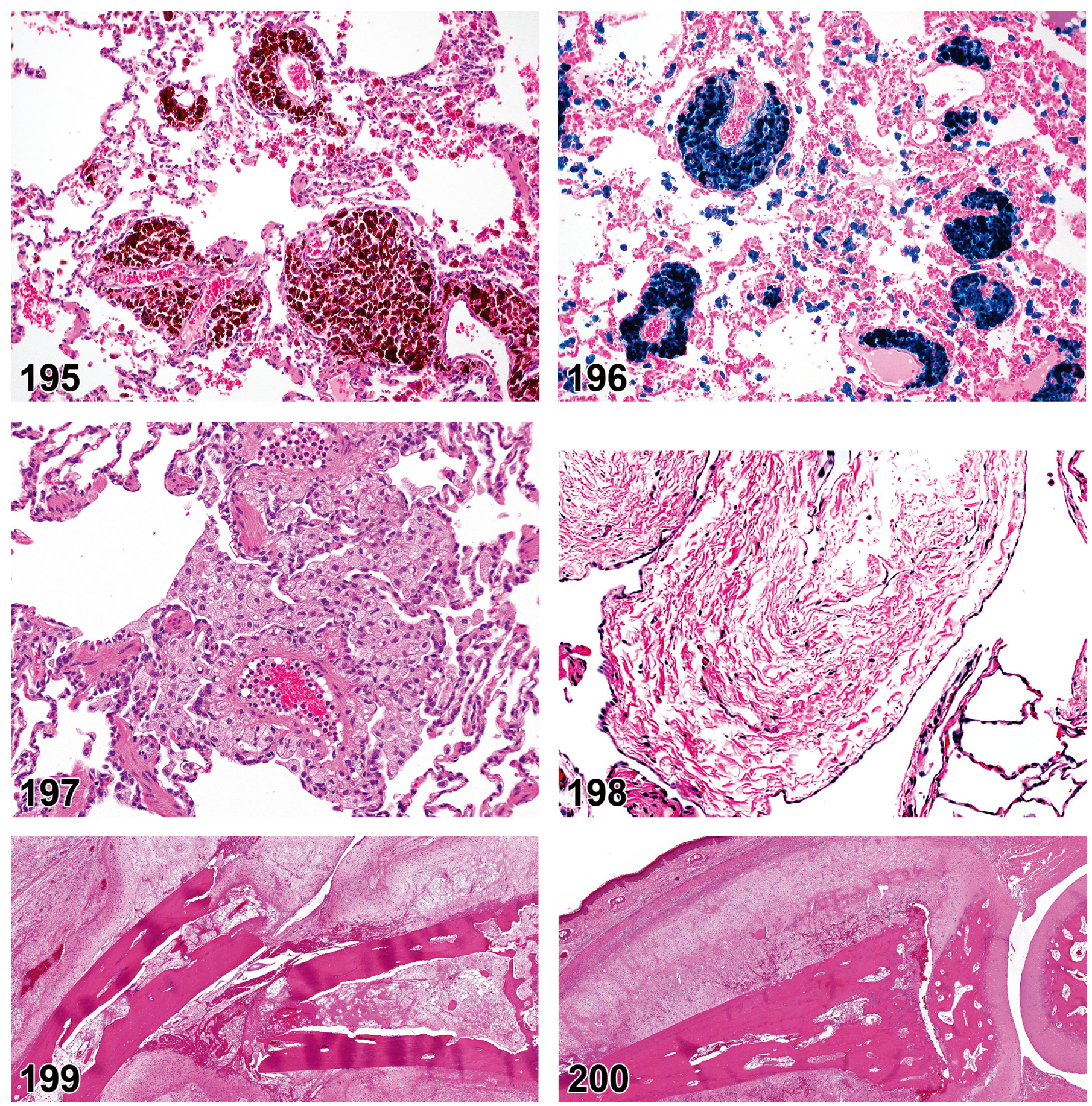

FIGURE 195.-Cynomolgus, Lung, Accumulation, pigmented macrophages, hemosiderin, H\&E.

Figure 196.-Cynomolgus, Lung, Accumulation, pigmented macrophages, hemosiderin, Perls.

Figure 197.-Cynomolgus, Lung, Macrophages increased, alveolar, H\&E.

Figure 198.-Cynomolgus, Pleura, Fibrous tag, H\&E; Courtesy of Dr. Molly Boyle.

FigURE 199.-NHP, Phalanx, Fracture, (diaphysis), with prominent callus, H\&E.

FIGURE 200.-NHP, Phalanx, Fracture, (physis), with prominent callus, H\&E. 

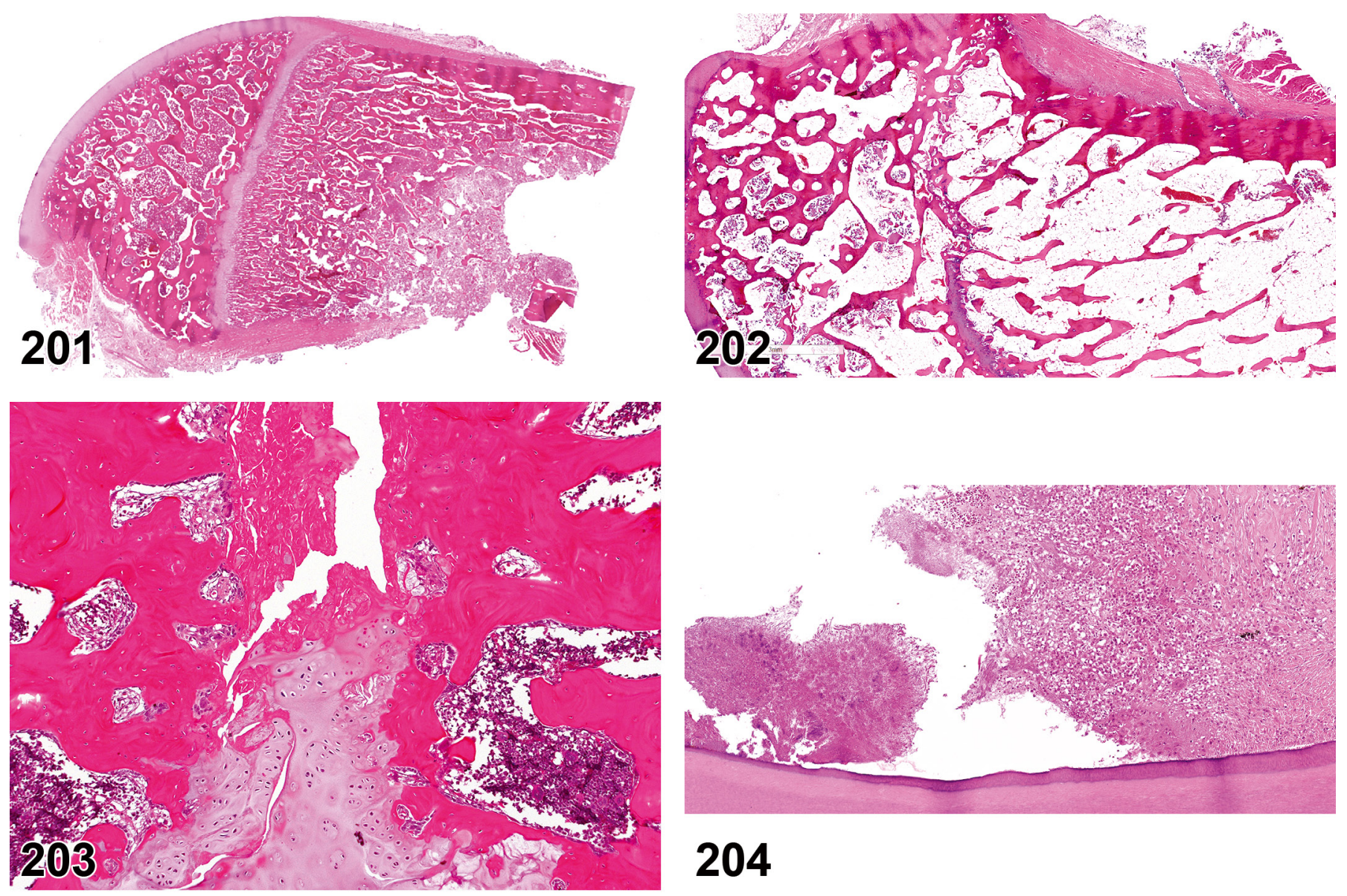

\section{4}

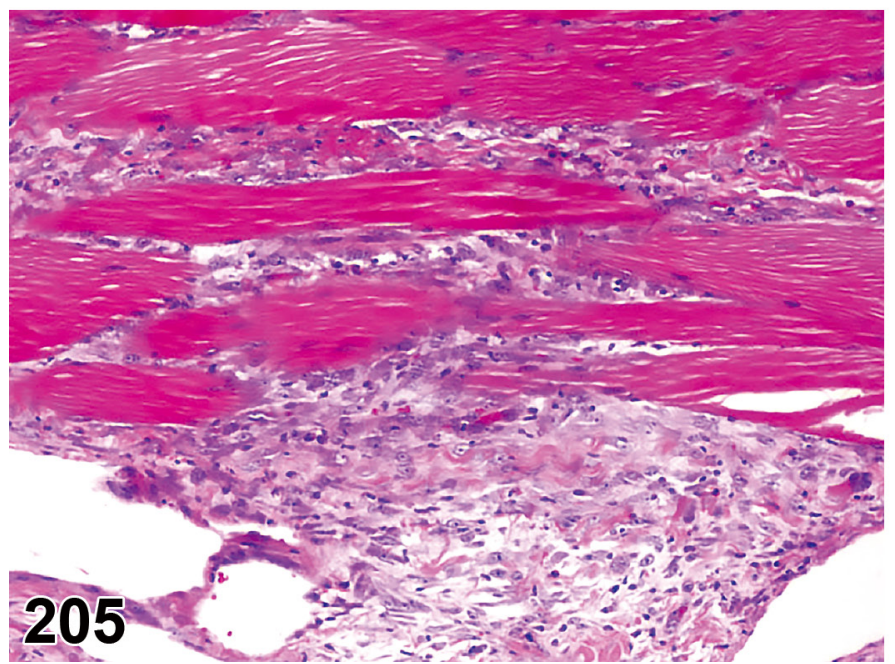

Figure 201.-Cynomolgus, Femur, open growth plate in a young adult from a 1-month toxicology study, H\&E.

Figure 202.-Cynomolgus, Femur, partially closed growth plate in a young adult, H\&E.

Figure 203.-Cynomolgus, Sternum, degeneration of cartilage, H\&E.

FIGURE 204.-Cynomolgus, Incisor tooth, Inflammation, periodontium, subacute (with a mixed inflammatory cell infiltrate and necrotic debris in the periodontal space), H\&E.

Figure 205.-Cynomolgus, Subcutis, Retroperitoneal fibromatosis, H\&E. 

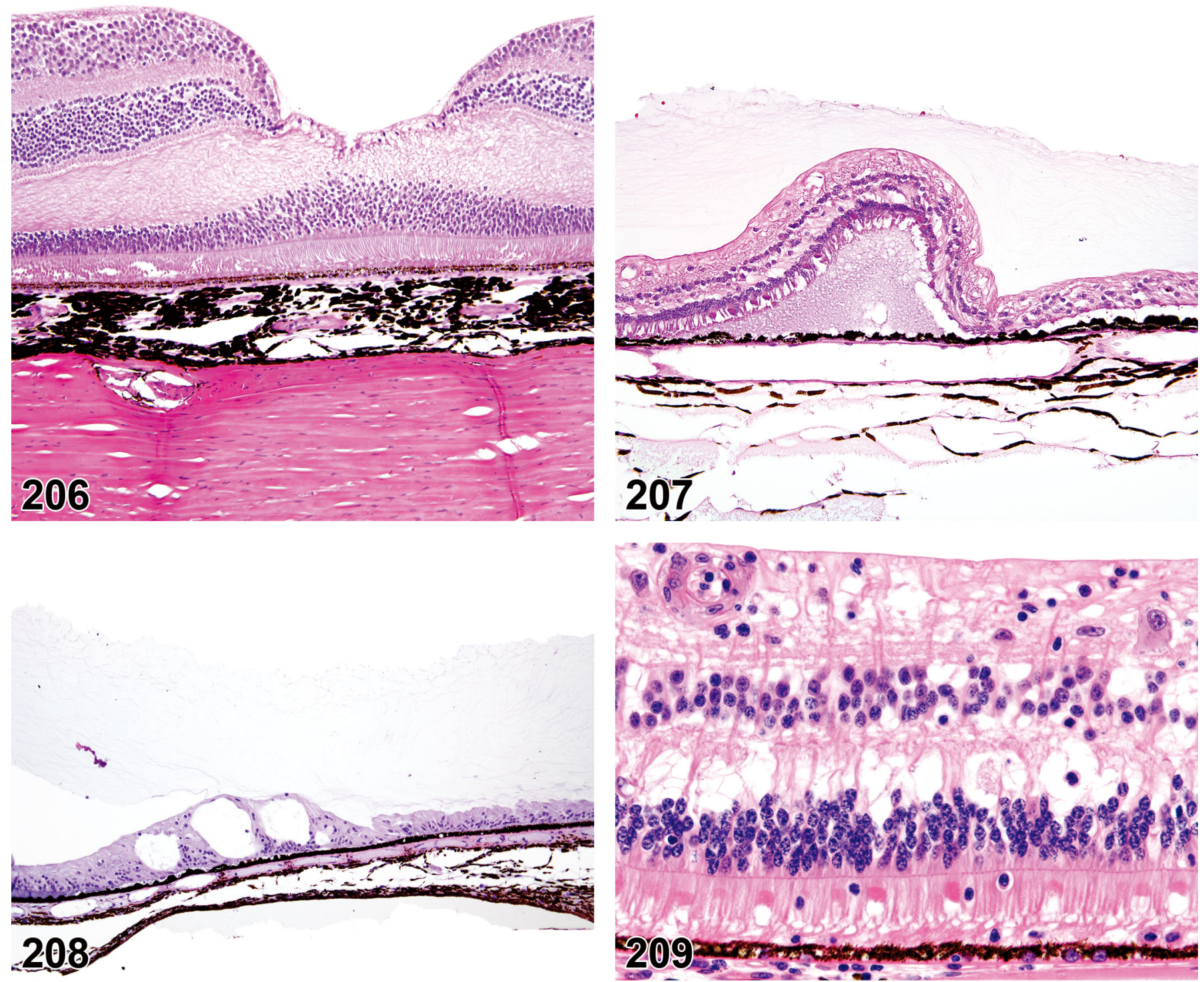

Figure 206.-Cynomolgus, Eye, Retina, normal fovea centralis and macula lutea, H\&E.

Figure 207.-Cynomolgus, Eye, Retina, Lange's fold, H\&E.

Figure 208.-Cynomolgus, Eye, Retina, Degeneration, microcystoid, peripheral, H\&E.

Figure 209.-Cynomolgus, Eye, Retina, Displacement, photoreceptor nuclei (rod/cone), and Edema, H\&E. 

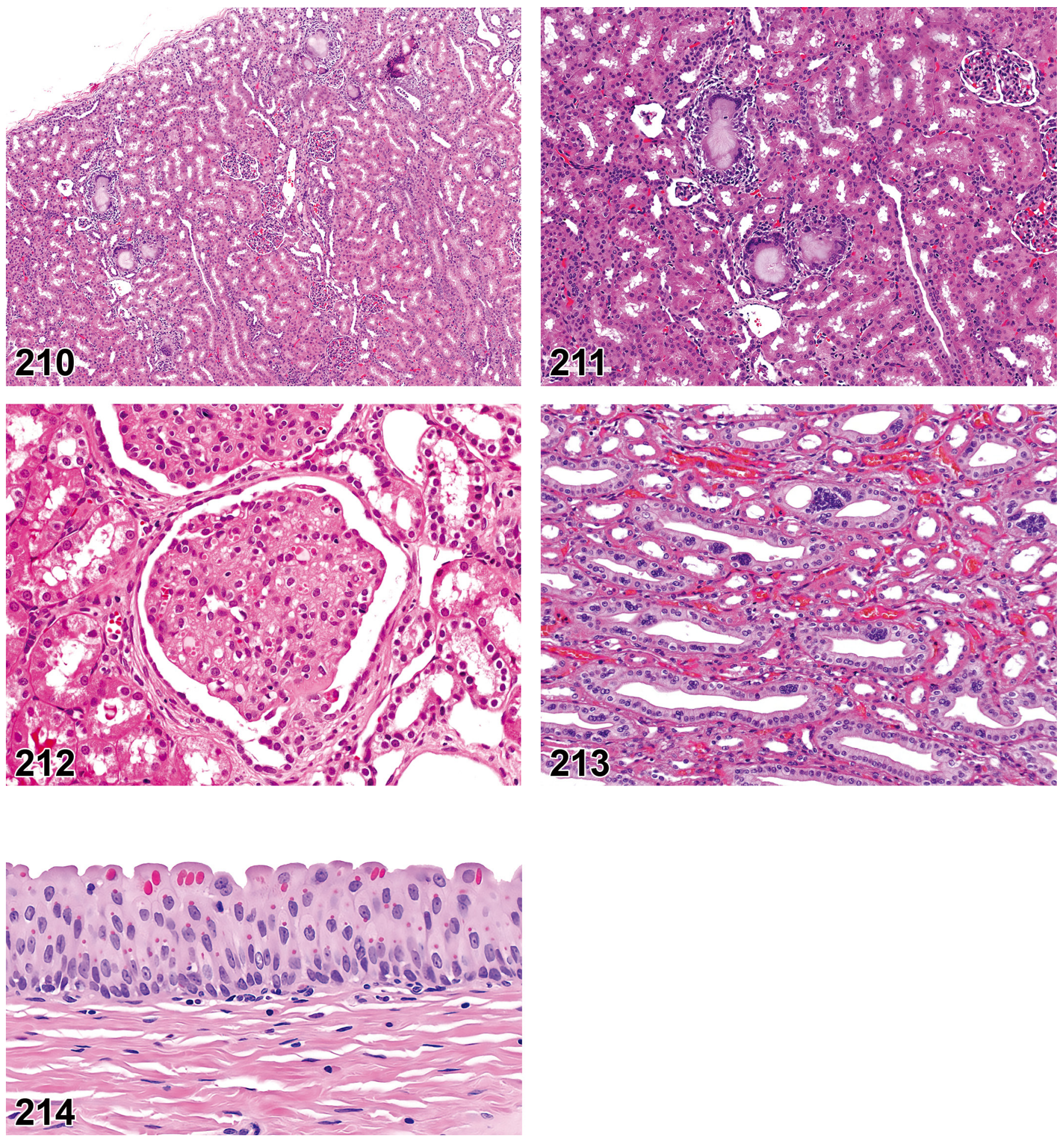

Figure 210.-Cynomolgus, Kidney, Crystals, H\&E, Low magnification. Figure 211.-Cynomolgus, Kidney, Crystals, H\&E, High magnification.

Figure 212.-Cynomolgus, Kidney, Eosinophilic droplets, glomerulus, H\&E; Courtesy of Dr. Kendall Frazier.

FIGURE 213.-Cynomolgus, Kidney, Multinucleated cells, papilla (collecting ducts), H\&E.

FIGURE 214.-Cynomolgus, Urinary bladder, Inclusions, eosinophilic cytoplasmic, H\&E. 


\section{REFERENCES}

1. Kaspareit J, Friderichs-Gromoll S, Buse E, and Habermann G. Spontaneous neoplasms observed in cynomolgus monkeys (Macaca fascicularis) during a 15-year period. Experimental and toxicologic pathology: official journal of the Gesellschaft fur Toxikologische Pathologie. 59:163-169. 2007.

2. Miller AD. Neoplasia and Proliferative Disorders of Nonhuman Primates. In: Nonhuman Primates in Biomedical Research (Second Edition). Vol 2. CR Abee, K Mansfield, S Tardif, T Morris (eds). Academic Press, Boston. 325-356. 2012.

3. Mann PC, Vahle J, Keenan CM, Baker JF, Bradley AE, Goodman DG, Harada T, Herbert R, Kaufmann W, Kellner R, Nolte T, Rittinghausen $\mathrm{S}$, and Tanaka T. International harmonization of toxicologic pathology nomenclature: an overview and review of basic principles. Toxicol Pathol. 40(Suppl): 7S-13S. 2012. [Medline] [CrossRef]

4. Elmore SA, Dixon D, Hailey JR, Harada T, Herbert RA, Maronpot RR, Nolte T, Rehg JE, Rittinghausen S, Rosol TJ, Satoh H, Vidal JD, Willard-Mack CL, and Creasy DM. Recommendations from the INHAND Apoptosis/Necrosis Working Group. Toxicol Pathol. 44: 173-188. 2016. [Medline] [CrossRef]

5. Greaves P, Chouinard L, Ernst H, Mecklenburg L, Pruimboom-Brees IM, Rinke M, Rittinghausen S, Thibault S, Von Erichsen J, and Yoshida T. Proliferative and non-proliferative lesions of the rat and mouse soft tissue, skeletal muscle and mesothelium. J Toxicol Pathol. 26(Suppl): 1S-26S. 2013. [Medline] [CrossRef]

6. Chamanza R, Marxfeld HA, Blanco AI, Naylor SW, and Bradley AE. Incidences and range of spontaneous findings in control cynomolgus monkeys (Macaca fascicularis) used in toxicity studies. Toxicol Pathol. 38: 642-657. 2010. [Medline] [CrossRef]

7. Haley PJ. The lymphoid system: a review of species differences. J Toxicol Pathol. 30: 111-123. 2017. [Medline] [CrossRef]

8. Kumar V, Abbas A, Fausto N, and Aster JC. Diseases of the Immune System. In: Robbins and Cotran Pathologic Basis of Disease, 8th ed. V Kumar, A Abbas, N Fausto, and JC Aster (eds). Saunders/Elsevier, Philadelphia. 2010.

9. Woldemeskel M. A concise review of amyloidosis in animals. Vet Med Int. 2012: 427296. 2012. [Medline] [CrossRef]

10. Abee CR, Mansfield K, Tardif S, and Morris T. Index. In: Nonhuman Primates in Biomedical Research (Second Edition) Vol 2. 2nd ed. CR Abee, K Mansfield, S Tardif, T Morris (eds). Academic Press, Boston. 841-852. 2012.

11. Sato J, Doi T, Kanno T, Wako Y, Tsuchitani M, and Narama I. Histopathology of incidental findings in cynomolgus monkeys ( macaca fascicularis ) used in toxicity studies. J Toxicol Pathol. 25: 63-101. 2012. [Medline] [CrossRef]

12. Wagner JD, Carlson CS, O'Brien TD, Anthony MS, Bullock BC, and Cefalu WT. Diabetes mellitus and islet amyloidosis in cynomolgus monkeys. Lab Anim Sci. 46: 36-41. 1996. [Medline]

13. Nakamura S, Nakayama H, Goto N, Ono F, Sakakibara I, and Yoshikawa Y. Histopathological studies of senile plaques and cerebral amyloidosis in cynomolgus monkeys. J Med Primatol. 27: 244-252. 1998. [Medline] [CrossRef]

14. Bailey C, and Mansfield K. Emerging and reemerging infectious diseases of nonhuman primates in the laboratory setting. Vet Pathol. 47: 462-481. 2010. [Medline] [CrossRef]

15. Irons RD. 16 - Blood and Bone Marrow. In: Handbook of Toxicologic Pathology. WM Haschek, and CG Rousseaux (eds). Academic Press, San Diego. 389-419. 1991.

16. Mense MG, Batey KL, Estep S, Armstrong K, Fleurie G, and Suttie AW. Disseminated Coccidioidomycosis in a Cynomolgus Monkey (Macaca fascicularis). Primatology. 2: 111. 2013.

17. Sasseville VG, and Mansfield KG. Overview of known non-human primate pathogens with potential to affect colonies used for toxicity testing. J Immunotoxicol. 7: 79-92. 2010. [Medline] [CrossRef]

18. Magden ER, Mansfield KG, Simmons JH, and Abee CR. Chapter 17
- Nonhuman Primates. In: Fox JG, Anderson LC, Otto GM, PritchettCorning KR, Whary MT, eds. Laboratory Animal Medicine (Third Edition). Boston: Academic Press; 2015:771-930.

19. Iverson WO, Karanth S, Wilcox A, Pham CD, Lockhart SR, and Nicholson SM. Talaromycosis (Penicilliosis) in a Cynomolgus Macaque. Vet Pathol. 55: 591-594. 2018. [Medline] [CrossRef]

20. Lerche NW, and Simmons JH. Beyond specific pathogen-free: biology and effect of common viruses in macaques. Comp Med. 58: 8-10. 2008. [Medline]

21. Sasseville VG, and Diters RW. Impact of infections and normal flora in nonhuman primates on drug development. ILAR J. 49: 179-190. 2008. [Medline] [CrossRef]

22. Mansfield KG, Sasseville VG, and Westmoreland SV. Molecular localization techniques in the diagnosis and characterization of nonhuman primate infectious diseases. Vet Pathol. 51: 110-126. 2014. [Medline] [CrossRef]

23. Osheroff MR, Uno H, and Bowman RE. Lead inclusion bodies in the anterior horn cells and neurons of the substantia nigra in the adult rhesus monkey. Toxicol Appl Pharmacol. 64: 570-576. 1982. [Medline] [CrossRef]

24. Karl SL, and Wong MM. A survey of Sarcocystis in non-human primates. Lab Anim Sci. 25: 641-645. 1975.

25. Abbott DP, and Majeed SK. A survey of parasitic lesions in wildcaught, laboratory-maintained primates: (rhesus, cynomolgus, and baboon). Vet Pathol. 21: 198-207. 1984. [Medline] [CrossRef]

26. Shoieb A, Radi ZA. Cerebral Baylisascaris larva migrans in a cynomolgus macaque (Macaca fascicularis). Experimental and toxicologic pathology: official journal of the Gesellschaft fur Toxikologische Pathologie. 2014;66(5-6):263-265.

27. Wachtman LM, and Mansfield KG. Opportunistic infections in immunologically compromised nonhuman primates. ILAR J. 49: 191-208. 2008. [Medline] [CrossRef]

28. Pisharath H, Zao CL, Kreeger J, Portugal S, Kawabe T, Burton T, Tomaeck L, Shoieb A, Campbell BM, and Franco J. Immunopathologic characterization of naturally acquired Trypanosoma cruzi infection and cardiac sequalae in cynomolgus macaques (Macaca fascicularis). $J$ Am Assoc Lab Anim Sci. 52: 545-552. 2013. [Medline]

29. Megill J, MacGuire J, Jackson RK, Janovitz EB, and Dubey JP. Transmission Electron Microscopy Used to Diagnose Acute Toxoplasmosis in a Quarantined, Captive Born Cynomolgus Macaque. Microsc Microanal. 20(S3): 1408-1409. 2014. [CrossRef]

30. Kumar V, Abbas AK, and Aster JC. Cellular Responses to Stress and Toxic Insults: Adaptation, Injury and Death. In: Robbins \& Cotran Pathologic Basis of Disease. 9th ed. Philadelphia, PA: Saunders Elsevier; 2015.

31. Miller MA, and Zachary JF. Mechanisms and Morphology of Cellular Injury, Adaptation, and Death. In: Zachary JF, McGavin MD, eds. Pathologic Basis of Veterinary Disease. 6th ed. St Louis, MO: Elsevier; 2017.

32. Yamakawa Y, Ide T, Mitori H, Oishi Y, and Matsumoto M. Accumulation of brown pigment-laden macrophages associated with vascular lesions in the lungs of cynomolgus monkeys(Macaca fascicularis). $J$ Toxicol Pathol. 29: 181-184. 2016. [Medline] [CrossRef]

33. Purcell JE, Philipp MT. Parasitic Diseases of Nonhuman Primates. 2005:579-593.

34. Fernandez-Flores A. Two new forms of hematoidin in the skin. J Cutan Pathol. 42: 1026-1030. 2015. [Medline] [CrossRef]

35. Ameri M. Laboratory diagnosis of malaria in nonhuman primates. Vet Clin Pathol. 39: 5-19. 2010. [Medline] [CrossRef]

36. Bronson RT, O’Connell M, Klepper-Kilgore N, Chalifoux LV, and Sehgal P. Fatal fasting syndrome of obese macaques. Lab Anim Sci. 32: 187-192. 1982. [Medline]

37. Laber-Laird KE, Jokinen MP, and Lehner ND. Fatal fatty liver-kidney syndrome in obese monkeys. Lab Anim Sci. 37: 205-209. 1987. [Medline]

38. Donnelly TM. Sudden death in an anorectic cynomolgus macaque. Fatal fasting syndrome of obese macaques. Lab Anim (NY). 32: 23-25. 
2003. [Medline]

39. Hukkanen RRVK, Gillen M, Murnane RD, and Kelley ST. Fatal Fasting Syndrome in the Pig-Tailed Macaque in: Combined 57th and 41st Annual Meetings of the American College of Veterinary Pathologists and the American Society for Veterinary Clinical Pathology. Vet Pathol. 43: 805-859. 2006. [CrossRef]

40. Christe KL, and Valverde CR. The Use of a Percutaneous Endoscopic Gastrotomy (PEG) Tube To Reverse Fatal Fasting Syndrome in a Cynomolgus Macaque (Macaca fascicularis). Contemp Top Lab Anim Sci. 38: 12-15. 1999. [Medline]

41. Fant P, Bellebeau F, and Howroyd P. TP14: Fatal fasting syndrome in a cynomolgus monkey (M. fascicularis). Paper presented at: Cutting Edge Pathology: 2nd Joint Europena Congress of the ESVP, ESTP and ECVP2014; Berlin, Germany.

42. Gliatto JM, and Bronson RT. Fatal Fasting Syndrome of Obese Macaques. In: Jones TC, Mohr U, Hunt RD, eds. Nonhuman Primates I. Berlin, Heidelberg: Springer Berlin Heidelberg; 1993:198-202.

43. Völker I, and Plesker R. Acute Pancreatic Necrosis in a Diabetic LongTailed Macaque (Macaca fascicularis). Laboratory Primate Newsletter. 50: 1-3. 2011.

44. Chandler FW, and McClure HM. Adenoviral pancreatitis in rhesus monkeys: current knowledge. Vet Pathol Suppl. 7: 171-180. 1982. [Medline] [CrossRef]

45. Longo DL. Part 4: Section 1: Neoplastic Disorders. In: Jameson JL, Fauci AS, Kasper DL, Hauser SL, Longo DL, Loscalzo J, eds. Harrison's Principles of Internal Medicine, 20e. New York, NY: McGrawHill Education; 2018.

46. Prencipe G, Bracaglia C, Caiello I, Pascarella A, Francalanci P, Pardeo M, Meneghel A, Martini G, Rossi MN, Insalaco A, Marucci G, Nobili V, Spada M, Zulian F, and De Benedetti F. The interferon-gamma pathway is selectively up-regulated in the liver of patients with secondary hemophagocytic lymphohistiocytosis. PLoS One. 14: e0226043. 2019. [Medline] [CrossRef]

47. Benveniste O, Dereuddre-Bosquet N, Clayette P, Leport C, Vildé JL, and Dormont D. High levels of IL-10 and determination of other cytokines and chemokines in HIV-associated haemophagocytic syndrome. Clin Exp Immunol. 121: 320-323. 2000. [Medline] [CrossRef]

48. Montiel NA, Todd PA, Yee J, and Lerche NW. Effects of simian betaretrovirus serotype 1 (SRV1) infection on the differentiation of hematopoietic progenitor cells $(\mathrm{CD} 34+)$ derived from bone marrow of rhesus macaques (Macaca mulatta). Comp Med. 62: 61-68. 2012. [Medline]

49. Wachtman L, and Mansfield K. Chapter 1 - Viral Diseases of Nonhuman Primates. In: Abee CR, Mansfield K, Tardif S, Morris T, eds. Nonhuman Primates in Biomedical Research (Second Edition). Boston: Academic Press; 2012:1-104.

50. Cotroneo TM, Colby LA, and Bergin IL. Hemophagocytic syndrome in a pancytopenic simian retrovirus-infected male rhesus macaque (Macaca mulatta). Vet Pathol. 48: 1138-1143. 2011. [Medline] [CrossRef]

51. Walton RM, Modiano JF, Thrall MA, and Wheeler SL. Bone marrow cytological findings in 4 dogs and a cat with hemophagocytic syndrome. J Vet Intern Med. 10: 7-14. 1996. [Medline] [CrossRef]

52. Weiss DJ. Hemophagocytic syndrome in dogs: 24 cases (1996-2005). $J$ Am Vet Med Assoc. 230: 697-701. 2007. [Medline] [CrossRef]

53. Harrington DS, Weisenburger DD, and Purtilo DT. Malignant lymphoma in the X-linked lymphoproliferative syndrome. Cancer. 59: 1419-1429. 1987. [Medline] [CrossRef]

54. Elazary AS, Wolf DG, Amir G, et al. Severe Epstein-Barr virus-associated hemophagocytic syndrome in six adult patients. Journal of clinical virology: the official publication of the Pan American Society for Clinical Virology. 2007;40(2):156-159.

55. Lenz B, Braendli-Baiocco A, Engelhardt J, Fant P, Fischer H, Francke S, Fukuda R, Gröters S, Harada T, Harleman H, Kaufmann W, Kustermann S, Nolte T, Palazzi X, Pohlmeyer-Esch G, Popp A, Romeike A, Schulte A, Lima BS, Tomlinson L, Willard J, Wood CE, and Yoshida M. Characterizing Adversity of Lysosomal Accumulation in Nonclinical Toxicity Studies: Results from the 5th ESTP International Expert Workshop. Toxicol Pathol. 46: 224-246. 2018. [Medline] [CrossRef]
56. Berridge BR, Mowat V, Nagai H, Nyska A, Okazaki Y, Clements PJ, Rinke M, Snyder PW, Boyle MC, and Wells MY. Non-proliferative and Proliferative Lesions of the Cardiovascular System of the Rat and Mouse. J Toxicol Pathol. 29(Suppl): 1S-47S. 2016. [Medline] [CrossRef]

57. Keenan CCT, and Mahler BW. Heart Trimming Protocol of the Laboratory Dog and Monkey. In:2005.

58. Register TC, Appt SE, and Clarkson TB. Atherosclerosis and Vascular Biologic Responses to Estrogens: Histologic, Immunohistochemical, Biochemical, and Molecular Methods. Methods Mol Biol. 1366: 517532. 2016. [Medline] [CrossRef]

59. Keenan CM, and Vidal JD. Standard morphologic evaluation of the heart in the laboratory dog and monkey. Toxicol Pathol. 34: 67-74. 2006. [Medline] [CrossRef]

60. Mueller RW, Gill SS, and Pulido OM. The monkey (Macaca fascicularis) heart neural structures and conducting system: an immunochemical study of selected neural biomarkers and glutamate receptors. Toxicol Pathol. 31: 227-234. 2003. [Medline]

61. Drevon-Gaillot E, Perron-Lepage MF, Clement C, Burnett R. A review of background findings in cynomolgus monkeys (Macaca fascicularis) from three different geographical origins. Experimental and toxicologic pathology: official journal of the Gesellschaft fur Toxikologische Pathologie. 2006;58(2-3):77-88.

62. Kaspareit J, Friderichs-Gromoll S, Buse E, Habermann G, Vogel F. Spontaneous squamous cysts and squamous epithelial plaques in the heart of cynomolgus monkeys (Macaca fascicularis). Experimental and toxicologic pathology: official journal of the Gesellschaft fur Toxikologische Pathologie. 2003;54(4):301-303.

63. Vidal JD, Drobatz LS, Holliday DF, Geiger LE, and Thomas HC. Spontaneous findings in the heart of Mauritian-origin cynomolgus macaques (Macaca fascicularis). Toxicol Pathol. 38: 297-302. 2010. [Medline] [CrossRef]

64. Agudelo CF, Svoboda M, Husnik R, and Dvir S. Heart lipomatosis in domestic animals: A review. Vet Med Veterinarni Medicina. 58: 252259. 2013. [CrossRef]

65. Harruff RC. Subendocardial hemorrhages in forensic pathology autopsies. Am J Forensic Med Pathol. 14: 284-288. 1993. [Medline] [CrossRef]

66. Charaschaisri W, Jongprasartsuk K, Rungruanghiranya S, and Kaufman L. Forensic aspect of cause of subendocardial hemorrhage in cardiopulmonary resuscitation cases: chest compression or adrenaline. Am J Forensic Med Pathol. 32: 58-60. 2011. [Medline] [CrossRef]

67. Reader JR, Canfield DR, Lane JF, Kanthaswamy S, Ardeshir A, Allen AM, and Tarara RP. Left Ventricular Hypertrophy in Rhesus Macaques (Macaca mulatta) at the California National Primate Research Center (1992-2014). Comp Med. 66: 162-169. 2016. [Medline]

68. Vogel P, and Fritz D. Cardiomyopathy associated with angiomatous pheochromocytoma in a rhesus macaque (Macaca mulatta). Vet Pathol. 40: 468-473. 2003. [Medline] [CrossRef]

69. Waites CR, Dominick MA, Sanderson TP, Schilling BE. Nonclinical safety evaluation of muraglitazar, a novel PPARalpha/gamma agonist. Toxicological sciences: an official journal of the Society of Toxicology. 2007;100(1):248-258

70. Papamitsou T, Barlagiannis D, Papaliagkas V, Kotanidou E, and Dermentzopoulou-Theodoridou M. Testosterone-induced hypertrophy, fibrosis and apoptosis of cardiac cells--an ultrastructural and immunohistochemical study. Med Sci Monit. 17: BR266-BR273. 2011. [Medline] [CrossRef]

71. Marsh JD, Lehmann MH, Ritchie RH, Gwathmey JK, Green GE, and Schiebinger RJ. Androgen receptors mediate hypertrophy in cardiac myocytes. Circulation. 98: 256-261. 1998. [Medline] [CrossRef]

72. Thorgeirsson UP, Farb A, Virmani R, and Adamson RH. Cardiac damage induced by 2-amino-3-methyl-imidazo[4,5-f]quinoline in nonhuman primates. Environ Health Perspect. 102: 194-199. 1994. [Medline]

73. Zhang XP, Vatner SF, Shen YT, Rossi F, Tian Y, Peppas A, Resuello RR, Natividad FF, and Vatner DE. Increased apoptosis and myocyte enlargement with decreased cardiac mass; distinctive features of the 
aging male, but not female, monkey heart. J Mol Cell Cardiol. 43: 487491. 2007. [Medline] [CrossRef]

74. Jasty V, Kowalski RL, Fonseca EH, Porter MC, Clemens GR, Bare JJ, and Hartnagel RE. An unusual case of generalized ceroid-lipofuscinosis in a cynomolgus monkey. Vet Pathol. 21: 46-50. 1984. [Medline] [CrossRef]

75. Jasty V, Jamison JR, and Hartnagel RE. Three types of cytoplasmic granules in cardiac muscle cells of cynomolgus monkeys (Macaca fascicularis). Vet Pathol. 21: 505-508. 1984. [Medline] [CrossRef]

76. Tay SS, Wong WC, and Ling EA. An ultrastructural study of small granule-containing cells in the heart of the monkey (Macaca fascicularis). J Anat. 136: 35-45. 1983. [Medline]

77. Chamanza R, Parry NM, Rogerson P, Nicol JR, and Bradley AE. Spontaneous lesions of the cardiovascular system in purpose-bred laboratory nonhuman primates. Toxicol Pathol. 34: 357-363. 2006. [Medline] [CrossRef]

78. Keenan CM, and Vidal JD. Standard morphologic evaluation of the heart in the laboratory dog and monkey. Toxicol Pathol. 34: 67-74. 2006. [Medline] [CrossRef]

79. Qureshi SR. Chronic interstitial myocarditis in primates. Vet Pathol. 16: 486-487. 1979. [Medline] [CrossRef]

80. Dunn ME, Coluccio D, Zabka TS, Gopalakrishnan G, Hirkaler G, Geng W, Nicklaus R, Lipshultz SE, Doessegger L, Saladino BH, Singer $\mathrm{T}$, and Mikaelian I. Myocardial mononuclear cell infiltrates are not associated with increased serum cardiac troponin I in cynomolgus monkeys. Toxicol Pathol. 40: 647-650. 2012. [Medline] [CrossRef]

81. Toft JD, and Eberhard ML. Chapter 3 - Parasitic Diseases. In: Bennett BT, Abee CR, Henrickson R, eds. Nonhuman Primates in Biomedical Research. New York: Academic Press; 1998:111-205.

82. April M, and Keith JC. Chapter 6 - Cardiovascular and Lymphoreticular Systems. In: Bennett BT, Abee CR, Henrickson R, eds. Nonhuman Primates in Biomedical Research. New York: Academic Press; 1998:245-262.

83. Osborn KG, Prahalada S, Lowenstine LJ, Gardner MB, Maul DH, and Henrickson RV. The pathology of an epizootic of acquired immunodeficiency in rhesus macaques. Am J Pathol. 114: 94-103. 1984. [Medline]

84. Zabka TS, Irwin M, and Albassam MA. Spontaneous cardiomyopathy in cynomolgus monkeys (Macaca fascicularis). Toxicol Pathol. 37: 814-818. 2009. [Medline] [CrossRef]

85. Konishi S, Kotera T, Koga M, and Ueda M. Spontaneous hypertrophic cardiomyopathy in a cynomolgus macaque (Macaca fascicularis). $J$ Toxicol Pathol. 31: 49-54. 2018. [Medline] [CrossRef]

86. Khullar M, Datta BN, Wahi PL, and Chakravarti RN. Catecholamineinduced experimental cardiomyopathy--a histopathological, histochemical and ultrastructural study. Indian Heart J. 41: 307-313. 1989. [Medline]

87. Mubiru JN, Yang A, Dick EJ Jr, Owston M, Sharp RM, VandeBerg JF, Shade RE, and VandeBerg JL. Correlation between presence of Trypanosoma cruzi DNA in heart tissue of baboons and cynomolgus monkeys, and lymphocytic myocarditis. Am J Trop Med Hyg. 90: 627-633. 2014. [Medline] [CrossRef]

88. Schillaci MA, Jones-Engel L, Heidrich JE, Benamore R, Pereira A, and Paul N. Thoracic radiography of pet macaques in Sulawesi, Indonesia. J Med Primatol. 37: 141-145. 2008. [Medline] [CrossRef]

89. Colman RJ, Anderson RM, Johnson SC, Kastman EK, Kosmatka KJ, Beasley TM, Allison DB, Cruzen C, Simmons HA, Kemnitz JW, and Weindruch R. Caloric restriction delays disease onset and mortality in rhesus monkeys. Science. 325: 201-204. 2009. [Medline] [CrossRef]

90. Freed LA, Levy D, Levine RA, Larson MG, Evans JC, Fuller DL, Lehman B, and Benjamin EJ. Prevalence and clinical outcome of mitral-valve prolapse. $N$ Engl J Med. 341: 1-7. 1999. [Medline] [CrossRef]

91. Swindle MM, Blum JR, Lima SD, and Weiss JL. Spontaneous mitral valve prolapse in a breeding colony of rhesus monkeys. Circulation. 71: 146-153. 1985. [Medline] [CrossRef]

92. Bendjama K, Guionaud S, Aras G, Arber N, Badimon L, Bamberger U, Bratfalean D, Brott D, David M, Doessegger L, Firat H, Gallas JF,
Gautier JC, Hoffmann P, Kraus S, Padro T, Saadoun D, Szczesny P, Thomann P, Vilahur G, Lawton M, and Cacoub P. Translation strategy for the qualification of drug-induced vascular injury biomarkers. Toxicol Pathol. 42: 658-671. 2014. [Medline] [CrossRef]

93. Frazier KS, Engelhardt JA, Fant P, Guionaud S, Henry SP, Leach MW, Louden C, Scicchitano MS, Weaver JL, Zabka TS. Society of Toxicologic Pathology Vascular Injury Working Group Scientific and Regulatory Policy Committee Points-to-consider Paper*: Drug-induced Vascular Injury Associated with Nonsmall Molecule Therapeutics in Preclinical Development: Part I. Biotherapeutics. Toxicol Pathol. 43: 915-934. 2015. [Medline] [CrossRef]

94. Engelhardt JA, Fant P, Guionaud S, Henry SP, Leach MW, Louden C, Scicchitano MS, Weaver JL, Zabka TS, Frazier KS. Society of Toxicologic Pathology Vascular Injury Working Group Scientific and Regulatory Policy Committee Points-to-consider Paper*: Drug-induced Vascular Injury Associated with Nonsmall Molecule Therapeutics in Preclinical Development: Part 2. Antisense Oligonucleotides. Toxicol Pathol. 43: 935-944. 2015. [Medline] [CrossRef]

95. Sasseville VG, Mansfield KG, Mankowski JL, Tremblay C, Terio KA, Mätz-Rensing K, Gruber-Dujardin E, Delaney MA, Schmidt LD, Liu D, Markovits JE, Owston M, Harbison C, Shanmukhappa S, Miller AD, Kaliyaperumal S, Assaf BT, Kattenhorn L, Macri SC, Simmons HA, Baldessari A, Sharma P, Courtney C, Bradley A, Cline JM, Reindel JF, Hutto DL, Montali RJ, and Lowenstine LJ. Meeting report: Spontaneous lesions and diseases in wild, captive-bred, and zoo-housed nonhuman primates and in nonhuman primate species used in drug safety studies. Vet Pathol. 49: 1057-1069. 2012. [Medline] [CrossRef]

96. Getz GS, and Reardon CA. Animal models of atherosclerosis. Arterioscler Thromb Vasc Biol. 32: 1104-1115. 2012. [Medline] [CrossRef]

97. Manning PJ, and Clarkson TB. Development, distribution, and lipid content of diet-induced atherosclerotic lesions of rhesus monkeys. Exp Mol Pathol. 17: 38-54. 1972. [Medline] [CrossRef]

98. Wilkinson IB, McEniery CM, and Cockcroft JR. Arteriosclerosis and atherosclerosis: guilty by association. Hypertension. 54: 1213-1215. 2009. [Medline] [CrossRef]

99. Rudijanto A. The role of vascular smooth muscle cells on the pathogenesis of atherosclerosis. Acta Med Indones. 39: 86-93. 2007. [Medline]

100. Russell DS. Comparative Pathology: Cardiovascular System. In: McManus LM, Mitchell RN, eds. Pathobiology of Human Disease. San Diego: Academic Press; 2014:1042-1070.

101. Seidman MA, Mitchell RN, and Stone JR. Chapter 12 - Pathophysiology of Atherosclerosis. In: Willis MS, Homeister JW, Stone JR, eds. Cellular and Molecular Pathobiology of Cardiovascular Disease. San Diego: Academic Press; 2014:221-237.

102. Bond MG, Bullock BC, Bellinger DA, and Hamm TE. Myocardial infarction in a large colony of nonhuman primates with coronary artery atherosclerosis. Am J Pathol. 101: 675-692. 1980. [Medline]

103. Porter BF, Frost P, and Hubbard GB. Polyarteritis nodosa in a cynomolgus macaque (Macaca fascicularis). Vet Pathol. 40: 570-573. 2003. [Medline] [CrossRef]

104. Frazier KS. Antisense oligonucleotide therapies: the promise and the challenges from a toxicologic pathologist's perspective. Toxicol Pathol. 43: 78-89. 2015. [Medline] [CrossRef]

105. Beach JE, Blair AM, Perani CL, Cox GE, and Dixon FJ. An unusual form of proliferative arteriopathy in macaque monkeys (Macacca sps). Exp Mol Pathol. 21: 322-338. 1974. [Medline] [CrossRef]

106. Lilbert J, and Burnett R. Main vascular changes seen in the saline controls of continuous infusion studies in the cynomolgus monkey over an eight-year period. Toxicol Pathol. 31: 273-280. 2003. [Medline] [CrossRef]

107. Kast A. Pulmonary hair embolism in monkeys. Experimental and toxicologic pathology: official journal of the Gesellschaft fur Toxikologische Pathologie. 1994;46(3):183-188.

108. Yanai T, Lackner AA, Sakai H, Masegi T, and Simon MA. Systemic arteriopathy in SIV-infected rhesus macaques (Macaca mulatta). J Med Primatol. 35: 106-112. 2006. [Medline] [CrossRef]

109. Chalifoux LV, Simon MA, Pauley DR, MacKey JJ, Wyand MS, and 
Ringler DJ. Arteriopathy in macaques infected with simian immunodeficiency virus. Lab Invest. 67: 338-349. 1992. [Medline]

110. George MP, Brower A, Kling H, Shipley T, Kristoff J, Reinhart TA, Murphey-Corb M, Gladwin MT, Champion HC, Morris A, and Norris KA. Pulmonary vascular lesions are common in SIV- and SHIV-envinfected macaques. AIDS Res Hum Retroviruses. 27: 103-111. 2011. [Medline] [CrossRef]

111. Lombardini ED, Virmani R, Blanchard TW, Lafond JF, Ménard S, and Doré M. Epithelioid hemangioendothelioma in the right auricle of an adult, male Rhesus macaque (Macaca mulatta). J Med Primatol. 39: 315-317. 2010. [Medline] [CrossRef]

112. Nolte T, Brander-Weber P, Dangler C, Deschl U, Elwell MR, Greaves P, Hailey R, Leach MW, Pandiri AR, Rogers A, Shackelford CC, Spencer A, Tanaka T, and Ward JM. Nonproliferative and Proliferative Lesions of the Gastrointestinal Tract, Pancreas and Salivary Glands of the Rat and Mouse. J Toxicol Pathol. 29(Suppl): 1S-125S. 2016. [Medline] [CrossRef]

113. Okazaki Y, Matsumoto M, Tsubota K, Nakatsuji S, Fujihira S, and Oishi Y. Foregut cyst of the oesophageal wall in a cynomolgus monkey (Macaca fascicularis). J Comp Pathol. 135: 259-262. 2006. [Medline] [CrossRef]

114. van Esch E, and Brennan S. Sebaceous gland metaplasia in the oesophagus of a cynomolgus monkey (Macaca fascicularis). J Comp Pathol. 147: 248-252. 2012. [Medline] [CrossRef]

115. Miles AEW. Absence of sebaceous glands from the oral mucosa of non-human primates. Primates Primates. 12: 95-96. 1971. [CrossRef]

116. Lowenstine LJ. A primer of primate pathology: lesions and nonlesions. Toxicol Pathol. 31(Suppl): 92-102. 2003. [Medline]

117. Brady AG, and Carville AAL. Chapter 12 - Digestive System Diseases of Nonhuman Primates. In: Abee CR, Mansfield K, Tardif S, Morris T, eds. Nonhuman Primates in Biomedical Research (Second Edition). Boston: Academic Press; 2012:589-627.

118. Lee JI, Kim KS, Oh BC, Kim NA, Kim IH, Park CG, and Kim SJ. Acute necrotic stomatitis (noma) associated with methicillin-resistant Staphylococcus aureus infection in a newly acquired rhesus macaque (Macaca mulatta). J Med Primatol. 40: 188-193. 2011. [Medline] [CrossRef]

119. Vidal JD, Mirabile RC, and Thomas HC. Evaluation of the cynomolgus monkey stomach: recommendations for standard sampling procedures in nonclinical safety studies. Toxicol Pathol. 36: 250-255. 2008. [Medline] [CrossRef]

120. Ito T, Chatani F, Sasaki S, Ando T, and Miyajima H. Spontaneous lesions in cynomolgus monkeys used in toxicity studies. Jikken Dobutsu. 41: 455-469. 1992. [Medline]

121. McKeag S, and McInnes EF. The Incidence of Lymphoplasmacytic Gastritis in the Fundus and Antrum of Cynomolgus Monkey ( Macaca fascicularis ) Stomachs. J Toxicol Pathol. 25: 249-256. 2012. [Medline] [CrossRef]

122. Shimoi A, Kakinuma C, Kuwayama C, and Watanabe M. Comparison of Spontaneous Minor Lesions in Wild-caught and Laboratory-bred Monkeys. J Toxicol Pathol. 11: 85-94. 1998. [CrossRef]

123. King NW. Nochtiasis. In: Jones TC, Mohr U, Hunt RD, eds. Nonhuman Primates. Berlin, Heidelberg: Springer Berlin Heidelberg; 1993:238240.

124. Scott GB. Mucosal microhernias in the nonhuman primate colon: their role in the pathogenesis of colonic disease. Vet Pathol Suppl. 19(Suppl 7): 134-140. 1982. [Medline] [CrossRef]

125. Fujisawa H, Zhang Z, Sun W, Huang M, Kobayashi J, Yasuda H, Kinoshita Y, Ando R, and Tamura K. Histopathological changes in the pancreas from a spontaneous hyperglycemic cynomolgus monkey. $J$ Toxicol Pathol. 25: 215-219. 2012. [Medline] [CrossRef]

126. Mansfield KG, Carville A, Shvetz D, MacKey J, Tzipori S, and Lackner AA. Identification of an Enterocytozoon bieneusi-like microsporidian parasite in simian-immunodeficiency-virus-inoculated macaques with hepatobiliary disease. Am J Pathol. 150: 1395-1405. 1997. [Medline]

127. Brändli-Baiocco A, Balme E, Bruder M, Chandra S, Hellmann J, Hoenerhoff MJ, Kambara T, Landes C, Lenz B, Mense M, Rittinghausen S,
Satoh H, Schorsch F, Seeliger F, Tanaka T, Tsuchitani M, Wojcinski Z, and Rosol TJ. Nonproliferative and Proliferative Lesions of the Rat and Mouse Endocrine System. J Toxicol Pathol. 31(Suppl): 1S-95S. 2018. [Medline] [CrossRef]

128. Remick AK, Wood CE, Cann JA, Gee MK, Feiste EA, Kock ND, and Cline JM. Histologic and immunohistochemical characterization of spontaneous pituitary adenomas in fourteen cynomolgus macaques (Macaca fascicularis). Vet Pathol. 43: 484-493. 2006. [Medline] [CrossRef]

129. Weber K, Ernst R, Fankhauser H, Hardisty JF, Heider W, and Stevens K. Thyroid Dysplasia in Wistar Hannover GALAS Rats. $J$ Toxicol Pathol. 22: 247-254. 2009. [Medline] [CrossRef]

130. Hatakeyama H, Takei Y, Cruz Y, Miyoshi S, Watanabe J, Koizumi H, Shimoi A, and Satoh H. Spontaneous vacuolar degeneration of the thyroid follicular epithelium in cynomolgus monkeys. J Toxicol Pathol. 24: 229-232. 2011. [Medline] [CrossRef]

131. Mousa S, and van Esch E. Two cases of adreno-hepatic fusion in cynomolgus monkeys (Macaca fascicularis). Toxicol Pathol. 32: 511-513. 2004. [Medline] [CrossRef]

132. Honma K. Adreno-hepatic fusion. An autopsy study. Zentralbl Pathol. 137: 117-122. 1991. [Medline]

133. Radi Z, Evans M. Retained fetal adrenal cortex in a cynomolgus macaque (Macaca fascicularis). Experimental and toxicologic pathology: official journal of the Gesellschaft fur Toxikologische Pathologie. 2014;66(8):357-359.

134. Ribelin WE. The effects of drugs and chemicals upon the structure of the adrenal gland. Fundamental and applied toxicology: official journal of the Society of Toxicology. 1984;4(1):105-119.

135. Reindel JF, Dominick MA, Bocan TM, Gough AW, and McGuire EJ. Toxicologic effects of a novel acyl-CoA:cholesterol acyltransferase inhibitor in cynomolgus monkeys. Toxicol Pathol. 22: 510-518. 1994. [Medline] [CrossRef]

136. Kaspareit J. Adrenal Gland Background Pathology of Primates in Toxicological Studies. In: Harvey PW, Everett DJ, Springall CJ, eds. Adrenal Toxicology. 1st ed. Boca Raton, FL: CRC Press; 2008.

137. Kaspareit J, Friderichs-Gromoll S, and Buse E. Background Lesions of the Adrenal Gland in Primates From Toxicological Studies. YJCPA Journal of Comparative Pathology. 141: 293. 2009. [CrossRef]

138. Tsuchitani M, Sato J, and Kokoshima H. A comparison of the anatomical structure of the pancreas in experimental animals. J Toxicol Pathol. 29: 147-154. 2016. [Medline] [CrossRef]

139. Willard-Mack CL, Elmore SA, Hall WC, Harleman J, Kuper CF, Losco P, Rehg JE, Rühl-Fehlert C, Ward JM, Weinstock D, Bradley A, Hosokawa S, Pearse G, Mahler BW, Herbert RA, and Keenan CM. Nonproliferative and Proliferative Lesions of the Rat and Mouse Hematolymphoid System. Toxicol Pathol. 47: 665-783. 2019. [Medline] [CrossRef]

140. Food and Drug Administration, HHS. International Conference on Harmonisation; Guidance on S8 Immunotoxicity Studies for Human Pharmaceuticals; availability. Notice. Fed Regist. 71: 19193-19194. 2006. [Medline]

141. Cesta MF. Normal structure, function, and histology of mucosa-associated lymphoid tissue. Toxicol Pathol. 34: 599-608. 2006. [Medline] [CrossRef]

142. Chamanza R, Taylor I, Gregori M, Hill C, Swan M, Goodchild J, Goodchild K, Schofield J, Aldous M, and Mowat V. Normal Anatomy, Histology, and Spontaneous Pathology of the Nasal Cavity of the Cynomolgus Monkey (Macaca fascicularis). Toxicol Pathol. 44: 636-654. 2016. [Medline] [CrossRef]

143. Haley P, Perry R, Ennulat D, Frame S, Johnson C, Lapointe JM, Nyska A, Snyder P, Walker D, Walter G. STP Immunotoxicology Working Group STP position paper: best practice guideline for the routine pathology evaluation of the immune system. Toxicol Pathol. 33: 404-407, discussion 408. 2005. [Medline] [CrossRef]

144. Parker GA, and Snyder PW. Pathology Evaluation for Detection of Immunomodulation. In: Parker GA, ed. Immunopathology in Toxicology and Drug Development: Volume 1, Immunobiology, Investigative 
Techniques, and Special Studies. Cham: Springer International Publishing; 2017:371-442.

145. Everds NE, Reindel J, Werner J, and Craven WA. Variability of Spleen and Mesenteric Lymph Node in Control Cynomolgus Monkeys ( Macaca fascicularis) from Nonclinical Safety Studies: A Retrospective Assessment. Toxicol Pathol. 47: 53-72. 2019. [Medline] [CrossRef]

146. Klumpp SA, Weaver DS, Jerome CP, and Jokinen MP. Salmonella osteomyelitis in a rhesus monkey. Vet Pathol. 23: 190-197. 1986. [Medline] [CrossRef]

147. Kamperschroer C, Tartaro K, and Kumpf SW. Quantitative PCR assays reveal high prevalence of lymphocryptovirus as well as lytic phase gene expression in peripheral blood cells of cynomolgus macaques. $J$ Virol Methods. 207: 220-225. 2014. [Medline] [CrossRef]

148. Kamperschroer C, Gosink MM, Kumpf SW, O'Donnell LM, and Tartaro KR. The genomic sequence of lymphocryptovirus from cynomolgus macaque. Virology. 488: 28-36. 2016. [Medline] [CrossRef]

149. Schmidtko J, Wang R, Wu CL, Mauiyyedi S, Harris NL, Della Pelle P, Brousaides N, Zagachin L, Ferry JA, Wang F, Kawai T, Sachs DH, Cosimi BA, and Colvin RB. Posttransplant lymphoproliferative disorder associated with an Epstein-Barr-related virus in cynomolgus monkeys. Transplantation. 73: 1431-1439. 2002. [Medline] [CrossRef]

150. Cavicchioli L, Ferraresso S, Westmoreland S, Kaliyaperumal S, Knight H, Crossan C, Scobie L, Danesi A, Vadori M, Trez D, Badin RA, Hantraye $\mathrm{P}$, and Cozzi E. Posttransplant Lymphoproliferative Disorders in Neuronal Xenotransplanted Macaques. Vet Pathol. 54: 336-344. 2017. [Medline] [CrossRef]

151. McInnes EF, and Mann P. Background lesions in laboratory animals: a color atlas. Edinburgh; New York: Saunders/Elsevier; 2012.

152. Zalusky R, Ghidoni JJ, McKinley J, Leffingwell TP, and Melville GS. Leukemia in the Rhesus Monkey (Macaca Mulata) Exposed to WholeBody Neutron Irradiation. Radiat Res. 25: 410-416. 1965. [Medline] [CrossRef]

153. Dzhikidze EK, and Iakovleva LA. [A case of leukemia in a monkey exposed to roentgen irradiation for a prolonged period of time]. Probl Gematol Pereliv Krovi. 10: 58-62. (in Russia) 1965. [Medline]

154. Siegal AM, Casey HW, Bowman RW, and Traynor JE. Leukemia in a rhesus monkey (Macaca mulatta) following exposure to whole-body proton irradiation. Blood. 32: 989-996. 1968. [Medline] [CrossRef]

155. Soshin T, Adachi K, Suzuki S, et al Histiocytic Sarcoma in a Cynomolgus Macaque $(<\mathrm{i}>$ Macaca fascicularis $</ \mathrm{i}>)$ Fed with a High-Fat Diet. $J$ Toxicol Pathol. 21: 69-72. 2008. [CrossRef]

156. Hirata A, Hashimoto K, Katoh Y, Sakai H, Bruce AG, Rose TM, Kaneko A, Suzuki J, Nikami H, and Yanai T. Characterization of spontaneous malignant lymphomas in Japanese macaques (Macaca fuscata). Vet Pathol. 52: 566-572. 2015. [Medline] [CrossRef]

157. Hutto DL. Opportunistic infections in non-human primates exposed to immunomodulatory biotherapeutics: considerations and case examples. J Immunotoxicol. 7: 120-127. 2010. [Medline] [CrossRef]

158. Arber DA, and Heerema-McKenney A. Acute Myeloid Leukemia. In: Jaffe ES, Harris NL, Vardiman JW, Campo E, Arber DA, eds. Hematopathology. St. Louis. MO: Elsevier; 2011:672-697.

159. Ioachim HL, Medeiros LJ. Ioachim's lymph node pathology. Philadelphia: Wolters Kluwer health - Lippincott Williams \& Wilkins; 2009.

160. Reagan WJ, Irizarry-Rovira A, Poitout-Belissent F, Bolliger AP, Ramaiah SK, Travlos G, Walker D, Bounous D, Walter G. Bone Marrow Working Group of ASVCP/STP Best practices for evaluation of bone marrow in nonclinical toxicity studies. Vet Clin Pathol. 40: 119-134. 2011. [Medline] [CrossRef]

161. Cunin P, and Nigrovic PA. Megakaryocyte emperipolesis: a new frontier in cell-in-cell interaction. Platelets. 31: 700-706. 2020. [Medline]

162. Rastogi V, Sharma R, Misra SR, Yadav L, and Sharma V. Emperipolesis - a review. J Clin Diagn Res. 8: ZM01-ZM02. 2014. [Medline]

163. Foucar K. Hematopoiesis and Morphological Review of Bone Marrow. In: Bone Marrow Pathology. Chicago: ASCP Press; 2001.

164. Spoor MS, Radi ZA, and Dunstan RW. Characterization of age- and gender-related changes in the spleen and thymus from control cynomolgus macaques used in toxicity studies. Toxicol Pathol. 36: 695-704.
2008. [Medline] [CrossRef]

165. Snyder PW, Everds NE, Craven WA, Werner J, Tannehill-Gregg SH, and Guzman RE. Maturity-related Variability of the Thymus in Cynomolgus Monkeys (Macaca fascicularis). Toxicol Pathol. 44: 874-891. 2016. [Medline] [CrossRef]

166. Mayer S. Zur Lehre von der Schilddrüse und Thymus bei den Amphibien. Anat Anz. 3: 97-103. 1888.

167. Bornemann A, Kirchner T. Thymic myoid cell turnover in myasthenia gravis patients and in normal controls. Virchows Archiv: an international journal of pathology. 1998;432(4):357-361.

168. Le Panse R, and Berrih-Aknin S. Thymic myoid cells protect thymocytes from apoptosis and modulate their differentiation: implication of the ERK and Akt signaling pathways. Cell Death Differ. 12: 463-472. 2005. [Medline] [CrossRef]

169. Kotani Y, Sato J, Wako Y, and Tsuchitani M. Mixed Thymoma in a Young Cynomolgus Monkey (Macaca fascicularis). J Toxicol Pathol. 23: 141-145. 2010. [Medline] [CrossRef]

170. Schwartz JA, Solomon JA, Henkelman K, Leininger JR, and Iverson WO. Spontaneous thymoma in a juvenile cynomolgus macaque (Macaca fascicularis). Toxicol Pathol. 39: 706-710. 2011. [Medline] [CrossRef]

171. Hong JJ, Villinger F, and Courtney CL. PAS-positive extracellular deposits within germinal centers of hyperplastic follicles during SIV infection in a rhesus macaque. J Med Primatol. 43: 374-377. 2014. [Medline] [CrossRef]

172. Kozlosky JC, Mysore J, Clark SP, Burr HN, Li J, Aranibar N, Vuppugalla R, West RC, Mangipudy RS, and Graziano MJ. Comparison of physiologic and pharmacologic parameters in Asian and mauritius cynomolgus macaques. Regul Toxicol Pharmacol. 73: 27-42. 2015. [Medline] [CrossRef]

173. Johns JL, and Christopher MM. Extramedullary hematopoiesis: a new look at the underlying stem cell niche, theories of development, and occurrence in animals. Vet Pathol. 49: 508-523. 2012. [Medline] [CrossRef]

174. Mueller SN, and Germain RN. Stromal cell contributions to the homeostasis and functionality of the immune system. Nat Rev Immunol. 9: 618-629. 2009. [Medline] [CrossRef]

175. Ruehl-Fehlert C, Hartmann E, Rinke M. Reactive and proliferative changes of splenic reticulum cells of rats investigated with special staining methods and immunohistochemistry. Experimental and toxicologic pathology: official journal of the Gesellschaft fur Toxikologische Pathologie. 2008;59(5):281-290.

176. Steiniger BS. Human spleen microanatomy: why mice do not suffice. Immunology. 145: 334-346. 2015. [Medline] [CrossRef]

177. Willard-Mack CL. Normal structure, function, and histology of lymph nodes. Toxicol Pathol. 34: 409-424. 2006. [Medline] [CrossRef]

178. Coates PT, Barratt-Boyes SM, Zhang L, Donnenberg VS, O'Connell PJ, Logar AJ, Duncan FJ, Murphey-Corb M, Donnenberg AD, Morelli AE, Maliszewski CR, and Thomson AW. Dendritic cell subsets in blood and lymphoid tissue of rhesus monkeys and their mobilization with Flt3 ligand. Blood. 102: 2513-2521. 2003. [Medline] [CrossRef]

179. Reeves RK, Wei Q, Stallworth J, and Fultz PN. Systemic dendritic cell mobilization associated with administration of FLT3 ligand to SIV- and SHIV-infected macaques. AIDS Res Hum Retroviruses. 25: 1313-1328. 2009. [Medline] [CrossRef]

180. Wijewardana V, Bouwer AL, Brown KN, Liu X, and Barratt-Boyes $\mathrm{SM}$. Accumulation of functionally immature myeloid dendritic cells in lymph nodes of rhesus macaques with acute pathogenic simian immunodeficiency virus infection. Immunology. 143: 146-154. 2014. [Medline] [CrossRef]

181. Guilliams M, Dutertre CA, Scott CL, McGovern N, Sichien D, Chakarov S, Van Gassen S, Chen J, Poidinger M, De Prijck S, Tavernier SJ, Low I, Irac SE, Mattar CN, Sumatoh HR, Low GHL, Chung TJK, Chan DKH, Tan KK, Hon TLK, Fossum E, Bogen B, Choolani M, Chan JKY, Larbi A, Luche H, Henri S, Saeys Y, Newell EW, Lambrecht BN, Malissen B, and Ginhoux F. Unsupervised High-Dimensional Analysis Aligns Dendritic Cells across Tissues and Species. Immunity. 45: 
669-684. 2016. [Medline] [CrossRef]

182. Guilliams M, Ginhoux F, Jakubzick C, Naik SH, Onai N, Schraml BU, Segura E, Tussiwand R, and Yona S. Dendritic cells, monocytes and macrophages: a unified nomenclature based on ontogeny. Nat Rev Immunol. 14: 571-578. 2014. [Medline] [CrossRef]

183. Schuh JCL. Letter to the Editor Regarding "Spontaneous Findings in the Eyes of Cynomolgus Monkeys ( Macaca fascicularis) of Mauritian Origin" by Woicke et al. ( Toxicol Pathol 46, 273-282, 2018). Toxicol Pathol. 46: 719-720. 2018. [Medline] [CrossRef]

184. Shi HN, and Walker WA. Development and Physiology of the Intestinal Mucosal Defence. In: Mestecky J, Strober W, Russell MW, Cheroutre H, Lambrecht N, Kelsall BL, eds. Mucosal Immunology. 4 ed. Waltham, MA: Elsevier Science; 2015.

185. Kuper CF. Histopathology of mucosa-associated lymphoid tissue. Toxicol Pathol. 34: 609-615. 2006. [Medline] [CrossRef]

186. Nair PN, and Rossinsky K. Organization of lymphoid tissue in the tonsilla lingualis. An ultrastructural study in Macaca fascicularis (Primates, Cercopithecoidea). Cell Tissue Res. 240: 233-242. 1985. [Medline] [CrossRef]

187. Kageyama M, Nakatsuka K, Yamaguchi T, Owen RL, and Shimada T. Ocular defense mechanisms with special reference to the demonstration and functional morphology of the conjunctiva-associated lymphoid tissue in Japanese monkeys. Arch Histol Cytol. 69: 311-322. 2006. [Medline] [CrossRef]

188. Woicke J, Haile S, Mysore J, Peden WM, Lejeune T, Sanderson T, and Brodie T. Spontaneous Findings in the Eyes of Cynomolgus Monkeys ( Macaca fascicularis) of Mauritian Origin. Toxicol Pathol. 46: 273-282. 2018. [Medline] [CrossRef]

189. Matsuda M, Ina K, Kitamura H, Fujikura Y, and Shimada T. Demonstration and organization of duct-associated lymphoid tissue (DALT) of the main excretory duct in the monkey parotid gland. Arch Histol Cytol. 60: 493-502. 1997. [Medline] [CrossRef]

190. Thoolen B, Maronpot RR, Harada T, Nyska A, Rousseaux C, Nolte T, Malarkey DE, Kaufmann W, Küttler K, Deschl U, Nakae D, Gregson R, Vinlove MP, Brix AE, Singh B, Belpoggi F, and Ward JM. Proliferative and nonproliferative lesions of the rat and mouse hepatobiliary system. Toxicol Pathol. 38(Suppl): 5S-81S. 2010. [Medline] [CrossRef]

191. Chamanza R. Chapter 1 - Non-human primates: cynomolgus (Macaca fascicularis) and rhesus (Macaca mulatta) macaques and the common marmoset (Callithrix jacchus). In: McInnes EF, Mann P, eds. Background Lesions in Laboratory Animals. Saint Louis: W.B. Saunders; 2012:1-15.

192. Foster JR. Spontaneous and drug-induced hepatic pathology of the laboratory beagle dog, the cynomolgus macaque and the marmoset. Toxicol Pathol. 33: 63-74. 2005. [Medline] [CrossRef]

193. Vons C, Beaudoin S, Helmy N, Dagher I, Weber A, and Franco D. First description of the surgical anatomy of the cynomolgus monkey liver. Am J Primatol. 71: 400-408. 2009. [Medline] [CrossRef]

194. Cattley RC, and Cullen JM. Chapter 45 - Liver and Gall Bladder. In: Haschek WM, Rousseaux CG, Wallig MA, eds. Haschek and Rousseaux's Handbook of Toxicologic Pathology (Third Edition). Boston: Academic Press; 2013:1509-1566.

195. Greaves P. Chapter 9 - Liver and Pancreas. In: Greaves P, ed. Histopathology of Preclinical Toxicity Studies (Fourth Edition). Boston: Academic Press; 2012:433-535.

196. Cullen JM, and Stalker MJ. Liver and Biliary System. In: Maxie MG, ed. Jubb, Kennedy \& Palmer's Pathology of Domestic Animals: Volume 2 (Sixth Edition). Vol 2. 6th ed. St. Louis, MO: Elsevier; 2016:258352.

197. Grieves JL, Dick EJ Jr, Schlabritz-Loutsevich NE, Butler SD, Leland MM, Price SE, Schmidt CR, Nathanielsz PW, and Hubbard GB. Barbiturate euthanasia solution-induced tissue artifact in nonhuman primates. J Med Primatol. 37: 154-161. 2008. [Medline] [CrossRef]

198. Loegering DJ, Commins LM, Minnear FL, Gary LA, and Hill LA. Effect of Kupffer cell phagocytosis of erythrocytes and erythrocyte ghosts on susceptibility to endotoxemia and bacteremia. Infect Immun. 55: 2074-2080. 1987. [Medline] [CrossRef]
199. Khansari N, and Fudenberg HH. Immune elimination of autologous senescent erythrocytes by Kupffer cells in vivo. Cell Immunol. 80: 426-430. 1983. [Medline] [CrossRef]

200. Hand WL, and King-Thompson NL. Effect of erythrocyte ingestion on macrophage antibacterial function. Infect Immun. 40: 917-923. 1983. [Medline] [CrossRef]

201. Bellows CF, Molina RM, and Brain JD. Diminished organelle motion in murine Kupffer cells during the erythrocytic stage of malaria. $J R$ Soc Interface. 8: 711-719. 2011. [Medline] [CrossRef]

202. Ohta E, Nagayama Y, Koyama N, Kakiuchi D, and Hosokawa S. Malaria in cynomolgus monkeys used in toxicity studies in Japan. $J$ Toxicol Pathol. 29: 31-38. 2016. [Medline] [CrossRef]

203. Strait K, Else JG, and Eberhard ML. Chapter 4 - Parasitic Diseases of Nonhuman Primates. In: Abee CR, Mansfield K, Tardif S, Morris T, eds. Nonhuman Primates in Biomedical Research (Second Edition). Boston: Academic Press; 2012:197-297.

204. Baskin GB, Murphey-Corb M, Watson EA, and Martin LN. Necropsy findings in rhesus monkeys experimentally infected with cultured simian immunodeficiency virus (SIV)/delta. Vet Pathol. 25: 456-467. 1988. [Medline] [CrossRef]

205. Dubey JP, Markovits JE, and Killary KA. Cryptosporidium muris-like infection in stomach of cynomolgus monkeys (Macaca fascicularis). Vet Pathol. 39: 363-371. 2002. [Medline] [CrossRef]

206. Yanai T, Chalifoux LV, Mansfield KG, Lackner AA, and Simon MA. Pulmonary cryptosporidiosis in simian immunodeficiency virusinfected rhesus macaques. Vet Pathol. 37: 472-475. 2000. [Medline] [CrossRef]

207. Wilson DW, Day PA, and Brummer ME. Diarrhea associated with Cryptosporidium spp in juvenile macaques. Vet Pathol. 21: 447-450. 1984. [Medline] [CrossRef]

208. Toft 2nd JD. The pathoparasitology of the alimentary tract and pancreas of nonhuman primates: a review. Vet Pathol Suppl. 19(Suppl 7): 44-92. 1982. [Medline] [CrossRef]

209. Kovatch RM, and White JD. Cryptosporidiosis in Two Juvenile Rhesus Monkeys. Vet Pathol. 9: 426-440. 1972. [Medline] [CrossRef]

210. Plesker R, Bauer C, Tackmann K, and Dinkel A. Hydatid echinococcosis (Echinococcus granulosus) in a laboratory colony of pig-tailed macaques (Macaca nemestrina). J Vet Med B Infect Dis Vet Public Health. 48: 367-372. 2001. [Medline] [CrossRef]

211. Bacciarini LN, Gottstein B, Pagan O, Rehmann P, and Gröne A. Hepatic alveolar echinococcosis in cynomolgus monkeys (Macaca fascicularis). Vet Pathol. 41: 229-234. 2004. [Medline] [CrossRef]

212. Helbig M, Frosch P, Kern P, and Frosch M. Serological differentiation between cystic and alveolar echinococcosis by use of recombinant larval antigens. J Clin Microbiol. 31: 3211-3215. 1993. [Medline] [CrossRef]

213. Muller-Eberhard U, Eiseman JL, Foidart M, and Alvares AP. Effect of heme on allylisopropylacetamide-induced changes in heme and drug metabolism in the rhesus monkey (Macaca mulatta). Biochem Pharmacol. 32: 3765-3769. 1983. [Medline] [CrossRef]

214. Karim MR, Wang R, Dong H, Zhang L, Li J, Zhang S, Rume FI, Qi M, Jian F, Sun M, Yang G, Zou F, Ning C, and Xiao L. Genetic polymorphism and zoonotic potential of Enterocytozoon bieneusi from nonhuman primates in China. Appl Environ Microbiol. 80: 1893-1898. 2014. [Medline] [CrossRef]

215. Chalifoux LV, Carville A, Pauley D, Thompson B, Lackner AA, and Mansfield KG. Enterocytozoon bieneusi as a cause of proliferative serositis in simian immunodeficiency virus-infected immunodeficient macaques (Macaca mulatta). Arch Pathol Lab Med. 124: 1480-1484. 2000. [Medline] [CrossRef]

216. Mecklenburg L, Kusewitt D, Kolly C, Treumann S, Adams ET, Diegel K, Yamate J, Kaufmann W, Müller S, Danilenko D, and Bradley A. Proliferative and non-proliferative lesions of the rat and mouse integument. J Toxicol Pathol. 26(Suppl): 27S-57S. 2013. [Medline] [CrossRef]

217. Fooden J. Field Museum of Natural H. Systematic review of Southeast Asian longtail macaques, Macaca fascicularis (Raffles, [1821]). Vol n.s. no.81 (1995). [Chicago, Ill.]: Field Museum of Natural History; 1995. 
218. Groves CP. Primate taxonomy. 2001.

219. Rowe N. The pictorial guide to the living primates. East Hampton, N.Y.: Pogonias Press; 1996.

220. Cline JM, Botts S, Lees CJ, Brommage R. Effects of lasofoxifene on the uterus, vagina, and breast in ovariectomized cynomolgus monkeys (Macaca fascicularis). American journal of obstetrics and gynecology. 2008;199(2):158 e151-158.

221. Engelhardt A, Hodges JK, Niemitz C, and Heistermann M. Female sexual behavior, but not sex skin swelling, reliably indicates the timing of the fertile phase in wild long-tailed macaques (Macaca fascicularis). Horm Behav. 47: 195-204. 2005. [Medline] [CrossRef]

222. Bernstein JA, and Didier PJ. Nonhuman primate dermatology: a literature review. Vet Dermatol. 20: 145-156. 2009. [Medline] [CrossRef]

223. Mecklenburg L, and Romeike A. Recommended Diagnostic Approach to Documenting and Reporting Skin Findings of Nonhuman Primates from Regulatory Toxicity Studies. Toxicol Pathol. 44: 591-600. 2016. [Medline] [CrossRef]

224. Mohamed RH, Sutoh Y, Itoh Y, Otsuka N, Miyatake Y, Ogasawara K, and Kasahara M. The SKINT1-like gene is inactivated in hominoids but not in all primate species: implications for the origin of dendritic epidermal T cells. PLoS One. 10: e0123258. 2015. [Medline] [CrossRef]

225. Adam L, Rosenbaum P, Cosma A, Le Grand R, and Martinon F. Identification of skin immune cells in non-human primates. J Immunol Methods. 426: 42-49. 2015. [Medline] [CrossRef]

226. Güçlü B, Mahoney GK, Pawson LJ, Pack AK, Smith RL, and Bolanowski SJ. Localization of Merkel cells in the monkey skin: an anatomical model. Somatosens Mot Res. 25: 123-138. 2008. [Medline] [CrossRef]

227. Cabo R, Gálvez MA, San José I, Laurà R, López-Muñiz A, GarcíaSuárez $\mathrm{O}$, Cobo T, Insausti R, and Vega JA. Immunohistochemical localization of acid-sensing ion channel 2 (ASIC2) in cutaneous Meissner and Pacinian corpuscles of Macaca fascicularis. Neurosci Lett. 516: 197-201. 2012. [Medline] [CrossRef]

228. Price KD. Bacterial infections in cynomolgus monkeys given small molecule immunomodulatory antagonists. J Immunotoxicol. 7: 128137. 2010. [Medline] [CrossRef]

229. Ruzek MC, Huang L, Zhang TT, Bryant S, Slivka PF, Cuff CA, Tripp $\mathrm{C}$, and Blaich $\mathrm{G}$. Dual Blockade of Interleukin-1 $\beta$ and Interleukin-17A Reduces Murine Arthritis Pathogenesis but Also Leads to Spontaneous Skin Infections in Nonhuman Primates. $J$ Pharmacol Exp Ther. 364: 474-484. 2018. [Medline] [CrossRef]

230. Horn TD. Cutaneous toxicities of drugs. In: Elder DE, Elenitsas R, Jaworsky C, Johnson Jr. BI, eds. Lever's Histopathology of the Skin. 8th ed. Philadelphia, PA: Lippincott-Raven; 1997.

231. Palanisamy GS, Marcek JM, Cappon GD, Whritenour J, Shaffer CL, Brady JT, and Houle C. Drug-induced Skin Lesions in Cynomolgus Macaques Treated with Metabotropic Glutamate Receptor 5 (mGluR5) Negative Allosteric Modulators. Toxicol Pathol. 43: 995-1003. 2015. [Medline] [CrossRef]

232. Hoffmann P, Bentley P, Sahota P, Schoenfeld H, Martin L, Longo L, Spaet R, Moulin P, Pantano S, Dubost V, Lapadula D, Burkey B, Kaushik V, Zhou W, Hayes M, Flavahan N, Chibout SD, and Busch $\mathrm{S}$. Vascular origin of vildagliptin-induced skin effects in Cynomolgus monkeys: pathomechanistic role of peripheral sympathetic system and neuropeptide Y. Toxicol Pathol. 42: 684-695. 2014. [Medline] [CrossRef]

233. Kobune F, Takahashi H, Terao K, Ohkawa T, Ami Y, Suzaki Y, Nagata N, Sakata H, Yamanouchi K, and Kai C. Nonhuman primate models of measles. Lab Anim Sci. 46: 315-320. 1996. [Medline]

234. Montagna W. The Skin of Nonhuman Primates. Am Zool American Zoologist. 12: 109-124. 1972. [CrossRef]

235. Dokter W, Ubink R, van der Lee M, van der Vleuten M, van Achterberg T, Jacobs D, Loosveld E, van den Dobbelsteen D, Egging D, Mattaar E, Groothuis P, Beusker P, Coumans R, Elgersma R, Menge W, Joosten J, Spijker H, Huijbregts T, de Groot V, Eppink M, de Roo G, Verheijden G, and Timmers M. Preclinical profile of the HER2-targeting ADC SYD983/SYD985: introduction of a new duocarmycin-based linker-drug platform. Mol Cancer Ther. 13: 2618-2629. 2014. [Medline] [CrossRef]

236. Skartved NJ, Jacobsen HJ, Pedersen MW, et al. Preclinical pharmacokinetics and safety of Sym004: a synergistic antibody mixture directed against epidermal growth factor receptor. Clinical cancer research: an official journal of the American Association for Cancer Research. 2011;17(18):5962-5972.

237. Kikkawa R. A Case of Drug-induced Cutaneous Toxicity Observed in Cynomolgus Monkeys. Toxicol Pathol. 44: 601-606. 2016. [Medline] [CrossRef]

238. Morris $\mathrm{J}$, and Etheridge M. A case of suspected contact dermatitis in a juvenile cynomolgus monkey (Macaca fascicularis). J Med Primatol. 37(Suppl 1): 56-59. 2008. [Medline] [CrossRef]

239. Saravanan C, Sasseville VG, and Mansfield KG. Chapter 10 - Nonhuman Primate Diseases of Relevance in Drug Development and their Impact on the Interpretation of Study Findings. In: Bluemel J, Korte S, Schenck E, Weinbauer GF, eds. The Nonhuman Primate in Nonclinical Drug Development and Safety Assessment. San Diego: Academic Press; 2015:187-213.

240. Gray WL. Simian varicella in old world monkeys. Comp Med. 58: $22-$ 30. 2008. [Medline]

241. Line SW, Ihrke PJ, and Prahalada S. Calcinosis circumscripta in two rhesus monkeys. Lab Anim Sci. 34: 616-618. 1984. [Medline]

242. Anderson WI, Boulay M, Lugo JR, Mischler SA, Pellegrini G, and Scott DW. Abstracts. Vet Pathol. 44: 732-785. 2007. [CrossRef]

243. Radi ZA, and Sato K. Bilateral dystrophic calcinosis circumscripta in a cynomolgus macaque (Macaca fascicularis). Toxicol Pathol. 38: 637-641. 2010. [Medline] [CrossRef]

244. Chen Y, Deng W, Zhu H, Li J, Xu Y, Dai X, Jia C, Kong Q, Huang L, Liu Y, Ma C, Xiao C, Liu Y, Li Q, Bezard E, and Qin C. The pathologic features of neurocutaneous melanosis in a cynomolgus macaque. Vet Pathol. 46: 773-775. 2009. [Medline] [CrossRef]

245. Wood CE, Tannehill-Gregg SH, Chen Z, Doorslaer K, Nelson DR, Cline JM, and Burk RD. Novel betapapillomavirus associated with hand and foot papillomas in a cynomolgus macaque. Vet Pathol. 48: 731-736. 2011. [Medline] [CrossRef]

246. Pellegrini G, Bienvenu JG, Meehan JT, Mischler SA, Perry RW, Scott DW, and Anderson WI. Cutaneous melanoma with metastasis in a cynomolgus monkey (Macaca fascicularis). J Med Primatol. 38: 444447. 2009. [Medline] [CrossRef]

247. Rudmann D, Cardiff R, Chouinard L, Goodman D, Küttler K, Marxfeld H, Molinolo A, Treumann S, Yoshizawa K. INHAND Mammary, Zymbal's, Preputial, and Clitoral Gland Organ Working Group Proliferative and nonproliferative lesions of the rat and mouse mammary, Zymbal's, preputial, and clitoral glands. Toxicol Pathol. 40(Suppl): 7S-39S. 2012. [Medline] [CrossRef]

248. Cline JM, and Wood CE. The Mammary Glands of Macaques. Toxicol Pathol. 36: 134s-141s. 2008. [Medline] [CrossRef]

249. Cline JM. Assessing the mammary gland of nonhuman primates: effects of endogenous hormones and exogenous hormonal agents and growth factors. Birth Defects Res B Dev Reprod Toxicol. 80: 126-146. 2007. [Medline] [CrossRef]

250. Speert $\mathrm{H}$. The normal and experimental development of the mammary gland of the rhesus monkey, with some pathological correlations. Contrib Embryol. 32: 9-65. 1948.

251. Schultz AH. Fetal growth and development of the rhesus monkey. Contrib Embryol. 26: 72-97. 1937.

252. Wood CE, Hester JM, and Cline JM. Mammary gland development in early pubertal female macaques. Toxicol Pathol. 35: 795-805. 2007. [Medline] [CrossRef]

253. Dewi FN, Wood CE, Lees CJ, Willson CJ, Register TC, Tooze JA, Franke AA, and Cline JM. Dietary soy effects on mammary gland development during the pubertal transition in nonhuman primates. Cancer Prev Res (Phila). 6: 832-842. 2013. [Medline] [CrossRef]

254. Lazala C, and Saenger P. Pubertal gynecomastia. J Pediatr Endocrinol Metab. 15: 553-560. 2002. [Medline] [CrossRef]

255. Stute P, Wood CE, Kaplan JR, and Cline JM. Cyclic changes in the 
mammary gland of cynomolgus macaques. Fertil Steril. 82(Suppl 3): 1160-1170. 2004. [Medline] [CrossRef]

256. Wood CE, Kaplan JR, Stute P, and Cline JM. Effects of soy on the mammary glands of premenopausal female monkeys. Fertil Steril. 85(Suppl 1): 1179-1186. 2006. [Medline] [CrossRef]

257. Neville MC. Regulation of Mammary Development and Lactation. In: Neville MC, Neifert MR, eds. Lactation: Physiology, Nutrition, and Breast-Feeding. Boston, MA: Springer US; 1983:103-140.

258. Stute P, Sielker S, Wood CE, Register TC, Lees CJ, Dewi FN, Williams JK, Wagner JD, Stefenelli U, and Cline JM. Life stage differences in mammary gland gene expression profile in non-human primates. Breast Cancer Res Treat. 133: 617-634. 2012. [Medline] [CrossRef]

259. Wilson ME, Walker ML, Pope NS, and Gordon TP. Prolonged lactational infertility in adolescent rhesus monkeys. Biol Reprod. 38: 163174. 1988. [Medline] [CrossRef]

260. Kunz C, and Lönnerdal B. Protein composition of rhesus monkey milk: comparison to human milk. Comp Biochem Physiol Part A Physiol. 104: 793-797. 1993. [Medline] [CrossRef]

261. Beck KL, Weber D, Phinney BS, Smilowitz JT, Hinde K, Lönnerdal B, Korf I, and Lemay DG. Comparative Proteomics of Human and Macaque Milk Reveals Species-Specific Nutrition during Postnatal Development. J Proteome Res. 14: 2143-2157. 2015. [Medline] [CrossRef]

262. Wood CE, Hester JM, Appt SE, Geisinger KR, and Cline JM. Estrogen effects on epithelial proliferation and benign proliferative lesions in the postmenopausal primate mammary gland. Lab Invest. 88: 938-948. 2008. [Medline] [CrossRef]

263. Wood CE, Usborne AL, Starost MF, Tarara RP, Hill LR, Wilkinson LM, Geisinger KR, Feiste EA, and Cline JM. Hyperplastic and neoplastic lesions of the mammary gland in macaques. Vet Pathol. 43: 471-483. 2006. [Medline] [CrossRef]

264. Daviau JS, and Trupkiewicz JG. Pituitary adenoma with galactorrhea in an adult male cynomolgus macaque (Macaca fascicularis). Contemp Top Lab Anim Sci. 40: 57-59. 2001. [Medline]

265. Malaivijitnond S, and Varavudhi P. Evidence for morphine-induced galactorrhea in male cynomolgus monkeys. J Med Primatol. 27: 1-9. 1998. [Medline] [CrossRef]

266. Ethun KF, Wood CE, Register TC, Cline JM, Appt SE, and Clarkson TB. Effects of bazedoxifene acetate with and without conjugated equine estrogens on the breast of postmenopausal monkeys. Menopause. 19: 1242-1252. 2012. [Medline] [CrossRef]

267. Fox JG, and Frost WW. Corynebacterium ulcerans mastitis in a bonnet macaque (Macaca radiata). Lab Anim Sci. 24: 820-822. 1974. [Medline]

268. Tavassoli FA, Casey HW, and Norris HJ. The morphologic effects of synthetic reproductive steroids on the mammary gland of rhesus monkeys. Mestranol, ethynerone, mestranol-ethynerone, chloroethynyl norgestrel-mestranol, and anagestone acetate-mestranol combinations. Am J Pathol. 131: 213-234. 1988. [Medline]

269. Wells CA, and El-Ayat GA. Non-operative breast pathology: apocrine lesions. J Clin Pathol. 60: 1313-1320. 2007. [Medline] [CrossRef]

270. Abdel-Fatah TM, Powe DG, Hodi Z, Lee AH, Reis-Filho JS, and Ellis IO. High frequency of coexistence of columnar cell lesions, lobular neoplasia, and low grade ductal carcinoma in situ with invasive tubular carcinoma and invasive lobular carcinoma. Am J Surg Pathol. 31: 417-426. 2007. [Medline] [CrossRef]

271. Folley SJ, Guthkelch AN, Zuckerman S, and Clark WELG. The mammary gland of the rhesus monkey under normal and experimental conditions. Proc R Soc Lond B Biol Sci. 126: 469-491. 1939. [CrossRef]

272. Kaufmann W, Bolon B, Bradley A, Butt M, Czasch S, Garman RH, George C, Gröters S, Krinke G, Little P, McKay J, Narama I, Rao D, Shibutani M, and Sills R. Proliferative and nonproliferative lesions of the rat and mouse central and peripheral nervous systems. Toxicol Pathol. 40(Suppl): 87S-157S. 2012. [Medline] [CrossRef]

273. Bolon B, Butt MT, Garman RH, and Dorman DC. Chapter 52 - Nervous System. In: Haschek WM, Rousseaux CG, Wallig MA, eds. Haschek and Rousseaux's Handbook of Toxicologic Pathology (Third Edition). Boston: Academic Press; 2013:2005-2093.

274. Bolon B. Comparative and correlative neuroanatomy for the toxicolog- ic pathologist. Toxicol Pathol. 28: 6-27. 2000. [Medline] [CrossRef]

275. Bolon B, Garman RH, Pardo ID, Jensen K, Sills RC, Roulois A, Radovsky A, Bradley A, Andrews-Jones L, Butt M, and Gumprecht L. STP position paper: Recommended practices for sampling and processing the nervous system (brain, spinal cord, nerve, and eye) during nonclinical general toxicity studies. Toxicol Pathol. 41: 1028-1048. 2013. [Medline] [CrossRef]

276. Pardo ID, Garman RH, Weber K, Bobrowski WF, Hardisty JF, and Morton D. Technical guide for nervous system sampling of the cynomolgus monkey for general toxicity studies. Toxicol Pathol. 40: 624636. 2012. [Medline] [CrossRef]

277. Bergin IL, Campbell B, and Agnew DW. Ependymal cyst in a cynomolgus macaque (Macaca fascicularis). J Med Primatol. 37: 239-244. 2008. [Medline] [CrossRef]

278. Chang KS, Lee SR, Kim SW, Cho ZH, Son HY, Kim D, and Chang KT. Ependymal cyst in the cerebrum of an African green monkey (Chlorocebus aethiops). J Comp Pathol. 145: 235-239. 2011. [Medline] [CrossRef]

279. Butt MT, Whitney KM, Davis W, Akella S, Parker S, and Foley GL. Microscopic background changes in brains of cynomolgus monkeys. Toxicol Pathol. 43: 513-518. 2015. [Medline] [CrossRef]

280. Myers RE, Valerio MG, Martin DP, and Nelson KB. Perinatal brain damage: porencephaly in a cynomolgous monkey. Biol Neonate. 22: 253-273. 1973. [Medline] [CrossRef]

281. Hirowatari C, Kodama R, Sasaki Y, Tanigawa Y, Fujishima J, Yoshikawa T, Yabuuchi K, Kuwamura Y, Hirakawa K, Kamimura Y, and Maeda H. Porencephaly in a cynomolgus monkey ( macaca fascicularis ). J Toxicol Pathol. 25: 45-49. 2012. [Medline] [CrossRef]

282. Butt M, Fuji R, Reichelt M, Sharma AK, and Cramer S. Autophagy of Sensory Neurons in the Trigeminal and Dorsal Root Ganglia of Cynomolgus Monkeys (Macaca fascicularis). Toxicol Pathol. 48: 238-243. 2020. [Medline] [CrossRef]

283. Butt MT, Bradley A, and Sills R. Nervous System. In: Sahota PS, Popp JA, Bouchard PR, Hardisty JF, Gopinath C, eds. Toxicologic Pathology: Nonclinical Safety Assessment. 2nd ed.: CRC Press; 2018:895930.

284. Abbott DP, and Edmondson NA. Neuronal ceroid-lipofuscin storage in a cynomolgus monkey (Macaca fascicularis). Lab Anim. 17: 18-20. 1983. [Medline] [CrossRef]

285. Haltia M. The neuronal ceroid-lipofuscinoses: from past to present. Biochim Biophys Acta. 1762: 850-856. 2006. [Medline] [CrossRef]

286. Fukuda T, Shimizu J, Furuhata H, Abe T, Shimizu K, Oishi T, Ogihara M, Kubota J, Sasaki A, Sasaki K, Azuma T, and Umemura S. Overexpression of heat shock proteins in pallido-nigral axonal spheroids of nonhuman aged primates. Acta Neuropathol. 110: 145-150. 2005. [Medline] [CrossRef]

287. Kodama R, Yang X, Saski Y, Iwashige S, Tanigawa Y, Yoshikawa T, Nagaoka T, Kamimura Y, and Maeda H. Age-related lesions in the cerebrum in middle-aged female cynomolgus monkeys. Toxicol Pathol. 38: 303-311. 2010. [Medline] [CrossRef]

288. Bradley AE, Bolon B, Butt MT, Cramer SD, Czasch S, Garman RH, George C, Gröters S, Kaufmann W, Kovi RC, Krinke G, Little PB, Narama I, Rao DB, Sharma AK, Shibutani M, and Sills R. Proliferative and Nonproliferative Lesions of the Rat and Mouse Central and Peripheral Nervous Systems: New and Revised INHAND Terms. Toxicol Pathol. 48: 827-844. 2020. [Medline]

289. Baskin GB, Ratterree M, Davison BB, Falkenstein KP, Clarke MR, England JD, Vanier MT, Luzi P, Rafi MA, and Wenger DA. Genetic galactocerebrosidase deficiency (globoid cell leukodystrophy, Krabbe disease) in rhesus monkeys (Macaca mulatta). Lab Anim Sci. 48: 476482. 1998. [Medline]

290. Suzuki K, and Grover WD. Krabbe's leukocystrophy (globoid cell leukodystrophy). An ultrastructural study. Am J Obstet Gynecol. 106: 385-396. 1970. [Medline]

291. Luzi P, Rafi MA, Victoria T, Baskin GB, and Wenger DA. Characterization of the rhesus monkey galactocerebrosidase (GALC) cDNA and gene and identification of the mutation causing globoid cell leukodys- 
trophy (Krabbe disease) in this primate. Genomics. 42: 319-324. 1997. [Medline] [CrossRef]

292. Borda JT, Alvarez X, Mohan M, Ratterree MS, Phillippi-Falkenstein $\mathrm{K}$, Lackner AA, and Bunnell BA. Clinical and immunopathologic alterations in rhesus macaques affected with globoid cell leukodystrophy. Am J Pathol. 172: 98-111. 2008. [Medline] [CrossRef]

293. Abuzayed B, Khreisat W, Maaiah W, and Agailat S. Supratentorial primitive neuroectodermal tumor presenting with intracranial hemorrhage in adult. J Neurosci Rural Pract. 5: 176-179. 2014. [Medline] [CrossRef]

294. Ostrom QT, de Blank PM, Kruchko C, et al. Alex's Lemonade Stand Foundation Infant and Childhood Primary Brain and Central Nervous System Tumors Diagnosed in the United States in 2007-2011. NeuroOncology. 2014;16(suppl_10):x1-x36.

295. Roberts RO, Lynch CF, Jones MP, and Hart MN. Medulloblastoma: a population-based study of 532 cases. J Neuropathol Exp Neurol. 50: 134-144. 1991. [Medline] [CrossRef]

296. Nicholson JC, Ross FM, Kohler JA, and Ellison DW. Comparative genomic hybridization and histological variation in primitive neuroectodermal tumours. Br J Cancer. 80: 1322-1331. 1999. [Medline] [CrossRef]

297. Russo C, Pellarin M, Tingby O, Bollen AW, Lamborn KR, Mohapatra G, Collins VP, and Feuerstein BG. Comparative genomic hybridization in patients with supratentorial and infratentorial primitive neuroectodermal tumors. Cancer. 86: 331-339. 1999. [Medline] [CrossRef]

298. Pomeroy SL, Tamayo P, Gaasenbeek M, Sturla LM, Angelo M, McLaughlin ME, Kim JY, Goumnerova LC, Black PM, Lau C, Allen JC, Zagzag D, Olson JM, Curran T, Wetmore C, Biegel JA, Poggio T, Mukherjee S, Rifkin R, Califano A, Stolovitzky G, Louis DN, Mesirov JP, Lander ES, and Golub TR. Prediction of central nervous system embryonal tumour outcome based on gene expression. Nature. 415: 436-442. 2002. [Medline] [CrossRef]

299. Chang Q, Pang JC, Li KK, Poon WS, Zhou L, and Ng HK. Promoter hypermethylation profile of RASSF1A, FHIT, and sFRP1 in intracranial primitive neuroectodermal tumors. Hum Pathol. 36: 1265-1272. 2005. [Medline] [CrossRef]

300. Pollack IF. Brain tumors in children. N Engl J Med. 331: 1500-1507. 1994. [Medline] [CrossRef]

301. Paulino AC, and Melian E. Medulloblastoma and supratentorial primitive neuroectodermal tumors: an institutional experience. Cancer. 86: 142-148. 1999. [Medline] [CrossRef]

302. Yanai T, Teranishi M, Manabe S, Takaoka M, Yamoto T, Matsunuma $\mathrm{N}$, and Goto N. Astrocytoma in a cynomolgus monkey (Macaca fascicularis). Vet Pathol. 29: 569-571. 1992. [Medline] [CrossRef]

303. Louis DN, Ohgaki H, Wiestler OD, Cavenee WK, Burger PC, Jouvet A, Scheithauer BW, and Kleihues P. The 2007 WHO classification of tumours of the central nervous system. Acta Neuropathol. 114: 97-109. 2007. [Medline] [CrossRef]

304. Ostrom QT, Gittleman H, Xu J, et al. CBTRUS Statistical Report: Primary Brain and Other Central Nervous System Tumors Diagnosed in the United States in 2009-2013. Neuro-oncology. 2016;18(suppl_5):v1v75.

305. Louis DN, Perry A, Reifenberger G, von Deimling A, Figarella-Branger D, Cavenee WK, Ohgaki H, Wiestler OD, Kleihues P, and Ellison DW. The 2016 World Health Organization Classification of Tumors of the Central Nervous System: a summary. Acta Neuropathol. 131: 803-820. 2016. [Medline] [CrossRef]

306. McClure HM. Tumors in nonhuman primates: observations during a six-year period in the Yerkes primate center colony. Am J Phys Anthropol. 38: 425-429. 1973. [Medline] [CrossRef]

307. Herring JM, Reyes M, Dochterman LW, and Bennett BT. Glioblastoma multiforme in a baboon. Lab Anim Sci. 40: 645-647. 1990. [Medline]

308. Burger PC, and Scheithauer BW. Tumors of the central nervous system. Washington; Bethesda, Maryland: Armed Forces Institute of Pathology; Universities Associated for Research and Education in Pathology; 1994.

309. Engelhard HH, Stelea A, and Cochran EJ. Oligodendroglioma: pathol- ogy and molecular biology. Surg Neurol. 58: 111-117, discussion 117. 2002. [Medline] [CrossRef]

310. Reifenberger G, Kros JM, Burger PC, Louis DN, and Collins VP. Oligodendroglioma. In: Kleihues P, Cavenee WK, eds. Pathology and Genetics of Tumors of the Nevous Sstem. Lyon: IARC Press; 2000:56-61.

311. Veandenberg SR, and Lopes MBS. Classification. In: Berger MS, Wilson CB, eds. The Gliomas. Philadelphia: W.B. Saunders; 1999:172-191.

312. Kleihues P, Louis DN, Scheithauer BW, Rorke LB, Reifenberger G, Burger PC, and Cavenee WK. The WHO classification of tumors of the nervous system. J Neuropathol Exp Neurol. 61: 215-225, discussion 226-229. 2002. [Medline] [CrossRef]

313. Dixon D, Alison R, Bach U, et al. Nonproliferative and proliferative lesions of the rat and mouse female reproductive system. Journal of toxicologic pathology. 2014;27(3-4 Suppl):1S-107S.

314. Cline JM, Wood CE, Vidal JD, Tarara RP, Buse E, Weinbauer GF, de Rijk EP, and van Esch E. Selected Background Findings and Interpretation of Common Lesions in the Female Reproductive System in Macaques. Toxicol Pathol. 36: 142s-163s. 2008. [Medline] [CrossRef]

315. van Esch E, Cline JM, Buse E, Weinbauer GF. The Macaque Endometrium, with Special Reference to the Cynomolgus Monkey (Macaca fascicularis). Toxicologic Pathology. 2008;36(7_suppl):67S-100S.

316. Van Esch E, Cline JM, Buse E, Wood CE, de Rijk EPCT, Weinbauer GF. Summary Comparison of Female Reproductive System in Human and the Cynomolgus Monkey (Macaca fascicularis). Toxicologic Pathology. 2008;36(7_suppl):171S-172S.

317. van Esch E, de Rijk EPCT, Buse E, Zöller M, Cline JM. Recommendations for Routine Sampling, Trimming, and Paraffin-Embedding of Female Reproductive Organs, Mammary Gland, and Placenta in the Cynomolgus Monkey. Toxicologic Pathology. 2008;36(7_suppl):164S$170 \mathrm{~S}$.

318. Weinbauer GF, Niehoff M, Niehaus M, Srivastav S, Fuchs A, Van Esch $\mathrm{E}$, and Cline JM. Physiology and Endocrinology of the Ovarian Cycle in Macaques. Toxicol Pathol. 36(7S): 7S-23S. 2008. [Medline] [CrossRef]

319. Wood CE. Morphologic and Immunohistochemical Features of the Cynomolgus Macaque Cervix. Toxicologic Pathology. 2008;36(7_ suppl):119S-129S.

320. Cooper TK, and Gabrielson KL. Spontaneous lesions in the reproductive tract and mammary gland of female non-human primates. Birth Defects Res B Dev Reprod Toxicol. 80: 149-170. 2007. [Medline] [CrossRef]

321. Sato J, Nasu M, and Tsuchitani M. Comparative histopathology of the estrous or menstrual cycle in laboratory animals. J Toxicol Pathol. 29: 155-162. 2016. [Medline] [CrossRef]

322. Ebeling M, Küng E, See A, Broger C, Steiner G, Berrera M, Heckel $\mathrm{T}$, Iniguez L, Albert T, Schmucki R, Biller H, Singer T, and Certa U. Genome-based analysis of the nonhuman primate Macaca fascicularis as a model for drug safety assessment. Genome Res. 21: 1746-1756. 2011. [Medline] [CrossRef]

323. Waterston RH, Lindblad-Toh K, Birney E, Rogers J, Abril JF, Agarwal P, Agarwala R, Ainscough R, Alexandersson M, An P, Antonarakis SE, Attwood J, Baertsch R, Bailey J, Barlow K, Beck S, Berry E, Birren B, Bloom T, Bork P, Botcherby M, Bray N, Brent MR, Brown DG, Brown SD, Bult C, Burton J, Butler J, Campbell RD, Carninci P, Cawley S, Chiaromonte F, Chinwalla AT, Church DM, Clamp M, Clee C, Collins FS, Cook LL, Copley RR, Coulson A, Couronne O, Cuff J, Curwen V, Cutts T, Daly M, David R, Davies J, Delehaunty KD, Deri J, Dermitzakis ET, Dewey C, Dickens NJ, Diekhans M, Dodge S, Dubchak I, Dunn DM, Eddy SR, Elnitski L, Emes RD, Eswara P, Eyras E, Felsenfeld A, Fewell GA, Flicek P, Foley K, Frankel WN, Fulton LA, Fulton RS, Furey TS, Gage D, Gibbs RA, Glusman G, Gnerre S, Goldman N, Goodstadt L, Grafham D, Graves TA, Green ED, Gregory S, Guigó R, Guyer M, Hardison RC, Haussler D, Hayashizaki Y, Hillier LW, Hinrichs A, Hlavina W, Holzer T, Hsu F, Hua A, Hubbard T, Hunt A, Jackson I, Jaffe DB, Johnson LS, Jones M, Jones TA, Joy A, Kamal M, Karlsson EK, Karolchik D, Kasprzyk A, Kawai J, Keibler E, Kells C, Kent WJ, Kirby A, Kolbe DL, Korf I, Kucherlapati RS, Kulbokas EJ, Kulp D, Landers T, Leger JP, Leonard S, Letunic I, Levine R, Li 
J, Li M, Lloyd C, Lucas S, Ma B, Maglott DR, Mardis ER, Matthews L, Mauceli E, Mayer JH, McCarthy M, McCombie WR, McLaren S, McLay K, McPherson JD, Meldrim J, Meredith B, Mesirov JP, Miller W, Miner TL, Mongin E, Montgomery KT, Morgan M, Mott R, Mullikin JC, Muzny DM, Nash WE, Nelson JO, Nhan MN, Nicol R, Ning Z, Nusbaum C, O'Connor MJ, Okazaki Y, Oliver K, Overton-Larty E, Pachter L, Parra G, Pepin KH, Peterson J, Pevzner P, Plumb R, Pohl CS, Poliakov A, Ponce TC, Ponting CP, Potter S, Quail M, Reymond A, Roe BA, Roskin KM, Rubin EM, Rust AG, Santos R, Sapojnikov V, Schultz B, Schultz J, Schwartz MS, Schwartz S, Scott C, Seaman S, Searle S, Sharpe T, Sheridan A, Shownkeen R, Sims S, Singer JB, Slater G, Smit A, Smith DR, Spencer B, Stabenau A, Stange-Thomann N, Sugnet C, Suyama M, Tesler G, Thompson J, Torrents D, Trevaskis E, Tromp J, Ucla C, Ureta-Vidal A, Vinson JP, Von Niederhausern AC, Wade CM, Wall M, Weber RJ, Weiss RB, Wendl MC, West AP, Wetterstrand K, Wheeler R, Whelan S, Wierzbowski J, Willey D, Williams S, Wilson RK, Winter E, Worley KC, Wyman D, Yang S, Yang SP, Zdobnov EM, Zody MC, Lander ES. Mouse Genome Sequencing Consortium Initial sequencing and comparative analysis of the mouse genome. Nature. 420: 520-562. 2002. [Medline] [CrossRef]

324. Kanthaswamy S, Ng J, Satkoski Trask J, George DA, Kou AJ, Hoffman LN, Doherty TB, Houghton P, and Smith DG. The genetic composition of populations of cynomolgus macaques (Macaca fascicularis) used in biomedical research. J Med Primatol. 42: 120-131. 2013. [Medline] [CrossRef]

325. Zhang X, Meng Y, Houghton P, Liu M, Kanthaswamy S, Oldt R, Ng J, Trask JS, Huang R, Singh B, Du H, and Smith DG. Ancestry, Plasmodium cynomolgi prevalence and rhesus macaque admixture in cynomolgus macaques (Macaca fascicularis) bred for export in Chinese breeding farms. J Med Primatol. 46: 31-41. 2017. [Medline] [CrossRef]

326. Du Y, Fan TY, Tan Y, Xiong Z, and Wang Z. Seasonal changes in the reproductive physiology of female rhesus macaques (Macaca mulatta). J Am Assoc Lab Anim Sci. 49: 289-293. 2010. [Medline]

327. Bartelmez GW. Cyclic changes in the endometrium of the rhesus monkey (Macaca mulatta). Washington: Carnegie Institution; 1951.

328. Koering MJ. Cyclic changes in ovarian morphology during the menstrual cycle in Macaca mulatta. Am J Anat. 126: 73-101. 1969. [Medline] [CrossRef]

329. Brenner RM, and Slayden OD. Cyclic changes in the primate oviduct and endometrium. In: Knobil E, Neill JD, eds. The physiology of reproduction. Vol 1. 2nd ed. New York, NY: Raven Press; 1994.

330. Poonia B, Walter L, Dufour J, Harrison R, Marx PA, and Veazey RS. Cyclic changes in the vaginal epithelium of normal rhesus macaques. $J$ Endocrinol. 190: 829-835. 2006. [Medline] [CrossRef]

331. Watanabe D, Hoshiya T, Sato J, et al Changes in the Reproductive Organs Depending on Phases of Reproductive Cycle and Aging in Female Cynomolgus Monkeys. J Toxicol Pathol. 19: 169-177. 2006. [CrossRef]

332. Buse E, Zöller M, Van Esch E. The Macaque Ovary, with Special Reference to the Cynomolgus Macaque (Macaca fascicularis). Toxicologic Pathology. 2008;36(7_suppl):24S-66S.

333. Halpern WG, Ameri M, Bowman CJ, Elwell MR, Mirsky ML, Oliver J, Regan KS, Remick AK, Sutherland VL, Thompson KE, Tremblay C, Yoshida M, and Tomlinson L. Scientific and Regulatory Policy Committee Points to Consider Review: Inclusion of Reproductive and Pathology End Points for Assessment of Reproductive and Developmental Toxicity in Pharmaceutical Drug Development. Toxicol Pathol. 44: 789-809. 2016. [Medline] [CrossRef]

334. Vidal JDWC, Colman K, Whitney KM, and Creasy DM. Reproductive System and Mammary Gland. In: Sahota PS, Popp JA, Hardisty JF, Gopinath C, Bouchard P, eds. Toxicologic pathology: nonclinical safety assessment. 2 ed. London, UK: CRC Press, Taylor and Francis; 2018.

335. Vidal JD. The Impact of Age on the Female Reproductive System. Toxicol Pathol. 45: 206-215. 2017. [Medline] [CrossRef]

336. Kavanagh K, Koudy Williams J, and Wagner JD. Naturally occurring menopause in cynomolgus monkeys: changes in hormone, lipid, and carbohydrate measures with hormonal status. J Med Primatol. 34:
171-177. 2005. [Medline] [CrossRef]

337. Nichols SM, Bavister BD, Brenner CA, Didier PJ, Harrison RM, and Kubisch HM. Ovarian senescence in the rhesus monkey (Macaca mulatta). Hum Reprod. 20: 79-83. 2005. [Medline] [CrossRef]

338. Atkins HM, Willson CJ, Silverstein M, Jorgensen M, Floyd E, Kaplan JR, and Appt SE. Characterization of ovarian aging and reproductive senescence in vervet monkeys (Chlorocebus aethiops sabaeus). Comp Med. 64: 55-62. 2014. [Medline]

339. Arifin E, Shively CA, Register TC, and Cline JM. Polycystic ovary syndrome with endometrial hyperplasia in a cynomolgus monkey (Macaca fascicularis). Vet Pathol. 45: 512-515. 2008. [Medline] [CrossRef]

340. Pasello-Legrand F, and Mowat V. Two cases of spontaneous pseudohermaphroditism in Cynomolgus monkeys (Macaca fascicularis). $\mathrm{J} \mathrm{Vet}$ Med A Physiol Pathol Clin Med. 51: 344-347. 2004. [Medline] [CrossRef]

341. van Wagenen G, and Simpson ME. Postnatal development of the ovary in Homo sapiens and Macaca mulatta and induction of ovulation in the macaque. New Haven1973.

342. Iguchi T, Takasugi N, Bern HA, and Mills KT. Frequent occurrence of polyovular follicles in ovaries of mice exposed neonatally to diethylstilbestrol. Teratology. 34: 29-35. 1986. [Medline] [CrossRef]

343. Iguchi T, Todoroki R, Takasugi N, and Petrow V. The effects of an aromatase inhibitor and a 5 alpha-reductase inhibitor upon the occurrence of polyovular follicles, persistent anovulation, and permanent vaginal stratification in mice treated neonatally with testosterone. Biol Reprod. 39: 689-697. 1988. [Medline] [CrossRef]

344. Kirigaya A, Kim H, Hayashi S, Chambon P, Watanabe H, Lguchi T, and Sato T. Involvement of estrogen receptor beta in the induction of polyovular follicles in mouse ovaries exposed neonatally to diethylstilbestrol. Zool Sci. 26: 704-712. 2009. [Medline] [CrossRef]

345. Mao TL, Kurman RJ, Huang CC, Lin MC, and Shih IM. Immunohistochemistry of choriocarcinoma: an aid in differential diagnosis and in elucidating pathogenesis. Am J Surg Pathol. 31: 1726-1732. 2007. [Medline] [CrossRef]

346. Farman CA, Benirschke K, Horner M, and Lappin P. Ovarian choriocarcinoma in a rhesus monkey associated with elevated serum chorionic gonadotropin levels. Vet Pathol. 42: 226-229. 2005. [Medline] [CrossRef]

347. Toyosawa K, Okimoto K, Koujitani T, and Kikawa E. Choriocarcinoma and teratoma in the ovary of a cynomolgus monkey. Vet Pathol. 37: 186-188. 2000. [Medline] [CrossRef]

348. Yokouchi Y, Imaoka M, Sayama A, and Sanbuissho A. Mixed germ cell tumor with embryonal carcinoma, choriocarcinoma, and epithelioid trophoblastic tumor in the ovary of a cynomolgus monkey. Toxicol Pathol. 39: 553-558. 2011. [Medline] [CrossRef]

349. Marr-Belvin AK, Bailey CC, Knight HL, Klumpp SA, Westmoreland $\mathrm{SV}$, and Miller AD. Ovarian pathology in rhesus macaques: a 12-year retrospective. J Med Primatol. 39: 170-176. 2010. [Medline] [CrossRef]

350. Kawano K, Kojima S, Hirakawa K, et al Mature Ovarian Teratoma in a Cynomolgus Monkey $(<\mathrm{i}>$ Macaca fascicularis $</ \mathrm{i}>)$. J Toxicol Pathol. 16: 283-285. 2003. [CrossRef]

351. de Silva KS, Kanumakala S, Grover SR, Chow CW, and Warne GL. Ovarian lesions in children and adolescents--an 11-year review. J Pediatr Endocrinol Metab. 17: 951-957. 2004. [Medline] [CrossRef]

352. Ethun KF, Wood CE, Cline JM, Register TC, Appt SE, and Clarkson TB. Endometrial profile of bazedoxifene acetate alone and in combination with conjugated equine estrogens in a primate model. Menopause. 20: 777-784. 2013. [Medline] [CrossRef]

353. Everds NE, Snyder PW, Bailey KL, Bolon B, Creasy DM, Foley GL, Rosol TJ, and Sellers T. Interpreting stress responses during routine toxicity studies: a review of the biology, impact, and assessment. Toxicol Pathol. 41: 560-614. 2013. [Medline] [CrossRef]

354. Cline JM, Söderqvist G, Register TC, Williams JK, Adams MR, and Von Schoultz B. Assessment of hormonally active agents in the reproductive tract of female nonhuman primates. Toxicol Pathol. 29: 84-90. 2001. [Medline] [CrossRef]

355. Vannuccini S, Tosti C, Carmona F, Huang SJ, Chapron C, Guo SW, 
and Petraglia F. Pathogenesis of adenomyosis: an update on molecular mechanisms. Reprod Biomed Online. 35: 592-601. 2017. [Medline] [CrossRef]

356. DiGiacomo RF. Gynecologic pathology in the rhesus monkey (Macaca mulatta). II. Findings in laboratory and free-ranging monkeys. Vet Pathol. 14: 539-546. 1977. [Medline] [CrossRef]

357. Baskin GB, Smith SM, and Marx PA. Endometrial hyperplasia, polyps, and adenomyosis associated with unopposed estrogen in rhesus monkeys (Macaca mulatta). Vet Pathol. 39: 572-575. 2002. [Medline] [CrossRef]

358. Beck AP, Erdelyi I, and Zeiss CJ. Endometrial decidualization and deciduosis in aged rhesus macaques (Macaca mulatta). Comp Med. 64: 148-156. 2014. [Medline]

359. Adams MR, Kaplan JR, and Koritnik DR. Psychosocial influences on ovarian endocrine and ovulatory function in Macaca fascicularis. Physiol Behav. 35: 935-940. 1985. [Medline] [CrossRef]

360. Miller MM, Hoffman DI, Creinin M, Levin JH, Chatterton RT Jr, Murad T, and Rebar RW. Comparison of endometrial biopsy and urinary pregnanediol glucuronide concentration in the diagnosis of luteal phase defect. Fertil Steril. 54: 1008-1011. 1990. [Medline] [CrossRef]

361. Sadekova ON, Nikitina LA, Rashidov TN, Voloschuk IN, Samokhodskaya LM, Demidova EM, and Tkachuk VA. Luteal phase defect is associated with impaired VEGF mRNA expression in the secretory phase endometrium. Reprod Biol. 15: 65-68. 2015. [Medline] [CrossRef]

362. Prakash A, Li TC, Laird S, Nargund G, and Ledger WL. Absence of follicular phase defect in women with recurrent miscarriage. Fertil Steril. 85: 1784-1790. 2006. [Medline] [CrossRef]

363. Bromer JG, Aldad TS, and Taylor HS. Defining the proliferative phase endometrial defect. Fertil Steril. 91: 698-704. 2009. [Medline] [CrossRef]

364. Slayden OD, Zelinski-Wooten MB, Chwalisz K, Stouffer RL, and Brenner RM. Chronic treatment of cycling rhesus monkeys with low doses of the antiprogestin ZK 137 316: morphometric assessment of the uterus and oviduct. Hum Reprod. 13: 269-277. 1998. [Medline] [CrossRef]

365. Brenner RM, Slayden OD, and Critchley HO. Anti-proliferative effects of progesterone antagonists in the primate endometrium: a potential role for the androgen receptor. Reproduction. 124: 167-172. 2002. [Medline] [CrossRef]

366. Chwalisz K, Garg R, Brenner R, Slayden O, Winkel C, and Elger W. Role of nonhuman primate models in the discovery and clinical development of selective progesterone receptor modulators (SPRMs). Reprod Biol Endocrinol. 4(Suppl 1): S8. 2006. [Medline] [CrossRef]

367. Pohl O, Williams AR, Bergeron C, and Gotteland JP. A 39-week oral toxicity study of ulipristal acetate in cynomolgus monkeys. Regul Toxicol Pharmacol. 66: 6-12. 2013. [Medline] [CrossRef]

368. Mutter GL, Bergeron C, Deligdisch L, et al The spectrum of endometrial pathology induced by progesterone receptor modulators. Modern pathology: an official journal of the United States and Canadian Academy of Pathology. Inc. 21: 591-598. 2008.

369. Slayden OD, and Brenner RM. Hormonal regulation and localization of estrogen, progestin and androgen receptors in the endometrium of nonhuman primates: effects of progesterone receptor antagonists. Arch Histol Cytol. 67: 393-409. 2004. [Medline] [CrossRef]

370. Brenner RM, and Slayden OD. Progesterone receptor antagonists and the endometrial antiproliferative effect. Semin Reprod Med. 23: 74-81. 2005. [Medline] [CrossRef]

371. Williams AR, Bergeron C, Barlow DH, Ferenczy A. Endometrial morphology after treatment of uterine fibroids with the selective progesterone receptor modulator, ulipristal acetate. International journal of gynecological pathology: official journal of the International Society of Gynecological Pathologists. 2012;31(6):556-569.

372. Kuwamura Y, Kakehi K, Hirakawa K, and Miyajima H. Ectopic uterine ovarian tissue in cynomolgus monkeys. Toxicol Pathol. 34: 220-222. 2006. [Medline] [CrossRef]

373. Cline JM, Register TC, and Clarkson TB. Comparative effects of tibolone and conjugated equine estrogens with and without medroxy- progesterone acetate on the reproductive tract of female cynomolgus monkeys. Menopause. 9: 242-252. 2002. [Medline] [CrossRef]

374. Sampson JA. Metastatic or Embolic Endometriosis, due to the Menstrual Dissemination of Endometrial Tissue into the Venous Circulation. The American journal of pathology. 1927;3(2):93-110 143.

375. Marsh EE, and Laufer MR. Endometriosis in premenarcheal girls who do not have an associated obstructive anomaly. Fertil Steril. 83: 758760. 2005. [Medline] [CrossRef]

376. Pinkert TC, Catlow CE, and Straus R. Endometriosis of the urinary bladder in a man with prostatic carcinoma. Cancer. 43: 1562-1567. 1979. [Medline] [CrossRef]

377. Burney RO, and Giudice LC. Pathogenesis and pathophysiology of endometriosis. Fertil Steril. 98: 511-519. 2012. [Medline] [CrossRef]

378. Sasson IE, and Taylor HS. Stem cells and the pathogenesis of endometriosis. Ann N Y Acad Sci. 1127: 106-115. 2008. [Medline] [CrossRef]

379. Ahn SH, Monsanto SP, Miller C, Singh SS, Thomas R, and Tayade C. Pathophysiology and Immune Dysfunction in Endometriosis. BioMed Res Int. 2015: 795976. 2015. [Medline] [CrossRef]

380. Atkins HM, Lombardini ED, Caudell DL, Appt SE, Dubois A, and Cline JM. Decidualization of Endometriosis in Macaques. Vet Pathol. 53: 1252-1258. 2016. [Medline] [CrossRef]

381. Zondervan KT, Weeks DE, Colman R, Cardon LR, Hadfield R, Schleffler J, Trainor AG, Coe CL, Kemnitz JW, and Kennedy SH. Familial aggregation of endometriosis in a large pedigree of rhesus macaques. Hum Reprod. 19: 448-455. 2004. [Medline] [CrossRef]

382. Yamanaka A, Kimura F, Takebayashi A, Kita N, Takahashi K, and Murakami T. Primate model research for endometriosis. Tohoku J Exp Med. 226: 95-99. 2012. [Medline] [CrossRef]

383. Assaf BT, and Miller AD. Pleural endometriosis in an aged rhesus macaque (Macaca mulatta): a histopathologic and immunohistochemical study. Vet Pathol. 49: 636-641. 2012. [Medline] [CrossRef]

384. Atkins HM, Caudell DL, Hutchison AR, LeGrande AC, and Kock ND. Abdominal Wall Endometriosis in a Rhesus Macaque (Macaca mulatta). Comp Med. 67: 277-280. 2017. [Medline]

385. Matthews ML. Abnormal uterine bleeding in reproductive-aged women. Obstet Gynecol Clin North Am. 42: 103-115. 2015. [Medline] [CrossRef]

386. Cline JMBF. Uterine vascular changes indicating prior pregnancy in macaques. Vet Pathol. 32: 585. 1995.

387. Greb RR, Kiesel L, Selbmann AK, Wehrmann M, Hodgen GD, Goodman AL, and Wallwiener D. Disparate actions of mifepristone (RU 486) on glands and stroma in the primate endometrium. Hum Reprod. 14: 198-206. 1999. [Medline] [CrossRef]

388. Trybus J, Bain FT, Fikes JD, Carlson CS, O'Sullivan MG, Jayo MJ, and Cline JM. Uterine infarctions in cynomolgus monkeys (Macaca fascicularis). Vet Pathol. 44: 309-313. 2007. [Medline] [CrossRef]

389. Enders AC. Implantation in the macaque: expansion of the implantation site during the first week of implantation. Placenta. 28: 794-802. 2007. [Medline] [CrossRef]

390. Ghosh D, and Sengupta J. Endometrial responses to a deciduogenic stimulus in ovariectomized rhesus monkeys treated with oestrogen and progesterone. J Endocrinol. 120: 51-58. 1989. [Medline] [CrossRef]

391. Valerio MG. Comparative aspects of contraceptive steroids: effects observed in the monkey. Toxicol Pathol. 17: 401-410. 1989. [Medline] [CrossRef]

392. Kaspareit J, Friderichs-Gromoll S, Buse E, Habermann G, Vogel F. Spontaneous epithelial plaques in the uterus of a non-pregnant cynomolgus monkey (Macaca fascicularis). Experimental and toxicologic pathology: official journal of the Gesellschaft fur Toxikologische Pathologie. 2004;56(1-2):9-12.

393. Padykula HA, Coles LG, McCracken JA, King NW Jr, Longcope C, and Kaiserman-Abramof IR. A zonal pattern of cell proliferation and differentiation in the rhesus endometrium during the estrogen surge. Biol Reprod. 31: 1103-1118. 1984. [Medline] [CrossRef]

394. Padykula HA, Coles LG, Okulicz WC, Rapaport SI, McCracken JA, King NW Jr, Longcope C, and Kaiserman-Abramof IR. The basalis of the primate endometrium: a bifunctional germinal compartment. Biol 
Reprod. 40: 681-690. 1989. [Medline] [CrossRef]

395. Kurman RJ, Kaminski PF, and Norris HJ. The behavior of endometrial hyperplasia. A long-term study of "untreated" hyperplasia in 170 patients. Cancer. 56: 403-412. 1985. [Medline] [CrossRef]

396. Hendrickx AG, and Binkerd PE. Congenital Malformations in Nonhuman Primates. In: Jones TC, Mohr U, Hunt RD, eds. Nonhuman Primates I. Berlin, Heidelberg: Springer Berlin Heidelberg; 1993:170-180.

397. Hendrickx AG, Prahalada S, and Binkerd PE. Long-term evaluation of the diethylstilbestrol (DES) syndrome in adult female rhesus monkeys (Macaca mulatta). Reprod Toxicol. 1: 253-261. 1987-1988. [Medline] [CrossRef]

398. Johnson LD. Lesions of the Female Genital System Caused by Diethylstilbestrol in Humans, Subhuman Primates, and Mice. In: Jones TC, Mohr U, Hunt RD, eds. Genital System. Berlin, Heidelberg: Springer Berlin Heidelberg; 1987:84-109.

399. Bergin IL, Bell JD, Chen Z, Zochowski MK, Chai D, Schmidt K, Culmer DL, Aronoff DM, Patton DL, Mwenda JM, Wood CE, and Burk RD. Novel genital alphapapillomaviruses in baboons (Papio hamadryas anubis) with cervical dysplasia. Vet Pathol. 50: 200-208. 2013. [Medline] [CrossRef]

400. Wood CE, Chen Z, Cline JM, Miller BE, and Burk RD. Characterization and experimental transmission of an oncogenic papillomavirus in female macaques. $J$ Virol. 81: 6339-6345. 2007. [Medline] [CrossRef]

401. Ostrow RS, McGlennen RC, Shaver MK, Kloster BE, Houser D, and Faras AJ. A rhesus monkey model for sexual transmission of a papillomavirus isolated from a squamous cell carcinoma. Proc Natl Acad Sci USA. 87: 8170-8174. 1990. [Medline] [CrossRef]

402. Wood CE, Borgerink H, Register TC, Scott L, and Cline JM. Cervical and vaginal epithelial neoplasms in cynomolgus monkeys. Vet Pathol. 41: 108-115. 2004. [Medline] [CrossRef]

403. Harari A, Wood CE, Van Doorslaer K, Chen Z, Domaingue MC, Elmore D, Koenig P, Wagner JD, Jennings RN, and Burk RD. Condylomatous genital lesions in cynomolgus macaques from Mauritius. Toxicol Pathol. 41: 893-901. 2013. [Medline] [CrossRef]

404. Creasy D, Bube A, de Rijk E, Kandori H, Kuwahara M, Masson R, Nolte T, Reams R, Regan K, Rehm S, Rogerson P, and Whitney K. Proliferative and nonproliferative lesions of the rat and mouse male reproductive system. Toxicol Pathol. 40(Suppl): 40S-121S. 2012. [Medline] [CrossRef]

405. Vidal JD, Mirsky ML, Colman K, Whitney KM, and Creasy DM. Reproductive System and Mammary Gland. In: Sahota PS, Popp JA, Hardisty JF, Gopinath C, eds. Toxicologic Pathology: Nonclinical Safety Assessment. 1st ed. Boca Raton, FL: CRC Press; 2013:717-830.

406. Smedley JV, Bailey SA, Perry RW, and O Rourke CM. Methods for predicting sexual maturity in male cynomolgus macaques on the basis of age, body weight, and histologic evaluation of the testes. Contemp Top Lab Anim Sci. 41: 18-20. 2002. [Medline]

407. Creasy DM, and Chapin RE. Chapter 59 - Male Reproductive System. In: Haschek WM, Rousseaux CG, Wallig MA, eds. Haschek and Rousseaux's Handbook of Toxicologic Pathology (Third Edition). Boston: Academic Press; 2013:2493-2598.

408. Kinzey WG. Male reproductive system and spermotegenesis. In: Hafez ESE, ed. Comparative reproduction of nonhuman primates. Springfield, IL: Charles C. Thomas; 1971:85-114.

409. de Rooij DG, van Alphen MM, and van de Kant HJ. Duration of the cycle of the seminiferous epithelium and its stages in the rhesus monkey (Macaca mulatta). Biol Reprod. 35: 587-591. 1986. [Medline] [CrossRef]

410. Dreef HC, Van Esch E, and De Rijk EP. Spermatogenesis in the cynomolgus monkey (Macaca fascicularis): a practical guide for routine morphological staging. Toxicol Pathol. 35: 395-404. 2007. [Medline] [CrossRef]

411. Hubbard GB, Eason RL, and Wood DH. Prostatic carcinoma in a rhesus monkey (Macaca mulatta). Vet Pathol. 22: 88-90. 1985. [Medline] [CrossRef]

412. McEntee MF, Epstein JI, Syring R, Tierney LA, and Strandberg JD. Characterization of prostatic basal cell hyperplasia and neoplasia in aged macaques: comparative pathology in human and nonhuman primates. Prostate. 29: 51-59. 1996. [Medline] [CrossRef]

413. Stout AP, and Engle ET. Spontaneous primary carcinoma of the prostate in a monkey (macaca mulatta). Am J Cancer. 39: 334-337. 1940.

414. Takayama S, Renwick AG, Johansson SL, et al. Long-term toxicity and carcinogenicity study of cyclamate in nonhuman primates. Toxicological sciences: an official journal of the Society of Toxicology. 2000;53(1):33-39.

415. Cline JM, Brignolo L, and Ford EW. Chapter 10 - Urogenital System. In: Abee CR, Mansfield K, Tardif S, Morris T, eds. Nonhuman Primates in Biomedical Research (Second Edition). Boston: Academic Press; 2012:483-562.

416. Gordon HP, Reim DA, and McClain SA. Condyloma acuminatum in a cynomolgus monkey (Macaca fascicularis). Contemp Top Lab Anim Sci. 39: 30-33. 2000. [Medline]

417. Zöller M, Friderichs-Gromoll S, and Kaspareit J. Testicular and epididymal appendages in the cynomolgus macaque (Macaca fascicularis). $J$ Med Primatol. 38: 448-454. 2009. [Medline] [CrossRef]

418. Zuckerman S, and Krohn PL. The Hydatids of Morgagni under Normal and Experimental Conditions. Philos Trans R Soc Lond B Biol Sci. 228: 147-172. 1937. [CrossRef]

419. Pereira Bacares ME, Vemireddi V, and Creasy D. Testicular Fibrous Hypoplasia in Cynomolgus Monkeys ( Macaca fascicularis): An Incidental, Congenital Lesion. Toxicol Pathol. 45: 536-543. 2017. [Medline] [CrossRef]

420. Zemjanis R, Gondos B, Adey WR, and Cockett AT. Testicular degeneration in Macaca nemestrina induced by immobilization. Fertil Steril. 21: 335-340. 1970. [Medline] [CrossRef]

421. Miller CJ, Vogel P, Alexander NJ, Dandekar S, Hendrickx AG, and Marx PA. Pathology and localization of simian immunodeficiency virus in the reproductive tract of chronically infected male rhesus macaques. Lab Invest. 70: 255-262. 1994. [Medline]

422. Sitzmann BD, Brown DI, Garyfallou VT, Kohama SG, Mattison JA, Ingram DK, Roth GS, Ottinger MA, and Urbanski HF. Impact of moderate calorie restriction on testicular morphology and endocrine function in adult rhesus macaques (Macaca mulatta). Age (Dordr). 36: 183-197. 2014. [Medline] [CrossRef]

423. O'Donnell L, Narula A, Balourdos G, Gu YQ, Wreford NG, Robertson DM, Bremner WJ, and McLachlan RI. Impairment of spermatogonial development and spermiation after testosterone-induced gonadotropin suppression in adult monkeys (Macaca fascicularis). J Clin Endocrinol Metab. 86: 1814-1822. 2001. [Medline] [CrossRef]

424. Ramaswamy S, and Weinbauer GF. Endocrine control of spermatogenesis: Role of FSH and LH/ testosterone. Spermatogenesis. 4: e996025. 2015. [Medline] [CrossRef]

425. Creasy D. Reproduction of the rat, mouse, dog, non-human primate and minipig. In: McInnes EF, Mann P, eds. Background Lesions in Laboratory Animals. Saint Louis: W.B. Saunders; 2012:101-122.

426. Haruyama E, Suda M, Ayukawa Y, Kamura K, Mizutamari M, Ooshima Y, and Tanimoto A. Testicular development in cynomolgus monkeys. Toxicol Pathol. 40: 935-942. 2012. [Medline] [CrossRef]

427. Vemireddi V, and Creasy D. Exacerbation of Seminiferous Tubule Dilatation by Fibrous Hypoplasia in Cynomolgus Monkey Testes. Paper presented at: STP 26th Annual Symposium2017; Montreal, Quebec.

428. Vidal JD, and Whitney KM. Morphologic manifestations of testicular and epididymal toxicity. Spermatogenesis. 4: e979099. 2014. [Medline] [CrossRef]

429. Reindel JF, Gough AW, Pilcher GD, Bobrowski WF, Sobocinski GP, and de la Iglesia FA. Systemic proliferative changes and clinical signs in cynomolgus monkeys administered a recombinant derivative of human epidermal growth factor. Toxicol Pathol. 29: 159-173. 2001. [Medline] [CrossRef]

430. Chapman ES, and Heidger PM Jr. Spermatic granuloma of vas deferens after vasectomy in rhesus monkeys and men: light and electron microscopic study. Urology. 13: 629-639. 1979. [Medline] [CrossRef]

431. Seppan P, and Krishnaswamy K. Long-term study of vasectomy in Macaca radiata--histological and ultrasonographic analysis of testis 
and duct system. Syst Biol Reprod Med. 60: 151-160. 2014. [Medline] [CrossRef]

432. Jeyaraj DA, Udayakumar TS, Rajalakshmi M, Pal PC, and Sharma RS. Effects of long-term administration of androgens and estrogen on rhesus monkey prostate: possible induction of benign prostatic hyperplasia. J Androl. 21: 833-841. 2000. [Medline]

433. Baskerville A, Cook RW, Dennis MJ, Cranage MP, and Greenaway PJ. Pathological changes in the reproductive tract of male rhesus monkeys associated with age and simian AIDS. J Comp Pathol. 107: 49-57. 1992. [Medline] [CrossRef]

434. Faltas NH. Urolithiasis in cynomolgus monkey (Macaca fascicularis): a case report. Contemp Top Lab Anim Sci. 39: 18-19. 2000. [Medline]

435. O'Rourke CM, Brammer DW, St Romain G, Peter GK, and Hofing GL. Calcium carbonate urolithiasis in an adult male cynomolgus monkey. Lab Anim Sci. 45: 222-224. 1995. [Medline]

436. Lees CJ, Carlson CS, and O'Sullivan MG. Urinary calculus caused by plant material in a cynomolgus monkey. Lab Anim Sci. 45: 441-442. 1995. [Medline]

437. Shirai N, and Evans MG. Testicular microlithiasis in a clinically healthy cynomolgus monkey (Macaca fascicularis). J Toxicol Pathol. 31: 147-150. 2018. [Medline] [CrossRef]

438. Chandolia RK, Luetjens CM, Wistuba J, Semjonow A, Pühse G, and Nieschlag E. Blockage of urine by intravesical ejaculate in cynomolgus monkeys. J Med Primatol. 36: 21-24. 2007. [Medline] [CrossRef]

439. Gumber S, Courtney CL, Strait KR, Sharma P, Freebersyser JE, Crane MM. Retrograde ejaculation associated spontaneous sperm cystolithiasis in four rhesus macaques (Macaca mulatta). Experimental and toxicologic pathology: official journal of the Gesellschaft fur Toxikologische Pathologie. 2013;65(7-8):1121-1125.

440. Frisk CS, Manning PJ, and Wagner JE. Semen matrix calculi in a rhesus monkey (Macaca mulatta). Lab Anim Sci. 24: 679-681. 1974. [Medline]

441. Udayakumar TS, Tyagi A, Rajalakshmi M, Das SN, Hashim S, and Bajaj JS. Changes in structure and functions of prostate by long-term administration of an androgen, testosterone enanthate, in rhesus monkey (Macaca mulatta). Anat Rec. 252: 637-645. 1998. [Medline] [CrossRef]

442. Simmons HA. Age-Associated Pathology in Rhesus Macaques (Macaca mulatta). Vet Pathol. 53: 399-416. 2016. [Medline] [CrossRef]

443. Renne R, Brix A, Harkema J, Herbert R, Kittel B, Lewis D, March T, Nagano K, Pino M, Rittinghausen S, Rosenbruch M, Tellier P, and Wohrmann T. Proliferative and nonproliferative lesions of the rat and mouse respiratory tract. Toxicol Pathol. 37(Suppl): 5S-73S. 2009. [Medline] [CrossRef]

444. Chamanza R, and Wright JA. A Review of the Comparative Anatomy, Histology, Physiology and Pathology of the Nasal Cavity of Rats, Mice, Dogs and Non-human Primates. Relevance to Inhalation Toxicology and Human Health Risk Assessment. J Comp Pathol. 153: 287-314. 2015. [Medline] [CrossRef]

445. Morgan KT, and Monticello TM. Airflow, gas deposition, and lesion distribution in the nasal passages. Environ Health Perspect. 85: 209218. 1990. [Medline]

446. Harkema JR. Wagner, James G., Carey, Stephan A. Comparative Anatomy of Nasal Airways: Relevance to Inhalation Toxicology and $\mathrm{Hu}-$ man Health. In: Morris JE, Shusterman, D. (Ed.)., ed. Toxicology of the Nose and Upper Airways. Boca Raton: CRC Press; 2010.

447. Geist FD. Nasal cavity, larynx, mouth and pharynx. In: Hartman CG, Straus WL, eds. The anatomy of the rhesus monkey (Macaca mulatta). New York: Hafner; 1933:193-194.

448. Hewitt G, MacLarnon A, Jones KE. The functions of laryngeal air sacs in primates: a new hypothesis. Folia primatologica; international journal of primatology. 2002;73(2-3):70-94.

449. Cullin CO, Colgin LM, and Lewis AD. Air sacculitis in three rhesus macaques (Macaca mulatta) and one Japanese macaque (M. fuscata). $J$ Med Primatol. 46: 48-50. 2017. [Medline] [CrossRef]

450. Mowat V, Alexander DJ, and Pilling AM. A Comparison of Rodent and Nonrodent Laryngeal and Tracheal Bifurcation Sensitivities in Inhalation Toxicity Studies and Their Relevance for Human Exposure. Toxicol Pathol. 45: 216-222. 2017. [Medline] [CrossRef]
451. Meyerholz DK, Suarez CJ, Dintzis SM, and Frevert CW. 9 - Respiratory System. In: Treuting PM, Dintzis SM, Montine KS, eds. Comparative Anatomy and Histology (Second Edition). San Diego: Academic Press; 2018:147-162.

452. Harkema JR, Nikula KJ, and Haschek WM. Chapter 51 - Respiratory System. In: Haschek WM, Rousseaux CG, Wallig MA, eds. Haschek and Rousseaux's Handbook of Toxicologic Pathology (Third Edition). Boston: Academic Press; 2013:1935-2003.

453. Keenan CBA, Vidal J, Covatta T, and Mahler BW. Respiratory Collection Protocol of the Laboratory Monkey. In:2010.

454. Taylor MA, Coop RL, and Wall RL. Parasites of Laboratory Animals. In: Veterinary parasitology. 4th ed. West Sussex, UK: John Wiley and Sons, LTD; 2016.

455. Van Scott MR, Hooker JL, Ehrmann D, Shibata Y, Kukoly C, Salleng K, Westergaard G, Sandrasagra A, and Nyce J. Dust mite-induced asthma in cynomolgus monkeys. J Appl Physiol (1985). 96: 1433-1444. 2004. [Medline] [CrossRef]

456. Shirai N, and Geoly FJ. Eosinophilic airway inflammation in a cynomolgus monkey. Vet Pathol. 47: 318-321. 2010. [Medline] [CrossRef]

457. Yamakawa Y, Ide T, Mitori H, Oishi Y, and Matsumoto M. Accumulation of brown pigment-laden macrophages associated with vascular lesions in the lungs of cynomolgus monkeys(Macaca fascicularis). $J$ Toxicol Pathol. 29: 181-184. 2016. [Medline] [CrossRef]

458. Nikula KJ, McCartney JE, McGovern T, Miller GK, Odin M, Pino $\mathrm{MV}$, and Reed MD. STP position paper: interpreting the significance of increased alveolar macrophages in rodents following inhalation of pharmaceutical materials. Toxicol Pathol. 42: 472-486. 2014. [Medline] [CrossRef]

459. Halliwell WH. Cationic amphiphilic drug-induced phospholipidosis. Toxicol Pathol. 25: 53-60. 1997. [Medline] [CrossRef]

460. Hook GE. Alveolar proteinosis and phospholipidoses of the lungs. Toxicol Pathol. 19: 482-513. 1991. [Medline]

461. Fossey S, Vahle J, Long P, Schelling S, Ernst H, Boyce RW, Jolette J, Bolon B, Bendele A, Rinke M, Healy L, High W, Roth DR, Boyle $\mathrm{M}$, and Leininger J. Nonproliferative and Proliferative Lesions of the Rat and Mouse Skeletal Tissues (Bones, Joints, and Teeth). J Toxicol Pathol. 29(Suppl): 49S-103S. 2016. [Medline] [CrossRef]

462. McCoy AM. Animal Models of Osteoarthritis: Comparisons and Key Considerations. Vet Pathol. 52: 803-818. 2015. [Medline] [CrossRef]

463. Vahle JL, Ma YL, and Burr DB. Chapter 32 - Skeletal Assessments in the Nonhuman Primate. In: Bluemel J, Korte S, Schenck E, Weinbauer GF, eds. The Nonhuman Primate in Nonclinical Drug Development and Safety Assessment. San Diego: Academic Press; 2015:605-625.

464. Fukuda S, Cho F, and Honjo S. Bone growth aud development of secondary ossification centers of extremities in the cynomolgus monkey (Macaca fascicularis). Jikken Dobutsu. 27: 387-397. 1978. [Medline]

465. Zoetis T, Tassinari MS, Bagi C, Walthall K, and Hurtt ME. Species comparison of postnatal bone growth and development. Birth Defects Res B Dev Reprod Toxicol. 68: 86-110. 2003. [Medline] [CrossRef]

466. Pritzker KPH, and Kessler MJ. Chapter 13 - Arthritis, Muscle, Adipose Tissue, and Bone Diseases of Nonhuman Primates. In: Abee CR, Mansfield K, Tardif S, Morris T, eds. Nonhuman Primates in Biomedical Research (Second Edition). Boston: Academic Press; 2012:629-697.

467. Gunson D, Gropp KE, and Varela A. Chapter 63 - Bone and Joints. In: Haschek WM, Rousseaux CG, Wallig MA, eds. Haschek and Rousseaux's Handbook of Toxicologic Pathology (Third Edition). Boston: Academic Press; 2013:2761-2858.

468. Schmelting B, Zöller M, and Kaspareit J. Peripheral ossifying fibroma and juxtacortical chondrosarcoma in cynomolgus monkeys (Macaca fascicularis). J Am Assoc Lab Anim Sci. 50: 98-104. 2011. [Medline]

469. Sieber SM, Correa P, Dalgard DW, and Adamson RH. Induction of osteogenic sarcomas and tumors of the hepatobiliary system in nonhuman primates with aflatoxin B1. Cancer Res. 39: 4545-4554. 1979. [Medline]

470. Jerome CP, Carlson CS, Register TC, et al. Bone functional changes in intact, ovariectomized, and ovariectomized, hormone-supplemented adult cynomolgus monkeys (Macaca fascicularis) evaluated by serum 
markers and dynamic histomorphometry. Journal of bone and mineral research: the official journal of the American Society for Bone and Mineral Research. 1994;9(4):527-540.

471. Smith SY, Jolette J, and Turner CH. Skeletal health: primate model of postmenopausal osteoporosis. Am J Primatol. 71: 752-765. 2009. [Medline] [CrossRef]

472. Ominsky MS, Vlasseros F, Jolette J, et al. Two doses of sclerostin antibody in cynomolgus monkeys increases bone formation, bone mineral density, and bone strength. Journal of bone and mineral research: the official journal of the American Society for Bone and Mineral Research. 2010;25(5):948-959.

473. Vahle JL, Zuehlke U, Schmidt A, Westmore M, Chen P, Sato M. Lack of bone neoplasms and persistence of bone efficacy in cynomolgus macaques after long-term treatment with teriparatide [rhPTH(1-34)]. Journal of bone and mineral research: the official journal of the American Society for Bone and Mineral Research. 2008;23(12):2033-2039.

474. Ettlin RA, Kuroda J, Plassmann S, Hayashi M, and Prentice DE. Successful drug development despite adverse preclinical findings part 2: examples. J Toxicol Pathol. 23: 213-234. 2010. [Medline] [CrossRef]

475. Salyusarenko M, Peeri D, Bibring U, Ranen E, Bdolah-Abram T, Aroch I. Hypertrophic Osteopathy: a Retrospective Case Control Study of 30 Dogs. Israel Journal of Veterinary Medicine - Vol 68 (3) - December 2013. 2013;68(3):209-217.

476. Sobry C, Flandre T, Forster R, and Palate B. Hypertrophic osteopathy in two cynomolgus monkeys secondary to opportunistic infections during a preclinical study with an immunomodulator. Paper presented at: Cutting Edge Pathology 2017, 3rd Joint European Congress of the ESVP, ESTP and ECVP2017; Lyon, France.

477. Bauer C, Dunn BG, Brothman AR, Dick EJ Jr, Christensen C, Voges A, and Moore CM. Polyostotic fibrous dysplasia in a cynomolgus Macaque (Macaca fascicularis). Comp Med. 62: 142-148. 2012. [Medline]

478. Cheverud JM. Epiphyseal union and dental eruption Macaca mulatta. Am J Phys Anthropol. 56: 157-167. 1981. [Medline] [CrossRef]

479. Frazier KS. Drug-induced Physeal Abnormalities in Preclinical Toxicity Studies. Toxicol Pathol. 45: 869-875. 2017. [Medline] [CrossRef]

480. Ryan AM, Eppler DB, Hagler KE, Bruner RH, Thomford PJ, Hall RL, Shopp GM, and O'Neill CA. Preclinical safety evaluation of rhuMAbVEGF, an antiangiogenic humanized monoclonal antibody. Toxicol Pathol. 27: 78-86. 1999. [Medline] [CrossRef]

481. Patyna S, Arrigoni C, Terron A, Kim TW, Heward JK, Vonderfecht SL, Denlinger R, Turnquist SE, and Evering W. Nonclinical safety evaluation of sunitinib: a potent inhibitor of VEGF, PDGF, KIT, FLT3, and RET receptors. Toxicol Pathol. 36: 905-916. 2008. [Medline] [CrossRef]

482. Burkhardt JE, Walterspiel JN, and Schaad UB. Quinolone arthropathy in animals versus children. Clin Infect Dis. 25: 1196-1204. 1997. [Medline] [CrossRef]

483. Sendzik J, Lode H, and Stahlmann R. Quinolone-induced arthropathy: an update focusing on new mechanistic and clinical data. Int J Antimicrob Agents. 33: 194-200. 2009. [Medline] [CrossRef]

484. Carlson CS, Loeser RF, Purser CB, Gardin JF, Jerome CP. Osteoarthritis in cynomolgus macaques. III: Effects of age, gender, and subchondral bone thickness on the severity of disease. Journal of bone and mineral research: the official journal of the American Society for Bone and Mineral Research. 1996;11(9):1209-1217.

485. Rao S, Bryant M, Herbert R, Sullivan N, Murray C, Bacher J, and Safdar N. Idiopathic chondrolysis condition in two young, wild-caught Cynomolgus monkeys (Macaca fascicularis) reared in captivity. Vet Pathol. 46: 509-513. 2009. [Medline] [CrossRef]

486. Tanaka M, Yamada H, Nishikawa S, Mori H, Ochi Y, Horai N, Li M, and Amizuka N. Joint Degradation in a Monkey Model of CollagenInduced Arthritis: Role of Cathepsin K Based on Biochemical Markers and Histological Evaluation. Int J Rheumatol. 2016: 8938916. 2016. [Medline] [CrossRef]

487. Hart BA, Bank RA, De Roos JA, Brok H, Jonker M, Theuns HM, Hakimi J, and Te Koppele JM. Collagen-induced arthritis in rhesus monkeys: evaluation of markers for inflammation and joint degradation. $\mathrm{Br}$
J Rheumatol. 37: 314-323. 1998. [Medline] [CrossRef]

488. Vierboom MP, Jonker M, Bontrop RE, and 't Hart B. Modeling human arthritic diseases in nonhuman primates. Arthritis Res Ther. 7: 145-154. 2005. [Medline] [CrossRef]

489. Takei S. A case report of spontaneous polyarthritis in cynomolgus monkeys. J Vet Med Sci. 75: 531-534. 2013. [Medline] [CrossRef]

490. Smith BH. Sequence of emergence of the permanent teeth in Macaca, Pan, Homo, and Australopithecus: Its evolutionary significance. American journal of human biology: the official journal of the Human Biology Council. 1994;6(1):61-76.

491. Oz HS, and Puleo DA. Animal models for periodontal disease. $J$ Biomed Biotechnol. 2011: 754857. 2011. [Medline] [CrossRef]

492. Ebersole JL, Brunsvold M, Steffensen B, Wood R, and Holt SC. Effects of immunization with Porphyromonas gingivalis and Prevotella intermedia on progression of ligature-induced periodontitis in the nonhuman primate Macaca fascicularis. Infect Immun. 59: 3351-3359. 1991. [Medline] [CrossRef]

493. Davis JA, Banks RE, and Young D. Ameloblastic odontoma in a cynomolgus monkey (Macaca fascicularis). Lab Anim Sci. 38: 312-315. 1988. [Medline]

494. Schou S, Holmstrup P, and Kornman KS. Non-human primates used in studies of periodontal disease pathogenesis: a review of the literature. J Periodontol. 64: 497-508. 1993. [Medline] [CrossRef]

495. Curtis JW Jr, Brodish DL, Weaver DS, and Brady AG. Periapical abscesses of cut canine teeth in cynomolgus macaques. Lab Anim. 20: 277-280. 1986. [Medline] [CrossRef]

496. Auskaps AM, and Shaw JH. Studies on the dentition of the cynomolgus monkey. J Dent Res. 36: 432-436. 1957. [Medline] [CrossRef]

497. Farges JC, Alliot-Licht B, Renard E, Ducret M, Gaudin A, Smith AJ, and Cooper PR. Dental Pulp Defence and Repair Mechanisms in Dental Caries. Mediators Inflamm. 2015: 230251. 2015. [Medline] [CrossRef]

498. Beighton D, Hayday H, and Walker J. The acquisition of Streptococcus mutans by infant monkeys (Macaca fascicularis) and its relationship to the initiation of dental caries. J Gen Microbiol. 128: 1881-1892. 1982. [Medline]

499. Loftin KC, Brown LR, and Levy BM. Comparison of the predominant cultivable microflora in the dental plaque of Macaca mulatta (rhesus) and Macaca fascicularis (cynomolgus). J Dent Res. 59: 1606-1612. 1980. [Medline] [CrossRef]

500. Bruce AG, Bakke AM, Bielefeldt-Ohmann H, Ryan JT, Thouless ME, Tsai CC, and Rose TM. High levels of retroperitoneal fibromatosis (RF)-associated herpesvirus in RF lesions in macaques are associated with ORF73 LANA expression in spindleoid tumour cells. J Gen Virol. 87: 3529-3538. 2006. [Medline] [CrossRef]

501. Westmoreland SV, and Mansfield KG. Comparative pathobiology of Kaposi sarcoma-associated herpesvirus and related primate rhadinoviruses. Comp Med. 58: 31-42. 2008. [Medline]

502. Giddens W, Bielitzski J, Morton W, et al Idiopathic retroperitoneal fibrosis-an enzootic disease in the pigtail monkey (macaca-nemestrina). Paper presented at: laboratory Investigation 1979.

503. Tsai CC, Giddens WE Jr, Morton WR, Rosenkranz SL, Ochs HD, and Benveniste RE. Retroperitoneal fibromatosis and acquired immunodeficiency syndrome in macaques: epidemiologic studies. Lab Anim Sci. 35: 460-464. 1985. [Medline]

504. Giddens WE Jr, Tsai CC, Morton WR, Ochs HD, Knitter GH, and Blakley GA. Retroperitoneal fibromatosis and acquired immunodeficiency syndrome in macaques. Pathologic observations and transmission studies. Am J Pathol. 119: 253-263. 1985. [Medline]

505. Rose TM, Strand KB, Schultz ER, Schaefer G, Rankin GW Jr, Thouless ME, Tsai CC, and Bosch ML. Identification of two homologs of the Kaposi's sarcoma-associated herpesvirus (human herpesvirus 8) in retroperitoneal fibromatosis of different macaque species. J Virol. 71: 4138-4144. 1997. [Medline] [CrossRef]

506. Gropp KE. Skeletal Muscle Toolbox. Toxicol Pathol. 45: 939-942. 2017. [Medline] [CrossRef]

507. Ramos MF, Baker J, Atzpodien EA, Bach U, Brassard J, Cartwright J, 
Farman C, Fishman C, Jacobsen M, Junker-Walker U, Kuper F, Moreno MCR, Rittinghausen S, Schafer K, Tanaka K, Teixeira L, Yoshizawa $\mathrm{K}$, and Zhang H. Nonproliferative and Proliferative Lesions of the Ratand Mouse Special Sense Organs(Ocular [eye and glands], Olfactory and Otic). J Toxicol Pathol. 31(Suppl): 97S-214S. 2018. [Medline] [CrossRef]

508. Kirk EC. Comparative morphology of the eye in primates. Anat Rec A Discov Mol Cell Evol Biol. 281: 1095-1103. 2004. [Medline] [CrossRef]

509. Komai Y, and Ushiki T. The three-dimensional organization of collagen fibrils in the human cornea and sclera. Invest Ophthalmol Vis Sci. 32: 2244-2258. 1991. [Medline]

510. Merindano Encina MD, Potau JM, Ruano D, and Costa J. A comparative study of Bowman's layer in some mammals: Relationships with other constituent corneal structures. Eur J Anat. 6: 133-139. 2002.

511. Wislocki GB. The anterior segment of the eye of the rhesus monkey investigated by histochemical means. Am J Anat. 91: 233-261. 1952. [Medline] [CrossRef]

512. Baroody RA, Bito LZ, DeRousseau CJ, and Kaufman PL. Ocular development and aging. 1. Corneal endothelial changes in cats and in free-ranging and caged rhesus monkeys. Exp Eye Res. 45: 607-622. 1987. [Medline] [CrossRef]

513. Van Horn DL, and Hyndiuk RA. Endothelial wound repair in primate cornea. Exp Eye Res. 21: 113-124. 1975. [Medline] [CrossRef]

514. Higa K, Shimmura S, Miyashita H, Shimazaki J, and Tsubota K. Melanocytes in the corneal limbus interact with K19-positive basal epithelial cells. Exp Eye Res. 81: 218-223. 2005. [Medline] [CrossRef]

515. Short BG. Safety evaluation of ocular drug delivery formulations: techniques and practical considerations. Toxicol Pathol. 36: 49-62. 2008. [Medline] [CrossRef]

516. Kuszak JR. The ultrastructure of epithelial and fiber cells in the crystalline lens. Int Rev Cytol. 163: 305-350. 1995. [Medline] [CrossRef]

517. Lütjen-Drecoll E. Functional morphology of the trabecular meshwork in primate eyes. Prog Retin Eye Res. 18: 91-119. 1999. [Medline] [CrossRef]

518. Almazan A, Tsai S, Miller PE, Lee SS, Vilupuru AS, Burke JA, and Robinson MR. Iridocorneal angle measurements in mammalian species: normative data by optical coherence tomography. Vet Ophthalmol. 16: 163-166. 2013. [Medline] [CrossRef]

519. Fischer AJ, Hendrickson A, and Reh TA. Immunocytochemical characterization of cysts in the peripheral retina and pars plana of the adult primate. Invest Ophthalmol Vis Sci. 42: 3256-3263. 2001. [Medline]

520. May-Simera H, Nagel-Wolfrum K, and Wolfrum U. Cilia - The sensory antennae in the eye. Prog Retin Eye Res. 60: 144-180. 2017. [Medline] [CrossRef]

521. Sand A, Schmidt TM, and Kofuji P. Diverse types of ganglion cell photoreceptors in the mammalian retina. Prog Retin Eye Res. 31: 287-302. 2012. [Medline] [CrossRef]

522. Curcio CA, Sloan KR, Kalina RE, and Hendrickson AE. Human photoreceptor topography. J Comp Neurol. 292: 497-523. 1990. [Medline] [CrossRef]

523. Kim JW, Yang HJ, Oel AP, Brooks MJ, Jia L, Plachetzki DC, Li W, Allison WT, and Swaroop A. Recruitment of Rod Photoreceptors from Short-Wavelength-Sensitive Cones during the Evolution of Nocturnal Vision in Mammals. Dev Cell. 37: 520-532. 2016. [Medline] [CrossRef]

524. Packer O, Hendrickson AE, and Curcio CA. Photoreceptor topography of the retina in the adult pigtail macaque (Macaca nemestrina). J Comp Neurol. 288: 165-183. 1989. [Medline] [CrossRef]

525. Wikler KC, and Rakic P. Distribution of photoreceptor subtypes in the retina of diurnal and nocturnal primates. $J$ Neurosci. 10: 3390-3401. 1990. [Medline] [CrossRef]

526. Borwein B. Scanning electron microscopy of monkey foveal photoreceptors. Anat Rec. 205: 363-373. 1983. [Medline] [CrossRef]

527. Bringmann A, Syrbe S, Görner K, Kacza J, Francke M, Wiedemann P, and Reichenbach A. The primate fovea: Structure, function and development. Prog Retin Eye Res. 66: 49-84. 2018. [Medline] [CrossRef]

528. Provis JM, Dubis AM, Maddess T, and Carroll J. Adaptation of the central retina for high acuity vision: cones, the fovea and the avascular zone. Prog Retin Eye Res. 35: 63-81. 2013. [Medline] [CrossRef]

529. Wolin LR, and Massopust LC Jr. Characteristics of the ocular fundus in primates. J Anat. 101: 693-699. 1967. [Medline]

530. Borwein B, Borwein D, Medeiros J, and McGowan JW. The ultrastructure of monkey foveal photoreceptors, with special reference to the structure, shape, size, and spacing of the foveal cones. Am J Anat. 159: 125-146. 1980. [Medline] [CrossRef]

531. Krebs W, and Krebs IP. Quantitative morphology of the central fovea in the primate retina. Am J Anat. 184: 225-236. 1989. [Medline] [CrossRef]

532. Hendrickson A, Troilo D, Possin D, and Springer A. Development of the neural retina and its vasculature in the marmoset Callithrix jacchus. J Comp Neurol. 497: 270-286. 2006. [Medline] [CrossRef]

533. Perry VH, and Cowey A. The lengths of the fibres of Henle in the retina of macaque monkeys: implications for vision. Neuroscience. 25: 225236. 1988. [Medline] [CrossRef]

534. Bernstein PS, Li B, Vachali PP, Gorusupudi A, Shyam R, Henriksen BS, and Nolan JM. Lutein, zeaxanthin, and meso-zeaxanthin: The basic and clinical science underlying carotenoid-based nutritional interventions against ocular disease. Prog Retin Eye Res. 50: 34-66. 2016. [Medline] [CrossRef]

535. El-Mofty AA, Eisner G, Balazs EA, Denlinger JL, and Gouras P. Retinal degeneration in rhesus monkeys, Macaca mulatta. Survey of three seminatural free-breeding colonies. Exp Eye Res. 31: 147-166. 1980. [Medline] [CrossRef]

536. Zeiss CJ. Animals as models of age-related macular degeneration: an imperfect measure of the truth. Vet Pathol. 47: 396-413. 2010. [Medline] [CrossRef]

537. Ogden TE. The nerve-fiber layer of the primate retina: an autoradiographic study. Invest Ophthalmol. 13: 95-100. 1974. [Medline]

538. Sanchez RM, Dunkelberger GR, and Quigley HA. The number and diameter distribution of axons in the monkey optic nerve. Invest Ophthalmol Vis Sci. 27: 1342-1350. 1986. [Medline]

539. Vecino E, Rodriguez FD, Ruzafa N, Pereiro X, and Sharma SC. Glianeuron interactions in the mammalian retina. Prog Retin Eye Res. 51: 1-40. 2016. [Medline] [CrossRef]

540. Booij JC, Baas DC, Beisekeeva J, Gorgels TG, and Bergen AA. The dynamic nature of Bruch's membrane. Prog Retin Eye Res. 29: 1-18. 2010. [Medline] [CrossRef]

541. Ferrara D, Waheed NK, and Duker JS. Investigating the choriocapillaris and choroidal vasculature with new optical coherence tomography technologies. Prog Retin Eye Res. 52: 130-155. 2016. [Medline] [CrossRef]

542. Khan KN, Mahroo OA, Khan RS, Mohamed MD, McKibbin M, Bird A, Michaelides M, Tufail A, and Moore AT. Differentiating drusen: Drusen and drusen-like appearances associated with ageing, age-related macular degeneration, inherited eye disease and other pathological processes. Prog Retin Eye Res. 53: 70-106. 2016. [Medline] [CrossRef]

543. Kong X, Wang K, Sun X, and Witt RE. Comparative study of the retinal vessel anatomy of rhesus monkeys and humans. Clin Exp Ophthalmol. 38: 629-634. 2010. [Medline] [CrossRef]

544. Kur J, Newman EA, and Chan-Ling T. Cellular and physiological mechanisms underlying blood flow regulation in the retina and choroid in health and disease. Prog Retin Eye Res. 31: 377-406. 2012. [Medline] [CrossRef]

545. Brooks DE, Komàromy AM, and Källberg ME. Comparative retinal ganglion cell and optic nerve morphology. Vet Ophthalmol. 2: 3-11. 1999. [Medline] [CrossRef]

546. Sandell JH, and Peters A. Effects of age on nerve fibers in the rhesus monkey optic nerve. J Comp Neurol. 429: 541-553. 2001. [Medline] [CrossRef]

547. Lockwood H, Reynaud J, Gardiner S, Grimm J, Libertiaux V, Downs JC, Yang H, and Burgoyne CF. Lamina cribrosa microarchitecture in normal monkey eyes part 1: methods and initial results. Invest Ophthalmol Vis Sci. 56: 1618-1637. 2015. [Medline] [CrossRef]

548. Quigley HA. The contribution of the sclera and lamina cribrosa to the pathogenesis of glaucoma: Diagnostic and treatment implications. 
Prog Brain Res. 220: 59-86. 2015. [Medline] [CrossRef]

549. Burgoyne CF. The non-human primate experimental glaucoma model. Exp Eye Res. 141: 57-73. 2015. [Medline] [CrossRef]

550. Kobayashi $\mathrm{H}$, and Kohshima S. Unique morphology of the human eye and its adaptive meaning: comparative studies on external morphology of the primate eye. J Hum Evol. 40: 419-435. 2001. [Medline] [CrossRef]

551. Mayhew JA, and Gómez JC. Gorillas with white sclera: A naturally occurring variation in a morphological trait linked to social cognitive functions. Am J Primatol. 77: 869-877. 2015. [Medline] [CrossRef]

552. Watson PG, and Young RD. Scleral structure, organisation and disease. A review. Exp Eye Res. 78: 609-623. 2004. [Medline] [CrossRef]

553. Ponsioen TL, Hooymans JM, and Los LI. Remodelling of the human vitreous and vitreoretinal interface--a dynamic process. Prog Retin Eye Res. 29: 580-595. 2010. [Medline] [CrossRef]

554. de Smet MD, Gad Elkareem AM, and Zwinderman AH. The vitreous, the retinal interface in ocular health and disease. Ophthalmologica Journal international d'ophtalmologie International journal of ophthalmology. Z Augenheilkd. 230: 165-178. 2013.

555. Qiao H, Hisatomi T, Sonoda KH, Kura S, Sassa Y, Kinoshita S, Nakamura T, Sakamoto T, and Ishibashi T. The characterisation of hyalocytes: the origin, phenotype, and turnover. Br J Ophthalmol. 89: 513-517. 2005. [Medline] [CrossRef]

556. Worst JG, and Los LI. Comparative anatomy of the vitreous body in rhesus monkeys and man. Doc Ophthalmol. 82: 169-178. 1992. [Medline] [CrossRef]

557. Denlinger JL, Eisner G, and Balazs EA. Age-related changes in the vitreus and lens of rhesus monkeys (Macaca mulatta). Exp Eye Res. 31: 67-79. 1980. [Medline] [CrossRef]

558. Chodosh J, Nordquist RE, and Kennedy RC. Comparative anatomy of mammalian conjunctival lymphoid tissue: a putative mucosal immune site. Dev Comp Immunol. 22: 621-630. 1998. [Medline] [CrossRef]

559. Ruskell GL. Organization and cytology of lymphoid tissue in the cynomolgus monkey conjunctiva. Anat Rec. 243: 153-164. 1995. [Medline] [CrossRef]

560. Ruskell GL, and VanderWerf F. Sensory innervation of conjunctival lymph follicles in cynomolgus monkeys. Invest Ophthalmol Vis Sci. $\mathbf{3 8}$ : 884-892. 1997. [Medline]

561. Bajpai RN, and Verma BM. Heterotropic cartilage in the upper eyelid of monkey. Indian J Ophthalmol. 28: 227-228. 1981. [Medline]

562. Bellhorn RW, Friedman AH, and Henkind P. Racemose (cirsoid) hemangioma in rhesus monkey retina. Am J Ophthalmol. 74: 517-522. 1972. [Medline] [CrossRef]

563. Hayreh SS. Structure of the Optic Nerve. In: Ischemic Optic Neuropathies. Berlin, Heidelberg: Springer Berlin Heidelberg; 2011:7-34.

564. Horiuchi T, Gass DM, and David NJ. Arteriovenous malformation in the retina of a monkey. Am J Ophthalmol. 82: 896-904. 1976. [Medline] [CrossRef]

565. Lin CC, Tso MO, and Vygantas CM. Coloboma of optic nerve associated with serous maculopathy. A clinicopathologic correlative study. Arch Ophthalmol. 102: 1651-1654. 1984. [Medline] [CrossRef]

566. Schmidt RE. Colobomas in non-human primates. Folia primatologica; international journal of primatology. 1971;14(3):256-262.

567. Mohr EL, Block LN, Newman CM, Stewart LM, Koenig M, Semler M, Breitbach ME, Teixeira LBC, Zeng X, Weiler AM, Barry GL, Thoong TH, Wiepz GJ, Dudley DM, Simmons HA, Mejia A, Morgan TK, Salamat MS, Kohn S, Antony KM, Aliota MT, Mohns MS, Hayes JM, Schultz-Darken N, Schotzko ML, Peterson E, Capuano S 3rd, Osorio JE, O'Connor SL, Friedrich TC, O'Connor DH, and Golos TG. Ocular and uteroplacental pathology in a macaque pregnancy with congenital Zika virus infection. PLoS One. 13: e190617. 2018. [Medline] [CrossRef]

568. Saxena S, Meyer CH. Peripapillary astrocytic hamartomas evolve from the optic nerve. BMJ case reports. 2015;2015.

569. Hudson LE, Mendoza PR, Yan J, and Grossniklaus HE. Reactive Retinal Astrocytic Tumor (Focal Nodular Gliosis): A Case Report. Ocul Oncol Pathol. 3: 1-7. 2017. [Medline] [CrossRef]
570. Jakobiec FA, Thanos A, Stagner AM, Grossniklaus HE, and Proia AD. So-called massive retinal gliosis: A critical review and reappraisal. Surv Ophthalmol. 61: 339-356. 2016. [Medline] [CrossRef]

571. Alvarado RG, and Horton JC. Myelination of the Nerve Fiber Layer Associated with Local Retinal Dysgenesis. Invest Ophthalmol Vis Sci. 45: 1621-1621. 2004.

572. Bellhorn RW, Hirano A, Henkind P, and Johnson PT. Schwann cell proliferations mimicking medullated retinal nerve fibers. Am J Ophthalmol. 87: 469-473. 1979. [Medline] [CrossRef]

573. Shirai N, and Evans MG. Characterization of ectopic myelinated nerve fibers in the retina of a cynomolgus monkey (Macaca fascicularis). $J$ Med Primatol. 47: 198-200. 2018. [Medline] [CrossRef]

574. Korshunov A, Sturm D, Ryzhova M, Hovestadt V, Gessi M, Jones DT, Remke M, Northcott P, Perry A, Picard D, Rosenblum M, Antonelli M, Aronica E, Schüller U, Hasselblatt M, Woehrer A, Zheludkova O, Kumirova E, Puget S, Taylor MD, Giangaspero F, Peter Collins V, von Deimling A, Lichter P, Huang A, Pietsch T, Pfister SM, and Kool M. Embryonal tumor with abundant neuropil and true rosettes (ETANTR), ependymoblastoma, and medulloepithelioma share molecular similarity and comprise a single clinicopathological entity. Acta Neuropathol. 128: 279-289. 2014. [Medline] [CrossRef]

575. Yanoff M, and Sassani JW. 18 - Retinoblastoma and Simulating Lesions. In: Yanoff M, Sassani JW, eds. Ocular Pathology (Eighth Edition). London: Elsevier; 2020:726-759.e725.

576. Cleary PE, Minckler DS, and Ryan SJ. Ultrastructure of traction retinal detachment in rhesus monkey eyes after a posterior penetrating ocular injury. Am J Ophthalmol. 90: 829-845. 1980. [Medline] [CrossRef]

577. Hida T, Sheta SM, Proia AD, and McCuen BW 2nd. Experimental transvitreal cyanoacrylate retinopexy in a primate model. Am J Ophthalmol. 103: 782-789. 1987. [Medline] [CrossRef]

578. Litts KM, Ach T, Hammack KM, Sloan KR, Zhang Y, Freund KB, and Curcio CA. Quantitative Analysis of Outer Retinal Tubulation in Age-Related Macular Degeneration From Spectral-Domain Optical Coherence Tomography and Histology. Invest Ophthalmol Vis Sci. 57: 2647-2656. 2016. [Medline] [CrossRef]

579. Gartner S, and Henkind P. Lange's folds: a meaningful ocular artifact. Ophthalmology. 88: 1307-1310. 1981. [Medline] [CrossRef]

580. Herwig MC, Müller AM, Holz FG, and Loeffler KU. Morphologic analysis of artifacts in human fetal eyes confounding histopathologic investigations. Invest Ophthalmol Vis Sci. 52: 2712-2718. 2011. [Medline] [CrossRef]

581. Schmidt RE. Ophthalmic lesions in non-human primates. Vet Pathol. 8 28-36. 1971. [Medline] [CrossRef]

582. Sinha DP, Cartwright ME, and Johnson RC. Incidental mononuclear cell infiltrate in the uvea of cynomolgus monkeys. Toxicol Pathol. 34: 148-151. 2006. [Medline] [CrossRef]

583. Hirose S, Kuwabara T, Nussenblatt RB, Wiggert B, Redmond TM, and Gery I. Uveitis induced in primates by interphotoreceptor retinoidbinding protein. Arch Ophthalmol. 104: 1698-1702. 1986. [Medline] [CrossRef]

584. Smith RE, Macy JI, Parrett C, and Irvine J. Variations in acute multifocal histoplasmic choroiditis in the primate. Invest Ophthalmol Vis Sci. 17: 1005-1018. 1978. [Medline]

585. Kodama R, Yang X, Araki T, Sato M, Iwashige S, Tanigawa Y, Uchimura M, Ishimaru K, Sasaki Y, Kamimura Y, and Maeda H. Keratoconus in a cynomolgus monkey. J Toxicol Pathol. 23: 53-58. 2010. [Medline] [CrossRef]

586. Peiffer RL Jr, Werblin TP, and Patel AS. Keratoconus in a rhesus monkey. J Med Primatol. 16: 403-406. 1987. [Medline] [CrossRef]

587. Lindquist NG, Larsson BS, and Stjernschantz J. Increased pigmentation of iridial melanocytes in primates induced by a prostaglandin analogue. Exp Eye Res. 69: 431-436. 1999. [Medline] [CrossRef]

588. Prota G, Vincensi MR, Napolitano A, Selen G, and Stjernschantz J. Latanoprost stimulates eumelanogenesis in iridial melanocytes of cynomolgus monkeys. Pigment Cell Res. 13: 147-150. 2000. [Medline] [CrossRef]

589. Grierson I, Jonsson M, and Cracknell K. Latanoprost and pigmenta- 
tion. Jpn J Ophthalmol. 48: 602-612. 2004. [Medline] [CrossRef]

590. Stjernschantz J, Ocklind A, Wentzel P, Lake S, and Hu DN. Latanoprostinduced increase of tyrosinase transcription in iridial melanocytes. Acta Ophthalmol Scand. 78: 618-622. 2000. [Medline] [CrossRef]

591. Cotlier E. Rubella in animals and experimental ocular aspects of congenital rubella. Int Ophthalmol Clin. 12: 137-146. 1972. [Medline]

592. Kessler MJ, and Rawlins RG. Congenital cataracts in a free-ranging rhesus monkey. J Med Primatol. 14: 225-228. 1985. [Medline] [CrossRef]

593. London WT, Levitt NH, Kent SG, Wong VG, and Sever JL. Congenital cerebral and ocular malformations induced in rhesus monkeys by Venezuelan equine encephalitis virus. Teratology. 16: 285-5. 1977. [Medline] [CrossRef]

594. Oishi T, Imai H, Go Y, Imamura M, Hirai H, and Takada M. Sporadic premature aging in a Japanese monkey: a primate model for progeria. PLoS One. 9: e111867. 2014. [Medline] [CrossRef]

595. Kaufman PL, and Bito LZ. The occurrence of senile cataracts, ocular hypertension and glaucoma in rhesus monkeys. Exp Eye Res. 34: 287-291. 1982. [Medline] [CrossRef]

596. Sasaki Y, Kodama R, Iwashige S, Fujishima J, Yoshikawa T, Kamimura $\mathrm{Y}$, and Maeda $\mathrm{H}$. Bilateral cataract in a cynomolgus monkey. $J$ Toxicol Pathol. 24: 69-73. 2011. [Medline] [CrossRef]

597. Uno H. Age-related pathology and biosenescent markers in captive rhesus macaques. Age (Omaha). 20: 1-13. 1997. [Medline] [CrossRef]

598. Cromeens DM, and Stephens LC. Insular amyloidosis and diabetes mellitus in a crab-eating macaque (Macaca fascicularis). Lab Anim Sci. 35: 642-645. 1985. [Medline]

599. Gibbs GE, Wilson RB, and Gifford H. Glomerulosclerosis in the longterm alloxan diabetic monkey. Diabetes. 15: 258-261. 1966. [Medline] [CrossRef]

600. Johnson MA, Lutty GA, McLeod DS, Otsuji T, Flower RW, Sandagar G, Alexander T, Steidl SM, and Hansen BC. Ocular structure and function in an aged monkey with spontaneous diabetes mellitus. Exp Eye Res. 80: 37-42. 2005. [Medline] [CrossRef]

601. Kaufman PL, Axelsson U, and Bárány EH. Induction of subcapsular cataracts in cynomolgus monkeys by echothiophate. Arch Ophthalmol. 95: 499-504. 1977. [Medline] [CrossRef]

602. Sonneveld P, Peperkamp E, and van Bekkum DW. Incidence of cataracts in rhesus monkeys treated with whole-body irradiation. Radiology. 133: 227-229. 1979. [Medline] [CrossRef]

603. Latendresse JR, Warbrittion AR, Jonassen H, and Creasy DM. Fixation of testes and eyes using a modified Davidson's fluid: comparison with Bouin's fluid and conventional Davidson's fluid. Toxicol Pathol. 30: 524-533. 2002. [Medline] [CrossRef]

604. Margo CE, and Lee A. Fixation of whole eyes: the role of fixative osmolarity in the production of tissue artifact. Graefe's archive for clinical and experimental ophthalmology =. Albrecht Von Graefes Arch Klin Exp Ophthalmol. 233: 366-370. 1995. [CrossRef]

605. Schafer KA, and Render JA. Toxicologic Pathology of the Eye: Alterations of the Lens and Posterior Segment. In: Weir AB, Collins M, eds. Assessing Ocular Toxicology in Laboratory Animals. Totowa, NJ: Humana Press; 2013:219-257.

606. Lai YL. Outward movement of photoreceptor cells in normal rat retina. Invest Ophthalmol Vis Sci. 19: 849-856. 1980. [Medline]

607. Lai YL, Masuda K, Mangum MD, Lug R, Macrae DW, Fletcher G, and Liu YP. Subretinal displacement of photoreceptor nuclei in human retina. Exp Eye Res. 34: 219-228. 1982. [Medline] [CrossRef]

608. Bringmann A, Reichenbach A, and Wiedemann P. Pathomechanisms of cystoid macular edema. Ophthalmic Res. 36: 241-249. 2004. [Medline] [CrossRef]

609. Bringmann A, and Wiedemann P. Involvement of Muller glial cells in epiretinal membrane formation. Graefe's archive for clinical and experimental ophthalmology =. Albrecht Von Graefes Arch Klin Exp Ophthalmol. 247: 865-883. 2009. [CrossRef]

610. Spaide RF. RETINAL VASCULAR CYSTOID MACULAR EDEMA: Review and New Theory. Retina. 36: 1823-1842. 2016. [Medline] [CrossRef]
611. Chester EM, Agamanolis DP, Harris JW, Victor M, Hines JD, and Kark JA. Optic atrophy in experimental vitamin B12 deficiency in monkeys. Acta Neurol Scand. 61: 9-26. 1980. [Medline] [CrossRef]

612. Cioffi GA, Fortune B, Wang L, Grant C, Bui BV, and Dong J. Idiopathic bilateral optic nerve atrophy in rhesus macaque (Macaca mulatta). Invest Ophthalmol Vis Sci. 45: 1115-1115. 2004.

613. Dubielzig RR, Leedle R, Nork TM, VerHoeve JA, and Christian BJ. Bilateral Optic Atrophy: A Background Finding in Cynomolgus Macaques Used in Toxicologic Research. Invest Ophthalmol Vis Sci. 50: 5344-5344. 2009.

614. Fortune B, Wang L, Bui BV, Burgoyne CF, and Cioffi GA. Idiopathic bilateral optic atrophy in the rhesus macaque. Invest Ophthalmol Vis Sci. 46: 3943-3956. 2005. [Medline] [CrossRef]

615. Leedle R, Dubielzig R, and Christian B. Annual Meeting Abstracts: Bilateral optic atrophy in cynomolgus monkey. Vet Pathol. 45: 781. 2008.

616. Piper C, Fortune B, Cull G, Cioffi GA, and Wang L. Basal blood flow and autoregulation changes in the optic nerve of rhesus monkeys with idiopathic bilateral optic atrophy. Invest Ophthalmol Vis Sci. 54: 714721. 2013. [Medline] [CrossRef]

617. Ribka EP, and Dubielzig RR. Multiple ophthalmic abnormalities in an infant rhesus macaque (Macaca mulatta). J Med Primatol. 37(Suppl 1): 16-19. 2008. [Medline] [CrossRef]

618. Francis PJ, Appukuttan B, Simmons E, Landauer N, Stoddard J, Hamon S, Ott J, Ferguson B, Klein M, Stout JT, and Neuringer M. Rhesus monkeys and humans share common susceptibility genes for age-related macular disease. Hum Mol Genet. 17: 2673-2680. 2008. [Medline] [CrossRef]

619. Yiu G, Tieu E, Munevar C, Wong B, Cunefare D, Farsiu S, Garzel L, Roberts J, and Thomasy SM. In Vivo Multimodal Imaging of Drusenoid Lesions in Rhesus Macaques. Sci Rep. 7: 15013. 2017. [Medline] [CrossRef]

620. Dawson WW, Dawson JC, Lake KP, and Gonzalez-Martinez J. Maculas, monkeys, models, AMD and aging. Vision Res. 48: 360-365. 2008. [Medline] [CrossRef]

621. Hope GM, Dawson WW, Engel HM, Ulshafer RJ, Kessler MJ, and Sherwood MB. A primate model for age related macular drusen. $\mathrm{Br} J$ Ophthalmol. 76: 11-16. 1992. [Medline] [CrossRef]

622. Suzuki MT, Terao K, Yoshikawa Y. Familial early onset macular degeneration in cynomolgus monkeys (Macaca fascicularis). Primates; journal of primatology. 2003;44(3):291-294.

623. Umeda S, Ayyagari R, Allikmets R, Suzuki MT, Karoukis AJ, Ambasudhan R, Zernant J, Okamoto H, Ono F, Terao K, Mizota A, Yoshikawa Y, Tanaka Y, and Iwata T. Early-onset macular degeneration with drusen in a cynomolgus monkey (Macaca fascicularis) pedigree: exclusion of 13 candidate genes and loci. Invest Ophthalmol Vis Sci. 46: 683-691. 2005. [Medline] [CrossRef]

624. Feeney-Burns L, Malinow R, Klein ML, and Neuringer M. Maculopathy in cynomolgus monkeys. A correlated fluorescein angiographic and ultrastructural study. Arch Ophthalmol. 99: 664-672. 1981. [Medline] [CrossRef]

625. Gouras P, Ivert L, Neuringer M, and Mattison JA. Topographic and age-related changes of the retinal epithelium and Bruch's membrane of rhesus monkeys. Graefe's archive for clinical and experimental ophthalmology =. Albrecht Von Graefes Arch Klin Exp Ophthalmol. 248: 973-984. 2010. [CrossRef]

626. McBride JL, Neuringer M, Ferguson B, Kohama SG, Tagge IJ, Zweig RC, Renner LM, McGill TJ, Stoddard J, Peterson S, Su W, Sherman LS, Domire JS, Ducore RM, Colgin LM, and Lewis AD. Discovery of a CLN7 model of Batten disease in non-human primates. Neurobiol Dis. 119: 65-78. 2018. [Medline] [CrossRef]

627. Rabiolo A, Sacconi R, Cicinelli MV, Querques L, Bandello F, and Querques G. Spotlight on reticular pseudodrusen. Clin Ophthalmol. 11: 1707-1718. 2017. [Medline] [CrossRef]

628. Pennesi ME, Neuringer M, and Courtney RJ. Animal models of age related macular degeneration. Mol Aspects Med. 33: 487-509. 2012. [Medline] [CrossRef] 
629. Anderson M, Dawson WW, Gonzalez-Martinez J, and Curcio CA. Drusen and lipid-filled retinal pigment epithelium cells in a rhesus macula. Vet Ophthalmol. 9: 201-207. 2006. [Medline] [CrossRef]

630. Fine BS, and Kwapien RP. Pigment epithelial windows and drusen: an animal model. Invest Ophthalmol Vis Sci. 17: 1059-1068. 1978. [Medline]

631. Ishibashi T, Sorgente N, Patterson R, and Ryan SJ. Pathogenesis of drusen in the primate. Invest Ophthalmol Vis Sci. 27: 184-193. 1986. [Medline]

632. Stafford TJ, Anness SH, and Fine BS. Spontaneous degenerative maculopathy in the monkey. Ophthalmology. 91: 513-521. 1984. [Medline] [CrossRef]

633. Monaco WA, Wormington CM. The rhesus monkey as an animal model for age-related maculopathy. Optometry and vision science: official publication of the American Academy of Optometry. 1990;67(7):532537.

634. Nishiguchi KM, Yokoyama Y, Fujii Y, Fujita K, Tomiyama Y, Kawasaki R, Furukawa T, Ono F, Shimozawa N, Togo M, Suzuki M, and Nakazawa T. Analysis of Macular Drusen and Blood Test Results in 945 Macaca fascicularis. PLoS One. 11: e0164899. 2016. [Medline] [CrossRef]

635. Albert DM, Dubielzig RR, Li Y, Lin T, Neekhra A, Orilla W, Ramos $\mathrm{M}$, Salamat MS, and Burke JA. Choroidal melanoma occurring in a nonhuman primate. Arch Ophthalmol. 127: 1080-1082. 2009. [Medline] [CrossRef]

636. Ramos M, Albert D, Dubielzig R, et al Uveal Melanoma in a NonHuman Primate. Invest Ophthalmol Vis Sci. 50: 3395-3395. 2009.

637. Rennie IG. Don't it make my blue eyes brown: heterochromia and other abnormalities of the iris. Eye (Lond). 26: 29-50. 2012. [Medline] [CrossRef]

638. Shields CL, Shields PW, Manalac J, Jumroendararasame C, and Shields JA. Review of cystic and solid tumors of the iris. Oman J Ophthalmol. 6: 159-164. 2013. [Medline] [CrossRef]

639. Arends G, Schramm U. The structure of the human semilunar plica at different stages of its development--a morphological and morphometric study. Annals of anatomy $=$ Anatomischer Anzeiger: official organ of the Anatomische Gesellschaft. 2004;186(3):195-207.

640. Schlegel T, Brehm H, Amselgruber WM. The cartilage of the third eyelid: a comparative macroscopical and histological study in domestic animals. Annals of anatomy = Anatomischer Anzeiger: official organ of the Anatomische Gesellschaft. 2001;183(2):165-169.

641. Jester JV, Nicolaides N, and Smith RE. Meibomian gland studies: histologic and ultrastructural investigations. Invest Ophthalmol Vis Sci. 20: 537-547. 1981. [Medline]

642. Ohnishi Y, and Kohno T. Polychlorinated biphenyls poisoning in monkey eye. Invest Ophthalmol Vis Sci. 18: 981-984. 1979. [Medline]

643. Stoeckelhuber M, Stoeckelhuber BM, and Welsch U. Apocrine glands in the eyelid of primates contribute to the ocular host defense. Cells Tissues Organs. 176: 187-194. 2004. [Medline] [CrossRef]

644. Frame NJ, and Burkat CN. Identifying an appropriate animal model for the nasolacrimal drainage system. Ophthal Plast Reconstr Surg. 25: 354-358. 2009. [Medline] [CrossRef]

645. Bast TH. The eye and the ear. In: Hartman C, Straus W, eds. Anatomy of the Rhesus Monkey: Macaca Mulatta. New York: Hafner; 1933:339359.

646. Boothe RG, Quick MW, Joosse MV, Abbas MA, and Anderson DC. Accessory lateral rectus orbital geometry in normal and naturally strabismic monkeys. Invest Ophthalmol Vis Sci. 31: 1168-1174. 1990. [Medline]

647. Schnyder $H$. The innervation of the monkey accessory lateral rectus muscle. Brain Res. 296: 139-144. 1984. [Medline] [CrossRef]

648. Mwasi LM, and Raviola G. Morphology and permeability properties of blood capillaries in extraocular muscles of macaque monkeys. Graefe's archive for clinical and experimental ophthalmology $=$. Albrecht Von Graefes Arch Klin Exp Ophthalmol. 223: 9-15. 1985. [CrossRef]

649. Waller BM, Parr LA, Gothard KM, Burrows AM, and Fuglevand AJ. Mapping the contribution of single muscles to facial movements in the rhesus macaque. Physiol Behav. 95: 93-100. 2008. [Medline] [CrossRef]

650. Stephens LC, Schultheiss TE, Price RE, Ang KK, and Peters LJ. Radiation apoptosis of serous acinar cells of salivary and lacrimal glands. Cancer. 67: 1539-1543. 1991. [Medline] [CrossRef]

651. Munday JS, Rodriguez NA, and Thomas DA. Lacrimal gland myoepithelioma in a rhesus macaque (Macaca mulatta). Comp Med. 54: 443-446. 2004. [Medline]

652. Simmons HA, Mattison JA. The incidence of spontaneous neoplasia in two populations of captive rhesus macaques (Macaca mulatta). Antioxidants \& redox signaling. 2011;14(2):221-227.

653. Kumar V, Sankhyan V, and Thakur A. Ophthalmic diseases and disorders in free-ranging rhesus macaque (Macaca mulatta) of Shivalik hill area of Himachal Pradesh, Northern India. J Med Primatol. 44: 89-96. 2015. [Medline] [CrossRef]

654. Frazier KS, Seely JC, Hard GC, Betton G, Burnett R, Nakatsuji S, Nishikawa A, Durchfeld-Meyer B, and Bube A. Proliferative and nonproliferative lesions of the rat and mouse urinary system. Toxicol Pathol. 40(Suppl): 14S-86S. 2012. [Medline] [CrossRef]

655. Tomson FN, Keller GL, and Knapke FB. Nutritional secondary hyperparathyroidism in a group of lemurs. Paper presented at: Symposia of the National Zoological, Park; 1980, 1978; Washington, D.C.

656. Snyder SB. OJL, Law D. H., Froelich J. W. Osteomalacia and nutritional secondary hyperparathyroidism is a semi-free-ranging troop of Japanese monkeys. In: Montali RJ, Migaki G, eds. Symposium on the Comparative Pathology of Zoo, Animals. Vol The comparative pathology of zoo animals: proceedings of a symposium held at the National Zoological Park, Smithsonian Institution, October 2-4, 1978. Washington: Smithsonian Institution Press; 1978:51-57.

657. Symposium on the Comparative Pathology of Zoo A. The comparative pathology of zoo animals: proceedings of a symposium held at the National Zoological Park, Smithsonian Institution, October 2-4, 1978 / Richard J. Montali and George Migaki, editors, with section editors, Kurt Benirschke ... [et al.]. Washington: Smithsonian Institution Press; 1980.

658. Venkatesan R, Dick EJ Jr, and Hubbard GB. Pathology of the male baboon (Papio spp.) urogenital system. J Med Primatol. 37: 245-249. 2008. [Medline] [CrossRef]

659. Drach GW, and Boyce WH. Nephrocalcinosis as a source for renal stone nuclei. Observations on humans and squirrel monkeys and on hyperparathyroidism in the squirrel monkey. J Urol. 107: 897-904. 1972. [Medline] [CrossRef]

660. Roberts JA. The Urinary System. Basel: Karger; 1972.

661. Seaman WJ. Multinucleated epithelial cells of renal collecting tubules in macaques. Vet Pathol. 23: 87-88. 1986. [Medline] [CrossRef]

662. Jones SR, and Casey HW. Primary renal tumors in nonhuman primates. Vet Pathol. 18(Suppl 6): 89-104. 1981. [Medline] [CrossRef]

663. Goens SD, Moore CM, Brasky KM, Frost PA, Leland MM, and Hubbard GB. Nephroblastomatosis and nephroblastoma in nonhuman primates. J Med Primatol. 34: 165-170. 2005. [Medline] [CrossRef]

664. McInnes EF. Background lesions in laboratory animals: a color atlas. Saunders Elsevier; 2012.

665. Burek JD, Van Zwieten MJ, and Stookey JL. Cytoplasmic inclusions in urinary bladder epithelium of the rhesus monkey. A histochemical, light-and electron-microscopic study. Vet Pathol. 9: 212-220. 1972. [Medline] [CrossRef] 